OPEN ACCESS

UWS Academic Portal

\title{
Physical fitness training for stroke patients
}

Saunders, David H.; Sanderson, Mark; Hayes, Sara; Johnson, Liam; Kramer, Sharon; Carter, Daniel D.; Jarvis, Hannah; Brazzelli, Miriam; Mead, Gillian E.

Published in:

The Cochrane Database of Systematic Reviews

DOI:

10.1002/14651858.CD003316.pub7

Published: 20/03/2020

Document Version

Publisher's PDF, also known as Version of record

Link to publication on the UWS Academic Portal

Citation for published version (APA):

Saunders, D. H., Sanderson, M., Hayes, S., Johnson, L., Kramer, S., Carter, D. D., Jarvis, H., Brazzelli, M., \& Mead, G. E. (2020). Physical fitness training for stroke patients. The Cochrane Database of Systematic Reviews, 3, [CD003316]. https://doi.org/10.1002/14651858.CD003316.pub7

\section{General rights}

Copyright and moral rights for the publications made accessible in the UWS Academic Portal are retained by the authors and/or other copyright owners and it is a condition of accessing publications that users recognise and abide by the legal requirements associated with these rights.

Take down policy

If you believe that this document breaches copyright please contact pure@uws.ac.uk providing details, and we will remove access to the work immediately and investigate your claim. 


\section{Physical fitness training for stroke patients (Review)}

Saunders DH, Sanderson M, Hayes S, Johnson L, Kramer S, Carter DD, Jarvis H, Brazzelli M, Mead GE

Saunders DH, Sanderson M, Hayes S, Johnson L, Kramer S, Carter DD, Jarvis H, Brazzelli M, Mead GE.

Physical fitness training for stroke patients.

Cochrane Database of Systematic Reviews 2020, Issue 3. Art. No.: CD003316.

DOI: 10.1002/14651858.CD003316.pub7.

www.cochranelibrary.com 
TABLE OF CONTENTS

HEADER

ABSTRACT

PLAIN LANGUAGE SUMMARY

SUMMARY OF FINDINGS

BACKGROUND

OBJECTIVES

METHODS

Figure 1.

RESULTS

Figure 2.

Figure 3.

Figure 4.

Figure 5.

Figure 6.

DISCUSSION

AUTHORS' CONCLUSIONS

ACKNOWLEDGEMENTS

REFERENCES

CHARACTERISTICS OF STUDIES

DATA AND ANALYSES

Analysis 1.1. Comparison 1 Cardiorespiratory training versus control - end of intervention, Outcome 1 Death.

Analysis 1.2. Comparison 1 Cardiorespiratory training versus control - end of intervention, Outcome 2 Disability - Functional Independence Measure.

Analysis 1.3. Comparison 1 Cardiorespiratory training versus control - end of intervention, Outcome 3 Disability - Barthel Index.

Analysis 1.4. Comparison 1 Cardiorespiratory training versus control - end of intervention, Outcome 4 Disability - Rivermead Mobility Index (scale 0 to 15).

Analysis 1.5. Comparison 1 Cardiorespiratory training versus control - end of intervention, Outcome 5 Disability - combined disability scales.

Analysis 1.6. Comparison 1 Cardiorespiratory training versus control - end of intervention, Outcome 6 Risk factors - blood pressure, systolic.

Analysis 1.7. Comparison 1 Cardiorespiratory training versus control - end of intervention, Outcome 7 Risk factors - blood pressure, diastolic.

Analysis 1.8. Comparison 1 Cardiorespiratory training versus control - end of intervention, Outcome 8 Risk factors - body mass index (BMI).

Analysis 1.9. Comparison 1 Cardiorespiratory training versus control - end of intervention, Outcome 9 Physical fitness - peak VO2.

Analysis 1.10. Comparison 1 Cardiorespiratory training versus control - end of intervention, Outcome 10 Physical fitness maximum cycling work rate.

Analysis 1.11. Comparison 1 Cardiorespiratory training versus control - end of intervention, Outcome 11 Mobility - functional ambulation categories.

Analysis 1.12. Comparison 1 Cardiorespiratory training versus control - end of intervention, Outcome 12 Mobility - walking maximal speed (over 5 to 10 metres).

Analysis 1.13. Comparison 1 Cardiorespiratory training versus control - end of intervention, Outcome 13 Mobility - walking preferred speed.

Analysis 1.14. Comparison 1 Cardiorespiratory training versus control - end of intervention, Outcome 14 Mobility - walking capacity (6-MWT metres).

Analysis 1.15. Comparison 1 Cardiorespiratory training versus control - end of intervention, Outcome 15 Mobility - walking capacity ( $\mathrm{m} / \mathrm{min})$.

Analysis 1.16. Comparison 1 Cardiorespiratory training versus control - end of intervention, Outcome 16 Mobility - community walk (min).

Analysis 1.17. Comparison 1 Cardiorespiratory training versus control - end of intervention, Outcome 17 Physical function Berg Balance Scale (score 0 to 56 ). 
Analysis 1.18. Comparison 1 Cardiorespiratory training versus control - end of intervention, Outcome 18 Physical function Timed Up and Go (sec).

Analysis 1.19. Comparison 1 Cardiorespiratory training versus control - end of intervention, Outcome 19 Health-related QoL SF-36 \& SF-12 Physical Health Component.

Analysis 1.20. Comparison 1 Cardiorespiratory training versus control - end of intervention, Outcome 20 Health-related QoL SF-36 \& SF-12 Mental Health Component.

Analysis 1.21. Comparison 1 Cardiorespiratory training versus control - end of intervention, Outcome 21 Health-related QoL EuroQol EQ-5D.

Analysis 1.22. Comparison 1 Cardiorespiratory training versus control - end of intervention, Outcome 22 Mood - Beck Depression Index.

Analysis 2.1. Comparison 2 Cardiorespiratory training versus control - end of retention follow-up, Outcome 1 Death. ............. Analysis 2.2. Comparison 2 Cardiorespiratory training versus control - end of retention follow-up, Outcome 2 Disability combined disability scales.

Analysis 2.3. Comparison 2 Cardiorespiratory training versus control - end of retention follow-up, Outcome 3 Mobility - walking maximal speed $(\mathrm{m} / \mathrm{min})$.

Analysis 2.4. Comparison 2 Cardiorespiratory training versus control - end of retention follow-up, Outcome 4 Mobility - walking preferred speed ( $\mathrm{m} / \mathrm{min})$.

Analysis 2.5. Comparison 2 Cardiorespiratory training versus control - end of retention follow-up, Outcome 5 Mobility - walking capacity (6-MWT metres).

Analysis 2.6. Comparison 2 Cardiorespiratory training versus control - end of retention follow-up, Outcome 6 Physical function - Berg Balance scale.

Analysis 2.7. Comparison 2 Cardiorespiratory training versus control - end of retention follow-up, Outcome 7 Health-related QoL - EuroQol EQ-5D.

Analysis 3.1. Comparison 3 Resistance training versus control - end of intervention, Outcome 1 Death.

Analysis 3.2. Comparison 3 Resistance training versus control - end of intervention, Outcome 2 Physical fitness - composite measure of muscle strength.

Analysis 3.3. Comparison 3 Resistance training versus control - end of intervention, Outcome 3 Physical fitness - muscle strength, paretic knee flexion.

Analysis 3.4. Comparison 3 Resistance training versus control - end of intervention, Outcome 4 Physical fitness - muscle strength, paretic knee extension.

Analysis 3.5. Comparison 3 Resistance training versus control - end of intervention, Outcome 5 Mobility - walking maximal speed $(\mathrm{m} / \mathrm{min})$.

Analysis 3.6. Comparison 3 Resistance training versus control - end of intervention, Outcome 6 Mobility - walking preferred speed $(\mathrm{m} / \mathrm{min})$.

Analysis 3.7. Comparison 3 Resistance training versus control - end of intervention, Outcome 7 Mobility - walking capacity (6MWT metres).

Analysis 3.8. Comparison 3 Resistance training versus control - end of intervention, Outcome 8 Physical function - Berg Balance Scale (score 0 to 56 ).

Analysis 3.9. Comparison 3 Resistance training versus control - end of intervention, Outcome 9 Physical function - stair climbing, maximal (sec/step).

Analysis 3.10. Comparison 3 Resistance training versus control - end of intervention, Outcome 10 Physical function - Timed Up and Go (sec).

Analysis 3.11. Comparison 3 Resistance training versus control - end of intervention, Outcome 11 Health-related QoL - SF-36 physical functioning (PF) scale.

Analysis 3.12. Comparison 3 Resistance training versus control - end of intervention, Outcome 12 Health-related QoL - SF- 36 mental health $(\mathrm{MH})$ scale.

Analysis 3.13. Comparison 3 Resistance training versus control - end of intervention, Outcome 13 Mood - Centre for Epidemiologic Studies for Depression scale (CES-D).

Analysis 3.14. Comparison 3 Resistance training versus control - end of intervention, Outcome 14 Mood - combined depression scales.

Analysis 4.1. Comparison 4 Resistance training versus control - end of retention follow-up, Outcome 1 Death. ....................... Analysis 4.2. Comparison 4 Resistance training versus control - end of retention follow-up, Outcome 2 Mobility - walking maximal speed ( $\mathrm{m} / \mathrm{min})$.

Analysis 4.3. Comparison 4 Resistance training versus control - end of retention follow-up, Outcome 3 Mobility - walking capacity (6-MWT metres). 
Analysis 4.4. Comparison 4 Resistance training versus control - end of retention follow-up, Outcome 4 Physical function - Timed Up and Go (sec).

Analysis 5.1. Comparison 5 Mixed training versus control - end of intervention, Outcome 1 Death.

Analysis 5.2. Comparison 5 Mixed training versus control - end of intervention, Outcome 2 Disability - Barthel Index (BI). ....... Analysis 5.3. Comparison 5 Mixed training versus control - end of intervention, Outcome 3 Disability - Lawton IADL. ............... Analysis 5.4. Comparison 5 Mixed training versus control - end of intervention, Outcome 4 Disability - Rivermead Mobility Index (RMI).

Analysis 5.5. Comparison 5 Mixed training versus control - end of intervention, Outcome 5 Disability - combined disability scales.

Analysis 5.6. Comparison 5 Mixed training versus control - end of intervention, Outcome 6 Risk factors - blood pressure, systolic.

Analysis 5.7. Comparison 5 Mixed training versus control - end of intervention, Outcome 7 Risk factors - blood pressure, diastolic.

Analysis 5.8. Comparison 5 Mixed training versus control - end of intervention, Outcome 8 Physical fitness - peak VO2 (mL/kg/ $\min )$.

Analysis 5.9. Comparison 5 Mixed training versus control - end of intervention, Outcome 9 Physical fitness - gait economy, VO2 $(\mathrm{mL} / \mathrm{kg} / \mathrm{metre})$.

Analysis 5.10. Comparison 5 Mixed training versus control - end of intervention, Outcome 10 Physical fitness - muscle strength, ankle dorsiflexion*.

Analysis 5.11. Comparison 5 Mixed training versus control - end of intervention, Outcome 11 Physical fitness - muscle strength, knee extension*.

Analysis 5.12. Comparison 5 Mixed training versus control - end of intervention, Outcome 12 Physical fitness - muscle strength, grip strength (paretic hand).

Analysis 5.13. Comparison 5 Mixed training versus control - end of intervention, Outcome 13 Mobility - walking maximum speed.

Analysis 5.14. Comparison 5 Mixed training versus control - end of intervention, Outcome 14 Mobility - walking preferred speed (m/min).

Analysis 5.15. Comparison 5 Mixed training versus control - end of intervention, Outcome 15 Mobility - walking capacity (6-MWT metres).

Analysis 5.16. Comparison 5 Mixed training versus control - end of intervention, Outcome 16 Mobility - Community Ambulation Speed (>0.8 m/sec).

Analysis 5.17. Comparison 5 Mixed training versus control - end of intervention, Outcome 17 Physical function - balance - Berg Balance scale.

Analysis 5.18. Comparison 5 Mixed training versus control - end of intervention, Outcome 18 Physical function - balance functional reach.

Analysis 5.19. Comparison 5 Mixed training versus control - end of intervention, Outcome 19 Physical function - balance combined outcome data.

Analysis 5.20. Comparison 5 Mixed training versus control - end of intervention, Outcome 20 Physical function - Timed Up and Go (sec).

Analysis 5.21. Comparison 5 Mixed training versus control - end of intervention, Outcome 21 Health-related QoL - SF-36 physical functioning.

Analysis 5.22. Comparison 5 Mixed training versus control - end of intervention, Outcome 22 Health-related QoL - SF-36 physical role functioning.

Analysis 5.23. Comparison 5 Mixed training versus control - end of intervention, Outcome 23 Health-related QoL - SF-36 social role functioning.

Analysis 5.24. Comparison 5 Mixed training versus control - end of intervention, Outcome 24 Mood - Hospital Anxiety and Depression Scale (HADS) - anxiety score.

Analysis 5.25. Comparison 5 Mixed training versus control - end of intervention, Outcome 25 Mood - Hospital Anxiety and Depression Scale (HADS) - depression score.

Analysis 5.26. Comparison 5 Mixed training versus control - end of intervention, Outcome 26 Mood - Stroke Impact Scale emotion score.

Analysis 5.27. Comparison 5 Mixed training versus control - end of intervention, Outcome 27 Mood - combined depression scales.

Analysis 5.28. Comparison 5 Mixed training versus control - end of intervention, Outcome 28 Cognitive function - FIM cognitive score. 
Analysis 5.29. Comparison 5 Mixed training versus control - end of intervention, Outcome 29 Cognitive function - SIS memory and thinking.

Analysis 5.30. Comparison 5 Mixed training versus control - end of intervention, Outcome 30 Cognitive function - SIS communication.

Analysis 6.1. Comparison 6 Mixed training versus control - end of retention follow-up, Outcome 1 Death.

Analysis 6.2. Comparison 6 Mixed training versus control - end of retention follow-up, Outcome 2 Disability - Barthel Index (BI).

Analysis 6.3. Comparison 6 Mixed training versus control - end of retention follow-up, Outcome 3 Disability - Nottingham Extended ADL.

Analysis 6.4. Comparison 6 Mixed training versus control - end of retention follow-up, Outcome 4 Disability - Rivermead Mobility Index (RMI).

Analysis 6.5. Comparison 6 Mixed training versus control - end of retention follow-up, Outcome 5 Disability - combined disability scales.

Analysis 6.6. Comparison 6 Mixed training versus control - end of retention follow-up, Outcome 6 Mobility - Functional Ambulation Categories.

Analysis 6.7. Comparison 6 Mixed training versus control - end of retention follow-up, Outcome 7 Mobility - walking preferred speed ( $\mathrm{m} / \mathrm{min})$.

Analysis 6.8. Comparison 6 Mixed training versus control - end of retention follow-up, Outcome 8 Mobility - walking capacity (6-MWT metres).

Analysis 6.9. Comparison 6 Mixed training versus control - end of retention follow-up, Outcome 9 Mobility - community ambulation speed $(>0.8 \mathrm{~m} / \mathrm{sec}$ ).

Analysis 6.10. Comparison 6 Mixed training versus control - end of retention follow-up, Outcome 10 Physical function - balance - Berg Balance Scale.

Analysis 6.11. Comparison 6 Mixed training versus control - end of retention follow-up, Outcome 11 Physical function - balance - functional reach.

Analysis 6.12. Comparison 6 Mixed training versus control - end of retention follow-up, Outcome 12 Physical function - Timed Up and Go (sec).

Analysis 6.13. Comparison 6 Mixed training versus control - end of retention follow-up, Outcome 13 Health-related QoL - SF-36 physical functioning.

Analysis 6.14. Comparison 6 Mixed training versus control - end of retention follow-up, Outcome 14 Health-related QoL - SF-36 physical role functioning.

Analysis 6.15. Comparison 6 Mixed training versus control - end of retention follow-up, Outcome 15 Mood - Stroke Impact Scale emotion score.

Analysis 6.16. Comparison 6 Mixed training versus control - end of retention follow-up, Outcome 16 Mood - Geriatric Depression Scale.

Analysis 6.17. Comparison 6 Mixed training versus control - end of retention follow-up, Outcome 17 Mood - Hospital Anxiety and Depression Scale (HADS) - anxiety score.

Analysis 6.18. Comparison 6 Mixed training versus control - end of retention follow-up, Outcome 18 Mood - Hospital Anxiety and Depression Scale (HADS) - depression score.

Analysis 6.19. Comparison 6 Mixed training versus control - end of retention follow-up, Outcome 19 Mood - combined depression scales.

Analysis 6.20. Comparison 6 Mixed training versus control - end of retention follow-up, Outcome 20 Cognitive function - FIM cognitive score.

Analysis 6.21. Comparison 6 Mixed training versus control - end of retention follow-up, Outcome 21 Cognitive function - SIS memory and thinking.

Analysis 6.22. Comparison 6 Mixed training versus control - end of retention follow-up, Outcome 22 Cognitive function - SIS communication.

Analysis 7.1. Comparison 7 Cardiorespiratory versus resistance versus mixed training, Outcome 1 Disability - combined disability scales.

Analysis 7.2. Comparison 7 Cardiorespiratory versus resistance versus mixed training, Outcome 2 Mobility - walking maximal speed.

Analysis 7.3. Comparison 7 Cardiorespiratory versus resistance versus mixed training, Outcome 3 Mobility - walking preferred speed.

Analysis 7.4. Comparison 7 Cardiorespiratory versus resistance versus mixed training, Outcome 4 Mobility - walking capacity (6-MWT distance).

Analysis 7.5. Comparison 7 Cardiorespiratory versus resistance versus mixed training, Outcome 5 Physical Function - Balance - Berg Balance Scale. 
Analysis 7.6. Comparison 7 Cardiorespiratory versus resistance versus mixed training, Outcome 6 Physical function - Timed up and go.

ADDITIONAL TABLES

APPENDICES

WHAT'S NEW

HISTORY

303

CONTRIBU 
[Intervention Review]

\section{Physical fitness training for stroke patients}

David H Saunders ${ }^{1}$, Mark Sanderson², Sara Hayes ${ }^{3}$, Liam Johnson 4 , Sharon Kramer ${ }^{4}$, Daniel D Carter ${ }^{5}$, Hannah Jarvis 6 , Miriam Brazzelli7, Gillian E Mead8

1Physical Activity for Health Research Centre (PAHRC), University of Edinburgh, Edinburgh, UK. 2Institute of Clinical Exercise and Health Science, University of the West of Scotland, Hamilton, UK. ${ }^{3}$ School of Allied Health, Ageing Research Centre, Health Research Institute, University of Limerick, Limerick, Ireland. ${ }^{4}$ The Florey Institute of Neuroscience and Mental Health, University of Melbourne, Heidelberg, Australia. ${ }^{5}$ School of Allied Health, Faculty of Education and Health Sciences, University of Limerick, Limerick, Ireland. ${ }^{6}$ Research Centre for Musculoskeletal Science and Sports Medicine, Faculty of Science and Engineering, Manchester Metropolitan University, Manchester, UK. ${ }^{7}$ Health Services Research Unit, University of Aberdeen, Aberdeen, UK. ${ }^{8}$ Centre for Clinical Brain Sciences, University of Edinburgh, Edinburgh, UK

Contact address: David H Saunders, Physical Activity for Health Research Centre (PAHRC), University of Edinburgh, St Leonards Land, Holyrood Road, Edinburgh, Midlothian, EH8 8AQ, UK. Dave.Saunders@ed.ac.uk.

Editorial group: Cochrane Stroke Group.

Publication status and date: New search for studies and content updated (conclusions changed), published in Issue 3, 2020.

Citation: Saunders DH, Sanderson M, Hayes S, Johnson L, Kramer S, Carter DD, Jarvis H, Brazzelli M, Mead GE. Physical fitness training for stroke patients. Cochrane Database of Systematic Reviews 2020, Issue 3. Art. No.: CD003316. DOI: 10.1002/14651858.CD003316.pub7.

Copyright @ 2020 The Cochrane Collaboration. Published by John Wiley \& Sons, Ltd.

\section{A B S T R A C T}

\section{Background}

Levels of physical activity and physical fitness are low after stroke. Interventions to increase physical fitness could reduce mortality and reduce disability through increased function.

\section{Objectives}

The primary objectives of this updated review were to determine whether fitness training after stroke reduces death, death or dependence, and disability. The secondary objectives were to determine the effects of training on adverse events, risk factors, physical fitness, mobility, physical function, health status and quality of life, mood, and cognitive function.

\section{Search methods}

In July 2018 we searched the Cochrane Stroke Trials Register, CENTRAL, MEDLINE, Embase, CINAHL, SPORTDiscus, PsycINFO, and four additional databases. We also searched ongoing trials registers and conference proceedings, screened reference lists, and contacted experts in the field.

\section{Selection criteria}

Randomised trials comparing either cardiorespiratory training or resistance training, or both (mixed training), with usual care, no intervention, or a non-exercise intervention in stroke survivors.

\section{Data collection and analysis}

Two review authors independently selected studies, assessed quality and risk of bias, and extracted data. We analysed data using randomeffects meta-analyses and assessed the quality of the evidence using the GRADE approach. Diverse outcome measures limited the intended analyses. 


\section{Main results}

We included 75 studies, involving 3017 mostly ambulatory participants, which comprised cardiorespiratory (32 studies, 1631 participants), resistance (20 studies, 779 participants), and mixed training interventions (23 studies, 1207 participants).

Death was not influenced by any intervention; risk differences were all 0.00 (low-certainty evidence). There were few deaths overall (19/3017 at end of intervention and 19/1469 at end of follow-up). None of the studies assessed death or dependence as a composite outcome. Disability scores were improved at end of intervention by cardiorespiratory training (standardised mean difference (SMD) 0.52 , $95 \% \mathrm{Cl} 0.19$ to $0.84 ; 8$ studies, 462 participants; $\mathrm{P}=0.002$; moderate-certainty evidence) and mixed training (SMD $0.23,95 \% \mathrm{Cl} 0.03$ to $0.42 ; 9$ studies, 604 participants; $P=0.02$; low-certainty evidence). There were too few data to assess the effects of resistance training on disability.

Secondary outcomes showed multiple benefits for physical fitness $\left(\mathrm{VO}_{2}\right.$ peak and strength), mobility (walking speed) and physical function (balance). These physical effects tended to be intervention-specific with the evidence mostly low or moderate certainty. Risk factor data were limited or showed no effects apart from cardiorespiratory fitness $\left(\mathrm{VO}_{2}\right.$ peak), which increased after cardiorespiratory training (mean difference (MD) $3.40 \mathrm{~mL} / \mathrm{kg} / \mathrm{min}, 95 \% \mathrm{Cl} 2.98$ to 3.83; 9 studies, 438 participants; moderate-certainty evidence). There was no evidence of any serious adverse events. Lack of data prevents conclusions about effects of training on mood, quality of life, and cognition. Lack of data also meant benefits at follow-up (i.e. after training had stopped) were unclear but some mobility benefits did persist. Risk of bias varied across studies but imbalanced amounts of exposure in control and intervention groups was a common issue affecting many comparisons.

\section{Authors' conclusions}

Few deaths overall suggest exercise is a safe intervention but means we cannot determine whether exercise reduces mortality or the chance of death or dependency. Cardiorespiratory training and, to a lesser extent mixed training, reduce disability during or after usual stroke care; this could be mediated by improved mobility and balance. There is sufficient evidence to incorporate cardiorespiratory and mixed training, involving walking, within post-stroke rehabilitation programmes to improve fitness, balance and the speed and capacity of walking. The magnitude of $\mathrm{VO}_{2}$ peak increase after cardiorespiratory training has been suggested to reduce risk of stroke hospitalisation by $\sim 7 \%$. Cognitive function is under-investigated despite being a key outcome of interest for patients. Further well-designed randomised trials are needed to determine the optimal exercise prescription, the range of benefits and any long-term benefits.

\section{PLAIN LANGUAGE SUMMARY}

\section{Physical fitness training for stroke survivors}

\section{Review question}

We reviewed the evidence that examines whether physical fitness training is beneficial for health and function in people who have had a stroke.

\section{Background}

Physical fitness is important to allow people to carry out everyday activities such as walking and climbing stairs. Physical fitness varies among everyone. For example, fitness in men tends to be a little higher than in women and everyone's fitness declines as we get older and if we become less physically active. In particular, in stroke survivors' physical fitness is often low. This may limit their ability to perform everyday activities and also worsen stroke-related disability. For this reason fitness training has been proposed as a beneficial approach for people with stroke. However, taking part in fitness training could have a range of other benefits important to people with stroke such as improving cognitive function (thinking skills), improving mood, and quality of life, and it could reduce the chance of having another stroke.

\section{Study characteristics}

In July 2018 we identified 75 studies for inclusion in the review. The studies involved a total of 3617 participants at all stages of care including being in hospital or back living at home. Most of the people who took part were able to walk on their own. The studies tested different forms of fitness training; these included cardiorespiratory or 'endurance' training, resistance or 'strength' training, or mixed training, which is a combination of cardiorespiratory plus resistance training.

\section{Key results}

We found that cardiorespiratory fitness training, particularly involving walking, can improve fitness, balance and walking after stroke. The improvements in cardiorespiratory fitness may reduce the chance of stroke hospitalisation by $7 \%$. Mixed training improves walking ability and improves balance. Strength training may have a role in improving balance. So, overall it seems likely that people with stroke are likely to benefit the most from training that involves cardiorespiratory training and that involves some walking. However, there was not enough information to draw reliable conclusions about the impact of fitness training on other areas such as quality of life, mood, or cognitive function. Cognitive function is under-investigated despite being a key outcome of interest for stroke survivors. There was no evidence that any of the different types of fitness training caused injuries or other health problems; exercise appears to be safe. We need more studies to examine the benefits that are most important to stroke survivors, in particular for those with more severe stroke who may be unable to walk.

\section{Quality of the evidence}


Studies of fitness training can be difficult to carry out. We have the highest confidence in the estimates of benefit from cardiorespiratory training (moderate/high). The evidence for other training types is moderate to low. However, some consistent findings emerged with different studies all tending to show similar effects in different groups of participants. 


\begin{tabular}{|c|c|c|c|c|c|}
\hline \multicolumn{6}{|c|}{ Summary of findings for the main comparison. Cardiorespiratory training compared to control for people with stroke: end of intervention } \\
\hline \multicolumn{6}{|c|}{ Cardiorespiratory training compared to control for people with stroke: end of intervention } \\
\hline \multicolumn{6}{|c|}{$\begin{array}{l}\text { Patient or population: people with stroke } \\
\text { Setting: during and after usual care } \\
\text { Intervention: cardiorespiratory training } \\
\text { Comparison: control; end of intervention }\end{array}$} \\
\hline Outcomes & & $\begin{array}{l}\text { Relative effect } \\
(95 \% \mathrm{Cl})\end{array}$ & $\begin{array}{l}\text { Number of } \\
\text { participants } \\
\text { (studies) }\end{array}$ & $\begin{array}{l}\text { Certainty of } \\
\text { the evidence } \\
\text { (GRADE) }\end{array}$ & Comments \\
\hline \multicolumn{2}{|l|}{ Death } & $\begin{array}{l}\text { Risk difference } 0.00 \\
(-0.01 \text { to } 0.01)\end{array}$ & $\begin{array}{l}1631 \\
(32 \mathrm{RCTs})\end{array}$ & $\begin{array}{l}\oplus \oplus \ominus \ominus \\
\operatorname{Low}^{a}\end{array}$ & $\begin{array}{l}\text { Death is very uncommon, with only } 4 \text { deaths; } 2 \text { deaths in the con- } \\
\text { trol group and } 2 \text { in the intervention group of a single study (Gor- } \\
\text { don 2013). }\end{array}$ \\
\hline \multicolumn{2}{|c|}{ Dead or dependent } & - & 0 (0 RCTs) & - & $\begin{array}{l}\text { No studies reported the composite outcome of death or depen- } \\
\text { dency. }\end{array}$ \\
\hline Disability & $\begin{array}{l}\text { Pooled functional } \\
\text { scales } \\
\text { Analysis } 1.5\end{array}$ & $\begin{array}{l}\text { SMD } 0.52 \text { higher } \\
\text { ( } 0.19 \text { higher to } 0.84 \text { high- } \\
\text { er) }\end{array}$ & $\begin{array}{l}462 \\
\text { (8 RCTs) }\end{array}$ & $\begin{array}{l}\oplus \oplus \oplus \ominus \\
\text { Moderateb }\end{array}$ & $\begin{array}{l}\text { A SMD of global scales of disability is difficult to interpret. The } \\
\text { magnitude of increase observed }(>0.5) \text { can generally be cate- } \\
\text { gorised as a 'moderate' effect. Any improvement may be reflect- } \\
\text { ing improved mobility since mobility items are commonly includ- } \\
\text { ed in these assessment tools. }\end{array}$ \\
\hline \multirow[t]{2}{*}{$\begin{array}{l}\text { Physical fit- } \\
\text { ness }\end{array}$} & $\begin{array}{l}\mathrm{VO}_{2} \text { peak }(\mathrm{mL} / \mathrm{kg} / \\
\text { min) } \\
\text { Analysis } 1.9\end{array}$ & $\begin{array}{l}\text { MD } 3.4 \mathrm{~mL} / \mathrm{kg} / \mathrm{min} \text { higher } \\
\text { (2.98 higher to } 3.83 \text { high- } \\
\text { er) }\end{array}$ & $\begin{array}{l}438 \\
\text { (9 RCTs) }\end{array}$ & $\begin{array}{l}\oplus \oplus \oplus \odot \\
\text { Moderatec }\end{array}$ & $\begin{array}{l}\text { An increase of } 1 \mathrm{MET} \text { ( } 3.3 \mathrm{~mL} / \mathrm{kg} / \mathrm{min} \text { ) is associated with a } 7 \% \text { risk } \\
\text { reduction in stroke hospitalisation (Pandey 2016). The effect here } \\
\text { is of similar magnitude and suggests secondary prevention tar- } \\
\text { gets could be achieved within short periods of training. }\end{array}$ \\
\hline & Muscle strength & - & - & - & No data; specificity of training gives little rationale to investigate \\
\hline \multirow[t]{2}{*}{ Mobility } & $\begin{array}{l}\text { Preferred gait speed } \\
\text { (m/min) } \\
\text { Analysis } 1.13\end{array}$ & $\begin{array}{l}\text { MD } 4.47 \mathrm{~m} / \mathrm{min} \text { faster } \\
\text { (2.07 faster to } 6.87 \text { faster) }\end{array}$ & $\begin{array}{l}588 \\
(12 \mathrm{RCTs})\end{array}$ & $\begin{array}{l}\oplus \oplus \oplus \oplus \\
\text { Highd }\end{array}$ & \multirow{2}{*}{$\begin{array}{l}\text { These increases in preferred walking speed and walking capaci- } \\
\text { ty are relevant to community ambulation. The interventions are } \\
\text { mostly those with a walking mode of exercise. These functional } \\
\text { benefits are also maintained after the end of the training inter- } \\
\text { ventions. Maximal speed and other indices of gait also improve. }\end{array}$} \\
\hline & $\begin{array}{l}\text { Gait endurance (6- } \\
\text { MWT metres) } \\
\text { Analysis } 1.14\end{array}$ & $\begin{array}{l}\text { MD } 33.41 \text { m further } \\
\text { (19.04 further to } 47.78 \text { fur- } \\
\text { ther) }\end{array}$ & $\begin{array}{l}882 \\
(16 \mathrm{RCTs})\end{array}$ & $\begin{array}{l}\oplus \oplus \oplus \oplus \\
\text { Highe }\end{array}$ & \\
\hline
\end{tabular}




\begin{tabular}{|c|c|c|c|}
\hline $\begin{array}{l}\text { Berg Balance Scale } \\
\text { ( } 0 \text { to } 56 \text {, best balance } \\
=56 \text { ) } \\
\text { Analysis } 1.17\end{array}$ & $\begin{array}{l}\text { MD } 1.92 \text { units higher } \\
\text { ( } 0.16 \text { higher to } 3.68 \text { high- } \\
\text { er) }\end{array}$ & $\begin{array}{l}471 \\
\text { (8 RCTs) }\end{array}$ & $\begin{array}{l}\oplus \oplus \oplus \odot \\
\text { Moderate }\end{array}$ \\
\hline $\begin{array}{l}\text { 3-metre Timed Up } \\
\text { and Go (seconds) } \\
\text { Analysis } 1.18\end{array}$ & $\begin{array}{l}\text { MD } 3.42 \mathrm{~s} \text { faster } \\
\text { (2.05 faster to } 4.78 \text { faster) }\end{array}$ & $\begin{array}{l}223 \\
\text { (5 RCTs) }\end{array}$ & $\begin{array}{l}\oplus \oplus \oplus \ominus \\
\text { Moderateg }\end{array}$ \\
\hline
\end{tabular}

*The risk in the intervention group (and its 95\% confidence interval) is based on the assumed risk in the comparison group and the relative effect of the intervention (and its $95 \% \mathrm{Cl})$.

6-MWT: 6-Minute Walk Test; Cl: confidence interval; MD: mean difference; MET: metabolic equivalent; RCT: randomised controlled trial; RR: risk ratio; SMD: standardised mean difference

\section{GRADE Working Group grades of evidence}

High certainty: we are very confident that the true effect lies close to that of the estimate of the effect.

Moderate certainty: we are moderately confident in the effect estimate: the true effect is likely to be close to the estimate of the effect, but there is a possibility that it is substantially different.

Low certainty: our confidence in the effect estimate is limited: the true effect may be substantially different from the estimate of the effect.

Very low certainty: we have very little confidence in the effect estimate: the true effect is likely to be substantially different from the estimate of effect.

a Most participants were high-functioning; risk of death was low among this group. There is some risk of bias from imbalanced exposure in 13 of the 20 included studies and some reporting uncertainties concerning reasons for dropouts in six of the 32 studies.

bThere is some heterogeneity ( $12=61 \%)$; other issues with individual studies account for this heterogeneity.

'There are some 'Risk of bias' items recorded as 'high' but these are among studies with a low weighting. There is uncertainty in the data of one study (Jin 2013). If we exclude this study, a clear effect is still present (MD $2.80 \mathrm{~mL} / \mathrm{kg} / \mathrm{min}$ higher (1.66 higher to 3.95 higher) with high-certainty evidence).

dAlthough five out of 13 studies are confounded for exposure time the effect is still apparent when these are excluded.

eSome heterogeneity is present $(12=30 \%)$ but sensitivity analysis of confounded studies and those using non-walking (cycling) exercise modes reduces this and a similar beneficial effect remains.

fThere is some heterogeneity $\left(1^{2}=57 \%\right)$.

gThree out of five studies are confounded for exposure time.

\section{Summary of findings 2. Resistance training compared to control for people with stroke: end of intervention}

\section{Resistance training compared to control for people with stroke: end of intervention}

Patient or population: people with stroke

Setting: during and after usual care

Intervention: resistance training 


\begin{tabular}{|c|c|c|c|c|c|}
\hline \multicolumn{6}{|c|}{ Comparison: control; end of intervention } \\
\hline \multicolumn{2}{|l|}{ Outcomes } & $\begin{array}{l}\text { Relative effect } \\
(95 \% \mathrm{Cl})\end{array}$ & $\begin{array}{l}\text { Number of } \\
\text { participants } \\
\text { (studies) }\end{array}$ & $\begin{array}{l}\text { Certainty of } \\
\text { the evidence } \\
\text { (GRADE) }\end{array}$ & Comments \\
\hline \multirow{2}{*}{\multicolumn{2}{|c|}{$\begin{array}{l}\text { Death } \\
\text { Analysis } 3.1\end{array}$}} & Risk difference 0.00 & & $\oplus \oplus \ominus \ominus$ & Death is very uncommon, with only 2 deaths; 1 in the control group \\
\hline & & & & & \\
\hline \multicolumn{2}{|c|}{ Dead or dependent } & - & 0 (0 RCTs) & - & No studies reported the composite outcome of death or dependen- \\
\hline Disability & $\begin{array}{l}\text { Pooled functional } \\
\text { scales }\end{array}$ & - & - & - & $\begin{array}{l}\text { Too few data reporting global indices of disability to establish any } \\
\text { consensus effects. }\end{array}$ \\
\hline \multirow[t]{4}{*}{$\begin{array}{l}\text { Physical Fit- } \\
\text { ness }\end{array}$} & $\mathrm{VO}_{2}$ peak $(\mathrm{mL} / \mathrm{kg} / \mathrm{min})$ & - & - & - & $\begin{array}{l}\text { The rationale for resistance training is to increase muscle strength } \\
\text { and not cardiorespiratory fitness therefore there is little rationale to } \\
\text { investigate this outcome. Only } 1 \text { study measured } \mathrm{VO}_{2} \text { peak and this } \\
\text { indicated a } 6 \% \text { improvement. }\end{array}$ \\
\hline & $\begin{array}{l}\text { Muscle strength - com- } \\
\text { posite measure } \\
\text { Analysis } 3.2\end{array}$ & $\begin{array}{l}\text { SMD } 0.58 \text { higher } \\
\text { (0.06 higher to } 1.1 \\
\text { higher) }\end{array}$ & 60 (2 RCTs) & $\begin{array}{l}\oplus \oplus \odot \ominus \\
L^{\circ} w^{b}\end{array}$ & \multirow{3}{*}{$\begin{array}{l}\text { Overall, } 11 \text { RCTs reported muscle strength outcomes. } 10 / 11 \text { studies, } \\
\text { mostly examining lower limb fitness, demonstrated that resistance } \\
\text { training can improve muscle strength and some studies also indicat- } \\
\text { ed that indices such as local muscular endurance and power output } \\
\text { can be improved. } \\
\text { Variation in the method for measuring strength restricts the pooling } \\
\text { of data however three small groups of outcomes could be pooled. } \\
\text { These show medium-sized effects (SMD }>0.5 \text { ) only for one outcome, } \\
\text { a composite measure of strength. }\end{array}$} \\
\hline & $\begin{array}{l}\text { Muscle strength - } \\
\text { paretic knee flexion } \\
\text { Analysis } 3.3\end{array}$ & $\begin{array}{l}\text { SMD } 0.72 \text { higher } \\
\text { ( } 0.10 \text { higher to } 1.34 \\
\text { higher) }\end{array}$ & 93 (3 RCTs) & $\begin{array}{l}\oplus \oplus \oplus \ominus \\
\text { Moderate }\end{array}$ & \\
\hline & $\begin{array}{l}\text { Muscle strength - } \\
\text { paretic knee extension } \\
\text { Analysis } 3.4\end{array}$ & $\begin{array}{l}\text { SMD } 1.09 \text { higher } \\
\text { ( } 0.23 \text { lower to } 2.41 \\
\text { higher) }\end{array}$ & 93 (3 RCTs) & $\begin{array}{l}\oplus \oplus \oplus \ominus \\
\text { Lowd }\end{array}$ & \\
\hline \multirow[t]{2}{*}{ Mobility } & $\begin{array}{l}\text { Preferred gait speed } \\
(\mathrm{m} / \mathrm{min}) \\
\text { Analysis } 3.6\end{array}$ & $\begin{array}{l}\text { MD } 2.15 \mathrm{~m} / \mathrm{min} \text { faster } \\
\text { ( } 3.57 \text { slower to } 7.87 \\
\text { faster) }\end{array}$ & $\begin{array}{l}203 \\
\text { (5 RCTs) }\end{array}$ & $\begin{array}{l}\oplus \oplus \oplus \odot \\
\text { Moderate }\end{array}$ & \multirow{2}{*}{$\begin{array}{l}\text { There was no statistically significant effect of training on preferred } \\
\text { walking speed or on other indices of gait such as maximal walking } \\
\text { speed. Only 6-MWT data showed a benefit but this and all other gait } \\
\text { measures showed no statistically significant effect after a follow-up } \\
\text { period. These training interventions were not based on a walking } \\
\text { mode of exercise. }\end{array}$} \\
\hline & $\begin{array}{l}\text { Gait endurance (6- } \\
\text { MWT metres) } \\
\text { Analysis } 3.7\end{array}$ & $\begin{array}{l}\text { MD } 24.98 \text { m further } \\
\text { (11.98 further to } \\
37.98 \text { further) }\end{array}$ & $\begin{array}{l}238 \\
(5 \mathrm{RCTS})\end{array}$ & $\begin{array}{l}\oplus \oplus \odot \odot \\
\text { Low }^{f}\end{array}$ & \\
\hline
\end{tabular}




\begin{tabular}{|c|c|c|c|c|c|}
\hline 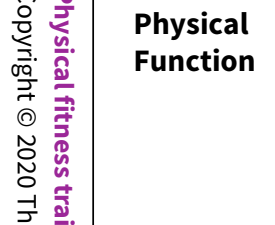 & $\begin{array}{l}\text { Berg Balance Scale } \\
(0 \text { to } 56 \text {, best balance = } \\
56) \\
\text { Analysis } 3.8\end{array}$ & $\begin{array}{l}\text { MD } 3.27 \text { higher } \\
\text { ( } 2.15 \text { higher to } 4.38 \\
\text { higher) }\end{array}$ & $\begin{array}{l}220 \\
\text { (5 RCTs) }\end{array}$ & $\begin{array}{l}\oplus \oplus \ominus \ominus \\
\text { Lowg }\end{array}$ & $\begin{array}{l}\text { Both of these outcomes are indices of balance. } \\
\text { The training only showed a statistically significant improvement in } \\
\text { balance measured with the Berg Balance Scale. }\end{array}$ \\
\hline 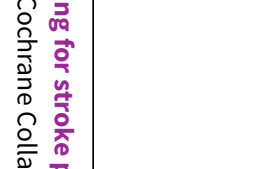 & $\begin{array}{l}\text { 3-metre Timed Up and } \\
\text { Go (seconds) } \\
\text { Analysis } 3.10\end{array}$ & $\begin{array}{l}\text { MD } 3.46 \text { s faster } \\
(0.02 \text { slower to } 6.94 \\
\text { faster) }\end{array}$ & $\begin{array}{l}224 \\
\text { (5 RCTs) }\end{array}$ & $\begin{array}{l}\oplus \oplus \ominus \ominus \\
\text { Lowh }\end{array}$ & \\
\hline
\end{tabular}

${ }^{*}$ The risk in the intervention group (and its 95\% confidence interval) is based on the assumed risk in the comparison group and the relative effect of the intervention (and its $95 \% \mathrm{Cl})$.

6-MWT: 6-Minute Walk Test; CI: confidence interval; MD: mean difference; RCT: randomised controlled trial; SMD: standardised mean difference

GRADE Working Group grades of evidence

High certainty: We are very confident that the true effect lies close to that of the estimate of the effect

Moderate certainty: We are moderately confident in the effect estimate: The true effect is likely to be close to the estimate of the effect, but there is a possibility that it is

substantially different

Low certainty: Our confidence in the effect estimate is limited: The true effect may be substantially different from the estimate of the effect

Very low certainty: We have very little confidence in the effect estimate: The true effect is likely to be substantially different from the estimate of effect

a Most participants were high-functioning; risk of death was low among this group. There is serious risk of bias from imbalanced exposure in 12 of the 20 included studies.

bone of the two included studies has major risk of bias issues and the composite measure of muscle strength is indirect in nature.

cOne of the three included studies has risk of bias issues. There is some heterogeneity $(12=47 \%)$.

dOne of the three included studies has risk of bias issues. There is high heterogeneity $(12=87 \%)$.

eHigh degree of heterogeneity $(12=76 \%)$.

fVery serious risk of bias including three out of five studies confounded for exposure time; key 'Risk of bias' items affecting highest weighted studies.

gVery serious risk of bias including three out of five studies confounded for exposure time.

hHigh degree of heterogeneity $\left(I^{2}=89 \%\right)$ and four out of five studies confounded for exposure time.

\section{Summary of findings 3. Mixed training compared to control for people with stroke: end of intervention}

\section{Mixed training compared to control for people with stroke: end of intervention}

Patient or population: people with stroke

Setting: during and after usual care

Intervention: mixed training

Comparison: control; end of intervention 


\begin{tabular}{|c|c|c|c|c|c|}
\hline \multicolumn{2}{|l|}{ Outcomes } & $\begin{array}{l}\text { Relative effect } \\
(95 \% \mathrm{CI})\end{array}$ & $\begin{array}{l}\text { Number of } \\
\text { participants } \\
\text { (studies) }\end{array}$ & $\begin{array}{l}\text { Certainty of } \\
\text { the evidence } \\
\text { (GRADE) }\end{array}$ & Comments \\
\hline \multicolumn{2}{|l|}{ Death } & Risk difference 0.00 & 1231 & $\oplus \oplus \ominus \ominus$ & Although there were more deaths in the control group \\
\hline \multicolumn{2}{|l|}{ Analysis 5.1} & $(-0.02$ to 0.01$)$ & $(203 \pi(15)$ & & groups. \\
\hline \multicolumn{2}{|c|}{ Dead or dependent } & - & 0 (0 RCTs) & - & No studies reported the composite outcome of death or de- \\
\hline Disability & $\begin{array}{l}\text { Pooled functional scales } \\
\text { Analysis } 5.5\end{array}$ & $\begin{array}{l}\text { SMD } 0.23 \text { higher } \\
\text { ( } 0.03 \text { higher to } 0.42 \text { higher) }\end{array}$ & $\begin{array}{l}604 \\
(9 \mathrm{RCTS})\end{array}$ & $\begin{array}{l}\oplus \oplus \ominus \ominus \\
\text { Low }^{b}\end{array}$ & $\begin{array}{l}\text { A SMD of global scales of disability is difficult to interpret. } \\
\text { The magnitude of increase observed ( } 0.2 \text { to } 0.5) \text { can gen- } \\
\text { erally be categorised as a 'small' effect. Any improvement } \\
\text { may be reflecting improved mobility since mobility items } \\
\text { are commonly included in these assessment tools. } 7 / 9 \text { stud- } \\
\text { ies were confounded for exposure time so any effects may } \\
\text { be exaggerated. Any improvement may be connected to im- } \\
\text { proved mobility since mobility items are a common feature } \\
\text { of these scales. }\end{array}$ \\
\hline \multirow[t]{6}{*}{$\begin{array}{l}\text { Physical fit- } \\
\text { ness }\end{array}$} & $\begin{array}{l}\mathrm{VO}_{2} \text { peak }(\mathrm{mL} / \mathrm{kg} / \mathrm{min}) \\
\text { Analysis } 5.8\end{array}$ & $\begin{array}{l}\text { MD } 1.4 \mathrm{~mL} / \mathrm{kg} / \mathrm{min} \text { higher } \\
(-0.19 \text { lower to } 2.99 \text { higher })\end{array}$ & $\begin{array}{l}140 \\
(2 \mathrm{RCTS})\end{array}$ & $\begin{array}{l}\oplus \oplus \ominus \ominus \\
\operatorname{LoW}^{c}\end{array}$ & $\begin{array}{l}\text { There were too few cardiorespiratory fitness data reported } \\
\text { to establish any consensus effects for mixed training. }\end{array}$ \\
\hline & $\begin{array}{l}\text { Muscle strength - ankle } \\
\text { dorsiflexion }\end{array}$ & $\begin{array}{l}\text { SMD } 0.8 \text { stronger ( }-0.82 \\
\text { weaker to } 2.41 \text { stronger) }\end{array}$ & 148 (2 RCTs) & $\begin{array}{l}\oplus \ominus \ominus \ominus \\
\text { Very lowd }\end{array}$ & \multirow{5}{*}{$\begin{array}{l}\text { Overall } 6 \text { RCTs reported muscle strength outcomes. } \\
\text { Variation in the method for measuring strength restrict- } \\
\text { ed the pooling of data; however, } 3 \text { groups of strength out- } \\
\text { comes could be pooled. } \\
\text { Only knee extensor strength showed a small }(>0.2) \text { benefi- } \\
\text { cial effect on knee extension strength. }\end{array}$} \\
\hline & & & & & \\
\hline & $\begin{array}{l}\text { Muscle strength - knee } \\
\text { extension } \\
\text { Analysis } 5.11\end{array}$ & $\begin{array}{l}\text { SMD } 0.33 \text { stronger ( } 0.05 \\
\text { stronger to } 0.61 \text { stronger) }\end{array}$ & 202 (3 RCTs) & $\begin{array}{l}\oplus \oplus \ominus \ominus \\
\text { Lowe }\end{array}$ & \\
\hline & $\begin{array}{l}\text { Muscle strength - paretic } \\
\text { grip strength }(\mathrm{kg})\end{array}$ & $\begin{array}{l}\text { MD } 0.32 \mathrm{Kg} \text { stronger }(-0.88 \\
\text { weaker to } 1.52 \text { stronger })\end{array}$ & 147 (3 RCTs) & $\begin{array}{l}\oplus \oplus \ominus \ominus \\
\text { Low }^{f}\end{array}$ & \\
\hline & Analysis 5.12 & & & & \\
\hline Mobility & $\begin{array}{l}\text { Preferred gait speed }(\mathrm{m} / \\
\mathrm{min}) \\
\text { Analysis } 5.14\end{array}$ & $\begin{array}{l}\text { MD } 4.71 \mathrm{~m} / \mathrm{min} \text { faster } \\
\text { (1.32 faster to } 8.1 \text { faster) }\end{array}$ & $\begin{array}{l}738 \\
(10 \mathrm{RCTs})\end{array}$ & $\begin{array}{l}\oplus \oplus \oplus \ominus \\
\text { Moderateg }\end{array}$ & $\begin{array}{l}\text { These increases in preferred walking speed and walking ca- } \\
\text { pacity are relevant to community ambulation. The inter- } \\
\text { ventions are mostly those with a walking mode of exercise. } \\
\text { These functional benefits in the 6-MWT were also main- } \\
\text { tained after the end of the training interventions. }\end{array}$ \\
\hline
\end{tabular}




\begin{tabular}{|c|c|c|c|c|c|}
\hline & $\begin{array}{l}\text { Gait endurance (6-MWT } \\
\text { metres) } \\
\text { Analysis } 5.15\end{array}$ & $\begin{array}{l}\text { MD } 35 \text { m further } \\
\text { (15.91 further to } 54.09 \text { fur- } \\
\text { ther) }\end{array}$ & $\begin{array}{l}720 \\
(10 \mathrm{RCTs})\end{array}$ & $\begin{array}{l}\oplus \oplus \Theta \ominus \\
\text { Lowh }\end{array}$ & \\
\hline \multirow[t]{3}{*}{$\begin{array}{l}\text { Physical } \\
\text { function }\end{array}$} & $\begin{array}{l}\text { Berg Balance Scale } \\
(0 \text { to } 56 \text {, best balance }= \\
56)\end{array}$ & $\begin{array}{l}\text { MD } 2.12 \text { higher } \\
\text { ( } 0.82 \text { higher to } 3.41 \text { higher) }\end{array}$ & $\begin{array}{l}419 \\
\text { (9 RCTs) }\end{array}$ & $\begin{array}{l}\oplus \oplus \Theta \ominus \\
\text { Low }^{i}\end{array}$ & \multirow[t]{3}{*}{$\begin{array}{l}\text { Both of these outcomes are indices of balance, only the } \\
\text { Berg Balance Scale demonstrated a statistically significant } \\
\text { improvement. }\end{array}$} \\
\hline & Analysis 5.17 & & & & \\
\hline & $\begin{array}{l}\text { 3-metre Timed Up and } \\
\text { Go (seconds) } \\
\text { Analysis } 5.20\end{array}$ & $\begin{array}{l}\text { MD } 2.21 \text { sec faster } \\
\text { ( } 0.02 \text { slower to } 4.43 \text { faster) }\end{array}$ & $\begin{array}{l}586 \\
(7 \mathrm{RCTs})\end{array}$ & $\begin{array}{l}\oplus \oplus \odot \ominus \\
\text { Lowj }\end{array}$ & \\
\hline
\end{tabular}

${ }^{*}$ The risk in the intervention group (and its $95 \%$ confidence interval) is based on the assumed risk in the comparison group and the relative effect of the intervention (and its $95 \% \mathrm{Cl})$.

6-MWT: 6-Minute Walk Test; Cl: confidence interval; MD: mean difference; OR: odds ratio; RCT: randomised controlled trial; RR: risk ratio; SMD: standardised mean difference

\section{GRADE Working Group grades of evidence}

High certainty: We are very confident that the true effect lies close to that of the estimate of the effect

Moderate certainty: We are moderately confident in the effect estimate: The true effect is likely to be close to the estimate of the effect, but there is a possibility that it is substantially different

Low certainty: Our confidence in the effect estimate is limited: The true effect may be substantially different from the estimate of the effect

Very low certainty: We have very little confidence in the effect estimate: The true effect is likely to be substantially different from the estimate of effect

a Most participants were high-functioning; risk of death was low among this group. There is serious risk of bias from imbalanced exposure in 16 of the 23 included studies.

bThere is heterogeneity $(12=21 \%)$ in addition to very serious risk of bias from imbalanced exposure in seven of the nine included studies.

CThere is heterogeneity $\left(I^{2}=35 \%\right)$ in addition to very serious risk of bias from imbalanced exposure in the study weighted $79.6 \%$.

dSubstantial heterogeneity $\left(I^{2}=76 \%\right)$; very serious risk of bias; both studies with imbalanced exposure.

eVery serious risk of bias; all three studies with imbalanced exposure.

fVery serious risk of bias with $3 / 3$ studies with imbalanced exposure.

gSubstantial heterogeneity $(12=76 \%)$.

hVery serious risk of bias with nine out of 10 studies with imbalanced exposure times.

iVery serious risk of bias with five out of nine studies with imbalanced exposure; there is no beneficial effect if these five studies are excluded.

jThere is heterogeneity $\left(I^{2}=45 \%\right)$ in addition to serious risk of bias from imbalanced exposure in seven of the nine included studies. 


\section{B A C K G R O U N D}

Physical activity and exercise recommendations exist for a wide range of healthy, older, and patient populations (O'Donovan 2010; Powell 2019), including those with specific health problems such as stroke (Billinger 2014). Exercise and physical activity are widely promoted during life after stroke. The current evidence in the previous version of this review indicates that exercise can reduce disability and improve physical fitness and aspects of physical function (Saunders 2016). More evidence is needed to examine the effects of different types and doses of exercise and to examine the effects on a wide spectrum of outcome measures.

\section{What is physical fitness training?}

Exercise refers to a subset of physical activity that is planned, structured, repetitive, and deliberately performed to train (improve) one or more components of physical fitness, physical performance, or health (USDHHS 2018). Since the term 'exercise' is used more generically within stroke care we will refer to exercise as 'physical fitness training'.

\section{What is physical fitness?}

Physical fitness can be defined as, "The ability to carry out daily tasks with vigor and alertness, without undue fatigue and with ample energy to enjoy leisure-time pursuits and meet unforeseen emergencies" (USDHHS 2018). The most important components of physical fitness are those directly connected to energy expenditure and muscular work.

- Cardiorespiratory fitness: this is the ability to transport and use oxygen and is usually expressed as maximal oxygen uptake $\left(\mathrm{VO}_{2}\right.$ max). Cardiorespiratory fitness confers 'endurance', that is the ability to perform physical activity for an extended period.

- Musculoskeletal fitness: the ability of muscle to generate force can be expressed in terms of strength and power. Muscle strength refers to the ability of a specific muscle or muscle group to exert force. Strength is associated with the ability to perform forceful movements such as pushing or lifting. Muscle power refers to the rate at which muscular work can be performed during a single explosive contraction. Power is associated with the ability to carry out forceful movements, in particular those that are dynamic.

In addition, other components of fitness can influence the ability to perform physical activities, including flexibility (range of motion about a specific joint), balance (ability to maintain stability and posture), and body composition (e.g. relative amounts of fat and fat-free mass).

\section{Determinants of fitness}

Sex has a direct influence on indices of physical fitness, which tends to be lower in women compared with men. For example, in women cardiorespiratory fitness is around $27 \%$ lower than men of the same age throughout the lifespan (Kaminsky 2015).

Increasing age results in deterioration of fitness; this is a normal consequence of healthy ageing. For example, cardiorespiratory fitness shows a linear decline of approximately $1 \%$ per year throughout adulthood (20 to 80 years of age; Kaminsky 2015). Muscle strength is well retained up to the age of around 50 to 60 years, after which it deteriorates quickly at approximately $1.6 \%$ per year (Kemmler 2018).

Physical inactivity causes rapid loss of musculoskeletal fitness and cardiorespiratory fitness. For example, just 10 days of bed rest causes rapid loss of muscle strength $(-13.2 \%)$, muscle power $(-14 \%)$, and cardiorespiratory fitness (-12\%) (Kortebein 2008).

Secondary consequences of chronic disease, such as inflammation, are also associated with loss of muscle strength and muscle mass (Degens 2006; Westbury 2018).

\section{Functional importance of fitness}

When the level of fitness is low (irrespective of the reason) physical activities may either become limited by fatigue or impossible to perform (Young 2001). Levels of fitness below the level needed to perform instrumental activities of daily living (ADL) may mean that these become impossible and this then may risk loss of independence. For example, cardiorespiratory fitness below 15 to $18 \mathrm{~mL} / \mathrm{kg} / \mathrm{min}$ is associated with loss of independence (Shephard 2009). Similarly muscle strength of various lower limb muscles below threshold values prevent older people performing ADL (Hasegawa 2008). Even without dropping below such threshold values reduced fitness is associated with reduced function. For example, low muscle power output in older people is associated with reduced mobility and an increased risk of falls (McKinnon 2017).

\section{Description of the condition}

The classic definition of stroke is "rapidly developing clinical signs of focal (at times global) disturbance of cerebral function, lasting more than 24 hours or leading to death with no apparent cause other than that of vascular origin" (Hatano 1976). A stroke is caused by an interruption to the circulation of the brain, either by a clot (ischaemic stroke) or a bleed (haemorrhagic stroke). Stroke can lead to death or cause disability; it can affect the way people move, think, feel, and behave. Globally, in 2016, stroke was the second leading cause of death and second leading cause of disabilityadjusted life years (WHO 2018), with around $50 \%$ of stroke survivors experiencing long-term disability (Mackay 2004).

A common neurological consequence of stroke is unilateral loss or limitation of muscle function; the direct consequence can be limitation or loss of movement, mobility, and functional ability. In addition, a whole range of indirect physical and psychological complications occur after stroke (Indredavik 2008; Langhorne 2000). Levels of physical activity are low in people with stroke in both inpatients (Bernhardt 2004; Bernhardt 2007), and also in community-dwelling stroke survivors (English 2014). In community-dwelling stroke survivors cardiorespiratory fitness ranges from $26 \%$ to $87 \%$ of the value expected in age- and gendermatched healthy people (Smith 2012). Muscle strength (Gerrits 2009; Horstman 2008), and muscle power (Saunders 2008), are also impaired with bilateral deficits, which suggest the influence of physical inactivity in addition to hemiparesis.

The level of post-stroke fitness may be low due to a range of factors directly and indirectly connected to stroke.

- Pre-stroke fitness levels may already be low because of physical inactivity (Lee 2002), and low levels of fitness (Kurl 2003), and are both risk factors for stroke. Stroke is not a disease restricted 
to elderly people; two-thirds of strokes are in people aged under 70 years (Feigin 2017); however, the effects of increasing age will have a part to play as will the presence of comorbid conditions.

- Direct neurological effects of stroke reduce the muscle mass available for activation (e.g. hemiparesis).

- Post-stroke physical inactivity will cause a longitudinal loss of fitness alongside the effects of comorbid diseases and increasing age. Limitation or loss of functional abilities after stroke (e.g. walking, stair climbing, chair rising) are associated with low cardiorespiratory fitness levels, muscle strength, and muscle power (Flansbjer 2006; Patterson 2007; Saunders 2008).

Therefore, when levels of physical fitness are low after stroke (for whatever reason) this may exacerbate or cause some common post-stroke physical limitations (Saunders 2013a). Restoration of motor function in order to improve functional ability is a key focus within stroke rehabilitation and a number of interventions have been investigated that involve physical activities and physical fitness training (Langhorne 2009).

\section{Description of the intervention}

The basic structure and content of all physical fitness training interventions are guided by a common set of well-established principles (ACSM 2011). The design of physical fitness training interventions varies across healthy people, older people, and different patient groups.

\section{Type of training}

Most physical fitness training programmes are classified as either:

- cardiorespiratory training (to improve cardiorespiratory fitness);

- resistance training (to improve muscular strength and muscle power); or

- mixed training, which combines cardiorespiratory and resistance training.

With regard to other aspects of fitness, all types of training programme have the potential to influence body composition (increase lean mass and reduce adiposity) and some may also incorporate elements that improve flexibility (stretching exercises) and balance.

\section{Mode of training}

The type of fitness training influences the mode(s) of exercise. For example, cardiorespiratory training commonly employs walking and cycling, whilst resistance training employs activities involving muscle contractions resisted by weights, body mass, or elastic devices.

\section{Dose of training}

The dose of training is controlled by influencing, firstly, the amount of training (e.g. programme length (weeks, months), frequency (days/week), and duration (minutes) of sessions), and secondly, the intensity of training (rate of work or effort made).

It is the manipulation of type, mode, and dose that defines an exercise prescription; however, the effectiveness is also influenced by some other critically important principles of training (ACSM 2011), including progression of training, whether training is task- related (specific), and the fact that training effects are reversible if training is reduced or stopped.

Physical fitness training is, therefore, very much a complex intervention with numerous component parts and this can give rise to variation in plausible benefits.

\section{How the intervention might work}

Regular physical activity is currently recommended where possible to people of all ages, including those with disabilities, in order to promote and maintain health (Haskell 2007; USDHHS 2018). The dose-response relationship means additional benefits exist if physical fitness training is employed, in particular with regard to physical function. Physical fitness training interventions improve physical function in healthy elderly people (Bangsbo 2019; Chodzko-Zajko 2009).

Post-stroke physical activity and fitness levels are low, and these low levels are associated with common post-stroke functional limitations. Increased fitness and physical function could benefit a range of other common post-stroke problems, for example by reducing fatigue, reducing the incidence of falls and fractures, compensating for the increased energetic cost of a hemiparetic gait, reducing disability and improving independence, and improving quality of life and mood.

Physical therapies are known to promote structural brain remodelling (Gauthier 2008), and this can influence post-stroke motor deficits. There is systematic review evidence that repetitive practice of some common day-to-day activities produces some modest improvements in mobility and ADL in people with stroke (French 2016). Fitness training is repetitive in nature and is commonly task-related in nature, therefore participation in it may lead to functional benefits even when fitness is not improved.

Engagement with group training activities may have some psychosocial benefits in people with stroke (Carin-Levy 2009; Mead 2005; Patterson 2009). Fitness training is commonly delivered in a group setting; therefore, in these instances simply participating in physical fitness training may be beneficial, particularly when group activities are delivered in a non-medical environment (Young 2019).

Cognitive function impairments are common after stroke and are predicted by low indices of physical fitness (Lee 2014). In older adults (aged over 65 years) with cognitive impairment, exercise interventions have been shown to improve cognitive function (Heyn 2004). Further, in people with stroke and traumatic brain injury, systematic review evidence suggests that exercise exerts a positive effect on global cognitive function, though further studies are required (Vanderbeken 2017). Therefore, there is some rationale that fitness training interventions could benefit cognition in people with stroke.

Physical fitness training is known to be beneficial for people with a number of conditions that are comorbid conditions or risk factors for stroke. Systematic review evidence shows that exercise interventions can reduce blood pressure (Cornelissen 2013; Smart 2019); improve vascular risk factors in obesity (Shaw 2006), and type II diabetes (Thomas 2006); improve cardiovascular mortality (Anderson 2016); and improve depressive symptoms regardless of health status (Gordon 2018). Therefore, post-stroke cardiorespiratory and resistance training could reduce morbidity 
and mortality through secondary prevention of stroke and comorbid disease

In summary, physical fitness training does not simply offer a mechanism to increase fitness, it has multiple mechanisms of action and has a spectrum of plausible benefits that are relevant to many people with stroke. However, as well as benefits there may also be risks associated with fitness training such as traininginduced soft tissue injuries, altered muscle tone, falls, and vascular events.

\section{Why it is important to do this review}

Physical fitness training for stroke survivors remains underinvestigated in three key areas.

- The range of possible benefits is not fully explored. The top 10 most important research priorities for 'life after stroke' have been defined by a partnership of patients, carers, and clinicians (Pollock 2012). These include, in order of priority, the need to identify interventions for 1) cognition, 2) coming to terms with long-term consequences, 3) aphasia, 4) arm function, 5) vision, 6) fatigue, 7) balance, gait and mobility, 8) speech, 9) confidence, and 10) exercise interventions for function, quality of life, and secondary prevention. Exercise interventions may have a beneficial role in at least four of these domains $(1,4,7$ and 10; Saunders 2014a).

- Enough evidence is available to implement fitness training for stroke, the optimal exercise prescription has yet to be defined (Mead 2011).

- Fitness training has a plausible benefit for secondary stroke prevention but there still remains a lack of direct evidence about secondary stroke and cardiovascular risk.

There has been sustained interest in physical fitness interventions for stroke evidenced by the studies included in previous updates of this review: Saunders 2004a (12 studies), Saunders 2009 (24 studies), Brazzelli 2011b (32 studies), Saunders 2013b (45 studies), and Saunders 2016 (58 studies). Considering the degree of incomplete knowledge, the high level of interest, and the clinical relevance of this review for improving patient care, we believe it is essential to continue updating the earlier version of this review.

\section{O B JECTIVES}

The primary objectives of this updated review were to determine whether fitness training after stroke reduces death, death or dependence, and disability.

The secondary objectives were to determine the effects of training on adverse events, risk factors, physical fitness, mobility, physical function, health status and quality of life, mood, and cognitive function.

\section{MET HODS}

\section{Criteria for considering studies for this review}

\section{Types of studies}

All studies described as randomised controlled trials (RCTs), singleblinded or open, which examined the effects of cardiorespiratory, resistance, or mixed training using any of the following six comparisons:
- cardiorespiratory training versus control: 1 ) at the end of intervention, 2) at the end of follow-up;

- resistance training versus control: 3 ) at the end of intervention, 4) at the end of follow-up;

- mixed training (cardiorespiratory plus resistance training) versus control: 5) at the end of intervention, 6) at the end of follow-up.

In this review 'end of intervention' refers to the time point when a training programme finishes; 'end of follow-up' refers to any time point occurring after the end of the intervention. Measures at the end of follow-up allow us to examine whether training effects (if any) are retained after training is completed. For studies with multiple follow-up phases we analysed data from the longest follow-up period.

We included studies in which controls were exposed to either physical activity occurring during usual care or no training after usual care. By 'no training' we meant either no intervention or a non-exercise intervention (e.g. cognitive tasks or sham training). Therefore, we deemed the following comparisons suitable for inclusion where 'usual care' refers to inpatient hospital care or other standard rehabilitation given to all stroke patients delivered as a normal part of stroke care in the region in which the studies were performed:

- training plus usual care versus usual care (during usual care);

- training versus no training (after usual care).

We included full-text reports of published and unpublished studies. Studies communicated via conference proceedings alone or by abstract alone provide only limited data and do not allow full assessment of study quality. Therefore, we did not exclude any studies that were potentially relevant but we retained them as 'awaiting classification' pending further information or full publication. We did not exclude studies on the basis of their sample size. We included studies published in languages other than English only when a translation could be arranged. Where investigators published several reports based on data from a single study population, we selected the most recent or most complete report for data extraction and we listed the other reports as additional publications.

\section{Types of participants}

Adult stroke survivors who were considered suitable for fitness training by the studies' authors; we used the study authors' definition of stroke. Participants were considered eligible irrespective of the time since stroke onset.

\section{Types of interventions}

We included the following interventions.

\section{Cardiorespiratory training interventions}

The aim of this type of training is to improve the cardiorespiratory component of physical fitness. It is typically performed for extended periods of time on devices or ergometers (e.g. treadmill, cycling, rowing) or by utilising modes of activity such as walking or climbing stairs. 


\section{Resistance training interventions}

This type of training is performed primarily to improve muscle strength and muscular endurance or muscle power output, or both. It is typically carried out by making repeated muscle contractions resisted by body weight, elastic devices, masses, free weights or specialised machine weights, and isokinetic devices.

\section{Mixed training interventions}

This describes training interventions that comprise different activity components, some intended to improve cardiorespiratory fitness and others to improve strength, power or muscular endurance; for example, a training programme comprising both cycling and weight training.

We only included studies that aimed at training stroke survivors. We defined 'training' as a systematic, progressive increase in the intensity or resistance, frequency, or duration of the physical activity throughout a scheduled programme. We sought measures of adherence to training since this can modify the dose of training received by study participants. For the purposes of this review, adherence included both: 1) attendance at training sessions, and 2) compliance with exercise instructions during training sessions.

We excluded studies that focused on different types of standard rehabilitation techniques but did not include a physical fitness training component. We also excluded studies that combined fitness training with assistive technologies, such as robotic and electromechanical-assisted gait training devices during body weight-supported locomotor training, as well as studies investigating virtual reality approaches.

We excluded studies that compared upper and lower body training if an additional non-exercise control group was not considered.

If any description of a training regimen was unclear, we contacted the authors for further information.

\section{Control interventions}

The control interventions included: 1) usual care; 2) no intervention or waiting-list control; or 3) attention control, sham intervention, or adjunct intervention. The types of comparison are as follows.

- Physical fitness training interventions versus no intervention or waiting-list control

- Physical fitness training interventions versus attention control, sham intervention or adjunct intervention

- Physical fitness training interventions plus usual care versus no intervention or waiting-list control plus usual care

- Physical fitness training interventions plus usual care versus attention control, sham intervention or adjunct intervention plus usual care

\section{Types of outcome measures}

We anticipated that existing studies in the literature would use different measures to assess outcomes relevant to this review; in particular they would use a variety of rating scales. For each outcome of interest we tried, therefore, to list the most common and relevant measures or tools. We only included rating scales that had been described in peer-reviewed journals.

\section{Primary outcomes}

- Death: numbers of deaths from all causes

- Death or dependence: composite outcome where dependence is classified as having a Barthel Index score of less than 20 or modified Rankin Scale score of 3, 4, or 5 (Lindley 1994).

- Disability: assessed by functional scales such as the Functional Independence Measure (Hamilton 1994); Barthel Index (Collin 1988); Rivermead Mobility Index (Collen 1991); Nottingham Extended Activities of Daily Living Scale (Wade 1992); Lawton Index of Activities of Daily Living (Lawton 1969); and the Stroke Impact Scale (Duncan 1999).

In the International Classification of Functional Disability and Handicap (ICF) classification the term 'disability' is an umbrella term for impairments and activity limitations (WHO 2001). In this review the primary outcome measure 'disability' refers to 'global indices of activity limitation'. Secondary outcome measures of mobility and physical function refer to 'specific activity limitations'.

\section{Secondary outcomes}

- Adverse effects: recurrent non-fatal cardiovascular or cerebrovascular events; altered muscle tone; training-induced injury; incidence of falls; incidence of fractures

- Vascular risk factors: resting systolic and diastolic blood pressure; resting heart rate; total cholesterol; glucose tolerance; body mass index (BMI)

- Physical fitness: maximum or peak oxygen uptake (peak $\mathrm{VO}_{2}$ ); muscle strength and power output

- Mobility: gait speed (maximum or preferred speed); gait capacity (e.g. Six-Minute Walk Test (6-MWT)); Functional Ambulation Categories

- Physical function: balance; stair climbing; weight bearing; Timed Up and Go test

- Health status and quality of life: any relevant scale such as the Short Form 36 Health Survey Questionnaire (Ware 1992), and the Nottingham Health Profile (Hunt 1980)

- Mood: any relevant scale such as the Hospital Anxiety and Depression Scale (HADS; Zigmond 1983); the Beck Depression Index (Beck 1961)

- Cognitive function: any subscale of disability or health status outcomes that relate to cognitive function, or any specific cognition instrument, for example the Repeatable Battery for the Assessment of Neuropsychological Status (Randolph 1998); the Montreal Cognitive Assessment (MOCA; Nasreddine 2005).

\section{Search methods for identification of studies}

See the methods for the Cochrane Stroke Specialised Register. We searched for relevant studies in all languages and arranged translation of relevant papers where necessary.

\section{Electronic searches}

The search strategies used for this review have been significantly revised and updated to account for newly identified relevant terms and to improve sensitivity and specificity. All discontinued versions of search strategies used are still available in the previous version of this review (Saunders 2016).

We searched the Cochrane Stroke Specialised Register and the following electronic databases. 
- Cochrane Central Register of Controlled Trials (CENTRAL; 2018, Issue 1; Appendix 1) in the Cochrane Library;

- MEDLINE Ovid (from 1946 to July 2018; Appendix 2);

- Embase Ovid (from 1974 to July 2018; Appendix 3);

- CINAHL EBSCO (Cumulative Index to Nursing and Allied Health Literature; from 1937 to July 2018; Appendix 4);

- SPORTDiscus EBSCO (from 1949 to July 2018; Appendix 5);

- PsycINFO Ovid (from 1806 to July 2018; Appendix 6);

- Conference Proceedings Citation Index- Science (Web of Science; from 1990 to July 2018; Appendix 7);

- PEDro (Physiotherapy Evidence database (www.pedro.fhs.usyd.edu.au/index.html) July 2018; Appendix 8).

The search strategy includes Cochrane Highly Sensitive Search Strategies for identification of RCTs, as described in the Cochrane Handbook for Systematic Reviews of Interventions (Lefebvre 2011), and Cochrane Stroke's search strategies for the identification of 'stroke' studies in respective databases and other resources. These are supplemented with strategies to identify exercise interventions. We have added PsycINFO to the search resources in this update in recognition of the increasing interest in cognition outcomes.

In order to identify other published, unpublished, and ongoing studies we searched for ongoing studies using the following registries.

- US National Institutes of Health Ongoing Trials Register ClinicalTrials.gov (clinicaltrials.gov/)

- World Health Organization International Clinical Trials Registry Platform (ICTRP; apps.who.int/trialsearch)

\section{Searching other resources}

We searched for theses in the following (using the search terms in Appendix 9):

- ProQuest Dissertations and Theses Global (www.proquest.com/ products-services/pqdtglobal.html);
- British Library EThOS (e-theses online service; www.bl.uk/ ethos-and-theses);

- DART-Europe E-theses PortAL (www.dart-europe.eu/basicsearch.php).

We searched grey literature using:

- Google Scholar

We checked the bibliographies of included studies and performed forward citation tracking of all included studies (and other relevant studies) using Google Scholar (scholar.google.co.uk/) for further references to relevant studies.

We contacted researchers in the field to obtain additional information on relevant studies and contacted original authors for clarification and further data if study reports were unclear.

\section{Data collection and analysis}

\section{Selection of studies}

Two review authors (DS or MS or SH or DC or SK or LJ or $\mathrm{HJ}$ ) independently screened titles and abstracts of the unique references obtained as a result of our searching activities. We excluded studies that two review authors classified as 'exclude'; we retained all other studies for full-text screening.

We retrieved the full-text articles for the remaining references and two review authors (DS or MS or SH or DC or SK or LJ or HJ) independently screened the full-text articles to identify studies for inclusion, and identify and record reasons for exclusion of the ineligible studies. We resolved any disagreements through discussion or, if required, we consulted a third review author (GM or MB). We collated multiple reports of the same study so that each study, not each reference, was the unit of interest in this review.

We used the Covidence tool to carry out the selection process and to record this in sufficient detail to complete a PRISMA flow chart (Moher 2009; Figure 1), and the 'Characteristics of excluded studies' table. 


\section{Figure 1. Study flow diagram for the current update of this review}

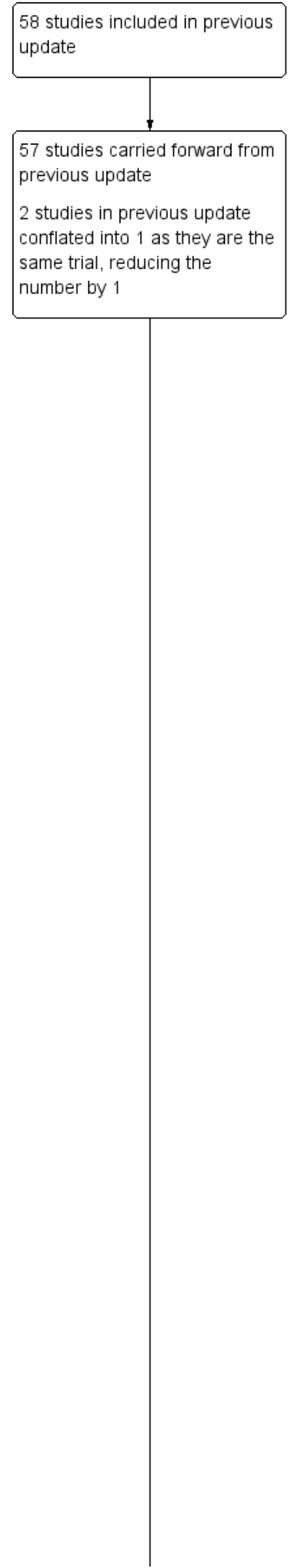

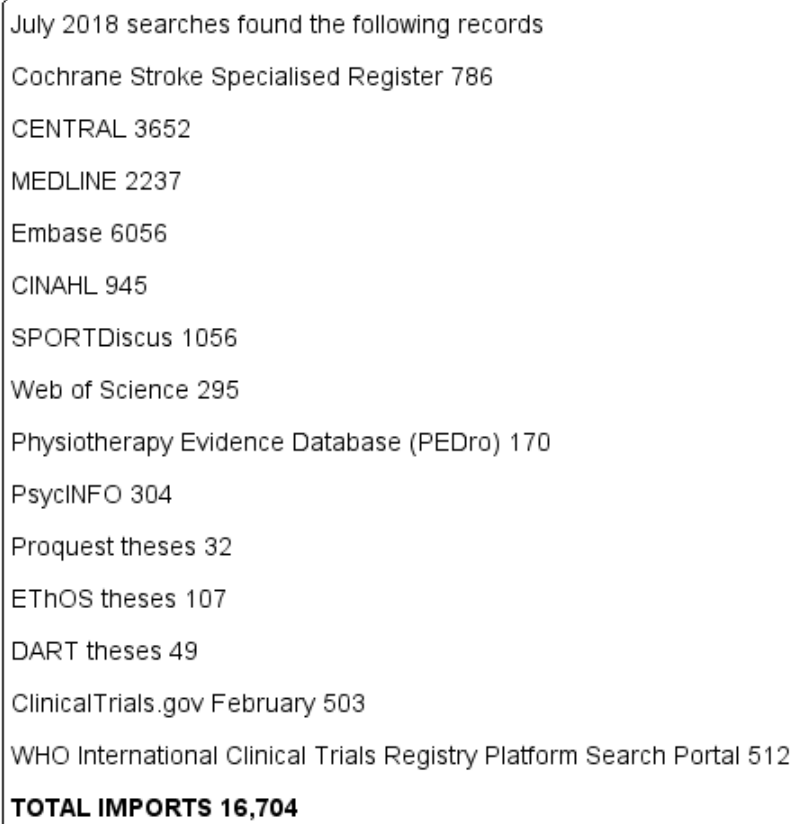

10,767 records excluded based on title, abstract, or full text when required. Includes exclusion of any remaining duplicates 
Figure 1. (Continued)

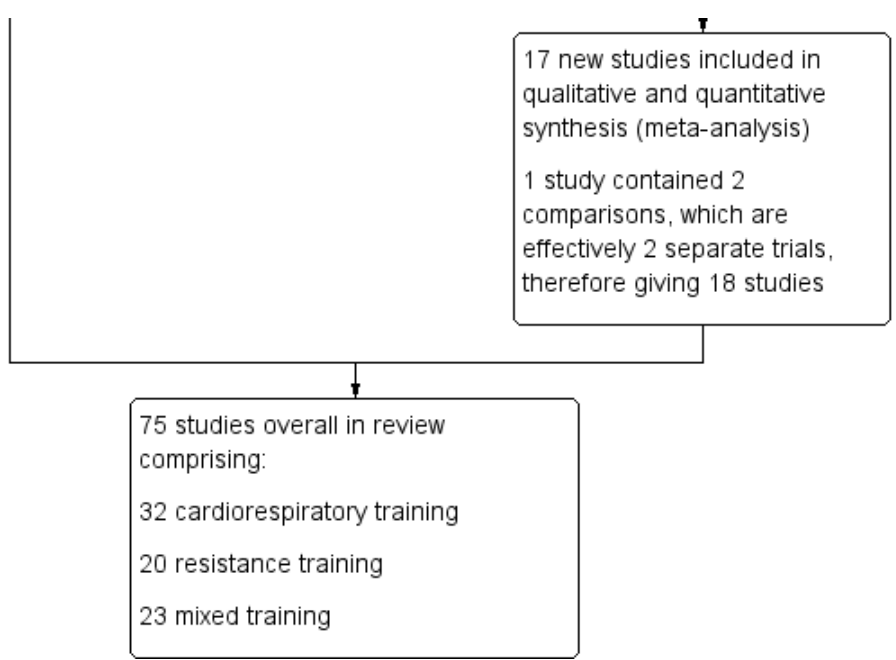

We included studies irrespective of publication status providing available reports had sufficient detail to apply eligibility criteria and perform quality assessment.

We retained potentially relevant studies with insufficient information to either include or exclude in the 'Characteristics of studies awaiting classification' table.

\section{Data extraction and management}

One review author (DS or MS or SH or DC or SK or LJ or HJ) extracted data from each included study and entered the outcome data directly into Review Manager 5 (Review Manager 2014). A second review author (DS or MS or SH or DC or SK or LJ or HJ) then crosschecked all entered data. We contacted study authors to obtain any missing data if required.

The domains for data extraction for each study included but were not limited to:

- publication details: authors, year of publication, publication status (published, unpublished, or ongoing), citation of other relevant studies;

- details of study conduct: study design, method of recruitment, inclusion and exclusion criteria, number of participants enrolled, number of participants excluded, number of participants assessed, losses to follow-up, geographical location of the study, setting in which the study was conducted (e.g. hospital, community);

- characteristics of participants: total number, age, gender, stage of care, severity of stroke, time since stroke onset, co-morbidity, walking ability;

- details of intervention: total number of intervention groups, type of training (i.e. cardiorespiratory, resistance, or mixed), training mode (e.g. treadmill walking, weight training), dose (i.e. intensity, frequency of delivery), timing (i.e. during or after usual care), length of training (i.e. duration and programme length), adherence to intervention (i.e. attendance, compliance);

- details of outcome measures: choice of outcomes (i.e. death, dependence, disability, physical fitness measures, gait assessment, physical function measures, health status and quality of life, mood, adverse events, risk factors), outcome data, reported outcomes, missing outcomes.

We classified all outcome data as being from time points at either the end of intervention or the end of follow-up, which we defined as any period of time after the training intervention was completed. We resolved any disagreement by consensus or arbitration.

\section{Assessment of risk of bias in included studies}

Two review authors (DS, MS, SH and either LJ, SH, MK, $\mathrm{HJ}$ ) independently assessed each study using Cochrane's tool for assessing risk of bias (Higgins 2017). We resolved any disagreements by discussion or by involving another review author (GM or MB). We assessed the risk of bias for each of the standard domains in the Cochrane 'Risk of bias' tool with the following exceptions and amendments.

\section{Blinding of participants (performance bias and detection bias)}

Participant blinding is often impossible to achieve in behavioural interventions. However, we considered studies to be at low risk of bias if the study authors described some attempt to disguise the true purpose of the comparisons being made (e.g. describing a study as a comparison of two different interventions or some kind of 'sham' intervention). We considered studies to be at high risk of bias if there was an imbalanced exposure such as would occur with no control intervention or a waiting-list control.

\section{Incomplete outcome data (attrition bias)}

We assessed this domain twice, once at the end of intervention and once at the end of follow-up. We considered studies to be at high risk of bias where we judged imbalanced losses to have occurred coupled with a per-protocol analysis. If overall participant attrition was $20 \%$ or greater of those randomised, we considered a study at high risk of bias (Schulz 2002), irrespective of distribution of losses, reasons given or analytical approach (e.g. imputations, intentionto-treat).

\section{Other bias}

We considered 'Risk of bias' items relevant to cluster-RCTs in this domain. 


\section{Imbalanced exposures}

We included this additional 'Risk of bias' item because an imbalanced exposure could exaggerate benefits (or harms) in a way where it is impossible to separate the effects of the intervention content from the effects of attention. Therefore, strictly speaking, this is a confounding effect rather than a bias effect, but it is appropriate to record it and analyse it in the same way as other bias items. We considered studies to be at low risk of bias if a 'dose' of exposure or attention was provided in the control group that matched that in the intervention groups (e.g. attention control or sham intervention). We considered studies to be at high risk of bias if the control group received no control intervention including being allocated to a waiting-list control.

In all categories when there was insufficient information to assign either a 'low risk' or 'high risk' of bias, we contacted the study authors and asked them for clarification. Where we could not obtain missing supplementary information, we recorded an 'unclear' risk of bias. We recorded 'high', 'low' or 'unclear' risk of bias along with a descriptive justification for our judgment in the 'Risk of bias' tables. We presented the data in 'Risk of bias' summary graphs.

\section{Measures of treatment effect}

\section{Dichotomous data}

For dichotomous outcome data we calculated odds ratios (OR) and 95\% confidence intervals (Cls).

\section{Continuous data}

Where possible, we presented the effects of interventions on all continuous outcome data as a mean difference (MD) and 95\% Cls. In instances where studies used different scales to measure the same clinical outcome, we presented the data as standardised mean difference (SMD) and 95\% Cls.

\section{Unit of analysis issues}

Cluster-RCTs: if clustering as a unit of allocation was not controlled by the study authors, we implemented this, where appropriate, during meta-analysis using the methods described in the Cochrane Handbook for Systematic Reviews of Interventions (Deeks 2017).

Cross-over studies: the data can be truncated after the first iteration of a cross-over study and treated as an RCT. We ignored subsequent iterations because of the risk of carry-over effects.

Lag-control or waiting-list studies: we dealt with these in the same way as cross-over studies. We ignored the delayed or waiting-list iteration of the study because of the risk of carry-over effects.

In studies with more than one relevant control group, we used only one control group within a meta-analysis. We performed sensitivity analysis to examine the relative influence of selecting each group on meta-analysis results. Where data from multiple control groups were similar we considered combining the control group data using the methods described in section 16.5.4 of the Cochrane Handbook for Systematic Reviews of Interventions (Higgins 2011).

In studies with more than one relevant intervention group, we included all intervention groups as separate comparisons within a meta-analysis, with the control group data replicated across all comparisons, but with the control group sample size divided evenly across among the comparisons to prevent inflation of overall sample size.

The principal time points for outcome measurement were: 1) at the end of intervention, and 2) at the end of follow-up.

\section{Dealing with missing data}

Missing participants: we account for the nature and extent of missing participant data (e.g. losses to follow-up) and how the study authors dealt with this (e.g. intention-to-treat analysis) via one of the 'Risk of bias' assessments (Assessment of risk of bias in included studies; incomplete outcome data).

Incomplete reporting: if RCTs had missing information we contacted the study authors to request this. Where there was insufficient information to include or exclude a potentially relevant study and this could not be retrieved we retained the study in the 'Characteristics of studies awaiting classification' section in case the information emerges at a later date.

\section{Assessment of heterogeneity}

We assessed heterogeneity using the $I^{2}$ statistic (Higgins 2003), presented as part of the forest plots in Review Manager 2014. We interpreted values of 12 statistic exceeding $50 \%$ as indicating substantial heterogeneity. In these cases we investigated potential causes of variation by inspecting study effects and by using subgroup and sensitivity analysis if appropriate.

\section{Assessment of reporting biases}

The comprehensive search strategy helped ameliorate reporting biases.

When meta-analyses included a minimum of 10 studies, we used a funnel plot (treatment effect versus study size).

\section{Data synthesis}

Where we considered studies to be sufficiently similar, we conducted a meta-analysis by pooling the appropriate data using Review Manager 2014.

We used random-effects meta-analysis models to calculate measures of effect and $95 \% \mathrm{Cls}$ at the end of intervention and the end of follow-up for each outcome measure with sufficient suitable data to pool.

\section{Summary of findings and assessment of the certainty of the evidence}

We used GRADE to assess the evidence for the primary outcomes death, death or dependence, and disability, plus the secondary outcomes relating to physical fitness (cardiorespiratory fitness, muscle strength), mobility (preferred walking speed, gait endurance), and physical function (Berg balance scores, threemetre Timed Up and Go). We performed these analyses and presented the results in a 'Summary of findings' table generated using GRADEpro GDT software.

\section{Subgroup analysis and investigation of heterogeneity}

When sufficient data were available, we planned to investigate heterogeneity between included studies (both clinical and statistical) by means of subgroup analyses. We attempted to 
compare effect estimates for all outcomes in the following main subgroups:

- type of training (cardiorespiratory versus resistance training versus mixed training);

- time of training (during usual care versus after usual care).

The complexity of exercise interventions and low numbers of studies in the meta-analyses meant that subgroup analyses were difficult to perform and difficult to interpret.

\section{Sensitivity analysis}

We planned to explore for all outcomes the influence of studies that were confounded by increased training time.

\section{RES U L T S}

\section{Description of studies}

\section{Results of the search}

The previous version of this review included 58 studies, involving 2797 participants (Saunders 2016). In this updated version we used the updated electronic searches and other relevant searches in July 2018. We screened a total of 16,704 citations; this includes duplicates.

We identified 29 new systematic reviews of exercise interventions and screened these for relevant studies (Abbasian 2018; Ammann 2014; Austin 2014; Baldwin 2016; Bonini-Rocha 2018; Boyne 2017; Chen 2016; Crozier 2018; D'Isabella 2017; Dorsch 2018; English 2017; Hasan 2016; latridou 2018; Ilunga Tshiswaka 2018; Jeon 2015; Kendall 2016; Mehrholz 2017; Oberlin 2017; Plummer 2018; Salter 2016; Saltychev 2016; Tally 2017; Van Criekinge 2019; Van Duijnhoven 2016; Vanderbeken 2017; Vloothuis 2016; Wang 2018; Wist 2016; Zheng 2016).

The results of our searching activities are summarised in Figure 1. We applied the eligibility criteria, with the following results.

- We excluded 86 studies that did not meet the eligibility criteria (see Characteristics of excluded studies table).

- We identified 56 new ongoing studies (see Characteristics of ongoing studies table).

- We identified 35 studies for which we require more information to establish eligibility, including those for which only the abstract is currently available (see Characteristics of studies awaiting classification table).

- We identified one study that was a secondary analysis of two studies already included in the previous update. These two previously included studies, authored by Aidar in 2012 and in 2014, have since been recognised as the being same trial and are now conflated along with their more recent publication to be collectively referred to as Aidar 2016.

- We identified one study that was a secondary analysis of a study included in the previous update (Aidar 2018).

- We identified 17 new studies that met the eligibility criteria.

With regard to ongoing studies and those awaiting classification in the previous version of this review:

- of the 13 ongoing studies, four have been completed and met the eligibility criteria and we have added them to the included studies (Dean 2018; Ivey 2017; Sandberg 2016; Vanroy 2017). The remainder either completed but we excluded them as irrelevant, were terminated, or the data were never published or made available;

- of the nine abstract-only studies awaiting classification, one is now included (Buyukvural 2015). The remainder have been excluded or have no full-text paper published or made available;

- of the three full publication studies awaiting classification that need further information to include or exclude, only one author team responded and we were able to exclude the data.

\section{Included studies}

We included 17 new studies in this update (Arabzadeh 2018; Buyukvural 2015; Coroian 2018; Dean 2018; Fernandez-Gonzalo 2016; Furnari 2014; Ivey 2017; Kim 2016a; Kim 2017a; Knox 2018; Mao 2015; Moore 2015; Sandberg 2016; Taylor-Pilliae 2014; Topcuoglu 2015; Vanroy 2017; Zou 2015).

Two separate studies in the previous update (Saunders 2016), have emerged as being the same study. These have now been conflated with a further recent publication and together these now form Aidar 2016.

One of the 17 included studies (Knox 2018), includes a strength training intervention group and a mixed training intervention group, both of which we included and analysed as two separate studies each sharing the same control group (effectively giving 18 new studies). For clarity, we counted the control group $(n=48)$ once to calculate the total number of unique participants in the review $(\mathrm{n}=3617)$. We split the control participants across the resistance training $(n=24)$ and mixed training $(n=24)$ groups where we were comparing them in a meta-analysis. In other meta-analyses, we used all 48 control participants; we ensured that there was no double counting at any point.

In summary, when these adjustments are reconciled, this update includes a total of 75 studies comprising 3617 participants (see Characteristics of included studies table).

\section{Participants}

\section{Characteristics}

A total of 3617 stroke survivors (sample range 13 to 250 individuals) were randomised to physical fitness training or control interventions in the 75 included clinical studies. The mean age of the participants was approximately 62 years. The mean time since onset of symptoms ranged from 8.8 days in studies assessing participants before discharge from hospital (Richards 1993), to 7.7 years in studies assessing participants after hospital discharge (Teixeira 1999).

Two studies recruited non-ambulatory stroke survivors (Richards 1993; Wang 2014), three studies recruited both ambulatory and non-ambulatory participants (Bateman 2001; Cooke 2010; Lennon 2008), six studies did not report this information (Donaldson 2009; Lee 2013a; Verheyden 2009; Winstein 2004; Topcuoglu 2015; Zou 2015), and all the remaining studies recruited ambulatory stroke survivors.

\section{Sample size}

Of the 75 included studies: 
- 16 studies had 20 participants or fewer (Arabzadeh 2018; Bale 2008; Coroian 2018; Cuviello-Palmer 1988; da Cunha 2002; Donaldson 2009; Duncan 1998; Glasser 1986; James 2002; Kim 2001; Kim 2016a; Letombe 2010; Moore 2010; Richards 1993; Smith 2008; Teixeira 1999);

- four studies had over 100 participants (Ada 2013102 participants; Gordon 2013128 participants; Jin 2013128 participants; Van de Port 2012250 participants);

- one publication had over 100 participants (Knox 2018144 participants) but is counted as two studies in this review;

- 54 remaining studies recruited between 21 and 100 participants.

\section{Interventions}

\section{Cardiorespiratory training}

Thirty-two studies with a total of 1631 randomised participants (range 15 to 128 individuals) examined cardiorespiratory training (Ada 2013; Aidar 2018; Bateman 2001; Cuviello-Palmer 1988; da Cunha 2002; Eich 2004; Glasser 1986; Globas 2012; Gordon 2013; Ivey 2010; Ivey 2011; Jin 2013; Kang 2012; Katz-Leurer 2003; Kim 2014; Kuys 2011; Lennon 2008; MacKay-Lyons 2013; Mao 2015; Moore 2010; Mudge 2009; Park 2011; Pohl 2002; Potempa 1995; Salbach 2004; Sandberg 2016; Smith 2008; Takami 2010; Topcuoglu 2015; Vanroy 2017; Wang 2014; Yang 2014). Details of the nature and dose of the cardiorespiratory interventions are summarised in Table 1.

Two of these studies assessed circuit training (Mudge 2009; Salbach 2004).

One study assessed aquatic training (Aidar 2018).

Twelve studies used some form of ergometry: seven assessed cycle ergometry (Bateman 2001; Jin 2013; Katz-Leurer 2003; Lennon 2008; Potempa 1995; Sandberg 2016; Yang 2014), two assessed seated/recumbent cycle ergometry (Vanroy 2017; Wang 2014), two assessed a 'Kinetron' ergometer (Cuviello-Palmer 1988; Glasser 1986), and one assessed arm cranking ergometer (Topcuoglu 2015).

Seventeen studies focused on walking using treadmills (da Cunha 2002; Eich 2004; Globas 2012; Ivey 2010; Ivey 2011; Kang 2012; Kuys 2011; MacKay-Lyons 2013; Mao 2015; Moore 2010; Pohl 2002; Smith 2008; Takami 2010), overground walking (Gordon 2013; Kim 2014; Park 2011), or a combination of treadmill and overground walking (Ada 2013).

The training programmes comprised regular weekly sessions of sufficient duration (usually longer than 20 minutes), the exercise intensity was clearly described in 20 of the 32 included studies. In 16 studies the cardiorespiratory training started after usual care, while in 16 studies it started during usual care. In five of these studies participants were recruited in the acute phase of stroke, less than one month post-stroke (Cuviello-Palmer 1988; da Cunha 2002; MacKay-Lyons 2013; Sandberg 2016; Takami 2010).

Three of the included cardiorespiratory training studies had more than one intervention group that met the eligibility criteria; these compare two different durations, intensities, and modes of training. Each of these studies therefore has two entries when included in any meta-analyses, each sharing $50 \%$ of the number of participants in the single control group from each study.
- Ada 2013: Group 1 - duration four months' training; Group 2 duration two months' training

- Pohl 2002: Group 1 - intensity high due to rapid progression; Group 2 - intensity lower due to limited progression

- Takami 2010: Group 1 - mode: backward walking on treadmill; Group 2 - mode: forward walking on treadmill

\section{Resistance training}

Twenty studies with a total of 779 randomised participants (range 18 to 93 individuals) assessed the effects of resistance training (Aidar 2016; Arabzadeh 2018; Bale 2008; Buyukvural 2015; Coroian 2018; Fernandez-Gonzalo 2016; Flansbjer 2008; Inaba 1973; Ivey 2017; Kim 2001; Knox 2018; Lee 2013a; Lee 2013b; Ouellette 2004; Sims 2009; Son 2014; Taylor-Pilliae 2014; Verheyden 2009; Winstein 2004; Zou 2015). Details of the nature and dose of the resistance training intervention studies are summarised in Table 2.

All employed muscle contractions resisted by weights, exercise machines, or elastic devices. One study trained the upper limbs (Winstein 2004), one trained the trunk (Verheyden 2009), two studies trained both the upper and lower limbs (Aidar 2016; Sims 2009), one was unclear (Taylor-Pilliae 2014), and the remaining studies involved the lower limbs only. Most programmes were short (less than 12 weeks) apart from Aidar 2016, Fernandez-Gonzalo 2016, Ivey 2017, Knox 2018, Ouellette 2004, and Taylor-Pilliae 2014 (12 weeks). Twelve studies started resistance training after usual care (Aidar 2016; Fernandez-Gonzalo 2016; Flansbjer 2008; Ivey 2017; Kim 2001; Knox 2018; Lee 2013a; Lee 2013b; Ouellette 2004; Sims 2009; Son 2014; Taylor-Pilliae 2014; Zou 2015), whilst eight studies started it during usual care (Arabzadeh 2018; Bale 2008; Buyukvural 2015; Coroian 2018; Inaba 1973; Verheyden 2009; Winstein 2004). Only Winstein 2004 recruited participants during the acute phase of stroke (less than one month post-onset).

Three of the studies appear similar in terms of participants and interventions and have a shared authorship (Lee 2013a; Lee 2013b; Son 2014). Although the sample sizes are different there is a possibility that these three publications share some of the same participants. We have not had a response to queries to establish this.

\section{Mixed training}

Twenty-three studies with a total of 1207 randomised participants (range 13 to 250 individuals) assessed the effects of mixed training (Cooke 2010; Dean 2018; Donaldson 2009; Duncan 1998; Duncan 2003; Furnari 2014; Galvin 2011; James 2002; Kim 2016a; Kim 2017a; Knox 2018; Langhammer 2007; Letombe 2010; Mead 2007; Moore 2015; Richards 1993; Richards 2004; Shin 2011; Teixeira 1999; Toledano-Zarhi 2011; Van de Port 2012; Yang 2006; Zedlitz 2012). Details of the nature and dose of the mixed training interventions are summarised in Table 3.

The modes of exercise are quite diverse since these comprise circuit training or various combinations of walking, treadmill training, and resistance training. All interventions contained one or more functionally relevant activity (such as walking). Most programmes were short, with six studies meeting or exceeding 12 weeks (Duncan 1998; Knox 2018; Mead 2007; Moore 2015; Van de Port 2012; Zedlitz 2012). Nine studies occurred during usual care; five of these recruited participants in the acute phase of stroke, less than one month post-onset (Galvin 2011; Kim 2016a; Letombe 2010; Richards 
1993; Toledano-Zarhi 2011), and four at a later stage of care (Furnari 2014; Kim 2017a; Richards 2004; Shin 2011).

\section{Adherence to the intervention}

We defined adherence to the interventions in terms of: 1) attendance at the planned training sessions, and 2) compliance with the planned content of the training sessions.

\section{Attendance}

Rate of attendance (\%) could be clearly determined in 33 of the 75 included studies (Ada 2013; Aidar 2016; Bateman 2001; Duncan 1998; Duncan 2003; Eich 2004; Fernandez-Gonzalo 2016; Flansbjer 2008; Globas 2012; Ivey 2017; Kim 2016a; Kuys 2011; Langhammer 2007; MacKay-Lyons 2013; Mead 2007; Moore 2015; Mudge 2009; Park 2011; Ouellette 2004; Pohl 2002; Richards 1993; Richards 2004; Salbach 2004; Sandberg 2016; Sims 2009; Taylor-Pilliae 2014; Toledano-Zarhi 2011; Van de Port 2012; Wang 2014; Winstein 2004; Yang 2006; Zedlitz 2012; Zou 2015). The proportion of attended training sessions ranged from $65 \%$ up to $100 \%$. Seven studies measured attendance for the training and the control groups separately and showed similar rates between groups (Bateman 2001; Langhammer 2007; MacKay-Lyons 2013; Mead 2007; Ouellette 2004; Salbach 2004; Taylor-Pilliae 2014). A few other studies described attempts to facilitate attendance and make up missed sessions, or reported that "attendance did not differ between intervention groups" but did not provide attendance rates (Bale 2008; Cooke 2010; Teixeira 1999). One study specifically excluded those participants who attended fewer than nine training sessions from the statistical analyses (da Cunha 2002); this prevents an intention-to-treat assessment of results.

\section{Compliance}

Compliance with the scheduled exercise programme during training sessions was described in only a few studies.

For cardiorespiratory training interventions, Langhammer 2007 stated that the compliance with the individualised training levels was 'high'; other studies reported that participants 'tolerated' training (Globas 2012; MacKay-Lyons 2013; Pohl 2002; Kim 2016a), or showed no discomfort (Jin 2013). Salbach 2004 maintained that most of the participants completed nine out of 10 circuit training exercises. Mao 2015 recorded compliance data but this was not reported.

For mixed training, Duncan 1998 reported 'good compliance' with home-based training, and Yang 2006 stated that mixed circuit training was "performed as planned". Mead 2007 reported $94 \%$ to $99 \%$ compliance with circuit training exercises 'tailored' to individual requirements. Dean 2018 reported that $70 \%$ of the participants received an "appropriate dose" of training. Information on compliance was not available for the remaining studies. Zedlitz 2012 described the compliance of participants with training as 'good'. Two studies reported good compliance of therapists in delivery of the content of the planned protocol (MacKay-Lyons 2013; Zedlitz 2012).

\section{Comparisons}

The included studies compared training interventions with control interventions in different ways. We identified seven different types of comparison, which has implications for establishing the effects of fitness training.

\section{Balanced comparisons}

The nature of some of these comparisons allows intervention and control groups to be comparable in terms of exposure time (both groups are exposed to an intervention, the frequency and duration of which is similar between groups) and the 'attention' received by the therapists. Therefore, these comparisons allow one to separate the specific effects of fitness training from those of usual rehabilitation interventions.

- Training plus a proportion of usual care versus usual care

- Training plus usual care versus non-exercise intervention plus usual care

- Training versus non-exercise intervention - after usual care

- Training versus usual outpatient care

\section{Confounded comparisons}

Other comparisons make it impossible to have a comparable intervention and control group exposure time (e.g. the 'training versus no intervention' comparison). We described these comparisons in the review as 'confounded by additional training time'. With regard to interventions involving physical exercise, a greater exposure to the intervention has a known effect on rehabilitation outcomes ('augmented therapy time'; Kwakkel 2004). Therefore, although these comparisons allow comment on the overall effect of training programmes, they make it difficult to attribute any benefits to the content of the exercise prescription itself.

- Training plus usual care versus usual care

- Training plus non-exercise intervention versus non-exercise intervention - after usual care

- Training versus no intervention - after usual care

\section{Outcome measures}

The included studies recorded outcome measures at the end of the training period (end of intervention), or at any other defined point either within the study duration or after completion of the training programme, or both (scheduled end of follow-up).

Some outcome measures involved continuous data (e.g. assessment scales) with skewed distributions. Due to time and resource constraints we did not attempt to transform these data. We therefore combined continuous skewed data and continuous normal-distributed data.

\section{Excluded studies}

The most common reasons for exclusion were interventions that either did not meet the criteria for fitness training ('wrong intervention') or were confounded by other intervention components ('co-intervention') along with inappropriate control groups, such as those with an active physical intervention ('wrong control'). These are documented in the Characteristics of excluded studies table.

\section{Risk of bias in included studies}

Details and justifications for 'Risk of bias' assessments in individual studies are shown in the Characteristics of included studies table. For Knox 2018, the risk of bias scores are included twice as there are two entirely separate classes of intervention in this study giving a total of 75 studies. As this is a complex review we decided to apply 
the 'Risk of bias' assessments to 'all outcomes' for simplicity apart from incomplete outcome data, for which we assessed bias at both the end of the intervention and the end of follow-up. We present the summary results in Figure 2 and Figure 3. 
Figure 2. 'Risk of bias' summary: review authors' judgements about each 'Risk of bias' item for each included study. In studies with no follow-up measurement we did not assess risk of bias for the item labelled 'Incomplete outcome data (attrition bias): end of follow-up'; this results in some blank spaces

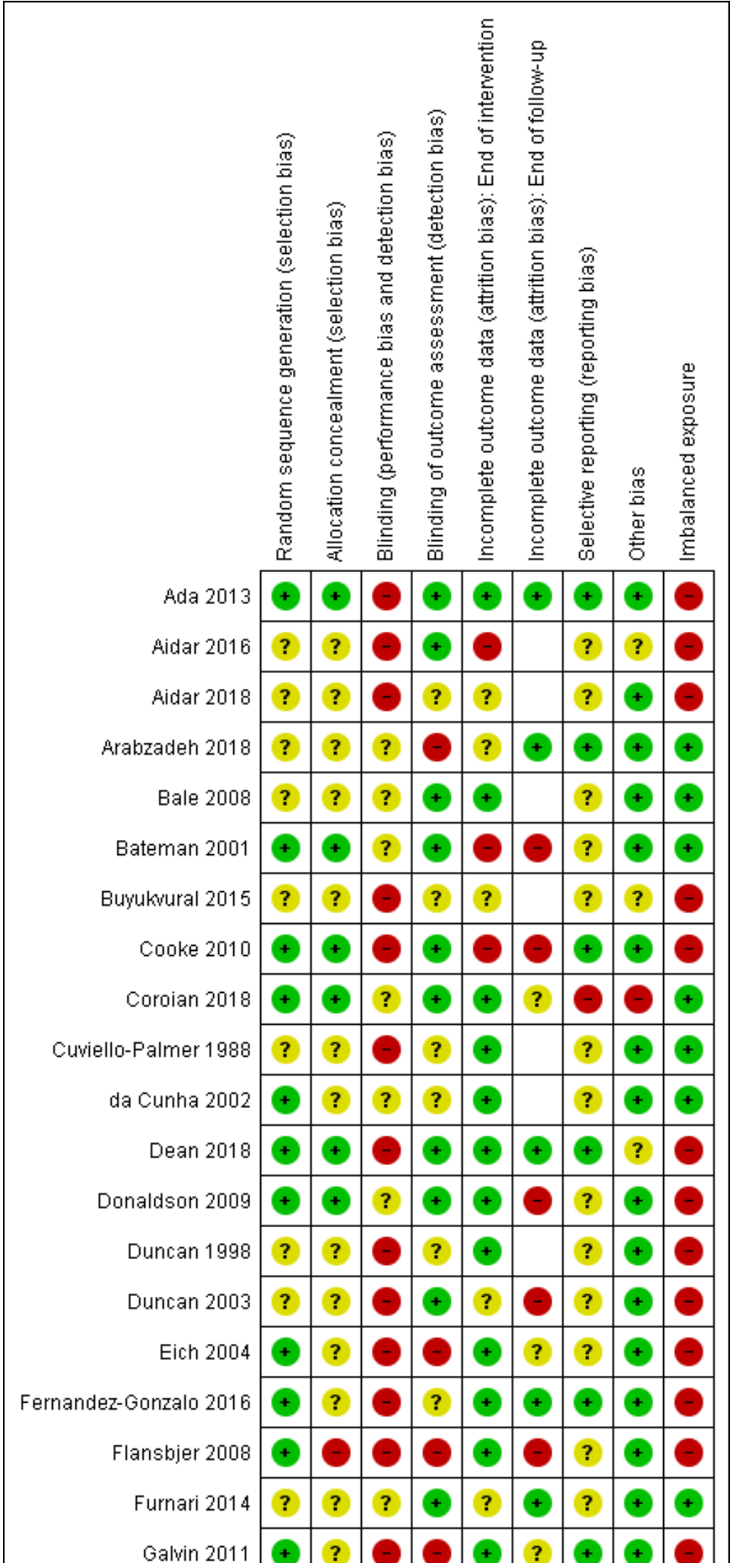


Figure 2. (Continued)

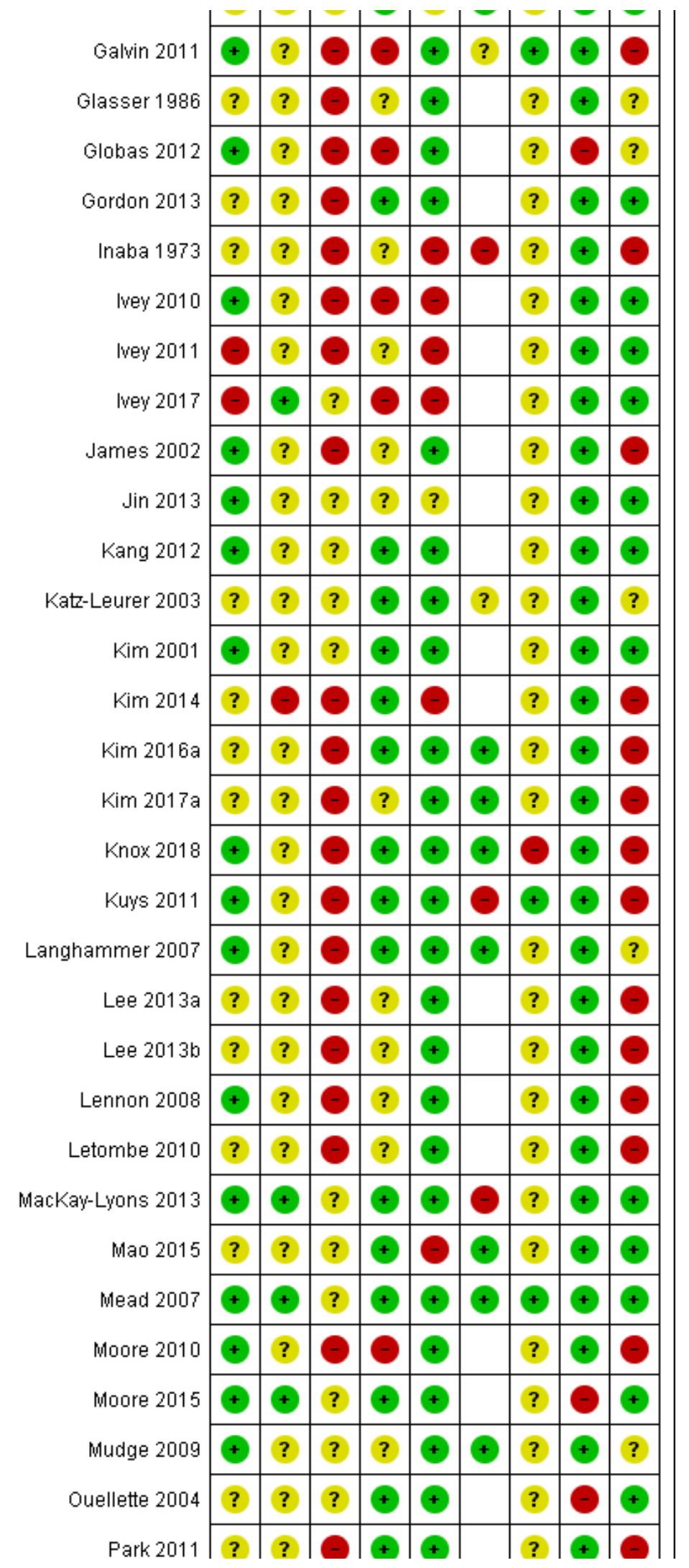


Figure 2. (Continued)

\begin{tabular}{|c|c|c|c|c|c|c|c|c|c|}
\hline Park 2011 & $?$ & $?$ & - & + & + & & $?$ & + & 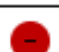 \\
\hline Pohl 2002 & $?$ & $?$ & $?$ & $?$ & + & & $?$ & + & $?$ \\
\hline Potempa 1995 & $?$ & $?$ & ? & ? & + & & $?$ & + & + \\
\hline Richards 1993 & $?$ & $?$ & ? & + & - & & $?$ & + & + \\
\hline Richards 2004 & $?$ & ? & $?$ & + & + & - & $?$ & + & + \\
\hline Salbach 2004 & + & $?$ & $?$ & - & + & & $?$ & + & + \\
\hline Sandberg 2016 & + & $?$ & - & $?$ & $?$ & $\odot$ & + & $\odot$ & - \\
\hline Shin 2011 & $?$ & ? & $?$ & ? & + & & $?$ & + & + \\
\hline Sims 2009 & + & + & - & $?$ & $\odot$ & $\odot$ & $?$ & $\odot$ & $\Theta$ \\
\hline Smith 2008 & $?$ & ? & - & - & + & + & $?$ & + & - \\
\hline Son 2014 & + & $?$ & ? & $?$ & $\odot$ & & $?$ & $\odot$ & + \\
\hline Takami 2010 & ? & ? & $?$ & ? & ? & & $?$ & + & + \\
\hline Taylor-Pilliae 2014 & $?$ & $?$ & - & + & + & + & $?$ & ? & $\odot$ \\
\hline Teixeira 1999 & $?$ & $?$ & $\odot$ & ? & $\odot$ & & $?$ & $\odot$ & $\Theta$ \\
\hline Toledano-Zarhi 2011 & $?$ & $?$ & - & ? & + & & ? & + & $\theta$ \\
\hline Topcuoglu 2015 & + & $?$ & - & $\odot$ & $\odot$ & & $?$ & + & $\Theta$ \\
\hline Van de Port 2012 & + & + & ? & $\odot$ & $\odot$ & + & $?$ & + & $\Theta$ \\
\hline Vanroy 2017 & + & + & - & + & + & & ? & + & + \\
\hline Verheyden 2009 & $?$ & $?$ & $\odot$ & + & + & & $?$ & $\odot$ & - \\
\hline Wang 2014 & $?$ & $?$ & $\odot$ & + & - & & $?$ & + & - \\
\hline Winstein 2004 & $?$ & $?$ & $\odot$ & $\Theta$ & + & $\odot$ & $?$ & + & $\odot$ \\
\hline Yang 2006 & $?$ & ? & - & + & $\odot$ & & $?$ & + & $\odot$ \\
\hline Yang 2014 & + & ? & - & + & + & & $?$ & + & - \\
\hline Zedlitz 2012 & ? & ? & - & + & $?$ & - & + & - & $\odot$ \\
\hline Zou 2015 & $?$ & - & $?$ & + & $\odot$ & & $?$ & $?$ & + \\
\hline
\end{tabular}


Figure 3. 'Risk of bias' graph: review authors' judgements about each 'Risk of bias' item presented as percentages across all included studies. In studies with no follow-up measurement, we did not assess risk of bias for the item labelled 'Incomplete outcome data (attrition bias): end of follow-up'; this results in some blank spaces

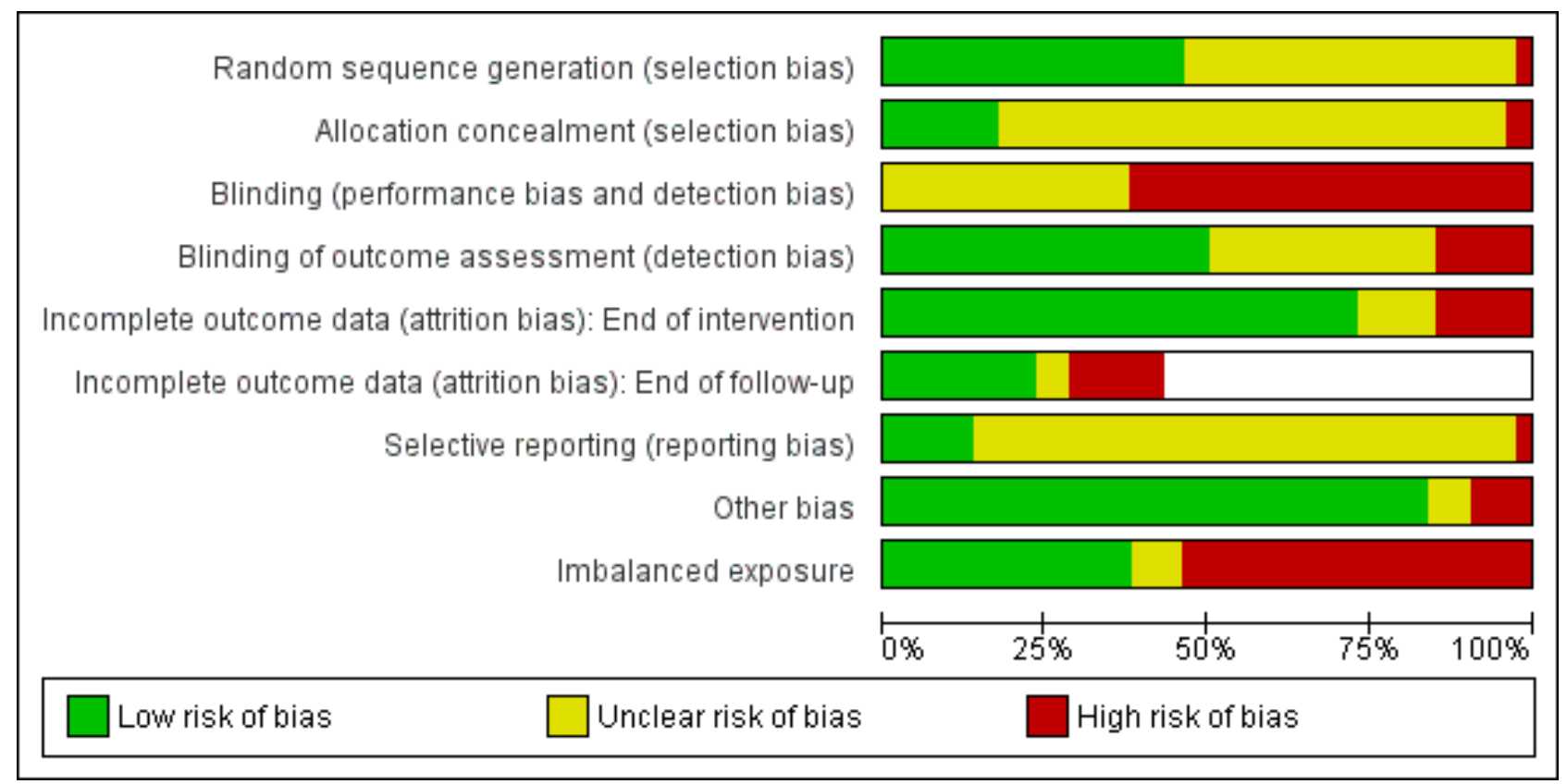

\section{Allocation}

\section{Randomisation}

We assessed 35 out of $75(47 \%)$ of the included studies as having a low risk of selection bias (Ada 2013; Bateman 2001; Cooke 2010; Coroian 2018; da Cunha 2002; Dean 2018; Donaldson 2009; Eich 2004; Fernandez-Gonzalo 2016; Flansbjer 2008; Galvin 2011; Globas 2012; Ivey 2010; James 2002; Jin 2013; Kang 2012; Kim 2001; Knox 2018 (both comparisons); Kuys 2011; Langhammer 2007; Lennon 2008; MacKay-Lyons 2013; Mead 2007; Moore 2010; Moore 2015; Mudge 2009; Salbach 2004; Sandberg 2016; Sims 2009; Son 2014; Topcuoglu 2015; Van de Port 2012; Vanroy 2017; Yang 2014. We assessed 2 out of $75(3 \%)$ of studies as being at high risk of bias (Ivey 2011; Ivey 2017) and the remaining 39 out of 75 (52\%) of studies were assessed as unclear risk of bias because there was not enough information to make a judgement. All studies identified that randomisation had occurred but many did not describe the actual mechanism of how they had achieved this. Therefore, uncertainties remain among a number of studies. Most studies of fitness training are small; therefore, the use of techniques to balance participant numbers (e.g. block randomisation) and participant characteristics (e.g. stratification or minimisation based on age, gender, or outcomes of interest recorded at baseline) is quite common.

\section{Allocation concealment}

We assessed 13 out of 75 (17\%) of the included studies at low risk of bias (Ada 2013; Bateman 2001; Cooke 2010; Coroian 2018; Dean 2018; Donaldson 2009; Ivey 2017; MacKay-Lyons 2013; Mead 2007; Moore 2015; Sims 2009; Van de Port 2012; Vanroy 2017). We assessed 2 out of $75(3 \%)$ of studies being at high risk of bias (Flansbjer 2008; Kim 2014; Zou 2015) and the remaining 60 out of 75 studies $(80 \%)$ of studies were assessed as unclear risk of bias because there was not enough information to make a judgement.
Mechanisms of allocation concealment were poorly reported. There are instances when centralised assignment mechanisms are used where allocation concealment is automatic (e.g. Mead 2007), in which case we rated the risk of bias as low. Other studies, where allocation concealment mechanisms were needed, frequently used envelopes. Numbered, sealed, opaque envelopes (e.g. Cooke 2010; Donaldson 2009), are appropriate. Many studies reporting the use of 'sealed envelopes' did not specify whether they were sequentially numbered or opaque, therefore we were unable to exclude potential selection bias with certainty.

Taking these together, only 12 of 75 studies (16\%) were at low risk of bias issues relating to allocation.

\section{Blinding}

\section{Participant blinding}

We assessed none of the 75 included studies as being at low risk of performance bias. We assessed 47 out of $75(63 \%)$ as being at high risk of bias (Ada 2013; Aidar 2016; Aidar 2018; Buyukvural 2015; Cooke 2010; Cuviello-Palmer 1988; Dean 2018; Duncan 2003; Eich 2004; Fernandez-Gonzalo 2016; Flansbjer 2008; Galvin 2011; Glasser 1986; Globas 2012; Gordon 2013; Inaba 1973; Ivey 2010; Ivey 2011; James 2002; Kim 2014; Kim 2016a; Kim 2017a; Knox 2018 (both comparisons); Kuys 2011; Langhammer 2007; Lee 2013a; Lee 2013b; Lennon 2008; Letombe 2010; Moore 2010; Park 2011; Sandberg 2016; Sims 2009; Smith 2008; Taylor-Pilliae 2014; Teixeira 1999; Toledano-Zarhi 2011; Topcuoglu 2015; Vanroy 2017; Verheyden 2009; Wang 2014; Winstein 2004; Yang 2006; Yang 2014; Zedlitz 2012) and the remaining 28 out of 75 studies (37\%) of studies were assessed as unclear risk of bias because there was not enough information to make a judgement.

Participants cannot be blinded to physical interventions such as fitness training; therefore, we judged no studies to be at low risk of 
bias. However, some studies utilised an attention control or other means to disguise the 'true nature' of the comparison.

\section{Investigator blinding}

We assessed the outcome assessment to be at low risk of detection bias in 38 out of 75 (50\%) of the included studies (Ada 2013; Aidar 2016; Bale 2008; Bateman 2001; Cooke 2010; Coroian 2018; Dean 2018; Donaldson 2009; Duncan 2003; Furnari 2014; Gordon 2013; Kang 2012; Katz-Leurer 2003; Kim 2001; Kim 2014; Kim 2016a; Knox 2018 (both comparisons); Kuys 2011; Langhammer 2007; MacKayLyons 2013; Mao 2015; Mead 2007; Moore 2015; Ouellette 2004; Park 2011; Richards 1993; Richards 2004; Taylor-Pilliae 2014; Topcuoglu 2015; Van de Port 2012; Vanroy 2017; Verheyden 2009; Wang 2014; Yang 2006; Yang 2014; Zedlitz 2012; Zou 2015). We assessed 11 out of $75(15 \%)$ as being at high risk of detection bias because outcome assessment was not blinded (Arabzadeh 2018; Eich 2004; Flansbjer 2008; Galvin 2011; Globas 2012; Ivey 2010; Ivey 2017; Moore 2010; Salbach 2004; Smith 2008; Winstein 2004) and the remaining 26 out of 75 (35\%) were assessed as unclear risk of bias because there was not enough information to make a judgement. Among studies that used blinded outcome assessment some instructed participants not to reveal group assignments (Bateman 2001; Duncan 2003; Flansbjer 2008; Mead 2007). However, some degree of unmasking can easily occur and was documented in some studies (e.g. Eich 2004; Mudge 2009; Salbach 2004).

\section{Incomplete outcome data}

We assessed risk of attrition bias at the end of intervention (75 studies) and at the end of follow-up where data existed (33 studies only).

We assessed 55 out of $75(73 \%)$ included studies as being at low risk of attrition bias at the end of intervention (Ada 2013; Bale 2008; Coroian 2018; Cuviello-Palmer 1988; da Cunha 2002; Dean 2018; Donaldson 2009; Duncan 1998; Eich 2004; Fernandez-Gonzalo 2016; Flansbjer 2008; Galvin 2011; Glasser 1986; Globas 2012; Gordon 2013; James 2002; Kang 2012; Katz-Leurer 2003; Kim 2001; Kim 2016a; Kim 2017a; Knox 2018 (both comparisons); Kuys 2011; Langhammer 2007; Lee 2013a; Lee 2013b; Lennon 2008; Letombe 2010; MacKay-Lyons 2013; Mead 2007; Moore 2010; Moore 2015; Mudge 2009; Ouellette 2004; Park 2011; Pohl 2002; Potempa 1995; Richards 2004; Salbach 2004; Shin 2011; Sims 2009; Smith 2008; Son 2014; Taylor-Pilliae 2014; Teixeira 1999; Toledano-Zarhi 2011; Topcuoglu 2015; Van de Port 2012; Vanroy 2017; Verheyden 2009; Winstein 2004; Yang 2006; Yang 2014; Zou 2015). We assessed 11 out of $75(15 \%)$ studies as being at high risk of attrition bias at the end of intervention (Aidar 2016; Bateman 2001; Cooke 2010; Inaba 1973; Ivey 2010; Ivey 2011; Ivey 2017; Kim 2014; Mao 2015; Richards 1993; Wang 2014). The remaining 9 out of 75 studies (12\%) were assessed as unclear risk of bias because there was not enough information to make a judgement based on the nature and extent of dropouts or on whether an intention-to-treat approach was used or not.

We assessed 18 out of 33 (55\%) included studies as being at low risk of attrition bias at the end of follow-up (Ada 2013; Arabzadeh 2018; Dean 2018; Fernandez-Gonzalo 2016; Furnari 2014; Kim 2016a; Kim 2017a; Knox 2018 (both comparisons); Langhammer 2007; Mao 2015; Mead 2007; Mudge 2009; Sandberg 2016; Sims 2009; Smith 2008; Taylor-Pilliae 2014; Van de Port 2012). We assessed 11 out of 33 (33\%) as being at high risk of bias (Bateman 2001; Cooke 2010; Donaldson 2009; Duncan 2003; Flansbjer 2008; Inaba 1973; Kuys 2011; MacKay-Lyons 2013; Richards 2004; Winstein 2004; Zedlitz
2012) and the remaining four out of 33 studies (12\%) of studies were assessed as unclear risk of bias because there was not enough information to make a judgement based on the nature and extent of dropouts or on whether an intention-to-treat.

One of the included studies did not analyse data for the participants who dropped out but we were able to impute sometimes large numbers of missing values in individual participant data obtained from Bateman 2001. This did not influence any of the findings; therefore, we included only the imputed data in this review for simplicity.

The bias assessment could not be applied to the 42 out of $75(56 \%)$ studies with no end of follow-up measurement. Therefore, there are 42 blank spaces in Figure 2 since a 'Risk of bias' judgement was not possible.

\section{Selective reporting}

We assessed nine out of $75(12 \%)$ studies as being at low risk of reporting bias (Ada 2013; Arabzadeh 2018; Cooke 2010; FernandezGonzalo 2016; Galvin 2011; Kuys 2011; Mead 2007; Sandberg 2016; Zedlitz 2012). We assessed three out of 75 (4\%) of studies as being at high risk of reporting bias (Coroian 2018; Knox 2018 both comparisons). The remaining 63 out of 75 studies (84\%) of studies were assessed as unclear risk of bias because there was not enough information to make a judgement. This is because the majority of studies did not have readily available protocols. In most cases, where these were available, there was no evidence of selective reporting of outcomes relevant to this review.

\section{Other potential sources of bias}

We assessed 63 out of 75 (84\%) studies as being at low risk of other biases (Ada 2013; Aidar 2018; Arabzadeh 2018; Bale 2008; Bateman 2001; Cooke 2010; Cuviello-Palmer 1988; da Cunha 2002; Donaldson 2009; Duncan 1998; Duncan 2003; Eich 2004; FernandezGonzalo 2016; Flansbjer 2008; Furnari 2014; Galvin 2011; Glasser 1986; Gordon 2013; Inaba 1973; Ivey 2010; Ivey 2011; Ivey 2017; James 2002; Jin 2013; Kang 2012; Katz-Leurer 2003; Kim 2001; Kim 2014; Kim 2016a; Kim 2017a; Knox 2018 (both comparisons); Kuys 2011; Langhammer 2007; Lee 2013a; Lee 2013b; Lennon 2008; Letombe 2010; MacKay-Lyons 2013; Mao 2015; Mead 2007; Moore 2010; Mudge 2009; Park 2011; Pohl 2002; Potempa 1995; Richards 1993; Richards 2004; Salbach 2004; Sandberg 2016; Shin 2011; Smith 2008; Son 2014; Takami 2010; Toledano-Zarhi 2011; Topcuoglu 2015; Van de Port 2012; Vanroy 2017; Verheyden 2009; Wang 2014; Winstein 2004; Yang 2006; Yang 2014). We assessed 7 out of $75(9 \%)$ of studies as being at high risk of other biases. These included those that recruited via media advertisements (Globas 2012; Ouellette 2004; Teixeira 1999; Zedlitz 2012), involved different travel demands between intervention and control exposures (Moore 2015), had baseline differences in outcomes of interest (Sims 2009), and reluctance to recruit some participants (Coroian 2018). The remaining 5 out of 75 studies $(7 \%)$ of studies were assessed as unclear risk of bias because there was not enough information to make a judgement.

\section{Confounded by additional training time (imbalanced exposure)}

We judged studies in which the participants received an unequal amount of exposure to the intervention and comparison arms of the study to be at high risk of bias. Technically this could be 
described as a source of confounding rather than bias but it is appropriate to record it here

.We assessed 28 out of 75 studies (37\%) as being at low risk of confounding as they had balanced exposures in control and intervention groups (Arabzadeh 2018; Bale 2008; Bateman 2001; Coroian 2018; Cuviello-Palmer 1988; da Cunha 2002; Furnari 2014; Gordon 2013; Ivey 2010; Ivey 2011; Ivey 2017; Jin 2013; Kang 2012; Kim 2001; MacKay-Lyons 2013; Mao 2015; Mead 2007; Moore 2015; Ouellette 2004; Potempa 1995; Richards 1993; Richards 2004; Salbach 2004; Shin 2011; Son 2014; Takami 2010; Vanroy 2017; Zou 2015). We assessed 41 out of 75 studies (55\%) as being at high risk of confounding as they had imbalanced exposure across the control and intervention groups (Ada 2013; Aidar 2016; Aidar 2018; Buyukvural 2015; Cooke 2010; Dean 2018; Donaldson 2009; Duncan 1998; Duncan 2003; Eich 2004; Fernandez-Gonzalo 2016; Flansbjer 2008; Galvin 2011; Inaba 1973; James 2002; Kim 2014; Kim 2016a; Kim 2017a; Knox 2018 (both comparisons); Kuys 2011; Lee 2013a; Lee 2013b; Lennon 2008; Letombe 2010; Moore 2010; Park 2011; Sandberg 2016; Sims 2009; Smith 2008; Taylor-Pilliae 2014; Teixeira 1999; Toledano-Zarhi 2011; Topcuoglu 2015; Van de Port 2012; Verheyden 2009; Wang 2014; Winstein 2004; Yang 2006; Yang 2014; Zedlitz 2012). The remaining six out of 75 (8\%) were assessed as unclear risk of confounding because there was not enough information to make a judgement. In summary, the design of more than half of the studies in this review meant that in 41 out of 75 studies (55\%) the effects of fitness training could be exaggerated because the training intervention groups received greater time of exposure irrespective of the content of the training programme.

\section{Effects of interventions}

See: Summary of findings for the main comparison Cardiorespiratory training compared to control for people with stroke: end of intervention; Summary of findings 2 Resistance training compared to control for people with stroke: end of intervention; Summary of findings $\mathbf{3}$ Mixed training compared to control for people with stroke: end of intervention

\section{Effect of training on primary outcome measures \\ Death}

Overall there were few deaths; there were 19 deaths in 3017 participants $(0.53 \%)$ before end of intervention. At the end of follow-up there were 19 deaths out of 1469 participants (1.29\%), 10 of these 19 occurred between end of intervention and the end of follow-up.

\section{Cardiorespiratory training (comparisons 1 and 2)}

\section{End of intervention}

Out of the 32 studies of cardiorespiratory training (1631 participants) only Gordon 2013 reported death ( $n=2$ in each study arm) as a reason for participant losses. There was no statistically significant overall effect $\left(\mathrm{RD} 0.00,95 \% \mathrm{Cl}-0.01\right.$ to $0.01 ; 1^{2}=$ $0 \%$; Analysis 1.1). There is low certainty in this estimate due to indirectness, imbalanced exposure (13/32 studies), and the fact that $6 / 32$ studies in this analysis did report dropouts but could either not contact participants (Kuys 2011: $n=1$ ), or did not fully describe all reasons for dropouts (Aidar 2016; Bateman 2001; Ivey 2011; Jin 2013; Sandberg 2016).

\section{End of follow-up}

One out of six studies (Katz-Leurer 2003), reported that one participant died in the training group (1/46) compared with one participant in the control group (1/46) with no statistically significant effect (RD $0.00,95 \% \mathrm{Cl}-0.03$ to $0.03 ; 1^{2}=0 \% ; 360$ participants; Analysis 2.1).

\section{Resistance training (comparisons 3 and 4) \\ End of intervention}

One of the 20 studies (Knox 2018), reported two deaths in the intervention group (1/45) and the control group (1/48). Overall, there is no statistically significant effect (RD $0.00,95 \% \mathrm{Cl}$ -0.02 to $0.02 ; 1^{2}=0 \% ; 803$ participants; Analysis 3.1 ); however, there is low certainty in this estimate due to indirectness, imbalanced exposures in 12 out of 20 studies, and because four studies had undocumented attrition (Aidar 2016; Arabzadeh 2018; Buyukvural 2015; Inaba 1973), including one with a large number of undocumented dropouts (Inaba 1973).

\section{End of follow-up}

One out of five studies (Knox 2018), reported four deaths in the intervention group (2/45) and the control group (2/48) with no statistically significant effect (RD $0.00,95 \% \mathrm{Cl}-0.04$ to $0.04 ; 12=0 \%$; 251 participants; Analysis 4.1). One study had a large number of undocumented dropouts (Inaba 1973).

\section{Mixed training (comparisons 5 and 6 )}

\section{End of intervention}

Three of the 23 studies (1231 participants) reported 13 deaths between the baseline and the end of intervention assessments: Knox 2018 (3/51 training, 1/48 control); Langhammer 2007 (1/32 training, 6/35 control); and Van de Port 2012 (0/126 training, 2/124 control). Overall, there was no statistically significant effect (RD $-0.00,95 \% \mathrm{Cl}-0.02$ to $0.01 ; 1^{2}=0 \%$; Analysis 5.1 ). There is low certainty in this estimate due to indirectness and imbalanced exposures affecting 16 out of 23 studies. Also, in Langhammer 2007, three of the six deaths in the control group and the one death in the training group occurred before discharge and before the intervention began; after excluding these data, the effect of training was still not statistically significant. The other 20 studies reported no deaths; however, two described undocumented losses: Richards 1993 (2 control); and Richards 2004 (5 training, 7 control) mentioning only that some participants were not available.

\section{End of follow-up}

Five of the 13 studies reported a total of 14 deaths (Cooke 2010; Duncan 2003; Galvin 2011; Knox 2018; Van de Port 2012), with no statistically significant effect (RD $-0.01,95 \% \mathrm{Cl}-0.03$ to $0.01 ; \mathrm{I}^{2}=$ $0 \%$; 906 participants; Analysis 6.1). The other eight mixed training studies reported that no losses to follow-up were attributable to death apart from Richards 1993 (2 control), Richards 2004 (5 training, 7 control), and Zedlitz 2012 (4 control), which describe only that some participants were lost or not available for follow-up.

\section{Death or dependence}

None of the studies reported the composite outcome of death or dependence. 


\section{Disability}

\section{Cardiorespiratory training (comparisons 1 and 2)}

\section{End of intervention}

Three studies assessed Functional Independence Measure (FIM) score, one during usual care (Bateman 2001), and two after usual care (Cuviello-Palmer 1988; Katz-Leurer 2003). Overall, there was no statistically significant effect of training (SMD 0.21, 95\% Cl -0.10 to $0.52 ; \mathrm{P}=0.18$; Analysis 1.2). However, the Bateman 2001 data were problematic because the procedures for obtaining FIM data at the end of intervention were not uniform and there was a high proportion of missing FIM data at the end of intervention (38\%); exclusion of this study does not change the result (SMD 0.17, 95\% $\mathrm{Cl}-0.29$ to $0.63 ; \mathrm{P}=0.46)$.

Three studies assessed Barthel Index scores, two during usual care (Bateman 2001; Wang 2014), and one after usual care (Gordon 2013), and there was no overall effect with (MD 6.65, 95\% Cl -0.67 to 13.98; Analysis 1.3) or without the problematic data from Bateman 2001. The high heterogeneity within this analysis could stem from the data from Wang 2014 whose participants were non-ambulatory.

Two studies assessed Rivermead Mobility Index (RMI) scores during usual care (Bateman 2001; Takami 2010), and one study after usual care (Globas 2012). There was a small overall improvement in scores (MD 1.56, 95\% $\mathrm{Cl} 0.20$ to 2.92; $\mathrm{P}=0.02$; Analysis 1.4). When we excluded the data from Bateman 2001 (risk of bias) the effect was strengthened ( $M D 2.18,95 \% \mathrm{Cl} 0.99$ to $3.37 ; \mathrm{P}=0.0003$ ).

- One study reported Physical Activity and Disability Scale scores (Mudge 2009).

- One study reported Older Americans Resources and Services Questionnaire (Gordon 2013).

- One study reported that FIM scores were better than the control but no data were reported (Topcuoglu 2015).

We combined all the available disability scale data from these individual outcomes (using FIM data from Bateman 2001), and can be moderately certain of an overall effect in favour of cardiorespiratory training (SMD $0.52,95 \% \mathrm{Cl} 0.19$ to $0.84 ; \mathrm{P}=$ 0.002 ; 462 participants; moderate-certainty evidence; Analysis 1.5). Exclusion of the Bateman 2001 data made a trivial difference. One of the included studies was confounded for exposure time and had multiple bias concerns (Wang 2014); if excluded, the heterogeneity disappeared and the overall beneficial effect remained (SMD 0.35 0.15 to $0.55 ; P=0.0007$ ). This study, of non-ambulatory stroke survivors, had the largest individual effect size.

\section{End of follow-up}

Studies reported a range of different global scales at followup including RMI scores (Bateman 2001), Nottingham Extended ADL (Bateman 2001), Physical Activity and Disability Scale scores (Mudge 2009), and the Frenchay Activities Index (FAl; Katz-Leurer 2003). When we combined all the disability scale data from these individual outcomes (Nottingham Extended ADL data from Bateman 2001), there was no statistically significant effect of cardiorespiratory training at the end of follow-up (SMD 0.20, 95\% $\mathrm{Cl}-0.07$ to $0.46 ; \mathrm{P}=0.14$; Analysis 2.2 . When the analysis was repeated using RMI data from Bateman 2001 instead of Nottingham Extended ADL data there was still no statistically significant effect. There was a considerable proportion of interpolated missing data
(21\%) and therefore the data from Bateman 2001 should be treated with caution; their exclusion does not change the findings.

Resistance training (comparisons 3 and 4)

End of intervention

There were no resistance training data suitable for pooling.

- One study reported the various subscales Late Life Function and Disability Instrument (Ouellette 2004). Those who received resistance training felt less self-perceived limitation; however, there was no detectable effect on overall disability or function components of this tool.

- One study reported an effect favouring improved Rivermead Mobility Index score (Buyukvural 2015).

- Three studies reported subscales or specific dimensions of existing functional scales and were not considered (Inaba 1973; Winstein 2004; Buyukvural 2015).

\section{End of follow-up}

There were no resistance training data suitable for pooling.

\section{Mixed training (comparisons 5 and 6 )}

\section{End of intervention}

Nine studies assessed the effects of mixed training at the end of the treatment phase or at follow-up using a variety of scales that measured disability outcomes: Lawton Instrumental Activities of Daily Living (IADL) scores reported by Duncan 1998 and Duncan 2003 at the end of intervention showed no statistically significant effect (MD 0.83, 95\% $\mathrm{Cl}-0.51$ to $2.17 ; \mathrm{P}=0.22$; Analysis 5.3).

Six studies assessed the Barthel Index during usual care (Galvin 2011; Kim 2016a; Letombe 2010), and after usual care (Duncan 1998; Duncan 2003; Langhammer 2007), at the end of intervention and showed no statistically significant effect (MD $2.84,95 \% \mathrm{Cl}-0.48$ to $6.17 ; \mathrm{P}=0.09$; Analysis 5.2). Barthel Index scores reached ceiling level in five out of 20 participants at baseline and 10 out of 20 participants at end of intervention (Duncan 1998); excluding this study reduces heterogeneity ( 12 statistic from $21 \%$ to $10 \%$ ) and gives a statistically significant beneficial effect (MD 4.02, 95\% $\mathrm{Cl} 0.16$ to 7.88; $P=0.04)$.

RMI was assessed by three studies after usual care (Dean 2018; Mead 2007; Van de Port 2012). The direction of benefit favoured training but the effect was not significant (MD $0.41,95 \% \mathrm{Cl}-0.02$, 0.84; Analysis 5.4).

- One study reported Nottingham Extended Activities of Daily Living (EADL; Mead 2007). In addition, Van de Port 2012 separately reported four subscales of the Nottingham EADL scale; only one was significantly affected in favour of the usual care rather than mixed training; all other subscales showed no statistically significant effect.

- One study reported FIM data (Mead 2007), and showed no statistically significant effect at the end of intervention.

- One study reported the Stroke Impact Scale (Duncan 2003), showing a marginal benefit. In addition, Van de Port 2012 separately reported 11 subscales of the Stroke Impact Scale. One subscale was significantly affected in favour of the usual care rather than mixed training; all other subscales were unaffected. 
- One study reported the Katz ADL scale (Letombe 2010), and showed no statistically significant effect at the end of intervention.

- One study reported the Modified Patient-Specific Functional Scale (Dean 2018), with no statistically significant effect shown at end of intervention.

We combined all available studies with disability scale data (nine studies, 604 participants) from the end of intervention, including the Barthel Index (Duncan 1998; Duncan 2003; Galvin 2011; Kim 2016a; Langhammer 2007; Letombe 2010), FIM (Mead 2007), and RMI (Dean 2018; Van de Port 2012). There was very low certainty in the small significant effect of mixed training at the end of the intervention (SMD $0.23,95 \% \mathrm{Cl} 0.03$ to $0.42 ; \mathrm{P}=0.02$; Analysis 5.5). There were several potential combinations of data that could be included in this analysis as individual studies reported more than one disability scale; we presented Barthel Index, FIM and RMI data. We observed moderate inconsistency among studies' heterogeneity: $\left(I^{2}=21 \%\right)$, and this may relate to the different specific domains each tool addresses. Seven of the nine studies included in these analyses were confounded by increased training time whereby the amount of contact with therapists in the experimental groups was greater than in the control groups (Dean 2018; Duncan 1998; Duncan 2003; Galvin 2011; Kim 2016a; Letombe 2010; Van de Port 2012). The remaining two studies without this source of confounding were among the smallest individual effects (Langhammer 2007; Mead 2007).

\section{End of follow-up}

Two studies reported the Barthel Index (Galvin 2011; Langhammer 2007); there was no statistically significant effect at the end of follow-up (MD 1.82, 95\% Cl-13.69 to 17.33; $\mathrm{P}=0.82$; Analysis 6.2).

Two studies reported Nottingham EADL (Galvin 2011; Mead 2007); there was no statistically significant effect at the end of follow-up ( $M D$ 3.10, $95 \% \mathrm{Cl}-5.20$ to $11.40 ; \mathrm{P}=0.46$; Analysis 6.3 ).

Three studies reported RMI (Dean 2018; Mead 2007; Van de Port 2012); there was a statistically significant benefit at the end of three to four months of follow-up (MD $0.35,95 \% \mathrm{Cl} 0.02$ to $0.69 ; \mathrm{P}=0.04$; Analysis 6.4). However, two of the three studies were confounded for increased training time (Dean 2018; Van de Port 2012).

When we combined all studies with disability scale data from the end of follow-up, including Barthel Index (Galvin 2011; Langhammer 2007), Modified Patient-Specific Functional Scale (Dean 2018), FIM (Mead 2007), and RMI (Van de Port 2012), there was no statistically significant effect $(\mathrm{SMD} 0.10,95 \% \mathrm{Cl}-0.17$ to $0.37 ; \mathrm{P}=$ 0.45 ; Analysis 6.5). It is worth noting that three studies included in these analyses were confounded by increased training time (Dean 2018; Galvin 2011; Van de Port 2012). There were several potential combinations of data that could be included in this analysis as individual studies reported more than one disability scale; we presented Barthel Index, FIM and RMI data

\section{Comparison of cardiorespiratory training, resistance training, and mixed training (comparison 7)}

We performed a subgroup analysis to directly compare the effects of the different types of training (cardiorespiratory training versus resistance training versus mixed training) on pooled disability outcomes at the end of the intervention (Analysis 7.1). There was only one includable study for resistance training (Ouellette 2004), but there were enough data to compare cardiorespiratory training and mixed training. Both of these show beneficial effects although there is some overall heterogeneity $(12=22 \%)$. In summary, cardiorespiratory training, with or without resistance training included, improves scores of global disability.

\section{Effect of training on secondary outcomes}

\section{Adverse events}

Adverse events were not typically sought (a priori) as an outcome measure but were instead reported in a more ad hoc fashion. However, a number of studies specifically reported no serious adverse events (Coroian 2018; Ivey 2017; Kim 2016a; Kim 2017a; Moore 2015; Sandberg 2016). One study reported serious adverse events $(n=6)$ that were mostly considered unconnected to the intervention (Dean 2018). One study reported excessive fatigue among participants after strength training (Coroian 2018).

The following studies reported falls.

- Mead 2007 reported 11 falls in eight of the 32 participants allocated to mixed training and five falls in four of the 34 participants in the control group ( $P=0.21$, non-significant). None of these falls occurred within training sessions.

- Van de Port 2012 reported 29 falls in participants allocated to mixed training and 26 falls in those allocated to usual care $(P=$ 0.93 , non-significant); one fall occurred during exercise training.

- Dean 2018 reported 10 falls during the intervention; one fall occurred at the training venue.

- Taylor-Pilliae 2014 reported 29 falls; 14 in the intervention group and 15 in the control group.

- Vanroy 2017 reported one fall in the intervention and one in the control group.

With regard to secondary cerebrovascular events; 11 participants (seven participants receiving the training intervention and four control participants) were reported to have had a cerebrovascular event between baseline and the end of the training intervention. In the studies that included a follow-up assessment, 11 participants (five participants receiving the training intervention and six control participants) were reported to have had a cerebrovascular event between the end of intervention and the end of follow-up.

With regard to cerebrovascular events, three participants (one participant receiving the training intervention and two control participants) were reported to have suffered a cardiovascular event between baseline and the end of the training intervention.

\section{Cardiometabolic risk factors}

Few studies within each type of training reported cardiometabolic risk factor data so meta-analysis was limited. Cardiorespiratory fitness, discussed in the next section, is also a risk factor predictive of stroke.

\section{Cardiorespiratory training (comparisons 1 and 2)}

Five studies of cardiorespiratory training, with a total of 318 participants, showed no statistically significant training effects on systolic ( $M D-0.20,95 \% \mathrm{Cl}-6.00$ to $5.60 ; \mathrm{P}=0.95$; Analysis 1.6 ), or diastolic blood pressure (MD $-0.15,95 \% \mathrm{Cl}-2.28$ to $1.98 ; \mathrm{P}=$ 0.89; Analysis 1.7) at the end of intervention (da Cunha 2002; Jin 2013; Katz-Leurer 2003; Lennon 2008; Potempa 1995). One study 
(da Cunha 2002), had an unusually high systolic blood pressure in the intervention group; this introduced heterogeneity but had little effect on the pooled effect size. One study stated that there was an effect of cardiorespiratory training on blood pressure but did not provide data (Ivey 2011).

Two studies (174 participants) reported body mass index (BMI) data at the end of cardiorespiratory training interventions with no evidence of effect (MD 1.19, $95 \% \mathrm{Cl}-0.38$ to 2.76; Analysis 1.8).

One study (Lennon 2008) reported waist girth measures but these were not affected by cardiorespiratory training.

One study of cardiorespiratory fitness training reported that glucose control (two-hour glucose), fasting insulin, insulin sensitivity (HOMA: Homeostatic Model Assessment), and total triglycerides improved after training (Wang 2014). There was no statistically significant effect on cholesterol levels (high-density lipoprotein (HDL), low-density lipoprotein (LDL)) or fasting glucose levels.

\section{Resistance training (comparisons 3 and 4)}

One study of resistance training (Zou 2015), reported post-training improvements in glucose control (two-hour glucose), fasting insulin, insulin sensitivity (HOMA), and in cholesterol levels (total, HDL, LDL). There was no statistically significant effect on fasting glucose or total triglycerides.

One resistance training study measured $\mathrm{BMI}$; there was no statistically significant effect (Zou 2015).

No resistance training studies reported blood pressure outcomes.

\section{Mixed training (comparisons 5 and 6 )}

Two studies of mixed training examined blood pressure (Moore 2015; Toledano-Zarhi 2011), and meta-analysis showed no effects on systolic (Analysis 5.6), or diastolic blood pressure (Analysis 5.7).

One study reported no effect of mixed training on body composition outcomes (body mass index and fat mass) (Moore 2015).

One study of mixed training reported post-training improvements in HDL cholesterol (representing a risk reduction) (Moore 2015). There was no statistically significant effect on glucose control (two-hour glucose), insulin sensitivity (HOMA), and total and LDL cholesterol levels.

\section{Physical fitness}

Cardiorespiratory training (comparisons 1 and 2)

\section{Cardiorespiratory fitness}

Nine studies (317 participants) assessed cardiorespiratory fitness using directly measured peak $\mathrm{VO}_{2}(\mathrm{~mL} / \mathrm{kg} /$ minute) at the end of the intervention. Most of the studies took place after usual care and there was a consistent pattern of improvement in peak $\mathrm{VO}_{2}$ measures. We can be moderately certain that cardiorespiratory fitness increased significantly in the training groups (MD $3.40 \mathrm{~mL}$ / $\mathrm{kg} /$ minute, $95 \% \mathrm{Cl} 2.98$ to $3.83 ; \mathrm{I}^{2}=0 \%$; $\mathrm{P}=0.00001$; Analysis 1.9 ). Doses of training varied between four weeks and six months among the studies. All studies demonstrated the same beneficial direction of effect; the effect was similar for interventions delivered during or after usual care. One study had unusually small values reported for the standard deviation (Jin 2013), and this study dominates the weighting (86\%). If excluded, a slightly smaller effect occurs (MD $2.80 \mathrm{~mL} / \mathrm{kg} /$ minute, $95 \% \mathrm{Cl} 1.66$ to $3.95 ; \mathrm{I}^{2}=0 \% ; \mathrm{P}=0.00001$ ). If we assumed that the reported values were standard error values incorrectly reported and we then converted them to SD, the effect was again similar (MD $2.86 \mathrm{~mL} / \mathrm{kg} /$ minute, $95 \% \mathrm{Cl} 1.77$ to 3.96 ; $\mathrm{I}^{2}$ $=0 \% ; \mathrm{P}=0.00001)$ and the weighting becomes comparable to the other studies (8.8\%).

- One study estimated peak $\mathrm{VO}_{2}$ indirectly from workload and showed a beneficial effect of training at the end of intervention (Lennon 2008).

- One study assessed peak $\mathrm{VO}_{2}$ after a 12-month follow-up and suggests a training-induced benefit still remained (MackayLyons 2013). This study is small $(n=50)$ but at low risk of bias.

- One study assessed $\mathrm{VO}_{2}$ cost during the 12-MWT and did not show any significant training effect at the end of intervention (Moore 2010).

- One study planned oxygen uptake measures but did not report them (Kuys 2011).

Six studies (336 participants) assessed maximum cycling work rate at the end of intervention. This indicated that cardiorespiratory fitness improved significantly in participants who received the training intervention (MD 10.60 watts, $95 \% \mathrm{Cl} 1.88$ to $19.33 ; \mathrm{I}^{2}=85 \%$; $P=0.02$; Analysis 1.10). The large number of dropouts in Bateman 2001 means these data are at risk of bias; if excluded, the overall effect was strengthened and all the heterogeneity disappeared (MD 12.90 watts, $95 \% \mathrm{Cl} 8.39$ to $17.42 ; \mathrm{I}^{2}=0 \%$ ). Data from Bateman 2001 suggested that the improvement measured by maximal cycling work rate was not maintained at follow-up.

\section{Musculoskeletal fitness}

No studies reported indices of musculoskeletal fitness.

\section{Resistance training (comparisons 3 and 4)}

\section{Cardiorespiratory fitness}

One study showed a small increase in cardiorespiratory fitness $\left(\mathrm{VO}_{2}\right.$ peak: 6\%) after strength training (Ivey 2017).

\section{Musculoskeletal fitness}

Musculoskeletal fitness data, including muscle strength data, were awkward to synthesise because data can be collected from different muscle groups, using different equipment, different muscle contraction types (e.g. isometric, concentric), and reported as different data dimensions (e.g. force, torque, power). A total of 11 studies examined the effects of resistance training on indices relating to muscle strength.

Two studies with a total of 60 participants assessed the effects of resistance training on a composite measure of muscle strength at the end of intervention, during and after usual care (Kim 2001; Winstein 2004). Kim 2001 used a composite measure (i.e. the sum of the percentage change in six muscle groups) to assess the strength of the lower limbs, while Winstein 2004 used a composite measure (i.e. the sum of the torque of the extensors and flexors of the wrist, elbow, and shoulder) to assess the strength of the upper limbs. We have low certainty in the pooled estimate of effect in favour of the resistance training group (SMD $0.58,95 \% \mathrm{Cl} 0.06$ to 1.10 ; $\mathrm{P}$ $=0.03$; Analysis 3.2). However, Winstein 2004 was biased by lack 
of blinding and the use of a dynamometer that was hand-held by the investigator, and confounded by increased training time in the intervention group.

Three studies with a total of 93 participants showed that training could increase knee flexion strength in the affected leg; we have moderate certainty in this effect (SMD $0.72,95 \% \mathrm{Cl} 0.10$ to 1.34 ; $\mathrm{P}$ $=0.02$; Analysis 3.3). The same studies showed no increase in knee extension strength of the affected leg; we have low certainty in this effect (SMD 1.09, $95 \% \mathrm{Cl}-0.23$ to $2.41 ; \mathrm{I}^{2}=87 \%$; Analysis 3.4 ). Only one of these studies (Flansbjer 2008), included any follow-up data.

- One study examined strength bilaterally in the lower limb extensors and unilaterally in the knee extensors and the ankle flexors (plantar and dorsi; Ouellette 2004). They reported that all strength measures improved significantly after resistance training compared with the control group except for ankle dorsiflexion on the unaffected side. They presented the data as graphs and we could not extract them satisfactorily for further analyses.

- One study reported that participants allocated to resistance training of the lower limbs achieved significantly greater gains in the 10-repetition maximum exercise compared with controls (12.18 versus $8.58 \mathrm{~kg}, \mathrm{P}<0.02)$ after one month of intervention (Inaba 1973). No statistically significant differences were observed between groups after two months of training. Inaba 1973 did not report any measures of variance and therefore we were not able to include these data in our analyses.

- One study reported significant gains in maximal strength (bilateral one-repetition maximum (1-RM)) in a range of upper and lower body muscle groups after resistance training compared with the control group (Aidar 2016).

- One study reported no statistically significant gains in elbow and wrist strength (3-RM; peak torque of flexors and extensors) at either the end of intervention (six weeks) or the end of follow-up (six months; Coroian 2018).

- One study reported increases in static and dynamic strength (1-RM, Newtons) of the extensors of the whole lower limb (hip and knee) in the trained (more affected) leg (Fernandez-Gonzalo 2016). This was accompanied by muscle hypertrophy (+9.4\%). In addition, there were strength gains in the less affected (untrained) leg.

- One study showed that maximum strength (1-RM, pounds) of extensors of the whole lower limb (hip and knee) improved in the paretic leg (143\%) and non-paretic leg (121\%) plus there was a small increase in cardiorespiratory fitness $\left(\mathrm{VO}_{2}\right.$ peak; $6 \%$; Ivey 2017).

One study showed that submaximal muscular endurance (number of repetitions) of extensors of the whole lower limb (hip and knee) increased after strength training with an increase in total number of repetitions in the paretic leg (178\%) and non-paretic leg (161\%; Ivey 2017).

Two studies reported peak explosive power output measures. Fernandez-Gonzalo 2016 reported increases in power output (watts) of the extensors of the whole lower limb (hip and knee) in the trained (more affected) leg. This was accompanied by muscle hypertrophy (+9.4\%). In addition there were power gains in the less affected (untrained) leg. Ouellette 2004 suggested that peak power was improved during unilateral knee extensions but not during bilateral extension of the whole lower limb; they presented the data as graphs and we could not extracted them satisfactorily to pool in meta-analysis.

\section{Mixed training (comparisons 5 and 6) \\ Cardiorespiratory fitness}

Two studies (140 participants) reported cardiorespiratory fitness data as $\mathrm{VO}_{2}$ peak scores and showed effect of cardiorespiratory fitness; we have low certainty in this effect (MD 1.40, $95 \% \mathrm{Cl}-0.19$ to 2.99; 12 = 35\%; Analysis 5.8). Letombe 2010 also reported beneficial differences in $\mathrm{VO}_{2}$ peak (+30\%) but incomplete reporting prevented incorporation in this meta-analysis.

- Two studies measured peak work rate (watts) as an index of cardiorespiratory fitness: Moore 2015 reported that peak work rate increased after training. Letombe 2010 also reported changes in peak work rate (watts: $+20 \%$ ) but incomplete reporting prevented meta-analysis of these two studies.

- One study examined gait economy (net $\mathrm{VO}_{2} \mathrm{~mL} / \mathrm{kg}$ per metre walked; Mead 2007). A small beneficial effect at the end of intervention disappeared after a three-month follow-up.

- One study examined walking performance (time or metabolic equivalents (METS)) during a Modified Bruce treadmill protocol and reported no statistically significant effect of mixed training (Toledano-Zarhi 2011).

\section{Musculoskeltal fitness}

A total of six studies examined the effects of mixed training on indices relating to muscle strength.

Two studies (148 participants) assessed ankle dorsiflexion strength but did not show any effect of training; we have very low certainty in this effect (SMD $0.80,95 \% \mathrm{Cl}-0.82$ to $2.41 ; 1^{2}=94 \%$; Analysis 5.10). There was considerable heterogeneity between their results and both studies were confounded by increased training time. Yang 2006 also reported a range of other lower limb strength improvements, but all measurements were potentially biased as they were obtained by means of a hand-held dynamometer, which is not a reliable, objective method of measurement.

Three studies (202 participants) assessed knee extension strength. We have moderate certainty in the small effect size in favour of the mixed training group at the end of intervention (SMD 0.33, 95\% Cl 0.05 to $0.61 ; \mathrm{P}=0.02$; Analysis 5.11). One of the studies showed that this training effect was not retained at the end of the scheduled follow-up (Cooke 2010).

Three studies (147 participants) that assessed grip strength of the paretic hand did not show any significant improvement after mixed training at the end of intervention; we have low certainty in this effect (MD 0.32, $95 \% \mathrm{Cl}-0.88$ to $1.52 ; \mathrm{I}^{2}=0 \%$; Analysis 5.12). One other study reported grip strength data that we could not pool in this meta-analysis, and showed no statistically significant effect of training at end of intervention or after a range of follow-up time points (Langhammer 2007).

- One study assessed knee flexion strength and showed no statistically significant effect at the end of intervention or end of follow-up (Cooke 2010).

- One study assessed the effect of mixed training on elbow extension, elbow flexion, and pinch force at the end of 
intervention but did not detect any significant training effect (Donaldson 2009).

- One study assessed the maximum explosive extensor power of the affected and unaffected lower limb and showed no statistically significant effect at the end of intervention or end of follow-up (Mead 2007).

\section{Mobility}

\section{Cardiorespiratory training (comparisons 1 and 2)}

\section{Functional Ambulation Category}

Two studies, which included three relevant comparisons and 73 participants, measured the effect of treadmill gait training using the Functional Ambulation Category (FAC) scale (da Cunha 2002; Pohl 2002). The pooled MD showed that the FAC score measured at the end of intervention was significantly better in stroke survivors who received cardiorespiratory training during usual care (MD 0.53, 95\% $\mathrm{Cl} 0.21$ to $0.85 ; \mathrm{P}=0.001$; Analysis 1.11 ). We could not pool the FAC data reported by one study due to the way it was reported; there was no evidence of an effect of training on FAC in this study (Vanroy 2017).

\section{Maximum walking speed (MWS)}

Seventeen studies with 20 comparisons and a total of 782 participants measured maximum walking speed (metres per minute) at the end of intervention. The pooled MD showed significant consensus in favour of the training group (MD +7.66 $\mathrm{m} /$ minute, $95 \% \mathrm{Cl} 3.65$ to $11.68 ; \mathrm{P}=0.0002$; Analysis 1.12 ). This analysis also shows a consistent effect across the studies as a whole and a similar magnitude of effect arising from training delivered during or after usual care. The mode of cardiorespiratory training in all these studies was walking-specific apart from cycle ergometry (Bateman 2001; Sandberg 2016; Vanroy 2017), circuit type-training (Mudge 2009), and aquatic exercise (Aidar 2018). Nine of the 20 comparisons were confounded for imbalanced exposure time. If the exposure-confounded studies and non-walking studies were excluded seven out of eight of the remaining studies show a beneficial direction of effect and together show a substantial consensus of benefit (MD $+12.49 \mathrm{~m} /$ minute, $95 \% \mathrm{Cl} 4.02$ to 20.95). A funnel plot of the complete data in this analysis showed a tendency toward asymmetry, suggesting potential publication bias and this is focused on those studies occurring during usual care (Figure 4).

Figure 4. Funnel plot of comparison 1. Cardiorespiratory training versus control - end of intervention, outcome: 1.12 mobility - walking maximal speed (over 5 to 10 metres; $\mathrm{m} / \mathrm{min}$ )

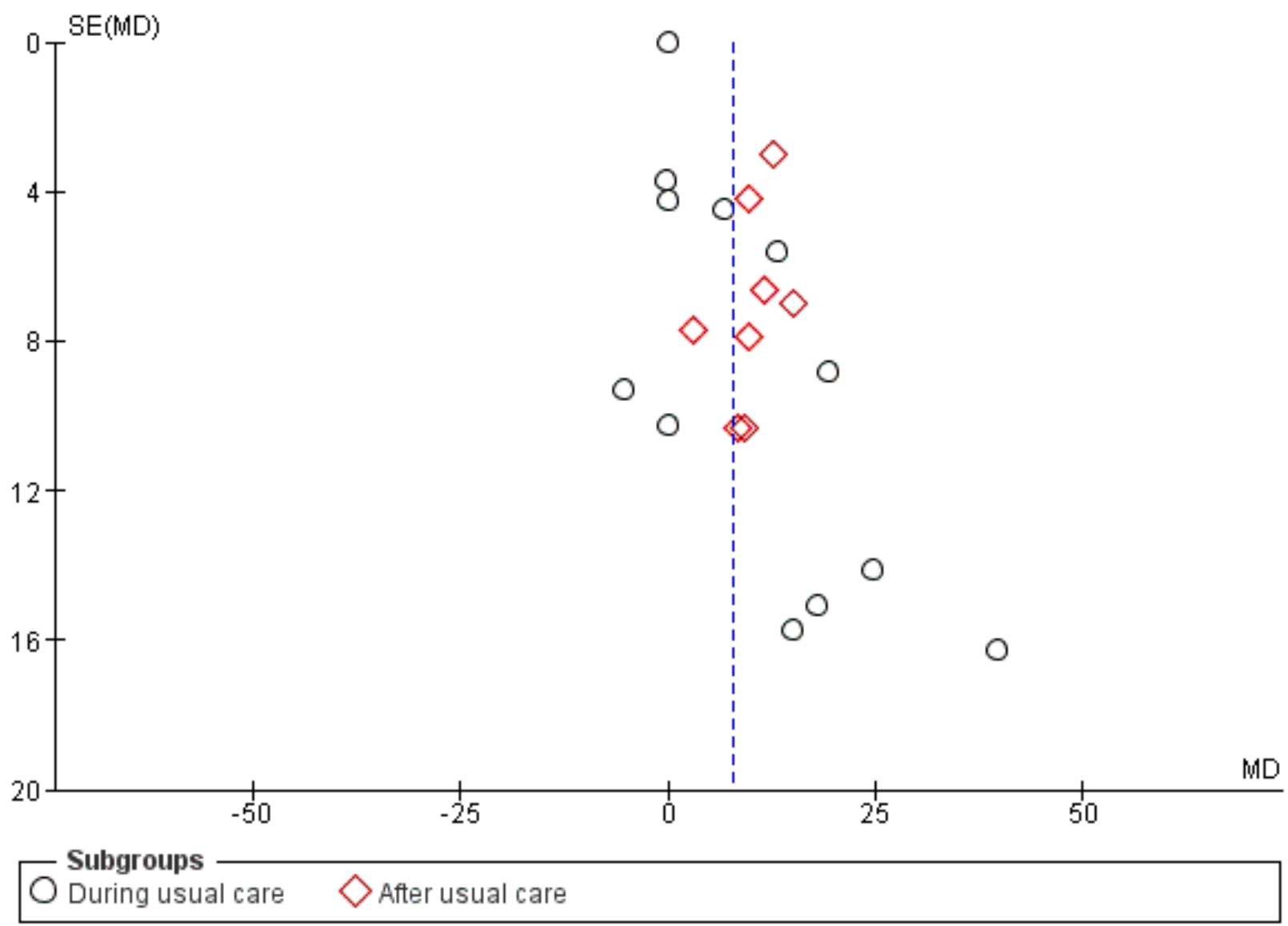

Five studies with six comparisons (312 participants) also provided follow-up data on maximum walking speed and observed a significant training effect at the end of follow-up (MD $6.71 \mathrm{~m} /$ minute, $95 \% \mathrm{Cl} 2.40$ to $11.02 ; \mathrm{P}=0.002$; Analysis 2.3). Although the overall effect is consistent, the two comparisons of Ada 2013 show the smallest effect. Ada 2013 used a 12-month follow-up whilst all the others used a three-month follow-up period. If we excluded 
the data, heterogeneity was reduced and the confidence in the treatment effect strengthened.

\section{Preferred walking speed (PWS)}

Twelve studies with 13 comparisons (588 participants) measured the preferred gait speed (metres per minute) at the end of intervention. We have high certainty in the pooled MD, which indicates a significant effect in favour of training (MD $4.47 \mathrm{~m} /$ minute, $95 \% \mathrm{Cl} 2.07$ to $6.87 ; \mathrm{P}=0.0003$; Analysis 1.13 ). This pooled effect is contributed to mostly by the consistent positive directions of effect among the studies taking place after usual care. The mode of cardiorespiratory training in all these studies was walkingspecific, apart from three studies that used cycle ergometry (KatzLeurer 2003; Vanroy 2017; Yang 2014). Five of the 13 comparisons are confounded for exposure time. If confounded and non-walking studies are excluded, the consistent beneficial effect among the remaining six studies is still apparent (MD $+5.83 \mathrm{~m} /$ minute, $95 \% \mathrm{Cl}$ 2.32 to 9.34). A funnel plot of the complete data shows no evidence of asymmetry (Figure 5).

Figure 5. Funnel plot of comparison 1. Cardiorespiratory training versus control - end of intervention, outcome: 1.13 mobility - walking preferred speed $(\mathrm{m} / \mathrm{min})$

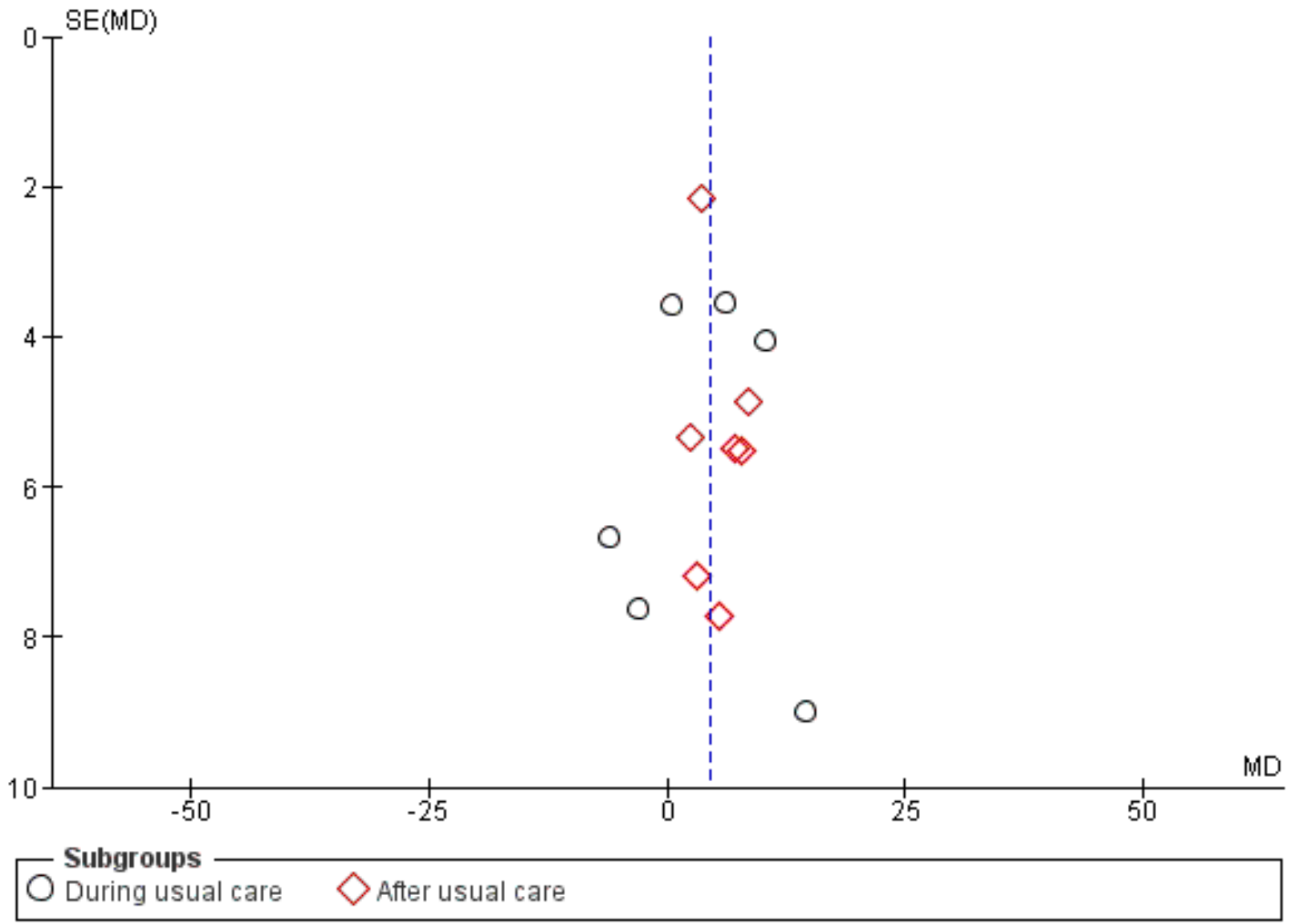

Three studies provided follow-up data at three months (Kuys 2011), and 12 months (Ada 2013; MacKay-Lyons 2013), after the intervention. Pooling these data showed no evidence of retention (Analysis 2.4).

\section{Six-Minute Walk Test (6-MWT)}

Sixteen studies with 17 comparisons (882 participants) assessed walking endurance using the 6-MWT (total metres walked in six minutes). We have high certainty that cardiorespiratory training significantly increased the walking capacity at the end of intervention (MD +33.41 metres/6 minutes, $95 \% \mathrm{Cl} 19.04,47.78$; $\mathrm{P}=0.00001$; Analysis 1.14). A consistent direction of effect was demonstrated at all stages of care although some heterogeneity is present $(12=30 \%)$. Nine of the 17 comparisons are confounded for exposure time. All studies contained walking-specific interventions apart from two that used cycle ergometry (Jin 2013; Yang 2014). If studies confounded for exposure and those using cycle ergometry are excluded then six of the remaining seven studies each show beneficial directions of effect and together these give a clear consensus effect in favour of training (MD +40.30 metres/ 6 minutes, $95 \% \mathrm{Cl} 13.24$ to 67.37 ); this is dominated by studies occurring after usual care. A funnel plot of the complete data shows no evidence of asymmetry (Figure 6). 
Figure 6. Funnel plot of comparison 1. Cardiorespiratory training versus control - end of intervention, outcome: 1.14 mobility - walking capacity (6-Minute Walk Test (metres))

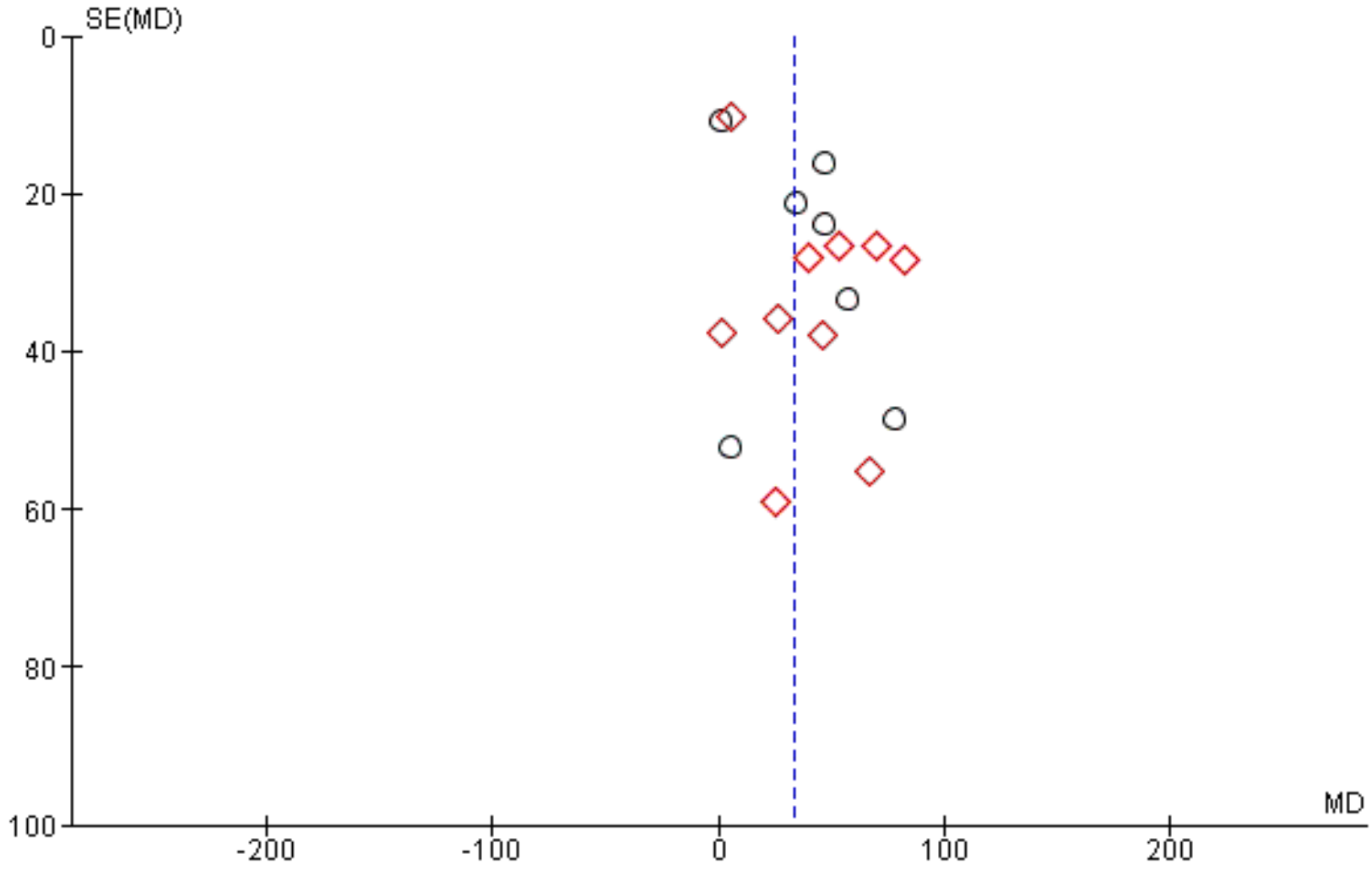

Subgroups

During usual care $\diamond$ After usual care

Five studies provided follow-up data at three months (Eich 2004; Kuys 2011; Mudge 2009), and 12 months (Ada 2013; MacKay-Lyons 2013), after the intervention. When pooled these data show some evidence of retention (MD 38.29 metres, $95 \% \mathrm{Cl} 7.19$ to 69.39 ; $\mathrm{P}=$ 0.02 ; Analysis 2.5). Although overall heterogeneity is low, the effects are variable and not obviously associated to either the shorter or longer follow-up periods.

\section{Other mobility outcomes}

Similar to the 6-MWT data, three studies measured walking endurance (reported as metres per minute) in 154 stroke survivors at the end of intervention, during (da Cunha 2002; Eich 2004), and after (Salbach 2004), usual care. Walking capacity increased significantly in participants who received cardiorespiratory training (MD 8.87 metres/minute, $95 \% \mathrm{Cl} 1.35$ to $16.40 ; \mathrm{P}=0.02$; Analysis 1.15).

Two studies reported time taken for the community walk test (Kim 2014; Park 2011). There was a small difference between participants who received community ambulation training and controls at the end of intervention (MD -10.54 minutes, $95 \% \mathrm{Cl}-14.11$ to -6.98 ; $\mathrm{P}$ $=0.00001$; Analysis 1.16).

- One study measured the time taken by stroke participants to walk a six-metre distance and did not find any significant difference between participants who received Kinetron walking training and controls (Glasser 1986).

\section{Resistance training (comparisons 3 and 4)}

Maximal walking speed (MWS)

Six studies (274 participants) measured maximal walking speed (metres per minute). Resistance training did not increase the walking velocity at the end of intervention (MD $2.83 \mathrm{~m} /$ minute, $95 \% \mathrm{Cl}-0.49$ to 6.14 ; Analysis 3.5 ). There was some moderate heterogeneity; studies showing positive directions of effect either involved walking-related exercise (Bale 2008), or were problematic due to either use of interpolated data (Buyukvural 2015), or due to being confounded for exposure time (Buyukvural 2015; Knox 2018). Follow-up data pooled from two studies did not show any effect of retained training effects (Flansbjer 2008; Knox 2018; Analysis 4.2).

\section{Preferred walking speed (PWS)}

Five studies (203 participants) measured preferred gait speed (metres per minute) but failed to demonstrate any effect of resistance training at the end of intervention; we have moderate certainty in this effect (MD $2.15 \mathrm{~m} / \mathrm{min}, 95 \% \mathrm{Cl}-3.57$ to 7.87 ; Analysis 3.6). One study measured comfortable walking speed after follow-up; the intervention effect exceeded the control group (Knox 2018). 


\section{Six-Minute Walk Test (6-MWT)}

Five studies (238 participants) assessed walking capacity as metres walked in six minutes. We have low certainty in the effect of resistance training at the end of intervention (MD 24.98 metres, $95 \% \mathrm{Cl} 11.98$ to 37.98; $\mathrm{P}=0.0002$; Analysis 3.7). Three of the five studies are confounded for exposure time, or have multiple risk of bias concerns (Ivey 2017). Two studies provided follow-up data that showed no training effect on walking capacity at the end of followup (Analysis 4.3).

\section{Mixed training (comparisons 5 and 6)}

\section{Maximum walking speed (MWS)}

Three studies (168 participants) reported an increase in maximum walking speed (MD $+8.48 \mathrm{~m} /$ minute, $95 \% \mathrm{Cl} 1.76$ to 15.20 ; Analysis 5.13). Two of the included studies are confounded for exposure time, which could exaggerate the response.

- Only one included follow-up data and suggested that end of intervention benefit was retained after 12 weeks of follow-up (Knox 2018).

\section{Preferred walking speed (PWS)}

Ten studies (738 participants) measured the effects of mixed training on preferred walking speed (metres per minute). We have moderate certainty in the walking speed increase at the end of intervention in stroke survivors who received mixed training (MD $4.71 \mathrm{~m} /$ minute, $95 \% \mathrm{Cl} 1.32$ to $8.10 ; \mathrm{P}=0.006$; Analysis 5.14 ). The effect is influenced mostly by data from interventions delivered after usual care and there is significant heterogeneity within this subgroup as well as overall. Only the interventions in three of the 10 studies had balanced control and training exposures and these show no statistically significant effect overall (Mead 2007; Richards 1993; Richards 2004). A funnel plot of these data (not included) did not show any evidence of asymmetry.

Five studies (542 participants) that provided follow-up data for preferred gait speed did not show a training effect at the end of the scheduled follow-up (Analysis 6.7).

- One study showed some indication of dose-response, where the improvement in preferred gait speed was positively associated with the amount of time spent on the gait training component (Richards 1993).

\section{Six-Minute Walk Test (6-MWT)}

Ten studies (720 participants) measured walking capacity (metres walked in six minutes). We have low certainty in the significant increase shown after mixed training (MD 35.00 metres, $95 \% \mathrm{Cl}$ 15.91 to 54.09; $\mathrm{P}<0.0003$; Analysis 5.15). All studies in this metaanalysis, apart from Moore 2015, were confounded for exposure time. A funnel plot of these data (not included) did not show any asymmetry.

Four studies (464 participants) included a follow-up and showed that walking capacity remained significantly greater in the groups who had participated in training (MD 47.48 metres, $95 \% \mathrm{Cl} 23.72$ to 71.23; $\mathrm{P}=0.0001$; Analysis 6.8). It is worth noting, however, that in all studies in this analysis the intervention groups were confounded by additional training time, which could exaggerate the effect.

\section{Other mobility outcomes}

Three studies (232 participants) measured community ambulation speed (the ability to walk at 0.8 metres per second or more) and did not demonstrate any significant training effects either at the end of intervention (Analysis 5.16), or at follow-up (Analysis 6.9).

- One study examined FAC scores and detected no statistically significant effect of training at the end of intervention or at end of three-month follow-up (Van de Port 2012).

\section{Comparison of cardiorespiratory, resistance training, and mixed training (comparison 7)}

Where sufficient data existed we performed a subgroup analysis to compare the effects of the different types combined with sensitivity analyses to examine combinations of training types on mobility outcomes at the end of intervention. The effect sizes described below arise from the sensitivity analyses below and are not shown in Analysis 7.2, Analysis 7.3, or Analysis 7.4.

\section{Maximal walking speed}

Maximal walking speed increased significantly after cardiorespiratory training and mixed training but not after resistance training (Analysis 7.2). We examined pair-wise combination of these subgroups. Excluding the resistance training subgroup showed that cardiorespiratory training with or without a resistance training element (mixed) benefits maximal walking speed (MD $7.77 \mathrm{~m} / \mathrm{min}, 95 \% \mathrm{Cl} 4.11$ to $11.44 ; \mathrm{P}=0.00001$ ); the heterogeneity in this analysis was greatly reduced when we removed studies confounded for intervention exposure. Excluding the cardiorespiratory training subgroup showed that resistance training with or without a cardiorespiratory training element (mixed) also appears to benefit maximal walking speed (MD $3.63 \mathrm{~m} /$ $\min , 95 \% \mathrm{Cl} 0.64$ to $6.63 ; \mathrm{P}=0.02$ ); however, this benefit is due to mixed training interventions not the resistance training.

\section{Preferred walking speed (PWS)}

Preferred walking speed increased significantly after cardiorespiratory and mixed training but not after resistance training (Analysis 7.3). We examined pair-wise combination of these subgroups. Excluding the resistance training subgroup showed that cardiorespiratory training with or without a resistance training element benefits preferred walking speed (MD $4.56 \mathrm{~m} / \mathrm{min}, 95 \% \mathrm{Cl}$ 2.35 to 6.77 ; P value $<0.0001$ ); excluding studies confounded for intervention time preserves a benefit whilst greatly reducing the heterogeneity. Excluding the cardiorespiratory training subgroup showed that resistance training with or without a cardiorespiratory element benefits preferred walking speed (MD $3.80 \mathrm{~m} / \mathrm{min}, 95 \% \mathrm{Cl}$ 1.07 to $6.53 ; \mathrm{P}=0.006$ ). All this benefit arises from the mixed training subgroup; however, seven out of 10 studies were confounded for intervention time.

\section{Six-Minute Walk Test (6-MWT)}

Gait endurance increased significantly after cardiorespiratory and mixed training but not after resistance training (Analysis 7.4). We examined pair-wise combination of these subgroups. Excluding the resistance training subgroup showed that cardiorespiratory training, with or without a resistance training element, benefits gait endurance (MD 33.97 metres, $95 \% \mathrm{Cl} 22.59$ to 45.35 ; $\mathrm{P}$ value $\mathrm{P}<0.00001$ ). There is a similar contribution from both subgroups but the majority of studies were confounded for intervention time. Excluding the cardiorespiratory training subgroup showed that 
resistance training, with or without a cardiorespiratory element, benefits gait endurance ( 29.53 metres $95 \% \mathrm{Cl} 18.87$, 40.18; $\mathrm{P}$ value $<0.00001)$; the majority of studies $(12 / 15)$ were confounded for intervention time.

\section{Physical function}

The included studies assessed participants' physical function using a variety of different measures including rating scales (e.g. Berg Balance Scale) and specific measures of functional performance (e.g. functional reach, Timed Up and Go test, stair climbing).

\section{Cardiorespiratory training (comparisons 1 and 2)}

\section{Balance outcomes}

Seven studies with eight comparisons (471 participants) assessed the effects of cardiorespiratory training on balance using the Berg Balance Scale. We have moderate certainty in the increase in balance scores (MD 1.92, $95 \% \mathrm{Cl} 0.16$ to 3.68; $\mathrm{P}=0.03$; Analysis 1.17). All studies except Bateman 2001 and Jin 2013 included a walking component; when we excluded these two studies the effect was strengthened (MD 2.99, 95\% $\mathrm{Cl} 0.95$ to 5.02: $\mathrm{P}=0.004$ ). The backwards-walking group of Takami 2010 appeared to produce a larger benefit compared with the forwards-walking group from the same study. Bateman 2001 and MacKay-Lyons 2013 also assessed participants at the end of the follow-up period but did not show any effect (Analysis 2.6).

- One study assessed balance using a single leg stance test (left and right legs, with and without eyes closed) and demonstrated a beneficial direction of effect in all (Sandberg 2016).

- One study showed Brunel Balance Scores did not change after training (Mao 2015).

\section{Other outcomes}

Five studies (223 participants) showed that performance of the Timed Up and Go test improved; we have moderate certainty in this effect (MD -3.42 sec, $95 \% \mathrm{Cl}-4.78$ to -2.05 ; P value 0.00001 ; Analysis 1.18).

- One study showed that functional reach was higher after training (Kang 2012).

- One study showed that Fugl-Meyer scores did not change after training (Mao 2015).

- One study demonstrated a medium beneficial effect of training on time taken to rise from a chair (Aidar 2018).

\section{Resistance training (comparisons 3 and 4)}

\section{Balance outcomes}

Five studies (220 participants) assessed balance using the Berg Balance scale. We have low certainty in the pooled after training (MD 3.27, 95\% Cl 2.15 to 4.38; $\mathrm{P}=0.00001$; Analysis 3.8). Only Knox 2018 reported Berg Balance data again after follow-up (at three months) and showed a small positive direction of benefit.

- One study assessed the maximum weight-bearing on the affected leg (\% body weight) and showed a beneficial change after training (Bale 2008).

- One study examined the effect on indices of postural sway (centre of pressure). This could not be pooled but suggested a beneficial effect of training (Arabzadeh 2018).
- One study assessed the effect on antero-posterior and mediolateral sway velocity and showed a beneficial effect of training (Lee 2013a).

- One study assessed the effect on antero-posterior and mediolateral sway distance and showed a beneficial effect of training (Son 2014).

\section{Step tests}

Four studies examined the effect of resistance training on stair climbing speed using different protocols.

Two studies (91 participants) reported time needed to ascend stairs using similar protocols (10 steps; Ouellette 2004; 11 steps Buyukvural 2015), and showed a beneficial effect of intervention at the end of the training period (MD $-2.07 \mathrm{sec}, 95 \% \mathrm{Cl}-3.18$ to -0.96 ; $P$ value 0.0003; Analysis 3.9).

Two other studies reported data not suitable to pool due to protocol differences. We could not use SMD as there would be a mix of endscore and change-score data.

- One study reported time to climb four steps. Change scores showed no statistically significant effect on maximal or chosen speed (Kim 2001).

- One study used a two-minute step test and showed beneficial effect on stair climbing (Taylor-Pilliae 2014).

\section{Timed Up and Go}

Five studies (224 participants) examined the effect of resistance training on the Timed Up and Go test. There was no benefit and we have low certainty in the estimate (MD $-3.46 \mathrm{sec}, 95 \%$ $\mathrm{Cl}-6.94$ to $0.02 ; \mathrm{I}^{2}=89 \%$; Analysis 3.10 ). The during- and afterusual-care subgroups did each show a beneficial effect; however, four out of five studies were confounded for intervention time, there is substantial heterogeneity, and Buyukvural 2015 is based on variance data interpolated from published $P$ values.

Two studies (117 participants) examined Timed Up and Go data at follow-up and this shows no statistically significant effect of retained benefits (Analysis 4.4)

\section{Other outcomes}

- One study examined the effect of resistance training on the Trunk Impairment Scale; there was no statistically significant effect size (Verheyden 2009).

- One study concluded there was no benefit of isokinetic upper limb training to upper limb function (upper limb Fugl-Meyer scores and Box and Block tests; Coroian 2018).

- One study showed trivial direction benefit from lower limb training on lower limb and total Fugl-Meyer scores (Zou 2015).

- One study showed no beneficial effect of training on the Short Physical Performance Battery score; only the 'strength' subscale showed a beneficial direction of change (Taylor-Pilliae 2014).

\section{Mixed training (comparisons 5 and 6)}

\section{Balance outcomes}

Nine studies (419 participants) assessed balance using the Berg Balance Scale. We have low certainty in the beneficial effect (MD 2.12, $95 \% \mathrm{Cl} 0.82$ to $3.41 ; \mathrm{P}=0.001$; Analysis 5.17). Heterogeneity is low in this meta-analysis; however, five of the nine studies were 
confounded for increased exposure time in the intervention groups; excluding these studies extinguishes the beneficial effect. Followup data from three studies (201 participants) did not show any significant retention of training effects (Analysis 6.10).

Two studies with a total of 166 participants measured balance using the functional reach test but did not show any benefit of mixed training at the end of intervention (Duncan 2003; Mead 2007; Analysis 5.18). One study provided follow-up data (Mead 2007); this did not show any retention effects.

Other study balance data included;

- One study measured the Four Square Step Test; however, these data were very different at baseline in a way that benefited the control group (Toledano-Zarhi 2011).

- One study measured balance using the timed balance test after intervention and after three-month follow-up (Van de Port 2012).

- One study measured postural sway (static balance) in a range of conditions and planes of movement; however, the study authors concluded there was no statistically significant effect of mixed training (Shin 2011).

- One study reported multiple benefits of aquatic exercise to a range of balance and stance outcomes measured using a 'baropodometric' system (Furnari 2014).

There were sufficient data among the all different measures of balance described above to justify pooling using SMD meta-analysis (12 studies, 755 participants). This showed an overall beneficial improvement in balance at the end of intervention (SMD 0.28 , $95 \% \mathrm{Cl} 0.11$ to $0.45 ; \mathrm{P}=0.001$; Analysis 5.19). However, seven of the 12 included studies were confounded by additional training time; when we excluded these data there was no statistically significant effect of training on balance. A funnel plot of these data (not included) appears asymmetrical; however, the asymmetry is caused by two small studies each showing negative effects (Kim 2016a; Toledano-Zarhi 2011).

\section{Other outcomes}

Seven studies (586 participants) measured the time to complete the Timed Up and Go test and showed no statistically significant effect of training; we have low certainty in this effect (MD $-2.21 \mathrm{sec}, 95 \% \mathrm{Cl}$ -4.43 to $0.02 ;\left.\right|^{2}=45 \%$; Analysis 5.20). Five of these seven included studies were confounded by additional training time. Follow-up data in five studies (510 participants) did not show a significant retention of mixed training benefits (Analysis 6.12); these data are also dominated by studies confounded for intervention time.

- One study assessed upper extremity functional performance using the Action Research Arm test and showed no difference at end of intervention (Donaldson 2009).

- One study recorded physical activity data (diary and accelerometer) at end of intervention and end of follow-up but did not analyse this (pilot study; Dean 2018). We calculated a MD and $95 \% \mathrm{Cl}$ and the data show no beneficial direction of effect for any domain of physical activity (total, light, moderate, vigorous (MVPA)) at either the end of intervention or end of follow up.

- One study reported Fugl-Meyer scores for the paretic lower limb; there was no evidence of an intervention effect (Kim 2016a).
Comparison of cardiorespiratory, resistance training, and mixed training (comparison 7)

Where sufficient data existed we performed a subgroup analysis to compare the effects of the different types combined with sensitivity analyses to examine combinations of training types on the Berg Balance Scale and Timed Up and Go data at the end of intervention. The effect sizes described below arise from the sensitivity analyses below and are not shown in Analysis 7.5 or Analysis 7.6.

\section{Balance}

Excluding the resistance training subgroup showed that cardiorespiratory training with or without a resistance training element benefits balance (MD $1.87,95 \% \mathrm{Cl} 0.76$ to 2.99; $\mathrm{P}$ $=0.0009$; Analysis 7.5); this is contributed to similarly for both training types but exclusion of the studies confounded for intervention time cancels out evidence of beneficial effect. Excluding the cardiorespiratory training subgroup showed that resistance training with or without a cardiorespiratory training element benefits balance (MD 2.75, 95\% Cl 1.92 to 3.58; P value $<0.00001)$. Both intervention types contribute in a similar and consistent way and the benefit is still evident when the studies confounded for intervention time are excluded (MD 2.81, 95\% Cl 1.47 to 4.15 ; $P$ value $<0.0001$ ).

\section{Timed Up and Go}

Excluding the resistance training subgroup showed that cardiorespiratory training with or without a resistance training element benefits Timed Up and Go (MD $-2.61 \mathrm{sec}, 95 \% \mathrm{Cl}$ -3.93 to -1.29 ; $\mathrm{P}$ value $<0.0001$; Analysis 7.6 ). Excluding the cardiorespiratory training subgroup showed that resistance training with or without a cardiorespiratory training element benefits Timed Up and Go (MD $-2.88 \mathrm{sec}, 95 \% \mathrm{Cl}-5.02$ to -0.75 ; $P$ value $<0.00001$ ). Taking into account these data as a whole the magnitude of effect across the three training types was very similar and was consistent, with 15 out of 17 studies showing a positive direction of benefit. However, many of the studies (12/17) are confounded for intervention time.

\section{Health status and quality of life}

\section{Cardiorespiratory training (comparisons 1 and 2)}

Two studies (164 participants) examined effects of training on the component summary measures shared by the SF-12 scale (Globas 2012), and the SF-36 scale (Gordon 2013). This showed that cardiorespiratory training benefited the 'physical health' component (SMD 0.51, 95\% Cl 0.20 to 0.82; $\mathrm{P}=0.001$; Analysis 1.19) but not the 'mental health' component (Analysis 1.20).

Two studies with three comparisons (158 participants) reported EuroQoL scores and this showed no pooled effect of training at the end of intervention (Analysis 1.21), or the end of follow-up (Analysis 2.7).

- One study reported the SF-36 'physical functioning' scale and the 'emotional role' scale showing the scores to be significantly better after training (Aidar 2018).

- One study reported hand and shoulder pain being improved in the intervention group (Topcuoglu 2015). 


\section{Resistance training (comparisons 3 and 4)}

Three studies (70 participants) assessed the 'physical functioning' or 'mental health' scales of the SF-36 at the end of intervention. There was no statistically significant effect on 'physical functioning' (Analysis 3.11) but there was evidence of benefit for 'mental health' (MD 7.69, 95\% Cl 1.56 to 13.83; Analysis 3.12). The two studies with the largest contributing effects were confounded for intervention exposure time.

- One study reported no training effect in the two 'physical health' and 'mental health' composite scores of the SF-36 at the end of intervention (Taylor-Pilliae 2014).

- One study showed that all eight scales of the SF-36 improved after training (but did not report the two composite scores); this intervention is confounded for exposure time (Aidar 2016).

- One study reported a benefit in Stroke Specific Quality of Life scale (Buyukvural 2015).

\section{Mixed training (comparisons 5 and 6)}

\section{SF-36 data}

Two studies (112 participants) showed significantly better scores in the SF-36 'physical functioning' scale in the mixed training group at the end of intervention (SMD $0.48,95 \% \mathrm{Cl} 0.10$ to $0.85 ; 0.01$; Analysis 5.21), but not after follow-up (Analysis 6.13). There was no statistically significant effect on the 'social role functioning' scale at the end of intervention (Analysis 5.23).

Three studies (178 participants) showed significantly better scores in the SF-36 'physical role functioning' scale for the mixed training group at the end of intervention (SMD $0.56,95 \% \mathrm{Cl} 0.26$ to 0.86 ; $\mathrm{P}$ $=0.0003$; Analysis 5.22). This effect was retained at follow-up (MD $11.61,95 \% \mathrm{Cl} 2.38$ to $20.84 ; \mathrm{P}=0.01$; Analysis 6.14$)$.

- One study showed that participants receiving mixed training had significantly better results in the 'emotional role functioning' scale of the SF-36 compared with controls at the end of the training period (Duncan 2003).

\section{Other outcome data}

- One study reported the two components of the EuroQol scale (health state and perceived health state). Although not significant there was a positive direction of effect after intervention but not at follow-up (Cooke 2010).

- One study assessed the effect of mixed training on the StrokeAdapted Sickness Impact profile and showed no statistically significant effect at the end of intervention or end of six-month follow-up (Zedlitz 2012).

- One study recorded quality of life using various metrics (Stroke QoL Scale, EQ-5D-5L, SF-12 physical and mental health components) at end of follow-up only but did not analyse this (pilot study; Dean 2018). We calculated MDs and $95 \% \mathrm{Cl}$ and these showed no effects. The direction of effect was beneficial for SF-12 scores but not for the Stroke QoL Scale or EQ-5D-5L.

- One study reported the domains of the Stroke Impact Scale as quality of life with only two out of 10 reported domains showing a beneficial direction of effect (Moore 2015).

Seven of the nine studies for mixed training in comparison 5 and 6 were confounded by additional training time, leaving only Mead 2007 and Moore 2010 unaffected.

\section{Mood}

\section{Cardiorespiratory training (comparisons 1 and 2)}

Two studies (56 participants) reported Beck Depression Index data at the end of intervention (Analysis 1.22) and showed no beneficial effect. Only one of these studies reported follow-up data too (Smith 2008).

- One study used the Beck Depression Index and stated depression was improved in the intervention group, but did not report any data (Topcuoglu 2015).

- One study using HADS reported that the depression score improved in the intervention group but not in the control group (Lennon 2008). We were, however, unable to include these study data in our analyses as they were presented in a format not suitable for Review Manager 2014.

- One study reported anxiety using HADS (Bateman 2001). The anxiety score decreased immediately after cardiorespiratory training but not at follow-up. This study had, however, substantial missing values at the end of intervention $(29 \%)$ and end of follow-up (37\%) and, therefore, we did not pool these with other depression scores.

- One study reported medium-sized beneficial effects in Anxiety Trait and Anxiety State measures (Aidar 2018).

\section{Resistance training (comparisons 3 and 4)}

Two studies (180 participants) reported the Centre for Epidemiological Studies for Depression scale (CES-D). The mood in the resistance training group was significantly better at the end of intervention (MD $-3.76,95 \% \mathrm{Cl}-6.98$ to -0.54 ; Analysis 3.13). One of these studies demonstrated that the effect was retained after a follow-up (Sims 2009).

One study reported a significant benefit in measures of the Beck Depression Inventory at the end of intervention (Aidar 2016). Combining data from the different depression scales reported in three studies (209 participants) showed a significant benefit at the end of intervention (SMD $-0.36,95 \% \mathrm{Cl}-0.64$ to $-0.09 ; \mathrm{P}=$ 0.01 ; Analysis 3.14). All three of these studies have major bias and confounding issues

- One study used the Brazilian translation of the State-Trait Anxiety Inventory and showed no effects (Aidar 2016).

\section{Mixed training (comparisons 5 and 6 )}

Three studies (391 participants) used the anxiety and depression components of the HADS (Mead 2007; Van de Port 2012; Zedlitz 2012). No immediate training effects were observed on either HADS component at the end of the intervention (Analysis 5.24; Analysis 5.25). No retained training effects were observed on either HADS component at the end of follow-up (Analysis 6.17; Analysis 6.18).

Two studies (Duncan 2003; Van de Port 2012; 335 participants) assessed mood using the 'emotion' domain of the Stroke Impact Scale (SIS) and showed no statistically significant effect at the end of intervention (Analysis 5.26), or after three-month follow-up (Analysis 6.15).

One study reported Geriatric Depression Scale scores (Duncan 2003). Combining all available depression data from four studies (484 participants) showed no statistically significant effect of 
training at the end of intervention (Analysis 5.27), or the end of follow-up (Analysis 6.19).

\section{Cognitive function}

\section{Cardiorespiratory training (comparisons 1 and 2)}

One study of cardiorespiratory training showed no statistically significant effect on FIM cognitive score (memory, problem-solving questions) at the end of intervention (Bateman 2001). We did not consider end of follow-up data due to the considerable proportions of missing data.

\section{Resistance training (comparisons 3 and 4)}

One study showed benefits of short intense training on attention, working memory, information processing, and executive function (Fernandez-Gonzalo 2016). Some cognition data is incomplete in this study for participants with expressive aphasia or upper limb spasticity.

\section{Mixed training (comparisons 5 and 6 )}

Two studies (159 participants) showed no statistically significant effect on FIM cognitive score (memory, problem-solving questions) at the end of intervention (Analysis 5.28), or end of follow-up (Analysis 6.20).

Two studies (133 participants) used SIS domains of 'communication' and 'memory and thinking' to assess cognitive function. The meta-analyses showed no statistically significant effects at the end of intervention (Analysis 5.29; Analysis 5.30), or the end of the six-month follow-up (Analysis 6.21; Analysis 6.22).

- One study showed that mixed training benefited cognitive function assessed by the Korean version of the Montreal Cognitive Assessment but not the Trail Making Test or Stroop test (Kim 2017a).

- One study showed that mixed training improved cognition measured using the ACE-R tool (Moore 2015).

\section{'Summary of findings' tables}

We have presented the results for the primary outcome measures 'Summary of findings' tables for cardiorespiratory training (Summary of findings for the main comparison), resistance training (Summary of findings 2), and mixed training (Summary of findings 3). In addition we made a post-hoc decision to include domains relating to physical fitness, mobility, and physical function.

\section{DISCUSSION}

\section{Summary of main results}

\section{Effect of training on primary outcome measures}

\section{Death}

There were no statistically significant effects on mortality, which suggests that there were no benefits but also that there were no adverse effects on mortality. Although there is low certainty in these analyses, death from any cause was a very uncommon event among the participants. At the end of intervention only 19 out of the total 3617 participants had died and these were restricted to five out of 75 studies. At the end of follow-up 19 out of 1469 participants had died; these were restricted to seven out of 24 studies with follow-up time points.
The observed numbers of deaths in this review may be low because the included participants were at lower risk of death compared with the wider stroke population. This may occur, first, because the inclusion criteria of the studies of exercise select participants with milder strokes (most were ambulatory), and reduced risk factors (such as blood pressure ceiling criteria). In other words, those at higher risk of dying were probably systematically excluded due to contraindications to exercise. Second, there may be selfselection by participants who are physically active with higher fitness. Higher physical activity and cardiorespiratory fitness are known to be associated with reduced stroke risk and stroke mortality (Hooker 2019). In addition, the majority of the training programmes in this review were of short duration (12 weeks or less). A systematic review of the effect of exercise-based cardiac rehabilitation showed reduced cardiovascular mortality in people with coronary heart disease (Anderson 2016), but the training programmes were longer (median six months) than those in this review. Since many stroke patients have coexisting heart disease, training might influence post-stroke mortality if it contains cardiorespiratory training delivered over long periods of time. With regard to longer-term interventions, currently two small studies of cardiorespiratory training (Ivey 2010; Ivey 2011), and one of mixed training (Dean 2018), exist; these used a six-month intervention duration. Current evidence lacks the interventions most likely to influence mortality.

Although higher physical activity and higher cardiorespiratory fitness are linked to the primary prevention of stroke, there is a lack of data on the role of fitness training interventions in the secondary prevention of stroke. This gap in knowledge is currently a research priority and requires investigation (Pollock 2012).

\section{Death or dependence}

There were no data available to allow us to draw conclusions about the influence of training on the composite outcome of death or dependence after stroke. Death is infrequent and measures of dependency, such as those based on simple questions, a Barthel Index score of less than 20, or a modified Rankin Scale score of 3, 4 , or 5, are lacking (Lindley 1994). Both elements of this composite outcome are likely to be rare in stroke survivors who are eligible for physical fitness training.

\section{Disability}

We assessed a number of different global indices of disability. Data using the same scales were limited, which restricted the metaanalyses to the use of standardised mean differences. In addition, a number of methodological issues weakened and biased the available data.

After cardiorespiratory training there was no improvement in Functional Independence Instrument scores (Analysis 1.2), Barthel Index scores (Analysis 1.3), or other individually-reported outcomes. However, there was an improvement in Rivermead Mobility Index scores (Analysis 1.4). Pooling all available disability scale data from different scales showed a moderate beneficial effect (SMD; Analysis 1.5). This pattern of findings could occur because training influences the physical/mobility items of these various scales; such items dominate the scoring in tools like the Rivermead Mobility Index (eight out of 15 items) whereas they are less influential in more 'global' tools like the Functional Independence Measure (two out of 18 items). Since walking is a common mode of cardiorespiratory exercise, these findings could 
be precipitated by improvements in walking and mobility rather than more 'global' effects on disability.

There were too few data to allow for any comment on the effect of resistance training and there was no evidence of intervention effects at the end of follow-up where data existed.

Studies of mixed training used various disability measurement instruments, which individually showed no clear consensus effects. Pooling all available data from different scales showed a small effect at the end of intervention (Analysis 5.5). Like cardiorespiratory training these significant effects could be driven principally by changes in mobility. Most pooled studies were confounded by additional training time; when these were excluded the benefits vanished. This means that participation in mixed training appeared effective but it is impossible to attribute any benefits to the actual content of the mixed training programmes.

The effects of cardiorespiratory training (moderate-certainty evidence) and mixed training (low-certainty evidence) are similar in magnitude at the end of intervention (Analysis 7.1). Overall, the findings show that interventions containing cardiorespiratory training, with or without resistance training content (i.e. mixed training), improve global measures of disability after stroke. These effects may be driven by improvements in mobility rather than being indicative of a change in more 'global' disability status. This would agree with the findings among the secondary outcomes (mobility).

\section{Effect of training on secondary outcome measures}

\section{Adverse events}

There was no evidence of any serious adverse events arising from training in people who participated in physical fitness training programmes. However, this finding cannot be generalised to the wider stroke population as only a few studies specifically recorded or reported adverse events. There is a clear need to improve the reporting of adverse events in physical fitness training studies.

\section{Risk factors}

Few studies reported vascular risk factors, and the variety of outcomes restricted meta-analysis. There were no consensus effects on blood pressure. In individual studies, which reported metabolic measures (glucose tolerance, insulin sensitivity, lipids), there were some beneficial directions of effect but no consensus can be reached. Blood pressure remained an uncommon outcome reported in studies of exercise after stroke and yet it could be an important, plausible benefit. Other recent systematic reviews have examined the effect of exercise on cardiovascular risk factors and reported beneficial effects on systolic blood pressure, fasting glucose and insulin, and HDL cholesterol (D'Isabella 2017); however, this included a number of studies that did not meet our eligibility criteria. The review of D'Isabella 2017 did not include cardiorespiratory fitness as an outcome.

There was an increase in cardiorespiratory fitness after cardiorespiratory training; this is discussed in the next section below from a functional perspective. However, as well as having functional implications, low $\mathrm{VO}_{2}$ peak values are associated with increased stroke risk and stroke mortality (Hooker 2019). An increase in $\mathrm{VO}_{2}$ peak of $1 \mathrm{MET}$ (equivalent to $+3.3 \mathrm{~mL} / \mathrm{kg} / \mathrm{min}$ ) equates to a $7 \%$ risk reduction in stroke hospitalisation (Pandey
2016). We have moderate certainty that our observed effect of +3.40 $\mathrm{mL} / \mathrm{kg} / \mathrm{min}(95 \% \mathrm{Cl} 2.98$ to 3.83$)$ is of similar magnitude to this and this indicates that meaningful risk reduction may be achievable with short cardiorespiratory training interventions either during or after usual care.

\section{Physical fitness}

\section{Cardiorespiratory fitness}

Cardiorespiratory training significantly improved physiological indicators $\left(\mathrm{VO}_{2}\right.$ peak), and performance indicators (exercise tolerance) of cardiorespiratory fitness. This improvement may be beneficial because a low $\mathrm{VO}_{2}$ peak is associated with functional limitation in elderly people (Young 2001). In people with stroke the functional benefits are, however, less clear (see for example the contradictory data by Michael 2007 and Patterson 2007).

A limited 'fitness reserve' caused by a low $\mathrm{VO}_{2}$ peak coupled with poor walking economy (high oxygen cost of walking) is a common post-stroke problem (Blokland 2018; Macko 2001). Training to improve either component $\left(\mathrm{VO}_{2}\right.$ peak and/or walking economy) could benefit walking performance and exercise tolerance after stroke. Although there were too few data to determine whether economy can be improved, there is clear consensus that $\mathrm{VO}_{2}$ peak can be improved. This increases the 'fitness reserve', meaning that walking at a given speed is less demanding. Our consensus effect $(+3.40 \mathrm{~mL} / \mathrm{kg} / \mathrm{min} 95 \% \mathrm{Cl} 2.98$ to 3.83$)$ is very similar to other recent systematic reviews (+2.2 mL/kg/min 95\% Cl 1.3 to 3.1; Boyne 2017).

There were too few other data to draw conclusions on the effects of the other training types or the post-training retention of cardiorespiratory fitness.

\section{Musculoskeletal fitness}

Consensus effects using meta-analysis measures of musculoskeletal fitness (muscle strength and power) are difficult to achieve in a meaningful way because there are so many different testing approaches, protocols, outcome dimensions, and muscle groups that can be used. Resistance training and mixed training studies included in this review that measured musculoskeletal fitness did so via a range of outcomes, including not just maximal strength but also other dimensions such as local muscular endurance and maximal explosive power output.

Most resistance training studies individually showed improvements in musculoskeletal fitness and in some cases these were substantial in magnitude and dominated by lower limb muscle groups.

Mixed training studies reported a range of outcomes with most of the examples of a beneficial direction of effect within lower limb rather than upper limb musculature.

In this review update there is evidence of increased interest in resistance training and thus musculoskeletal fitness measures occur more commonly. However, the certainty of the evidence is still low to moderate.

Several studies examine dynamic expressions of musculoskeletal fitness such as explosive power output (Fernandez-Gonzalo 2016; Mead 2007; Ouellette 2004). In people with stroke, explosive power is associated with function and disability after stroke (Saunders 2008), and in elderly people explosive power output may be more 
important than strength for function and disability (Puthoff 2007). Interventions to improve explosive power after stroke remain under-investigated.

\section{Mobility}

All the meta-analyses of walking performance outcomes are summarised in Table 4 and this shows a clear pattern of findings.

Cardiorespiratory training increased maximal walking speed, preferred walking speed, and walking capacity (6-MWT) at the end of the training period (Analysis 1.12; Analysis 1.13; Analysis 1.14). When these analyses are restricted to studies that have a balanced control group exposure, and that use walking as a mode of exercise, all three magnitudes of beneficial effect increase, suggesting the importance of exercise being task-related. Benefits were retained in both maximum walking speed (Analysis 2.3), and 6-MWT (Analysis 2.5). Benefits to walking performance also emerge when walking in a community setting outside the research environment (Analysis 1.16). There is evidence that suggests that cardiorespiratory training, as well as improving walking speed, may reduce the reliance of stroke survivors on other people to assist with ambulation (Functional Ambulation Categories score; Analysis 1.11). There is high certainty of the evidence for cardiorespiratory training.

Resistance training benefited only walking capacity (6-MWT) and not maximal or preferred walking speed. It is worth noting that most of the resistance training interventions did not incorporate walking as a mode of exercise. Improvements noted in muscle strength may not necessarily produce functional benefits (Kim 2001), which translate into a better walking performance. The relationships between 'fitness' and 'function' is indeed very complex and may arise from factors such as non-linear associations (Buchner 1991), or the interaction of 'co-impairments' such as lack of balance and low muscle strength (Rantanen 2001). In this review it could also be that the intramuscular metabolic adaptations arising from resistance training increased the tolerance of participants to any fatiguing effects of the performance-based 6-MWT. However, there is low certainty of the evidence for this outcome.

Mixed training increased maximal walking speed, preferred walking speed, and walking capacity (6-MWT) at the end of the training period (Analysis 5.14; Analysis 5.15). These effect sizes are all similar in magnitude to those for cardiorespiratory training and they are also of lower certainty (low to moderate). However, these consensus effects are more tenuous because of heterogeneity, some of which can be explained by the fact that all are dominated by imbalanced exposures, which could exaggerate the effects. Benefits were retained only in the 6-MWT performance (Analysis 6.8). Moreover, all studies, except Yang 2006, included specific walking training.

When comparing the effects of the different interventions (comparison 7) it is cardiorespiratory training with or without resistance training (mixed training) that benefits walking speed outcomes. Within this analysis it is clear that the cardiorespiratory element is key and this is most effective when presented via a walking mode of exercise. Resistance training with or without a cardiorespiratory element (mixed) also benefits walking speed but these data are dominated by the effect of mixed training and they are less trustworthy.
Walking speed improvements after training could be connected to an increased fitness reserve (arising from an increased $\mathrm{VO}_{2}$ peak and/or improved gait economy). Walking-based training interventions dominate the effects of both cardiorespiratory and mixed training and these are by definition task-related and repetitive in nature. These elements by themselves may facilitate motor learning and benefit gait performance even in the absence of an obvious improvement in physical fitness parameters.

Therefore, on the whole, there is consistent evidence that measures of walking performance improve after both cardiorespiratory training and mixed training, but not after resistance training alone. Although the improvements are clear, one could still question whether they are clinically important. For example, Fulk 2011 concluded that a clinically important increase in preferred walking speed after stroke would be $10.5 \mathrm{~m} /$ minute; this is greater than the upper $95 \%$ confidence interval $(\mathrm{Cl})$ margin of the effect sizes for preferred walking speed in this review. Fulk 2018 suggested that the minimum clinically important difference for 6 -MWT is +71 to +130 metres based on patients who are initially fast walkers $(\geq 0.4 \mathrm{~m} /$ $\mathrm{sec})$; these exceed the effects in this review. Baseline gait speed will remain an important consideration for making judgements about magnitude of effects in walking speed outcomes.

\section{Physical function}

A variety of measures to assess motor function were used in the included studies; balance data and Timed Up and Go data could each be pooled and were recorded across all training types.

All three main intervention types collected Berg Balance score data and each showed beneficial effect sizes. Most cardiorespiratory training studies involved walking, and these contributed most of the beneficial effect (moderate certainty). Mixed training data were less trustworthy and possibly affected by confounding (low certainty). Resistance training interventions demonstrated the largest overall effect but were of low certainty. Taken all together interventions involving cardiorespiratory training with or without resistance training (mixed) improved balance. Likewise resistance training with or without cardiorespiratory training (mixed) improved balance. These data suggest that there is more to training balance that just specific 'balance training' interventions as demonstrated by the similar effects of resistance training and of walking-type exercise. Other systematic reviews have examined cardiorespiratory training effects (Pang 2013), and trunk training effects (Cabanas-Valdés 2013; Sorinola 2014), on balance after stroke. Although Sorinola 2014 reported a significant effect of trunk exercise on standing balance (SMD $0.72,95 \% \mathrm{Cl}-0.01$ to 1.45 ; P $=0.05)$ the balance data as a whole are not convincing and these reviews have different eligibility criteria to the current review. The systematic review of Van Duijnhoven 2016 examined the effects of different exercise therapy interventions and showed that these benefited balance; the overall effect size on the Berg Balance scale was 2.22 points ( $95 \% \mathrm{Cl} 1.26$ to 3.17 ), which is very similar to our data. In addition, Van Duijnhoven 2016 noted that it was specific balance and/or weight-shifting training that gave the largest effect size ( 3.75 points $95 \% \mathrm{Cl} 1.71$ to 5.78 ), whereas in this review, the largest effect was after resistance training and this is of similar magnitude (MD 3.27, 95\% $\mathrm{Cl} 2.15$ to 4.38 ).

All three training types appear to give a similar overall effect on Timed Up and Go performance. While there is no clear minimum clinically-important difference established for this outcome, it does 
relates to mobility, balance and risk of falls and is therefore relevant to people with stroke.

\section{Health status and quality of life}

Data do exist that examine quality-of-life measures; however, studies use a range of different tools to report different subscales and summary statistics, which makes meaningful pooling of these data difficult. Therefore, few conclusions can be drawn on whether training can improve self-perceived health status and quality of life after stroke. Taking into account all the data, there is also no discernible pattern in terms of studies showing or not showing a beneficial direction of effect across physical, mental, or emotional domains of the scales used. In addition, 14 out of 19 studies that included quality-of-life outcomes were confounded for additional intervention time, which means it is difficult to attribute any effects to the content of training. A systematic review of exercise after stroke included quality of life outcomes and also concluded that there was no consistent effect (Pang 2013).

\section{Mood}

Few studies of variable methodological quality were available to assess the effects of training on mood. There is no consistent pattern of effects and the pool of studies are at risk of bias due to attrition and confounding due to imbalanced exposures.

A recent systematic review of exercise for depressive symptoms after stroke combined data from 13 studies, with a total of 1022 participants, and showed a small effect at the end of exercise (SMD $-0.13,95 \% \mathrm{Cl}-0.26$ to $0.01 ; \mathrm{P}=0.03$ ), but not follow-up (Eng 2014). These findings may differ from the current review, since Eng 2014 pooled different exercise types; only five out of 13 RCTs met our eligibility criteria, and we made a methodological decision not to pool the data from Lennon 2008 in our meta-analyses (as this would have involved estimating mean and standard deviation from median and range).

In both reviews small benefits could be exaggerated due to confounded exposure. Also, any lack of effect could arise from the fact that the symptoms of depression are relatively mild; this could be due to confounding by antidepressant medications, which were not reported.

\section{Cognitive function}

Only six studies have examined the effect of fitness training interventions on cognitive function outcomes; currently no conclusions can be drawn. There are other systematic reviews that have examined the effects of exercise on cognitive function (Cumming 2012; Garcia-Soto 2013; Zheng 2016). The review of Cumming 2012 showed that physical activity and exercise interventions produced significant improvements in cognitive function at the end of the intervention (SMD $0.20,95 \% \mathrm{Cl} 0.04$ to $0.36 ; \mathrm{P}=0.015$; nine studies, 716 participants). Although six out of 13 of the studies involved exercise interventions only three out of 13 included studies meet our inclusion criteria (Bateman 2001; Duncan 2003 (cited as Studentski); Mead 2007). Garcia-Soto 2013 reviewed the effects of cardiorespiratory and resistance training interventions on cognitive function after stroke. None of the five included studies met our criteria for inclusion. Likewise, Zheng 2016 examined the effect of cardiorespiratory exercises and none of the 10 included studies met our eligibility criteria.
There is a rationale as to why cognitive function may be improved by fitness training (Heyn 2004), and interventions to improve cognitive function have emerged as the highest rated research priority with regard to life after stroke (Pollock 2012). There are still very few data to determine whether exercise is beneficial, therefore this remains an important knowledge gap.

\section{Factors influencing primary and secondary outcome measures}

Performing subgroup analyses is challenging when the number of studies is small; the consequences are reduced power and the influence of characteristics unrelated to the grouping factors.

\section{Dose of training}

All the training interventions in this review occurred regularly and were progressive in nature. The interventions differed in the dose of training, quantified in terms of the intensity of the exercise, and overall volume of training time.

With regard to cardiorespiratory training interventions (Table 1), and studies that together reported a clear improvement in cardiorespiratory fitness (Analysis 1.9), nine out of the 10 studies reported exercise intensity as $60 \%$ to $80 \%$ heart rate reserve, $50 \%$ to $85 \%$ maximum heart rate, or $30 \%$ to $50 \%$ maximum effort; rate of perceived exertion was reported as 13 to 15 in two of 10 studies; there is some variation. Likewise, programme length varied from less than 12 weeks in four out of 10 studies to 12 weeks or longer in six out of 10 studies. There is heterogeneity in the analysis of cardiorespiratory fitness but this gives no obvious explanation to magnitude of effects. It is worth noting that all these studies involved cardiorespiratory training, which was regular (mostly 3 to 5 days/week) and progressive. The undeniable tendency is for an improved fitness response across all the studies irrespective of dose, so perhaps precise prescription of intensity is less important compared to being able to engage with exercise, which is regular and progressive; this elicits a physiological adaptation.

With regard to resistance training interventions (Table 2), eight out of 20 studies reported intensity of $70 \%$ to $80 \%$ maximal strength, and five out of 20 studies stated that a maximum effort was required over a target number of repetitions. Overall, 12 out of 20 studies required six to 15 repetitions of each exercise used; nine out of 20 studies reported that three to six sets of each exercise were performed. Programme length varied from less than 12 weeks in 14 out of 20 studies to 12 weeks or longer in six out of 10 studies. Like cardiorespiratory training, there is variation in overall dose and some studies activated more muscle groups/used differing numbers of exercises than others. Although we synthesised data without meta-analysis, there is a clear pattern of improved muscle strength arising from resistance training interventions. Regular resistance training can stimulate musculoskeletal adaptations in relatively short exercise programmes, and although these vary, they are regular and progressive.

With regard to mixed training interventions (Table 3), where the dose parameters for the cardiorespiratory and resistance training components are reported, these are similar in nature to those described above.

With regard to dose, the reality of progression is that dose is not fixed and should constantly change to drive adaptations. In those who respond more, progression may occur faster whilst being more conservative in those who adapt slower. A 'one-size-fits-all' dose, 
particularly in terms of intensity and progression, does not seem realistic and should be instead be personalised. With regard to a starting dose, perhaps this is less important because progression will move things on quickly; just doing something will be a good start and help familiarise patients with what is involved.

Underestimation of benefits may arise if interventions are poorly attended or complied with. Few included studies had full attendance, where interventions occurred partly or completely during inpatient care, were home-based, or were of very short duration (four weeks). Overall, there were no real threats to planned dose among the included studies as a whole. This is a reflection of there being few adverse events and indicates that these interventions are acceptable to those who participated. However, the patient mix of included participants may have restricted generalisability the wider stroke population.

Overestimation of benefits may arise in studies where the intervention group is potentially confounded by increased training time compared with the control group; this is adding an undefined element of 'dose', which may involve physical activity. In these studies with no attention control, additional benefits could arise from non-specific effects of therapist input, psychosocial effects of contact with other participants, and factors such as travel to and from a training location that could amount to a substantial dose of physical activity from which a real training effect could arise.

Although benefits have emerged in programmes less than 12 weeks in length, there is some evidence to suggesting that four- to sixweek programmes of physical rehabilitation are less effective than those of eight to 14 weeks (Pollock 2014). There is no reason why longer programmes of fitness training would not be more effective, likewise increased physical activity during the whole of life after stroke could achieve or maintain some of the same benefits as exercise.

Overall, the findings of this systematic review indicate that stroke survivors may successfully participate in, and complete, a variety of short-term training interventions. An 'optimal' dose for the content of training for people with stroke has yet to be established. In reality, this is likely to be highly individual and therefore a personalised, stratified approach to physical fitness training seems more appropriate.

\section{Type of training}

We were able to compare the effects of the different types of training on gait speed. Walking speed increased significantly after cardiorespiratory training and mixed training, but not after resistance training. Both cardiorespiratory interventions and mixed interventions comprised specific gait-related training, which resulted in positive training effects.

Overall, the findings of this review show that benefits reflect the concept of specificity, the task-related nature of the training response. In particular, cardiorespiratory fitness $\left(\mathrm{VO}_{2}\right.$ peak) improved after interventions including cardiorespiratory training; muscle strength improved after interventions including resistance training; walking performance improved in particular after training interventions based on walking or walking-like modes of exercise.

Balance was improved by gait-containing cardiorespiratory training content but also by resistance training content; the different stimuli and adaptations reflect the multi-dimensional nature of balance.

Overall, there is no one 'optimal' training type. Most patients are likely to benefit from a mixed programme of cardiorespiratory plus resistance training for secondary prevention, to improve fitness, improve mobility, and improve balance.

\section{Timing of training}

All our meta-analyses were divided into 'during usual care' and 'after usual care' subgroups. However, these analyses should be interpreted with caution as for many comparisons only a limited number of studies was available.

\section{Overall completeness and applicability of evidence}

\section{We include patient-centred outcomes: still a lack of key outcomes}

A priority-setting exercise undertaken in 2012 identified the top 10 research priorities for life after stroke (Pollock 2012). Among the priorities identified by people with stroke and those who care for them are a substantial proportion of areas for which fitness training could be beneficial:

- cognitive function;

- upper limb function;

- mobility, balance and gait; and

- the role of exercise in physical function, quality of life, and secondary stroke prevention (Saunders 2014a).

Apart from mobility, balance, and some aspects of function, there is little evidence relating to the other areas, which patients identified as relevant. In particular, cognitive function lacks investigation despite being ranked the most important research priority.

To measure disability and dependence in stroke is problematic. A variety of disability and assessment scales are usually reported in studies of physical rehabilitation and fitness training. These scales do not always assess the same functional domain and therefore pose the problem of the validity and reliability of combining their results in a meta-analysis. Furthermore, some of these scales are not validated in stroke survivors and, therefore, may lack specificity. Rating scales are also prone to a 'ceiling effect' and to skewed distributions. It would be useful if only well-known, validated scales were used in future studies for the assessment of participants' functional performance and if study investigators would clearly address the problems related to the use of these scales.

Stroke survivors who are eligible for fitness training have typically mild levels of disability. Mild impairments may be difficult to assess and many of the existing disability scales may fail to detect them. However, functional decline over time that is simply due to increasing age and inactivity could mean that mild disability may progress quickly to more serious levels. Therefore, it would be useful to assess long-term outcomes in mild stroke survivors using pre-clinical disability measures (e.g. Fried 1996).

\section{Some lack of follow-up data}

Both improvements in physical fitness after training and improvements in physical function after rehabilitation are transient. Since physical fitness may be linked to functional status, 
the long-term retention of benefit should be routinely examined in studies assessing the effects of fitness training. Fitness and function parameters are known to deteriorate with physical inactivity and to decrease with increasing age. Therefore, it is plausible that shortterm effects of training only emerge as being beneficial after a period of functional decline. There is a need to examine strategies aimed at promoting physical activity (including reduction of sedentary behaviour) and maintaining physical fitness in the long term after stroke.

Functional advantages observed at the end of rehabilitation interventions are known to be transient, disappearing at a later stage (Kwakkel 2002). This is probably due to continued improvements in the control group rather than deterioration in function (Langhorne 2002). Fitness improvements observed at the end of training interventions are also known to deteriorate. Few studies included in this systematic review assessed possible retention of benefits over time. Those that did were at increased risk of attrition bias. Most of the functional improvements observed at the end of the training period were not sustained at later assessments. We found, however, that cardiorespiratory and mixed training effects on some measures of walking performance were retained at the end of the follow-up period. This retention effect could have arisen from an increase in habitual levels of physical activity (including walking) facilitated by participation in a training intervention. The extent to which short-term fitness training influences longer-term, habitual physical activity after stroke is still unknown. Currently, there are no data examining either longterm fitness training interventions or interventions to facilitate continued exercise after the training intervention is completed. Long-term assessments should be incorporated into future studies of physical fitness training. Long-term uptake of exercise could benefit from additional behaviour change support rather than just a finite exercise intervention (e.g. eight-week programme).

\section{Interventions are typically short}

While programmes of exercise may be finite, patients are likely to benefit from activity (including exercise) during the whole of life after stroke. Other interventions, such as being more physically active and being less sedentary, are, like exercise, also 'energy expending' in nature and may provide some similar benefits to exercise. It is plausible that these approaches may contribute to what exercise can offer and facilitate long-term retention and benefits.

\section{Participant characteristics}

Most participants were ambulatory and were higher functioning. Only five out of 75 studies included participants who were nonambulatory, so there are difficulties in generalising the review findings to people with stroke who cannot walk. Included studies used eligibility criteria relating to contraindications to exercise; this means that participants are more likely have better function and health status. Although this restricts the population, the data are still generalisable to people who are screened as being able to safely participate in exercise interventions. The average age of participants was approximately 62 years. The findings are considered generalisable since two-thirds of all strokes are among those aged under 70 years (Feigin 2017).

\section{Increase in studies including resistance training elements}

Compared with previous updates of this systematic review, we have observed an increase in studies with a resistance training element. This means the data about fitness training are now more complete. The increase in interest mirrors that in other areas of public health (Steele 2017).

\section{Nature of control groups: confounding}

More than half of the included studies (41/75 studies; $55 \%$ ) have a design without attention control, meaning that the exposure time is not equivalent. This confounds these data such that the benefits can only be attributed to the whole exposure rather than the content and design of the fitness training itself.

\section{Quality of the evidence}

All the data we have reviewed are from RCTs. The main threat concerning conclusions about the content and design of the exercise programmes is due to imbalanced exposure. Our sensitivity analyses identify where this may be happening.

\section{Potential biases in the review process}

At the search stage it is possible that some relevant studies were missed. However, we used a very comprehensive search strategy and ensured that every stage of inclusion involved an independent consensus decision by two review authors.

At the study selection stage it is possible that the interventions were misclassified resulting in either exclusion or resulting in misclassification of primary subgroup (cardiorespiratory, resistance, or mixed training). However, we used well-known definitions for fitness training that allowed clear criteria to be used (USDHHS 2018).

At the data extraction stage most included studies did not formally include adverse events as a pre-planned outcome. However, we screened all studies for adverse and serious adverse events.

At the data extraction stage two review authors extracted all study characteristics and 'Risk of bias' items independently and reached a consensus. One review author extracted outcome data but all the analysed data in Review Manager 2014 were double checked by a second review author.

At the data analysis stage missing data from one included study were interpolated before analysis (Bateman 2001). Where heterogeneity was introduced we tested the effect of including this study with a sensitivity analysis.

At the data analysis stage there could be publication bias and smallstudy biases that affected the conclusions. We did test for evidence of publication bias where a meta-analysis included 10 or more studies. There was no evidence of problematic publication biases.

At the data analysis stage we examined a range of different classes of outcomes; this is a broad review with that aim. However, small feasibility or pilot studies with high risk of bias and multiple outcomes may be more 'visible' in this review as the data may appear in multiple meta-analyses of different outcomes. Conversely, large studies focused on limited numbers of outcomes may be less 'visible'. Small studies with multiple outcome measures 
are also more vulnerable to showing positive effects by chance if not adjusted for multiple comparisons.

At the reporting stage we made post hoc decisions about which outcomes to include in the tables of studies. We included the findings for the primary outcomes and we did this for all three training types irrespective of effect. Secondary outcomes that were not included are clearly stated as being incomplete data on which a consensus cannot be reached.

\section{Agreements and disagreements with other studies or reviews}

We retrieved 29 systematic reviews relating to exercise after stroke during the searches for this update. On the whole these differ from the architecture of this broad review because most tend to focus on one type of exercise (e.g. cardiorespiratory or resistance training), one mode of exercise (e.g. walking/treadmill), or report a restricted range of types of outcome measures. Therefore, this review is much more comprehensive in terms of capturing both the complexity of intervention and also the range of potential benefits from exercise as a whole.

Outcome by outcome there are some patterns of similarity. These include that cardiorespiratory fitness can be improved (Baldwin 2016; Boyne 2017; Saltychev 2016); balance can be improved by cardiorespiratory, resistance, and mixed training (Chen 2016; Hasan 2016; latridou 2018; Tally 2017; Van Duijnhoven 2016; Vloothuis 2016; Wist 2016); and walking/mobility can be improved by mixed and cardiorespiratory training (Baldwin 2016; Bonini-Rocha 2018; Boyne 2017; English 2017; Ilunga Tshiswaka 2018; Jeon 2015; Kendall 2016; Mehrholz 2017), particularly if walking is used as an exercise mode. There are also some shared concerns among other systematic reviews about the benefits of resistance training alone in terms of carry over of functional benefits (Dorsch 2018; Salter 2016; Wist 2016).

There are some differences, with several reviews showing positive effects on cognitive outcomes (Hasan 2016; Oberlin 2017; Vanderbeken 2017; Zheng 2016), whereas our analyses were inconclusive. One reason why our findings and those of other reviews may differ is that some reviews included other cointervention elements that we would automatically exclude, and they may also have included studies that do not meet our criteria for being defined as exercise. One review author team shared our concerns about inadequate reporting, and the nature and dose of exercise interventions (Ammann 2014).

\section{AUTHORS' CONCLUSIONS}

\section{Implications for practice}

Cardiorespiratory training alone can improve cardiorespiratory fitness. As well as benefiting functional capacity this may have a risk reduction effect for secondary events.

Cardiorespiratory training alone or combined with a resistance training element (mixed training) improves the speed and capacity of walking when a walking mode of exercise is used; some of these effects are retained

Cardiorespiratory training or resistance training alone or in combination (mixed training) improves indices of balance. The largest effects relate to resistance training. These effects may reduce risk of falls.

Therefore, for people with stroke who are able to take part in exercise there are good reasons to want to combine different training types in order to maximise benefits directly relating to physical fitness and mobility, and indirectly relating to reduction of risk of falls and secondary prevention.

A range of initiatives including practitioner training, best practice guidelines, and recommendations have been developed worldwide that help facilitate the flow of research information into practice. For example:

- UK (Exercise and Fitness Training after Stroke Instructor course; www.laterlifetraining.co.uk/courses/exercisefor-stroke-instructor/);

- Canada (Canadian Best Practice Recommendations for Stroke Care; www.strokebestpractices.ca/);

- USA (APA Physical Activity and Exercise Recommendations for Stroke Survivors; Billinger 2014).

These initiatives are based on existing evidence about the benefits of exercise after stroke and the needs of stroke survivors to have ongoing access to rehabilitation after discharge from hospital; they can inform service delivery.

There are now a range of service delivery models in place in different countries around the world (Australia, Brazil, Canada, India, Nigeria, Singapore, Sweden, UK, and USA; Van Wijck 2019). The European Stroke Action Plan 2018-2030 gives a clear mandate for implementation of services including fitness training, stating a target for 2030 of "Offering physical fitness programmes to all stroke survivors living in the community" (Norrving 2018).

The findings of this systematic review update will contribute to evidence-based pathways aimed at improving life after stroke.

\section{Implications for research}

Larger, well-designed clinical studies are needed to assess the effects of physical fitness training after stroke and to determine the optimal regimen for improving fitness.

Future studies should:

- comply with the current CONSORT guidelines for reporting of randomised clinical studies (CONSORT 2010);

- report exercise and control interventions more clearly; intervention reporting guidelines do exist (TIDiER; Hoffmann 2014) including some specific to exercise (CERT Consensus Exercise Reporting Template; Slade 2014);

- include a broader population of stroke survivors (including nonambulatory stroke survivors) to allow stratification by gender, level of impairment, and functional ability;

- assess the effects of physical fitness training in people with specific post-stroke problems, for example people with depression or post-stroke fatigue;

- be of longer duration (12 weeks or longer);

- have a long-term follow-up; and

- have some type of attention control to reduce confounding. 


\section{ACK N OWLEDGEMENTS}

Parts of the Background and Methods sections of this review include sections of verbatim template text because the approaches used correspond to the protocol of a connected review by some of the same author team investigating interventions to reduce sedentary behaviour after stroke (Saunders 2018). The approach is permitted by The Cochrane Publication Policy.
We thank the Cochrane Stroke editorial team for their assistance in preparing the protocol. We are grateful to Josh Cheyne for his assistance in developing the search strategies. We also thank all investigators who provided further information about existing studies.

We would be grateful if people who are aware of studies potentially relevant for this review could contact David Saunders. 


\section{R E F E R E N C E S}

\section{References to studies included in this review}

Ada 2013 \{published data only\}

* Ada L, Dean CM, Lindley R. Randomized trial of treadmill training to improve walking in community-dwelling people after stroke: the AMBULATE trial. International Journal of Stroke 2013;8(6):436-44.

Dean CM, Ada L, Lindley RI. Treadmill training provides greater benefit to the subgroup of community-dwelling people after stroke who walk faster than $0.4 \mathrm{~m} / \mathrm{s}$ : a randomised trial. Journal of Physiotherapy 2014;60(2):97-101.

\section{Aidar 2016 \{published data only\}}

Aidar FJ, Gama de Matos D, Jacó de Oliveira R, Carneiro AL, Cabral BG, Dantas PM, et al. Relationship between depression and strength training in survivors of the ischemic stroke. Journal of Human Kinetics 2014;43:7-15.

Aidar FJ, Jacó de Oliveira R, Silva AJ, Gama de Matos D, Mazini Filho ML, Hickner RC, et al. The influence of resistance exercise training on the levels of anxiety in ischemic stroke. Stroke Research and Treatment 2012;2012:298-375.

* Aidar FJ, Jacó de Oliveira RJ, Gama de Matos D, Mazini Filho ML, Moreira OC, de Oliveira CE, et al. A randomized trial investigating the influence of strength training on quality of life in ischemic stroke. Topics in Stroke Rehabilitation 2016;23(2):84-9. [DOI: 10.1080/10749357.2015.1110307]

\section{Aidar 2018 \{published data only\}}

* Aidar FJ, Jacó de Oliveira R, Gama de Matos D, Chilibeck PD, Carneiro AL, Machado Reis V. A randomized trial of the effects of an aquatic exercise program on depression, anxiety levels, and functional capacity of people who suffered an ischemic stroke. Journal of Sports Medicine and Physical Fitness 2018;58(7-8):1171-7. [DOI: 10.23736/S0022-4707.17.07284-X]

Aidar FJ, Silva AJ, Machado Reis V, Carniero A, Carniero-Cotta S. A study on the quality of life in ischaemic vascular accidents and its relation to physical activity [Estudio de la calidad de vida en el accidente vascular isquémico y su relación con la acividad física]. Revista de Neurología 2007;45:518-22.

\section{Arabzadeh 2018 \{published data only\}}

Arabzadeh S, Goljaryan S, Salahzadeh Z, Oskouei AE, Somee AS. Effects of a task-oriented exercise program on balance in patients with hemiplegia following stroke. Iranian Red Crescent Medical Journal 2018; Vol. 20, issue 1:e38429.

\section{Bale 2008 \{published data only\}}

Bale M, Strand LI. Does functional strength training of the leg in subacute stroke improve physical performance? A pilot randomized controlled trial. Clinical Rehabilitation 2008;22(10-11):911-21.

\section{Bateman 2001 \{published and unpublished data\}}

Bateman A, Culpan FJ, Pickering AD, Powell JH, Scott OM, Greenwood RJ. The effect of aerobic training on rehabilitation outcomes after recent severe brain injury: a randomized controlled evaluation. Archives of Physical Medicine and Rehabilitation 2001;82(2):174-82.

\section{Buyukvural 2015 \{published data only\}}

Büyükvural SS, Ozbudak DS, Ekiz T, Ozgirgin N. Effects of the bilateral isokinetic strengthening training on functional parameters, gait, and the quality of life in patients with stroke. International Journal of Clinical and Experimental Medicine 2015;8(9):16871-9.

\section{Cooke 2010 \{published data only\}}

Cooke E, Pomeroy VM, Miller S, Tallis RC. The effects of functional strength training on lower limb strength and function after stroke. Cerebrovascular Diseases 2006;21 Suppl 4:104

Cooke E, Tallis R, Miller S, Pomeroy V. The effects of functional strength training on lower limb strength and function after stroke. UK Stroke Forum Conference. 2006.

Cooke E, Tallis R, Pomeroy V. The effects of different intensity of exercise-based physiotherapy on motor recovery in the lower limb after stroke: a meta-analysis. UK Stroke Forum Conference 4-6 December 2007. The Stroke Association, 2007.

* Cooke EV, Tallis RC, Clark A, Pomeroy VM. Efficacy of functional strength training on restoration of lower-limb motor function early after stroke: phase I randomized controlled trial. Neurorehabilitation and Neural Repair 2010;24(1):88-96.

Cooke EV, Tallis RC, Miller S, Pomeroy VM. The effects of functional strength training on lower limb strength and function after stroke. Cerebrovascular Diseases 2008;25 Suppl 2:45-6.

Cooke EV, Tallis RC, Miller S, Pomeroy VM. The effects of type and intensity of physiotherapy on lower limb strength and function after stroke. Cerebrovascular Diseases 2007;23 Suppl 2:129.

Kerr A, Clark A, Cooke EV, Rowe P, Pomeroy VM. Functional strength training and movement performance therapy produce analogous improvement in sit-to-stand early after stroke: early-phase randomised controlled trial. Physiotherapy 2017;103(3):259-65.

\section{Coroian 2018 \{published data only\}}

Coroian F, Froger J, Koang HY, Jourdan C, Bakhti K, Julia M, et al. Isokinetic muscular strengthening of upper limb versus passive movement in chronic stroke patients. Randomised controlled trial. Annals of Physical and Rehabilitation Medicine 2016;59:e72.

* Coroian F, Jourdan C, Bakhti K, Palayer C, Jaussent A, Picot MC, et al. Upper limb isokinetic strengthening versus passive mobilization in patients with chronic stroke: a randomized controlled trial. Archives of Physical Medicine and Rehabilitation 2018;99(2):321-8.

\section{Cuviello-Palmer 1988 \{published data only\}}

Cuviello-Palmer ED. Effect of the Kinetron II on Gait and Functional Outcome in Hemiplegic Subjects. Texas, USA: Texas Women's University, 1988. 
da Cunha 2002 \{published data only\}

Lim PA, Henson H, Cunha I, Qureshy H, Monga TN, Protas EJ. Body weight-supported gait training in stroke patients. American Journal of Physical Medicine and Rehabilitation 2000;79(2):203.

da Cunha IT, Lim PA, Qureshy H, Henson H, Monga T, Protas EJ. A comparison of regular rehabilitation with supported treadmill ambulation training for acute stroke patients. Journal of Rehabilitation Research and Development 2001;38(2):245-55.

* da Cunha IT, Lim PA, Qureshy H, Henson H, Monga T, Protas EJ. Gait outcomes after acute stroke rehabilitation with supported treadmill training: a randomized controlled pilot study. Archives of Physical Medicine and Rehabilitation 2002;83(9):1258-65.

\section{Dean 2018 \{published data only\}}

Dean S, Calitri R, Shepherd A, Hollands L, Poltawski L, James M, et al. Community-based rehabilitation training after stroke (ReTrain): results of a pilot randomised control trial (RCT). International Journal of Stroke 2016;11(4 Suppl 1):13.

Dean SG, Poltawski L, Forster A, Taylor RS, Spencer A, James M, et al. Community-based rehabilitation training after stroke: protocol of a pilot randomised controlled trial (ReTrain). BMJ Open 2016;6(10):e012375.

* Dean SG, Poltawski L, Forster A, Taylor RS, Spencer A, James $\mathrm{M}$, et al. Community-based rehabilitation training after stroke: results of a pilot randomised controlled trial (ReTrain) investigating acceptability and feasibility. BMJ Open 2018;8(2):e018409.

Hollands H, Calitri R, Shepherd A, Poltawski L, Norris M, Taylor R, et al. The ReTrain trial: evaluating intervention fidelity via video analysis of the independently getting up off the floor (IGO) technique. International Journal of Stroke 2016;11(4 Suppl 1):58-9.

\section{Donaldson 2009 \{published data only\}}

* Donaldson C, Tallis R, Miller S, Sunderland A, Lemon R, Pomeroy V. Effects of conventional physical therapy and functional strength training on upper limb motor recovery after stroke: a randomized phase II study. Neurorehabilitation and Neural Repair 2009;23(4):389-97.

Donaldson C, Tallis R, Miller S, Sunderland A, Lemon R, Pomeroy $V$. The effects of current physical therapy and functional strength training on upper limb function and neuromuscular weakness after stroke: a pilot study (Abst. poster 156). UK Stroke Forum Conference. The Stroke Association, 4-6 December 2007.

Donaldson C, Tallis R, Pomeroy V. The effects of current physical therapy and functional strength training on upper limb function and neuromuscular weakness after stroke: a pilot study. Cerebrovascular Diseases 2007;23 Suppl 2:130.

\section{Duncan 1998 \{published data only\}}

Duncan P, Richards L, Wallace D, Stoker-Yates J, Pohl P, Luchies $C$, et al. A randomized, controlled pilot study of a home- based exercise program for individuals with mild and moderate stroke. Stroke 1998;29(10):2055-60.

Duncan 2003 \{published data only\}

* Duncan P, Studenski S, Richards L, Gollub S, Lai SM, Reker D, et al. Randomized clinical trial of therapeutic exercise in subacute stroke. Stroke 2003;34(9):2173-80.

Lai SM, Studenski S, Richards L, Perera S, Reker D, Rigler S, et al. Therapeutic exercise and depressive symptoms after stroke. Journal of the American Geriatric Society 2006;54:240-7.

Perera S, Mody SH, Woodman RC, Studenski SA. Meaningful change and responsiveness in common physical performance measures in older adults. Journal of the American Geriatrics Society 2006;54:743-9.

Schmid A, Duncan PW, Studenski S, Lai SM, Richards L, Perera S, et al. Improvements in speed-based gait classifications are meaningful. Stroke 2007;38:2096-100.

Studenski S, Duncan PW, Perera S, Reker D, Lai SM, Richards L. Daily functioning and quality of life in a randomized controlled trial of therapeutic exercise for subacute stroke survivors. Stroke 2005;36(8):1764-70.

\section{Eich 2004 \{published data only\}}

Eich HJ, Hesse S, Mach H. Aerobic endurance training of hemiparetic patients who are able to walk. Results of a prospective randomised study. Deutsche Zeitschrift für Sportmedizin 2003;54(7/8):S98.

* Eich HJ, Mach H, Werner C, Hesse S. Aerobic treadmill plus Bobath walking training improves walking in subacute stroke: a randomized controlled trial. Clinical Rehabilitation 2004;18(6):640-51.

Eich HJ, Parchmann H, Hesse S, Mach H, Werner C. Aerobic treadmill training plus physiotherapy improves walking ability in subacute stroke patients. A randomized controlled study. Neurologie und Rehabilitation 2004;10(4):187-216.

Fitch S, Elkins M, Moseley A. Was CAP summary faithful?. Australian Journal of Physiotherapy 2005;51:265-6.

Hesse S, Eich HJ, Mach H, Parchmann H, Werner C. Aerobic treadmill training plus physiotherapy improves walking speed and capacity in subacute, moderately affected patients after stroke. Neurologie und Rehabilitation 2005;11(1):7-12.

Moseley A. Treadmill training more effective than Bobath training in improving walking following stroke. Australian Journal of Physiotherapy 2005;51(3):192.

\section{Fernandez-Gonzalo 2016 \{published data only\}}

Fernandez-Gonzalo R, Fernandez-Gonzalo S, Turon M, Prieto C, Tesch PA, Garcia-Carreira MD. Muscle, functional and cognitive adaptations after flywheel resistance training in stroke patients: a pilot randomized controlled trial. Journal of NeuroEngineering and Rehabilitation 2016;13(1):37. 


\section{Flansbjer 2008 \{published data only\}}

Flansbjer UB, Lexell J, Brogardh C. Long-term benefits of progressive resistance training in chronic stroke: a 4-year follow-up. Journal of Rehabilitation Medicine 2012;44(3):218-21.

* Flansbjer UB, Miller M, Downham D, Lexell J. Progressive resistance training after stroke: effects on muscle strength, muscle tone, gait performance and perceived participation. Journal of Rehabilitation Medicine 2008;40(1):42-8.

Flansbjer UB, Miller M, Lexell J. Progressive resistance training after stroke: effects on muscle function, gait performance and perceived participation. Stroke Rehabilitation 2006. Evidence for Stroke Rehabilitation - Bridging into the Future. Goteborg, 2006.

\section{Furnari 2014 \{published data only\}}

Furnari A, Calabro RS, Gervasi G, La Fauci-Belponer F, Marzo A, Berbiglia $F$, et al. Is hydrokinesitherapy effective on gait and balance in patients with stroke? A clinical and baropodometric investigation. Brain Injury 2014;28(8):1109-14.

\section{Galvin 2011 \{published data only\}}

* Galvin R, Cusack T, O'Grady E, Murphy TB, Stokes E. Familymediated exercise intervention (FAME): evaluation of a novel form of exercise delivery after stroke. Stroke 2011;42(3):681-6.

Galvin R, Stokes E, Cusack T. Family-mediated exercises (FAME): an exploration of participant's involvement in a novel form of exercise delivery after stroke. Topics in Stroke Rehabilitation 2014;21(1):63-74.

\section{Glasser 1986 \{published data only\}}

Glasser L. Effects of isokinetic training on the rate of movement during ambulation in hemiparetic patients. Physical Therapy 1986;66(5):673-6.

\section{Globas 2012 \{published data only\}}

* Globas C, Becker C, Cerny J, Lam JM, Lindemann U, Forrester LW, et al. Chronic stroke survivors benefit from highintensity aerobic treadmill exercise: a randomized control trial. Neurorehabilitation and Neural Repair 2012;26(1):85-95.

Mackay-Lyons M. Aerobic treadmill training effectively enhances cardiovascular fitness and gait function for older persons with chronic stroke. Journal of Physiotherapy 2012;58(4):271.

\section{Gordon 2013 \{published data only\}}

Gordon CD, Wilks R, McCaw-Binns A. Effect of aerobic exercise (walking) training on functional status and health-related quality of life in chronic stroke survivors: a randomized controlled trial. Stroke 2013;44(4):1179-81.

Inaba 1973 \{published data only\}

Inaba M, Edberg E, Montgomery J, Gillis MK. Effectiveness of functional training, active exercise, and resistive exercise for patients with hemiplegia. Physical Therapy 1973;53(1):28-35.

\section{Ivey 2010 \{published data only\}}

Ivey FM, Hafer-Macko CE, Ryan AS, Macko RF. Impaired leg vasodilatory function after stroke: adaptations with treadmill exercise training. Stroke 2010;41(12):2913-7.

Ivey 2011 \{published data only\}

Ivey FM, Ryan AS, Hafer-Macko CE, Macko RF. Improved cerebral vasomotor reactivity after exercise training in hemiparetic stroke survivors. Stroke 2011;42(7):1994-2000.

\section{Ivey 2017 \{published data only\}}

Ivey FM, Prior SJ, Hafer-Macko CE, Katzel LI, Macko RF, Ryan AS. Strength training for skeletal muscle endurance after stroke. Journal of Stroke and Cerebrovascular Diseases 2017;26(4):787-94.

\section{James 2002 \{unpublished data only\}}

James JE. Closed kinetic chain training to enhance muscle power, control and retrain dynamic balance under task specific conditions improves functional walking ability in chronic stroke survivors. Dublin, Ireland: National University of Ireland, 2002.

\section{Jin 2013 \{published data only\}}

Jin $\mathrm{H}$, Jiang Y, Wei Q, Chen L, Ma G. Effects of aerobic cycling training on cardiovascular fitness and heart rate recovery in patients with chronic stroke. NeuroRehabilitation 2013;32(2):327-35

\section{Kang 2012 \{published data only\}}

Kang HK, Kim Y, Chung Y, Hwang S. Effects of treadmill training with optic flow on balance and gait in individuals following stroke: randomized controlled trials. Clinical Rehabilitation 2012;26(3):246-55.

Katz-Leurer 2003 \{published data only\}

* Katz-Leurer M, Carmeli E, Shochina M. The effect of early aerobic training on independence six months post stroke. Clinical Rehabilitation 2003;17(7):735.

Katz-Leurer M, Seren I, Keren O, Dvir Z. The influence of early cycling training on balance in stroke patients at the subacute stage. Results of a preliminary trial. Clinical Rehabilitation 2006;20:398-405.

Katz-Leurer M, Shochina M. The influence of autonomic impairment on aerobic exercise outcome in stroke patients. NeuroRehabilitation 2007;22(4):267-72.

Katz-Leurer M, Shochina M, Carmeli E, Friedlander Y. The influence of early aerobic training on the functional capacity in patients with cerebrovascular accident at the subacute stage. Archives of Physical Medicine and Rehabilitation 2003;84:1609-14.

\section{Kim 2001 \{published data only\}}

Kim CM, Eng JJ, MacIntyre DL, Dawson AS. Effects of isokinetic strength training on walking in persons with stroke: a double-blind controlled pilot study. Journal of Stroke and Cerebrovascular Diseases 2001;10(6):265-73. 
Kim 2014 \{published data only\}

Kim M, Cho K, Lee W. Community walking training program improves walking function and social participation in chronic stroke patients. Tohoku Journal of Experimental Medicine 2014;234(4):281-6.

\section{Kim 2016a \{published data only\}}

Kim SM, Han EY, Kim BR, Hyun CW. Clinical application of circuit training for subacute stroke patients: a preliminary study. Journal of Physical Therapy Science 2016;28(1):169-74.

\section{Kim 2017a \{published data only\}}

Kim J, Yim J. Effects of an exercise protocol for improving handgrip strength and walking speed on cognitive function in patients with chronic stroke. Medical Science Monitor 2017;23:5402-9.

\section{Knox 2018 \{published data only\}}

Knox M, Stewart A, Richards CL. Six hours of task-oriented training optimizes walking competency post stroke: a randomized controlled trial in the public health-care system of South Africa. Clinical Rehabilitation 2018;32(8):1057-68.

\section{Kuys 2011 \{published data only\}}

Kuys SS, Brauer SG, Ada L. Higher-intensity treadmill walking during rehabilitation after stroke in feasible and not detrimental to walking pattern or quality: a pilot randomized trial. Clinical Rehabilitation 2011;25(4):316-26.

\section{Langhammer 2007 \{published data only\}}

* Langhammer B, Lindmark B, Stanghelle JK. Stroke patients and long-term training: is it worthwhile? A randomized comparison of two different training strategies after rehabilitation. Clinical Rehabilitation 2007;21(6):495-510.

Langhammer B, Lindmark B, Stanghelle KJ. Activity and exercise in a long perspective. A solution for chronic stroke patients?. Stroke Rehab 2006. Evidence for Stroke Rehabilitation - Bridging into the Future. Goteborg, 2006.

\section{Lee 2013a \{published data only\}}

Lee NK, Kwon JW, Son SM, Kang KW, Kim K, Hyun-Nam S. The effects of closed and open kinetic chain exercises on lower limb muscle activity and balance in stroke survivors. NeuroRehabilitation 2013;33(1):177-83.

\section{Lee 2013b \{published data only\}}

Lee NK, Kwon JW, Son SM, Nam SH, Choi YW, Kim CS. Changes of plantar pressure distributions following open and closed kinetic chain exercise in patients with stroke. NeuroRehabilitation 2013;32(2):385-90.

\section{Lennon 2008 \{published data only\}}

Lennon O, Carey A, Gaffney N, Stephenson J, Blake C. A pilot randomized controlled trial to evaluate the benefit of the cardiac rehabilitation paradigm for the non-acute ischaemic stroke population. Clinical Rehabilitation 2008;22(2):125-33.

\section{Letombe 2010 \{published data only\}}

Letombe A, Cornille C, Delahaye H, Khaled A, Morice O, Tomaszewski A, et al. Early post-stroke physical conditioning in hemiplegic patients: a preliminary study. Annals of Physical and Rehabilitation Medicine 2010;53(10):632-42.

MacKay-Lyons 2013 \{published data only\}

MacKay-Lyons M, McDonald A, Matheson J, Eskes G, Klus MA. Dual effects of body-weight supported treadmill training on cardiovascular fitness and walking ability early after stroke: a randomized controlled trial. Neurorehabilitation and Neural Repair 2013;27(7):644-53.

Mao 2015 \{published data only\}

Mao YR, Lo WL, Lin Q, Li L, Xiao X, Raghavan P, et al. The effect of body weight support treadmill training on gait recovery, proximal lower limb motor pattern, and balance in patients with subacute stroke. BioMed Research International 2015;2015:175719.

Mead 2007 \{published data only\}

Mead G. Exercise or relaxation after stroke?. BMJ 2005;330(7503):1337.

* Mead GE, Greig CA, Cunningham I, Lewis SJ, Dinan S, Saunders DH, et al. Stroke: a randomised trial of exercise or relaxation. Journal of the American Geriatrics Society 2007;55(6):892-9.

Mead GE, Greig CA, Cunningham I, Lewis SJ, Dinan S, Saunders DH, et al. Stroke: a randomised trial of exercise or relaxation. UK Stroke Forum Conference 4-6 December 2007. Harrogate, UK: The Stroke Association, 2007.

\section{Moore 2010 \{published data only\}}

Moore JL, Roth EJ, Killian C, Hornby TG. Locomotor training improves daily stepping activity and gait efficiency in individuals poststroke who have reached a "plateau" in recovery. Stroke 2010;41(1):129-35.

\section{Moore 2015 \{published data only\}}

Moore S, Hallsworth K, Jakovljevic D, Blamire A, He J, Ford G, et al. Effects of exercise therapy on metabolic risk factors, brain atrophy and cerebral blood flow following stroke: a randomised controlled trial. International Journal of Stroke 2014;9 Suppl $4: 32$

* Moore SA, Hallsworth K, Jakovljevic DG, Blamire AM, He J, Ford GA, et al. Effects of community exercise therapy on metabolic, brain, physical, and cognitive function following stroke: a randomized controlled pilot trial. Neurorehabilitation and Neural Repair 2015;29(7):623-35.

Moore SA, Jakovljevic DG, Ford GA, Rochester L, Trenell MI. Exercise induces peripheral muscle but not cardiac output adaptations after stroke. A randomised controlled pilot trial. Archives of Physical Medicine and Rehabilitation 2016;97(4):596-603.

\section{Mudge 2009 \{published data only\}}

ACTRN12607000081415. The impact of a group exercise programme on usual walking performance in adults who are at least 6 months post stroke: a single blinded randomised controlled trial. www.anzctr.org.au/Trial/Registration/ 
TrialReview.aspx?id=81826\&isReview=true (first received 24 January 2007).

* Mudge S, Barber PA, Stott NS. Circuit-based rehabilitation improves gait endurance but not usual walking activity in chronic stroke: a randomized controlled trial. Archives of Physical Medicine and Rehabilitation 2009;90(12):1989-96.

\section{Ouellette 2004 \{published data only\}}

Ouellette MM, LeBrasseur NK, Bean JF, Phillips E, Stein J, Frontera WR, et al. High-intensity resistance training improves muscle strength, self-reported function, and disability in longterm stroke survivors. Stroke 2004;35(6):1404-9.

\section{Park 2011 \{published data only\}}

Park HJ, Oh DW, Kim SY, Choi JD. Effectiveness of communitybased ambulation training for walking function of post-stroke hemiparesis: a randomized controlled pilot trial. Clinical Rehabilitation 2011;25(5):451-9.

\section{Pohl 2002 \{published data only\}}

Pohl M, Mehrholz J, Ritschel C, Ruckriem S. Speed-dependent treadmill training in ambulatory hemiparetic stroke patients: a randomized controlled trial. Stroke 2002;33(2):553-8.

\section{Potempa 1995 \{published data only\}}

Potempa K, Lopez M, Braun LT, Szidon JP, Fogg L, Tincknell T. Physiological outcomes of aerobic exercise training in hemiparetic stroke patients. Stroke 1995;26(1):101-5.

\section{Richards 1993 \{published data only\}}

Malouin F, Richards CL, Wood-Dauphinee S, Williams JI. A randomized controlled trial comparing early and intensive task-specific therapy to conventional therapy in acute stroke patients. Canadian Journal of Rehabilitation 1993;7(1):27-8.

* Richards CL, Malouin F, Wood-Dauphinee S, Williams JI, Bouchard JP, Brunet D. Task-specific physical therapy for optimization of gait recovery in acute stroke patients. Archives of Physical Medicine and Rehabilitation 1993;74(6):612-20.

\section{Richards 2004 \{published data only\}}

Richards CL, Malouin F, Bravo G, Dumas F, Wood-Dauphinee S. The role of technology in task-oriented training in persons with subacute stroke: a randomized controlled trial. Neurorehabilitation and Neural Repair 2004;18(4):199-211.

\section{Salbach 2004 \{published data only\}}

* Salbach NM, Mayo NE, Robichaud-Ekstrand S, Hanley JA, Richards CL, Wood-Dauphinee S. The effect of a task-oriented walking intervention on improving balance self-efficacy poststroke: a randomized, controlled trial. Journal of the American Geriatrics Society 2005;53(4):576-82.

Salbach NM, Mayo NE, Wood-Dauphinee S, Hanley JA, Richards CL, Côté R. A task-orientated intervention enhances walking distance and speed in the first year post stroke: a randomized controlled trial. Clinical Rehabilitation 2004;18(5):509-19.

\section{Sandberg 2016 \{published data only\}}

* Sandberg K, Kleist M, Falk L, Enthoven P. Effects of twiceweekly intense aerobic exercise in early subacute stroke: a randomized controlled trial. Archives of Physical Medicine \& Rehabilitation 2016;97(8):1244-53.

Wijkman MO, Sandberg K, Kleist M, Falk L, Enthoven P. The exaggerated blood pressure response to exercise in the subacute phase after stroke is not affected by aerobic exercise. Journal of Clinical Hypertension 2018;20(1):56-64.

Shin 2011 \{published data only\}

Shin WS, Lee SW, Lee YW, Choi SB, Song CH. Effects of combined exercise training on balance of hemiplegic stroke patients. Journal of Physical Therapy Science 2011;23(4):639-43.

\section{Sims 2009 \{published data only\}}

Sims J. Regenerate: a strength training program to enhance the physical and mental health of chronic post stroke patients with depression. Australian New Zealand Clinical Trials Registry (ANZCTR) www.anzctr.org.au/. 2005

* Sims J, Galea M, Taylor N, Dodd K, Jespersen S, Joubert L, et al. Regenerate: assessing the feasibility of a strength-training program to enhance the physical and mental health of chronic post stroke patients with depression. International Journal of Geriatric Psychiatry 2009;24(1):76-83.

Smith 2008 \{published data only\}

Smith PS, Thompson M. Treadmill training post stroke: are there any secondary benefits? A pilot study. Clinical Rehabilitation 2008;22(10-11):997-1002.

\section{Son 2014 \{published data only\}}

Son SM, Park MK, Lee NK. Influence of resistance exercise training to strengthen muscles across multiple joints of the lower limbs on dynamic balance functions of stroke patients. Journal of Physical Therapy Science 2014;26(8):1267-9.

Takami 2010 \{published data only\}

Takami A, Wakayama S. Effects of partial body weight support while training acute stroke patients to walk backwards on a treadmill: a controlled clinical trial using randomized allocation. Journal of Physical Therapy Science 2010;22(2):177-87.

Taylor-Pilliae 2014 \{published data only\}

Taylor-Piliae RE, Coull BM. Community-based Yang-style Tai Chi is safe and feasible in chronic stroke: a pilot study. Clinical Rehabilitation 2012;26(2):121-31.

* Taylor-Pilliae RE, Hoke TM, Hepworth JT, Latt LD, Najafi B, Coull BM. Effect of Tai Chi on physical function, fall rates and quality of life among older stroke survivors. Archives of Physical Medicine and Rehabilitation 2014;95:816-24. [DOI: 10.1016/ j.apmr.2014.01.001]

Teixeira 1999 \{published data only\}

Teixeira L, Nadeau S, Olney S, McBride I, Culham E, Zee B. The impact of a muscle strengthening and physical conditioning program on gait and stair climbing performance in chronic stroke subjects. Gait and Posture 1998;7(2):144-5. 
Teixeira-Salmela LF, Nadeau S, McBride I, Olney SJ. Effects of muscle strengthening and physical conditioning training on temporal, kinematic and kinetic variables during gait in chronic stroke survivors. Journal of Rehabilitation Medicine 2001;33(2):53-60.

* Teixeira-Salmela LF, Olney SJ, Nadeau S, Brouwer B. Muscle strengthening and physical conditioning to reduce impairment and disability in chronic stroke survivors. Archives of Physical Medicine and Rehabilitation 1999;80(10):1211-8.

\section{Toledano-Zarhi 2011 \{published data only\}}

Toledano-Zarhi A, Tanne D, Carmeli E, Katz-Leurer M. Feasibility, safety and efficacy of an early aerobic rehabilitation program for patients after minor ischemic stroke: a pilot randomized controlled trial. NeuroRehabilitation 2011;28(2):85-90.

\section{Topcuoglu 2015 \{published data only\}}

Topcuoglu A, Gokkaya NK, Ucan H, Karaku D. The effect of upper-extremity aerobic exercise on complex regional pain syndrome type I: a randomized controlled study on subacute stroke. Topics in Stroke Rehabilitation 2015; Vol. 22, issue 4:253-61.

\section{Van de Port 2012 \{published data only\}}

Dean C. Group task-specific circuit training for patients discharged home after stroke may be as effective as individualised physiotherapy in improving mobility. Journal of Physiotherapy 2012;58(4):269.

* Van de Port IG, Wevers LE, Lindeman E, Kwakkel G. Effects of circuit training as alternative to usual physiotherapy after stroke: randomised controlled trial. BMJ 2012;344:e2672.

Van der Port IG, Wevers L, Roelse H, Van Kats L, Lindeman E, Kwakkel G. Cost-effectiveness of a structured progressive taskoriented circuit class training programme to enhance walking competency after stroke: the protocol of the FIT-Stroke trial. BMC Neurology 2009;9:43.

\section{Vanroy 2017 \{published data only\}}

* Vanroy C, Feys H, Swinnen A, Vanlandewijck Y, Truijen S, Vissers D, et al. Effectiveness of active cycling in subacute stroke rehabilitation: a randomized controlled trial. Archives of Physical Medicine and Rehabilitation 2017;98(8):1576-85.

Vanroy C, Vanlandewijck Y, Cras P, Truijen S, Vissers D, Swinnen $A$, et al. Does a cycling program combined with education and followed by coaching promote physical activity in subacute stroke patients? A randomized controlled trial. Disability and Rehabilitation 2019;41(4):413-21.

\section{Verheyden 2009 \{published data only\}}

Verheyden G, Vereeck L, Truijen S, Troch M, Lafosse C, Saeys W, et al. Additional exercises improve trunk performance after stroke: a pilot randomized controlled trial. Neurorehabilitation and Neural Repair 2009;23(3):281-6.

\section{Wang 2014 \{published data only\}}

Wang Z, Wang L, Fan H, Lu X, Wang T. Effect of low-intensity ergometer aerobic training on glucose tolerance in severely impaired nondiabetic stroke patients. Journal of Stroke and Cerebrovascular Diseases 2014;23(3):e187-93.

Winstein 2004 \{published data only\}

Rose DK, Winstein CJ, Tan SM, Azen SP, Chui HC. Comparison of upper extremity intervention strategies at six and nine months post-stroke. Neurology Report 2001;25(4):130.

Rose DK, Winstein CJ, Yang AN, Weiss WB, Tan SM, Azen SP, et al. Relationship between upper extremity function and impairment in individuals with unilateral stroke. Neurology Report 1999;23(5):186.

Winstein CJ, Rose DK, Chui HC, Yang AN, Weiss WB, Tan SM, et al. Recovery and rehabilitation of arm use after stroke. Journal of Stroke and Cerebrovascular Diseases 2001;10(4):197.

* Winstein CJ, Rose DK, Tan SM, Lewthwaite R, Chui HC, Azen SP. A randomized controlled comparison of upperextremity rehabilitation strategies in acute stroke: a pilot study of immediate and long-term outcomes. Archives of Physical Medicine and Rehabilitation 2004;85(4):620-8.

Yang 2006 \{published data only\}

Yang YR, Wang RY, Lin KH, Chu MY, Chan RC. Task-oriented progressive resistance strength training improves muscle strength and functional performance in individuals with stroke. Clinical Rehabilitation 2006;20:860-70.

Yang 2014 \{published data only\}

Yang HC, Lee CL, Lin R, Hsu MJ, Chen CH, Lin JH, et al. Effect of biofeedback cycling training on functional recovery and walking ability of lower extremity in patients with stroke. Kaohsiung Journal of Medical Sciences 2014;30(1):35-42.

Zedlitz 2012 \{published data only\}

Zedlitz AM, Rietveld TC, Geurts AC, Fasotti L. Cognitive and graded activity training can alleviate persistent fatigue after stroke: a randomized, controlled trial. Stroke 2012;43(4):1046-51.

\section{Zou 2015 \{published data only\}}

Zou J, Wang Z, Qu Q, Wang L. Resistance training improves hyperglycemia and dyslipidemia, highly prevalent among nonelderly, nondiabetic, chronically disabled stroke patients. Archives of Physical Medicine and Rehabilitation 2015; Vol. 96, issue 7:1291-6.

\section{References to studies excluded from this review}

\section{Alabdulwahab 2015 \{published data only\}}

Alabdulwahab SS, Ahmad F, Singh H. Effects of functional limb overloading on symmetrical weight bearing, walking speed, perceived mobility, and community participation among patients with chronic stroke. Rehabilitation Research and Practice 2015;2015:241519. [DOI: 10.1155/2015/241519]

\section{Askim 2018 \{published data only\}}

Askim T, Langhammer B, Gunnes M, Ihle-Hansen H, Indredavik B. Life after stroke-the LAST study. International 
Journal of Stroke 2015;10 Suppl 2:419. [DOI: http://

dx.doi.org/10.1111/ijs.12479]

* Askim T, Langhammer B, Ihle-Hansen H, Gunnes M, Lydersen St, Indredavik B. Efficacy and safety of individualized coaching after stroke: the LAST study (Life After Stroke): a pragmatic randomized controlled trial. Stroke 2018;49(2):426-32.

Askim T, Langhammer B, Ihle-Hansen H, Lydersen S, Gunnes M, Indredavik B. Effect of an individualized prolonged follow up programme for maintenance of motor function after strokethe LAST study. A multisite randomised controlled trial. International Journal of Stroke 2016;11 Suppl 3:21. [DOI: http:// dx.doi.org/10.1177/1747493016670567]

Askim T, Langhammer B, Ihle-Hansen H, Mari G, Stian L, Bent I. A randomized controlled trial assessing the effect of a longterm follow-up programme aiming to maintain motor function after stroke: the life after stroke (LAST) study. European Stroke Journal 2016;1 Suppl 1:727. [DOI: 10.1177/2396987316642910]

Gunnes M, Langhammer B, Aamot IL, Lydersen S, Schroeter W, Reneflot $\mathrm{K}$, et al. How well do stroke survivors adhere to an 18 month physical activity and exercise programme? secondary results from a randomised controlled trial. Gait and Posture 2017;57 Suppl 1:166. [DOI: 10.1016/j.gaitpost.2017.06.367]

\section{Awad 2015 \{published data only\}}

Awad A, Shaker H, Shendy W. Effect of shoulder girdle strengthening on trunk alignment in patients with stroke. Physiotherapy 2015;101 Suppl 1:eS1378-eS1379. [DOI: 10.1016/ j.physio.2015.03.1320]

\section{Baer 2018 \{published data only\}}

Baer GD, Salisbury LG, Smith MT, Pitman J, Dennis M. Treadmill training to improve mobility for people with subacute stroke: a phase II feasibility randomized controlled trial. Clinical Rehabilitation 2018;32(2):201-12. [DOI: 10.1177/0269215517720486]

\section{Bang 2014 \{published data only\}}

Bang D-H, Shin W-S, Noh H-J, Song M-S. Effect of unstable surface training on walking ability in stroke patients. Journal of Physical Therapy Science 2014;26(11):1689-91.

\section{Bernhardt 2018 \{published data only\}}

* Bernhardt J, Churilov L, Langhorne P, Pandian J, Dewey H, Shrikanth $\mathrm{V}$, et al. Determining optimal early rehabilitation after stroke (avertdose): a multi-arm covariate-adjusted, responseadaptive randomised controlled trial. European Stroke Journal 2018;3 Suppl 1:612. [DOI: 10.1177/2396987318773967]

Bernhardt J, Dewey H, Langhorne P, Pandian J, Thijs V, Churilov L, et al. Avert-dose (determining optimal early rehabilitation after stroke): a planned international randomised controlled trial. International Journal of Stroke 2016;11 Suppl 3:226-7. [DOI: 10.1177/1747493016670567]

\section{Boss 2017 \{published data only\}}

* Boss HM, Deijle IA, Van Schaik SM, De Melker EC, Van den Berg BT, Weinstein HC, et al. Cardiorespiratory fitness after transient ischemic attack and minor ischemic stroke: baseline data of the MovelT study. Journal of Stroke and Cerebrovascular Diseases 2017;26(5):1114-20. [DOI: 10.1016/ j.jstrokecerebrovasdis.2016.12.029]

Boss HM, Schaik MS, Deijle IA, Melker EC, Berg BT, Scherder EJA, et al. A randomised controlled trial of aerobic exercise after transient ischaemic attack or minor stroke to prevent cognitive decline: the Movelt study protocol. BMJ Open 2015;4(12):e007065. [DOI: 10.1136/bmjopen-2014-007065]

Boss HM, Van Schaik SM, Deijle IA, De Melker EC, Van den Berg BT, Scherder EJ, et al. A randomised control trial of aerobic exercise after transient ischaemic attack or minor stroke to prevent cognitive decline: the MOVEIT study protocol. BMJ Open 2014;4(12):e007065.

Boss HM, Van Schaik SM, Deijle IA, De Melker EC, Van den Berg BT, Scherder EJ, et al. A randomised controlled trial of aerobic exercise after transient ischaemic attack or minor stroke to prevent cognitive decline: the MovelT study. European Stroke Journal 2018;3 Suppl 1:91. [DOI: 10.1177/2396987318770127]

Boss HM, Van Schaik SM, Deijle IA, De Melker EC, Van den Berg BT, Scherder EJ, et al. Safety and feasibility of poststroke care and exercise after minor ischemic stroke or transient ischemic attack: MotiveS \& MovelT. NeuroRehabilitation 2014;34:401-7.

\section{Brauer 2018 \{published data only\}}

* Brauer SG, Kuys SS, Paratz JD, Ada L. Improving physical activity after stroke via treadmill training and self management (IMPACT): a protocol for a randomised controlled trial. BMC Neurology 2018;18:1-8. [DOI: 10.1186/s12883-018-1015-6]

Brauer SG, Kuys SS, Waters L, Paratz JD, Ada L. The strokeIMPACT trial (improving physical activity via treadmill training): a single blinded randomised controlled trial protocol. International Journal of Stroke 2014;9 Suppl 1:46.

Brouwer 2018 \{published data only\}

Brouwer B, Bryant D, Garland JS. Effectiveness of clientcentered "tune-ups" on community reintegration, mobility, and quality of life after stroke: a randomized controlled trial. Archives of Physical Medicine and Rehabilitation 2018;99(7):1325-32.

\section{Buyukavci 2016 \{published data only\}}

Buyukavci R, Sahin F, Sag S, Dogu B, Kuran B. The impact of additional trunk balance exercises on balance, functional condition and ambulation in early stroke patients: randomized controlled trial. Turkiye Fiziksel Tip ve Rehabilitasyon Dergisi 2016;62(3):248-56. [DOI: 10.5606/tftrd.2016.84770]

\section{Cabanas-Valdés 2017 \{published data only\}}

Cabanas Valdes R, German Romero A, Girabent Farres M, Bagur Calafat C, Caballero Gomez MF, Gerard UC. Long-term follow-up effects of additional core stability exercises training on improving dynamic sitting balance for stroke patients. A randomized controlled trial. European Stroke Journal 2016;1 Suppl 1:341. [DOI: 10.1177/2396987316642909] 
Cabanas-Valdés R, Bagur-Calafat C, Girabent-Farrés M, Caballero-Gómez FM, Hernández-Valiño M, Urrutia Cuchi G. The effect of additional core stability exercises on improving dynamic sitting balance and trunk control for subacute stroke patients: a randomized controlled trial. Clinical Rehabilitation 2016;30(10):1024-33.

* Cabanas-Valdés R, Bagur-Calafat C, Girabent-Farrés M, Caballero-Gómez FM, du Port de Pontcharra-Serra H, GermanRomero A, et al. Long-term follow-up of a randomized controlled trial on additional core stability exercises training for improving dynamic sitting balance and trunk control in stroke patients. Clinical Rehabilitation 2017;31(11):1492-9. [DOI: 10.1177/026921551770180]

NCT01864382. "Core stability" exercises to improve sitting balance in stroke patients (Fisionet) [Effects of the inclusion of exercises "Core Stability" in the treatment of inpatient physiotherapy to improve balance in post-stroke patients sitting in subacute phase. Randomized clinical trial]. clinicaltrials.gov/ ct2/show/NCT01864382 (first received 29 May 2013).

\section{Choi 2015 \{published data only\}}

Choi JU, Kang SH. The effects of patient-centered taskoriented training on balance activities of daily living and selfefficacy following stroke. Journal of Physical Therapy Science 2015;27(9):2985-8. [DOI: 10.1589/jpts.27.2985]

\section{Choi 2017 \{published data only\}}

Choi YK, Kim K, Choi, JU. Effects of stair task training on walking ability in stroke patients. Journal of Physical Therapy Science 2017;29(2):235-7. [DOI: 10.1589/jpts.29.235]

\section{Chua 2016 \{published data only\}}

Chua J, Culpan J, Menon E. Efficacy of an electromechanical gait trainer poststroke in Singapore: a randomized controlled trial. Archives of Physical Medicine and Rehabilitation 2016;97(5):683-90. [DOI: 10.1016/j.apmr.2015.12.025]

\section{Dong Hyun 2016 \{published data only\}}

Kim DH, Jang SH. Effects of an upper-limb exercise program for improving muscular strength and range of movement on respiratory function of stroke patients. Journal of Physical Therapy Science 2016;28(10):2785-8.

\section{Dubey 2018 \{published data only\}}

Dubey L, Karthikbabu S, Mohan, D. Effects of pelvic stability training on movement control, hip muscles strength, walking speed and daily activities after stroke: a randomized controlled trial. Annals of Neurosciences 2018;25:80-9. [DOI: 10.1159/000486273]

\section{English 2015 \{published data only\}}

* English C, Bernhardt J, Crotty M, Esterman A, Segal L, Hillier S. Circuit class therapy or seven-day week therapy for increasing rehabilitation intensity of therapy after stroke (CIRCIT): a randomized controlled trial. International Journal of Stroke 2015;10(4):594-602. [DOI: 10.1111/ijs.12470]

English C, Bernhardt J, Crotty M, Esterman A, Segal L, Watts J, et al. Circuit class therapy reduces length of rehabilitation stay, but weekend therapy does not. An exploratory secondary analysis of the CIRCIT trial. Cerebrovascular Diseases 2016;42 Suppl 1:6. [DOI: 10.1159/000447732]

Faulkner 2014 \{published data only\}

Faulkner J, Lanford J, Lambrick D, Stoner L, Woolley B, Donnell $\mathrm{T}$, et al. The efficacy of early exercise engagement as a secondary prevention strategy for stroke and TIA: study protocol and recruitment feasibility. International Journal of Stroke 2014;9 Suppl 1:36.

Faulkner 2015 \{published data only\}

Faulkner J, McGonigal G, Woolley B, Stoner L, Wong L, Lambrick D. A randomized controlled trial to assess the psychosocial effects of early exercise engagement in patients diagnosed with transient ischaemic attack and mild, nondisabling stroke. Clinical Rehabilitation 2015;29(8):783-94. [DOI: 10.1177/0269215514555729]

\section{Faulkner 2017a \{published data only\}}

Faulkner J, Stoner L, Lanford J, Jolliffe E, Mitchelmore A, Lambrick $D$. Long-term effect of participation in an early exercise and education program on clinical outcomes and cost implications, in patients with TIA and minor, non-disabling stroke. Translational Stroke Research 2017;8(3):220-7. [DOI: 10.1007/s12975-016-0510-6]

\section{Graef 2016 \{published data only\}}

Graef P, Michaelsen SM, Dadalt ML, Rodrigues DA, Pereira F, Pagnussat AS. Effects of functional and analytical strength training on upper-extremity activity after stroke: a randomized controlled trial. Brazilian Journal of Physical Therapy 2016;20(6):543-52. [DOI: 10.1590/bjpt-rbf.2014.0187]

\section{Gunnes 2017 \{published data only\}}

Gunnes M, Langhammer B, Aamot IL, Lydersen S, Schroeter W, Reneflot K, et al. How well do stroke survivors adhere to an 18month physical activity and exercise programme? Secondary results from a randomised controlled trial. Gait and Posture 2017;57 Suppl 1:199.

\section{Hahn 2015 \{published data only\}}

Hahn J, Shin S, Lee W. The effect of modified trampoline training on balance, gait, and falls efficacy of stroke patients. Journal of Physical Therapy Science 2015;27(11):3351-4. [DOI: 10.1589/jpts.27.3351]

\section{Haruyama 2017 \{published data only\}}

Haruyama K, Kawakami M, Otsuka T. Effect of core stability training on trunk function, standing balance, and mobility in stroke patients: a randomized controlled trial. Neurorehabilitation and Neural Repair 2017;31(3):240-9.

\section{Hendrey 2018 \{published data only\}}

Hendrey G, Clark RA, Holland AE, Mentiplay BF, Davis C, Windfeld-Lund $C$, et al. Feasibility of ballistic strength training in sub-acute stroke: a randomized, controlled, assessor-blinded pilot study. Archives of Physical Medicine and Rehabilitation 2018;99(12):2430-46. [DOI: 10.1016/j.apmr.2018.04.032] 
Heron 2017 \{published data only\}

Heron N, Kee F, Mant J, Reilly PM, Cupples M, Tully M, et al. Stroke Prevention Rehabilitation Intervention Trial of Exercise (SPRITE) - a randomised feasibility study. BMC Cardiovascular Disorders 2017;17(1):290. [DOI: 10.1186/s12872-017-0717-9]

Hillier 2014 \{published data only\}

Hillier S, English C, Bernhardt J, Crotty M, Esterman A, Segal L. Circuit class and 7-day week therapy for increasing rehabilitation intensity of therapy after stroke (CIRCIT): six month follow-up and cost analysis of the CIRCIT RCT. International Journal of Stroke 2014;9 Suppl 3:22.

\section{Hornby 2016 \{published data only\}}

* Hornby TG, Holleran CL, Hennessy PW, Leddy AL, Connolly M, Camardo J, et al. Variable Intensive Early Walking postStroke (VIEWS): a randomized controlled trial. Neurorehabilitation and Neural Repair 2016;30(5):440-50.

Leddy AL, Connolly M, Holleran CL, Hennessy PW, Woodward J, Arena RA, et al. Alterations in aerobic exercise performance and gait economy following high-intensity dynamic stepping training in persons with subacute stroke. Journal of Neurologic Physical Therapy 2016;40(4):239-48. [DOI: 10.1097/ NPT.0000000000000147]

Mahtani GB, Kinnaird CR, Connolly M, Holleran CL, Hennessy PW, Woodward J, et al. Altered sagittal- and frontalplane kinematics following high-intensity stepping training versus conventional interventions in subacute stroke. Physical Therapy 2017;97(3):320-9. [DOI: 10.2522/ptj.20160281]

\section{Hubbard 2015 \{published data only\}}

Hubbard IJ, Carey LM, Budd TW, Levi C, McElduff P, Hudson S, et al. A randomized controlled trial of the effect of early upper-limb training on stroke recovery and brain activation. Neurorehabilitation and Neural Repair 2015;29(8):703-13. [DOI: 10.1177/1545968314562647]

\section{Hunter 2018 \{published data only\}}

Hunter SM, Johansen-Berg H, Ward N, Kennedy NC, Chandler E, Weir $\mathrm{CJ}$, et al. Functional strength training and movement performance therapy for upper limb recovery early poststrokeefficacy, neural correlates, predictive markers, and costeffectiveness: FAST-INdiCATE trial. Frontiers in Neurology 2018;25(8):733. [DOI: 10.3389/fneur.2017.00733]

Immink 2014 \{published data only\} Immink MA, Hillier S, Petkov J. Randomized controlled trial of yoga for chronic poststroke hemiparesis: motor function, mental health, and quality of life outcomes. Topics in Stroke Rehabilitation 2014;21(3):256-71. [DOI: 10.1310/tsr2103-256]

\section{Kim 2016b \{published data only\}}

Kim N, Lee B, Kim Y, Min W. Effects of virtual reality treadmill training on community balance confidence and gait in people post-stroke: a randomized controlled trial. Journal of Experimental Stroke and Translational Medicine 2016;9(1):1-7.

\section{Kim 2017b \{published data only\}}

Kim CY, Lee JS, Kim HD. Comparison of the effect of lateral and backward walking training on walking function in patients with poststroke hemiplegia: a pilot randomized controlled trial. American Journal of Physical Medicine and Rehabilitation 2017;96(2):61-7. [DOI: 10.1097/PHM.0000000000000541]

Kwon 2015 \{published data only\}

Kwon OH, Woo Y, Lee JS, Kim KH. Effects of task-oriented treadmill-walking training on walking ability of stroke patients. Topics in Stroke Rehabilitation 2015;22(6):444-52. [DOI: 10.1179/1074935715Z.00000000057]

Lee 2015 \{published data only\}

Lee YH, Park SH, Yoon ES, Lee CD, Wee SO, Fernhall B, et al. Effects of combined aerobic and resistance exercise on central arterial stiffness and gait velocity in patients with chronic poststroke hemiparesis. American Journal of Physical Medicine and Rehabilitation 2015;94(9):687-95. [DOI: 10.1097/ PHM.0000000000000233]

Lee 2016 \{published data only\}

Lee MM, Shin DC, Song CH. Canoe game-based virtual reality training to improve trunk postural stability, balance, and upper limb motor function in subacute stroke patients: a randomized controlled pilot study. Journal of Physical Therapy Science 2016;28(7):2019-24. [DOI: doi.org/10.1589/jpts.28.2019]

Lee 2017a \{published data only\}

Lee DH, Park SH, Han JW. Effect of bilateral upper extremity exercise on trunk performance in patients with stroke. Journal of Physical Therapy Science 2017;29(4):625-8. [DOI: 10.1589/ jpts.29.625]

\section{Lee 2017b \{published data only\}}

Lee MJ, Lee JH, Koo HM, Lee SM. Effectiveness of bilateral arm training for improving extremity function and activities of daily living performance in hemiplegic patients. Journal of Stroke and Cerebrovascular Diseases 2017;26(5):1020-5. [DOI: 10.1016/ j.jstrokecerebrovasdis.2016.12.008]

\section{Lee 2018a \{published data only\}}

Lee MJ, Yoon S, Kang JJ, Kim J, Kim JM, Han JY. Efficacy and safety of caregiver-mediated exercise in poststroke rehabilitation. Annals of Rehabilitation Medicine 2018;42(3):406-15. [DOI: 10.5535/arm.2018.42.3.406]

\section{Lee 2018b \{published data only\}}

Lee MM, Lee KJ, Song CH. Game-based virtual reality canoe paddling training to improve postural balance and upper extremity function: a preliminary randomized controlled study of 30 patients with subacute stroke. Medical Science Monitor 2018;24:2590-8. [DOI: 10.12659/MSM.906451]

\section{Lim 2016 \{published data only\}}

Lim HS, Kim YL, Lee SM. The effects of Pilates exercise training on static and dynamic balance in chronic stroke patients: a randomized controlled trial. Journal of Physical Therapy Science 2016;28(6):1819-24. [DOI: 10.1589/jpts.28.1819] 
Lim 2017 \{published data only\}

Lim HS, Yoon S. The effects of Pilates exercise on cardiopulmonary function in the chronic stroke patients: a randomized controlled trials. Journal of Physical Therapy Science 2017;29(5):959-63.

\section{Lin 2015 \{published data only\}}

Lin CH, Chou LW, Luo HJ, Tsai PY, Lieu FK, Chiang SL, et al. Effects of computer-aided interlimb force coupling training on paretic hand and arm motor control following chronic stroke: a randomized controlled trial. PloS One 2015;10(7):e0131048. [DOI: 10.1371/journal.pone.0131048]

\section{Linder 2017 \{published data only\}}

Linder S, Rosenfeldt A, Alberts J. Forced aerobic exercise enhances upper extremity task practice in patients with stroke. Stroke 2015;46 Suppl 1:A144.

Linder S, Rosenfeldt A, Bazyk A, Lee J, Blohm M, Penko A, et al. Forced- and voluntary-rate aerobic exercise training improve cardiovascular function in individuals with chronic stroke. Stroke 2017;48 Suppl 1:AWP154.

Linder S, Rosenfeldt A, Penko A, Alberts J. The utilization of forced aerobic exercise to augment the recovery of motor function following stroke: a randomized clinical trial. Neurology 2014;82 Suppl 10:P2.016.

* Linder SM, Rosenfeldt AB, Dey T, Alberts JL. Forced aerobic exercise preceding task practice improves motor recovery poststroke. American Journal of Occupational Therapy 2017;71(2):1-9. [DOI: 10.5014/ajot.2017.020297]

\section{Lund 2018 \{published data only\}}

Lund C, Dalgas U, Grønborg TK, Andersen H, Severinsen K, Riemenschneider $\mathrm{M}$, et al. Balance and walking performance are improved after resistance and aerobic training in persons with chronic stroke. Disability and Rehabilitation 2018;40(20):2408-15. [DOI: 10.1080/09638288.2017.1336646]

\section{Malagoni 2016 \{published data only\}}

Malagoni AM, Cavazza S, Ferraresi G, Grassi G, Felisatti M, Lamberti N, et al. Effects of a "test in-train out" walking program versus supervised standard rehabilitation in chronic stroke patients: a feasibility and pilot randomized study. European Journal of Physical and Rehabilitation Medicine 2016;52(3):279-87.

\section{Malik 2018 \{published data only\}}

Malik AN, Amjad I, ul-Ain Q. Effect of circuit gait training vs traditional gait training on mobility performance in stroke. Journal of the Pakistan Medical Association 2018;68(3):455-8.

\section{Marryam 2017 \{published data only\}}

Marryam M, Umar M. Effectiveness of task oriented training in improving upper limb function after stroke. Rawal Medical Journal 2017;42(3):341-3.

\section{Martins 2017 \{published data only\}}

Martins JC, Aguiar LT, Nadeau S, Scianni AA, Teixeira-Salmela LF, Faria CD. Efficacy of task-specific training on physical activity levels of people with stroke: protocol for a randomized controlled trial. Physical Therapy 2017;97(6):640-8. [DOI:

10.1093/physth/pzx032]

McDonnell 2017 \{published data only\}

McDonnell MN, Serranda I, Hiller SL. Increasing the amount of upper extremity rehabilitation in the first four weeks following stroke: a feasibility study. Stroke 2017;48 Suppl 1:ATMP39.

Meng 2018 \{published data only\}

Meng G, Meng X, Tan Y, Yu J, Jin A, Zhao Y, et al. Short-term efficacy of hand-arm bimanual intensive training on upper arm function in acute stroke patients: a randomized controlled trial. Frontiers in Neurology 2018;8:726. [DOI: 10.3389/ fneur.2017.00726]

\section{Ordahan 2015 \{published data only\}}

Ordahan B, Karahan AY, Basaran A, Turkoglu G, Kucuksarac S, Cubukcu M, et al. Impact of exercises administered to stroke patients with balance trainer on rehabilitation results: a randomized controlled study. Hippokratia 2015;19(2):125-30.

\section{Pandian 2015 \{published data only\}}

Pandian S, Arya KN, Kumar D. Effect of motor training involving the less-affected side (MTLA) in post-stroke subjects: a pilot randomized controlled trial. Topics in Stroke Rehabilitation 2015;22(5):357-67. [DOI: 10.1179/1074935714Z.0000000022]

\section{Park 2015a \{published data only\}}

Park BS, Noh JW, Kim MY, Lee LK, Yang SM, Lee WD, et al. The effects of aquatic trunk exercise on gait and muscle activity in stroke patients: a randomized controlled pilot study. Journal of Physical Therapy Science 2015;27(11):3549-53. [DOI: 10.1589/ jpts.27.3549]

\section{Park 2015b \{published data only\}}

Park BS, Kim MY, Lee LK, Yang SM, Lee WD, Noh JW, et al. Effects of conventional overground gait training and a gait trainer with partial body weight support on spatiotemporal gait parameters of patients after stroke. Journal of Physical Therapy Science 2015;27(5):1603-7. [DOI: 10.1589/jpts.27.1603]

Park 2015c \{published data only\}

Park KH, Kim DY, Kim TH. The effect of step climbing exercise on balance and step length in chronic stroke patients. Journal of Physical Therapy Science 2015;27(11):3515-8. [DOI: 10.1589/ jpts.27.3515]

Park 2016a \{published data only\}

Park KH, Lim JY, Kim TH. The effects of ankle strategy exercises on unstable surfaces on dynamic balance and changes in the COP. Journal of Physical Therapy Science 2016;28(2):456-9. [DOI: 10.1589/jpts.28.456]

Park 2016b \{published data only\}

Park GD, Choi JU, Kim YM. The effects of multidirectional stepping training on balance, gait ability, and falls efficacy following stroke. Journal of Physical Therapy Science 2016;28(1):82-6. [DOI: 10.1589/jpts.28.82] 
Park 2016c \{published data only\}

Park KT, Kim HJ. Effect of the a circuit training program using obstacles on the walking and balance abilities of stroke patients. Journal of Physical Therapy Science 2016;28(4):1194-8. [DOI: 10.1589/jpts.28.1194]

\section{Park 2016d \{published data only\}}

Park T-Y, Cha Y-J, Son S-M. Comparison of body composition of trunk and extremities of affected sides at different aerobic exercise intensities in chronic stroke patients: a randomized controlled preliminary report. Iranian Journal of Public Health 2016;45(8):1085-6.

\section{Park 2017a \{published data only\}}

Park J, Gong J, Yim J. Effects of a sitting boxing program on upper limb function, balance, gait, and quality of life in stroke patients. NeuroRehabilitation 2017;40(1):77-86. [DOI: 10.3233/ NRE-161392]

\section{Park 2017b \{published data only\}}

Park YK, Kim JH. Effects of kinetic chain exercise using EMG-biofeedback on balance and lower extremity muscle activation in stroke patients. Journal of Physical Therapy Science 2017;29(8):1390-3. [DOI: 10.1589/jpts.29.1390]

\section{Paul 2016 \{published data only\}}

Paul L, Wyke S, Brewster S, Sattar N, Gill JM, Alexander G, et al. Increasing physical activity in stroke survivors using STARFISH, an interactive mobile phone application: a pilot study. Topics in Stroke Rehabilitation 2016;23(3):170-7. [DOI: 10.1080/10749357.2015.1122266]

\section{Rand 2015 \{published data only\}}

Rand D, Yacoby A, Weiss R, Reif S, Malka R, Weingarden $\mathrm{H}$, et al. Home-based self-training using video-games: preliminary data from a randomised controlled trial. IEEE 2015 International Conference on Virtual Rehabilitation (ICVR). 2015:86-91.

\section{Redzuan 2012 \{published data only\}}

Redzuan NS, Engkasan JP, Mazlan M, Abdullah SJ. Effectiveness of a video-based therapy program at home after acute stroke: a randomized controlled trial. Archives of Physical Medicine and Rehabilitation 2012;93(12):2177-83. [DOI: 10.1016/ j.apmr.2012.06.025]

\section{Ribeiro 2017 \{published data only\}}

* Ribeiro TS, Silva EM, Silva IA, Costa MF, Cavalcanti FA, Lindquist AR. Effects of treadmill training with load addition on non-paretic lower limb on gait parameters after stroke: a randomized controlled clinical trial. Gait and Posture 2017;54:229-35. [DOI: 10.1016/j.gaitpost.2017.03.008]

Ribeiro TS, da Silva C, Camyla T, Carlos R, de Souza e Silva $\mathrm{EM}$, Lacerda MO, et al. Is there influence of the load addition during treadmill training on cardiovascular parameters and gait performance in patients with stroke? A randomized clinical trial. NeuroRehabilitation 2017;40(3):345-54. [DOI: 10.3233/ NRE-161422]

\section{Roh 2016 \{published data only\}}

Roh S, Gil HJ, Yoon S. Effects of 8 weeks of mat-based Pilates exercise on gait in chronic stroke patients. Journal of Physical Therapy Science 2016;28(9):2615-9. [DOI: 10.1589/jpts.28.2615]

\section{Rose 2017 \{published data only\}}

Rose DK, Nadeau SE, Wu SS, Tilson JK, Dobkin BH, Pei Q, et al. Locomotor training and strength and balance exercises for walking recovery after stroke: response to number of training sessions. Physical Therapy 2017;97(11):1066-74. [DOI: 10.1093/ $\mathrm{ptj} / \mathrm{pzx} 079]$

\section{Rose 2018 \{published data only\}}

Rose DK, DeMark L, Fox EJ, Clark DJ, Wludyka P. A backward walking training program to improve balance and mobility in acute stroke: a pilot randomized controlled trial. Journal of Neurologic Physical Therapy 2018;42(1):12-21. [DOI: 10.1097/ NPT.0000000000000210]

\section{Ru 2017 \{published data only\}}

Ru X, Dai H, Jiang B, Li N, Zhao X, Hong Z, et al. Communitybased rehabilitation to improve stroke survivors' rehabilitation participation and functional recovery. American Journal of Physical Medicine and Rehabilitation 2017;96(7):e123-e129. [DOI: 10.1097/PHM.0000000000000650]

Schachten 2015 \{published data only\}

Schachten T, Jansen P. The effects of golf training in patients with stroke: a pilot study. International Psychogeriatrics 2015;27(5):865-73. [DOI: 10.1017/S1041610214002452]

\section{Seo 2015 \{published data only\}}

Seo KC, Kim HA. The effects of ramp gait exercise with PNF on stroke patients' dynamic balance. Journal of Physical Therapy Science 2015;27(6):1747-9. [DOI: 10.1589/jpts.27.1747]

\section{Sharma 2017 \{published data only\}}

Sharma V, Kaur J. Effect of core strengthening with pelvic proprioceptive neuromuscular facilitation on trunk, balance, gait, and function in chronic stroke. Journal of exercise rehabilitation 2017;13(2):200-5. [DOI: 10.12965/jer.1734892.446]

Shin 2016 \{published data only\}

Shin JW, Kim KD. The effect of enhanced trunk control on balance and falls through bilateral upper extremity exercises among chronic stroke patients in a standing position. Journal of Physical Therapy Science 2016;28(1):194-7. [EMBASE: 10.1589/ jpts.28.194]

\section{Sun 2016 \{published data only\}}

Sun X, Gao Q, Dou H, Tang S. Which is better in the rehabilitation of stroke patients, core stability exercises or conventional exercises?. Journal of Physical Therapy Science 2016;28(4):1131-3. [DOI: 10.1589/jpts.28.1131]

\section{Tang 2014 \{published data only\}}

Tang A, Eng JJ, Krassioukov AV, Madden KM, Mohammadi A, Tsang MY, et al. Exercise-induced changes in cardiovascular function after stroke: a randomized controlled trial. International Journal of Stroke 2014;9:883-9. [DOI: 10.1111/ ijs.12156] 
Vahlberg 2017 \{published data only\}

Vahlberg B, Cederholm T, Hellström K, Zetterberg L, Lindmark B. Body composition and training after stroke. European Geriatric Medicine 2015;6 Suppl 1:S112-S113. [DOI: 10.1016/ S1878-7649(15)30402-2]

Vahlberg B, Cederholm T, Lindmark B, Zetterberg L, Hellstrom K. Progressive resistance and balance training in circuit classes about a year after stroke: a randomized controlled trial. Cerebrovascular Diseases 2014;37 Suppl 1:428.

Vahlberg B, Cederholm T, Lindmark B, Zetterberg L, Hellström K. Effects of progressive resistance and balance training 1-3 years after stroke: a randomized controlled trial. FASEB Journal 2014;28 Suppl 1:706.14.

* Vahlberg B, Cederholm T, Lindmark B, Zetterberg L, Hellström K. Short-term and long-term effects of a progressive resistance and balance exercise program in individuals with chronic stroke: a randomized controlled trial. Disability and Rehabilitation 2017;39(16):1615-22. [DOI: 10.1080/09638288.2016.1206631]

Vahlberg B, Cederholm T, Zetterberg L, Lindmark B, Hellstrom K. Progressive resistance and balance training in circuit classes about one year after stroke: a randomized controlled trial. Cerebrovascular Diseases 2014;37 Suppl 1:334.

Vahlberg B, Lindmark B, Zetterberg L, Hellström K, Cederholm T. Body composition and physical function after progressive resistance and balance training among older adults after stroke: an exploratory randomized controlled trial. Disability and Rehabilitation 2017;39(12):1207-14. [DOI: 10.1080/09638288.2016.1191551]

\section{Valkenborghs 2016 \{published data only\}}

Valkenborghs S, Callister R, Nilsson M, Erickson K, Visser M, Dunn A, et al. Aerobic exercise to increase efficacy of taskspecific training for the upper limb after stroke: a pilot study. International Journal of Stroke 2016;11 Suppl 1:12.

* Valkenborghs S, Callister R, Nilsson M, Erickson K, Visser M, Dunn A, et al. Aerobic exercise to increase efficacy of taskspecific training for the upper limb after stroke: a pilot study protocol. Cerebrovascular Diseases 2016;42 Suppl 1:113-4.

\section{Valkenborghs 2017 \{published data only\}}

Valkenborghs SR, Visser MM, Dunn A, Erickson KI, Nilsson M, Callister R, et al. AExaCTT - Aerobic Exercise and Consecutive Task-specific Training for the upper limb after stroke: protocol for a randomised controlled pilot study. Contemporary Clinical Trials Communications 2017;7:179-85. [DOI: 10.1016/ j.conctc.2017.07.009]

\section{Van Criekinge 2017 \{published data only\}}

Van Criekinge T, Saeys W, Hallemans A, Vereeck L, De Hertogh W, Van de Walle $P$, et al. Effectiveness of additional trunk exercises on gait performance: study protocol for a randomized controlled trial. Trials 2017;18(1):1-12. [DOI: 10.1186/ s13063-017-1989-1]

\section{Vasileva 2017 \{published data only\}}

Vasileva D, Izov N, Maznev I, Lubenova D, Mihova M, Markovski V, et al. Changes in kinetic parameters of gait in patients with supratentorial unilateral stroke in chronic period. Open Access Macedonian Journal of Medical Sciences 2017;5(2):201-6. [DOI: 10.3889/oamjms.2017.053]

Wang 2015 \{published data only\}

Wang TC, Tsai AC, Wang JY, Lin YT, Lin KL, Chen JJ, et al. Caregiver-mediated intervention can improve physical functional recovery of patients with chronic stroke: a randomized controlled trial. Neurorehabilitation and Neural Repair 2015;29(1):3-12. [DOI: 10.1177/1545968314532030]

Wright 2018 \{published data only\}

Wright H, Wright T, Pohlig RT, Kasner SE, Raser-Schramm J, Reisman D. Protocol for promoting recovery optimization of walking activity in stroke (PROWALKS): a randomized controlled trial. BMC Neurology 2018;18(1):39. [DOI: 10.1186/ s12883-018-1044-1]

\section{Zhang 2016 \{published data only\}}

Zhang Y, Wang YZ, Huang LP, Bai B, Zhou S, Yin MM, et al. Aquatic therapy improves outcomes for subacute stroke patients by enhancing muscular strength of paretic lower limbs without increasing spasticity: a randomized controlled trial. American Journal of Physical Medicine and Rehabilitation 2016;95(11):840-9. [DOI: 10.1097/PHM.0000000000000512]

\section{Zhiyan 2017 \{published data only\}}

Zhiyan H, Li NI, Baoyun C, Zunke G, Qinghong W, Lange F. Rehabilitation nursing for cerebral stroke patients within a suitable recovery empty period. Iranian Journal of Public Health 2017;46(2):180-5.

\section{Zhu 2016 \{published data only\}}

Zhu Z, Cui L, Yin M, Yu Y, Zhou X, Wang H, et al. Hydrotherapy vs. conventional land-based exercise for improving walking and balance after stroke: a randomized controlled trial. Clinical Rehabilitation 2016;30(6):587-93. [DOI: 10.1177/0269215515593392]

\section{References to studies awaiting assessment}

Brands Guendling 2017 \{published data only\}

Brands-Guendling N, Guendling PW. Equipmentbased movement therapy in stroke rehabilitation. BMC Complementary and Alternative Medicine 2017;17 Suppl 1:P22. [DOI: 10.1186/s12906-017-1782-4]

Chan 2017 \{published data only\}

Chan K, Phadke CP, Stremler D, Suter L, Pauley T, Ismail F, et al. The effect of water-based exercises on balance in persons post-stroke: a randomized controlled trial. Topics in Stroke Rehabilitation 2017;24(4):228-35. [DOI: 10.1080/10749357.2016.1251742]

Chan 2018 \{published data only\}

Chan WN, Tsang WW. Effect of Tai Chi training on dual-tasking performance that involves stepping down among stroke 
survivors: a pilot study. Evidence-based Complementary and Alternative Medicine : ECAM 2017;2017:Article ID 9134173. [DOI: $10.1155 / 2017 / 9134173]$

* Chan WN, Tsang WW. The effect of Tai Chi training on the dual-tasking performance of stroke survivors: a randomized controlled trial. Clinical Rehabilitation 2018;32(8):1076-85. [DOI: 10.1177/0269215518777872]

\section{Chen 2014 \{published data only\}}

Chen YJ, Huang HC, Tasi SY, Lin CW, Lin SY. Exercise interventions increase lower extremity muscle strength in patients with stroke. Cerebrovascular Diseases 2014;38 Suppl 1:102.

\section{Deshpande 2018 \{published data only\}}

CTRI/2017/05/008482. The effect of task oriented circuit training on hand function in people with one-sided weakness living in the society [Influence of task oriented circuit training on upper limb function in community dwelling stroke survivors]. ctri.nic.in/Clinicaltrials/pmaindet2.php? trialid=17978\&EncHid=\&userName=CTRI/2017/05/008482 (first received 4 May 2017).

* Deshpande SA, Girish N, Mohapatra S. Influence of task oriented circuit training on upper limb function among community dwelling stroke survivors. Neurorehabilitation and Neural Repair 2018;32(4-5):408-9. [DOI: 10.1177/1545968318765498]

\section{Ellis 2018 \{published data only\}}

* Ellis MD, Carmona C, Drogos J, Dewald J. Progressive abduction loading therapy with horizontal-plane viscous resistance targeting weakness and flexion synergy to treat upper limb function in chronic hemiparetic stroke: a randomized clinical trial. Frontiers in Neurology 2018;9:71. [DOI: 10.3389/fneur.2018.00071]

Ellis MD, Carmona C, Drogos J, Traxel S, Dewald JP. Progressive abduction loading therapy targeting flexion synergy to regain reaching function in chronic stroke: preliminary results from an RCT. Conference Proceedings - IEEE Engineering in Medicine and Biology Society 2016:5837-40.

\section{Faulkner 2017b \{published data only\}}

Faulkner J, Tzeng YC, Lambrick D, Woolley B, Allan PD, $O$ 'Donnell $T$, et al. A randomized controlled trial to assess the central hemodynamic response to exercise in patients with transient ischaemic attack and minor stroke. Journal of Human Hypertension 2017;31(3):172-7. [DOI: 10.1038/jhh.2016.72]

\section{Floel 2018 \{published data only\}}

* Flöel A, Nave A, Rackoll T, Grittner U, Blasing H, Gorsler A, et al. Physical activity in subacute stroke - phys-stroke. European Stroke Journal 2018;3 Suppl 1:593. [DOI: $10.1177 / 2396987318773967]$

Rackoll T, Nave A, Grittner U, Meisel A, Endres M, Ebinger M, et al. A heart for running-safety considerations on aerobic exercise in the subacute phase of stroke-data from the physstroke trial. European Stroke Journal 2018;3(Suppl 1):129. [DOI: 10.1177/2396987318770127]

\section{Frimpong 2014 \{published data only\}}

Frimpong E, Olawale OA, Antwi DA, Antwi-Boasiako C, Dzudzor B. Task-oriented circuit training improves ambulatory functions in acute stroke: a randomized controlled trial. Journal of Medicine and Medical Sciences 2014;5(8):169-75.

\section{Gezer 2018 \{published data only\}}

Gezer H, Karaahmet OZ, Gurcay E, Dulgeroglu D, Cakci A. The effect of aerobic exercise on stroke rehabilitation. Irish Journal of Medical Science 2018:1-5.

\section{Hwang 2015 \{published data only\}}

Hwang G-Y. The Effects of Aquatic Exercise Program on Cardiorespiratory Function, Motor Fitness, and Physical Activity Affect for Poststroke Adults [PhD Thesis]. Denton, Texas USA: Texas Womens University, 2015.

\section{Jeon 2016 \{published data only\}}

Jeon HJ, An S, Yoo J, Park NH, Lee KH. The effect of monkey chair and band exercise system on shoulder range of motion and pain in post-stroke patients with hemiplegia. Journal of Physical Therapy Science 2016;28(8):2232-7. [DOI: 10.1589/ jpts.28.2232]

Kim 2015 \{published data only\}

Kim SJ, Cho HY, Kim YL, Lee SM. Effects of stationary cycling exercise on the balance and gait abilities of chronic stroke patients. Journal of Physical Therapy Science 2015;27(11):3529-31. [DOI: 10.1589/jpts.27.3529]

\section{Kim 2017c \{published data only\}}

Kim K, Jung SI, Lee DK. Effects of task-oriented circuit training on balance and gait ability in subacute stroke patients: a randomized controlled trial. Journal of Physical Therapy Science 2017;29(6):989-92. [DOI: 10.1589/jpts.29.989]

Koç 2015 \{published data only\}

Koç A. Exercise in patients with subacute stroke: a randomized, controlled pilot study of home-based exercise in subacute stroke. Work 2015;52(3):541-7. [DOI: 10.3233/WOR-152156]

\section{Kumaran 2016 \{published data only\}}

Kumaran DS, Rao BK, Rao SN, Kamath A. Effect of a task and context based exercise program (TCEP) on improving walking function in community dwelling stroke survivors: a randomized controlled trial. International Journal of Stroke 2016;11:59. [DOI: 10.1177/1747493016670567]

\section{Lawal 2016 \{published data only\}}

* Lawal I, Hillier S, Hamzat T, Rhoda A. Augmented duration of circuit class therapy in the rehabilitation of muscle strength and spasticity post stroke: a randomized controlled trial. International Journal of Stroke 2016;11 Suppl 3:188. [DOI: 10.1177/1747493016670567]

Lawal IU, Hillier SL, Hamzat TK, Rhoda A. Effectiveness of a structured circuit class therapy model in stroke rehabilitation: a protocol for a randomised controlled trial. BMC Neurology 2015;15:88. [DOI: 10.1186/s12883-015-0348-7] 
Lawal IU, Lawal I. Effectiveness of augmenting therapy time in circuit class therapy on mobility of upper/lower extremity post stroke: a randomized pilot study. Physiotherapy 2015;101:eS840-eS841. [DOI: 10.1016/j.physio.2015.03.1658]

Maheshwari 2018 \{published data only\}

Maheshwari PK, Kaushik S, Vishwas K. Comparison of strength training and task specific exercises to improve upper limb function in stroke patients. Neurorehabilitation and Neural Repair 2018;32(4-5):491-2. [DOI: 10.1177/1545968318765498]

\section{Matsumoto 2016 \{published data only\}}

Matsumoto S, Uema T, Ikeda K, Miyara K, Nishi T, Noma T, et al. Effect of underwater exercise on lower-extremity function and quality of life in post-stroke patients: a pilot controlled clinical trial. Journal of Alternative and Complementary Medicine 2016;22(8):635-41. [DOI: 10.1089/acm.2015.0387]

\section{Oh 2016 \{published data only\}}

Oh DS, Park SE. The effect of lumbar stabilization exercise on the pulmonary function of stroke patients. Journal of Physical Therapy Science 2016;28(6):1896-900. [DOI: 10.1589/ jpts.28.1896]

\section{Opara 2016 \{published data only\}}

Opara J, Szczygiel J. Effects of treadmill training with partial body-weight support on clinical parameters of GAIT early after stroke-preliminary results. Cerebrovascular Diseases 2016;41 Suppl 1:113.

\section{Ploughman 2017 \{published data only\}}

Ploughman M, Eskes GA, Kelly LP, Kirkland MC, Devasayaham AJ, Wallack EM, et al. Aerobic exercise enhances the beneficial effects of cognitive training and reopens the 'window of recovery' in chronic stroke via neurotrophins. International Journal of Stroke 2017;12(4 Suppl 1):18-9. [DOI: 10.1177/1747493017721569]

\section{Pudipeddi 2016 \{published data only\}}

Pudipeddi BK. Effect of strength training on normalizing the tone and strength of spastic elbow flexors in subjects with stroke with isokinetic analyser [BIODEX]. International Journal of Stroke 2016;11 Suppl 3:135. [DOI: 10.1177/1747493016670567]

\section{Ruescas-Nicolau 2015 \{published data only\}}

Ruescas-Nicolau MA, Sanchez-Sanchez ML, Espi-Lopez GV, Marques-Sule E, Perez-Miralles JA. Effect of a combined cardiovascular/task-oriented interval training programme on walking capacity in chronic stroke subjects. Cerebrovascular Diseases 2015;39 Suppl 2:322.

\section{Sanchez Sanchez 2015 \{published data only\}}

Sanchez-Sanchez ML, Ruescas-Nicolau MA, Espi-Lopez GV, Perez-Miralles JA, Morcillo-Fores JA, Marques-Sule E, et al. Effects on the upper limb sensoriomotor function of a circuit class strength training with elastic bands. Cerebrovascular Diseases 2015;39 Suppl 2:323.

\section{Song 2015a \{published data only\}}

Song GB, Hwangbo G. The effect of a rehabilitational sliding machine and conventional neurological physical therapy on the balance of patients with hemiplegia. Journal of Physical Therapy Science 2015;27(1):171-3. [DOI: 10.1589/jpts.27.171]

\section{Song 2015b \{published data only\}}

* Song HS, Kim JY, Park SD. Effect of the class and individual applications of task-oriented circuit training on gait ability in patients with chronic stroke. Journal of Physical Therapy Science 2015;27(1):187-9. [DOI: 10.1589/jpts.27.187]

Song HS, Kim JY, Park SD. The effect of class-based taskoriented circuit training on the self-satisfaction of patients with chronic stroke. Journal of Physical Therapy Science 2015;27(1):127-9. [DOI: 10.1589/jpts.27.127]

\section{Szczygiel 2015 \{published data only\}}

Szczygiel J, Opara J. Effects of treadmill training with partial body-weight support on biomechanical parameters of gait after stroke. Physiotherapy 2015;101 Suppl 1:eS1151. [DOI: 10.1016/ j.physio.2015.03.2062]

Van den Berg 2016 \{published data only\}

* Van Den Berg M, Crotty M, Liu E, Killington M, Kwakkel G, Van Wegen E. Early supported discharge by caregiver-mediated exercises and e-Health support after stroke: a proof-ofconcept trial. Stroke 2016;47(7):1885-92. [DOI: 10.1161/ STROKEAHA.116.013431]

Vloothuis J, Mulder M, Nijland RH, Konijnenbelt M, Mulder $\mathrm{H}$, Hertogh CM, et al. Caregiver-mediated exercises with e-health support for early supported discharge after stroke (CARE4STROKE): study protocol for a randomized controlled trial. BMC Neurology 2015;15(1):193. [DOI: 10.1186/ s12883-015-0440-z]

Vij 2015 \{published data only\}

Vij JS, Multani NK. NDT based gait training as compared to lower limb strengthening in correction of gait pattern of post-stroke hemiparetic patients. Physiotherapy 2015;101:eS1591-eS1592. [DOI: 10.1016/j.physio.2015.03.1599]

\section{Wu 2017 \{published data only\}}

Wu X-L. Effect of Qi-cultivating and mood-soothing bodybuilding exercise on psychological mood and degree of satisfaction with care in stroke patients with gastrointestinal bleeding. Shi Jie Hua Ren Xiao Hua Za Zhi 2017;25(9):837-40.

Xu 2015 \{published data only\}

Xu CW, Liu HL, Zhao D. Effect of acupuncture and exercise rehabilitation on motor function and activity of daily life among hemiplegia patients after stroke. Journal of Clinical Acupuncture and Moxibustion 2015;31(3):11-3.

Yang 2018 \{published data only\}

Yang ZH, Wu HY, Shen MY, Yang JQ, Cao LM. Clinical analysis of three kinds of exercise therapy on knee control in hemiplegic patients. Neurorehabilitation and Neural Repair 2018;32(4-5):392. [DOI: 10.1177/1545968318765498] 
Yelnik 2017 \{published data only\}

Schnitzler A, Yelnik A, Wanepain M, Reiner P, Devailly JP, Vicaut E. Active mobility early after stroke (AMOBES), 1 year follow-up. A randomised controlled trial. Annals of Physical and Rehabilitation Medicine 2018;61 Suppl:e20. [DOI: 10.1016/ j.rehab.2018.05.044]

Yelnik A, Andriantsifanetra C, Reinert P, Evrard M, Marneff H, Wanepain $\mathrm{M}$, et al. Active mobility early after stroke. A randomised controlled trial (AMOBES). Annals of Physical and Rehabilitation Medicine 2016;59 Suppl:e67. [DOI: 10.1016/ j.rehab.2016.07.155]

* Yelnik AP, Quintaine V, Andriantsifanetra C, Wannepain M, Reiner P, Marnef $\mathrm{H}$, et al. AMOBES (Active Mobility Very Early After Stroke): a randomized controlled trial. Stroke 2017;48(2):400-5. [DOI: 10.1161/STROKEAHA.116.014803]

\section{Zhang 2015 \{published data only\}}

Zhang T, Pan J. Effect of sling exercise therapy on lower limb function in patients with stroke. Journal of Shenyang Institute of Physical Education 2015;34(5):101-3.

\section{References to ongoing studies}

\section{ACTRN12615000728538 \{published data only\}}

ACTRN12615000728538. Home-based, tailored intervention for reducing falls after stroke: the Falls After Stroke Trial (FAST). www.anzctr.org.au/Trial/Registration/TrialReview.aspx? id=368847 (first received 15 July 2015).

\section{ACTRN12616000391471 \{published data only\}}

ACTRN12616000391471. A study on benefit of circuit class therapy on mobility, balance, reintegration into normal life and quality of life of people with stroke. [Outcomes of circuit class therapy on functional mobility, balance, community reintegration and quality of life of stroke survivors: randomised controlled trial]. www.anzctr.org.au/Trial/Registration/ TrialReview.aspx?id=369779 (first received 24 March 2016).

\section{ACTRN12616001288415 \{published data only\}}

ACTRN12616001288415. A comparison of two forms of physiotherapy on functional performance after acquired brain injury: a pilot randomised controlled trial. [Repetitive Exercise for People after Stroke (REPS): effects on functional performance]. www.anzctr.org.au/Trial/Registration/ TrialReview. aspx?id=363081 (first received 13 September 2016).

\section{ACTRN12617000452392 \{published data only\}}

ACTRN12617000452392. Core muscles strengthening for balance and gait performance in individuals with chronic stroke [Core muscles strengthening for balance and gait performance in individuals with chronic stroke]. www.anzctr.org.au/Trial/ Registration/TrialReview.aspx?id=372139 (first received 27 March 2017).

\section{ACTRN12617000746336 \{published data only\}}

ACTRN12617000746336. Walking away fatigue and disease after stroke [Feasibility of using accelerometers to reduce fatigue, improve beneficial activity behaviours and reduce risk of chronic disease after stroke: a pilot study]. www.anzctr.org.au/
Trial/Registration/TrialReview.aspx?id=372416 (first received 22 May 2017).

\section{ChiCTR ICR 15006362 \{published data only\}}

ChiCTR-ICR-15006362. Effect of early low-intensity aerobic training with ergometer on the activities of daily living among severely impaired post-stroke hemiplegic patients: a pilot study. www.chictr.org.cn/com/25/showprojen.aspx?proj=10911 (first received 3 May 2015).

ChicTR-IOR-17010821 \{published data only\}

ChiCTR-IOR-17010821. Effect of sling exercise training on the balance function of stroke patients with hemiplegia. www.chictr.org.cn/showprojen.aspx?proj=18374 (first received 9 March 2017).

\section{CTRI/2016/09/007258 \{published data only\}}

CTRI/2016/09/007258. Exercise using all four limbs in half-side paralysis [Interlimb coupling in post-stroke rehabilitation]. ctri.nic.in/Clinicaltrials/pmaindet2.php? trialid=11975\&EncHid=\&userName=CTRI/2016/09/007258 (first received 8 September 2016).

\section{CTRI/2016/10/007337 \{published data only\}}

CTRI/2016/10/007337. Effectiveness of physiotherapy training to improve control of sitting on floor [Improving deep knee flexion activities (squatting and sitting on the floor) in persons with stroke using functional exercise]. ctri.nic.in/Clinicaltrials/pmaindet2.php? trialid=12902\&EncHid=\&userName=CTRI/2016/10/007337 (first received 5 October 2016).

\section{CTRI/2017/03/008061 \{published data only\}}

CTRI/2017/03/008061. Comparison of home exercises verses exercises given in hospital set up in improving functional recovery of upper limbs in post stroke patients [Comparison of home based exercise program by care givers verses routine therapy at hospital setup in improving functional recovery of upper limb among stroke subjects - a randomised controlled trial]. ctri.nic.in/Clinicaltrials/showallp.php? mid1=13575\&EncHid=\&userName=CTRI/2017/03/008061 (first received 10 March 2017).

\section{CTRI/2018/01/011543 \{published data only\}}

CTRI/2018/01/011543. Efficacy of task-oriented training approach on trunk and hip musculature to improve balance in stroke subjects: a randomised controlled trial. ctri.nic.in/Clinicaltrials/pmaindet2.php? trialid=22039\&EncHid=\&userName=CTRI/2018/01/011543 (first received 18 January 2018).

\section{IRCT20150721023277N2 \{published data only\}}

IRCT20150721023277N2. The efficacy of occupation-based and exercise-based interventions in stroke subjects [The efficacy of occupation-based and exercise-based interventions on performance components and areas of occupation in subjects with chronic stroke (clinical trial)]. en.irct.ir/trial/19879 (first received 16 December 2017). 
IRCT2016102430477N1 \{published data only\}

IRCT2016102430477N1. Effect of rehabilitation in stroke recovery [Evaluation and comparison of the effect of cross education training of unaffected upper limb on neurophysiological parameters and functional indices of affected upper limb in sub-acute stroke patients]. en.irct.ir/ trial/24206 (first received 1 December 2016).

\section{IRCT20171105037256N4 \{published data only\}}

IRCT20171105037256N4. The effect of dual-task exercises on balance, falling and activities of daily living in stroke patients. www.irct.ir/trial/27608 (first received 20 May 2018).

\section{JPRN-UMIN000019380 \{published data only\}}

JPRN-UMIN000019380. The effect of walking exercise by Nordic pole on the aerobic performance for stroke patients. upload.umin.ac.jp/cgi-open-bin/ctr_e/ctr_view.cgi? recptno=R000022410 (first received 1 November 2015).

\section{Miller 2014 \{published data only\}}

* Miller KJ, Hunt MA, Pollock CL, Bryant D, Garland SJ. Protocol for a randomized controlled clinical trial investigating the effectiveness of Fast muscle Activation and Stepping Training (FAST) for improving balance and mobility in sub-acute stroke. BMC Neurology 2014;14(1):187. [DOI: 10.1186/s12883-014-0187y]

NCT01573585. Fast muscle activation and stepping training (FAST) post-stroke [Effectiveness of fast muscle activation and stepping training (FAST) on balance and mobility post-stroke]. clinicaltrials.gov/ct2/show/study/NCT01573585 (first received 9 April 2012)

\section{NCT01161329 \{published data only\}}

NCT01161329. Effect of intensified physical activity for patients with stroke - a combined physical and behavioural approach [Description of physical and psychosocial problems one year after stroke and the effect of intensified physical activity for patients with stroke - a combined physical and behavioural approach]. clinicaltrials.gov/ct2/show/NCT01161329 (first received 13 July 2010).

\section{NCT01915368 \{published data only\}}

Klassen T, Eng JJ, Bayley M, Benavente O, Krassioukov A, Piitz M, et al. Determining optimal post-stroke exercise (DOSE): study protocol for a randomized controlled trial investigating exercise intensity during inpatient rehabilitation. International Journal of Stroke 2017;12(4 Suppl 1):57-8. [DOI: 10.1177/1747493017721569]

Klassen TD, Eng JJ, Bayley M, Benavente O, Bennett J, Fraser J, et al. Implementing an extra hour of intensive, task-specific, physical therapy daily for individuals post-stroke during inpatient rehabilitation: feasibility data from the DOSE study. International Journal of Stroke 2015;10 Suppl 4:86. [DOI: 10.1111/ijs.12633-2]

* NCT01915368. Determining Optimal post-Stroke Exercise (DOSE). clinicaltrials.gov/ct2/show/NCT01915368 (first received 2 August 2013).

\section{NCT01916486 \{published data only\}}

NCT01916486. Vitality: promoting cognitive function in older adults with chronic stroke (vitality) [Complex mental and social activities to promote cognitive function in older adults with chronic stroke: a randomized controlled trial]. clinicaltrials.gov/ ct2/show/NCT01916486 (first received 5 August 2013).

NCT02272426 \{published data only\}

NCT02272426. The safety and tolerability of an aerobic and resistance exercise program with cognitive training post-stroke. clinicaltrials.gov/ct2/show/NCT02272426 (first received 23 October 2014).

\section{NCT02437006 \{published data only\}}

NCT02437006. Early intervention with a low-intensity leg cycling exercise program for individuals after stroke. clinicaltrials.gov/ ct2/show/record/NCT02437006 (first received 7 May 2015).

\section{NCT02494518 \{published data only\}}

NCT02494518. Forced aerobic exercise for stroke rehabilitation. clinicaltrials.gov/ct2/show/NCT02494518 (first received 10 July 2015).

\section{NCT02550015 \{published data only\}}

NCT02550015. High intensity interval training after stroke [The effect of high intensity interval training on maximal oxygen uptake and risk factors for recurrent stroke]. clinicaltrials.gov/ ct2/show/NCT02550015 (first received 15 September 2015).

\section{NCT02550990 \{published data only\}}

* NCT02550990. Synergistic effects of aerobic exercise and cognitive training on cognition in stroke patients with cognitive decline [Synergistic effects of aerobic exercise and cognitive training on cognition, physiological markers, daily function and quality of life in stroke patients with cognitive decline]. clinicaltrials.gov/ct2/show/NCT02550990 (first received 16 September 2015).

Wu CY, Yeh TT, Lee YY, Teng CH, Chang KC. Synergistic effects of aerobic exercise and cognitive training on cognition and quality of life in stroke patients with cognitive decline. Archives of Physical Medicine and Rehabilitation 2016;97(10):e12.

Yeh TT, Wu CY, Hsieh YW, Chang KC, Lee LC, Hung JW, et al. Synergistic effects of aerobic exercise and cognitive training on cognition, physiological markers, daily function, and quality of life in stroke survivors with cognitive decline: study protocol for a randomized controlled trial. Trials 2017;18(1):405. [DOI: 10.1186/s13063-017-2153-7]

\section{NCT02619110 \{published data only\}}

NCT02619110. The effect of backward walking treadmill training on balance in patient with chronic stroke [The purpose of this study was to discuss on the effects of backward walking treadmill training on balance ability, speed of walking and cardiopulmonary fitness for patient with chronic stroke]. clinicaltrials.gov/ct2/show/NCT02619110 (first received 2 December 2015). 


\section{NCT02703805 \{published data only\}}

* NCT02703805. Fit For Function: a community wellness program for persons with stroke. clinicaltrials.gov/ct2/show/ NCT02703805 (first received 9 March 2016).

Richardson J, Tang A, Guyatt G, Thabane L, Xie F, Sahlas D, et al. FIT for FUNCTION: study protocol for a randomized controlled trial. Trials 2018;19(1):39. [DOI: 10.1186/s13063-017-2416-3]

\section{NCT02710773 \{published data only\}}

NCT02710773. Backward treadmill training in patients with chronic stroke [Effects of backward treadmill training on spasticity and gait ability in patients with chronic stroke: a randomized controlled trial]. clinicaltrials.gov/ct2/show/ NCT02710773 (first received 17 March 2016).

\section{NCT02717715 \{published data only\}}

NCT02717715. SunRISe Study - Stroke rehabilitation In Suriname [The effect of a tele-supervised home based rehabilitation program on physical fitness, quality of gait, upper limb disability and quality of life in people after chronic stroke in a urban setting in Suriname. Randomised controlled trial]. clinicaltrials.gov/ct2/show/NCT02717715 (first received 24 March 2016).

\section{NCT02731235 \{published data only\}}

NCT02731235. The effect of aerobic exercise in patients with Lacunar stroke (HITPALS) [High-intensity training in patients with Lacunar stroke (HITPALS)]. clinicaltrials.gov/ct2/show/ NCT02731235 (first received 7 April 2016).

\section{NCT02753322 \{published data only\}}

NCT02753322. Training dual-task balance and walking in people with stroke [Training dual-task balance and walking in people with stroke: a randomized controlled trial]. clinicaltrials.gov/ ct2/show/NCT02753322 (first received 27 April 2016).

\section{NCT02855424 \{published data only\}}

NCT02855424. The effect of leg cycling exercise program at low or moderate intensity for individuals with subacute stroke. clinicaltrials.gov/ct2/show/NCT02855424 (first received 4 August 2016).

\section{NCT02902367 \{published data only\}}

NCT02902367. SMS-guided training after acute stroke or transient ischemic attack - a randomized controlled trial [Effects of SMS-guided outdoor walking and strength training after acute stroke and TIA - a randomized controlled trial]. clinicaltrials.gov/ct2/show/NCT02902367 (first received 15 September 2016).

\section{NCT02923765 \{published data only\}}

NCT02923765. Stepper aerobic training on fitness, disability, inflammation and thrombosis in stroke patients [Efficacy of stepper aerobic training on cardiopulmonary fitness, disability, systemic inflammation and thrombosis in stroke patients with hemiplegia]. clinicaltrials.gov/ct2/show/NCT02923765 (first received 5 October 2016).

\section{NCT02937480 \{published data only\}}

NCT02937480. Efficacy of task-specific training in physical activity level post-stroke [Efficacy of task-specific training in physical activity level of subjects with stroke: a randomized controlled trial]. clinicaltrials.gov/ct2/show/NCT02937480 (first received 18 October 2016 ).

\section{NCT02938000 \{published data only\}}

NCT02938000. E-Rehabilitation: aerobic resistance training for stroke survivors. clinicaltrials.gov/ct2/show/NCT02938000 (first received 19 October 2016).

\section{NCT02948725 \{published data only\}}

NCT02948725. Clinical application of cross-education during stroke rehabilitation. clinicaltrials.gov/ct2/show/NCT02948725 (first received 28 October 2016).

\section{NCT03122626 \{published data only\}}

NCT03122626. Does participation in a group, task-oriented community-based exercise program improve the ability to do everyday activities among people with stroke? [Increasing access to community-based task-oriented exercise programs through healthcare-recreation partnerships to improve function post-stroke: feasibility of a 2-group randomized controlled trial protocol]. clinicaltrials.gov/ct2/show/NCT03122626 (first received 21 April 2017).

\section{NCT03259932 \{published data only\}}

NCT03259932. Comparison of fatigue and recovery after stroke depending on the usual management with or without physical training [Personalised physical training associated with usual management versus usual management alone on fatigue and recovery after minor stroke: randomised controlled trial]. clinicaltrials.gov/show/NCT03259932 (first received 24 August 2017).

\section{NCT03425890 \{published data only\}}

NCT03425890. Effect of self-empowered upper limb repetitive engagement (SURE) program on upper limb recovery after stroke [The effect of the self-empowered upper limb repetitive engagement (SURE) program on upper limb recovery compared with education in people with stroke undergoing inpatient rehabilitation]. clinicaltrials.gov/show/NCT03425890 (first received 8 February 2018).

\section{NCT03458884 \{published data only\}}

NCT03458884. Does cardiorespiratory interval training improve post-stroke fatigue [Does a cardiorespiratory interval training program at home improve post-stroke fatigue?]. clinicaltrials.gov/ct2/show/NCT03458884 (first received 8 March 2018).

\section{NCT03479632 \{published data only\}}

NCT03479632. Aerobic walking exercise for non-ambulatory stroke survivors. clinicaltrials.gov/show/NCT03479632 (first received 27 March 2018).

\section{NCT03528148 \{published data only\}}

NCT03528148. The effects of elliptical cross training bike for stroke patients. clinicaltrials.gov/show/NCT03528148 (first received 17 May 2018). 


\section{NCT03548090 \{published data only\}}

NCT03548090. The effects of plantar flexion training in people with chronic stroke [The effects of plantar flexion training on plantar flexion activation, torque, and step length asymmetry in people with chronic stroke]. clinicaltrials.gov/ct2/show/ NCT03548090 (first received 7 June 2018).

\section{PACTR201511001359344 \{published data only\}}

PACTR201511001359344. The NIgerian Stroke Aerobic Study (NISAS) [Effects of interval and continuous aerobic exercise on clinical profiles of stroke survivors]. pactr.samrc.ac.za/ TrialDisplay.aspx?TrialID=1359 (first received 23 November 2015)

\section{PACTR201712002689193 \{published data only\}}

PACTR201712002689193. Effect of 6 weeks task-oriented circuit training on balance and quality of life of stroke [Effect of 6 weeks task-oriented circuit training on balance and quality of life of stroke survivors]. pactr.samrc.ac.za/TrialDisplay.aspx? TrialID=2689 (first received 13 October 2017).

\section{RBR-26q479 \{published data only\}}

RBR-26q4z9. Effects of exercises with action observation and aquatic physical therapy in arm recovery after stroke [Comparative study between action observation training and hydrotherapy in upper limb recovery after stroke: randomized clinical trial]. ensaiosclinicos.gov.br/rg/RBR-26q4z9/ (first received 23 March 2016).

\section{RBR-2mf595 \{published data only\}}

RBR-2mf595. Serious game for evaluation and treatment in stroke. ensaiosclinicos.gov.br/rg/RBR-2mf595/ (first received 15 January 2018).

\section{RBR-4g6fhf $\{$ published data only}

RBR-4g6fhf. The effects of exercise with cycle ergometer in patients after acute stroke. ensaiosclinicos.gov.br/rg/ RBR-4g6fhf/ (first received 5 December 2018).

\section{RBR-4wk4b3 \{published data only\}}

RBR-4wk4b3. Influence of an exercise program on cardiac function of patients with stroke. ensaiosclinicos.gov.br/rg/ RBR-4wk4b3/ (first received 19 September 2016).

\section{RBR-4wz3w3 \{published data only\}}

RBR-4wz3w3. Group therapy and self-monitored home exercises to increase exercise practice in the subacute phase after stroke - randomized clinical trial. ensaiosclinicos.gov.br/rg/ RBR-4wz3w3/ (first received 23 February 2018).

\section{RBR-5thjgv \{published data only\}}

RBR-5thjgv. Effects of physical training on metabolic, hemodynamic, autonomic and inflammatory parameters of post-stroke individuals. ensaiosclinicos.gov.br/rg/RBR-5thjgv/ (first received 7 November 2017).

\section{RBR-7699xz \{published data only\}}

RBR-7699xz. Analysis of walking training with partial weight support for post stroke patients. ensaiosclinicos.gov.br/rg/ RBR-7699xz/ (first received 5 April 2016).
RBR-7hqk8t \{published data only\}

RBR-7hqk8t. The effect of training functional activities on biological factors after stroke. ensaiosclinicos.gov.br/rg/ RBR-7hqk8t/ (first received 26 January 2018).

\section{TCTR20160601005 \{published data only\}}

TCTR20160601005. Effectiveness of antigravity treadmill training in improving walking capacity and balance in hemiparetic ischemic stroke patients. clinicaltrials.in.th/index.php? $\mathrm{tp}=$ regtrials\&menu=trialsearch\&smenu=fulltext\&task=search\&task2=view1 (first received 29 May 2016).

\section{TCTR20170615002 \{published data only\}}

TCTR20170615002. Effects of task-oriented training on upper extremity functional performance in patients with sub-acute stroke: a randomized controlled trial. www.clinicaltrials.in.th/index.php? $\mathrm{t} p=$ regtrials\&menu=trialsearch\&smenu=fulltext\&task=search\&task2=view1 (first received 15 June 2017).

\section{Vahlberg 2018 \{published data only\}}

Vahlberg B, Holmbäck U, Eriksson S, Cederholm T. Protocol and pilot study of a short message service-guided training after acute stroke/transient ischemic attack to increase walking capacity and physical activity. Preventive Medicine Reports 2018;11:109-14. [DOI: 10.1016/j.pmedr.2018.05.016]

\section{Additional references}

\section{Abbasian 2018}

Abbasian S, Rastegar MM. Is the intensity or duration of treadmill training important for stroke patients? A meta-analysis. Journal of Stroke and Cerebrovascular Diseases 2018;27(1):32-43. [DOI: 10.1016/ j.jstrokecerebrovasdis.2017.09.061]

\section{ACSM 1998}

American College of Sports Medicine. The recommended quantity and quality of exercise for developing and maintaining cardiorespiratory and muscular fitness, and flexibility in healthy adults. Medicine \& Science in Sports \& Exercise 1998;30(6):975-91.

\section{ACSM 2011}

Garber CE, Blissmer B, Deschenes MR, Franklin BA, LaMonte MJ, Lee IM, et al. Quantity and quality of exercise for developing and maintaining cardiorespiratory, musculoskeletal, and neuromotor fitness in apparently healthy adults: guidance for prescribing exercise. Medicine \& Science in Sports \& Exercise 2011;43(7):1334-59.

\section{Ammann 2014}

Ammann BC, Knols RH, Baschung P, De Bie RA, de Bruin ED. Application of principles of exercise training in sub-acute and chronic stroke survivors: a systematic review. BMC Neurology 2014;14(1):167. 


\section{Anderson 2016}

Anderson L, Oldridge N, Thompson DR, Zwisler AD, Rees K, Martin N, et al. Exercise-based cardiac rehabilitation for coronary heart disease: Cochrane systematic review and meta-analysis. Journal of the American College of Cardiology 2016;67(1):1-12.

\section{Austin 2014}

Austin MW, Ploughman M, Glynn L, Corbett D. Aerobic exercise effects on neuroprotection and brain repair following stroke: a systematic review and perspective. Neuroscience Research 2014;87:8-15.

\section{Baldwin 2016}

Baldwin J, Plummer L, Joshi S, Sangave A. Effectiveness of aerobic training in individuals with chronic stroke: a systematic review. Archives of Physical Medicine and Rehabilitation 2016;97(10):e141.

\section{Bangsbo 2019}

Bangsbo J, Blackwell J, Boraxbekk CJ, Caserotti P, Dela F, Evans AB, et al. Copenhagen Consensus Statement 2019: physical activity and ageing. British Journal of Sports Medicine 2019; Vol. bjsports-2018.

\section{Beck 1961}

Beck AT, Ward CH, Mendelson M, Mock J, Erbaugh J. An inventory for measuring depression. Archives of General Psychiatry 1961;4:561-71.

\section{Bernhardt 2004}

Bernhardt J, Dewey H, Thrift A, Donnan G. Inactive and alone: physical activity within the first 14 days of acute stroke unit care. Stroke 2004;35(4):1005-9.

\section{Bernhardt 2007}

Bernhardt J, Chan J, Nicola I, Collier JM. Little therapy, little physical activity: rehabilitation within the first 14 days of organized stroke unit care. Journal of Rehabilitation Medicine 2007;39:43-8.

\section{Billinger 2014}

Billinger SA, Arena R, Bernhardt J, Eng JJ, Franklin BA, Johnson CM, et al. Physical activity and exercise recommendations for stroke survivors: a statement for healthcare professionals from the American Heart Association/ American Stroke Association. Stroke 2014;45(8):2532-53.

\section{Blokland 2018}

Blokland IJ, IJmker T, Houdijk H. Aerobic capacity and load of activities of daily living after stroke. In: Müller B. Wolf SI editor(s). Handbook of Human Motion. Springer International Publishing, 2017:1-22.

\section{Bonini-Rocha 2018}

Bonini-Rocha AC, de Andrade AL, Moraes AM, Matheus LB, Diniz LR, Martins WR. Effectiveness of circuit-based exercises on gait speed, balance, and functional mobility in people affected by stroke: a meta-analysis. Physical Medicine and Rehabilitation 2018;10(4):398-409.

\section{Boyne 2017}

Boyne P, Welge J, Kissela B, Dunning K. Factors influencing the efficacy of aerobic exercise for improving fitness and walking capacity after stroke: a meta-analysis with metaregression. Archives of Physical Medicine and Rehabilitation 2017;98(3):581-95.

\section{Buchner 1991}

Buchner DM, De Lateur BJ. The importance of skeletal muscle strength to physical function in older adults. Annals of Behavioral Medicine 1991;13(3):91-8.

\section{Cabanas-Valdés 2013}

Cabanas-Valdés R, Cuchi G, Bagur-Calafat C. Trunk training exercises approaches for improving trunk performance and functional sitting balance in patients with stroke: a systematic review. NeuroRehabilitation 2013;33(4):575-92.

\section{Carin-Levy 2009}

Carin-Levy G, Kendall M, Young A, Mead G. The psychosocial effects of exercise and relaxation classes for persons surviving a stroke. Canadian Journal of Occupational Therapy 2009;76:73-80.

\section{Chen 2016}

Chen L, Peng Q, Chen J, Zou Y, Liu G. Effect of sling exercise training on balance in patients with stroke: a meta-analysis. PloS One 2016;11(10):e0163351.

\section{Chodzko-Zajko 2009}

Chodzko-Zajko WJ, Proctor DN, Fiatarone Singh MA, Minson CT, Nigg CR, Salem GJ, et al. Exercise and physical activity for older adults. Medicine and Science in Sports and Exercise 2009;41(7):1510-30.

\section{Collen 1991}

Collen FM, Wade DT, Robb GF, Bradshaw CM. The Rivermead Mobility Index: a further development of the Rivermead Motor Assessment. International Disability Studies 1991;13:50-4.

\section{Collin 1988}

Collin C, Wade DR, Davies S, Horne V. The Barthel ADL Index: a reliability study. International Disability Studies 1988;10:61-3.

\section{CONSORT 2010}

Schulz KF, Altman DG, Moher D, for the CONSORT Group. CONSORT 2010 Statement: updated guidelines for reporting parallel group randomised trials. BMJ 2010;340:c332.

\section{Cornelissen 2013}

Cornelissen VA, Buys R, Smart NA. Endurance exercise beneficially affects ambulatory blood pressure: a systematic review and meta-analysis. Journal of Hypertension 2013;31(4):639-48.

\section{Covidence [Computer program]}

Veritas Health Innovation. Covidence. Version accessed 14 July 2018. Melbourne, Australia: Veritas Health Innovation. 


\section{Crozier 2018}

Crozier J, Roig M, Eng JJ, MacKay-Lyons M, Fung J, Ploughman M, et al. High-intensity interval training after stroke: an opportunity to promote functional recovery, cardiovascular health, and neuroplasticity. Neurorehabilitation and Neural Repair 2018;32(6-7):543-56.

\section{Cumming 2012}

Cumming TB, Tyedin K, Churilov L, Morris ME, Bernhardt J. The effect of physical activity on cognitive function after stroke: a systematic review. International Psychogeriatrics 2012;24(4):557-67.

\section{D'Isabella 2017}

D'Isabella NT, Shkredova DA, Richardson JA, Tang A. Effects of exercise on cardiovascular risk factors following stroke or transient ischemic attack: a systematic review and metaanalysis. Clinical Rehabilitation 2017;31(12):1561-72.

\section{Deeks 2017}

Deeks JJ, Higgins JP, Altman DG (editors), on behalf ofthe Cochrane Statistical Methods Group. Chapter 9: Analysing data and undertaking meta-analyses. In: Higgins JPT, Churchill R, Chandler J, Cumpston MS (editors), Cochrane Handbook for Systematic Reviews of Interventions version 5.2.0 (updated June 2017), Cochrane, 2017. Available from www.training.cochrane.org/handbook.

\section{Degens 2006}

Degens H, Alway SE. Control of muscle size during disuse, disease, and aging. International Journal of Sports Medicine 2006;27(2):94-9.

\section{Dorsch 2018}

Dorsch S, Ada L, Alloggia D. Progressive resistance training increases strength after stroke but this may not carry over to activity: a systematic review. Journal of Physiotherapy 2018;64(2):84-90.

\section{Duncan 1999}

Duncan PW, Wallace D, Lai SM, Johnson D, Embretson S, Laster LJ. The Stroke Impact Scale version 2.0. Evaluation of reliability, validity, and sensitivity to change. Stroke 1999;30:2131-40.

\section{Eng 2014}

Eng JJ, Reime B. Exercise for depressive symptoms in stroke patients: a systematic review and meta-analysis. Clinical Rehabilitation 2014;28(8):731-9.

\section{English 2014}

English C, Manns PJ, Tucak C, Bernhardt J. Physical activity and sedentary behaviors in people with stroke living in the community: a systematic review. Physical Therapy 2014;94(2):185-96.

\section{English 2017}

English C, Hillier SL, Lynch EA. Circuit class therapy for improving mobility after stroke. Cochrane Database of Systematic Reviews 2017, Issue 6. [DOI: 10.1002/14651858.CD007513.pub3]

\section{Feigin 2017}

Feigin VL, Norrving B, Mensah GA. Global burden of stroke. Circulation Research 2017;120(3):439-48.

\section{Flansbjer 2006}

Flansbjer UB, Downham D, Lexell J. Knee muscle strength, gait performance, and perceived participation after stroke. Archives of Physical Medicine and Rehabilitation 2006;87(7):974-80.

\section{French 2016}

French B, Thomas LH, Coupe J, McMahon NE, Connell L, Harrison J, et al. Repetitive task training for improving functional ability after stroke. Cochrane Database of Systematic Reviews 2016, Issue 11. [DOI: 10.1002/14651858.CD006073.pub3]

\section{Fried 1996}

Fried LP, Bandeen-Roche K, Williamson JD, PrasadaRao P, Chee E, Tepper S, et al. Functional decline in older adults: expanding methods of ascertainment. Journals of Gerontology Series A - Biological Sciences and Medical Sciences 1996;51(5):M206-14.

\section{Fulk 2011}

Fulk GD, Ludwig M, Dunning K, Golden S, Boyne P, West T. Estimating clinically important change in gait speed in people with stroke undergoing outpatient rehabilitation. Journal of Neurologic Physical Therapy 2011;35:82-9.

\section{Fulk 2018}

Fulk GD, He Y. Minimal clinically important difference of the 6Minute Walk Test in people with stroke. Journal of Neurologic Physical Therapy 2018;42(4):235-40.

\section{Garcia-Soto 2013}

Garcia-Soto E, Lourdes Lopez de Munain M, Santibanez M. Effects of combined aerobic and resistance training on cognition following stroke: a systematic review. Revista de Neurologia 2013;57(12):535-41.

\section{Gauthier 2008}

Gauthier LV, Taub E, Perkins C, Ortmann M, Mark VW, Uswatte GI. Remodeling the brain: plastic structural brain changes produced by different motor therapies after stroke. Stroke 2008;39(5):1520-5.

\section{Gerrits 2009}

Gerrits KH, Beltman MJ, Koppe PA, Konijnenbelt H, Elich PD, De Haan A, et al. Isometric muscle function of knee extensors and the relation with functional performance in patients with stroke. Archives of Physical Medicine and Rehabilitation 2009;90(3):480-7.

\section{Gordon 2018}

Gordon BR, McDowell CP, Hallgren M, Meyer JD, Lyons M, Herring MP. Association of efficacy of resistance exercise training with depressive symptoms: meta-analysis and meta-regression analysis of randomized clinical trials. JAMA Psychiatry 2018;75(6):566-76. 


\section{GRADEpro GDT [Computer program]}

MCMaster University (developed by Evidence Prime). GRADEpro GDT. Version accessed 01 August 2019. Hamilton (ON): McMaster University (developed by Evidence Prime).

\section{Hamilton 1994}

Hamilton BB, Laughlin JA, Fiedler RC, Granger CV. Interrater reliability of the 7-level Functional Independence Measure (FIM). Scandinavian Journal of Rehabilitation Medicine 1994;26:115-9.

\section{Hasan 2016}

Hasan SM, Rancourt SN, Austin MW, Ploughman M. Defining optimal aerobic exercise parameters to affect complex motor and cognitive outcomes after stroke: a systematic review and synthesis. Neural Plasticity 2016;2016:2961573.

\section{Hasegawa 2008}

Hasegawa R, Islam MM, Sung CL, Koizumi D, Rogers ME, Takeshima N. Threshold of lower body muscular strength necessary to perform ADL independently in community-dwelling older adults. Clinical Rehabilitation 2008;22(10-11):902-10.

\section{Haskell 2007}

Haskell WL, Lee IM, Pate RR, Powell KE, Blair SN, Franklin BA, et al. Physical activity and public health: updated recommendation for adults from the American College of Sports Medicine and the American Heart Association. Medicine and Science in Sports and Exercise 2007;39:1423-34.

\section{Hatano 1976}

Hatano S. Experience from a multicentre stroke register: a preliminary report. Bulletin of the World Health Organization 1976;54(5):541-3.

\section{Heyn 2004}

Heyn P, Abreu BC, Ottenbacher KJ. The effects of exercise training on elderly persons with cognitive impairment and dementia: a meta-analysis. Archives of Physical Medicine and Rehabilitation 2004;85(10):1694-704.

\section{Higgins 2003}

Higgins JP, Thompson SG, Deeks JJ, Altman DG. Measuring inconsistency in meta-analyses. BMJ 2003;327:557-60.

\section{Higgins 2011}

Higgins JP, Deeks JJ, Altman DG (editors). Chapter 16: Special topics in statistics. In: Higgins JPT, Green S (editors). Cochrane Handbook for Systematic Reviews of Interventions. Version 5.1.0 (updated March 2011). The Cochrane Collaboration, 2011. Available from www.training.cochrane.org/handbook.

\section{Higgins 2017}

Higgins JP, Altman DG, Sterne JA (editors). Chapter 8: Assessing risk of bias in included studies. In: Higgins JPT, Churchill R, Chandler J, Cumpston MS (editors), Cochrane Handbook for Systematic Reviews of Interventions version 5.2.0 (updated June 2017), Cochrane, 2017. Available from www.training.cochrane.org/handbook.

\section{Hoffmann 2014}

Hoffmann TC, Glasziou PP, Boutron I, Milne R, Perera R, Moher D, et al. Better reporting of interventions: template for intervention description and replication (TIDieR) checklist and guide. BMJ 2014;348:1687.

\section{Hooker 2019}

Hooker SP, McDonnell MN. Cardiorespiratory fitness, physical activity, and stroke. In: Kokkinos P, Narayan P editor(s). Cardiorespiratory Fitness in Cardiometabolic Diseases. Cham: Springer, 2019.

\section{Horstman 2008}

Horstman AM, Beltman MJ, Gerrits KH, Koppe P, Janssen TW, Elich $\mathrm{P}$, et al. Intrinsic muscle strength and voluntary activation of both lower limbs and functional performance after stroke. Clinical Physiology and Functional Imaging 2008;28(4):251-61.

\section{Hunt 1980}

Hunt SM, McKenna SP, McEwen J, Backett EM, Williams J, Papp E. A quantitative approach to perceived health status: a validation study. Journal of Epidemiology and Community Health 1980;34(4):281-6.

\section{latridou 2018}

latridou G, Pelidou HS, Varvarousis D, Stergiou A, Beris A, Givissis $P$, et al. The effectiveness of hydrokinesiotherapy on postural balance of hemiplegic patients after stroke: a systematic review and meta-analysis. Clinical Rehabilitation 2018;32(5):583-93.

\section{Ilunga Tshiswaka 2018}

Ilunga Tshiswaka D, Bennett C, Franklin C. Effects of walking trainings on walking function among stroke survivors: a systematic review. International Journal of Rehabilitation Research 2018;41(1):1-13.

\section{Indredavik 2008}

Indredavik B, Rohweder GI, Naalsund E, Lydersen S. Medical complications in a comprehensive stroke unit and an early supported discharge service. Stroke 2008;39(2):414-20.

\section{Jeon 2015}

Jeon BJ, Kim WH, Park EY. Effect of task-oriented training for people with stroke: a meta-analysis focused on repetitive or circuit training. Topics in Stroke Rehabilitation 2015;22(1):34-43.

\section{Kaminsky 2015}

Kaminsky LA, Arena R, Myers J. Reference standards for cardiorespiratory fitness measured with cardiopulmonary exercise testing: data from the Fitness Registry and the Importance of Exercise National Database. Mayo Clinic Proceedings 2015;90(11):1515-23.

\section{Kemmler 2018}

Kemmler W, Von Stengel S, Schoene D, Kohl M. Changes of maximum leg strength indices during adulthood: a crosssectional study with non-athletic men aged 19-9. Frontiers in Physiology 2018; Vol. 9. 


\section{Kendall 2016}

Kendall BJ, Gothe NP. Effect of aerobic exercise interventions on mobility among stroke patients: a systematic review. American Journal of Physical Medicine and Rehabilitation 2016;95(3):214-24.

\section{Kortebein 2008}

Kortebein P, Symons TB, Ferrando A, Paddon-Jones D, Ronsen $\mathrm{O}$, Protas $\mathrm{E}$, et al. Functional impact of 10 days of bed rest in healthy older adults. Journals of Gerontology Series A Biological Sciences and Medical Sciences 2008;63(10):1076-81.

\section{Kurl 2003}

Kurl S, Laukkanen JA, Rauramaa R, Lakka TA, Sivenius J, Salonen JT. Cardiorespiratory fitness and the risk for stroke in men. Archives of Internal Medicine 2003;163(14):1682-8.

\section{Kwakkel 2002}

Kwakkel G, Kollen BJ, Wagenaar RC. Long term effects of intensity of upper and lower limb training after stroke: a randomised trial. Journal of Neurology, Neurosurgery, and Psychiatry 2002;72(4):473-9.

\section{Kwakkel 2004}

Kwakkel G, Van Peppen R, Wagenaar RC, Wood Dauphinee S, Richards C, Ashburn A, et al. Effects of augmented exercise therapy time after stroke: a meta-analysis. Stroke 2004;35(11):2529-39.

\section{Langhorne 2000}

Langhorne P, Stott DJ, Robertson L, MacDonald J, Jones L, McAlpine C, et al. Medical complications after stroke: a multicenter study. Stroke 2000;31(6):1223-9.

\section{Langhorne 2002}

Langhorne P. Intensity of rehabilitation: some answers and more questions?. Journal of Neurology, Neurosurgery, and Psychiatry 2002;72(4):430-1.

\section{Langhorne 2009}

Langhorne P, Coupar F, Pollock A. Motor recovery after stroke: a systematic review. Lancet Neurology 2009;8(8):741-54.

\section{Lawton 1969}

Lawton MP, Brody EM. Assessment of older people: selfmaintaining and instrumental activities of daily living. Gerontologist 1969;9(3):179-86.

\section{Lee 2002}

Lee CD, Blair SN. Cardiorespiratory fitness and stroke mortality in men. Medicine and Science in Sports and Exercise 2002;34(4):592-5.

\section{Lee 2014}

Lee YH, Yoon ES, Park SH, Heffernan KS, Lee C, Jae SY. Associations of arterial stiffness and cognitive function with physical fitness in patients with chronic stroke. Journal of Rehabilitation Medicine 2014;46(5):413-7.

\section{Lefebvre 2011}

Lefebvre C, Manheimer E, Glanville J. Chapter 6: Searching for studies. In: Higgins JPT, Green S (editors). Cochrane Handbook for Systematic Reviews of Interventions Version 5.1.0 (updated March 2011). The Cochrane Collaboration, 2011. Available from www.handbook.cochrane.org.

\section{Lindley 1994}

Lindley R, Waddell F, Livingstone M. Can simple questions assess outcome after stroke?. Cerebrovascular Diseases 1994;4:314-24.

\section{Mackay 2004}

Mackay J, Mensah GA, Greenlund K. The atlas of heart disease and stroke. Geneva: World Health Organization 2004.

\section{Macko 2001}

Macko RF, Smith GV, Dobrovolny CL, Sorkin JD, Goldberg AP, Silver $\mathrm{KH}$. Treadmill training improves fitness reserve in chronic stroke patients. Archives of Physical Medicine and Rehabilitation 2001;82(7):879-84.

\section{McKinnon 2017}

McKinnon NB, Connelly DM, Rice CL, Hunter SW, Doherty TJ. Neuromuscular contributions to the age-related reduction in muscle power: mechanisms and potential role of high velocity power training. Ageing Research Reviews 2017;35:147-54.

\section{Mead 2005}

Mead G. Exercise or relaxation after stroke?. BMJ 2005;330:1337.

\section{Mead 2011}

Mead G, Bernhardt J. Physical fitness training after stroke, time to implement what we know: more research is needed. International Journal of Stroke 2011;6:506-8.

\section{Mehrholz 2017}

Mehrholz J, Thomas S, Elsner B. Treadmill training and body weight support for walking after stroke. Cochrane Database of Systematic Reviews 2017, Issue 8. [DOI: 10.1002/14651858.CD002840.pub4]

\section{Michael 2007}

Michael K, Macko RF. Ambulatory activity intensity profiles, fitness, and fatigue in chronic stroke. Topics in Stroke Rehabilitation 2007;14(2):5-12.

\section{Moher 2009}

Moher D, Liberati A, Tetzlaff J, Altman DG, The PRISMA Group (2009). Preferred reporting items for systematic reviews and meta-analyses: the PRISMA Statement. PLoS Medicine 2009;6(7):e1000097. [DOI: 10.1371/journal.pmed1000097]

\section{Nasreddine 2005}

Nasreddine ZS, Phillips NA, Bédirian V, Charbonneau S, Whitehead V, Collin I, et al. The Montreal Cognitive Assessment, MoCA: a brief screening tool for mild cognitive impairment. Journal of the American Geriatrics Society 2005;53(4):695-9. 


\section{Norrving 2018}

Norrving B, Barrick J, Davalos A, Dichgans M, Cordonnier C, Guekht A, et al. Action plan for stroke in Europe 20182030. European Stroke Journal 2018;3(4):309-36. [DOI: $10.1177 / 2396987318808719]$

\section{O'Donovan 2010}

O'Donovan G, Blazevich AJ, Boreham C, Cooper AR, Crank H, Ekelund $U$, et al. The $A B C$ of physical activity for health: a consensus statement from the British Association of Sport and Exercise Sciences. Journal of Sports Sciences 2010;28(6):573-91.

\section{Oberlin 2017}

Oberlin LE, Waiwood AM, Cumming TB, Marsland AL, Bernhardt J, Erickson KI. Effects of physical activity on poststroke cognitive function: a meta-analysis of randomized controlled trials. Stroke 2017;48(11):3093-100.

\section{Pandey 2016}

Pandey A, Patel MR, Willis B, Gao A, Leonard D, Das SR, et al. Association between midlife cardiorespiratory fitness and risk of stroke: the Cooper Center Longitudinal Study. Stroke 2016;47(7):1720-6.

\section{Pang 2013}

Pang MY, Charlesworth SA, Lau RW, Chung RC. Using aerobic exercise to improve health outcomes and quality of life in stroke: evidence-based exercise prescription recommendations. Cerebrovascular Diseases 2013;35(1):7-22.

\section{Patterson 2007}

Patterson SL, Forrester LW, Rodgers MM, Ryan AS, Ivey FM, Sorkin JD, et al. Determinants of walking function after stroke: differences by deficit severity. Archives of Physical Medicine and Rehabilitation 2007;88(1):115-9.

\section{Patterson 2009}

Patterson S, Ross-Edwards B. Long-term stroke survivors' needs and perceptions of an exercise maintenance model of care. International Journal of Therapy and Rehabilitation 2009;16(12):659-69.

\section{Plummer 2018}

Plummer P, Iyigun G. Effects of physical exercise interventions on dual-task gait speed following stroke: a systematic review and meta-analysis. Archives of Physical Medicine and Rehabilitation 2018;99(12):2548-60.

\section{Pollock 2012}

Pollock A, St George B, Fenton M, Firkins L. Top ten research priorities relating to life after stroke. Lancet Neurology 2012;11:209.

\section{Pollock 2014}

Pollock A, Baer G, Campbell P, Choo PL, Forster A, Morris J, et al. Physical rehabilitation approaches for the recovery of function and mobility following stroke. Cochrane Database of Systematic Reviews 2014, Issue 4. [DOI: 10.1002/14651858.CD001920.pub3]

\section{Powell 2019}

Powell KE, King AC, Buchner DM, Campbell WW, DiPietro L, Erickson Kl, et al. The scientific foundation for the Physical Activity Guidelines for Americans. Journal of Physical Activity and Health 2019; Vol. 16, issue 1:1-11.

\section{Puthoff 2007}

Puthoff ML, Nielsen DH. Relationships among impairments in lower-extremity strength and power, functional limitations, and disability in older adults. Physical Therapy 2007;87(10):1334-47.

\section{Randolph 1998}

Randolph C, Tierney MC, Mohr E, Chase TN. The Repeatable Battery for the Assessment of Neuropsychological Status (RBANS): preliminary clinical validity. Journal of Clinical and Experimental Neuropsychology 1998;20(3):310-9.

\section{Rantanen 2001}

Rantanen T, Guralnik JM, Ferrucci L, Penninx BW, Leveille S, Sipila S, et al. Coimpairments as predictors of severe walking disability in older women. Journal of the American Geriatrics Society 2001;49(1):21-7.

\section{Review Manager 2014 [Computer program]}

Nordic Cochrane Centre, The Cochrane Collaboration. Review Manager 5 (RevMan 5). Version 5.3. Copenhagen: Nordic Cochrane Centre, The Cochrane Collaboration, 2014.

\section{Salter 2016}

Salter K, Musovic A, Taylor NF. In the first 3 months after stroke is progressive resistance training safe and does it improve activity? A systematic review. Topics in Stroke Rehabilitation 2016;23(5):366-75.

\section{Saltychev 2016}

Saltychev M, Sjogren T, Barlund E, Laimi K, Paltamaa J. Do aerobic exercises really improve aerobic capacity of stroke survivors? A systematic review and meta-analysis. European Journal of Physical and Rehabilitation Medicine 2016;52(2):233-43.

\section{Saunders 2008}

Saunders DH, Greig CA, Young A, Mead GE. Association of activity limitations and lower-limb explosive extensor power in ambulatory people with stroke. Archives of Physical Medicine and Rehabilitation 2008;89(4):677-83.

\section{Saunders 2013a}

Saunders DH, Greig CA. Physical fitness and function after stroke. In: Mead GE, Van Wijck F editor(s). Exercise and Fitness Training after Stroke - A Handbook for Evidence-based Practice. 1st Edition. London: Churchill Livingston Elsevier, 2013:77-91.

\section{Saunders 2014a}

Saunders DH, Greig CA, Mead GE. Physical activity and exercise after stroke: review of multiple meaningful benefits. Stroke 2014;45(12):3742-7.

\section{Saunders 2018}

Saunders DH, Mead GE, Fitzsimons C, Kelly P, Van Wijck F, Verschuren $\mathrm{O}$, et al. Interventions for reducing 
sedentary behaviour in people with stroke. Cochrane Database of Systematic Reviews 2018, Issue 4. [DOI: 10.1002/14651858.CD012996]

\section{Schulz 2002}

Schulz KF, Grimes DA. Sample size slippages in randomised trials: exclusions and the lost and wayward. Lancet 2002;359(9308):781-5.

\section{Shaw 2006}

Shaw KA, Gennat HC, O'Rourke P, Del Mar C. Exercise for overweight or obesity. Cochrane Database of Systematic Reviews 2006, Issue 4. [DOI: 10.1002/14651858.CD003817.pub3]

\section{Shephard 2009}

Shephard RJ. Maximal oxygen intake and independence in old age. British Journal of Sports Medicine 2009;43(5):342-6.

\section{Slade 2014}

Slade SC, Dionne CE, Underwood M, Buchbinder R. Standardised method for reporting exercise programmes: protocol for a modified Delphi study. BMJ Open 2014;4(12):e00668.

\section{Smart 2019}

Smart NA, Way D, Carlson D, Millar P, McGowan C, Swaine I, et al. Effects of isometric resistance training on resting blood pressure: individual participant data meta-analysis. Journal of Hypertension 2019; Vol. 37, issue 10:1927.

\section{Smith 2012}

Smith AC, Saunders DH, Mead G. Cardiorespiratory fitness after stroke: a systematic review. International Journal of Stroke 2012;7:499-510.

\section{Sorinola 2014}

Sorinola IO, Powis I, White CM. Does additional exercise improve trunk function recovery in stroke patients? A meta-analysis. NeuroRehabilitation 2014;35(2):205-13.

\section{Steele 2017}

Steele J, Fisher J, Skivington M, Dunn C, Arnold J, Tew G, et al. A higher effort-based paradigm in physical activity and exercise for public health: making the case for a greater emphasis on resistance training. BMC Public Health 2017;17(1):300.

\section{Tally 2017}

Tally Z, Boetefuer L, Kauk C, Perez G, Schrand L, Hoder J. The efficacy of treadmill training on balance dysfunction in individuals with chronic stroke: a systematic review. Topics in Stroke Rehabilitation 2017;24(7):539-46.

\section{Thomas 2006}

Thomas DE, Elliott EJ, Naughton GA. Exercise for type 2 diabetes mellitus. Cochrane Database of Systematic Reviews 2006, Issue 3. [DOI: 10.1002/14651858.CD002968.pub2]

\section{USDHHS 2018}

US Department of Health and Human Services Centers for Disease Control and Prevention, National Center for Chronic Disease Prevention and Health Promotion. Physical Activity
Guidelines Advisory Committee Report. Washington, DC: US Department of Health and Human Services, 2018.

\section{Van Criekinge 2019}

Van Criekinge T, Truijen S, Verbruggen C, Van de Venis L, Saeys W. The effect of trunk training on muscle thickness and muscle activity: a systematic review. Disability and Rehabilitation 2019;41(15):1751-9.

\section{Van Duijnhoven 2016}

Van Duijnhoven HJ, Heeren A, Peters MA, Veerbeek JM, Kwakkel G, Geurts AC, et al. Effects of exercise therapy on balance capacity in chronic stroke: systematic review and metaanalysis. Stroke 2016;47(10):2603-10.

\section{Van Wijck 2019}

Van Wijck F, Bernhardt J, Billinger SA, Bird ML, Eng J, English C, et al. Improving life after stroke needs global efforts to implement evidence-based physical activity pathways. International Journal of Stroke 2019;11:1747493019840930. [DOI: 10.1177/1747493019840930]

\section{Vanderbeken 2017}

Vanderbeken I, Kerckhofs E. A systematic review of the effect of physical exercise on cognition in stroke and traumatic brain injury patients. NeuroRehabilitation 2017;40(1):33-48.

\section{Vloothuis 2016}

Vloothuis JD, Mulder M, Veerbeek JM, Konijnenbelt M, Visser-Meily JM, Ket JC, et al. Caregiver-mediated exercises for improving outcomes after stroke. Cochrane Database of Systematic Reviews 2016, Issue 12. [DOI: 10.1002/14651858.CD011058.pub2]

\section{Wade 1992}

Wade DT. Measurement in Neurological Rehabilitation. Oxford University Press, 1992

\section{Wang 2018}

Wang C, Redgrave J, Shafizadeh M, Majid A, Kilner, Ali AN. Aerobic exercise interventions reduce blood pressure in patients after stroke or transient ischaemic attack: a systematic review and meta-analysis. British Journal of Sports Medicine 2018;Dec 53(24):1515-25.

\section{Ware 1992}

Ware Jr JE, Sherbourne CD. The MOS 36-item short-form health survey (SF-36): I. Conceptual framework and item selection. Medical Care 1992;30(6):473-83.

\section{Westbury 2018}

Westbury LD, Fuggle NR, Syddall HE, Duggal NA, Shaw SC, Maslin K, et al. Relationships between markers of inflammation and muscle mass, strength and function: findings from the Hertfordshire Cohort Study. Calcified Tissue International 2018;102(3):287-95.

\section{WHO 2001}

World Health Organization (WHO). International classification of functional disability and handicap. World Health Organization 
(WHO). Fifty-Fourth World Health Assembly WHA54.21, 2001. www.who.int/classifications/icf/en/ (accessed November 2010).

\section{WHO 2018}

World Health Organization (WHO). Global health estimates 2016: deaths by cause, age, sex, by country and by region, 2000-2016. Geneva, World Health Organization 2018.

\section{Wist 2016}

Wist S, Clivaz J, Sattelmayer M. Muscle strengthening for hemiparesis after stroke: a meta-analysis. Annals of Physical and Rehabilitation Medicine 2016;59(2):114-24.

\section{Young 2001}

Young A. For a healthier old age. In: Young A, Harries M editor(s). Physical Activity for Patients: An Exercise Prescription. London: Royal College of Physicians, 2001.

\section{Young 2019}

Young RE, Broom D, Sage K, Crossland K, Smith C. Experiences of venue based exercise interventions for people with stroke in the UK: a systematic review and thematic synthesis of qualitative research. Physiotherapy (in press).

\section{Zheng 2016}

Zheng G, Zhou W, Xia R, Tao J, Chen L. Aerobic exercises for cognition rehabilitation following stroke: a systematic review. Journal of Stroke and Cerebrovascular Diseases 2016;25(11):2780-9.

\section{Zigmond 1983}

Zigmond AS, Snaith PR. The Hospital Anxiety and Depression Scale. Acta Psychiatrica Scandinavica 1983;67:361-70.

\section{References to other published versions of this review \\ Brazzelli 2011a}

Brazzelli M, Saunders DH, Greig CA, Mead GE.

Physical fitness training for stroke patients. Cochrane Database of Systematic Reviews 2011, Issue 11. [DOI: 10.1002/14651858.CD003316.pub4]

\section{Brazzelli 2011b}

Brazzelli M, Saunders DH, Greig CA, Mead GE. Physical fitness training for stroke patients: a systematic review of the evidence. International Journal of Stroke 2011; Vol. 6:11.

\section{CHARACTERISTICS OF STUDIES}

Characteristics of included studies [ordered by study ID]

\section{Brazzelli 2012}

Brazzelli M, Saunders DH, Greig CA, Mead GE. Physical fitness training for patients with stroke updated review. Stroke 2012;43(4):E39-40.

\section{Saunders 2002}

Saunders DH, Greig CA, Young A, Mead GE. Physical fitness training for acute stroke patients - a systematic review. Cerebrovascular Diseases 2002;13 Suppl 3:63.

\section{Saunders 2004a}

Saunders DH, Greig C, Young A, Mead G. Physical fitness training for stroke patients. Cochrane Database of Systematic Reviews 2004, Issue 1. [DOI: 10.1002/14651858.CD003316.pub2]

\section{Saunders 2004b}

Saunders DH, Greig C, Young A, Mead G. Physical fitness training for stroke patients. Stroke 2004;35:2235.

\section{Saunders 2009}

Saunders DH, Greig CA, Mead GE, Young A. Physical fitness training for stroke patients. Cochrane Database of Systematic Reviews 2009, Issue 4. [DOI: 10.1002/14651858.CD003316.pub3]

\section{Saunders 2013b}

Saunders David H, Sanderson Mark, Brazzelli Miriam, Greig Carolyn A, Mead Gillian E. Physical fitness training for stroke patients. Cochrane Database of Systematic Reviews 2013, Issue 10. [DOI: 10.1002/14651858.CD003316.pub5]

\section{Saunders 2014b}

Saunders DH, Sanderson M, Brazzelli M, Greig CA, Mead GE. Physical fitness training for patients with stroke: an updated review. Stroke 2014;45:e45-e55.

\section{Saunders 2016}

Saunders DH, Sanderson M, Hayes S, Kilrane M, Greig CA, Brazzelli $M$, et al. Physical fitness training for stroke patients. Cochrane Database of Systematic Reviews 2016, Issue 3. [DOI: 10.1002/14651858.CD003316.pub6]

* Indicates the major publication for the study

Ada 2013

Methods Design: randomised trial of cardiorespiratory training vs no intervention - after UC

Randomised: computer-generated randomisation stratified on walking disability by independent researcher

Allocation concealment: not applicable

Blinding: assessors blind to group allocation 
Ada 2013 (Continued)

\section{ITT: yes}

Measurements: end of interventions ( 2 and 4 months) and 6- and 12-month follow-up

Withdrawals: 2 months treadmill training group: 1 participant withdrew; control group: 3 participants withdrew - reasons unclear

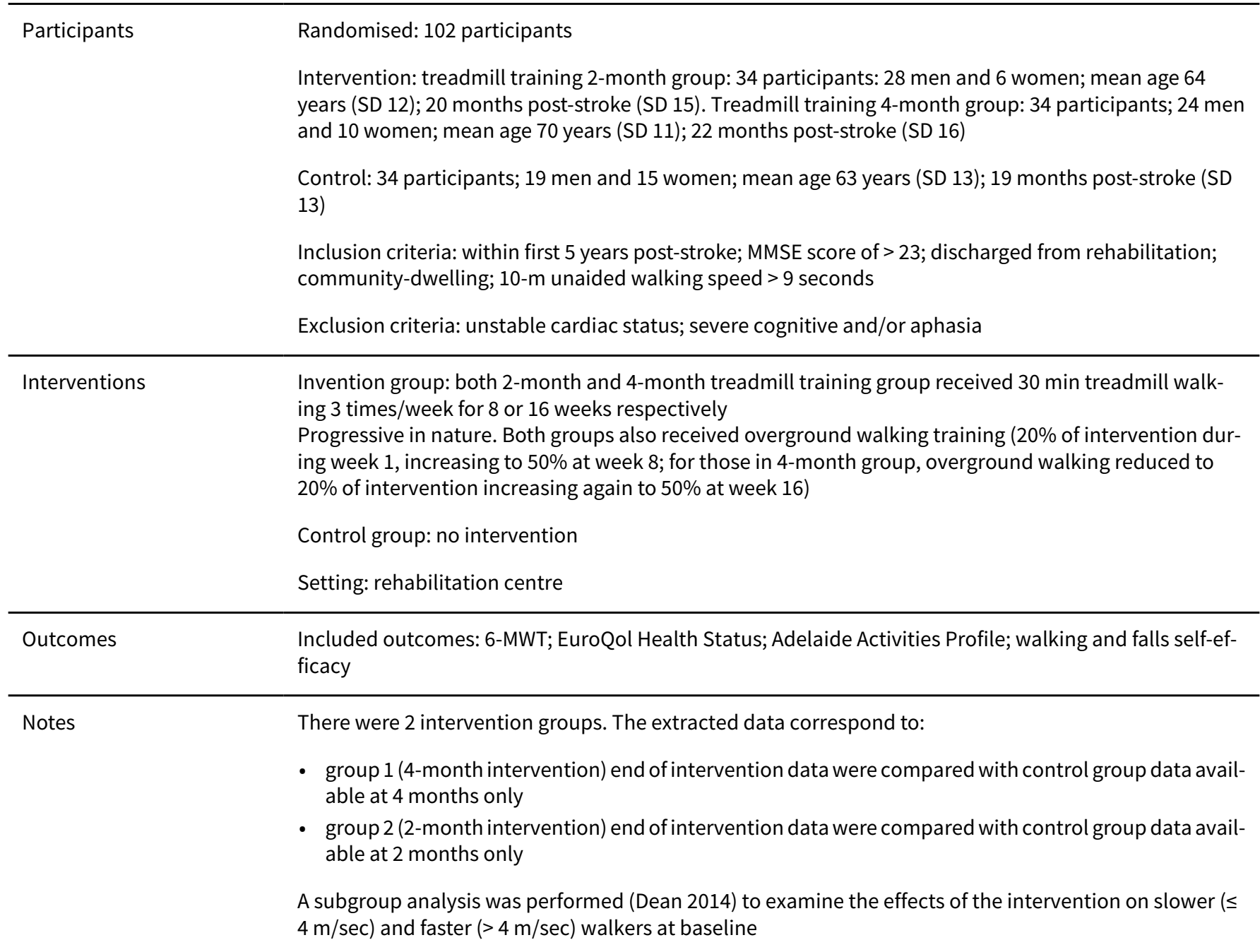

\section{Risk of bias}

\begin{tabular}{lll}
\hline Bias & Authors' judgement & Support for judgement \\
\hline $\begin{array}{l}\text { Random sequence genera- } \\
\text { tion (selection bias) }\end{array}$ & Low risk & $\begin{array}{l}\text { Computer-generated randomisation stratified on walking disability by inde- } \\
\text { pendent researcher }\end{array}$ \\
\hline $\begin{array}{l}\text { Allocation concealment } \\
\text { (selection bias) }\end{array}$ & Low risk & $\begin{array}{l}\text { Allocation concealment ensured because all available participants allocated in } \\
\text { groups of 15 to blocks of } 3 \text { after baseline measures recorded }\end{array}$ \\
\hline $\begin{array}{l}\text { Blinding (performance } \\
\text { bias and detection bias) } \\
\text { All outcomes }\end{array}$ & High risk & No attention control \\
\hline $\begin{array}{l}\text { Blinding of outcome as- } \\
\text { sessment (detection bias) } \\
\text { All outcomes }\end{array}$ & Low risk & Assessor blinded \\
\hline
\end{tabular}

Physical fitness training for stroke patients (Review) 
Ada 2013 (Continued)

Incomplete outcome data Low risk (attrition bias)

End of intervention
ITT analysis performed

Few (2/102) losses; 2-month treadmill training group: 1 participant withdrew; control group: 3 participants withdrew

Reasons and timing unclear

\section{Incomplete outcome data Low risk \\ ITT analysis performed}

(attrition bias)

End of follow-up
Few losses (2/102); 2-month treadmill training group: 1 participant withdrew; control group: 3 participants withdrew

Reasons and timing unclear

Reported outcomes correspond to trial registry ACTRN12607000227493

Selective reporting (re- Low risk $\quad$ Reported outcomes correspond to trial registry ACTRN12607000227493
porting bias)

\begin{tabular}{lll}
\hline Other bias & Low risk & None known \\
\hline Imbalanced exposure & High risk & Intervention group has uncontrolled exposure \\
\hline
\end{tabular}

Aidar 2016

Mesign: randomised trial of resistance training vs no intervention after UC
Randomised mechanism: lottery allocation into groups
Allocation concealment: not reported
Blinding: assessor blinded to group allocation
Measurements: end of intervention (12 weeks)
Withdrawals: 3 participants from intervention group during 2nd week of intervention and 2 participants
from control group were not assessed at the end of the intervention

\section{Participants}

Randomised: 24 participants

Intervention: 11 participants; 6 men and 5 women; mean age 51.7 years (SD 8.0); days after stroke unknown (> 1 year)

Control: 13 participants; 9 men and 4 women; mean age 52.5 years (SD 7.7); days after stroke unknown (>1 year)

Inclusion criteria: below 'per-capita' income, below minimum wage, medical authorisation 'clinically healthy people', stroke > 1 year ago, clinically stable, presence of hemiplegia or hemiparesis

Exclusion criteria: aphasia, no recurrent strokes, asymptomatic with a non-disabling deficit or with severe disabilities

Interventions Intervention group: resistance training; 12-week intervention, 3 times/week, each session lasting 60
min - conducted in the morning. Minimum of $48 \mathrm{~h}$ rest between sessions. Warm-up including $10-15$ min
walking followed by upper and lower body strengthening exercises: bar-guided squat, machine bench
press, horizontal leg press, military press machine, abdominal crunch, front lateral pull downs and bar-
guided lunges. 3 sets of 8-10 reps with 2-min rest between sets
Control group: no intervention
Setting: unclear; community-based project, indoor basketball court 
Aidar 2016 (Continued)
Outcomes
Included outcomes:
- 2012 paper: State-Trait Anxiety Inventory; muscle strength (1-RM kg for various lifts; squat, bench press, leg press, military press, lateral pull downs, lunges)
- 2014 paper: depression (BDI), muscle strength
- 2016 paper: SF-36

Notes Two separate publications 2012 and 2016

\title{
Risk of bias
}

\begin{tabular}{|c|c|c|}
\hline Bias & Authors' judgement & Support for judgement \\
\hline $\begin{array}{l}\text { Random sequence genera- } \\
\text { tion (selection bias) }\end{array}$ & Unclear risk & "Lottery" allocation into groups; still unclear exactly what was done \\
\hline $\begin{array}{l}\text { Allocation concealment } \\
\text { (selection bias) }\end{array}$ & Unclear risk & Allocation concealment: not reported \\
\hline $\begin{array}{l}\text { Blinding (performance } \\
\text { bias and detection bias) } \\
\text { All outcomes }\end{array}$ & High risk & No attention control \\
\hline $\begin{array}{l}\text { Blinding of outcome as- } \\
\text { sessment (detection bias) } \\
\text { All outcomes }\end{array}$ & Low risk & Blinded outcome assessors \\
\hline $\begin{array}{l}\text { Incomplete outcome data } \\
\text { (attrition bias) } \\
\text { End of intervention }\end{array}$ & High risk & 2/13 control group not analysed in 2016 paper; no ITT analysis \\
\hline $\begin{array}{l}\text { Selective reporting (re- } \\
\text { porting bias) }\end{array}$ & Unclear risk & No protocol available \\
\hline Other bias & Unclear risk & Self-selection bias may occur as advertisements were used \\
\hline Imbalanced exposure & High risk & Intervention group has uncontrolled exposure \\
\hline
\end{tabular}

\section{Aidar 2018}

\section{Methods}

\author{
Design: randomised trial of cardiorespiratory training (aquatic physical exercises) vs no intervention - \\ after UC \\ Randomisation: stated 'random' but no further details provided \\ Allocation concealment: not reported \\ Blinding: not reported \\ ITT: no \\ Measurements: at the end of intervention (12 weeks) \\ Withdrawals: (2007) 1 intervention group participant refused training programme; 2 control partici- \\ pants were not assessed at the end of the intervention
}


Aidar 2018 (Continued)

Withdrawals: (2018) 3 intervention group participants dropped out of training programme; 4 control participants were not assessed at the end of the intervention

Randomised (2007): 31 participants; assessed 28 (15 intervention 13 control)
Randomised (2018): $31+12=43$ participants; assessed 36 (19 intervention 17 control)
Intervention: (2007) 15 participants; 10 men and 5 women; mean age 50.3 years (SD 9.1)
Intervention: (2018) 19 participants; 10 men and 9 women; mean age 51.8 years (SD 8.5)
Control: (2007) 13 participants; 9 men and 4 women; mean age 52.5 years (SD 7.7)
Control: (2018) 17 participants; 9 men and 8 women; mean age 52.7 years (SD 6.7)
Inclusion criteria: ischaemic cerebrovascular accident; hemiplegia or hemiparesis
Exclusion criteria: cognitive impairment; significant co-morbidities

Interventions Intervention group: aquatic physical sessions (e.g. walking activity and physical exercises in the water; swimming) 45-60 min each session; 2 times/week for 12 weeks

Control group: no intervention - delayed started of the same programme

Setting: community setting

\begin{tabular}{ll}
\hline Outcomes & Included outcomes (2007): SF-36 (with 2007 sample size) \\
& - Included outcomes (2018): BDI, State Trait Anxiety Inventory (Portuguese version), TUG, maximum \\
& walking speed (7.62 m), timed sit to stand, BBS
\end{tabular}

Notes

Content of the intervention not very detailed. It appears that the Aidar 2018 paper is an extension of this same study published in 2007 with 8 new women participants added and different outcome reported

\begin{tabular}{|c|c|c|}
\hline \multicolumn{3}{|l|}{ Risk of bias } \\
\hline Bias & Authors' judgement & Support for judgement \\
\hline $\begin{array}{l}\text { Random sequence genera- } \\
\text { tion (selection bias) }\end{array}$ & Unclear risk & Stated 'random' but no further details provided \\
\hline $\begin{array}{l}\text { Allocation concealment } \\
\text { (selection bias) }\end{array}$ & Unclear risk & Not reported \\
\hline $\begin{array}{l}\text { Blinding (performance } \\
\text { bias and detection bias) } \\
\text { All outcomes }\end{array}$ & High risk & No attention control \\
\hline $\begin{array}{l}\text { Blinding of outcome as- } \\
\text { sessment (detection bias) } \\
\text { All outcomes }\end{array}$ & Unclear risk & Not reported \\
\hline $\begin{array}{l}\text { Incomplete outcome data } \\
\text { (attrition bias) } \\
\text { End of intervention }\end{array}$ & Unclear risk & $1 / 16$ lost from intervention and $2 / 15$ from control group. No ITT analysis \\
\hline $\begin{array}{l}\text { Selective reporting (re- } \\
\text { porting bias) }\end{array}$ & Unclear risk & No protocol available \\
\hline Other bias & Low risk & None known \\
\hline
\end{tabular}


Aidar 2018 (Continued)

Imbalanced exposure High risk Imbalanced exposure

Arabzadeh 2018

Design: open-label, randomised clinical trial; task-orientated resistance training (and balance) vs CPT
during UC
Randomisation: unclear; states that the randomisation procedure was performed by a person who was
not involved in the assessment
Allocation concealment: independent person prepared sealed envelopes, which were picked up by par-
ticipants in the order in which they entered the study
Blinding: participants in both groups were treated and assessed by the same physiotherapist; bias due
to "non blinding" was noted in the studies limitations; participants and assessors mave under-
stood nature of group assignments
ITT: not stated
Measurements: end of intervention (4 weeks)
Withdrawals: not reported

Participants

Randomised: 20 participants were randomised

Intervention: 10 participants; 8 men (80\%) and 2 women (20\%); mean age: 58.9 (SD 26.8); time since stroke onset: 32.80 days (SD 16.71)

Control: 10 participants; 7 men (70\%) and 3 women (30\%); mean age: 59.6 (SD 7.16); time since stroke onset: 32.5 days (SD 15.78)

Inclusion criteria: hemiplegia following stroke within 3 months, observational clear asymmetry in weight bearing, ability to walk $10 \mathrm{~m}$ independently without an assistive device, and BBS in the range of $30-40$

Exclusion criteria: impaired vision, neurological or orthopaedic diseases affecting postural function, and other diseases preventing participation

Intervention: 10 task-oriented exercises aimed at promoting balance, some of which incorporated strengthening. Progression was achieved by increasing repetitions, height of exercise step and adding weights around ankles; and somatosensory and vision manipulations (open/closed eyes and hard/soft surface). Frequency of intervention: $50 \mathrm{~min} / \mathrm{session} 3$ days/week for 4 weeks

Control: CPT (mat exercises, range of motion exercises, and walking education). The control group matched the intervention group in terms of time and number of exercises. Frequency of intervention: $50 \mathrm{~min} / \mathrm{session} 3$ days/week for 4 weeks

Setting: unclear, patients referred from 2 hospital outpatient clinics

Included outcomes: Persian version of BBS
Other outcomes: plantar pressure distribution, centre of pressure path length, and the centre of pres-
sure confidence ellipse area

\section{Risk of bias}


Arabzadeh 2018 (Continued)

Random sequence genera- Unclear risk Report states "randomisation procedure" but no further details provided on tion (selection bias) how order of envelopes was achieved

\begin{tabular}{|c|c|c|}
\hline $\begin{array}{l}\text { Allocation concealment } \\
\text { (selection bias) }\end{array}$ & Unclear risk & $\begin{array}{l}\text { Performed by an independent person but unclear whether envelopes were } \\
\text { opaque and sequentially numbered }\end{array}$ \\
\hline
\end{tabular}

\begin{tabular}{|c|c|c|}
\hline $\begin{array}{l}\text { Blinding (performance } \\
\text { bias and detection bias) } \\
\text { All outcomes }\end{array}$ & Unclear risk & $\begin{array}{l}\text { Comparison of } 2 \text { different interventions (task-oriented exercise program vs } \\
\text { CPT) }\end{array}$ \\
\hline
\end{tabular}

All outcomes

\begin{tabular}{|c|c|c|}
\hline $\begin{array}{l}\text { Blinding of outcome as- } \\
\text { sessment (detection bias) } \\
\text { All outcomes }\end{array}$ & High risk & Both the article and trial registry indicate that blinding was not used \\
\hline $\begin{array}{l}\text { Incomplete outcome data } \\
\text { (attrition bias) } \\
\text { End of intervention }\end{array}$ & Unclear risk & ITT not reported and no information about extent of dropouts or retention \\
\hline $\begin{array}{l}\text { Incomplete outcome data } \\
\text { (attrition bias) } \\
\text { End of follow-up }\end{array}$ & Low risk & \\
\hline $\begin{array}{l}\text { Selective reporting (re- } \\
\text { porting bias) }\end{array}$ & Low risk & Included outcomes correspond with protocol IRCT2015100224297N1 \\
\hline Other bias & Low risk & None known \\
\hline Imbalanced exposure & Low risk & Matched exposures \\
\hline
\end{tabular}

Bale 2008

Design: randomised trial of resistance training plus \% UC vs UC - during UC
Sample size calculation reported
Randomisation: drawing lots - not clearly described
Allocation concealment: unclear
Blinding: outcome assessors blinded
ITT: planned but no withdrawals
Measurements: at the end of intervention (4 weeks)
Withdrawals: none

Participants Randomised: 18 participants

Intervention: 8 participants; 3 men and 5 women; mean age 68.0 years (SD 13); time since stroke 49.4 (SD 22.1) days

Control: 10 participants; 4 men and 6 women; mean age 64.9 years (SD 8.8); time since stroke 32.0 (SD 18.5) days

Inclusion criteria: first onset of stroke with reduced muscle strength in the affected leg; ability to understand verbal information; ability to sit without support 
Bale 2008 (Continued)

Exclusion criteria: significant sensory or cognitive sequels; arrhythmia; uncontrolled angina pectoris or hypertension; co-morbidities that could mask the sequels from the stroke; lack of motor control of the affected leg

Interventions

Intervention group: resistance training $50 \mathrm{~min} /$ day 3 days/week for 4 weeks. 8 individually tailored exercises for the affected lower limb involving weight bearing, stepping, sit-to-stand, heel/toe raising, and bridging. Tailored progression included using weights, reducing speed, adding more sets, etc. Other functional activities sometimes included too (walking, stair climbing, sit-to-stand). 1 set of 10-15 reps to moderate fatigue

Control group: UC (Bobath) $50 \mathrm{~min} /$ day 3 days/week for 4 weeks, plus UC (other) $50 \mathrm{~min} /$ day, 2 days/ week for 4 weeks. Total training: $50 \mathrm{~min} /$ day 5 days/week for 4 weeks

Setting: 2 rehabilitation units

\begin{tabular}{ll} 
Outcomes & Included outcomes: isometric muscle strength; preferred walking speed; maximal walking speed \\
& $\begin{array}{l}\text { Other outcomes: maximum weight bearing; } 2 \text { items of the MAS; Patient Global Impression of Change } \\
\text { tool }\end{array}$ \\
\hline Notes & Very small sample size \\
& Poor external validity
\end{tabular}

\section{Risk of bias}

\begin{tabular}{|c|c|c|}
\hline Bias & Authors' judgement & Support for judgement \\
\hline $\begin{array}{l}\text { Random sequence genera- } \\
\text { tion (selection bias) }\end{array}$ & Unclear risk & Drawing lots - not clearly described \\
\hline $\begin{array}{l}\text { Allocation concealment } \\
\text { (selection bias) }\end{array}$ & Unclear risk & Poorly reported \\
\hline $\begin{array}{l}\text { Blinding (performance } \\
\text { bias and detection bias) } \\
\text { All outcomes }\end{array}$ & Unclear risk & Attention control exposure \\
\hline $\begin{array}{l}\text { Blinding of outcome as- } \\
\text { sessment (detection bias) } \\
\text { All outcomes }\end{array}$ & Low risk & Outcome assessor \\
\hline $\begin{array}{l}\text { Incomplete outcome data } \\
\text { (attrition bias) } \\
\text { End of intervention }\end{array}$ & Low risk & ITT planned but no withdrawals \\
\hline $\begin{array}{l}\text { Selective reporting (re- } \\
\text { porting bias) }\end{array}$ & Unclear risk & No protocol available \\
\hline Other bias & Low risk & Unclear \\
\hline Imbalanced exposure & Low risk & Balanced exposure \\
\hline
\end{tabular}

\section{Bateman 2001}

$\begin{array}{ll}\text { Methods } & \text { Design: multicentre randomised trial of cardiorespiratory training plus UC vs non-exercise intervention } \\ \text { plus UC - during UC }\end{array}$


Randomisation: mechanism - computer; method - blocks size of 10 participants

Allocation concealment: numbered, sealed envelopes

Blinding: investigator blinded; participants encouraged to maintain blinding; efficacy unknown

ITT: yes, but participants were excluded after recruitment and baseline assessments due to discharge

Measurements: end of intervention (12 weeks) and at follow-up

Withdrawals: intervention group (12 participants: 4 before and 8 after the 12-week assessment); con-

trol group (12 participants: 2 before and 10 after the 12-week assessment)

Reasons unclear but included early discharge

Randomised: 84 participants
Intervention: 40 participants; men 20, women 20; age 47.0 years (SD 13.1); 144 days (SD 84) post-stroke
Control: 44 participants; men 29, women 14; age 50.3 years (SD 10.1); 184 days (SD 127) day post-stroke
Inclusion criteria: single stroke; could comply with planned interventions; could sit on a cycle ergome-
ter
Exclusion criteria: likely to be inpatient for < 3 months; impairments severe enough to limit training
compliance and participation; cardiac disease; co-morbidities contraindicated for exercise

Intervention: cardiorespiratory training; cycle ergometry at $60 \%-80 \%$ of age-related HR maximum for
up to $30 \mathrm{~min} /$ day 3 days/week for 12 weeks
Control: relaxation - programme individualised: included breathing exercises, progressive muscle re-
laxation, autogenic exercises, visualisation techniques
Setting: multicentre, 4 rehabilitation units

Outcomes Included outcomes: FIM; BI (0-20 scale); NEADL; RMI; HADS; BBS; gait maximum speed; maximum cycling workload (data transformed to Log base e); BMI

Other outcomes: fatigue questionnaire

Notes Mixed brain injury data provided by study authors; stroke-only data retained and re-analysed. High rate of missing data made statistical analyses difficult

\section{Risk of bias}

Bias Authors' judgement Support for judgement

Random sequence genera- Low risk $\quad$ Computer-based block $(n=10)$ randomisation
tion (selection bias)

\begin{tabular}{lll}
\hline $\begin{array}{l}\text { Allocation concealment } \\
\text { (selection bias) }\end{array}$ & Low risk & Numbered, sealed envelopes \\
\hline $\begin{array}{l}\text { Blinding (performance } \\
\text { bias and detection bias) } \\
\text { All outcomes }\end{array}$ & Unclear risk & Attention control \\
\hline
\end{tabular}

\begin{tabular}{|c|c|c|}
\hline $\begin{array}{l}\text { Blinding of outcome as- } \\
\text { sessment (detection bias) }\end{array}$ & Low risk & $\begin{array}{l}\text { Investigator blinded; participants encouraged to maintain blinding; efficacy } \\
\text { unknown }\end{array}$ \\
\hline
\end{tabular}

All outcomes Investigator blinded; participants encouraged to maintain blinding; efficacy
unknown

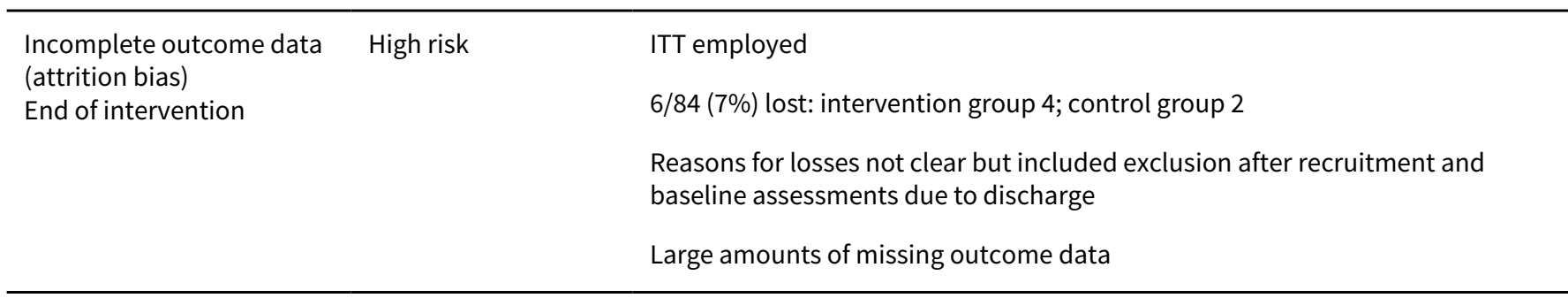

Incomplete outcome data High risk ITT employed
(attrition bias)


Bateman 2001 (Continued)

Selective reporting (re- Unclear risk $\quad$ Protocol not available
porting bias)

\begin{tabular}{lll}
\hline Other bias & Low risk & None known \\
\hline Imbalanced exposure & Low risk & Balanced exposure \\
\hline
\end{tabular}

\section{Buyukvural 2015}

\begin{tabular}{|c|c|}
\hline \multirow[t]{8}{*}{ Methods } & $\begin{array}{l}\text { Design: prospective RCT; resistance training + conventional rehabilitation (isokinetic group) vs conven- } \\
\text { tional rehabilitation alone (control group) - during UC }\end{array}$ \\
\hline & $\begin{array}{l}\text { A group of healthy controls performed the isokinetic test protocol (healthy controls) only for compari- } \\
\text { son of healthy vs non-paretic side peak torque measurements pre-treatment (not discussed in this re- } \\
\text { view) }\end{array}$ \\
\hline & Randomisation: not reported \\
\hline & Allocation concealment: not reported \\
\hline & Blinding: not reported \\
\hline & ITT: not reported \\
\hline & Measurements: before (baseline) and at the end of intervention (3 weeks) \\
\hline & Withdrawals: not reported \\
\hline \multirow[t]{6}{*}{ Participants } & $\begin{array}{l}\text { Randomised: } 50 \text { stroke participants. } 25 \text { participants were randomised to resistance training, and } 25 \text { to } \\
\text { the control group }\end{array}$ \\
\hline & Number randomised in comparisons used in this review this review $=50$ \\
\hline & $\begin{array}{l}\text { Intervention: } 25 \text { participants; } 17 \text { men (68\%) and } 8 \text { women (32\%); mean age: } 51.3 \text { (SD 12.0); mean } \\
\text { months post-stroke: } 3 \text { (range } 2-8 \text { ) }\end{array}$ \\
\hline & $\begin{array}{l}\text { Control: } 25 \text { participants; } 16 \text { men (64\%) and } 9 \text { women (36\%); mean age: } 55.4 \text { (SD 10.5); mean months } \\
\text { post-stroke: } 3 \text { (range 2-9) }\end{array}$ \\
\hline & Inclusion criteria: subacute-chronic stroke \\
\hline & $\begin{array}{l}\text { Exclusion criteria: not co-operative, with a previous history of stroke, concomitant disorders preventing } \\
\text { rehabilitation programme such as severe cardiovascular and pulmonary diseases, uncontrolled hyper- } \\
\text { tension, stroke due to tumour or trauma, major sensorimotor aphasia, orthopaedic disorders can affect } \\
\text { isokinetic assessments, musculoskeletal pain of lower limbs, and vestibular disorders }\end{array}$ \\
\hline
\end{tabular}

Interventions

Intervention: conventional rehabilitation + isokinetic strengthening. Isokinetic strengthening consisted of maximal concentric isokinetic training to the bilateral knee-ankle muscles according to the following protocols: knee exercise protocol: 5 repetitions at $60^{\circ} / \mathrm{s}, 90^{\circ} / \mathrm{s}, 120^{\circ} / \mathrm{s}, 150^{\circ} / \mathrm{s}$ angular velocities; $10 \mathrm{~s}$ rest period; 10 repetitions at $180^{\circ} / \mathrm{s}$

Ankle exercise protocol: 5 repetitions at $60^{\circ} / \mathrm{s}, 90^{\circ} / \mathrm{s}, 120^{\circ} / \mathrm{s}$ angular velocities; $10 \mathrm{~s}$ rest period; 10 repetitions at $150^{\circ} / \mathrm{s}$ angular velocity. Frequency of intervention: 5 days/week for 3 weeks

Control: conventional rehabilitation. Frequency of intervention: not reported

Setting: hospital 
Buyukvural 2015 (Continued)

Outcomes

Included outcomes: muscle strength; paretic and non-paretic knee peak torque (at $60 \% \mathrm{~s}$ and $180 \% \mathrm{~s}$ ) and ankle peak torque (at $60^{\circ} / \mathrm{s}$ and $120^{\circ} / \mathrm{s}$ ), Stroke Specific QoL Scale, timed 10-metre walk test, 6 MWT, stair-climbing test, TUG test, BBI, and RMI

Other outcomes: FIM (motor subscale)

Notes It is unclear what the conventional rehabilitation consisted of, in terms of content and dose
No variance/SD data available for outcomes; requested from author. SD of change scores interpolated
from P values

\section{Risk of bias}

\begin{tabular}{|c|c|c|}
\hline Bias & Authors' judgement & Support for judgement \\
\hline $\begin{array}{l}\text { Random sequence genera- } \\
\text { tion (selection bias) }\end{array}$ & Unclear risk & No information on randomisation procedure. Groups appear balanced \\
\hline $\begin{array}{l}\text { Allocation concealment } \\
\text { (selection bias) }\end{array}$ & Unclear risk & Not reported \\
\hline $\begin{array}{l}\text { Blinding (performance } \\
\text { bias and detection bias) } \\
\text { All outcomes }\end{array}$ & High risk & $\begin{array}{l}\text { Not attention control for amount of exposure therefore no opportunity to con- } \\
\text { ceal purpose etc }\end{array}$ \\
\hline $\begin{array}{l}\text { Blinding of outcome as- } \\
\text { sessment (detection bias) } \\
\text { All outcomes }\end{array}$ & Unclear risk & Not described \\
\hline $\begin{array}{l}\text { Incomplete outcome data } \\
\text { (attrition bias) } \\
\text { End of intervention }\end{array}$ & Unclear risk & Extent of missing outcome data not reported \\
\hline $\begin{array}{l}\text { Selective reporting (re- } \\
\text { porting bias) }\end{array}$ & Unclear risk & No trial registry or protocol information \\
\hline Other bias & Unclear risk & $\begin{array}{l}\text { Inadequate reporting makes it difficult to assess other potential biases in this } \\
\text { study }\end{array}$ \\
\hline Imbalanced exposure & High risk & No attention control \\
\hline
\end{tabular}

Cooke 2010

Methods

Design: phase I multicentre trial; 4 centres; mixed training plus UC vs UC - during UC - i.e. FST + CPT vs CPT alone and vs CPT + CPT

Randomisation: computer-generated random allocation in blocks of 9 per study centre (stratified allocation by baseline scores for visual spatial neglect)

Allocation concealment: sequentially numbered, sealed, opaque envelopes

Blinding: assessor blinded to group allocation

ITT: attempt to measure participants at outcome and follow-up even if they withdrew but analyses were not performed according to ITT principle

Measurements: at the end of intervention ( 6 weeks) and 12 weeks later (follow-up) 
Withdrawals: at outcome $7 / 74$ (9\%) participants were lost at outcome in the control CPT group (3 unwell, 3 withdrew, 1 moved abroad). At follow-up, a further 21 participants had withdrawn (total 28/74 26\%). 14 participants were lost in the CPT group (5 unwell, 4 withdrew, 1 moved abroad, 2 housebound, 2 died) and 7 in the CPT + FST group (5 unwell, 2 withdrew)

Participants

Randomised: total 109 participants. 38 participants were randomised to CPT, 35 to CPT + CPT, and 36 to FST + CPT (only the results from the CPT and the CPT + FST groups were included in this review)

Number randomised in comparisons used in this review this review $=74$

Intervention: FST + CPT = 36 participants; 22 men (61\%) and 14 women (39\%); mean age: 71.17 (SD 10.6); 33.86 (SD 16.50) days after stroke

Control: CPT = 38 participants; 21 men (55\%) and 17 women (45\%); mean age: 66.37 (SD 13.7); 36.76 (SD 22.41) days after stroke

Inclusion criteria: inpatients between 1 and 13 weeks after anterior circulation stroke (ischaemic and haemorrhagic); independently mobile; some voluntary contraction in the lower affected limb; no orthopaedic surgery or trauma affecting the lower limb in the last 8 weeks; no previous history of neurological diseases; able to follow a 1-stage command

Exclusion criteria: not reported

Interventions

Intervention: FST/mixed training + CPT. FST consisted of increasing the amount of body weight the participants needed to move; increasing movement resistance; reducing amount of body weight support during treadmill training. Frequency of intervention: $1 \mathrm{~h}$ for 4 days/week for 6 weeks

Control: CPT included soft tissue mobilisation, facilitation of muscle activity, facilitation of co-ordinated multi-joint movement; tactile and proprioceptive input, resistive exercise, and functional retraining. Frequency of intervention: $1 \mathrm{~h}$ for 4 days/week for 6 weeks

Setting: hospital

\begin{tabular}{lll}
\hline Outcomes & $\begin{array}{l}\text { Included outcomes: walking speed; HRQoL measures (e.g. EuroQol) } \\
\text { Other outcomes: gait parameters; paretic knee torque force analysis; modified RMI }\end{array}$ \\
\hline Notes & Study authors stated 'strength training' but intervention was actually mixed training \\
\hline Risk of bias & Authors' judgement & Support for judgement \\
\hline Bias & Low risk & $\begin{array}{l}\text { Computer-generated random allocation in blocks of 9 per study centre (strati- } \\
\text { fied allocation by baseline scores for visual spatial neglect) }\end{array}$ \\
\hline $\begin{array}{l}\text { Random sequence genera- } \\
\text { tion (selection bias) }\end{array}$ & Sequentially numbered, sealed, opaque envelopes \\
\hline $\begin{array}{l}\text { Allocation concealment } \\
\text { (selection bias) }\end{array}$ & Low risk & \\
\hline
\end{tabular}

Blinding (performance High risk Comparison used means no attention control

bias and detection bias)

All outcomes

Blinding of outcome as- Low risk Assessor blinded to group allocation

Incomplete outcome data High risk (attrition bias)

End of intervention
Attempt to measure participants at outcome and follow-up even if they withdrew but analyses were not performed according to ITT principle. Imbalanced losses at the end of intervention 
Incomplete outcome data High risk

(attrition bias)

End of follow-up
Attempt to measure participants at outcome and follow-up even if they withdrew but analyses were not performed according to ITT principle. Imbalanced large losses at the end of follow-up

28/74 (38\%) total losses: 14 participants were lost from the CPT group (5 unwell, 4 withdrew, 1 moved abroad, 2 housebound, 2 died) and 7 in the intervention group CPT + FST group (5 unwell, 2 withdrew)

\begin{tabular}{lll}
\hline $\begin{array}{l}\text { Selective reporting (re- } \\
\text { porting bias) }\end{array}$ & Low risk & Reported outcome correspond with those in trial register NCT00322192 \\
\hline Other bias & Low risk & None known \\
\hline Imbalanced exposure & High risk & $\begin{array}{l}\text { Imbalanced exposure (CPT + CPT group although balanced does not meet in- } \\
\text { clusion criteria) }\end{array}$ \\
\hline
\end{tabular}

\section{Coroian 2018}

\section{Methods}

Design: RCT; resistance training plus UC vs non-exercise (sham) intervention plus UC to compare isokinetic strengthening of elbow and wrist muscles to passive mobilisation of the same joints in a population of chronic stroke

Randomisation: randomly assigned in a 1:1 ratio. Randomisation sequence was centralised and computed in permuted blocks of 4 participants by a statistician (using Talena)

Allocation concealment: not listed

Blinding: physiotherapists and occupational therapists were blinded as to which participants had been included in which group. Provision of upper limb strengthening exercises was provided by a therapist not involved with participant's care

ITT: ITT analysis performed but not described

Measurements: baseline, end of intervention (variable, between 45 days and 60 days) and follow-up at 3 months and 6 months

Withdrawals: intervention group: $2 / 10$ (20\%) discontinued after the end of the intervention ( 2 moved to another region). Control group: 2/10 (20\%) discontinued after the 3-month follow-up (gastric ulcer = 1; lost to follow up $n=1$ ) the elbow and wrist $>2 / 5$, Modified Ashworth Scale score $<3 / 5$ for the wrist and elbow muscles, no limitations in upper limb range of motion (at shoulder, elbow, metatarsophalangeal joint)

Exclusion criteria: cognitive impairment (Boston Aphasia Quotient Score $<4 / 5$ ), Catherine Bergego Scale $>14 / 20$, MMSE $<22$ and participant (or their legal team) refusal to take part in the study

Interventions

Intervention: 18-day rehabilitation programme spread over 6 consecutive weeks (3 days/week). Each day: $2 \times 30$ min of PT (passive and active mobilisation of upper and lower limbs, balance exercises, manual strengthening of weak muscles, global motor reinforcement, task training, cardiorespiratory and walking training. Plus $1 \times 45 \mathrm{~min}$ OT (focused on hand grasp). Upper limb isokinetic muscle 
strengthening exercises using the dynamometer (for 30 min with 6 sets of 8 repetitions) intensity progressing from $40 \%-70 \%$ of maximal baseline torque values

Control: 18-day rehabilitation programme spread over 6 consecutive weeks ( 3 days/week). Each day: $2 \times 30$ min of PT (passive and active mobilisation of upper and lower limbs, balance exercises, manual strengthening of weak muscles, global motor reinforcement, task training, cardiorespiratory and walking training. Plus 1 x 45 min OT (focused on hand grasp). Performed only passive mobilisation of the elbow and wrist in the allowed range of motion

Setting: not reported

Outcomes Included outcomes: upper limb Fugl-Meyer Assessment (sensorimotor impairment), Box and Block test (grasping capacities), Modified Ashworth scale (spasticity), muscle strength of wrist and elbow flexors and extensors (maximum isokinetic reciprocal flexion/extension contractions, plus highest peak torques)

Notes Clinical Trials Registration Number: NCT01554137

\section{Risk of bias}

\begin{tabular}{|c|c|c|}
\hline Bias & Authors' judgement & Support for judgement \\
\hline $\begin{array}{l}\text { Random sequence genera- } \\
\text { tion (selection bias) }\end{array}$ & Low risk & Block randomisation mechanism described \\
\hline $\begin{array}{l}\text { Allocation concealment } \\
\text { (selection bias) }\end{array}$ & Low risk & Centralised system; randomisation sequence likely to be secure \\
\hline $\begin{array}{l}\text { Blinding (performance } \\
\text { bias and detection bias) } \\
\text { All outcomes }\end{array}$ & Unclear risk & $\begin{array}{l}\text { Attempts to blind those involved in delivering and receiving the interventions } \\
\text { and care, to the exact nature of the overall exposures }\end{array}$ \\
\hline $\begin{array}{l}\text { Blinding of outcome as- } \\
\text { sessment (detection bias) } \\
\text { All outcomes }\end{array}$ & Low risk & Blinded outcome assessment \\
\hline $\begin{array}{l}\text { Incomplete outcome data } \\
\text { (attrition bias) } \\
\text { End of intervention }\end{array}$ & Low risk & No dropouts at end intervention (45d). ITT analysis performed \\
\hline $\begin{array}{l}\text { Incomplete outcome data } \\
\text { (attrition bias) } \\
\text { End of follow-up }\end{array}$ & Unclear risk & $\begin{array}{l}2 / 10(20 \%) \text { lost from intervention at } 3 \text { months and } 2 / 10 \text { lost from control at } 6 \\
\text { months. Therefore, at final follow-up ( } 6 \text { months) both groups lost } 2 / 10 \text { but ITT } \\
\text { analysis used }\end{array}$ \\
\hline $\begin{array}{l}\text { Selective reporting (re- } \\
\text { porting bias) }\end{array}$ & High risk & $\begin{array}{l}\text { Only the Fugl Meyer outcome measure was reported on the NCT01554137 trial } \\
\text { registry }\end{array}$ \\
\hline Other bias & High risk & $\begin{array}{l}\text { Anecdotal assessment of fatigue resulted in changes and reluctance to recruit } \\
\text { participants }\end{array}$ \\
\hline Imbalanced exposure & Low risk & Sham passive mobilisation attention control \\
\hline
\end{tabular}

\section{Cuviello-Palmer 1988}

\begin{tabular}{ll}
\hline Methods & Design: randomised trial of cardiorespiratory training plus \% UC vs UC - after UC \\
& Randomisation: unknown \\
& Allocation concealment: unknown
\end{tabular}


Cuviello-Palmer 1988 (Continued)

Blinding: unknown

ITT: no

Measurements: end of intervention (3 weeks)

Withdrawals: none

Rarticipants
Intervention: 10 participants; 6 men and 4 women; age 69.5 years (SD 14.1); 20.7 days post-stroke (SD
13.2)
Control: 10 participants; 7 men and 3 women; age 71.8 years (SD 12.0); 12.0 days post-stroke (SD 16.8)
Inclusion criteria: unknown
Exclusion criteria: unknown

Interventions

Intervention: cardiorespiratory training: isokinetic ergometer allowing resisted reciprocal leg movements (Kinetron II); commencing at $2 \times 7 \mathrm{~min} /$ day for 5 days/week and $1 \times 7 \mathrm{~min} /$ day for 1 day/week (total 6 days/week) for 3 weeks progressing to $10 \mathrm{~min} / \mathrm{session}$ in week 2 and $12 \mathrm{~min}$ in week 3

Exercise intensity maintained at a $\mathrm{HR}$ of $<20 \mathrm{bpm}$ above resting

Control: UC: 2 × $45 \mathrm{~min} /$ day for 5 days/week and 1 x $45 \mathrm{~min} /$ day for 1 day/week (total 6 days/week) for 3 weeks

Gait training, mat exercises, and transfer training achieved via strengthening exercises, post neuromuscular facilitation (PNF), functional electrical stimulation (FES), Brunnstrom, Rood, and neurodevelopment techniques

Setting: rehabilitation centre

\begin{tabular}{ll}
\hline Outcomes & $\begin{array}{l}\text { Included outcomes: FIM (old version); preferred gait speed (7 seconds) } \\
\text { Other outcomes: stance symmetry; contact time (seconds); stride cadence steps/min and other biome- } \\
\text { chanical gait parameters }\end{array}$ \\
\hline Notes & -
\end{tabular}

\section{Risk of bias}

\begin{tabular}{|c|c|c|}
\hline Bias & Authors' judgement & Support for judgement \\
\hline $\begin{array}{l}\text { Random sequence genera- } \\
\text { tion (selection bias) }\end{array}$ & Unclear risk & Not reported \\
\hline $\begin{array}{l}\text { Allocation concealment } \\
\text { (selection bias) }\end{array}$ & Unclear risk & Not reported \\
\hline $\begin{array}{l}\text { Blinding (performance } \\
\text { bias and detection bias) } \\
\text { All outcomes }\end{array}$ & High risk & Some degree of attention control \\
\hline $\begin{array}{l}\text { Blinding of outcome as- } \\
\text { sessment (detection bias) } \\
\text { All outcomes }\end{array}$ & Unclear risk & Not reported \\
\hline $\begin{array}{l}\text { Incomplete outcome data } \\
\text { (attrition bias) } \\
\text { End of intervention }\end{array}$ & Low risk & No withdrawals, no planned ITT \\
\hline $\begin{array}{l}\text { Selective reporting (re- } \\
\text { porting bias) }\end{array}$ & Unclear risk & No protocol available \\
\hline Other bias & Low risk & Not known \\
\hline Imbalanced exposure & Low risk & Exposure balanced \\
\hline
\end{tabular}


da Cunha 2002

Design: randomised trial of cardiorespiratory training plus \% UC vs UC - during UC
Randomisation mechanism: random number table
Allocation concealment: unknown
Blinding: unknown
ITT: no
Measurements: end of intervention (2/3 weeks - until discharge)
Withdrawals: none

\begin{tabular}{|c|c|}
\hline Participants & $\begin{array}{l}\text { Randomised: } 15 \text { participants } \\
\text { Intervention: } 7 \text { participants; } 6 \text { men and } 1 \text { women; age } 57.8 \text { years (SD 5.5); } 15.7 \text { days post-stroke (SD } 7.7 \text { ) } \\
\text { Control: } 8 \text { participants; } 7 \text { men and } 1 \text { women; age } 58.9 \text { years (SD } 12.9) ; 19.0 \text { days post-stroke (SD } 12.7 \text { ) } \\
\text { Inclusion criteria: recent stroke (onset }<6 \text { weeks); significant gait deficit ( }<36 \mathrm{~m} / \mathrm{min} \text {; FAC score of } 0,1 \\
\text { or 2); sufficient cognition to participate in training (MMSE } \geq 21 \text { ); able to stand and take } \geq 1 \text { steps without } \\
\text { assistance } \\
\text { Exclusion criteria: co-morbidity or disability other than hemiparesis; recent MI; any uncontrolled } \\
\text { health condition; joint disease or rheumatoid arthritis; obesity ( }>110 \mathrm{~kg} \text { ); cognitive impairment (MMSE } \\
<21 \text { ) }\end{array}$ \\
\hline
\end{tabular}

Interventions

Intervention: cardiorespiratory training: treadmill walking with body weight support $20 \mathrm{~min} /$ day 6 days/week for 2-3 weeks (until discharge); intensity unknown but rapid progression imposed by increasing speed and reducing body weight support; the 20-min training replaced the 20-min gait training component of the control

Control: UC $3 \mathrm{~h}$ /day for 6 days/week for 2-3 weeks until discharge; included kinesitherapy (1 h/day), OT $(1 \mathrm{~h} /$ day), and physical therapy $(1 \mathrm{~h} /$ day $)$ : the physical therapist included $20 \mathrm{~min}$ of gait training comprising stepping, standing, turning, etc, but not continuous walking Setting: rehabilitation centre

Included outcomes: cycle performance work rate (watts); $\mathrm{VO}_{2}$ peak; BP; FAC; FIM (lower limb); gait
speed maximal $(5 \mathrm{~m})$; gait endurance $(5 \mathrm{~min})$; gait economy
Other outcomes: stance symmetry; contact time $(\mathrm{s}) ;$ stride cadence steps/min and other biomechani-
cal gait parameters

\section{Notes}

\section{Risk of bias}

\begin{tabular}{lll}
\hline Bias & Authors' judgement & Support for judgement \\
\hline $\begin{array}{l}\text { Random sequence genera- } \\
\text { tion (selection bias) }\end{array}$ & Low risk & $\begin{array}{l}\text { Randomisation by using random numbers to pre-assign participants based on } \\
\text { recruitment order }\end{array}$ \\
\hline $\begin{array}{l}\text { Allocation concealment } \\
\text { (selection bias) }\end{array}$ & Unclear risk & Not reported \\
\hline $\begin{array}{l}\text { Blinding (performance } \\
\text { bias and detection bias) } \\
\text { All outcomes }\end{array}$ & Unclear risk & Some degree of attention control \\
\hline $\begin{array}{l}\text { Blinding of outcome as- } \\
\text { sessment (detection bias) } \\
\text { All outcomes }\end{array}$ & Unclear risk & Not reported \\
\hline
\end{tabular}

$\begin{aligned} & \text { Incomplete outcome data } \\ & \text { (attrition bias) }\end{aligned}$
Low risk $\quad$ No withdrawals, no planned ITT

End of intervention 
da Cunha 2002 (Continued)

Selective reporting (re- $\quad$ Unclear risk $\quad$ No protocol available
porting bias)

\begin{tabular}{lll}
\hline Other bias & Low risk & None known \\
\hline Imbalanced exposure & Low risk & Balanced exposure \\
\hline
\end{tabular}

ReTRAIN i.e. mixed training plus UC vs UC alone - after UC (> 1 month post-discharge)

Randomisation: the random sequence was computer-generated with minimisation for time since stroke ( $\leq 3$ months vs $>3$ months) and level of functional disability ( $m R S$ score $\leq 2$ vs $>2$ )

Allocation concealment: using a password-protected validated web-based remote randomisation service supported by the Peninsula Clinical Trials Unit. The Trial Manager requested randomisation only after a cohort of participants had been consented

Blinding: assessor blinded to group allocation; participants were reminded not to reveal their allocation to assessors but any unblinding was recorded; after assessments assessors were asked to guess participant allocation

Participants, trainers providing the intervention and researchers conducting the process and economic evaluations could not be blinded to allocation

ITT: missing data were reported but no attempts were made to analyse according to ITT

Measurements: baseline, 6-month and 9-month follow-up

Withdrawals: before start of intervention 2/23 allocated to ReTRAIN withdrew (1 unwell, 1 family crisis); $2 / 22$ control withdrew before intervention ( 1 unwell, 1 wanted to be in the experimental group). At 6 month follow-up there were missing data for 1 participant in the control group; none in the experimental group. At 9 months there were no missing data (ReTRAIN $n=21$; control $n=20$ )

Participants

Randomised: 45 participants

Intervention: ReTRAIN + UC 23 participants; 16 men (70\%) and 7 women (30\%); mean age: 70 (SD 12); time since stroke $\leq 3$ months $n=1(4 \%),>3$ months $n=22(96 \%)$

Control: UC 22 participants; 14 men (67\%) and 8 women (33\%); mean age: 71 (SD 10); time since stroke $\leq 3$ months $\mathrm{n}=0(0 \%),>3$ months $\mathrm{n}=22(100 \%)$

Inclusion criteria: diagnosis of stroke, any time since stroke but at least 1 month since discharge from NHS physical rehabilitation services, able to walk independently indoors with or without mobility aids, but with self-reported difficulty with stairs, slopes or uneven surfaces, willingness to be randomised and attend the training venue and cognitive capacity and communication ability sufficient to participate

Exclusion criteria: < 18 years old, currently (or within 1 month of) receiving ARNI training or have contraindications to moderate to vigorous physical activity (adapted from ACSM guidelines)

1. an introductory one-to-one session;

2. $10 \times$ twice-weekly group classes (duration $2 \mathrm{~h}$ ) with up to 2 trainers and 8 clients;

3. a closing one-to-one session (over 3 months), followed by 3 drop-in sessions (once/month over the subsequent 3 months). 
Dean 2018 (Continued)

Participants received a bespoke home-based training programme (duration unclear). All participants received treatment as usual.

Control: all participants received treatment as usual. The control group received a UK Stroke Association booklet on exercise after stroke.

Setting: community setting

Outcomes Included outcomes: RMI, TUG Test, modified Patient-Specific Functional Scale, Stroke QoL, adverse events at 6 and 9 months, SF12, EQ-5D-5L, EQ5 (QoL)

Other outcomes: feasibility, acceptability and process outcome, acceptability of randomisation, outcome measurement burden and the intervention assessments). Intervention fidelity, physical activity subjective and objective (accelerometry and a physical activity diary). Stroke Self-Efficacy Questionnaire, Fatigue Assessment Scale, Exercise Beliefs and Self-questionnaires, Carer Burden Index and Health and Social Service use through a Service Receipt Inventory

Notes ReTRAIN trial

NCT02429180

\section{Risk of bias}

\begin{tabular}{|c|c|c|}
\hline Bias & Authors' judgement & Support for judgement \\
\hline $\begin{array}{l}\text { Random sequence genera- } \\
\text { tion (selection bias) }\end{array}$ & Low risk & Computer-generated randomisation \\
\hline $\begin{array}{l}\text { Allocation concealment } \\
\text { (selection bias) }\end{array}$ & Low risk & Remote randomisation service \\
\hline $\begin{array}{l}\text { Blinding (performance } \\
\text { bias and detection bias) } \\
\text { All outcomes }\end{array}$ & High risk & The control group did not receive an active or sham treatment \\
\hline $\begin{array}{l}\text { Blinding of outcome as- } \\
\text { sessment (detection bias) } \\
\text { All outcomes }\end{array}$ & Low risk & $\begin{array}{l}\text { Outcomes were assessed by independent researchers who were blinded to } \\
\text { group allocation. Participants were also reminded not to reveal their alloca- } \\
\text { tion }\end{array}$ \\
\hline $\begin{array}{l}\text { Incomplete outcome data } \\
\text { (attrition bias) } \\
\text { End of intervention }\end{array}$ & Low risk & $\begin{array}{l}\text { Very few dropouts: } 2 \text { in each group. Intervention (2/23): reasons } 1 \text { unwell, } 1 \\
\text { family crisis; control (2/22): } 1 \text { unwell, } 1 \text { wanted ReTRAIN intervention. At end of } \\
\text { intervention data was available for } 21 / 21 \text { in the intervention group and } 19 / 22 \\
\text { in the control. ITT not mentioned }\end{array}$ \\
\hline $\begin{array}{l}\text { Incomplete outcome data } \\
\text { (attrition bias) } \\
\text { End of follow-up }\end{array}$ & Low risk & $\begin{array}{l}\text { Outcome data available at } 12 \text {-month follow-up for } 21 / 23 \text { in the intervention } \\
\text { and } 20 / 22 \text { in the control group. ITT not mentioned }\end{array}$ \\
\hline $\begin{array}{l}\text { Selective reporting (re- } \\
\text { porting bias) }\end{array}$ & Low risk & $\begin{array}{l}\text { The primary outcomes as reported in the study registration, feasibility, accept- } \\
\text { ability and fidelity were not mentioned in the study registration (outcomes not } \\
\text { included in review) }\end{array}$ \\
\hline Other bias & Unclear risk & Exercise after stroke booklet given to control group \\
\hline Imbalanced exposure & High risk & $\begin{array}{l}\text { The intervention received active 'treatment' whereas the control group re- } \\
\text { ceived UC and this could vary from nothing or could meet or exceed that pro- } \\
\text { vided in the intervention }\end{array}$ \\
\hline
\end{tabular}




\begin{tabular}{|c|c|}
\hline \multirow[t]{7}{*}{ Methods } & $\begin{array}{l}\text { Design: phase II randomised multicentre trial; } 3 \text { centres; mixed training plus UC vs UC - during UC - i.e. } \\
\text { FST + CPT vs CPT alone and vs CPT plus CPT }\end{array}$ \\
\hline & $\begin{array}{l}\text { Randomisation: computer-generated random allocation. Allocation was stratified by baseline Action } \\
\text { Research Arm Test score in blocks of } 3 \text { within each stratum }\end{array}$ \\
\hline & $\begin{array}{l}\text { Allocation concealment: sequentially numbered, sealed, opaque envelopes held by an independent in- } \\
\text { vestigator }\end{array}$ \\
\hline & Blinding: assessor blinded to group allocation \\
\hline & ITT: yes \\
\hline & Measurements: at the end of intervention ( 6 weeks) and 12 weeks after (follow-up) \\
\hline & $\begin{array}{l}\text { Withdrawals: } 2 \text { participants were lost at outcome in the CPT group (new stroke }=1 \text {; dropped out }=1 \text { ). } \\
\text { A further } 11 \text { participants were lost at follow-up. } 5 \text { participants in the CPT group ( } 3 \text { unwell, } 1 \text { moved } \\
\text { abroad, } 1 \text { dropped out) and } 2 \text { in the CPT + FST group ( } 1 \text { unwell, } 1 \text { moved abroad) }\end{array}$ \\
\hline \multirow[t]{5}{*}{ Participants } & $\begin{array}{l}\text { Randomised: total } 30 \text { participants. } 10 \text { participants were randomised to CPT, } 10 \text { to CPT + CPT, and } 10 \text { to } \\
\text { CPT + FST (only the results from the CPT and the CPT + FST groups were included in this review, total } \\
\text { 20) }\end{array}$ \\
\hline & Intervention: CPT + FST = 10 participants, 3 men and 7 women; mean age: 72.6 \\
\hline & Control: $\mathrm{CPT}=10$ participants, 5 men and 5 women; mean age: 72.6 \\
\hline & $\begin{array}{l}\text { Inclusion criteria: inpatients; infarction of the anterior cerebral circulation between } 1 \text { week and } 3 \\
\text { months after stroke; some voluntary contraction in the upper affected limb; no obvious unilateral visu- } \\
\text { ospatial neglect; ability, prior to the stroke, to use the paretic upper limb to lift a cup and drink; ability } \\
\text { to follow a 1-stage command }\end{array}$ \\
\hline & Exclusion criteria: not reported \\
\hline
\end{tabular}

Intervention: CPT + FST. FST = repetition and goal-directed functional activity of the upper limb; hand positioning; hand grip activities; hand manipulation involving objects; improving power of shoulder/elbow muscles to enable appropriate hand position. Frequency of intervention: $1 \mathrm{~h}$ for 4 days/week for 6 weeks

Control: CPT included soft issue mobilisation, facilitation of muscle activity/movement, positioning; joint alignment; tactile and proprioceptive input. Frequency of intervention: $1 \mathrm{~h}$ for 4 days/week for 6 weeks

Setting: hospital setting

Outcomes

Included outcomes: upper limb strength (hand grip force, pinch grip force; isometric elbow flexion and extension force); upper limb function (ARAT)

Other outcomes: dexterity (i.e. 9-HPT)

\section{Risk of bias}

\begin{tabular}{lll}
\hline Bias & Authors' judgement & Support for judgement \\
\hline $\begin{array}{ll}\text { Random sequence genera- } \\
\text { tion (selection bias) }\end{array}$ & Low risk & $\begin{array}{l}\text { Computer-generated random allocation } \\
\text { Allocation was stratified by baseline ARAT score in blocks of 3 within each stra- } \\
\text { tum }\end{array}$
\end{tabular}


Donaldson 2009 (Continued)

Allocation concealment Low risk Sequentially numbered, sealed, opaque envelopes held by an independent in(selection bias) vestigator

Blinding (performance

bias and detection bias)

All outcomes
Unclear risk

No attention control in the comparison, however

Quote: "The majority of subjects (68\%) who completed outcome measures were unsure as to which group they had been allocated (CPT 75\%, CPT + CPT $60 \%$, CPT + FST $70 \%$; Table 3). Only 4 of the 28 subjects (14\%) correctly identified the treatment they received. Even in the CPT group who had been told that they would receive no extra therapy, only 1 person correctly identified their grouping."

\begin{tabular}{|c|c|c|}
\hline $\begin{array}{l}\text { Blinding of outcome as- } \\
\text { sessment (detection bias) } \\
\text { All outcomes }\end{array}$ & Low risk & Assessor blinded to group allocation; efficacy unknown \\
\hline $\begin{array}{l}\text { Incomplete outcome data } \\
\text { (attrition bias) } \\
\text { End of intervention }\end{array}$ & Low risk & $\begin{array}{l}\text { ITT analysis planned } \\
2 / 20(10 \%) \text { lost at the end of intervention: control CPT group (new stroke = } 1 \text {; } \\
\text { bail }=1)\end{array}$ \\
\hline $\begin{array}{l}\text { Incomplete outcome data } \\
\text { (attrition bias) } \\
\text { End of follow-up }\end{array}$ & High risk & $\begin{array}{l}\text { ITT analysis planned } \\
9 / 20 \text { (45\%) total losses at the end of follow-up: additional } 5 \text { participants in the } \\
\text { control CPT group ( } 3 \text { unwell, } 1 \text { moved abroad, } 1 \text { bail) and } 2 \text { in the intervention } \\
\text { CPT + FST group ( } 1 \text { unwell, } 1 \text { moved abroad) }\end{array}$ \\
\hline $\begin{array}{l}\text { Selective reporting (re- } \\
\text { porting bias) }\end{array}$ & Unclear risk & Unclear how the study relates to NCT00322192; outcomes do not correspond \\
\hline Other bias & Low risk & None known \\
\hline Imbalanced exposure & High risk & Imbalanced exposure in comparison used CPT vs CPT + FST \\
\hline
\end{tabular}

Duncan 1998

$\begin{array}{ll}\text { Methods } & \text { Design: randomised trial of mixed training vS UC - after UC (outpatient) } \\ \text { Randomisation mechanism: unknown; method: blocks of } 10 \\ \text { Allocation concealment: third party involvement } \\ \text { Blinding: unclear } \\ \text { ITT: yes } \\ \text { Measurements: end of intervention (12 weeks) } \\ \text { Withdrawals: none } \\ \text { Randomised: } 20 \text { participants } \\ \text { Intervention: } 10 \text { participants; number of men and women unknown; age } 67.3 \text { years (SD 9.6); } 66 \text { days } \\ \text { post-stroke } \\ \text { Control: } 10 \text { participants; number of men and women unknown; age } 67.8 \text { years (SD 7.2); } 56 \text { days post- } \\ \text { stroke } \\ \text { Inclusion criteria: } 30-90 \text { days post-stroke; minimal/moderately impaired sensorimotor function; avail- } \\ \text { able to attend all training sessions; ambulatory with or without supervision or walking aids; living at } \\ \text { home within } 50 \text { miles } \\ \text { Exclusion criteria: medical condition that compromised outcome assessment or prevented fitness } \\ \text { training; MMSE score < } 18 \text { or receptive aphasia }\end{array}$


Duncan 1998 (Continued)

Interventions
Intervention: mixed training, performed approximately $90 \mathrm{~min} /$ day 3 days/week for 12 weeks (8 weeks supervised 1:1 with therapist and 4 weeks alone), functional exercises comprising assistive/resistive exercise, balance exercises, upper limb functional activities, walking or cycling; apart from some resisted exercise the training intensity was not quantified

Control: usual outpatient care, physical and OT as advised by the patient's physician, averaging 44 $\mathrm{min} /$ day, 3.25 days/week for 12 weeks, therapeutic interventions were during home or outpatient visits and comprised balance training (60\%), strength training (40\%), bimanual activities (50\%), and facilitative exercise (30\%); cardiorespiratory training was not provided (0\%) Setting: home-based, therapist-supervised for first 8 weeks

Included outcomes: BI; Lawton ADL; gait endurance (6-MWT); BBS; gait preferred speed (data lack vari-
ance measures)
Other outcomes: SF-36 (non-standard pooling of data), Jebsen Hand Test; Fugl Meyer (upper and lower
extremity)

\section{Notes}

$-$

\section{Risk of bias}

\begin{tabular}{|c|c|c|}
\hline Bias & Authors' judgement & Support for judgement \\
\hline $\begin{array}{l}\text { Random sequence genera- } \\
\text { tion (selection bias) }\end{array}$ & Unclear risk & Block randomisation used (blocks of 10 ), method unknown \\
\hline $\begin{array}{l}\text { Allocation concealment } \\
\text { (selection bias) }\end{array}$ & Unclear risk & Third party involvement \\
\hline $\begin{array}{l}\text { Blinding (performance } \\
\text { bias and detection bias) } \\
\text { All outcomes }\end{array}$ & High risk & Degree of attention control \\
\hline $\begin{array}{l}\text { Blinding of outcome as- } \\
\text { sessment (detection bias) } \\
\text { All outcomes }\end{array}$ & Unclear risk & Not reported \\
\hline $\begin{array}{l}\text { Incomplete outcome data } \\
\text { (attrition bias) } \\
\text { End of intervention }\end{array}$ & Low risk & Planned ITT; no losses \\
\hline $\begin{array}{l}\text { Selective reporting (re- } \\
\text { porting bias) }\end{array}$ & Unclear risk & Protocol not available \\
\hline Other bias & Low risk & None known \\
\hline Imbalanced exposure & High risk & Imbalanced exposure \\
\hline
\end{tabular}

Duncan 2003

Methods

Design: randomised trial of mixed training vs UC - after UC (outpatient)

Randomisation mechanism: unknown; method: blocks of 6

Allocation concealment: sealed envelopes

Blinding: investigator; participants asked to maintain blinding

ITT: yes

Measurements: end of intervention (12/14 weeks) and 6-month follow-up 
Withdrawals: intervention (10 participants: 6 before ( 1 renal insufficiency, 1 subclavian steal syndrome, 1 chose withdrawal, 3 recurrent stroke), 4 after the 3-month follow-up (1 died, 1 hospital, 2 recurrent stroke); control (11 participants: 2 before ( 1 withdrew, 1 non-return), 9 after 3-month follow-up ( 2 died, 2 hospital, 5 withdrew)

Randomised: 100 participants
Intervention: 50 participants; 23 men and 27 women; age 68.5 years (SD 9.0); 77.5 days post-stroke (SD
28.7 )
Control: 50 participants; men and 27 women 23; age 70.2 years (SD 11.4); 73.5 days post-stroke (SD
27.1 )
Inclusion criteria: $30-150$ days post-stroke; independent ambulation for 25 feet; Fugl-Meyer scores
27-90; Orpington Prognostic Scale $2.0-5.2$ ); Folstein Mini-Mental State score 16
Exclusion criteria: serious cardiac condition; oxygen dependence; severe weight-bearing pain; serious
organ system disease; life expectancy< 1 year

Interventions

Intervention: mixed training, performed approximately $90 \mathrm{~min} /$ day 3 days/week for $12-14$ weeks (36 sessions); training included range of motion and flexibility, strength training, balance, functional upper extremity practice, endurance training via interval training on cycle ergometer. All elements progressive but intensity not quantified

Control: usual outpatient care including PT and OT for participants who needed. All controls received 30 -min visit every 2 weeks including provision of health promotion information Setting: home-based, therapist-supervised for first 8 weeks

Included outcomes: cognitive and motor subscales of the FIM; SF-36 subscales; ankle dorsiflexion and
knee extension isometric strength $(\mathrm{Nm})$; isometric grip strength $(\mathrm{N}) ; \mathrm{BBS}$; functional reach; $\mathrm{VO}_{2}$ peak;
gait speed preferred ( 10 metre); $6-\mathrm{MWT}$; community ambulation $(>0.8 \mathrm{~m} / \mathrm{s})$
Other outcomes: Stroke Impact Scale; cycle duration; Fugl Meyer scores

Notes Some outcomes reported as change from baseline scores, others reported as means at the end of 6month follow-up

\section{Risk of bias}

\begin{tabular}{lll}
\hline Bias & Authors' judgement & Support for judgement \\
\hline $\begin{array}{l}\text { Random sequence genera- } \\
\text { tion (selection bias) }\end{array}$ & Unclear risk & Block randomisation used (blocks of 6), method unknown \\
\hline $\begin{array}{l}\text { Allocation concealment } \\
\text { (selection bias) }\end{array}$ & Unclear risk & Sealed envelopes \\
\hline
\end{tabular}

Blinding (performance High risk Degree of attention control
bias and detection bias)
All outcomes

Blinding of outcome as-
sessment (detection bias) Low risk Investigator blinded; participants asked to maintain blinding

All outcomes

\begin{tabular}{ll}
\hline $\begin{array}{l}\text { Incomplete outcome data } \\
\text { (attrition bias) }\end{array}$ & Unclear risk \\
$\begin{array}{l}\text { End of intervention } \\
\text { ITT used }\end{array}$ & $8 / 100(8 \%)$ losses before outcome assessment \\
& $\begin{array}{l}\text { Intervention } 6 \text { (1 renal insufficiency, } 1 \text { subclavian steal syndrome, } 1 \text { chose } \\
\text { withdrawal, } 3 \text { recurrent stroke) }\end{array}$ \\
Control 2 (1 withdrew, 1 non-return)
\end{tabular}

Incomplete outcome data High risk
(attrition bias)


Duncan 2003 (Continued) End of follow-up

\section{$21 / 100(21 \%)$ total losses at the end of follow-up}

Intervention 4 (1 died, 1 hospital, 2 recurrent stroke)

Control 9 (2 died, 2 hospital, 5 withdrew)

Selective reporting (re- Unclear risk $\quad$ Protocol not available
porting bias)

\begin{tabular}{lll}
\hline Other bias & Low risk & None known \\
\hline Imbalanced exposure & High risk & Imbalanced exposure \\
\hline
\end{tabular}

Eich 2004

\begin{tabular}{ll}
\hline Methods & Design: randomised trial of cardiorespiratory training plus UC vs UC - during UC \\
Randomisation mechanism: picking envelopes; method: restricted \\
Allocation concealment: sealed envelopes \\
Blinding: investigator; efficacy was compromised \\
ITT: yes \\
Measurements: end of intervention (6 weeks) and 3-month follow-up \\
Withdrawals: intervention 1 participant (refusal) after the 6-week training \\
\hline Randomised: 50 participants \\
Intervention: 25 participants; 17 men and 8 women; age 62.4 years (SD 4.8); 43 days post-stroke (SD 15) \\
Control: 25 participants; 16 men and 9 women; age 64 years (SD 9); 44 days post-stroke (SD 18) \\
Inclusion criteria: aged 50-75 years; first stroke; time since stroke <6 weeks; walk 12 m with/without as- \\
sistance; BI score 50-80; participating in 12-week comprehensive rehabilitation programme; stable car- \\
diovascular responses; no non-stroke walking impairments; able to understand purpose and content of \\
study
\end{tabular}

$\begin{array}{ll}\text { Interventions } & \text { Intervention: cardiorespiratory training, performed } 30 \text { min/day } 5 \text { days/week for } 6 \text { weeks; progressive } \\ \text { treadmill training with either no or minimal support of body weight; intensity was } 60 \% \text { of HR reserve } \\ \text { Control: both groups received UC comprising individual PT based on Bobath concept plus occupation- } \\ \text { al and speech therapy, and neuropsychology as required } \\ \text { Setting: rehabilitation unit - inpatient care }\end{array}$

Outcomes Included outcomes: gait speed maximal (10 m); gait endurance (6-MWT)

Other outcomes: RMA (non-normal data); walking quality scale (non-normal data)

Notes -

\section{Risk of bias}

\begin{tabular}{lll}
\hline Bias & Authors' judgement & Support for judgement \\
\hline $\begin{array}{l}\text { Random sequence genera- } \\
\text { tion (selection bias) }\end{array}$ & Low risk & $\begin{array}{l}\text { Restricted randomisation; independent person picking one of (initially) 50 } \\
\text { sealed envelopes }\end{array}$ \\
\hline $\begin{array}{l}\text { Allocation concealment } \\
\text { (selection bias) }\end{array}$ & Unclear risk & Sealed envelopes; opaque and numbered unknown \\
\hline $\begin{array}{l}\text { Blinding (performance } \\
\text { bias and detection bias) } \\
\text { All outcomes }\end{array}$ & High risk & No suitable attention control \\
\hline
\end{tabular}


Eich 2004 (Continued)

Blinding of outcome as- High risk Quote: "one could not fully exclude the possibility that the outcome observers sessment (detection bias)

All outcomes

$$
\text { were not totally blind" }
$$

\begin{tabular}{ll}
\hline $\begin{array}{l}\text { Incomplete outcome data } \\
\text { (attrition bias) }\end{array}$ & Low risk \\
$\begin{array}{l}\text { End of intervention } \\
\end{array}$ & Only 1/50 (2\%) lost: intervention 1 participant (refusal) after the 6-week train- \\
& ing
\end{tabular}

\begin{tabular}{|c|c|c|}
\hline $\begin{array}{l}\text { Incomplete outcome data } \\
\text { (attrition bias) } \\
\text { End of follow-up }\end{array}$ & Unclear risk & $\begin{array}{l}\text { ITT planned } \\
\text { Only } 1 / 50(2 \%) \text { lost overall }\end{array}$ \\
\hline
\end{tabular}

Selective reporting (re- Unclear risk $\quad$ Protocol not available
porting bias)

\begin{tabular}{lll}
\hline Other bias & Low risk & None known \\
\hline Imbalanced exposure & High risk & Imbalanced exposure \\
\hline
\end{tabular}

\section{Fernandez-Gonzalo 2016}

Design: RCT of resistance training vs no intervention (usual routines). Study commenced after UC
Randomisation: computerised block randomisation
Allocation concealment: unclear
Blinding: blinded outcome assessment for MRI and cognitive function; no blinded outcome assessment
stated for force, power, function and dual task outcomes
ITT: not done; per protocol analysis
Measurements: baseline and end of intervention (12 weeks)
Withdrawals: intervention group $n=2$ (unrelated medical issue); control group $n=1$ (unrelated medical
issue)

Participants

Randomised: total 32 participants ( $n=29$ reported in study; intervention $n=14$; control $n=15$ )

Intervention: 14 participants; 11 men (79\%) and 3 women (21\%); mean age 61.2 (SD 9.8) years; 3.5 (SD 3.6) years after stroke

Control: 15 participants; 11 men (73\%) and 4 women (27\%); mean age 65.7 (SD 12.7) years; 4.3 (SD 4.9) years after stroke

Inclusion criteria: stroke confirmed by CT or MRI, age $>40$ years, $\geq 6$ months post-stroke, able to walk 20 $\mathrm{m}$ with/without assistive device, ability to perform closed-chain exercise using the prescribed training device

Exclusion criteria: unstable angina, congestive heart failure, severe arterial disease, major depression, dementia ( $<24$ on the MMSE), failure to understand instructions, chronic pain
Interventions
Intervention: unilateral explosive resistance training of the more affected leg; real time feedback was given to participants. 4 sets of 7 maximal repetitions of the hip and leg extensors whilst seated on a fly- wheel-resisted leg press device. Each repetition included a resisted concentric and eccentric phase. Training occurred 2 days/week for 12 weeks
Peak power during sessions progressively increased throughout the 12 weeks


Fernandez-Gonzalo 2016 (Continued)

Control: maintain usual routines

Setting: unclear

Outcomes Included outcomes: muscle volume and cross-sectional area (MRI); maximal unilateral isometric force, maximal unilateral dynamic force, peak power output; BBS; TUG; cognitive function; QoL SF-36 'physical functioning (PF) and 'mental health' (MH) scales

Other outcomes: dual task performance

Notes NCT02120846

\section{Risk of bias}

\begin{tabular}{|c|c|c|}
\hline Bias & Authors' judgement & Support for judgement \\
\hline $\begin{array}{l}\text { Random sequence genera- } \\
\text { tion (selection bias) }\end{array}$ & Low risk & Computerised block randomisation to either a training group or control group \\
\hline $\begin{array}{l}\text { Allocation concealment } \\
\text { (selection bias) }\end{array}$ & Unclear risk & Unclear, no detail \\
\hline $\begin{array}{l}\text { Blinding (performance } \\
\text { bias and detection bias) } \\
\text { All outcomes }\end{array}$ & High risk & No attention control \\
\hline $\begin{array}{l}\text { Blinding of outcome as- } \\
\text { sessment (detection bias) } \\
\text { All outcomes }\end{array}$ & Unclear risk & $\begin{array}{l}\text { Mix of blind (MRI and cognition) and unblinded assessment (strength and pow- } \\
\text { er) }\end{array}$ \\
\hline $\begin{array}{l}\text { Incomplete outcome data } \\
\text { (attrition bias) } \\
\text { End of intervention }\end{array}$ & Low risk & No ITT analysis (per protocol analysis) but only $2 / 14$ and $1 / 15$ losses \\
\hline $\begin{array}{l}\text { Incomplete outcome data } \\
\text { (attrition bias) } \\
\text { End of follow-up }\end{array}$ & Low risk & \\
\hline $\begin{array}{l}\text { Selective reporting (re- } \\
\text { porting bias) }\end{array}$ & Low risk & Fidelity with NCT02120846 \\
\hline Other bias & Low risk & None known \\
\hline Imbalanced exposure & High risk & Control group followed their usual routines; no attention control \\
\hline
\end{tabular}

Flansbjer 2008

Methods

Design: randomised trial of resistance training vs no training - after UC

Randomisation: stratified unequal randomisation (2:1)

Allocation concealment: non-sealed envelopes

Blinding: physiotherapists who assessed isokinetic strength and gait performance outcomes were blinded to group assignment but the physiotherapist who assessed dynamic strength and muscle tone outcomes was not blinded; participants were not blinded but were told not to disclose group assignment 
Flansbjer 2008 (Continued)

\section{ITT: yes}

Measurements: at the end of intervention (10 weeks), 5-month follow-up, and a 4-year follow-up

Withdrawals: 1 participant dropped out from the intervention group due to an accident unrelated to strength training. 2 participants were unable to perform follow-up assessments due to new illness, 4 participants did not wish to continue at follow-up stage (but were reported in general good health)

Rarticipants
Intervention: 15 participants (16 randomised), 9 men and 6 women; mean age 61 (SD 5) years; time
since stroke 18.9 (SD 7.9) months
Control: 9 participants, 5 men and 4 women; mean age 60 (SD 5) years; time since stroke 20.0 (SD 11.6)
months
Inclusion criteria: age $40-70$ years; 6 months post-stroke; able to perform isolated extension and flexion
movements of the knee; at least $15 \%$ reduction in muscle strength in the paretic limb (mean isokinetic
peak torque at $60^{\circ} / \mathrm{s}$ ); walk unsupervised for 200 m with or without walking aid; no medication, physi-
cal, cognitive, or mental dysfunction that could impact upon knee muscle strength, gait performance,
or perceived participation; able to understand verbal and written information
Exclusion criteria: not reported

Interventions Intervention group: 10 weeks of dynamic and isokinetic knee muscle strength training. Each training session started with a warm-up of 5 min of stationary cycling, 5 repetitions without resistance and 5 repetitions at $25 \%$ of maximum load. The participants then performed $6-8$ repetitions at about $80 \%$ of their maximum load with a 2-min rest between each set. The participants performed as many repetitions as possible. The load was adjusted every 2 weeks to remain at $80 \%$ of their maximum load. Each training session lasted about $90 \mathrm{~min}$ but the actual progressive strength training time was $<6 \mathrm{~min}$ Control group: participants were encouraged to continue daily activities and training but not to engage in any progressive strength training

Setting: community dwelling; training in hospital

Outcomes Included outcomes: dynamic and isokinetic muscle strength; $3 \mathrm{~m}$ TUG; maximum walking speed; 6 MWT; SIS - Swedish version; muscle tone assessed with the MAS

Other outcomes: none

Notes

Maximum walking speed data obtained from study authors. The physiotherapist that supervised the resistance training was the same that assessed dynamic strength and muscle tone outcomes

4-year follow-up data available in secondary publication

\section{Risk of bias}

\begin{tabular}{lll}
\hline Bias & Authors' judgement & Support for judgement \\
\hline $\begin{array}{l}\text { Random sequence genera- } \\
\text { tion (selection bias) }\end{array}$ & Low risk & Stratified by gender unequal randomisation (2:1) \\
\hline $\begin{array}{l}\text { Allocation concealment } \\
\text { (selection bias) }\end{array}$ & High risk & Non-sealed envelopes \\
\hline $\begin{array}{l}\text { Blinding (performance } \\
\text { bias and detection bias) } \\
\text { All outcomes }\end{array}$ & High risk & No attention control \\
\hline
\end{tabular}

Blinding of outcome as- High risk sessment (detection bias)
Physiotherapists who assessed isokinetic strength and gait performance outcomes were blinded to group assignment but the physiotherapist who as- 
Flansbjer 2008 (Continued)

All outcomes sessed dynamic strength and muscle tone outcomes was not blinded; participants were not blinded but were told not to disclose group assignment

Therapists not blinded at 4-year follow-up

\begin{tabular}{ll}
\hline $\begin{array}{l}\text { Incomplete outcome data } \\
\text { (attrition bias) }\end{array}$ & Low risk \\
End of intervention & $\begin{array}{l}1 / 25(4 \%) \text { losses; } 1 \text { participant dropped out from the intervention group due to } \\
\text { an accident unrelated to strength training }\end{array}$
\end{tabular}

\begin{tabular}{ll}
\hline $\begin{array}{l}\text { Incomplete outcome data } \\
\text { (attrition bias) }\end{array}$ & High risk \\
$\begin{array}{ll}\text { End of follow-up } & 1 / 25(4 \%) \text { total losses at the end of 5-month follow-up, ITT analysis used } \\
& 7 / 25(28 \%) \text { total losses at the end of 4-year follow-up and no ITT analysis used. } \\
& 2 \text { participants were unable to perform follow-up assessments due to new ill- } \\
\text { ness, } 4 \text { participants did not wish to continue at follow-up stage (but were re- } \\
\text { ported in general good health) }\end{array}$ \\
\hline
\end{tabular}

Selective reporting (re- Unclear risk No study protocol available
porting bias)

\begin{tabular}{lll}
\hline Other bias & Low risk & None known \\
\hline Imbalanced exposure & High risk & Imbalanced exposure \\
\hline
\end{tabular}

\section{Furnari 2014}

Design: single-blinded RCT; mixed training (aquatic exercise) plus conventional physical therapy vs
conventional physical therapy alone - during UC
Randomisation: stratified randomisation; stratification based on age and gender
Allocation concealment: not reported, unclear
Blinding: assessor blinded to group allocation; participants may have understood nature of group as-
signments
ITT: not stated, withdrawals not outlined - unclear if analyses were performed according to ITT princi-
ple
Measurements: end of intervention (8 weeks)
Withdrawals: none reported

Participants

\section{Randomised: 40 participants}

Intervention: 8-week hydrokinesitherapy (aquatic therapy) and conventional physical therapy $=20$ participants; 10 men (50\%) and 10 women (50\%); mean age: 68 (SD 3); time since stroke onset: 7 months (SD 1.6)

Control: 8-week conventional physical therapy = 20 participants; 10 men (50\%) and 10 women (50\%); mean age: 72 (SD 5); time since stroke onset: 6 months (SD 1.4)

Inclusion criteria: paresis and spasticity secondary to a first stroke, clinically stable, absence of previous MI, ability to stand up without assistance for at least $51.2 \mathrm{~s}$ with closed eyes, ability to walk $10 \mathrm{~m}$ (with or without assistive device), time since stroke of at least 30 days, serological negativity to hepatitis $B$, hepatitis $C$ and HIV, absence of infectious diseases, absence of pressure ulcers, absence of urinary and/or faecal incontinence, and MMSE $\geq 24$

Exclusion criteria: previous vascular accident, muscle contractures of the lower limb joints, previous treatment with botulinum toxin, phenol or alcohol nerve blocks tor motor point injections for low- 
Furnari 2014 (Continued)

er limb spasticity, de novo treatment with anti-spasticity drugs, additional neurological and/or orthopaedic deficits that impaired ambulation or heart failure

Interventions

Intervention: aquatic therapy consisted of a 10-min light warming up in the water 15 min of Halliwick method (sagittal rotation control; transverse rotation control) and combined rotation control (a combination of transverse sagittal and longitudinal rotations), $15 \mathrm{~min}$ of rounding and balancing exercises according to the Ai Chi method focused on weight bearing on the more affected side, 10 min of lower-limb strength exercises using water resistance and 10 min of light cooling down (walking). Degree of difficulty was reviewed weekly to ensure participants made progress at their own pace. Frequency of intervention: aquatic therapy: $1 \mathrm{~h}, 3$ times/week for 8 weeks and conventional physical therapy: $1 \mathrm{~h}, 3$ times/ week for 8 weeks

Control: conventional physical therapy programme consisting of general conditioning exercises, including 10 min of warming up (e.g. calf, shoulder and hand passive range of motion exercises), 20 min of lower and upper extremity strengthening, $20 \mathrm{~min}$ of postural control exercise, including maintenance of standing and shifting the weight loads to the paretic side, and $10 \mathrm{~min}$ of gait training for the same amount of time as the aquatic therapy group spent in the pool. Exercises in this programme were designed with gradual increments to meet the capabilities of each participant. Frequency of intervention: 1 h, 6 times/week for 8 weeks

Setting: hospital

Outcomes Included outcomes: baropodometric outcomes: postural stability and spatio-temporal gait parameters Other outcomes: clinical data for baseline only (Modified Ashworth Scale, BI, FIM, Tinetti Test)

\section{Notes}

No pre-published trial registry entry

Study author confirmed that control exposure is UC

\section{Risk of bias}

\begin{tabular}{|c|c|c|}
\hline Bias & Authors' judgement & Support for judgement \\
\hline $\begin{array}{l}\text { Random sequence genera- } \\
\text { tion (selection bias) }\end{array}$ & Unclear risk & Stated 'randomised' but no further details provided \\
\hline $\begin{array}{l}\text { Allocation concealment } \\
\text { (selection bias) }\end{array}$ & Unclear risk & Not reported \\
\hline $\begin{array}{l}\text { Blinding (performance } \\
\text { bias and detection bias) } \\
\text { All outcomes }\end{array}$ & Unclear risk & Balanced exposure dose \\
\hline $\begin{array}{l}\text { Blinding of outcome as- } \\
\text { sessment (detection bias) } \\
\text { All outcomes }\end{array}$ & Low risk & Assessor blinded \\
\hline $\begin{array}{l}\text { Incomplete outcome data } \\
\text { (attrition bias) } \\
\text { End of intervention }\end{array}$ & Unclear risk & $\begin{array}{l}\text { No indication of whether dropouts occurred or what analytical approach to } \\
\text { this was used }\end{array}$ \\
\hline $\begin{array}{l}\text { Incomplete outcome data } \\
\text { (attrition bias) } \\
\text { End of follow-up }\end{array}$ & Low risk & \\
\hline $\begin{array}{l}\text { Selective reporting (re- } \\
\text { porting bias) }\end{array}$ & Unclear risk & No trial registry entry available \\
\hline
\end{tabular}


Furnari 2014 (Continued)

\begin{tabular}{lll} 
Other bias & Low risk & None known \\
\hline Imbalanced exposure & Low risk & $\begin{array}{l}\text { Control group received conventional physical therapy of equal time, frequency } \\
\text { and duration }\end{array}$ \\
\hline
\end{tabular}

Galvin 2011

Mesign: randomised trial of mixed FAME plus UC vs UC - during (and after UC)
Randomised mechanism: independent person using computer-generated random numbers
Allocation concealment: sealed envelopes
Blinding: assessor not blinded to group allocation
ITT: all randomised participants analysed using LOCF
Measurements: end of intervention (8 weeks) and at follow-up (3 months)
Withdrawals: 2 participants in the intervention group before outcome assessment (MI and stroke). In
the control group 1 withdrew before outcome assessment (1 unwell), 2 died before follow-up assess-
ment

Participants Randomised: 37 participants

Intervention: 19 participants; 7 men and 13 women; mean age 69.95 years (SD 11.7)

Control: 18 participants; 13 men and 7 women; mean age 63.15 years (SD 13.3)

Inclusion criteria: 2 weeks after stroke onset; diagnosed as first unilateral stroke; > 18 years of age; participating in a PT programme; medically stable family member willing to participate in the programme

Exclusion criteria: impairment of cognition, $<18$ years

\begin{tabular}{ll}
\hline Interventions & Invention group: individualised FAME programmes daily for 35 min for 8 weeks aiming to improve sta- \\
bility, gait velocity, and lower limb strength plus UC (CPT) & Control group: UC (CPT) \\
& Setting: rehabilitation unit \\
\hline Outcomes & Included outcome: lower limb Fugl-Meyer Assessment; MAS; BBS; 6-MWT \\
\hline Notes & - \\
\hline
\end{tabular}

\section{Risk of bias}

\begin{tabular}{lll}
\hline Bias & Authors' judgement & Support for judgement \\
\hline $\begin{array}{l}\text { Random sequence genera- } \\
\text { tion (selection bias) }\end{array}$ & Low risk & Independent person using computer-generated random numbers \\
\hline $\begin{array}{l}\text { Allocation concealment } \\
\text { (selection bias) }\end{array}$ & Unclear risk & Sealed envelope; whether opaque and numbered unknown \\
\hline $\begin{array}{l}\text { Blinding (performance } \\
\text { bias and detection bias) } \\
\text { All outcomes }\end{array}$ & High risk & No attention control \\
\hline
\end{tabular}


Galvin 2011 (Continued)
Blinding of outcome as-
High risk
Assessor not blinded to group allocation sessment (detection bias)
All outcomes

\begin{tabular}{|c|c|c|}
\hline $\begin{array}{l}\text { Incomplete outcome data } \\
\text { (attrition bias) } \\
\text { End of intervention }\end{array}$ & Low risk & $\begin{array}{l}\text { ITT used; all randomised participants analysed using LOCF } \\
3 / 37(8 \%) \text { lost from intervention group } 2 \text { (MI and stroke); control group } 1 \text { ( } 1 \text { un- } \\
\text { well), } 2 \text { died before follow-up assessment }\end{array}$ \\
\hline $\begin{array}{l}\text { Incomplete outcome data } \\
\text { (attrition bias) } \\
\text { End of follow-up }\end{array}$ & Unclear risk & $\begin{array}{l}\text { ITT used; all randomised participants analysed using LOCF } \\
5 / 37(14 \%) \text { total losses; control group } 2 \text { (died) }\end{array}$ \\
\hline $\begin{array}{l}\text { Selective reporting (re- } \\
\text { porting bias) }\end{array}$ & Low risk & Reported outcomes correspond to protocol NCT00666744 \\
\hline Other bias & Low risk & None known \\
\hline Imbalanced exposure & High risk & Imbalanced exposure \\
\hline
\end{tabular}

\section{Glasser 1986}

\begin{tabular}{|c|c|}
\hline Methods & $\begin{array}{l}\text { Design: randomised trial of cardiorespiratory training plus \% UC vs UC - during UC } \\
\text { Randomisation: unknown } \\
\text { Allocation concealment: unknown } \\
\text { Blinding: unknown } \\
\text { ITT: no withdrawals } \\
\text { Measurements: end of intervention (10 weeks) } \\
\text { Withdrawals: none }\end{array}$ \\
\hline Participants & $\begin{array}{l}\text { Randomised: } 20 \text { participants } \\
\text { Intervention: } 10 \text { participants; } 4 \text { men and } 6 \text { women } \\
\text { Control: } 10 \text { participants; } 6 \text { men and } 4 \text { women } \\
\text { All participants age } 40-75 \text { years and were } 3-6 \text { months post-stroke; all participants exhibited hemipare- } \\
\text { sis with upper and lower extremity motor dysfunction; some showed sensory deficits and mild expres- } \\
\text { sive or receptive aphasia } \\
\text { Inclusion criteria: unknown } \\
\text { Exclusion criteria: unknown }\end{array}$ \\
\hline Interventions & $\begin{array}{l}\text { Intervention: cardiorespiratory training: isokinetic ergometer (Kinetron) training twice a day } 5 \text { days/ } \\
\text { week for } 10 \text { weeks; the intensity was maintained at } 50-100 \text { psi and duration of each session progressed } \\
\text { from } 10-30 \text { min over the first } 5 \text { weeks } \\
\text { Control: therapeutic exercise and gait training } 1 \mathrm{~h} / \text { session } 2 \text { sessions/day, } 5 \text { days/week for } 5 \text { weeks } \\
\text { Setting: physical therapy department }\end{array}$ \\
\hline Outcomes & $\begin{array}{l}\text { Included outcomes: gait speed maximal ( } 6 \mathrm{~m}) \\
\text { Other outcomes: FAPS }\end{array}$ \\
\hline Notes & - \\
\hline
\end{tabular}

\section{Risk of bias}

Bias Authors' judgement Support for judgement


Glasser 1986 (Continued)

Random sequence genera- Unclear risk Not reported
tion (selection bias)

$\begin{array}{ll}\begin{array}{l}\text { Allocation concealment } \\ \text { (selection bias) }\end{array} & \text { Unclear risk }\end{array}$

Blinding (performance High risk Some attention control
bias and detection bias)
All outcomes

\begin{tabular}{|c|c|c|}
\hline $\begin{array}{l}\text { Blinding of outcome as- } \\
\text { sessment (detection bias) } \\
\text { All outcomes }\end{array}$ & Unclear risk & Not reported \\
\hline
\end{tabular}

\begin{tabular}{lll}
\hline $\begin{array}{l}\text { Incomplete outcome data } \\
\text { (attrition bias) } \\
\text { End of intervention }\end{array}$ & Low risk & No losses \\
\hline $\begin{array}{l}\text { Selective reporting (re- } \\
\text { porting bias) }\end{array}$ & Unclear risk & No protocol available \\
\hline Other bias & Low risk & None known \\
\hline Imbalanced exposure & Unclear risk & Some attention control; may be a balanced exposure \\
\hline
\end{tabular}

Globas 2012

\begin{tabular}{|c|c|}
\hline \multirow[t]{6}{*}{ Methods } & $\begin{array}{l}\text { Design: randomised, cross-over, controlled trial of high-intensity cardiorespiratory training plus UC vs } \\
\text { UC - after UC }\end{array}$ \\
\hline & $\begin{array}{l}\text { Randomised mechanism: computer-based pseudo-random number generator and Moses-Oakford as- } \\
\text { signment algorithm to perform stratified block allocation scheme ( } 3 \text { blocks, allocation 1:1) }\end{array}$ \\
\hline & Allocation concealment: not reported \\
\hline & Blinding: not blinded to participants; unknown if blinded to assessors \\
\hline & Measurements: end of intervention ( 3 months); follow-up data (12 months) not used \\
\hline & $\begin{array}{l}\text { Withdrawals: } 2 \text { participants in the intervention group, } 1 \text { due to recurrent stroke, } 1 \text { due to transport } \\
\text { problems. Other dropouts were reported but these occurred after the cross-over part of the study be- } \\
\text { gan and are therefore uncontrolled }\end{array}$ \\
\hline \multirow[t]{5}{*}{ Participants } & $\begin{array}{l}\text { Randomised: } 36 \text { participants completed endpoint investigation, } 32 \text { participants completed } 12 \text {-month } \\
\text { follow-up }\end{array}$ \\
\hline & Intervention: 18 participants; 14 men and 4 women; mean age 68.6 years (SD 6.7) \\
\hline & Control: 18 participants; 15 men and 3 women; mean age 68.7 years (SD 6.1) \\
\hline & $\begin{array}{l}\text { Inclusion criteria: > } 6 \text { months post-stroke, confirmed diagnosis of ischaemic stroke via CT and/or MRI } \\
\text { scans; hemiparetic gait as evaluated by a neurologist; at least } 1 \text { clinical sign for paresis, spasticity, or } \\
\text { circumduction during gait; ability to treadmill walk at }>0.3 \mathrm{~km} / \mathrm{h} \text { for } 3 \mathrm{~min}\end{array}$ \\
\hline & $\begin{array}{l}\text { Exclusion criteria: unstable angina pectoris; heart failure; haemodynamically significant valvular dys- } \\
\text { function; peripheral arterial occlusive disease; dementia; aphasia; major depression; already perform- } \\
\text { ing aerobic exercise training (> } 20 \mathrm{~min} / \text { day, > } 1 \text { day/week) }\end{array}$ \\
\hline
\end{tabular}


Globas 2012 (Continued)

Interventions

Invention group: 39 sessions of 30-50 min of treadmill training 3 times/week for 3 months. Training in tensity was $60 \%-80 \%$ maximum HR. Treadmill training was progressed as tolerated by $1-5 \mathrm{~min} /$ week and by $0.1-0.3 \mathrm{~km} / \mathrm{h}$ every $1-2$ weeks. Treadmill inclination was $0^{\circ}$

Control group: UC PT included passive, muscle tone-regulating exercises for upper and lower limbs with element of balance training. Performed for $1 \mathrm{~h}$ for 1-3 times/week. Control group also completed cross-over period of treadmill training, which was similar in protocol except for $2^{\circ}$ inclination

Setting: outpatients rehabilitation clinic

$\begin{array}{ll}\text { Outcomes } & \text { Included outcome: peak exercise capacity }\left(\mathrm{VO}_{2} \text { peak); 6-MWT; } 10 \mathrm{~m} \text { timed walks; 5-chair rise test; BBS; }\right. \\ \text { RMI; SF-12 }\end{array}$
RMI; SF-12

\begin{tabular}{ll}
\hline Notes & $\begin{array}{l}\text { Cross-over part of the study not included } \\
\text { Advertisements used for recruitment }\end{array}$
\end{tabular}

\section{Risk of bias}

\begin{tabular}{|c|c|c|}
\hline Bias & Authors' judgement & Support for judgement \\
\hline $\begin{array}{l}\text { Random sequence genera- } \\
\text { tion (selection bias) }\end{array}$ & Low risk & Computer-based, stratified, block randomisation \\
\hline $\begin{array}{l}\text { Allocation concealment } \\
\text { (selection bias) }\end{array}$ & Unclear risk & Not reported \\
\hline $\begin{array}{l}\text { Blinding (performance } \\
\text { bias and detection bias) } \\
\text { All outcomes }\end{array}$ & High risk & No attention control \\
\hline $\begin{array}{l}\text { Blinding of outcome as- } \\
\text { sessment (detection bias) } \\
\text { All outcomes }\end{array}$ & High risk & Outcome assessment not blinded \\
\hline $\begin{array}{l}\text { Incomplete outcome data } \\
\text { (attrition bias) } \\
\text { End of intervention }\end{array}$ & Low risk & $\begin{array}{l}\text { ITT (LOCF) used } \\
2 / 36(6 \%) \text { dropouts from intervention group ( } 1 \text { recurrent stroke, } 1 \text { transporta- } \\
\text { tion problems) }\end{array}$ \\
\hline $\begin{array}{l}\text { Selective reporting (re- } \\
\text { porting bias) }\end{array}$ & Unclear risk & No protocol available \\
\hline Other bias & High risk & May be self-selection bias due to use of newspaper adverts \\
\hline Imbalanced exposure & Unclear risk & Some attention control but time appears not to be balanced \\
\hline
\end{tabular}

Gordon 2013

Methods Design: RCT of aerobic (walking) training vs massage - after UC

Randomisation: block randomised

Allocation concealment: unclear

Blinding: blinded assessor

ITT: completed

Measurements: 6 weeks and 12 weeks (end of intervention)

Withdrawals: 7 participants from the intervention and 5 participants from the control dropped out 
Gordon 2013 (Continued)

Participants

Randomised: total 128 participants; 64 participants were randomised to intervention, 64 to control Intervention: 64 participants; 29 men (45.3\%) and 35 women (54.7\%); mean age: 63.4 (SD 9.4); 384 (SD 108) days after stroke

Control: 64 participants; 29 men (45.3\%) and 35 women (54.7\%); mean age: 64.9 (SD 11.1); 354 (SD 108) days after stroke

Inclusion criteria: $\geq 40$ years of age, community-dwelling, 6-24 months post-stroke, able to walk with or without an assistive device

Exclusion criteria: not currently in rehabilitation or regular exercise programme, not having any disorder that would compromise exercise training such as unstable cardiovascular diseases, not having any cognitive deficits

Interventions

Intervention: cardiorespiratory walking training. Participants were supervised by trained instructors to walk briskly along a prescribed course for $15 \mathrm{~min}, 3$ times/week, for 12 weeks, initially progressing by 5 min over week up to $30 \mathrm{~min}$ in their home or community. Target HR was $60 \%-85 \%$ of age-predicted maximum HR (220-age). Training progression was also carried out by increasing speed

Control: light massage to the affected limbs for $25 \mathrm{~min}, 3$ times/week, for 12 weeks, at home

Setting: community/home

Outcomes

Included outcomes: Physical and Mental Component Summary scores of the Medical Outcomes Survey, SF-36, BI, Instrumental ADL dimension of the Older Americans Resources and Services Questionnaire; 6-MWT

Other outcomes: resting HR, lower limb Motricity Index

Notes Study author provided details of dropouts and withdrawals

\section{Risk of bias}

\begin{tabular}{|c|c|c|}
\hline Bias & Authors' judgement & Support for judgement \\
\hline $\begin{array}{l}\text { Random sequence genera- } \\
\text { tion (selection bias) }\end{array}$ & Unclear risk & $\begin{array}{l}\text { Described as "block randomised" but with no further information on how this } \\
\text { was achieved }\end{array}$ \\
\hline $\begin{array}{l}\text { Allocation concealment } \\
\text { (selection bias) }\end{array}$ & Unclear risk & Not reported in paper \\
\hline $\begin{array}{l}\text { Blinding (performance } \\
\text { bias and detection bias) } \\
\text { All outcomes }\end{array}$ & High risk & $\begin{array}{l}\text { No blinding of participants or deliverers of intervention or control pro- } \\
\text { grammes }\end{array}$ \\
\hline $\begin{array}{l}\text { Blinding of outcome as- } \\
\text { sessment (detection bias) } \\
\text { All outcomes }\end{array}$ & Low risk & Assessor blinded to group allocation \\
\hline $\begin{array}{l}\text { Incomplete outcome data } \\
\text { (attrition bias) } \\
\text { End of intervention }\end{array}$ & Low risk & $\begin{array}{l}\text { Missing data have been imputed using appropriate methods } \\
\text { 12/128 (9.4\%) total losses: } \\
\text { - } 7 \text { participants were lost from the intervention group; death (2), recurrent } \\
\text { stroke (2), intervening comorbidity (1), programme too difficult (1), did not } \\
\text { like group assignment (1) } \\
\text { - } 5 \text { in the control group; death (2), intervening comorbidity (2), or violence in } \\
\text { community (1) }\end{array}$ \\
\hline $\begin{array}{l}\text { Selective reporting (re- } \\
\text { porting bias) }\end{array}$ & Unclear risk & $\begin{array}{l}\text { Trial register or protocol not available. "Trial was not registered as enrolment } \\
\text { commenced before } 2005 \text { " }\end{array}$ \\
\hline Other bias & Low risk & None known \\
\hline
\end{tabular}


Gordon 2013 (Continued)

$\begin{array}{ll}\text { Imbalanced exposure Low risk } & \begin{array}{l}\text { Balanced exposure: intervention and control groups were exposed to the same } \\ \text { frequency and duration of treatment }\end{array}\end{array}$

frequency and duration of treatment

Inaba 1973

Design: randomised trial of resistance training plus UC vS UC - during UC
Randomisation: unknown
Allocation concealment: unknown
Blinding: outcome assessor - unclear
ITT: no
Measurements: end of intervention (4-8 weeks) and 2-month follow-up
Withdrawals: unclear: $101 / 177$ participants lost to follow-up across the control and both intervention
groups; 54 participants completed the control vs strength training comparison; estimated dropouts ap-
proximately 60
1 reason given for dropouts was discharge before end of the study

Randomised: 54 participants
Intervention: 28 participants; 11 men and 17 women; age 55.6 years; <3 months post-stroke
Control: 26 participants; 15 men and 11 women; age 56.9 years; <3 months post-stroke
All participants had hemiparesis
Inclusion criteria: hemiparesis arising from cerebrovascular accident secondary to thrombosis; embo-
lus or haemorrhage; able to follow verbal or demonstrated directions; extend the involved lower limb
against a load of $1.1 \mathrm{~kg}$; independent ambulation
Exclusion criteria: aetiology of aneurysm or trauma

Interventions Intervention: progressive resistive exercise once/day for 4-8 weeks; extension of the affected lower limb from $90^{\circ}$ to full-knee extension whilst in the supine position on an Elgin table (machine weights), 5 repetitions at $50 \%$ maximum weight, and 10 at maximum

Control: UC: conventional functional training, including stretching, 4-8 weeks until discharge

Setting: rehabilitation centre

$\begin{array}{ll}\text { Outcomes } & \begin{array}{l}\text { Included outcomes: leg strength (10-RM) lacked variance measures number of participants able to per- } \\ \text { form } 10 \mathrm{ADL}\end{array}\end{array}$

Notes -

\section{Risk of bias}

\begin{tabular}{lll}
\hline Bias & Authors' judgement & Support for judgement \\
\hline $\begin{array}{l}\text { Random sequence genera- } \\
\text { tion (selection bias) }\end{array}$ & Unclear risk & Not reported \\
\hline $\begin{array}{l}\text { Allocation concealment } \\
\text { (selection bias) }\end{array}$ & Unclear risk & Not reported \\
\hline $\begin{array}{l}\text { Blinding (performance } \\
\text { bias and detection bias) } \\
\text { All outcomes }\end{array}$ & High risk & No attention control \\
\hline $\begin{array}{l}\text { Blinding of outcome as- } \\
\text { sessment (detection bias) } \\
\begin{array}{l}\text { All outcomes } \\
\hline\end{array}\end{array}$ & Unclear risk & Unclear \\
\hline
\end{tabular}


Inaba 1973 (Continued)

Incomplete outcome data High risk Large numbers of undocumented losses and no ITT analysis

(attrition bias)

Unclear: 101/177 participants lost to follow-up across the control and both in-

End of intervention tervention groups; 54 participants completed the control vs strength training comparison; estimated dropouts approximately 60

1 reason given for dropouts was discharge before end of the study

\section{Incomplete outcome data High risk}

(attrition bias)

End of follow-up

\section{Large numbers of undocumented losses and no ITT analysis}

Unclear: 101/177 participants lost to follow-up across the control and both intervention groups; 54 participants completed the control vs strength training comparison; estimated dropouts approximately 60

1 reason given for dropouts was discharge before end of the study

\begin{tabular}{lll}
\hline $\begin{array}{l}\text { Selective reporting (re- } \\
\text { porting bias) }\end{array}$ & Unclear risk & No protocol available \\
\hline Other bias & Low risk & None known \\
\hline Imbalanced exposure & High risk & Imbalanced exposure \\
\hline
\end{tabular}

Ivey 2010

Design: randomised trial of cardiorespiratory training vs UC - after UC
Randomised: blocked allocation schema and computer-based pseudo-random number generator
Allocation concealment: not reported
Blinding: assessors not blinded
ITT: no
Measurements: end of intervention (6 months)
Withdrawals: intervention group 10 participants and control group 17 participants lost to follow-up, 7
in both groups due to medical reasons unrelated to study procedures; 3 and 10 respectively due to gen-
eral compliance issues

Participants

Randomised: 53 participants

Intervention: 29 participants; 18 men and 11 women; mean age 62 years (SD 8)

Control: 24 participants; 11 men and 13 women; mean age 60 years (SD 8)

Inclusion criteria: chronic hemiparetic stroke (> 6 months); completed all conventional UC

Exclusion criteria: history of vascular surgery; vascular disorders of the lower limb; symptomatic peripheral arterial occlusive disease

Interventions

Invention group: treadmill training for $40 \mathrm{~min} 3$ times/week for 6 months at a target intensity of $60 \%-70 \%$ HR reserve, initially started with discontinuous training, which progressed to continuous

Control group: UC: 13 targeted active and passive supervised stretching movements of the upper and lower body for 30-40 $\min 3$ times/week for 6 months

Setting: rehabilitation unit 
Ivey 2010 (Continued)

Other outcomes: resting and reactive hyperaemic calf blood flow in both paretic and non-paretic legs

\begin{tabular}{|c|c|c|}
\hline Notes & - & \\
\hline \multicolumn{3}{|l|}{ Risk of bias } \\
\hline Bias & Authors' judgement & Support for judgement \\
\hline $\begin{array}{l}\text { Random sequence genera- } \\
\text { tion (selection bias) }\end{array}$ & Low risk & $\begin{array}{l}\text { Blocked allocation schema and computer-based pseudo-random number gen- } \\
\text { erator }\end{array}$ \\
\hline $\begin{array}{l}\text { Allocation concealment } \\
\text { (selection bias) }\end{array}$ & Unclear risk & Not reported \\
\hline $\begin{array}{l}\text { Blinding (performance } \\
\text { bias and detection bias) } \\
\text { All outcomes }\end{array}$ & High risk & $\begin{array}{l}\text { Participants described as not blinded, although there was matched exposure } \\
\text { to staff }\end{array}$ \\
\hline $\begin{array}{l}\text { Blinding of outcome as- } \\
\text { sessment (detection bias) } \\
\text { All outcomes }\end{array}$ & High risk & Outcome assessors not blinded \\
\hline $\begin{array}{l}\text { Incomplete outcome data } \\
\text { (attrition bias) } \\
\text { End of intervention }\end{array}$ & High risk & $\begin{array}{l}\text { ITT not reported } \\
27 / 53 \text { (51\%) losses; intervention group } 10 \text { and control group } 17 \text { due to medical } \\
\text { reasons unrelated to study procedures; } 3 \text { and } 10 \text { respectively due to general } \\
\text { compliance issues }\end{array}$ \\
\hline $\begin{array}{l}\text { Selective reporting (re- } \\
\text { porting bias) }\end{array}$ & Unclear risk & Relationship to trial register entries unclear \\
\hline Other bias & Low risk & None known \\
\hline Imbalanced exposure & Low risk & Matched exposure \\
\hline
\end{tabular}

Ivey 2011

Methods

Design: randomised trial of cardiorespiratory training vs UC - after UC

Randomised: mechanism unknown

Allocation concealment: unknown

Blinding: unknown

ITT: no

Measurements: end of intervention (6 months)

Withdrawals: 13 participants withdrew at the end of intervention, reasons unknown

Participants

Randomised: 38 participants completed study; 51 may have been randomised

Intervention: 19 participants; mean age 61 years (SD 8)

Control: 19 participants; mean age 62 years (SD 10)

Inclusion criteria: chronic hemiparetic stroke with mild to moderate hemiparetic gait; completed all conventional UC; still present with residual hemiparetic gait deficits $>6$ months post-stroke 
Ivey 2011 (Continued)

Exclusion criteria: inability for insonation of the middle cerebral artery bilaterally

Interventions Invention group: treadmill training for $40 \mathrm{~min} 3$ times/week for 6 months at a target intensity of 60\%-70\% HR reserve, initially started with discontinuous training, which progressed to continuous

Control group: UC: 13 targeted active and passive supervised stretching movements of the upper and lower body for 30-40 min 3 times/week for 6 months

Setting: rehabilitation unit

\begin{tabular}{ll}
\hline Outcomes & Included outcomes: 6-MWT, peak aerobic capacity during treadmill protocol \\
& $\begin{array}{l}\text { Other outcomes: middle cerebral artery blood flow velocity bilaterally during normocapnia and hyper- } \\
\text { capnia }\left(6 \% \mathrm{CO}_{2}\right)\end{array}$ \\
\hline Notes & -
\end{tabular}

\section{Risk of bias}

\begin{tabular}{|c|c|c|}
\hline Bias & Authors' judgement & Support for judgement \\
\hline $\begin{array}{l}\text { Random sequence genera- } \\
\text { tion (selection bias) }\end{array}$ & High risk & Mechanism not described, number randomised not clear \\
\hline $\begin{array}{l}\text { Allocation concealment } \\
\text { (selection bias) }\end{array}$ & Unclear risk & Not reported \\
\hline $\begin{array}{l}\text { Blinding (performance } \\
\text { bias and detection bias) } \\
\text { All outcomes }\end{array}$ & High risk & Attention control was included \\
\hline $\begin{array}{l}\text { Blinding of outcome as- } \\
\text { sessment (detection bias) } \\
\text { All outcomes }\end{array}$ & Unclear risk & Not reported \\
\hline $\begin{array}{l}\text { Incomplete outcome data } \\
\text { (attrition bias) } \\
\text { End of intervention }\end{array}$ & High risk & $\begin{array}{l}\text { ITT analysis not reported } \\
\text { There may have been losses after randomisation; up to } 13 / 51(25 \%)\end{array}$ \\
\hline $\begin{array}{l}\text { Selective reporting (re- } \\
\text { porting bias) }\end{array}$ & Unclear risk & Relationship to trial register entries unclear \\
\hline Other bias & Low risk & None known \\
\hline Imbalanced exposure & Low risk & Matched exposure \\
\hline
\end{tabular}

Ivey 2017

Methods

Design: prospective RCT; resistance training (intervention group) vs stretching (control group) - after UC

Randomisation: blocked allocation schema and a computer-based pseudorandom number generator. Separate blocked randomisations were performed according to age ( $<65 \mathrm{vs} \geq 65$ years) and ratio of leg press 1-RM strength in the paretic leg to 1-RM strength in the non-paretic leg ( $<0.64$ vs $\geq 0.64$ ) 
Ivey 2017 (Continued)

Randomisation was confounded toward the end of the study to achieve more even final group numbers and account for an uneven discontinuation rate during the study The entire final group of participants $(n=4)$ were assigned to the resistance training group

Allocation concealment: not reported

Blinding: outcome assessments not blinded

ITT: not reported; appears to be a per-protocol analysis

Measurements: before (baseline) and at the end of intervention (12 weeks)

Withdrawals: 25 participants withdrew prior to randomisation; eight $(n=8)$ participants assigned to resistance training withdrew due to medical reasons $(n=4)$ or other (i.e. compliance issues: $n=4)$. No participants from the control group withdrew

Participants

Randomised: 38 stroke participants. 22 participants were randomised to resistance training, and 16 to the control group

Number analysed $=30$

Intervention: 14 participants; 10 men (71\%) and 4 women (29\%); mean age: 57 (SD 14) years; mean years post-stroke: 5 (SD 4)

Control: 16 participants; 11 men (69\%) and 5 women (31\%); mean age: 55 (SD 9); mean years poststroke: 6 (SD 5)

Inclusion criteria: chronic hemiparesis (> 6 months post-stroke)

Exclusion criteria: participants with signs indicating myocardial ischaemia during baseline exercise testing or other contraindications for participating in exercise training

Interventions

Intervention: bilateral resistance training of the lower extremities, $45 \mathrm{~min} /$ day for 3 days/week for 12 weeks. Leg extension, leg curl and leg press were trained on a pneumatic resistance device. Participants performed 2 sets of 20 repetitions on each leg and each machine $(20 \times 2 \times 3=120$ repetitions/session). Resistance was initially set at 10-15-RM and then incrementally lowered within each set such that the full set of 20 repetitions could be completed

Control: 45 min of supervised, passive and active stretching primarily aimed at the lower extremity musculature. Frequency of intervention: not reported, but the control and intervention groups were matched for level of research staff attention

Setting: outpatient

Outcomes

Included outcomes: muscle endurance; maximum number of paretic and non-paretic leg press repetitions at $70 \% 1-\mathrm{RM}$ at a fixed cadence $\left(60 \mathrm{bpm}, 0^{\circ}-90^{\circ}\right)$. Muscle strength; paretic and non-paretic leg press 1-RM. 6-MWT, peak oxygen consumption $\left(\mathrm{VO}_{2}\right.$ peak) during a graded treadmill exercise text, Timed 10-metre walking speed test (self-selected walking speed and fastest comfortable walking speed)

Notes

It is unclear what the conventional rehabilitation consisted of, in terms of content and dose

\section{Risk of bias}

Bias Authors' judgement Support for judgement

Random sequence genera- High risk tion (selection bias)
Computer-based block pseudo-randomisation; unpredictable enough to be considered random. Non-random allocation of $4 / 38$ participants to intervention group to compensate for dropouts; $4 / 14$ of the intervention group 
Ivey 2017 (Continued)

\begin{tabular}{|c|c|c|}
\hline $\begin{array}{l}\text { Allocation concealment } \\
\text { (selection bias) }\end{array}$ & Low risk & Randomisation based on baseline data so allocation concealment not an issue \\
\hline $\begin{array}{l}\text { Blinding (performance } \\
\text { bias and detection bias) } \\
\text { All outcomes }\end{array}$ & Unclear risk & $\begin{array}{l}\text { Attention control will compensate for the fact that blinding participants not } \\
\text { possible }\end{array}$ \\
\hline $\begin{array}{l}\text { Blinding of outcome as- } \\
\text { sessment (detection bias) } \\
\text { All outcomes }\end{array}$ & High risk & Open unblinded assessment \\
\hline $\begin{array}{l}\text { Incomplete outcome data } \\
\text { (attrition bias) } \\
\text { End of intervention }\end{array}$ & High risk & $\begin{array}{l}8 / 22(36 \%) \text { dropout rate from intervention group; no dropouts from control } \\
\text { group. } \\
\text { No ITT analysis }\end{array}$ \\
\hline $\begin{array}{l}\text { Selective reporting (re- } \\
\text { porting bias) }\end{array}$ & Unclear risk & No available pre-published protocol or trial registry entry \\
\hline Other bias & Low risk & None known \\
\hline Imbalanced exposure & Low risk & Matched exposures \\
\hline
\end{tabular}

\section{James 2002}

Methods

Design: randomised trial of mixed training vs no intervention - after UC

Randomisation mechanism: computer; method: blocks of 4

Allocation concealment: sealed envelopes

Blinding: investigator

ITT: yes

Measurements: end of intervention (4 weeks)

Withdrawals: control group 2 dropped out (neurological problems)

Randomised: 20 participants
Intervention: 10 participants; 4 men and 6 women; age 76.1 years (SD 12.33); 1826 days post-stroke
Control: 10 participants; 2 men and 8 women; age 80.8 years (SD 9.0); 1845 days post-stroke
Inclusion criteria: stroke with hemiplegia; ability to give informed consent
Exclusion criteria: no complicating medical history (cardiac, pulmonary, or neurological); no severe
deficits in communication, memory or understanding; no painful orthopaedic conditions that could
limit participation

Interventions Intervention: mixed training, performed 90-120 min/day 3 days/week for 4 weeks

Warm-up followed by half squats; chair squats; small knee bends; standing on affected leg; single-leg half squat on affected leg; standing on unaffected leg and bending affected hip and knee; stair stepping; stepping on spot; walking indoors and outdoors; stepping forwards, backwards and sideways; opening and closing doors; walking and placing/lifting objects; placing objects on shelves. Finished with a cool down; progression achieved increasing pulse rate from $50 \%$ (first 2 weeks) to $60 \%$ (last 2 weeks) of HR reserve, increasing total distance walked, and increasing step height and repetition number

Control: no intervention

Setting: participants' homes

$\begin{array}{ll}\text { Outcomes } & \text { Included outcomes: gait speed preferred ( } 5 \mathrm{~m} \text { with mixed surfaces and a dead turn at } 2.5 \mathrm{~m} \text { ) } \\ \text { Other outcomes: functional walking ability questionnaire; upright motor control test; SF-36 - older ver- } \\ \text { sion }\end{array}$


James 2002 (Continued)
Notes
Unpublished thesis

\section{Risk of bias}

\begin{tabular}{|c|c|c|}
\hline Bias & Authors' judgement & Support for judgement \\
\hline $\begin{array}{l}\text { Random sequence genera- } \\
\text { tion (selection bias) }\end{array}$ & Low risk & Block randomisation (groups of 4) using computer software \\
\hline $\begin{array}{l}\text { Allocation concealment } \\
\text { (selection bias) }\end{array}$ & Unclear risk & Sealed envelopes; opaque and numbered unknown \\
\hline $\begin{array}{l}\text { Blinding (performance } \\
\text { bias and detection bias) } \\
\text { All outcomes }\end{array}$ & High risk & No attention control \\
\hline $\begin{array}{l}\text { Blinding of outcome as- } \\
\text { sessment (detection bias) } \\
\text { All outcomes }\end{array}$ & Unclear risk & Investigator blinded \\
\hline $\begin{array}{l}\text { Incomplete outcome data } \\
\text { (attrition bias) } \\
\text { End of intervention }\end{array}$ & Low risk & $\begin{array}{l}\text { ITT analysis used } \\
2 / 20(10 \%) \text { losses; } 2 / 10 \text { in control group (neurological problems) }\end{array}$ \\
\hline $\begin{array}{l}\text { Selective reporting (re- } \\
\text { porting bias) }\end{array}$ & Unclear risk & Protocol not available \\
\hline Other bias & Low risk & None known \\
\hline Imbalanced exposure & High risk & Imbalanced exposure \\
\hline
\end{tabular}

$\operatorname{Jin} 2013$

Design: RCT of progressive aerobic cycling training vs control
Randomisation: stratified randomisation; stratification based on age, gender and deficit severity
Allocation concealment: information not included
Blinding: "single blind" but does not outline in the paper who was blinded
ITT: not completed
Measurements: 12 weeks (end of intervention)
Withdrawals: before randomisation

Participants

Randomised: total 128 participants. 65 participants were randomised to intervention, 63 to control Intervention: 65 participants; 46 men (71\%) and 19 women (29\%); mean age: 57.6 (SD 6.6); 561 (SD 156) days after stroke

Control: 63 participants; 45 men (71\%) and 18 women (29\%); mean age: 56.3 (SD 6.5); 537 (SD 144) days after stroke

Inclusion criteria: 42-68 years, Chinese Han population, first ischaemic stroke ( $<6$ months), independent mobility with or without an assistive device

Exclusion criteria: haemorrhagic stroke, brainstem lesions and/or bilateral signs, diabetes mellitus or other concomitant nervous system disorders, cardiac or pulmonary disease possibly affecting the autonomic nervous system, any clinically relevant arrhythmia, heart failure, renal failure, unstable angina, uncontrolled hypertension, peripheral arterial occlusive disease, aphasia, dementia, untreated major depression, and other medical conditions that precluded participation in exercise training and conventional treatment 
Jin 2013 (Continued)

Interventions

Intervention: cardiorespiratory training: cycling training for $40 \mathrm{~min} /$ day, 5 times/week, target intensity of 50\%-70\% for 12 weeks. The training was started at a low intensity (40\%-50\% HR reserve) for $10-20$ min and increased by approximately 5 min every 2 weeks as tolerated. Aerobic intensity was similarly progressed by $5 \%$ HR reserve every 2 weeks. Participants pedaled for $6-10 \mathrm{~min}$ in each task condition, and 2-3 min of rest were provided between each task

Control: matched duration of conventional therapy including supervised stretching movements lasting $35 \mathrm{~min}$ and 5-min low-intensity over-ground walking training at 20\%-30\% HR reserve, 5 times/week

Setting: rehabilitation centre

\section{Outcomes}

Included outcomes: peak $\mathrm{VO}_{2}(\mathrm{~L} / \mathrm{min})$, peak $\mathrm{VO}_{2}, \mathrm{~mL} / \mathrm{kg} / \mathrm{min}$, resting SBP, resting DBP, BMI, 6 min Walk Distance, BBS, spasticity (modified Ashworth Scale), paretic knee strength, non-paretic knee strength

Other outcomes: resting HR, peak HR, peak SBP, peak DBP

\begin{tabular}{|c|c|c|}
\hline Notes & - & \\
\hline \multicolumn{3}{|l|}{ Risk of bias } \\
\hline Bias & Authors' judgement & Support for judgement \\
\hline $\begin{array}{l}\text { Random sequence genera- } \\
\text { tion (selection bias) }\end{array}$ & Low risk & $\begin{array}{l}\text { Quote: "After baseline testing, the subjects were stratified according to age, } \\
\text { gender and deficit severity, then, randomly assigned to either an aerobic cy- } \\
\text { cling training group or a control group by drawing lots." }\end{array}$ \\
\hline $\begin{array}{l}\text { Allocation concealment } \\
\text { (selection bias) }\end{array}$ & Unclear risk & Not reported in paper \\
\hline $\begin{array}{l}\text { Blinding (performance } \\
\text { bias and detection bias) } \\
\text { All outcomes }\end{array}$ & Unclear risk & Not clear who was blinded; exposure was balanced \\
\hline $\begin{array}{l}\text { Blinding of outcome as- } \\
\text { sessment (detection bias) } \\
\text { All outcomes }\end{array}$ & Unclear risk & Not reported in paper \\
\hline $\begin{array}{l}\text { Incomplete outcome data } \\
\text { (attrition bias) } \\
\text { End of intervention }\end{array}$ & Unclear risk & $\begin{array}{l}\text { No information on dropouts or incomplete outcome data after randomisation } \\
\text { and at end of intervention. ITT not carried out }\end{array}$ \\
\hline $\begin{array}{l}\text { Selective reporting (re- } \\
\text { porting bias) }\end{array}$ & Unclear risk & Trial register or protocol not available \\
\hline Other bias & Low risk & None known \\
\hline Imbalanced exposure & Low risk & $\begin{array}{l}\text { Balanced exposure: matched duration of treatment exposure between inter- } \\
\text { vention and control groups }\end{array}$ \\
\hline
\end{tabular}

Kang 2012

\section{Methods}

Design: randomised trial of cardiorespiratory training plus UC vs non-exercise intervention plus UC - after UC

Randomised: picking sealed envelopes

Allocation concealment: sealed envelopes 
Kang 2012 (Continued)

Blinding: assessor blinded to group allocation

ITT: not reported

Measurements: end of intervention (4 weeks)

Withdrawals: intervention group 1 participant due to lack of lack of participation

Randomised: 21 participants
Intervention: 10 participants; 6 men and 4 women, mean age 56.3 (SD 7.6); 13.5 days post-stroke (SD
$4.0)$
Control: 10 participants; 6 men and 4 women, mean age 56.1 (SD 7.8); 15.1 days post-stroke (SD 7.4)
Inclusion criteria: hemiparetic stroke 6 months after diagnosis; ability to walk for 15 min; without visual
disabilities; MMSE score of $\geq 21$; Brunnstrom stage $>4$
Exclusion criteria: cardiovascular problems, orthopaedic, and other neurological diseases except
stroke for influencing gait

Interventions Invention group: treadmill training for $30 \mathrm{~min} /$ day 3 times/week for 4 weeks, progressed by $0.1 \mathrm{~km} / \mathrm{h}$ each time stable walking for $20 \mathrm{~s}$ was achieved

Control group: non-exercise intervention of general stretching added range of motion exercises plus UC

Setting: rehabilitation centre

Outcomes Included outcomes: TUG; Functional Reach Test; 10-metre maximal walk test; 6-MWT

Notes 1 arm of this 3-group RCT was not used (treadmill with optic flow intervention)

10-metre maximal walk test data converted from $\mathrm{m} / \mathrm{sec}$ into $\mathrm{m} / \mathrm{min}$

\section{Risk of bias}

\begin{tabular}{|c|c|c|}
\hline Bias & Authors' judgement & Support for judgement \\
\hline $\begin{array}{l}\text { Random sequence genera- } \\
\text { tion (selection bias) }\end{array}$ & Low risk & Independent person picking sealed envelopes \\
\hline $\begin{array}{l}\text { Allocation concealment } \\
\text { (selection bias) }\end{array}$ & Unclear risk & Sealed envelopes; whether opaque or numbered unknown \\
\hline $\begin{array}{l}\text { Blinding (performance } \\
\text { bias and detection bias) } \\
\text { All outcomes }\end{array}$ & Unclear risk & Attention control was included \\
\hline $\begin{array}{l}\text { Blinding of outcome as- } \\
\text { sessment (detection bias) } \\
\text { All outcomes }\end{array}$ & Low risk & Blinded outcome assessors \\
\hline $\begin{array}{l}\text { Incomplete outcome data } \\
\text { (attrition bias) } \\
\text { End of intervention }\end{array}$ & Low risk & $\begin{array}{l}\text { ITT not reported } \\
1 / 21(5 \%) \text { losses; intervention group } 1 \text { participant (lack of participation) }\end{array}$ \\
\hline $\begin{array}{l}\text { Selective reporting (re- } \\
\text { porting bias) }\end{array}$ & Unclear risk & No protocol available \\
\hline Other bias & Low risk & None known \\
\hline
\end{tabular}


Kang 2012 (Continued)

Imbalanced exposure Low risk Balanced exposure

Katz-Leurer 2003

Design: randomised trial of cardiorespiratory training plus UC vs UC - during UC
Randomisation mechanism: unknown; method: blocks based on side of lesion
Allocation concealment: not reported
Blinding: investigator; efficacy unknown
ITT: unknown
Measurements: end of intervention and 6-month post-stroke follow-up
Withdrawals: intervention: no losses at the end of intervention, 5 losses at 6-month follow-up (4 not lo-
cated, 1 died); control: 2 discontinued intervention (1 acute MI, 1 deep vein thrombosis), 6 losses to fol-
low-up (3 not located, 1 died, 2 recurrent stroke)

Randomised: 92 participants
Intervention: 46 participants; 26 men and 20 women; age 62 years (SD 11); time since stroke unknown
Control: 46 participants; 23 men and 23 women; age 65 years (SD 11); time since stroke unknown
Inclusion criteria: age $>50$ years; $>6$ months after first ever stroke; walk 40 m with +/- rest, +/- assistive
device; $\geq$ stage 3 of Chedoke-McMaster Stroke Assessment: tolerate 45 min of exercise with rest inter-
vals; non-participation in other therapy programmes
Exclusion criteria: comprehensive aphasia; not medically stable; musculoskeletal problems not associ-
ated with stroke

Interventions Intervention: cardiorespiratory training: cycle ergometer; 8-week programme: (1) 20 min/day 5 days/ week for 2 weeks of intermittent ( $10 \times 1 \mathrm{~min}$ ) exercise progressing to $20 \mathrm{~min}$ continuous exercise by end of week 2; (2) $30 \mathrm{~min} /$ day 3 days/week for 6 weeks not exceeding $60 \%$ HR reserve; ACSM criteria for cardiorespiratory training met

Control: CPT, OT, speech therapy, and group activity/exercise

Setting: rehabilitation centre

\begin{tabular}{ll}
\hline Outcomes & $\begin{array}{l}\text { Included outcomes: FIM; BP; maximum cycle workload (watts); comfortable walking speed (10 m) gait } \\
\text { endurance; distance until fatigue; FAl; stair climbing } \\
\text { Other outcomes: SSS }\end{array}$ \\
\hline
\end{tabular}

Notes -

\section{Risk of bias}

\begin{tabular}{lll}
\hline Bias & Authors' judgement & Support for judgement \\
\hline $\begin{array}{l}\text { Random sequence genera- } \\
\text { tion (selection bias) }\end{array}$ & Unclear risk & Block randomisation based on side of lesion; mechanism not reported \\
\hline $\begin{array}{l}\text { Allocation concealment } \\
\text { (selection bias) }\end{array}$ & Unclear risk & Not reported \\
\hline $\begin{array}{l}\text { Blinding (performance } \\
\text { bias and detection bias) } \\
\text { All outcomes }\end{array}$ & Unclear risk & No attention control \\
\hline $\begin{array}{l}\text { Blinding of outcome as- } \\
\text { sessment (detection bias) } \\
\begin{array}{l}\text { All outcomes } \\
\hline\end{array}\end{array}$ & Low risk & Investigator blinded; efficacy unknown \\
\hline
\end{tabular}


Katz-Leurer 2003 (Continued)

\begin{tabular}{|c|c|c|}
\hline $\begin{array}{l}\text { Incomplete outcome data } \\
\text { (attrition bias) } \\
\text { End of intervention }\end{array}$ & Low risk & $\begin{array}{l}\text { ITT not reported } \\
2 / 96(2 \%) \text { lost at the end of intervention } \\
\text { Intervention: no losses, control: } 2 \text { discontinued ( } 1 \text { acute MI, } 1 \text { deep vein throm- } \\
\text { bosis) }\end{array}$ \\
\hline $\begin{array}{l}\text { Incomplete outcome data } \\
\text { (attrition bias) } \\
\text { End of follow-up }\end{array}$ & Unclear risk & $\begin{array}{l}\text { ITT not reported } \\
\text { 13/96 (14\%) total losses at the end of 6-month follow-up } \\
\text { Intervention: } 5 \text { ( } 4 \text { not located, } 1 \text { died); control } 6 \text { ( } 3 \text { not located, } 1 \text { died, } 2 \text { recur- } \\
\text { rent stroke) }\end{array}$ \\
\hline $\begin{array}{l}\text { Selective reporting (re- } \\
\text { porting bias) }\end{array}$ & Unclear risk & Protocol not available \\
\hline Other bias & Low risk & None known \\
\hline Imbalanced exposure & Unclear risk & Unclear \\
\hline
\end{tabular}

\section{Kim 2001}

Design: randomised trial of resistance training vs non-exercise intervention - after UC
Randomisation mechanism: unknown; method: stratified based on gender, age (50-59 or 60+ years),
and time since onset of stroke (6 months-2 years/2+ years)
Allocation concealment: unknown
Blinding: investigator; participants blinded to purpose of interventions
ITT: unknown
Measurements: end of intervention (6 weeks)
Withdrawals: none
Participants Randomised: 20 participants
Intervention: 10 participants; 7 men and 3 women; age 60.4 years (SD 9.5); 4.9 years post-stroke (SD 3.3)
Control: 10 participants; 7 men and 3 women; age 61.9 years (SD 7.5); 3.2 years post-stroke (SD 1.2) All participants had hemiparesis
Inclusion criteria: age > 50 years; > 6 months after first ever stroke; walk $40 \mathrm{~m}$ with +/- rest, +/- assistive device; stage 3 of Chedoke-McMaster Stroke Assessment; tolerate 45 min of exercise with rest intervals; non-participation in other therapy programmes
Exclusion criteria: comprehensive aphasia; not medically stable; musculoskeletal problems not associ- ated with stroke

Interventions

Intervention: isokinetic dynamometer (Kin-Com); 45 min/day, 3 days/week for 6 weeks; after a warmup this comprised 30 min of $3 \times 10$ resisted repetitions of maximal effort concentric hip flexion/extension, knee flexion/extension and ankle dorsiflexion/plantarflexion of the affected lower limb; progression in the resistance was achieved by increasing the preload on the Kin-Com device; ACSM criteria for resistance training met

Control: exactly the same as intervention except the resisted contractions replaced with passive range of motion movements

Setting: rehabilitation centre

Outcomes

Included outcomes: gait preferred speed ( $\mathrm{m} / \mathrm{min}$ over $8 \mathrm{~m})$; gait maximum speed ( $\mathrm{m} / \mathrm{min})$; stair climbing speed (stairs/s); composite strength score for the affected (trained) lower limb; stair walking performance ( 4 x $18 \mathrm{~cm}$ steps) self-selected and maximal; 'physical functioning' and 'mental health' scales of the SF-36 
Kim 2001 (Continued)

Other outcomes:

\begin{tabular}{|c|c|c|}
\hline Notes & Data reported as chan & e scores \\
\hline \multicolumn{3}{|l|}{ Risk of bias } \\
\hline Bias & Authors' judgement & Support for judgement \\
\hline $\begin{array}{l}\text { Random sequence genera- } \\
\text { tion (selection bias) }\end{array}$ & Low risk & $\begin{array}{l}\text { Mechanism unknown; method stratified based on gender, age ( } 50 \text { to } 59 \text { or } 60+ \\
\text { years), and time since onset of stroke ( } 6 \text { months to } 2 \text { years/2+ years). Likely } \\
\text { that software was used to achieve this complexity. }\end{array}$ \\
\hline $\begin{array}{l}\text { Allocation concealment } \\
\text { (selection bias) }\end{array}$ & Unclear risk & Not reported \\
\hline $\begin{array}{l}\text { Blinding (performance } \\
\text { bias and detection bias) } \\
\text { All outcomes }\end{array}$ & Unclear risk & Attention control used; participants blinded to purpose of interventions \\
\hline $\begin{array}{l}\text { Blinding of outcome as- } \\
\text { sessment (detection bias) } \\
\text { All outcomes }\end{array}$ & Low risk & Investigator blinded; efficacy unknown \\
\hline $\begin{array}{l}\text { Incomplete outcome data } \\
\text { (attrition bias) } \\
\text { End of intervention }\end{array}$ & Low risk & $\begin{array}{l}\text { ITT not reported } \\
\text { No losses }\end{array}$ \\
\hline $\begin{array}{l}\text { Selective reporting (re- } \\
\text { porting bias) }\end{array}$ & Unclear risk & Protocol not available \\
\hline Other bias & Low risk & None known \\
\hline Imbalanced exposure & Low risk & Balanced exposure \\
\hline
\end{tabular}

Kim 2014

Methods Design: randomised trial of community walking training programme plus UC vs UC (conventional PT and OT) during UC

Randomisation: sealed envelopes prepared in advance

Allocation concealment: sealed envelopes

Blinding: outcome assessment

ITT: no

Measurements: end of intervention (4 weeks)

Withdrawals: 4 dropouts - 2 from intervention group and 2 from control group

Randomised: total 26 participants; 13 to intervention and 13 to control
Intervention: 11 participants; 6 male, 5 women; mean age: 50.18 years (SD 10.29); 109 days post-stroke
(SD 108)
Control: 11 participants; 7 male, 4 women; mean age 50.73 years (SD 7.24); 273 days post-stroke (SD
108)
Inclusion criteria: hemiparesis from single stroke occurring at least 6 months previously; sufficient cog-
nition to comprehend study purpose; gait speed < $0.8 \mathrm{~m} / \mathrm{s} ;$ ability to walk 10 m independently without
device; no musculoskeletal condition that could potentially affect ability to walk safely; no hemispatial
neglect


Kim 2014 (Continued)

Exclusion criteria: participation in other studies or rehabilitation programmes; severe heart disease or other uncontrolled hypertension or pain

Interventions
Week $1-200 \mathrm{~m}$ route, walking near hospital
Week $2-300 \mathrm{~m}$ route, outside hospital/uneven ground
Week 3 - $400 \mathrm{~m}$ route, uneven ground, gradual slope, unpaved road, obstacles
Week 4 - walking around shopping centre
Control: conventional PT and OT
Setting: community-based intervention delivered during inpatient care

Outcomes Included outcomes: walking function measured using 10-metre walk test, 6-MWT and 'community
walking assessment' ( $300 \mathrm{~m}$ route, different terrains and gradients)

Other outcomes: social participation measured with SIS

Notes Change from baseline scores reported and used

\section{Risk of bias}

\begin{tabular}{|c|c|c|}
\hline Bias & Authors' judgement & Support for judgement \\
\hline $\begin{array}{l}\text { Random sequence genera- } \\
\text { tion (selection bias) }\end{array}$ & Unclear risk & $\begin{array}{l}\text { Sealed envelopes marked on the inside with an "O or X". Unclear how this was } \\
\text { administered }\end{array}$ \\
\hline $\begin{array}{l}\text { Allocation concealment } \\
\text { (selection bias) }\end{array}$ & High risk & $\begin{array}{l}\text { Unclear whether sealed envelopes were numbered, opaque, or opened se- } \\
\text { quentially. Also the envelopes were "... marked on the inside with an O or X". } \\
\text { Therefore concealment may be threatened }\end{array}$ \\
\hline $\begin{array}{l}\text { Blinding (performance } \\
\text { bias and detection bias) } \\
\text { All outcomes }\end{array}$ & High risk & No attention control \\
\hline $\begin{array}{l}\text { Blinding of outcome as- } \\
\text { sessment (detection bias) } \\
\text { All outcomes }\end{array}$ & Low risk & "... the assessor was blinded" \\
\hline $\begin{array}{l}\text { Incomplete outcome data } \\
\text { (attrition bias) } \\
\text { End of intervention }\end{array}$ & High risk & $\begin{array}{l}\text { ITT analysis not used } \\
4 / 26(15 \%) \text { losses; } 2 \text { participants lost from each group } \\
\text { Due to health, personal reasons or discharge; distribution between groups un- } \\
\text { known }\end{array}$ \\
\hline $\begin{array}{l}\text { Selective reporting (re- } \\
\text { porting bias) }\end{array}$ & Unclear risk & No study protocol available \\
\hline Other bias & Low risk & None known \\
\hline Imbalanced exposure & High risk & Imbalanced exposure \\
\hline
\end{tabular}


Design: single-centre randomised trial: mixed circuit training plus \% UC (excluding PT) to conventional PT based on neurodevelopmental treatment plus UC (excluding PT) - during UC (inpatient)

Randomisation: participants were randomised allocated in a 1:1 ratio, no further details were provided regarding methods of randomisation

Allocation concealment: no details provided

Blinding: assessor blinded to group allocation

ITT: 20 patients were randomised and analysed; the study authors did not explicitly report on dropouts, or missing data or ITT

Measurements: at the end of intervention (4 weeks)

Withdrawals: not reported; 20 participants were randomised and 20 were analysed

\section{Participants}

Randomised: 20 participants; 10 to the circuit class and 10 to conventional PT

Intervention: circuit training 10 participants; 6 men (60\%) and 4 women (40\%); mean age: 65.2 (SD

10.1); 30.1 (SD 21.8) days after stroke

Control: CPT 10 participants; 7 men (70\%) and 3 women (30\%); mean age: 66.0 (SD 8.8); 29.9 (SD 20.3) days after stroke

Inclusion criteria: a clinical diagnosis of a first stroke confirmed by neuroimaging (CT or MRI); a hemiparesis; a time interval between stroke and recruitment of $\leq 3$ months; the ability to comprehend the instructions for the testing procedures; and mild to moderate walking deficit, as indicated by FAC between 3 and 4

Exclusion criteria: a severe cognitive impairment (Korean-MMSE $\leq 10$ ) or aphasia; previous stroke history; not independent 'sit to stand' activity (BBS score < 18); acute systemic illness or infection; a significant orthopaedic condition or pain that limited participation in exercise; and visual impairment or vestibular system deficit that caused balance impairment 4 weeks. Training consisted of a 5-min warm-up period, 5 classes of 15-min duration of complex exercises interspersed by 1-min rest, and a 5-min cool-down period. The 5 categories were: trunk exercise and active sitting practice, sit-to-stand practice, standing and walking practice, aerobic treadmill training and lower-limb strength training (TheraBand). Treadmill speed and gradient were progressed and TherBand repetitions and resistance progressed

Control: individual CPT for $30 \mathrm{~min}$ twice a day (total $60 \mathrm{~min}$ ), 5 days a week for the 4 weeks. The content of the individual PT sessions was based on neurodevelopmental treatment for motor recovery

Setting: inpatient rehabilitation

Outcomes Included outcomes: motor function lower limb (lower limb score Fugl-Meyer assessment), gait endurance (6-MWT), balance (BBS), ADL function (Korean version of the Modified BI)

Other outcomes: none

\section{Notes}

\section{Risk of bias}

Bias Authors' judgement Support for judgement

\begin{tabular}{|c|c|c|}
\hline $\begin{array}{l}\text { Random sequence genera- } \\
\text { tion (selection bias) }\end{array}$ & Unclear risk & $\begin{array}{l}\text { Randomised in a 1:1 ratio, evenly matched via sealed envelope technique but } \\
\text { no further information provided }\end{array}$ \\
\hline
\end{tabular}


Kim 2016a (Continued)

$\begin{array}{ll}\begin{array}{l}\text { Allocation concealment } \\ \text { (selection bias) }\end{array} & \begin{array}{l}\text { Randomisation was performed using a sealed envelope technique, unclear if } \\ \text { envelopes were opaque }\end{array}\end{array}$

\begin{tabular}{|c|c|c|}
\hline $\begin{array}{l}\text { Blinding (performance } \\
\text { bias and detection bias) }\end{array}$ & High risk & $\begin{array}{l}\text { There was no description provided regarding information participants re- } \\
\text { ceived about the aim of the study and no sham control was provided }\end{array}$ \\
\hline
\end{tabular}

All outcomes

Blinding of outcome as- $\quad$ Low risk $\quad$ The outcome assessor was blinded to allocation
sessment (detection bias)
All outcomes

\begin{tabular}{|c|c|c|}
\hline $\begin{array}{l}\text { Incomplete outcome data } \\
\text { (attrition bias) } \\
\text { End of intervention }\end{array}$ & Low risk & $\begin{array}{l}\text { All participants who were randomised were analysed no details on dropouts or } \\
\text { missing data was reported }\end{array}$ \\
\hline $\begin{array}{l}\text { Incomplete outcome data } \\
\text { (attrition bias) } \\
\text { End of follow-up }\end{array}$ & Low risk & \\
\hline $\begin{array}{l}\text { Selective reporting (re- } \\
\text { porting bias) }\end{array}$ & Unclear risk & $\begin{array}{l}\text { All outcomes specified in the methods were reported in detail, the study proto- } \\
\text { col was not published and no reference was made to registration of the study }\end{array}$ \\
\hline Other bias & Low risk & None known \\
\hline Imbalanced exposure & High risk & No attention control; imbalanced exposure doses \\
\hline
\end{tabular}

Kim 2017a

Design: randomised trial of mixed training plus UC vs UC only - during UC
Randomisation: no description of method used
Allocation concealment: not described
Blinding: not described
ITT: not described; per-protocol analysis
Measurements: baseline and end of intervention (6 weeks)
Withdrawals: 1 dropout from exercise group (discharged home)

$\begin{array}{ll}\text { Participants } & \text { Randomised: total } 30 \text { participants (15 intervention, } 15 \text { control) } \\ \text { Intervention: } 14 \text { participants ( } 9 \text { men), mean age } 50.71 \text { years (14.81) and time since stroke 12.79 (7.34) } \\ \text { months } \\ \text { Control: } 15 \text { participants ( } 10 \text { men), mean age } 51.87 \text { years (17.42) and time since stroke 11.73 (8.02) } \\ \text { months } \\ \text { Inclusion criteria: at least } 3 \text { months post-stroke, cognitive ability allowed research instructions to be } \\ \text { followed, Korean-MMSE score } \geq 10 \text { points, ability to walk } \geq 10 \text { m independently, and adequate vision for } \\ \text { study tests } \\ \text { Exclusion criteria: bilateral stroke or previous stroke affecting the other side, insufficient gait ability for } \\ \text { using treadmill, risk of epilepsy, and use of anti-epileptics } \\ \text { Intervention: handgrip strength training; progressive } 15 \text { min grasping training with power web exercis- } \\ \text { er and digi-flex devices. Walking speed cardiorespiratory treadmill training (15 min) with 5\% body mass }\end{array}$


Kim 2017a (Continued)

loading of the unaffected side. Speed of treadmill increased in increments each week. Total exercise dose $30 \mathrm{~min} /$ day, $3 \mathrm{x}$ week for 6 weeks

Conventional therapy alone. Involved joint movements, mat movements and walking exercises 10 min each. $60 \mathrm{~min} / \mathrm{session}, 2$ sessions/day, 5 days/week for 6 weeks

Control: conventional therapy alone. Involved joint movements, mat movements and walking exercises $10 \mathrm{~min}$ each. $60 \mathrm{~min} / \mathrm{session}, 2$ sessions/day, 5 days/week for 6 weeks

Setting: hospital

Outcomes Included outcomes: cognitive function test (Korean version of Montreal Cognitive Assessment, trail
making test and Stroop test, handgrip strength test, 10 metre walking test, TUG

Notes Uncertainty on exercise frequency; sessions/day or/week

\section{Risk of bias}

\begin{tabular}{|c|c|c|}
\hline Bias & Authors' judgement & Support for judgement \\
\hline $\begin{array}{l}\text { Random sequence genera- } \\
\text { tion (selection bias) }\end{array}$ & Unclear risk & No mechanism described \\
\hline $\begin{array}{l}\text { Allocation concealment } \\
\text { (selection bias) }\end{array}$ & Unclear risk & No information provided \\
\hline $\begin{array}{l}\text { Blinding (performance } \\
\text { bias and detection bias) } \\
\text { All outcomes }\end{array}$ & High risk & $\begin{array}{l}\text { No description of who ran the intervention, unbalanced intervention between } \\
\text { groups }\end{array}$ \\
\hline $\begin{array}{l}\text { Blinding of outcome as- } \\
\text { sessment (detection bias) } \\
\text { All outcomes }\end{array}$ & Unclear risk & No information available \\
\hline $\begin{array}{l}\text { Incomplete outcome data } \\
\text { (attrition bias) } \\
\text { End of intervention }\end{array}$ & Low risk & Analysed per protocol but only $1 / 15$ dropped out in intervention group \\
\hline $\begin{array}{l}\text { Incomplete outcome data } \\
\text { (attrition bias) } \\
\text { End of follow-up }\end{array}$ & Low risk & \\
\hline $\begin{array}{l}\text { Selective reporting (re- } \\
\text { porting bias) }\end{array}$ & Unclear risk & No trial registration information \\
\hline Other bias & Low risk & $\begin{array}{l}\text { Muscle strength should be maximum value not an average of triplicate mea- } \\
\text { sures; but overall no substantive issues noted }\end{array}$ \\
\hline Imbalanced exposure & High risk & No attention control \\
\hline
\end{tabular}

Knox 2018

$\begin{array}{ll}\text { Methods } & \text { Design: single-blinded, RCT with } 2 \text { intervention groups } \\ & \text { - mixed training (task-oriented circuit gait training) vs no exercise (control) - after UC } \\ \text { - resistance training vs no exercise (control) - after UC }\end{array}$


Knox 2018 (Continued)

Randomisation: stratified randomisation; stratification based on comfortable gait speed mild $(\geq 0.8 \mathrm{~m} /$ $\mathrm{s})$, moderate $(\geq 0.4 \mathrm{~m} / \mathrm{s}$ and $<0.8 \mathrm{~m} / \mathrm{s})$, and severe $(<0.4 \mathrm{~m} / \mathrm{s})$ and then randomised into the 3 intervention arms using computer-generated random numbers

Allocation concealment: envelopes; unclear whether sequentially numbered, sealed or opaque

Blinding: outcome assessor blinded; participants may have understood nature of group assignments

ITT: analyses were not performed according to ITT principle

Measurements: 12 weeks post-intervention and at follow-up a further 12 weeks later

Withdrawals: 12 weeks post-intervention: task group: 6/51 (11\%) participants from task group (3 deceased, 2 moved away, 1 return to work), 5/45 (11\%) participants from strength group (1 deceased, 2 no transport, 2 moved away), 5/48 (10\%) participants from control group (1 deceased, 2 no transport, 1 return to work, 1 private healthcare)

Follow-up after a further 12 weeks: 10/51 (20\%) participants from task group ( 2 moved away, 1 second stroke, 1 no transport), 6/45 (13\%) participants from strength group (1 deceased), 8/48 (17\%) participants from control group (1 returned to work, 1 deceased, 1 no transport)

Participants

Randomised: 144 participants randomised

Mixed training group 51 participants; 25 men (49\%) and 26 women (51\%); mean age: 51 (SD 15); time since stroke: 10 weeks (SD 8)

Resistance training group $=45$ participants, 25 men (56\%) and 20 women (44\%); mean age: 51 (SD 12); time since stroke: 9 weeks (SD 7)

Control: 48 participants, 22 men (46\%) and 26 women (54\%); mean age: 48 (SD 14); time since stroke 8 weeks (SD 7)

Inclusion criteria: between 20-79 years of age, $<6$ months after the onset of a first stroke, medically stable (people who were HIV+ but on antiretroviral treatment and stable), and who could walk at least 10 $\mathrm{m}$ at $<1.1 \mathrm{~m} / \mathrm{s}$ without assistance but with the use of assistive devices or supervision, if needed, able to understand and follow instructions, and live near enough to attend sessions in the hospital with the help of caregivers

Exclusion criteria: medical, neurological, or orthopaedic conditions that could interfere with their poststroke recovery such as active HIV status, neurological disorders, or musculoskeletal injuries interfering with gait rehabilitation

Interventions

Mixed training group: (task group) 6 exercises focused on improving strength, balance, and task performance while standing and walking, and included an endurance walking station. Exercises were progressed by reducing the use of supports and increasing the complexity of the tasks. Participants' caregivers attended to give the correct assistance to the participant in the execution of the tasks and were directed to assist the participant in working on a progressively more difficult structured walking programme at home between the formal therapy sessions. Frequency of intervention: 6 sessions of $1 \mathrm{~h}$ each over a 12-week period

Resistance training group: (strength group) 3 sets of 10 repetitions; 10 exercises performed while sitting and lying, which targeted the major muscles in the lower extremities. Exercise progressed as per the participants' performance and feedback. Resistance was provided with gravity, free weights, elastic bands, and balls. Frequency of intervention: 6 sessions of $1 \mathrm{~h}$ each over a 12-week period

Control: 90-min educational session on stroke management that included 20 min of exercises, provided by an occupational or physiotherapist. Frequency of intervention: one 90-min session. Single session so not a training intervention

Setting: hospital

Outcomes

Included outcomes: walking endurance- six-min walk test, comfortable and fast gait speeds, $10 \mathrm{~m}$ gait speed, balance function, mobility 
Knox 2018 (Continued)

Other outcomes:

Notes Trial registered retrospectively (9 years after study start date): PACTR201802003054396

\section{Risk of bias}

\begin{tabular}{lll}
\hline Bias & Authors' judgement & Support for judgement \\
\hline $\begin{array}{ll}\text { Random sequence genera- } \\
\text { tion (selection bias) }\end{array}$ & Low risk & $\begin{array}{l}\text { Stratified by comfortable gait speed and randomised with computer-generat- } \\
\text { ed random numbers }\end{array}$ \\
\hline
\end{tabular}

\begin{tabular}{|c|c|c|}
\hline $\begin{array}{l}\text { Allocation concealment } \\
\text { (selection bias) }\end{array}$ & Unclear risk & $\begin{array}{l}\text { Computer-generated sequence was used by a physiotherapist blinded to } \\
\text { group allocation. However, the study authors outline that "stratification en- } \\
\text { velopes" were used but it is unclear whether they were sequentially num- } \\
\text { bered, sealed or opaque }\end{array}$ \\
\hline
\end{tabular}

\begin{tabular}{|c|c|c|}
\hline $\begin{array}{l}\text { Blinding (performance } \\
\text { bias and detection bias) } \\
\text { All outcomes }\end{array}$ & High risk & No attempt was made to blind participants to group assignment. \\
\hline $\begin{array}{l}\text { Blinding of outcome as- } \\
\text { sessment (detection bias) } \\
\text { All outcomes }\end{array}$ & Low risk & Outcome assessor blinded \\
\hline $\begin{array}{l}\text { Incomplete outcome data } \\
\text { (attrition bias) } \\
\text { End of intervention }\end{array}$ & Low risk & $\begin{array}{l}\text { Participants with missing outcome data were not excluded. Participants } \\
\text { with missing follow-up data were not excluded. Small, balanced number of } \\
\text { dropouts ( } 11 \% \text { task group, } 11 \% \text { strength group, and } 10 \% \text { control group). }\end{array}$ \\
\hline $\begin{array}{l}\text { Incomplete outcome data } \\
\text { (attrition bias) } \\
\text { End of follow-up }\end{array}$ & Low risk & $\begin{array}{l}\text { Balanced attrition across } 3 \text { groups post-intervention, } \leq 20 \% \text { attrition in all } \\
\text { groups }\end{array}$ \\
\hline $\begin{array}{l}\text { Selective reporting (re- } \\
\text { porting bias) }\end{array}$ & High risk & $\begin{array}{l}\text { Reported outcomes correspond to protocol PACTR201802003054396; howev- } \\
\text { er, protocol published retrospectively }\end{array}$ \\
\hline Other bias & Low risk & None known \\
\hline Imbalanced exposure & High risk & $\begin{array}{l}\text { No attempt to account for imbalanced exposure in experimental arms (both } \\
6 \times 1 \text {-h sessions over } 12 \text { weeks) and control arm ( } 1 \text { x 90-min session over } 12 \\
\text { weeks) }\end{array}$ \\
\hline
\end{tabular}

Kuys 2011

Methods

Design: randomised, single-blind trial of cardiorespiratory plus UC vs UC - during UC

Randomised: independent researcher generated random sequence in blocks of 4 using computer-generated random number sequence

Allocation concealment: consecutively numbered envelopes

Blinding: outcome assessors

ITT: yes

Measurements: end of intervention ( 6 weeks) and 3-month follow-up 
Kuys 2011 (Continued)

Withdrawals: intervention group ( 2 participants before end of intervention ( 1 withdrew, 1 due to fall); 2 participants before follow-up (1 moved, 1 medical condition); control group ( 3 participants before fol-

low-up (1 unable to be contacted, 1 medical condition, 1 moved)

Randomised: 30 participants
Intervention: 15 participants; 7 men and 8 women; mean age 63 years (SD 14); 52 days post-stroke (SD
32)
Control: 15 participants; 7 men and 8 women; mean age 72 years (SD 17); 49 days post-stroke (SD 30)
Inclusion criteria: first stroke diagnosed via CT; referred for PT rehabilitation; scored $\leq 2$ MAS; medically
stable; MMSE score of at least 24
Exclusion criteria: normal gait speed $(>1.2 \mathrm{~m} / \mathrm{s})$; cardiovascular problems

Invention group: treadmill walking for 30 min 3 times/week for 6 weeks at $40 \%-60 \%$ HR reserve (initially
starting at $40 \%$ HR reserve, progressing by $5 \%-10 \%$ increase each week until $60 \%$ reached)
Control group: CPT care
Setting: 2 rehabilitation units

Outcomes Included outcomes: 10-metre walk test; comfortable walking speed; 6-MWT

Other outcomes: walking kinematic data

\begin{tabular}{ll}
\hline Notes & - \\
\hline
\end{tabular}

\section{Risk of bias}

\begin{tabular}{|c|c|c|}
\hline Bias & Authors' judgement & Support for judgement \\
\hline $\begin{array}{l}\text { Random sequence genera- } \\
\text { tion (selection bias) }\end{array}$ & Low risk & $\begin{array}{l}\text { Independent researcher generated random sequence in blocks of } 4 \text { using com- } \\
\text { puter-generated random number sequence }\end{array}$ \\
\hline $\begin{array}{l}\text { Allocation concealment } \\
\text { (selection bias) }\end{array}$ & Unclear risk & $\begin{array}{l}\text { Consecutively numbered envelopes; not reported whether these were sealed } \\
\text { and opaque }\end{array}$ \\
\hline $\begin{array}{l}\text { Blinding (performance } \\
\text { bias and detection bias) } \\
\text { All outcomes }\end{array}$ & High risk & No attention control \\
\hline $\begin{array}{l}\text { Blinding of outcome as- } \\
\text { sessment (detection bias) } \\
\text { All outcomes }\end{array}$ & Low risk & Blinded outcome assessors \\
\hline $\begin{array}{l}\text { Incomplete outcome data } \\
\text { (attrition bias) } \\
\text { End of intervention }\end{array}$ & Low risk & $\begin{array}{l}\text { ITT analysis used } \\
2 / 30(7 \%) \text { losses } \\
\text { Intervention group } 2 \text { ( } 1 \text { withdrew, } 1 \text { due to fall) }\end{array}$ \\
\hline $\begin{array}{l}\text { Incomplete outcome data } \\
\text { (attrition bias) } \\
\text { End of follow-up }\end{array}$ & High risk & $\begin{array}{l}\text { ITT analysis used } \\
7 / 30(23 \%) \text { total losses } \\
\text { Intervention group } 2 \text { ( } 1 \text { moved, } 1 \text { medical condition); control group } 3 \text { (1 unable } \\
\text { to be contacted, } 1 \text { medical condition, } 1 \text { moved) }\end{array}$ \\
\hline $\begin{array}{l}\text { Selective reporting (re- } \\
\text { porting bias) }\end{array}$ & Low risk & $\begin{array}{l}\text { All included outcomes were described in trial registry ACTRN12607000412437. } \\
\text { Planned oxygen uptake measures not reported }\end{array}$ \\
\hline
\end{tabular}


Kuys 2011 (Continued)

\begin{tabular}{lll} 
Other bias & Low risk & None known \\
\hline Imbalanced exposure & High risk & Imbalanced exposure \\
\hline
\end{tabular}

\section{Methods}

Design: randomised trial of mixed training vs UC - after UC - i.e. intensive exercise (with emphasis on endurance, strength, and balance) vs regular exercise (no specific treatment was recommended) at discharge. Sample size calculation reported

Randomisation: stratified randomisation according to gender and hemisphere lesion (minimisation). Method of randomisation: dice (uneven numbers vs even numbers). Randomisation was performed by an investigator not involved with the participants or the treatment

Allocation concealment: unclear. Protocol was sealed for 1.5 years from the start of the study

Blinding procedure: outcome assessor blinded

ITT: planned but not performed

Measurements: 3, 6, and 12 months

Withdrawals: 3 participants in the intensive group at discharge ( 1 dead and 2 withdrawals) and 5 ( 3 dead and 2 withdrawals) in the regular exercise group at discharge. 1 dead and 1 withdrawal at 3 months and 2 dead at 6 months in the regular exercise control group

\section{Participants}

\section{Randomised: 75 participants}

Intervention: 35 participants, gender not reported; mean age 76 years (SD 12.7)

Control: 40 participants, gender not reported; mean age 72 years (SD 13.6)

Inclusion criteria: first-time stroke, confirmed by CT and voluntary participation

Exclusion criteria: > 1 stroke event, subarachnoid bleeding, tumour, other serious illness, brainstem, or cerebellar stroke

Intervention: intensive individualised training programme supervised by physiotherapists. Endurance = walking indoors and outdoors, stationary bicycling, stair walking, treadmill, etc, at 70\%-80\% maximal pulse. Strength = push-ups, sit-ups, weight lifting, pulley, etc, at 50\%-60\% calculated from 1-RM. Participants were also encouraged to maintain high activity level apart from that in the training sessions. Frequency: $2 / 3$ times/week (daily in rehabilitation ward); minimum $20 \mathrm{~h}$ every third month, in the first year after stroke

Control: rehabilitation and follow-up treatments according to participants' needs but not on regular basis. No specific treatment was recommended. Participants were, however, encouraged to maintain high activity level

Setting: general hospital, participants' homes, and community service centres

$\begin{array}{ll}\text { Outcomes } & \text { Included outcomes: MAS; BI; grip strength measured with a Martin Vigorimeter; occurrences of falls and } \\ \text { pain } & \\ \text { Other outcomes: none }\end{array}$

$$
\text { Notes }
$$$$
-
$$

\section{Risk of bias}


Langhammer 2007 (Continued)

\section{Bias Authors' judgement Support for judgement}

Random sequence genera- Low risk tion (selection bias)

Use of dice (uneven numbers vs even numbers). In addition, randomisation was stratified according to gender and hemisphere lesion (minimisation) Randomisation was performed by an investigator not involved with the participants or the treatment

\begin{tabular}{lll}
\hline $\begin{array}{l}\text { Allocation concealment } \\
\text { (selection bias) }\end{array}$ & Unclear risk & Unclear; protocol was sealed for 1.5 years from the start of the study \\
\hline $\begin{array}{l}\text { Blinding (performance } \\
\text { bias and detection bias) }\end{array}$ & High risk & $\begin{array}{l}\text { Some unstructured attention control "The amount of training was equal in the } \\
\text { two groups". However, the control intervention was not given on a regular ba- } \\
\text { sis outcomes }\end{array}$
\end{tabular}

\begin{tabular}{l}
\hline Blinding of outcome as- $\quad$ Low risk \\
sessment (detection bias) \\
All outcomes
\end{tabular}

$\begin{array}{ll}\begin{array}{l}\text { Incomplete outcome data } \\ \text { (attrition bias) }\end{array} & \text { Low risk } \\ \text { End of intervention } & \begin{array}{l}\text { ITT analysis } \\ 8 / 75(11 \%) \text { losses at the end of intervention; } 3 \text { participants in the intensive ex- } \\ \text { ercise group at discharge (1 dead and } 2 \text { withdrawals) and } 5 \text { ( } 3 \text { dead and } 2 \text { with- } \\ \text { drawals) in the control group at discharge }\end{array}\end{array}$

\begin{tabular}{|c|c|c|}
\hline $\begin{array}{l}\text { Incomplete outcome data } \\
\text { (attrition bias) } \\
\text { End of follow-up }\end{array}$ & Low risk & $\begin{array}{l}\text { ITT analysis } \\
12 / 75(16 \%) \text { losses at the end of follow-up; } 1 \text { dead and } 1 \text { withdrawal at } 3 \\
\text { months and } 2 \text { dead at } 6 \text { months in the control group }\end{array}$ \\
\hline $\begin{array}{l}\text { Selective reporting (re- } \\
\text { porting bias) }\end{array}$ & Unclear risk & Protocol not available \\
\hline Other bias & Low risk & None known \\
\hline Imbalanced exposure & Unclear risk & Imbalanced exposure \\
\hline
\end{tabular}

Lee $2013 a$

Methods Design: randomised trial of close kinetic chain resistance exercise vs open kinetic chain resistance exercise vs no intervention

Randomisation: unclear

Allocation concealment: information not included

Blinding: information not included

ITT: no ITT but no losses

Measurements: end of intervention ( 6 weeks)

Withdrawals: none

Participants

Randomised: total 33 participants. 11 to close kinetic chain exercise (CKC), 11 to open kinetic chain exercise (OKC) and 11 to control

Intervention 1 CKC: 11 participants; 7 men, 4 women; mean age: 59.3 (SD 8.87); months post-stroke 19.9 (SD 7.59)

Intervention 2 OKC 11 participants; 7 men, 4 women; mean age 58.8 (SD 6.81); months post-stroke 20.3 (SD 8.13)

Control: 11 participants: 6 men, 5 women; mean age 60.10 (SD 7.01); months post-stroke 19.70 (SD 9.42) 
Lee 2013a (Continued)

Inclusion criteria: age 30-65 years; stroke occurring at least 6 months before start of study; sufficient cognition to comprehend study purpose; one-sided hemiparesis of lower extremity

Exclusion criteria: severe cognitive, communicative, perceptual or sensory problems preventing understanding of study purpose; other neurologic or psychiatric problems causing difficulties in following programme; unstable cardiovascular/ventilatory problems

Warm-up - 4 reps at 25\% of 1-RM followed by 3 sets (8-10 reps) at 70\% of 1-RM adjusted weekly

Intervention 1 CKC: seated, paretic foot on pedal of a leg press machine with pneumatic resistance, extend leg and slowly flex

Intervention 2 OKC: sat in chair, back facing leg press exercise machine, knee maintained at $90^{\circ}$ of flexion with free distal extremity. Extend and slowly flex knee

Control: no intervention (maintained routine activity)

Setting: unclear

Other outcomes: muscle activation (limb muscle electromyography recordings)

Notes Very similar to Lee 2013b

\section{Risk of bias}

\begin{tabular}{|c|c|c|}
\hline Bias & Authors' judgement & Support for judgement \\
\hline $\begin{array}{l}\text { Random sequence genera- } \\
\text { tion (selection bias) }\end{array}$ & Unclear risk & Not reported in paper \\
\hline $\begin{array}{l}\text { Allocation concealment } \\
\text { (selection bias) }\end{array}$ & Unclear risk & Not reported in paper \\
\hline $\begin{array}{l}\text { Blinding (performance } \\
\text { bias and detection bias) } \\
\text { All outcomes }\end{array}$ & High risk & Imbalanced exposure \\
\hline $\begin{array}{l}\text { Blinding of outcome as- } \\
\text { sessment (detection bias) } \\
\text { All outcomes }\end{array}$ & Unclear risk & Not reported in paper \\
\hline $\begin{array}{l}\text { Incomplete outcome data } \\
\text { (attrition bias) } \\
\text { End of intervention }\end{array}$ & Low risk & No losses \\
\hline $\begin{array}{l}\text { Selective reporting (re- } \\
\text { porting bias) }\end{array}$ & Unclear risk & Protocol not available \\
\hline Other bias & Low risk & None known \\
\hline Imbalanced exposure & High risk & Imbalanced exposure between control group and both intervention groups \\
\hline
\end{tabular}


Design: randomised trial of close kinetic chain resistance exercise vs open kinetic chain resistance exercise vs no intervention

Randomisation: unclear

Allocation concealment: information not included

Blinding: information not included

ITT: no ITT but no losses

Measurements: end of intervention ( 6 weeks)

Withdrawals: none

Participants

Randomised: total 39 participants. 13 to close kinetic chain exercise (CKC), 13 to open kinetic chain exercise (OKC) and 13 to control

Intervention 1 CKC: 13 participants; 8 men, 5 women; mean age: 49.3 (SD 8.87); months after stroke

14.9 (SD 9.59)

Intervention 2 (OKC): 13 participants; 8 men, 5 women; mean age 50.8 (SD 6.81); months post-stroke

15.7 (SD 8.13)

Control: 13 participants; 9 men, 4 women; mean age 49.10 (SD 7.01); months after stroke 15.10 (SD 8.73) Inclusion criteria: hemiparesis secondary to single onset unilateral stroke; ability to ambulate independently over $10 \mathrm{~m}$ (with/without device); absence of significant lower extremity joint pain and major sensory deficits; absence of significant lower limb contractures; no significant cardiovascular or respiratory symptoms contradictive to walking

Exclusion criteria: none stated

Resistance training 5 times/week for 6 weeks

Warm-up - 4 reps at 25\% of 1-RM followed by 3 sets (8-10 reps) at 70\% of 1-RM adjusted weekly

Intervention 1 CKC: seated, paretic foot on pedal of a leg press machine with pneumatic resistance, extend leg and slowly flex

Intervention 2 OKC: sat in chair, back facing leg press exercise machine, knee maintained at $90^{\circ}$ of flexion with free distal extremity. Extend and slowly flex knee

Control: no intervention (maintained routine activity)

Setting: unclear

\begin{tabular}{ll}
\hline Outcomes & Included outcomes: none \\
& Other outcomes: barefoot plantar pressure distributions
\end{tabular}

Notes Very similar to Lee 2013b

\section{Risk of bias}

\begin{tabular}{lll}
\hline Bias & Authors' judgement & Support for judgement \\
\hline $\begin{array}{l}\text { Random sequence genera- } \\
\text { tion (selection bias) }\end{array}$ & Unclear risk & Not reported in paper \\
\hline $\begin{array}{l}\text { Allocation concealment } \\
\text { (selection bias) }\end{array}$ & Unclear risk & Not reported in paper \\
\hline $\begin{array}{l}\text { Blinding (performance } \\
\text { bias and detection bias) } \\
\text { All outcomes }\end{array}$ & High risk & Imbalanced exposure \\
\hline
\end{tabular}


Lee 2013b (Continued)

$\begin{array}{ll}\text { Blinding of outcome as- } & \text { Unclear risk }\end{array}$

All outcomes

\begin{tabular}{|c|c|c|}
\hline $\begin{array}{l}\text { Incomplete outcome data } \\
\text { (attrition bias) } \\
\text { End of intervention }\end{array}$ & Low risk & No losses \\
\hline $\begin{array}{l}\text { Selective reporting (re- } \\
\text { porting bias) }\end{array}$ & Unclear risk & Protocol not available \\
\hline Imbalanced exposure & High risk & Imbalanced exposure between control group and both intervention groups \\
\hline
\end{tabular}

Lennon 2008

\begin{tabular}{|c|c|}
\hline \multirow[t]{7}{*}{ Methods } & $\begin{array}{l}\text { Design: pilot randomised study of cardiorespiratory training vs UC - after UC. Sample size calculation } \\
\text { reported }\end{array}$ \\
\hline & $\begin{array}{l}\text { Randomisation: stratified randomisation (by age and sex) into } 4 \text { blocks of } 6 \text { using a sequence generator } \\
\text { by an independent party }\end{array}$ \\
\hline & Allocation concealment: opaque envelopes \\
\hline & Blinding: single-blinded; unclear who was blinded \\
\hline & ITT: no but only 1 participant dropped out in the control group \\
\hline & Measurements: end of intervention (10 weeks) \\
\hline & Withdrawals: 1 participant (refusal) in the control group \\
\hline
\end{tabular}

Participants

Randomised: total 48 participants. Participants were recruited from the Stroke Rehabilitation Database (Dublin). Volunteers contacted the research team for initial screening

Intervention: 24 participants; 14 men (58\%) and 10 women (42\%); mean age 59.0 years (SD 10.3); mean number of weeks from stroke 237.3 (SD 110.7)

Control: 24 participants; 14 men (58\%) and 10 women (42\%); mean age 60.5 years (SD 10.0); mean number of weeks from stroke 245.3 (SD 169.8)

Inclusion criteria: > 1 year post-ischaemic stroke and > 18 years of age; participants were recruited irrespective of their ability to ambulate independently

Exclusion criteria: $\mathrm{O}_{2}$ dependence, angina, unstable cardiac conditions, uncontrolled diabetes mellitus, major medical conditions, claudication, cognitive impairment, or beta blocker medication

Interventions Intervention: the Cardiac Rehabilitation Programme consisted of cycle ergometry training using either
the upper or lower limbs. Exercise load was set at 50\%-60\% of the participants' maximal HR. Resistance
and speed were adjusted daily to ensure progression. Frequency: participants trained twice weekly for
30 min each time, for 10 weeks. Measurements performed at week 1 and re-assessment at week 10 . All
sessions were supervised by a physiotherapist

Control: CPT and OT; no therapy contained an aerobic exercise component; measurements at week 1 and re-assessment at week 10 . No further details provided

Setting: outpatient rehabilitation 
Lennon 2008 (Continued)

Outcomes

Included outcomes: $\mathrm{VO}_{2}$; $\mathrm{BMI}$; maximum cycle workload; resting SBP; resting DBP; total cholesterol; FAI; HADS

Other outcomes: resting HR; cardiac risk score; RPE

Notes

The study authors maintained that their pilot study was too small for detecting functional benefits (a minimum of 120 participants in each group would have been required to show expected change in all primary outcomes); possible Hawthorn effect due to the fact that the control group did not receive the comparable non-exercise related attention to the intervention group

\section{Risk of bias}

\begin{tabular}{|c|c|c|}
\hline Bias & Authors' judgement & Support for judgement \\
\hline $\begin{array}{l}\text { Random sequence genera- } \\
\text { tion (selection bias) }\end{array}$ & Low risk & $\begin{array}{l}\text { Stratified randomisation (by age and sex) into } 4 \text { blocks of } 6 \text { using a sequence } \\
\text { generator by an independent party }\end{array}$ \\
\hline $\begin{array}{l}\text { Allocation concealment } \\
\text { (selection bias) }\end{array}$ & Unclear risk & Opaque envelopes; sealed and numbered unknown \\
\hline $\begin{array}{l}\text { Blinding (performance } \\
\text { bias and detection bias) } \\
\text { All outcomes }\end{array}$ & High risk & $\begin{array}{l}\text { Control group did not receive the comparable non-exercise related attention } \\
\text { to the intervention group }\end{array}$ \\
\hline $\begin{array}{l}\text { Blinding of outcome as- } \\
\text { sessment (detection bias) } \\
\text { All outcomes }\end{array}$ & Unclear risk & Unclear who was blinded \\
\hline $\begin{array}{l}\text { Incomplete outcome data } \\
\text { (attrition bias) } \\
\text { End of intervention }\end{array}$ & Low risk & $\begin{array}{l}\text { No ITT analysis } \\
1 / 48(2 \%) \text { participant dropped out } 1 \text { (refusal) in the control group }\end{array}$ \\
\hline $\begin{array}{l}\text { Selective reporting (re- } \\
\text { porting bias) }\end{array}$ & Unclear risk & Protocol not available \\
\hline Other bias & Low risk & None known \\
\hline Imbalanced exposure & High risk & Imbalanced exposure \\
\hline
\end{tabular}

Letombe 2010

Design: randomised trial of mixed training plus UC vs UC
Randomisation: information not included
Allocation concealment: information not included
Blinding: information not included
ITT: not completed
Measurements: before and end of intervention (4 weeks)
Withdrawals: none reported

Participants

Randomised: total 18 participants: 9 participants were randomised to intervention, 9 to control Intervention: 9 participants; 5 men, 4 women; mean age: (combined men and women) 59.1 years (SD 9.4); mean height: unknown; mean weight: unknown; type of stroke: ischaemic 5 , haemorrhagic 4; paretic side: right 4, left 5; time since stroke onset: 20 days Control: 9 participants; 6 men, 3 women: mean age: (combined men and women) 60.6 years (SD 8.2); mean height: unknown; mean weight: unknown; type of stroke: ischaemic 5 , haemorrhagic 4; paretic side: right 4, left 5; time since stroke onset: 20 days 
Inclusion criteria: right or left hemiplegia following ischaemic or haemorrhagic hemispheric stroke; a full set of aetiological data (CT and/or MRI scans, Holter ECG, Doppler, cardiac ultrasound); a stable clinical state; well-balanced treatment (particularly in terms of antihypertensives and anticoagulants)

Exclusion criteria: existence of disorders associated with hemiplegic motor damage, such as cognitive and memory disorders; hemisensory neglect; the existence of an intercurrent affection or unstable brain lesions

Interventions

Intervention: participants in the training group $(n=9)$ received conservative physical therapy for $3 \mathrm{~h} /$ day, 5 days/week, for a period of 4 weeks. Conservative physical therapy consisted of gait exercises, stance exercises, the treatment of orthopaedic disorders, balance work (with a view to subsequently withdrawing gait aids), use of support stockings and braces and maintenance of the freedom of movement of the proximal-distal limb joints. In addition general exercise training was implemented, with cardiorespiratory exercise (monitored by a HR monitor), muscle strengthening, gait exercises, and work focused on executive functions, lasting for between 40 and $60 \mathrm{~min} /$ day, 4 times a week. Aerobic exercise was included in the form of steady exercise on a semi-recumbent cycle ergometer (with both feet pedaling) was performed at between $70 \%$ and $80 \%$ of maximum power

Control: participants in the control group $(n=9)$ received conservative physical therapy for $3 \mathrm{~h} /$ day, 5 days/week, for a period of 4 weeks. Conservative physical therapy consisted of gait exercises, stance exercises, the treatment of orthopaedic disorders, balance work (with a view to subsequently withdrawing gait aids), use of support stockings and braces and maintenance of the freedom of movement of the proximal-distal limb joints

Setting: hospital setting

\begin{tabular}{ll}
\hline Outcomes & Included outcomes: triangular maximal aerobic power test using a cycle ergometer \\
\hline Notes & - \\
\hline
\end{tabular}

\section{Risk of bias}

\begin{tabular}{|c|c|c|}
\hline Bias & Authors' judgement & Support for judgement \\
\hline $\begin{array}{l}\text { Random sequence genera- } \\
\text { tion (selection bias) }\end{array}$ & Unclear risk & Described only as "randomized into two groups" \\
\hline $\begin{array}{l}\text { Allocation concealment } \\
\text { (selection bias) }\end{array}$ & Unclear risk & Not reported \\
\hline $\begin{array}{l}\text { Blinding (performance } \\
\text { bias and detection bias) } \\
\text { All outcomes }\end{array}$ & High risk & Not reported; not attention control \\
\hline $\begin{array}{l}\text { Blinding of outcome as- } \\
\text { sessment (detection bias) } \\
\text { All outcomes }\end{array}$ & Unclear risk & Not reported \\
\hline $\begin{array}{l}\text { Incomplete outcome data } \\
\text { (attrition bias) } \\
\text { End of intervention }\end{array}$ & Low risk & $\begin{array}{l}\text { ITT not reported } \\
\text { No participant losses }\end{array}$ \\
\hline $\begin{array}{l}\text { Selective reporting (re- } \\
\text { porting bias) }\end{array}$ & Unclear risk & Trial register or protocol not available \\
\hline Other bias & Low risk & None known \\
\hline Imbalanced exposure & High risk & Imbalanced exposure \\
\hline
\end{tabular}


Randomisation: computer-generated blocked randomisation.

Allocation concealment: yes

Blinding: yes

ITT: yes

Measurements: end of intervention (12 weeks), 6 months, 12 months

Withdrawals: 5 withdrawals by end of intervention - 3 from intervention and 2 from control; 3 more withdrawals by 6 -month follow-up - 1 from intervention, 2 from control; 5 more withdrawals by 12 month follow-up - 3 from intervention, 2 from control

\section{Participants}

Randomised: total 50 participants: 45 of these completed the exercise programme

24 randomised to intervention ( 22 completed intervention)

26 randomised to control ( 23 completed)

Intervention: 24 participants; 15 men, 9 women; mean age: 61.5 (SD 15.4); days after stroke 23.3 (SD

5.7)

Control: 26 participants; 14 men, 12 women; mean age 59.0 (SD 12.7); days after stroke 23.1 (SD 4.4) Inclusion criteria: > 18 years, within 1 month of first ischaemic stroke confirmed by neuroimaging, inpatients in stroke rehabilitation centre, able to walk $5 \mathrm{~m}$ with or without ambulatory aids, ankle orthoses, or stand-by assistance

Exclusion criteria: contraindications to maximal exercise stress testing; musculoskeletal or cognitive limitations that could preclude participation in the programme, involvement in other pharmacological or physical intervention studies

5-10 min active/passive stretching; 10-15 min upper extremity training (active exercises and stretching); 10-15 min lower extremity training (active exercises and stretching)

25-30 min treadmill gait training (initially treadmill speed 80\%-90\% of self-paced overground speed with $20 \%-30 \%$ body weight supported for ambulatory-independent participants and $70 \%-80 \%$ of overground speed with $40 \%$ body weight supported for ambulatory-dependent participants)

Control: 60 min, 5 days/week for 6 weeks then 3 days/week for 6 weeks

5-10 min active/passive stretching; 10-15 min upper extremity training (active exercises and stretching); 10-15 min lower extremity training (active exercises and stretching)

5-10 min of pre-gait activities in standing followed by 20-25 min overground walking at comfortable self-selected speeds

Setting: stroke rehabilitation unit

Included outcomes: cardiovascular fitness $\left(\mathrm{VO}_{2}\right.$ peak); 6-MWT; comfortable walking speed; BBS
Other outcomes: Chedoke-McMaster stages of Recovery Leg and Foot; participant satisfaction with pro-
gramme

\section{Risk of bias}


MacKay-Lyons 2013 (Continued)

$\begin{array}{ll}\begin{array}{l}\text { Random sequence genera- } \\ \text { tion (selection bias) }\end{array} & \text { Low risk } \\ & \text { status }\end{array}$

\begin{tabular}{|c|c|c|}
\hline $\begin{array}{l}\text { Allocation concealment } \\
\text { (selection bias) }\end{array}$ & Low risk & $\begin{array}{l}\text { Quote: "A person not involved in the study prepared and safeguarded individ- } \\
\text { ual, opaque sealed envelopes containing group and physiotherapist alloca- } \\
\text { tion, which were opened after completion of the baseline assessment" }\end{array}$ \\
\hline $\begin{array}{l}\text { Blinding (performance } \\
\text { bias and detection bias) } \\
\text { All outcomes }\end{array}$ & Unclear risk & $\begin{array}{l}\text { There was a similar dose of exposure across both groups. Participants were } \\
\text { informed they would be allocated to } 1 \text { of } 2 \text { 'intervention' groups. The groups } \\
\text { were kept separate }\end{array}$ \\
\hline
\end{tabular}

\begin{tabular}{|c|c|c|}
\hline $\begin{array}{l}\text { Blinding of outcome as- } \\
\text { sessment (detection bias) } \\
\text { All outcomes }\end{array}$ & Low risk & $\begin{array}{l}\text { Blinded outcome assessment. Participants instructed not to discuss their in- } \\
\text { tervention with outcome assessor. A test of blinding was also performed and } \\
\text { analysed statistically to demonstrate no significant unblinding }\end{array}$ \\
\hline
\end{tabular}

\begin{tabular}{ll}
\hline $\begin{array}{l}\text { Incomplete outcome data } \\
\text { (attrition bias) }\end{array}$ & Low risk \\
$\begin{array}{l}\text { End of intervention } \\
\text { ITT analysis }\end{array}$ & 2 lost from BSWTT (1 moved, 1 for medical reasons) \\
& 3 lost from UC group (2 for medical reasons, 1 dropped out as disinterested) \\
& $\begin{array}{l}\text { Quote: "All analyses were conducted on an intention-to treat basis, carrying } \\
\text { the last observation forward for those lost to follow-up" }\end{array}$
\end{tabular}

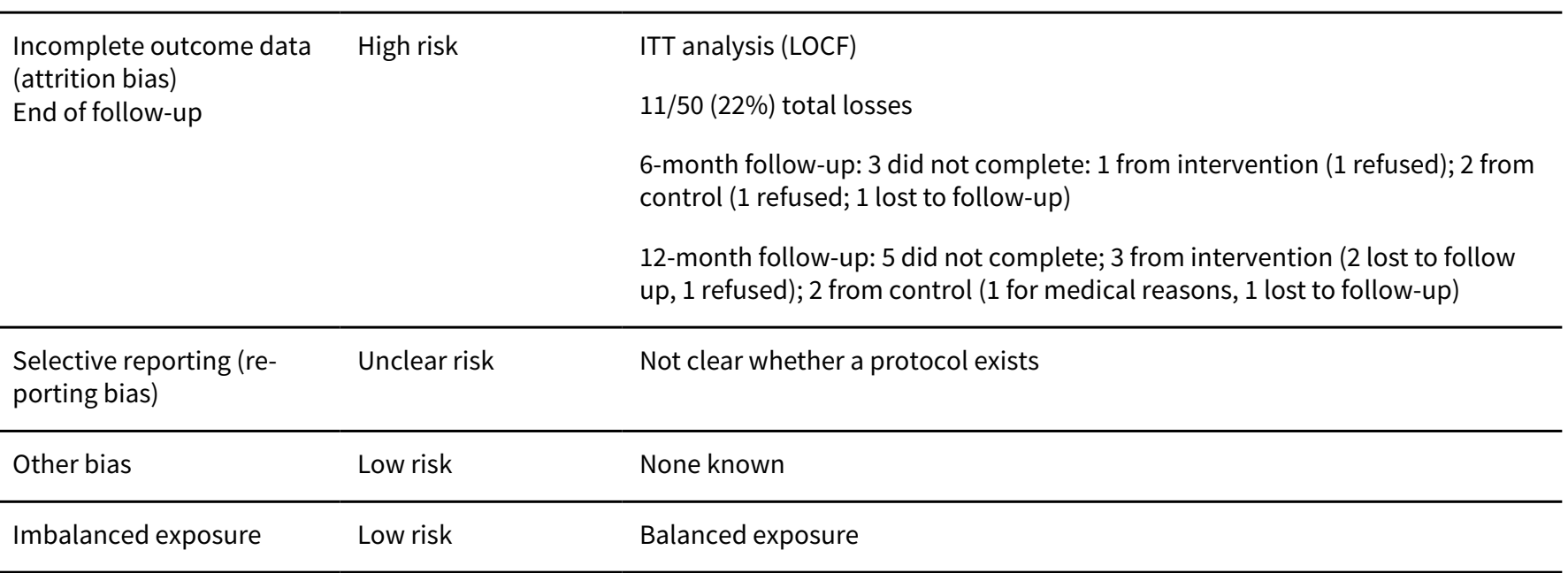

\section{Mao 2015}

\section{Methods}

Design: single hospital centre RCT. Cardiorespiratory training (BWSTT) plus UC vs conventional overground walking training plus UC (BWSTT replaces part of UC) - during UC

Randomisation: no details provided

Allocation concealment: no details provided

Blinding: baseline and end of intervention outcome measures completed by examiner blinded to group allocation

ITT: dropouts reported, no attempt to measure participants at outcome. No ITT reported

Measurements: baseline, at the end of intervention ( 3 weeks) 
Mao 2015 (Continued)

Withdrawals: after baseline but prior to outcome 3/15 (20\%) participants were lost in the intervention group (1 cardiovascular instability, 2 early discharged). After baseline but prior to outcome 2/14 (14\%) participants were lost in the control group (2 early discharged)

Participants

Randomised: total 29 participants. 15 participants were randomised to intervention, data from 12 were presented and analysed. 14 participants were randomised to control group, data from 12 were presented and analysed

Intervention: BWSTT plus UC = 12 participants; 2 men (17\%) and 10 women (83\%); mean age: 59.55 (SD 9.23) years; mean body mass 65.17 (SD 10.26) kg; 49.25 (SD 19.51) days after stroke

Control: conventional overground walking training plus UC $=12$ participants; 3 men (25\%) and 9 women (75\%); mean age: 60.82 (SD 10.7) years; mean body mass 65.25 (SD 11.42) kg; 47.67 (SD 16.78) days after stroke

Inclusion criteria: stroke confirmed by CT or MRI; unilateral hemiparesis for no more than 3 months resulting from first stroke; residual gait impairment, defined by an abnormal $10 \mathrm{~m}$ walk time according to age (age $<60=\geq 10 \mathrm{~s}$ or $1 \mathrm{~m} / \mathrm{s}$; age $60-69: \geq 12.5 \mathrm{~s}$ or $0.8 \mathrm{~m} / \mathrm{s}$; age $\geq 70: \geq 16.6 \mathrm{~s},<0.6 \mathrm{~m} / \mathrm{s}$ ); and adequate mental and physical capacity to attempt the tasks as instructed (MMSE score $\geq 27$, average modified Ashworth Scale score at hip, knee, and ankle $\leq 2$ )

Exclusion criteria: presence of significant medical complications or unstable vital signs that precluded participation in the study

Interventions

Intervention: BWSTT plus UC. Standardised BWSTT consisted of 30\%-40\% assisted body support treadmill walking. Progressive programme in which assisted body support was decreased and treadmill speed increased separately. No further detail for percentage assisted body support was provided. Frequency of intervention: initial at 0.5 miles per hour $(0.8 \mathrm{~km} / \mathrm{h})$ treadmill speed for $20 \mathrm{~min}, 5$ days/week for 3 weeks progressing to 2.5 miles per hour $(4 \mathrm{~km} / \mathrm{h}$ ) for $40 \mathrm{~min}, 5$ days/week for 3 weeks

Control: conventional overground walking training plus UC. Individualised overground gait training based on the principles of neurodevelopmental therapy (Bobath method). Frequency of control: 30 min, 5 days/week for 3 weeks

UC for both intervention and control groups consisted of 20-40 min, 5 days/week for 3 weeks of therapeutic exercises (range of movement; strengthening exercises; as well as facilitation techniques to recruit muscle activity on the paretic extremity. UC also consisted of $60 \mathrm{~min}, 5$ days/week for 3 weeks of OT session (functional stimulation and self-exercise programme). No further specific details were provided

Setting: hospital

Outcomes

Included outcomes: Brunel Balance Assessment; Fugl-Meyer Lower Extremity Assessment; self-selected walking speed

Other outcomes: 3-D motion analysis at self-selected walking speed (cadence, stride time and length, step time and length; kinematic and kinetic data included joint angles and moments)

Notes

\section{Risk of bias}

Bias Authors' judgement Support for judgement

Random sequence genera- Unclear risk Randomly assigned to the groups, no further details provided tion (selection bias)

Allocation concealment Unclear risk Not reported

(selection bias) 
Mao 2015 (Continued)

Blinding (performance Unclear risk Active attention control compensates for lack of blinding bias and detection bias)

All outcomes

\begin{tabular}{lll}
$\begin{array}{l}\text { Blinding of outcome as- } \\
\text { sessment (detection bias) } \\
\text { All outcomes }\end{array}$ & Low risk & Assessor blinded to group allocation \\
\hline $\begin{array}{l}\text { Incomplete outcome data } \\
\text { (attrition bias) }\end{array}$ & High risk & $\begin{array}{l}\text { Overall dropout rate 20\% in intervention and 14\% in control groups. No ITT } \\
\text { and of intervention }\end{array}$
\end{tabular}

End of intervention

\section{Incomplete outcome data Low risk}

(attrition bias)

End of follow-up

\begin{tabular}{lll}
\hline $\begin{array}{l}\text { Selective reporting (re- } \\
\text { porting bias) }\end{array}$ & Unclear risk & No trial registration details accessible \\
\hline Other bias & Low risk & None known \\
\hline Imbalanced exposure & Low risk & Attention control \\
\hline
\end{tabular}

\section{Mead 2007}

\section{Methods}

Design: explanatory randomised trial of mixed training vs non-exercise intervention - after UC

Randomisation mechanism: internet application; minimisation dichotomised on sex; FIM score (120);

age (70 years)

Allocation concealment: sequence generation and allocation occurred simultaneously

Blinding: investigator; participants encouraged to maintain blinding

ITT: yes

Measurements: end of intervention (12-14 weeks) and 4-month follow-up

Withdrawals: intervention 0; control 4: 1 withdrew before intervention; 3 after end of intervention follow-up (1 stroke-related illness, 1 fall, 1 recurrent stroke)

Randomised: 66 participants
Intervention: 32 participants; 18 men and 14 women; age 72.0 years (SD 10.4); median 171 (IQR 55 to
287) days post-stroke
Control: 34 participants; 18 men and 16 women; age 71.7 years (SD 9.6); median 147.5 (IQR 78.8 to
235.5) days post-stroke
Inclusion criteria: independently ambulatory; living within central or south Edinburgh
Exclusion criteria: dysphasia or confusion severe enough to prevent informed consent or impair safety
in exercise classes; medical contraindications to exercise training

Interventions

Intervention: mixed training: group circuit training performed 40-75 min/day 3 days/week for 12-14 weeks (36 sessions); after a warm-up the training comprised 2 components: (1) a cardiorespiratory circuit (cycle ergometry, raising and lowering an exercise ball, shuttle walking, standing chest press, and stair climbing and descending); (2) resistance training circuit (upper back exercise and triceps extension using TheraBand, lifting a weighted pole, a sit-to-stand exercise); progression in duration, repetition number, speed, mass of objects and resistance of TheraBand whilst maintaining a RPE (6-20 scale) of $13-60$

Control: non-exercise intervention; seated relaxation involving deep breathing and progressive muscular relaxation; no muscle contractions were involved Setting: rehabilitation hospital 
Mead 2007 (Continued)

Outcomes Included outcomes: FIM; NEADL; RMI; functional reach; TUG; sit-to-stand time; SF-36 - version 2; HADS; gait preferred speed; gait economy $\left(\mathrm{VO}_{2} \mathrm{~mL} / \mathrm{kg} / \mathrm{m}\right)$; lower limb extensor explosive power $(\mathrm{W} / \mathrm{kg})$

Other outcomes: EMS (ceiling effect); FAC (ceiling effect)

\begin{tabular}{|c|c|c|}
\hline Notes & - & \\
\hline \multicolumn{3}{|l|}{ Risk of bias } \\
\hline Bias & Authors' judgement & Support for judgement \\
\hline $\begin{array}{l}\text { Random sequence genera- } \\
\text { tion (selection bias) }\end{array}$ & Low risk & $\begin{array}{l}\text { Internet software-based minimisation dichotomised on sex; FIM score (120); } \\
\text { age (70 years) }\end{array}$ \\
\hline $\begin{array}{l}\text { Allocation concealment } \\
\text { (selection bias) }\end{array}$ & Low risk & Not applicable; sequence generation and allocation occurred simultaneously \\
\hline $\begin{array}{l}\text { Blinding (performance } \\
\text { bias and detection bias) } \\
\text { All outcomes }\end{array}$ & Unclear risk & $\begin{array}{l}\text { Suitable attention control } \\
\text { Quote: "Patients were blinded to the underlying hypothesis by reiterating the } \\
\text { possible benefits of both interventions" }\end{array}$ \\
\hline $\begin{array}{l}\text { Blinding of outcome as- } \\
\text { sessment (detection bias) } \\
\text { All outcomes }\end{array}$ & Low risk & $\begin{array}{l}\text { Outcome assessor blinded } \\
\text { Quote: "Outcome assessors were blinded by asking patients not to discuss } \\
\text { their allocated intervention" }\end{array}$ \\
\hline $\begin{array}{l}\text { Incomplete outcome data } \\
\text { (attrition bias) } \\
\text { End of intervention }\end{array}$ & Low risk & $\begin{array}{l}\text { ITT analysis } \\
1 / 66(2 \%) \text { lost at the end of intervention; intervention } 0 \text {; control } 1\end{array}$ \\
\hline $\begin{array}{l}\text { Incomplete outcome data } \\
\text { (attrition bias) } \\
\text { End of follow-up }\end{array}$ & Low risk & $\begin{array}{l}\text { ITT analysis } \\
4 / 66(6 \%) \text { total losses at the end of follow-up; intervention 0; control group ( } 1 \\
\text { stroke-related illness, } 1 \text { fall, } 1 \text { recurrent stroke) }\end{array}$ \\
\hline $\begin{array}{l}\text { Selective reporting (re- } \\
\text { porting bias) }\end{array}$ & Low risk & $\begin{array}{l}\text { Reported outcome correspond to proposal; Chief Scientist Office of the Scot- } \\
\text { tish Executive (CZB/4/46) }\end{array}$ \\
\hline Other bias & Low risk & None known \\
\hline Imbalanced exposure & Low risk & Balanced exposure \\
\hline
\end{tabular}

\section{Moore 2010}

Methods

Design: randomised, cross-over trial of cardiorespiratory training vs no intervention - after UC - (i.e. intensive locomotor training - including treadmill training - vs delayed cardiovascular training)

Randomisation: stratified randomisation according to severity of gait impairment

Allocation concealment: sealed envelopes

Blinding: investigators were not blinded

ITT: not reported

Measurements: end of intervention (4 weeks) 
Moore 2010 (Continued)

Withdrawals: none reported

Participants Randomised: 20 participants; mean age 50 years (SD 15); men 14, women 6; duration of post-stroke symptoms 13 months (SD 8); moderate/severe gait limitations 13/7

Intervention: the number of participants randomised to the immediate locomotor training group was not clearly reported

Control: the number of participants randomised to the delayed locomotor training group was not clearly reported

Inclusion criteria: patients with hemiparesis of $>6$ months' duration who were attending physical therapy after unilateral supratentorial stroke; all patients were required to walk $>10 \mathrm{~m}$ overground without physical assistance and medical clearance

Exclusion criteria: lower extremity contractures; significant osteoporosis; cardiovascular instability; previous history of peripheral or central nervous system injury, cognitive or communication impairment; inability to adhere to study requirements

Interventions

Intervention: the immediate locomotor training group received 4 weeks of intensive locomotor training after discharge from clinical physical therapy, which consisted of high-intensity stepping practice on a motorised treadmill while wearing an overhead harness attached to a safety system. Frequency: 2-5 days/week for 4 weeks. Intensity: highest tolerable speed with velocity increased in $0.5 \mathrm{~km} / \mathrm{h}$ increments until participants reached $80 \%-85 \%$ of predicted maximum HR or until the participants' RPE increased to 17 on the Borg scale. Partial weighted support was reduced in $10 \%$ increments as tolerated by participants who needed partial weighted support. Measurements were performed: 4 weeks before termination of usual physical therapy; soon after termination of usual physical therapy; after completion of the 4-week locomotor training; and again after a delay of 4 weeks after termination of locomotor training

Control: delayed locomotor training group. The delayed group was also assessed 4 weeks before and after termination of usual physical therapy, but did not receive locomotor training or any other interventions for 4 weeks after termination of usual physical therapy. After this 4-week delay the participants received locomotor training as described above

Setting: rehabilitation centre

Outcomes Included outcomes: preferred gait speed; fastest gait speed; $12-\mathrm{MWT} ; \mathrm{O}_{2}$ cost; peak treadmill speed;
$\mathrm{VO}_{2}$ peak, TUG; BBS

Notes

We used only data at the end of the first cross-over period for analyses

\section{Risk of bias}

\begin{tabular}{lll}
\hline Bias & Authors' judgement & Support for judgement \\
\hline $\begin{array}{l}\text { Random sequence genera- } \\
\text { tion (selection bias) }\end{array}$ & Low risk & Stratified randomisation according to severity of gait impairment \\
\hline $\begin{array}{l}\text { Allocation concealment } \\
\text { (selection bias) }\end{array}$ & Unclear risk & Sealed envelopes; opaque, numbered is unknown \\
\hline $\begin{array}{l}\text { Blinding (performance } \\
\text { bias and detection bias) } \\
\text { All outcomes }\end{array}$ & High risk & No attention control \\
\hline $\begin{array}{l}\text { Blinding of outcome as- } \\
\text { sessment (detection bias) } \\
\begin{array}{l}\text { All outcomes } \\
\hline\end{array}\end{array}$ & High risk & Investigators were not blinded \\
\hline
\end{tabular}


Moore 2010 (Continued)

\begin{tabular}{|c|c|c|}
\hline $\begin{array}{l}\text { Incomplete outcome data } \\
\text { (attrition bias) } \\
\text { End of intervention }\end{array}$ & Low risk & $\begin{array}{l}\text { ITT not reported } \\
\text { No dropouts }\end{array}$ \\
\hline
\end{tabular}

End of intervention

\begin{tabular}{lll}
\hline $\begin{array}{l}\text { Selective reporting (re- } \\
\text { porting bias) }\end{array}$ & Unclear risk & Protocol not available \\
\hline Other bias & Low risk & None known \\
\hline Imbalanced exposure & High risk & Imbalanced exposure \\
\hline
\end{tabular}

\section{Moore 2015}

\section{Methods}

Design: single-blind RCT. Mixed training vs attention control - after UC

Randomisation: randomised using computer software; method unclear

Allocation concealment: independent administrator used

Blinding: outcome assessors blinded to group allocation and hypotheses

ITT: no pre-planned ITT described

Measurements: at the end of intervention (19 weeks)

Withdrawals: 0 withdrawals

\section{Participants}

Randomised: 40 participants were randomised

Intervention: 20 participants; 18 men (90\%) and 2 women (10\%); mean age: 68 (SD 8); 21 (SD 34) months after stroke

Control: 20 participants; 16 men (80\%) and 4 women (20\%); mean age: 70 (SD 11); 16 (SD 12) months after stroke

Inclusion criteria: age $>50$ years, $>6$ months after stroke onset, could complete 6-MWT, living at home, completed CPT and were not undertaking moderate exercise $\geq 3$ days/week

Exclusion criteria: absolute/relative contraindications to exercise, type 1 diabetes, non-stroke neurological disorders, pain on walking, inability to follow commands, untreated depression, contraindications to MRI

\section{Interventions}

Intervention: mixed training. Group classes including warm-up, stretching, functional strengthening (increasing load and repetitions; 2 sets of 5 to 3 sets of 10), balance, agility and cardiorespiratory training (40\%-50\% maximum HR increasing to $70 \%-80 \%)$. Frequency of intervention: $40-60 \mathrm{~min} /$ day for 3 days/week for 19 weeks

Control: home-based stretching. 10 seated stretches for the upper and lower body. Frequency of intervention: $40-60 \mathrm{~min} /$ day for 3 days/week for 19 weeks

Setting: community leisure centre (intervention) and home (control)

Outcomes

Included outcomes: risk factors (BP, glucose control, lipid profile, body composition), cognition, mobility (10-MWT, 6-MWT), balance, QoL, physical fitness (peak oxygen consumption and peak work rate)

Other outcomes: brain atrophy and metabolism

Notes $\quad$ ISRCTN41026907


Moore 2015 (Continued)

Risk of bias

\begin{tabular}{|c|c|c|}
\hline Bias & Authors' judgement & Support for judgement \\
\hline $\begin{array}{l}\text { Random sequence genera- } \\
\text { tion (selection bias) }\end{array}$ & Low risk & Online tool used by independent administrator; method unknown \\
\hline $\begin{array}{l}\text { Allocation concealment } \\
\text { (selection bias) }\end{array}$ & Low risk & $\begin{array}{l}\text { Randomisation and allocation performed independently and remotely after } \\
\text { consent }\end{array}$ \\
\hline $\begin{array}{l}\text { Blinding (performance } \\
\text { bias and detection bias) } \\
\text { All outcomes }\end{array}$ & Unclear risk & Attention control used \\
\hline $\begin{array}{l}\text { Blinding of outcome as- } \\
\text { sessment (detection bias) } \\
\text { All outcomes }\end{array}$ & Low risk & $\begin{array}{l}\text { Outcome assessors were blinded to the study hypotheses and group assign- } \\
\text { ment }\end{array}$ \\
\hline $\begin{array}{l}\text { Incomplete outcome data } \\
\text { (attrition bias) } \\
\text { End of intervention }\end{array}$ & Low risk & No losses \\
\hline $\begin{array}{l}\text { Selective reporting (re- } \\
\text { porting bias) }\end{array}$ & Unclear risk & $\begin{array}{l}\text { Trial registry mentions a physical activity and a stroke impairment outcome } \\
\text { which are not present in the publications }\end{array}$ \\
\hline Other bias & High risk & $\begin{array}{l}\text { Intervention is leisure-centre based, the control is home-based - there would } \\
\text { be } 3 \times 2 \times 19=114 \text { journeys to and from the intervention which is potentially a } \\
\text { lot of un-quantified physical activity }\end{array}$ \\
\hline Imbalanced exposure & Low risk & $\begin{array}{l}\text { Stretching was provided to the control - study authors list that stretching does } \\
\text { not lower metabolic factors while other such as yoga does }\end{array}$ \\
\hline
\end{tabular}

Mudge 2009

Methods

Design: randomised trial of cardiorespiratory training vs non-exercise intervention training - after UC

(circuit-based rehabilitation vs social and educational sessions); power calculation reported

Randomisation: computer-generated random numbers by an individual not associated with the study

Allocation concealment: not reported

Blinding: assessor blinded (unmasking of the independent assessor occurred in 3 cases who inadver-

tently stated or implied their group allocation)

ITT: yes

Measurements: end of intervention (4 weeks) and 3-month follow-up

Withdrawals: 1 participant in the intervention group (disinterest) and 2 participants in the control group (too busy) withdrew at the end of intervention. 3 further participants withdrew from the intervention group (health problems $=2$; another stroke $=1$ ) and 2 from the control group (health problems $=1$; another stroke $=1$ ) before the end of follow-up

Participants

Randomised: 58 participants; median age 71.5 years (range 39.0-89.0 years); median 3.9 years after stroke (range 0.5-18.7 years); participants were recruited through the Stroke Foundation of New Zealand, stroke clubs, and the local hospital stroke service. Potential candidates were invited to contact the investigators if they wished to participate. All participants walked independently and 26 (45\%) used an assistive device. 55 participants completed the study 
Mudge 2009 (Continued)

Intervention: 31 participants were randomised to circuit training; 19 men and 12 women; median age 76.0 (range 39.0-89.0); median onset of stroke 3.33 years (range 0.6-13.3)

Control: 27 participants were randomised to social and educational sessions; 13 men and 14 women; median age 71.0 (range 44.0-86.0); median onset of stroke 5.8 years (range 0.5-18.7)

Inclusion criteria: participants with $\geq 1$ strokes $>6$ months earlier, had been discharged from rehabilitation and were able to walk independently (with an aid if necessary). Some residual gait difficulty was required, as defined by a score of $<2$ on at least 1 of the walking items of the physical functioning scale of the SF-36

Exclusion criteria: participants were excluded if they had progressive neurological diseases or significant health problems, $>2$ falls in the previous 6 months, unstable cardiac conditions, uncontrolled hypertension, or congestive heart failure

Interventions

Intervention: participants in the intervention group attended 12 group circuit sessions 3 times/week for 4 weeks. Groups were led by 1 of the principal investigators assisted by 2 physiotherapist students. There were 15 stations in the circuit that were graded to each participant's ability and progressed as tolerated. Each station contained either a task-oriented gait or standing balance activity (e.g. stepups, balance beam, marching in place) or strengthening of a lower extremity muscle with the purpose to improve gait (e.g. lunges, Swiss ball squats, side leg lifts). Total exercise time was 30 min including stretching. Measurements performed post-intervention and at 3-month follow-up

Control: participants in the control group attended 8 sessions - 4 social and 4 educational sessions (e.g. provide participants with relevant and useful information for everyday activities; provide intellectual stimulation and enjoyment sessions; play a game; cafe outing). Each session lasted $90 \mathrm{~min}$. The control group was led by an occupational therapist. Measurements performed post-intervention and at $3-$ month follow-up

Setting: rehabilitation clinic

Outcomes Included outcomes: mean number of steps a day measured by the StepWatch Activity Monitor; walking speed and walking endurance

Other outcomes: self-reported confidence during ADL and self-reported mobility assessed by the ABCS, the RMI, and the PADS

Notes

Randomisation was revealed to each participant by the principal investigator after the second baseline assessment. The study was limited by the small number of participants. Participants volunteered to participate and were likely to be highly motivated. The sample appeared in fact to be higher functioning in terms of gait speed. A gait endurance component was not included in the training circuit

\section{Risk of bias}

\begin{tabular}{lll}
\hline Bias & Authors' judgement & Support for judgement \\
\hline $\begin{array}{l}\text { Random sequence genera- } \\
\text { tion (selection bias) }\end{array}$ & Low risk & $\begin{array}{l}\text { Computer-generated random numbers by an individual not associated with } \\
\text { the study }\end{array}$ \\
\hline $\begin{array}{l}\text { Allocation concealment } \\
\text { (selection bias) }\end{array}$ & Unclear risk & Not reported \\
\hline $\begin{array}{l}\text { Blinding (performance } \\
\text { bias and detection bias) } \\
\text { All outcomes }\end{array}$ & Unclear risk & Attention control incorporated but not equivalent \\
\hline $\begin{array}{l}\text { Blinding of outcome as- } \\
\text { sessment (detection bias) } \\
\text { All outcomes }\end{array}$ & Unclear risk & $\begin{array}{l}\text { Assessor blinded; unmasking of the independent assessor occurred in 3 cases, } \\
\text { who inadvertently stated or implied their group allocation }\end{array}$ \\
\hline
\end{tabular}


Mudge 2009 (Continued)

Incomplete outcome data

(attrition bias)

End of intervention
Low risk ITT reported

3/58 (5\%) lost at the end of intervention: 1 participant in the intervention group (disinterest) and 2 participants in the control group (too busy) withdrew at the end of intervention

\section{Incomplete outcome data Low risk (attrition bias) \\ End of follow-up \\ ITT reported \\ 8/58 (14\%) lost overall at the end of follow-up: 3 further participants withdrew from the intervention group (health problems $=2$; another stroke $=1$ ) and 2 from the control group (health problems $=1$; another stroke $=1$ )}

\begin{tabular}{lll}
\hline $\begin{array}{l}\text { Selective reporting (re- } \\
\text { porting bias) }\end{array}$ & Unclear risk & Protocol not available \\
\hline Other bias & Low risk & None known \\
\hline Imbalanced exposure & Unclear risk & Attention control used but there is not an equivalent exposure \\
\hline
\end{tabular}

\section{Ouellette 2004}

$\begin{array}{ll}\text { Methods } & \text { Design: randomised trial of resistance training vs non-exercise intervention - after UC } \\ \text { Randomisation: unknown } \\ \text { Allocation concealment: unknown } \\ \text { Blinding: investigator } \\ \text { ITT: yes } \\ \text { Measurements: end of intervention (12 weeks) } \\ \text { Withdrawals: intervention: } 1 \text { withdrew (cardiac problem) and } 1 \text { was lost at follow-up (hernia); control: } \\ 2 \text { withdrew during intervention, } 1 \text { was lost at follow-up (abnormal ECG) } \\ \text { Randomised: } 42 \text { participants } \\ \text { Intervention: } 21 \text { participants; number of men and women unknown; age } 65.8 \text { years (SD 11.5); } 968 \text { days } \\ \text { post-stroke (SD } 460) \\ \text { Control: } 21 \text { participants; number of men and women unknown; age 66.1 years (SD 9.62); } 779 \text { days post- } \\ \text { stroke (SD 558) } \\ \text { Inclusion criteria: age } \geq 50 \text { years; } 6 \text { months-6 years after single unilateral mild/moderate stroke with } \\ \text { residual lower extremity hemiparesis; community-dwelling; independently ambulatory +/- walking } \\ \text { aids; report of } \geq 2 \text { limitations on the physical function subscale of the SF-36; ability to travel to the exer- } \\ \text { cise laboratory; willing to be randomised }\end{array}$

Interventions

Intervention: progressive resistance training of both lower limbs performed 3 days/week for 12 weeks comprising 3 sets of $8-10$ repetitions at $70 \%$ of $1-\mathrm{RM}$ ); exercises were (1) seated bilateral leg press, and (2) unilateral knee extension, both using pneumatic resistance, and unilateral ankle; dorsiflexion; plantarflexion, both using weights; progression achieved via weekly assessment of 1-RM; warm-up for each exercise was 4 repetitions of 25\% 1-RM

Control: non-exercise: bilateral range of motion and upper body flexibility exercises 3 days/week for 12 weeks

Setting: exercise laboratory

Outcomes

Included outcomes: muscle strength (bilateral lower limb extension force); muscle strength (unilateral knee extension, ankle dorsiflexion and ankle plantarflexion); gait endurance (6-MWT), preferred speed $(10 \mathrm{~m})$ and maximal speed $(10 \mathrm{~m})$; chair rise time (5 repetitions); stair climb time (10 steps); late life function and disability instrument scale; SF-36 physical function subscale Other outcomes: muscle power - bilateral lower limb extension and unilateral knee extension; geriatric depression scale (data not reported); sickness impact profile; Ewarts self efficacy scale 
Ouellette 2004 (Continued)

Notes Variance reported as standard error and converted to standard deviation

\section{Risk of bias}

\begin{tabular}{|c|c|c|}
\hline Bias & Authors' judgement & Support for judgement \\
\hline $\begin{array}{l}\text { Random sequence genera- } \\
\text { tion (selection bias) }\end{array}$ & Unclear risk & Not reported \\
\hline $\begin{array}{l}\text { Allocation concealment } \\
\text { (selection bias) }\end{array}$ & Unclear risk & Not reported \\
\hline $\begin{array}{l}\text { Blinding (performance } \\
\text { bias and detection bias) } \\
\text { All outcomes }\end{array}$ & Unclear risk & Attention control incorporated \\
\hline $\begin{array}{l}\text { Blinding of outcome as- } \\
\text { sessment (detection bias) } \\
\text { All outcomes }\end{array}$ & Low risk & Investigator blinded; efficacy unknown \\
\hline $\begin{array}{l}\text { Incomplete outcome data } \\
\text { (attrition bias) } \\
\text { End of intervention }\end{array}$ & Low risk & $\begin{array}{l}\text { ITT } \\
5 / 42(12 \%) \text { lost at the end of intervention: } \\
\text { Intervention: } 1 \text { withdrew (cardiac problem) and } 1 \text { was lost at follow-up (her- } \\
\text { nia); control: } 2 \text { withdrew during intervention, } 1 \text { was lost at follow-up (abnor- } \\
\text { mal ECG) }\end{array}$ \\
\hline $\begin{array}{l}\text { Selective reporting (re- } \\
\text { porting bias) }\end{array}$ & Unclear risk & Protocol not available \\
\hline Other bias & High risk & Newspaper advert recruitment \\
\hline Imbalanced exposure & Low risk & Balanced exposure \\
\hline
\end{tabular}

\section{Park 2011}

\section{Methods}

Design: randomised, single-blind trial of cardiorespiratory training plus UC vs UC - during UC

Randomisation mechanism: participants blindly pick 1 of 2 cards

Allocation concealment: envelopes used

Blinding: outcome assessor blind to group allocation

ITT: not reported

Measurements: end of intervention (4 weeks)

Withdrawals: 2 participants (1 from both intervention and control groups) not regularly participating

\section{Participants}

\section{Randomised: 27 participants}

Intervention: 14 participants; 7 men and 6 women; mean age 59.4 years (SD 8.5)

Control: 13 participants; 5 men and 7 women; mean age 56.9 years (SD 7.8)

Inclusion criteria: 6 months to 5 years post-first stroke; walking speed $<0.7 \mathrm{~m} / \mathrm{s}$ 
Park 2011 (Continued)

Exclusion criteria: auditory or visual deficits; no orthopaedic or cardiovascular conditions; cognitive impairment (> 25 MMSE score)

\begin{tabular}{|c|c|c|}
\hline \multirow[t]{3}{*}{ Interventions } & \multicolumn{2}{|c|}{$\begin{array}{l}\text { Invention group: 4-phased walking training programme (progressing } 150 \mathrm{~m}-200 \mathrm{~m}-300 \mathrm{~m}-500 \mathrm{~m} \text { ) } 1 \mathrm{~h} 3 \\
\text { times/week for } 4 \text { weeks }\end{array}$} \\
\hline & \multicolumn{2}{|c|}{ Control group: CPT care $1 \mathrm{~h}$ daily based on Bobath concept } \\
\hline & \multicolumn{2}{|c|}{ Setting: community based } \\
\hline Outcomes & \multicolumn{2}{|c|}{ Included outcomes: 10-metre walk test; 6-MWT; Community Walk test } \\
\hline & \multicolumn{2}{|c|}{ Other outcomes: walking ability questionnaire; activities-specific balance confidence scale } \\
\hline Notes & - & \\
\hline \multicolumn{3}{|l|}{ Risk of bias } \\
\hline Bias & Authors' judgement & Support for judgement \\
\hline $\begin{array}{l}\text { Random sequence genera- } \\
\text { tion (selection bias) }\end{array}$ & Unclear risk & Participants drew 1 of 2 cards from an envelope \\
\hline $\begin{array}{l}\text { Allocation concealment } \\
\text { (selection bias) }\end{array}$ & Unclear risk & Envelopes used; nature of concealment unclear \\
\hline $\begin{array}{l}\text { Blinding (performance } \\
\text { bias and detection bias) } \\
\text { All outcomes }\end{array}$ & High risk & No attention control \\
\hline $\begin{array}{l}\text { Blinding of outcome as- } \\
\text { sessment (detection bias) } \\
\text { All outcomes }\end{array}$ & Low risk & Person assessing outcome and analysing data blinded \\
\hline $\begin{array}{l}\text { Incomplete outcome data } \\
\text { (attrition bias) } \\
\text { End of intervention }\end{array}$ & Low risk & $\begin{array}{l}\text { ITT analysis not reported } \\
2 / 27(7 \%) \text { lost at the end of intervention: } 1 \text { from intervention and } 1 \text { from con- } \\
\text { trol groups (not regularly participating) }\end{array}$ \\
\hline $\begin{array}{l}\text { Selective reporting (re- } \\
\text { porting bias) }\end{array}$ & Unclear risk & No protocol available \\
\hline Other bias & Low risk & None known \\
\hline Imbalanced exposure & High risk & Imbalanced exposure \\
\hline
\end{tabular}

Pohl 2002

Design: randomised trial of cardiorespiratory training plus \% UC vs UC - during UC
Randomisation mechanism: unknown; method: equal block based on gait speed
Allocation concealment: unknown
Blinding: investigator; efficacy unknown
ITT: no
Measurements: end of intervention (4 weeks)
Withdrawals: none


Pohl 2002 (Continued)

Participants
Randomised: 60 participants. 20 participants were randomised to the speed-dependent treadmill training group (STT); 20 participants to the limited progressive treadmill training group (LTT) and 20 participants to a conventional gait training group (CGT)

Intervention: STT group = 20 participants; 14 men, 6 women; age 57.1 years (SD 13.9); 16.8 (20.5) weeks post-stroke. LTT group = 20 participants; 16 men, 4 women; age 58.2 years (SD 10.5); 16.2 (16.4) weeks post-stroke

Control: 20 participants; 13 men, 7 women; age 61.6 years (SD 10.6); 16.10 (SD 18.5) weeks post-stroke Inclusion criteria: left or right hemiparesis for $>4$ weeks; impaired gait; no or slight abnormal muscle tone (Ashworth Score 0 and 1); walk without assistance (FAC =3); $10 \mathrm{~m}$ walk time $>5 \mathrm{~s}$ and < $60 \mathrm{~s}$; class B exercise risk (ACSM 1998); absence of known heart disease; no evidence of heart failure, ischaemia or angina at rest or exercise; appropriate rise in SBP and absence of ventricular tachycardia during exercise

Exclusion criteria: previous treadmill training; class C or D exercise risk (ACSM 1998); cognitive deficits (MMSE < 26 of 30); movement disorders; orthopaedic or gait-influencing diseases

\section{Interventions Intervention:}

Group 1: STT; $30 \mathrm{~min} /$ day 3 days/week for 4 weeks; minimal body weight support (10\%) for first 3 sessions; speed was increased progressively to the highest speed at which the participant could walk safely. The maximum-achieved speed was held for $10 \mathrm{~s}$ followed by a recovery period. Each time the participant successfully completed $10 \mathrm{~s}$ of walking at the set speed, the speed was increased during the next phase by $10 \%$. Treadmill was run at $0 \%$ incline

Group 2: LTT; $30 \mathrm{~min} /$ day 3 days/week for 4 weeks; minimal body weight support for first 3 sessions; speed was increased by no more than $5 \%$ of the maximum initial speed each week ( $20 \%$ over 4 weeks); treadmill was run at $0 \%$ incline

Both intervention groups also received CPT 45 min/day 2 days/week for 4 weeks (included some gait training); total $12 \mathrm{~h}$ of treatment

Control: conventional gait training that comprised post neuromuscular facilitation and Bobath techniques; $30 \mathrm{~min} /$ day 3 days/week for 4 weeks. The control group also received CPT $45 \mathrm{~min} /$ day 2 days/ week for 4 weeks (included some gait training); total $15 \mathrm{~h}$ of treatment

Setting: rehabilitation centre

$\begin{array}{ll}\text { Outcomes } & \text { Included outcomes: gait maximum speed; FAC } \\ \text { Other outcomes: stride cadence (steps } / \mathrm{min}) ; \text { stride length }(\mathrm{m})\end{array}$

Notes

The control group (20 participants) was divided between the 2 relevant comparisons to avoid exaggeration of overall participant numbers in the analyses

\section{Risk of bias}

\begin{tabular}{lll}
\hline Bias & Authors' judgement & Support for judgement \\
\hline $\begin{array}{l}\text { Random sequence genera- } \\
\text { tion (selection bias) }\end{array}$ & Unclear risk & Mechanism unknown; randomised to equal blocks based on gait speed \\
\hline $\begin{array}{l}\text { Allocation concealment } \\
\text { (selection bias) }\end{array}$ & Unclear risk & Not reported \\
\hline $\begin{array}{l}\text { Blinding (performance } \\
\text { bias and detection bias) } \\
\text { All outcomes }\end{array}$ & Unclear risk & Attention control used, but not equivalent \\
\hline $\begin{array}{l}\text { Blinding of outcome as- } \\
\text { sessment (detection bias) } \\
\begin{array}{l}\text { All outcomes } \\
\hline\end{array}\end{array}$ & Unclear risk & Investigator; efficacy unknown \\
\hline
\end{tabular}


Pohl 2002 (Continued)
Incomplete outcome data
(attrition bias)
End of intervention
Low risk ITT no reported
No losses

\begin{tabular}{lll}
\hline $\begin{array}{l}\text { Selective reporting (re- } \\
\text { porting bias) }\end{array}$ & Unclear risk & No protocol available \\
\hline Other bias & Low risk & None known \\
\hline Imbalanced exposure & Unclear risk & Imbalanced exposure favouring training (control 15 $\mathrm{h}>$ intervention $12 \mathrm{~h}$ ) \\
\hline
\end{tabular}

\section{Potempa 1995}

\begin{tabular}{|c|c|}
\hline Methods & $\begin{array}{l}\text { Design: randomised trial of cardiorespiratory training vs non-exercise intervention - after UC } \\
\text { Randomisation: unknown } \\
\text { Allocation concealment: unknown } \\
\text { Blinding: unknown } \\
\text { ITT: no } \\
\text { Measurements: end of intervention (10 weeks) } \\
\text { Withdrawals: none }\end{array}$ \\
\hline Participants & $\begin{array}{l}\text { Randomised: } 42 \text { participants } \\
\text { Intervention: } 19 \text { participants; } 8 \text { men and } 11 \text { women } \\
\text { Control: } 23 \text { participants; } 15 \text { men and } 8 \text { women } \\
\text { All participants aged } 43-70 \text { years and were } 216 \text { days post-stroke (SD 43) } \\
\text { All participants had upper and lower limb hemiparesis } \\
\text { Inclusion criteria: medically stable; at least } 6 \text { months post-stroke; completed formal rehabilitation } \\
\text { Exclusion criteria: patients with brain stem lesions; any clinical evidence that would preclude maximal } \\
\text { exercise testing }\end{array}$ \\
\hline Interventions & $\begin{array}{l}\text { Intervention: cardiorespiratory training: cycle ergometer training for } 30 \mathrm{~min} / \text { day } 3 \text { days/week for } 10 \\
\text { weeks; intensity } 30 \%-50 \% \text { of maximal effort increasing to maximum sustainable over first } 4 \text { weeks } \\
\text { Control: non-exercise intervention: passive range of motion exercises for } 30 \mathrm{~min} / \text { day } 3 \text { days/week for } \\
10 \text { weeks } \\
\text { Setting: unknown }\end{array}$ \\
\hline
\end{tabular}

Outcomes Included outcomes: BP; maximum cycling work rate (watts)

Other outcomes: HR at rest and during maximal exercise; respiratory exchange rate and other respira-

tory variables; exercise duration; Fugl Meyer score

Notes Variance reported as standard error and converted to standard deviation

\section{Risk of bias}

\begin{tabular}{lll}
\hline Bias & Authors' judgement & Support for judgement \\
\hline $\begin{array}{l}\text { Random sequence genera- } \\
\text { tion (selection bias) }\end{array}$ & Unclear risk & Not reported \\
\hline $\begin{array}{l}\text { Allocation concealment } \\
\text { (selection bias) }\end{array}$ & Unclear risk & Not reported \\
\hline $\begin{array}{l}\text { Blinding (performance } \\
\text { bias and detection bias) }\end{array}$ & Unclear risk & Attention control \\
\hline
\end{tabular}


Potempa 1995 (Continued)

All outcomes

\begin{tabular}{lll}
$\begin{array}{l}\text { Blinding of outcome as- } \\
\text { sessment (detection bias) } \\
\text { All outcomes }\end{array}$ & Unclear risk & Not reported \\
\hline $\begin{array}{l}\text { Incomplete outcome data } \\
\text { (attrition bias) }\end{array}$ & Low risk & ITT not reported \\
End of intervention & & No losses
\end{tabular}

\begin{tabular}{lll}
\hline $\begin{array}{l}\text { Selective reporting (re- } \\
\text { porting bias) }\end{array}$ & Unclear risk & Protocol not available \\
\hline Other bias & Low risk & None known \\
\hline Imbalanced exposure & Low risk & Balanced exposure \\
\hline
\end{tabular}

Richards 1993

Design: randomised trial of mixed training plus UC vs UC - during UC
Randomisation mechanism: unknown; method: stratified on BI scores
Allocation concealment: unknown
Blinding: investigator; efficacy unknown
ITT: no
Measurements: end of intervention (5 weeks)
Withdrawals: control group 3 (1 refusal, 2 unknown)

Participants Randomised: 18 participants

Intervention: 10 participants; 5 men and 5 women; age 69.6 years (SD 7.4 years); 8.3 days post-stroke

(SD 1.4)

Control: 8 participants; 2 men and 6 women; age 67.3 years (SD 11.2); 8.8 days post-stroke (SD 1.5)

Inclusion criteria: within $50 \mathrm{~km}$ of treatment centre; men and women aged 40-80 years; 0-7 days af-

ter first stroke; middle cerebral artery syndrome identified by CT; under care of neurologist involved in

study; willing to sign informed consent

Exclusion criteria: other major medical conditions that would interfere with functional capacity or interfere with rehabilitation; patients who were independently ambulatory 1 week after stroke; patients who were unconscious at onset

Interventions
ercises using a Kinetron, and treadmill walking, 104 min/day 5 days/week for 5 weeks; progression
achieved via velocity and resistance (Kinetron) increments
Control: traditional neurophysical techniques 109 min/day 5 days/week for 5 weeks
Setting: hospital

Outcomes Included outcomes: Barthel Ambulation scores; BBS; gait velocity

Other outcomes: Fugl-Meyer balance; Fugl-Meyer upper and lower extremity scores

Notes

We did not use a second control group of early conventional therapy for comparison since it differed from the institution UC; it commenced earlier than usual during hospital care and had substantially longer contact time.

\section{Risk of bias}

\section{Bias}

\section{Authors' judgement Support for judgement}


Richards 1993 (Continued)

Random sequence genera- Unclear risk Stratified randomisation based on BI scores tion (selection bias)

\begin{tabular}{|c|c|c|}
\hline $\begin{array}{l}\text { Allocation concealment } \\
\text { (selection bias) }\end{array}$ & Unclear risk & Not reported \\
\hline $\begin{array}{l}\text { Blinding (performance } \\
\text { bias and detection bias) } \\
\text { All outcomes }\end{array}$ & Unclear risk & Suitable attention control \\
\hline $\begin{array}{l}\text { Blinding of outcome as- } \\
\text { sessment (detection bias) } \\
\text { All outcomes }\end{array}$ & Low risk & Investigator blinded; efficacy unknown \\
\hline $\begin{array}{l}\text { Incomplete outcome data } \\
\text { (attrition bias) } \\
\text { End of intervention }\end{array}$ & High risk & $\begin{array}{l}\text { No ITT analysis } \\
3 / 18(17 \%) \text { total losses at the end of intervention: intervention 0; control group } \\
3 \text { ( } 1 \text { refusal, } 2 \text { unknown) }\end{array}$ \\
\hline $\begin{array}{l}\text { Selective reporting (re- } \\
\text { porting bias) }\end{array}$ & Unclear risk & Protocol not available \\
\hline Other bias & Low risk & None known \\
\hline Imbalanced exposure & Low risk & Balanced exposure \\
\hline
\end{tabular}

\section{Richards 2004}

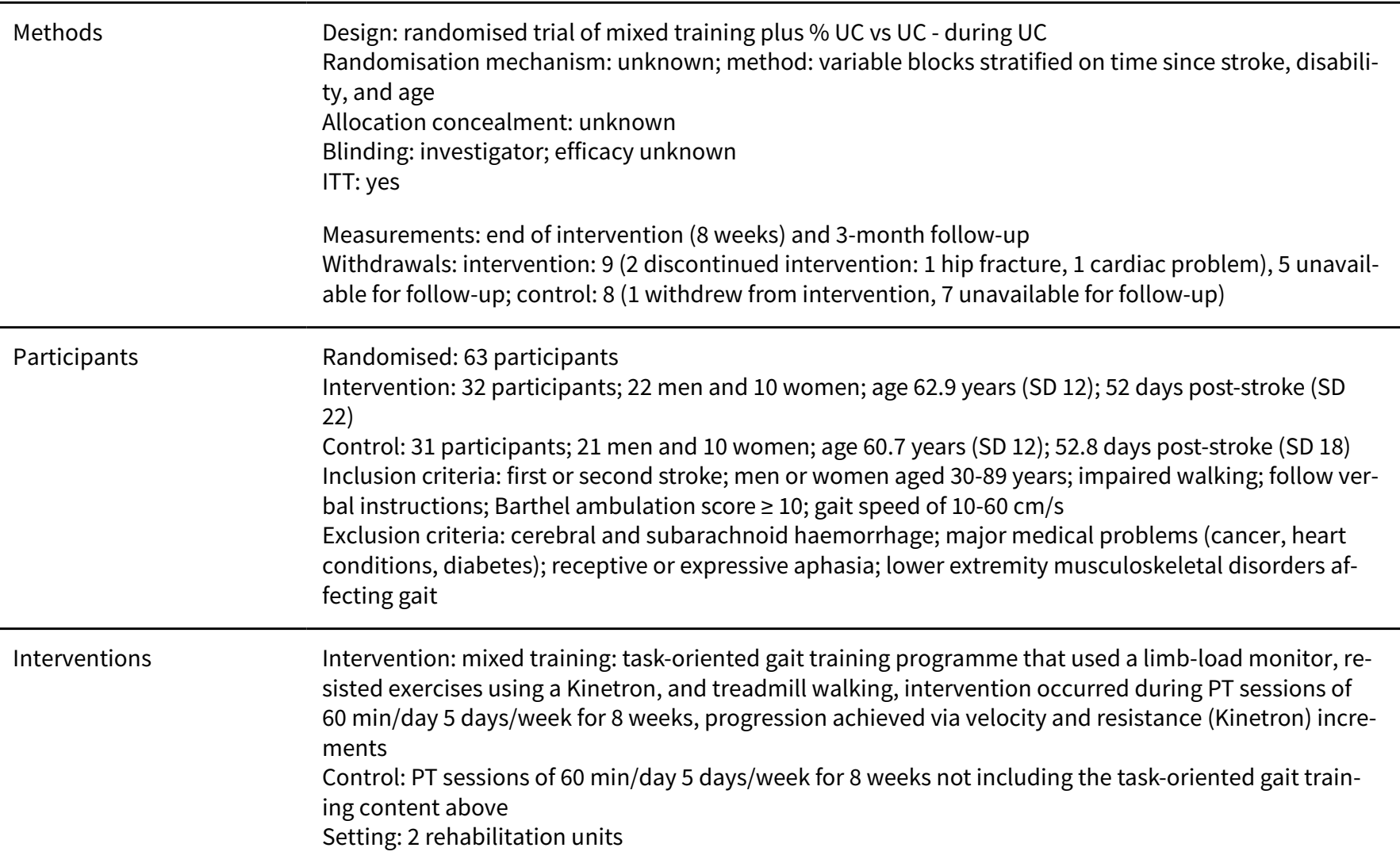


Richards 2004 (Continued)

Outcomes

Included outcomes: preferred walking speed; TUG; BI (ambulation subscore); BBS

Other outcomes: kinematic gait analysis weakened by missing data in 50\% participants; Fugl-Meyer leg and arm scores

Notes

A second control group of conventional therapy was not used for comparison since (1) it was much shorter in duration, and (2) started later than the training intervention

Outcome data imputed from graphs in publication

\section{Risk of bias}

\begin{tabular}{|c|c|c|}
\hline Bias & Authors' judgement & Support for judgement \\
\hline $\begin{array}{l}\text { Random sequence genera- } \\
\text { tion (selection bias) }\end{array}$ & Unclear risk & $\begin{array}{l}\text { Unclear; randomisation based on variable blocks stratified on time since } \\
\text { stroke, disability, and age }\end{array}$ \\
\hline $\begin{array}{l}\text { Allocation concealment } \\
\text { (selection bias) }\end{array}$ & Unclear risk & Not reported \\
\hline $\begin{array}{l}\text { Blinding (performance } \\
\text { bias and detection bias) } \\
\text { All outcomes }\end{array}$ & Unclear risk & Suitable attention control \\
\hline $\begin{array}{l}\text { Blinding of outcome as- } \\
\text { sessment (detection bias) } \\
\text { All outcomes }\end{array}$ & Low risk & Investigator blinded \\
\hline $\begin{array}{l}\text { Incomplete outcome data } \\
\text { (attrition bias) } \\
\text { End of intervention }\end{array}$ & Low risk & $\begin{array}{l}\text { ITT analysis } \\
5 / 63(8 \%) \text { losses at the end of intervention; intervention ( } 2 \text { discontinued inter- } \\
\text { vention: } 1 \text { hip fracture, } 1 \text { cardiac problem); control ( } 1 \text { withdrew from interven- } \\
\text { tion) }\end{array}$ \\
\hline $\begin{array}{l}\text { Incomplete outcome data } \\
\text { (attrition bias) } \\
\text { End of follow-up }\end{array}$ & High risk & $\begin{array}{l}\text { ITT analysis } \\
17 / 63(27 \%) \text { total losses at the end of follow-up; intervention ( } 5 \text { not available); } \\
\text { control ( } 7 \text { not available) }\end{array}$ \\
\hline $\begin{array}{l}\text { Selective reporting (re- } \\
\text { porting bias) }\end{array}$ & Unclear risk & Protocol not available \\
\hline Other bias & Low risk & None known \\
\hline Imbalanced exposure & Low risk & Balanced exposure \\
\hline
\end{tabular}

Salbach 2004

Methods

Design: randomised trial of cardiorespiratory training vs non-exercise intervention - after UC Randomisation mechanism: computer; method: stratified on gait speed

Allocation concealment: unknown

Blinding: investigator blinded (unblinded during assessment of intervention group 18/42 and control group 16/43)

ITT: yes

Measurements: end of intervention ( 6 weeks) 
Salbach 2004 (Continued)

Withdrawals: intervention: 3 discontinued (refused to travel, wanted both interventions, groin pain) with 2 of these lost to follow-up; control: 4 discontinued (MI, prostate cancer, fall + fracture, wanted other intervention) with 3 of these lost to follow-up

Randomised: 91 participants
Intervention: 44 participants; 26 men and 18 women; age 71 years (SD 12); 239 days post-stroke (SD 83)
Control: 47 participants; 30 men and 17 women; age 73 years (SD 8); 217 days post-stroke (SD 73)
Inclusion criteria: first or recurrent stroke; gait deficit from recent stroke; mental competency; inde-
pendently ambulatory for $10 \mathrm{~m}+/$ - aids or supervision; ability to comprehend instructions; resident in
community; discharged from rehabilitation; recent stroke $\leq 1$ year
Exclusion criteria: neurological deficit caused by metastatic disease; gait function (6-MWT) equivalent
to healthy norms; discharged to permanent care; comorbidity preventing participation in either inter-
vention

Interventions Intervention: cardiorespiratory training: task-oriented circuit training, performed 55 min/day 3 days/ week for 6 weeks, comprising a warm-up followed by 10 walking-related tasks (step ups, balance beam, kicking ball, stand up and walk, obstacle course, treadmill, walk and carry, speed walk, backward walking, stairs); progression of speed, load and degree of assistance Control: functional practice, whilst seated, of writing, keyboard use, and manipulating cards; some practice encouraged at home. 3 days/week for 6 weeks

Setting: 2 rehabilitation centres or hospitals

Outcomes Included outcomes: gait endurance 6-MWT; gait comfortable speed; gait maximal speed; TUG; BBS Other outcomes: activity-specific balance confidence scale

Notes -

\section{Risk of bias}

\begin{tabular}{lll}
\hline Bias & Authors' judgement & Support for judgement \\
\hline $\begin{array}{l}\text { Random sequence genera- } \\
\text { tion (selection bias) }\end{array}$ & Low risk & Computer-based randomisation stratified on gait speed \\
\hline $\begin{array}{l}\text { Allocation concealment } \\
\text { (selection bias) }\end{array}$ & Unclear risk & Not reported \\
\hline $\begin{array}{l}\text { Blinding (performance } \\
\text { bias and detection bias) } \\
\text { All outcomes }\end{array}$ & Unclear risk & Suitable attention control \\
\hline $\begin{array}{l}\text { Blinding of outcome as- } \\
\text { sessment (detection bias) } \\
\text { All outcomes }\end{array}$ & High risk & \\
\hline
\end{tabular}

$\begin{array}{ll}\begin{array}{l}\text { Incomplete outcome data } \\ \text { (attrition bias) }\end{array} & \text { Low risk } \\ \begin{array}{l}\text { End of intervention } \\ \end{array} & \text { ITT analysis } \\ 7 / 91(8 \%) \text { losses at the end of intervention assessment } \\ \text { Intervention: } 3 \text { discontinued (refused to travel, wanted both interventions, } \\ \begin{array}{l}\text { groin pain) with } 2 \text { of these lost to follow-up; control: } 4 \text { discontinued (MI, } \\ \text { prostate cancer, fall + fracture, wanted other intervention) with } 3 \text { of these lost } \\ \text { to follow-up }\end{array}\end{array}$

\begin{tabular}{lll}
\hline $\begin{array}{l}\text { Selective reporting (re- } \\
\text { porting bias) }\end{array}$ & Unclear risk & Protocol not available \\
\hline Other bias & Low risk & None known \\
\hline
\end{tabular}


Salbach 2004 (Continued)

Imbalanced exposure Low risk Balanced exposure

Sandberg 2016

\begin{tabular}{|c|c|}
\hline \multirow[t]{7}{*}{ Methods } & Design: RCT. Cardiorespiratory training vs no intervention - after UC \\
\hline & Randomisation: shuffling sealed envelopes \\
\hline & Allocation concealment: sealed envelopes \\
\hline & Blinding: cycle ergometer test stated as blind at baseline but blinding unclear for other outcome \\
\hline & ITT: no pre-planned ITT described \\
\hline & Measurements: at the end of intervention (12 weeks) \\
\hline & Withdrawals: 2/27 (7\%) withdrew from control group; 1 due to recurrent stroke, 1 unknown reason \\
\hline \multirow[t]{5}{*}{ Participants } & Randomised: 56 participants were randomised ( $n=53$ in second publication) \\
\hline & $\begin{array}{l}\text { Intervention: } 29 \text { participants; } 14 \text { men (52\%) and } 15 \text { women (52\%); mean age: } 73.1 \text { (SD 7); } 22.2 \text { (SD 10.1) } \\
\text { days after stroke (earlier for some outcomes) }\end{array}$ \\
\hline & $\begin{array}{l}\text { Control: } 27 \text { participants; } 14 \text { men (52\%) and } 13 \text { women (48\%); mean age: } 70.4 \text { (SD 8.1); } 22.8 \text { (SD 10.8) } \\
\text { days after stroke (earlier for some outcomes) }\end{array}$ \\
\hline & Inclusion criteria: age $\geq 50$ years, could walk $>5 \mathrm{~m}$, understand instructions, stroke diagnosis \\
\hline & $\begin{array}{l}\text { Exclusion criteria: medical or neurological diseases that were a risk or prevented delivery of training } \\
\text { programme }\end{array}$ \\
\hline
\end{tabular}

$\begin{array}{ll}\text { Interventions } & \text { Intervention: cardiorespiratory training } \\ & \text { Part 1: warm-up } 15 \text { min light to moderate (11-14 RPE); sitting, standing and walking } \\ & \text { Part 2: cycle ergometry } 8 \text { min (14-15 RPE; 75\% maximum oxygen consumption; } 80 \% \text { max HR) } \\ \text { Part 3: low-intensity exercises (10 min); flexibility while sitting, standing and walking } \\ \text { Part 4: cycle ergometry } 8 \text { min (14-15 RPE; 75\% maximum oxygen consumption; } 80 \% \text { max HR) } \\ \text { Part 5: cool-down } 15 \text { min; in different positions } \\ \text { 4 min to move between stations } \\ \text { Frequency of intervention: } 60 \text { min/day for } 2 \text { days/week for } 12 \text { weeks } \\ \text { Control: only advice about exercise and physical activity (usual practice in this setting) } \\ \text { Setting: unclear } \\ \text { Included outcomes: peak work rate (watts), 6-MWT, 10-metre walk test, TUG, balance (single leg stance } \\ \text { test), QoL (EQ-5D) } \\ \text { Other outcomes: SIS domains: } 8 \text { (participation) and 9 (recovery); BP response to exercise }\end{array}$

\begin{tabular}{ll}
\hline Notes & NCT02107768 \\
\hline Risk of bias &
\end{tabular}

\begin{tabular}{lll}
\hline Bias & Authors' judgement & Support for judgement \\
\hline $\begin{array}{l}\text { Random sequence genera- } \\
\text { tion (selection bias) }\end{array}$ & Low risk & Physical method probably adequate \\
\hline
\end{tabular}


Sandberg 2016 (Continued)

Allocation concealment Unclear risk Not clear whether envelopes had sufficient safeguards (opaque etc) (selection bias)

Blinding (performance

High risk

No attention control and intervention delivered by 2 of the study authors

bias and detection bias)

All outcomes

\begin{tabular}{lll}
\hline $\begin{array}{l}\text { Blinding of outcome as- } \\
\text { sessment (detection bias) } \\
\text { All outcomes }\end{array}$ & Unclear risk & $\begin{array}{l}\text { Some outcomes were not assessed by blind personnel and blinding seemed to } \\
\text { be applied differently at baseline and follow-up }\end{array}$ \\
\hline $\begin{array}{l}\text { Incomplete outcome data } \\
\text { (attrition bias) }\end{array}$ & Unclear risk & $\begin{array}{l}2 / 27(7 \%) \text { dropouts in } 2016 \text { paper }(n=56 \text { total) from control group plus no ITT } \\
\text { and of intervention }\end{array}$ \\
$\begin{array}{l}\text { ducing this to } n=24) \text { were missing BP data and were not included. Exact ef- } \\
\text { fects uncertain }\end{array}$
\end{tabular}

Incomplete outcome data Low risk

(attrition bias)

2/27 lost in control group due to unknown reasons and recurrent stroke. Not

End of follow-up likely attributed to intervention received

\begin{tabular}{lll}
\hline $\begin{array}{l}\text { Selective reporting (re- } \\
\text { porting bias) }\end{array}$ & Low risk & BP (2017 paper) was not in the pre-published protocol so might be questioned \\
\hline Other bias & Low risk & None known \\
\hline Imbalanced exposure & High risk & No attention control for exposure \\
\hline
\end{tabular}

\section{Shin 2011}

Design: randomised trial of mixed training vs UC
Randomisation: information not included
Allocation concealment: information not included
Blinding: information not included
ITT: not completed
Measurements: before and end of intervention (4 weeks)
Withdrawals: none reported

Participants

Randomised: total 21 participants. 11 participants were randomised to intervention, 10 to control Intervention: 11 participants; 5 men, 6 women; mean age: (combined men and women) 58.1 years (SD 4.6); mean height: $160.6 \mathrm{~cm}$ (SD 6.6); mean weight: $65.2 \mathrm{~kg}$ (SD 8.3); type of stroke: unknown; paretic side: right 8 , left 3; time since stroke onset: between 6 months and 5 years Control: 10 participants; 3 men, 7 women; mean age: (combined men and women) 57.3 years (SD 4.4); mean height: $164.5 \mathrm{~cm}$ (SD 7.1); mean weight: $65.0 \mathrm{~kg}$ (SD 7.5); type of stroke: unknown; paretic side: right 5, left 5; time since stroke onset: between 6 months and 5 years Inclusion criteria: 6 months to 5 years post-stroke with lower limb hemiplegia

Exclusion criteria: unable to ride a bicycle or perform functional exercise due to arthritis, low-back pain or degenerative joint disease; receiving treatment for other symptoms; unable to follow instructions due to low perceptive abilities, cognitive or communication disorder

Interventions

Intervention: participants in the training group $(n=11)$ received a total of $60 \mathrm{~min} /$ day combined exercise training, consisting of $30 \mathrm{~min}$ of functional strength training and $30 \mathrm{~min}$ of aerobic exercise 5 days/ week for 4 weeks. Functional strength training included bridging, stepping and stair exercises. Aerobic exercise was completed using a cycle ergometer and treadmill for $15 \mathrm{~min}$ each at $<40 \% \mathrm{HR}$ reserve based on age matched maximum HR 
Shin 2011 (Continued)

Control: participants in the control group $(n=10)$ received conservative physical therapy for $60 \mathrm{~min} /$ day, 5 days/week, for 4 weeks. Conservative physical therapy consisted of balance, postural control, and gait exercises

Setting: community setting

Outcomes Included outcomes: static and dynamic balance (force platform measurements), BBS (eyes open and eyes closed)

\begin{tabular}{|c|c|c|}
\hline Notes & - & \\
\hline \multicolumn{3}{|l|}{ Risk of bias } \\
\hline Bias & Authors' judgement & Support for judgement \\
\hline $\begin{array}{l}\text { Random sequence genera- } \\
\text { tion (selection bias) }\end{array}$ & Unclear risk & Described only as "randomly allocated" \\
\hline $\begin{array}{l}\text { Allocation concealment } \\
\text { (selection bias) }\end{array}$ & Unclear risk & Not reported \\
\hline $\begin{array}{l}\text { Blinding (performance } \\
\text { bias and detection bias) } \\
\text { All outcomes }\end{array}$ & Unclear risk & Not reported but there was an attention control \\
\hline $\begin{array}{l}\text { Blinding of outcome as- } \\
\text { sessment (detection bias) } \\
\text { All outcomes }\end{array}$ & Unclear risk & Not reported \\
\hline $\begin{array}{l}\text { Incomplete outcome data } \\
\text { (attrition bias) } \\
\text { End of intervention }\end{array}$ & Low risk & $\begin{array}{l}\text { ITT not reported } \\
\text { No participant losses }\end{array}$ \\
\hline $\begin{array}{l}\text { Selective reporting (re- } \\
\text { porting bias) }\end{array}$ & Unclear risk & Trial register or protocol not available \\
\hline Other bias & Low risk & None known \\
\hline Imbalanced exposure & Low risk & Balanced exposure \\
\hline
\end{tabular}

Sims 2009

Methods

Design: pilot randomised study of resistance training vs no intervention (i.e. a waiting list comparison group) - after UC. Sample size calculation reported

Randomisation: computer-generated block randomisation by an independent investigator - blocks of 6 stratified by gender

Allocation concealment: unclear

Blinding: unclear

ITT: yes

Measurements: at the end of the training programme (10 weeks) and at 6-month follow-up 
Sims 2009 (Continued)

Withdrawals: 1 participant did not complete the 10-week assessment; 5 participants ( 3 intervention, 2 control) did not complete the physical assessment at 10 weeks due to health reasons unrelated to the programme or time commitments. 43 participants completed the 6-month survey assessment

Rarticipants
since stroke 13.2 months (SD 4.95)
Intervention: 23 participants were allocated to the progressive resistance training group. 21 partici-
pants completed the 10-week programme (2 people became medically ineligible)
Control: 22 participants were allocated to the waiting list control group
Inclusion criteria: stroke survivors with depressive symptoms
Exclusion criteria: < 18 years; stroke < 6 months ago; inability to walk a distance of at least 20 m inde-
pendently with or without a gait assistive device; Prime-MD Patients Health Questionnaire score < 5;
depression with psychotic features; alcohol or drug-related depression, schizophrenia; bipolar disor-
der; other psychiatric diagnoses; suicidal ideation; dementia; terminally ill; uncontrolled hypertension;
unstable angina; and unstable insulin-dependent diabetes

Interventions

Intervention: participants in the intervention group attended a community gymnasium twice/week for 10 weeks and trained under the supervision of an accredited fitness trainer. The training programme entailed moderate strengthening exercises ( 3 sets of $8 / 10$ repetitions at a resistance of $80 \%$ of $1-\mathrm{RM}$ ) using machine weights for the major upper and lower limb muscle groups. Resistance was increased when participants were able to complete 3 sets of 10 repetitions of an exercise

Control: the waiting list controls received UC and were asked not to do any resistance-type exercise (content of the 'usual care' intervention not specified)

Setting: community-based setting

Outcomes Included outcomes: CES-D; AQoL SF-12

Other outcomes: SIS; SWLS; LOT-R; Self Esteem Scale; RLOC

Notes

Sample size calculation performed but sample obtained was smaller than that of the calculation (45 participants instead of 60). Small sample size. At baseline the intervention group had significantly lower depression scores than the comparison group. Impact of social interaction was not assessed

The participants in the control group received more attention than simply UC as they received a $10-$ week strength assessment

\section{Risk of bias}

\begin{tabular}{lll}
\hline Bias & Authors' judgement & Support for judgement \\
\hline $\begin{array}{l}\text { Random sequence genera- } \\
\text { tion (selection bias) }\end{array}$ & Low risk & $\begin{array}{l}\text { Quote: "Following the baseline assessments participants were randomly allo- } \\
\text { cated to the intervention or comparison group by a centrally located indepen- } \\
\text { dent person using a computer generated block randomisation list, with blocks } \\
\text { of six, stratified by gender." }\end{array}$ \\
\hline
\end{tabular}

\begin{tabular}{|c|c|c|}
\hline $\begin{array}{l}\text { Allocation concealment } \\
\text { (selection bias) }\end{array}$ & Low risk & $\begin{array}{l}\text { Not applicable as participants allocated in blocks after recruitment and base- } \\
\text { line assessment }\end{array}$ \\
\hline
\end{tabular}

\begin{tabular}{|c|c|c|}
\hline $\begin{array}{l}\text { Blinding (performance } \\
\text { bias and detection bias) } \\
\text { All outcomes }\end{array}$ & High risk & No attention control (waiting list comparison) \\
\hline
\end{tabular}

\begin{tabular}{|c|c|}
\hline $\begin{array}{l}\text { Blinding of outcome as- } \\
\text { sessment (detection bias) } \\
\text { All outcomes }\end{array}$ & Unclear risk \\
\hline
\end{tabular}


Sims 2009 (Continued)

$\begin{array}{ll}\begin{array}{l}\text { Incomplete outcome data } \\ \text { (attrition bias) }\end{array} & \text { ITT analysis } \\ \text { End of intervention } & \begin{array}{l}1 \text { participant did not complete the } 10 \text {-week assessment; } 5 \text { participants ( } 3 \text { inter- } \\ \text { vention, } 2 \text { control) did not complete the physical assessment at } 10 \text { weeks due } \\ \text { to health reasons unrelated to the programme or time commitments }\end{array}\end{array}$

\begin{tabular}{ll}
\hline $\begin{array}{l}\text { Incomplete outcome data } \\
\text { (attrition bias) }\end{array}$ & Low risk \\
End of follow-up & $43 / 45$ participants completed the 6-month survey assessment
\end{tabular}

\begin{tabular}{lll}
\hline $\begin{array}{l}\text { Selective reporting (re- } \\
\text { porting bias) }\end{array}$ & Unclear risk & Included outcomes correspond with protocol ACTRN12605000613606 \\
\hline Other bias & High risk & $\begin{array}{l}\text { At baseline the intervention group had significantly lower depression scores } \\
\text { than the comparison group }\end{array}$ \\
\hline Imbalanced exposure & High risk & Imbalanced exposure \\
\hline
\end{tabular}

Smith 2008

$\begin{array}{ll}\text { Methods } & \text { Design: randomised trial of cardiorespiratory training vs non-exercise intervention - after UC (i.e. tread- } \\ \text { mill gait training vs weekly telephone calls - the main purpose of the study was to explore the potential } \\ \text { additional benefits of treadmill training) }\end{array}$
additional benefits of treadmill training)

Randomisation: random matched-pair assignment. The investigator assigned a number to suitable participants and placed them in 1 of the intervention groups by 'the roll of a dice' (odd control, even treatment), or systematically allocated a participant to match a randomly assigned participant in the alternate group (minimisation?)

Allocation concealment: unclear

Blinding: clinical assessor not blinded

ITT: not reported, but no withdrawals

Measurements: at the end of the intervention (4 weeks) and then 6 weeks later

Withdrawals: none

Participants Randomised: 20 participants; age range 42-72 years

Intervention: 10 participants, 8 men and 2 women; mean age 57.8 years (SD 7.0); time from stroke: 8 participants $<1$ year and 2 participants $\geq 1$ year $<2$ years

Control: 10 participants, 4 men and 6 women; mean age 56 years (SD 8.3); time from stroke: 8 participants $<1$ year and 2 participants $\geq 1$ year $<2$ years

Inclusion criteria: stroke in the middle cerebral artery territory $>3$ months but $<2$ years prior to enrolling in the study; walking slower than pre-stroke

Exclusion criteria: cognitive impairment; unable to ambulate; concomitant pathology that prevented walking on a treadmill

Interventions

Intervention: participants in the intervention group received 12 sessions of treadmill training (20 min each session) over 4 weeks plus weekly calls from the investigator enquiring about the quality of their week and encouraging them to keep a QoL log. They wore a standard gait belt on the treadmill and had a practice session prior to the start of the study. The starting speed on the treadmill was the speed at which the participant could walk during the practice session for 5 min with a RPE $\leq 13$. The speed was increased by $0.2 \mathrm{mph}$ each time the participant walked for 10 consecutive min with a RPE $\leq 13$ 
Smith 2008 (Continued)

Control: participants in the control group received weekly calls from the investigator enquiring about the quality of their week and encouraging them to keep a QoL log only

Setting: community-based setting

\begin{tabular}{ll}
\hline Outcomes & Included outcomes: depression (BDI), mobility \\
& Other outcomes: social participation (SIS 3.0 subscales)
\end{tabular}

Notes Very small sample size. Fitness outcomes not considered

\section{Risk of bias}

\begin{tabular}{|c|c|c|}
\hline Bias & Authors' judgement & Support for judgement \\
\hline $\begin{array}{l}\text { Random sequence genera- } \\
\text { tion (selection bias) }\end{array}$ & Unclear risk & $\begin{array}{l}\text { Random matched-pair assignment. The investigator assigned a number to } \\
\text { suitable participants and placed them in } 1 \text { of the intervention groups by 'the } \\
\text { roll of a dice' (odd control, even treatment), or systematically allocated a par- } \\
\text { ticipant to match a randomly assigned participant in the alternate group (min- } \\
\text { imisation?) }\end{array}$ \\
\hline $\begin{array}{l}\text { Allocation concealment } \\
\text { (selection bias) }\end{array}$ & Unclear risk & Not reported \\
\hline $\begin{array}{l}\text { Blinding (performance } \\
\text { bias and detection bias) } \\
\text { All outcomes }\end{array}$ & High risk & No suitable attention control \\
\hline $\begin{array}{l}\text { Blinding of outcome as- } \\
\text { sessment (detection bias) } \\
\text { All outcomes }\end{array}$ & High risk & Clinical assessor not blinded \\
\hline $\begin{array}{l}\text { Incomplete outcome data } \\
\text { (attrition bias) } \\
\text { End of intervention }\end{array}$ & Low risk & $\begin{array}{l}\text { ITT analysis not reported } \\
\text { No withdrawals }\end{array}$ \\
\hline $\begin{array}{l}\text { Incomplete outcome data } \\
\text { (attrition bias) } \\
\text { End of follow-up }\end{array}$ & Low risk & $\begin{array}{l}\text { ITT analysis not reported } \\
\text { No withdrawals }\end{array}$ \\
\hline $\begin{array}{l}\text { Selective reporting (re- } \\
\text { porting bias) }\end{array}$ & Unclear risk & Protocol not available \\
\hline Other bias & Low risk & None known \\
\hline Imbalanced exposure & High risk & Imbalanced exposure \\
\hline
\end{tabular}

\section{Son 2014}

Design: randomised trial of resistance training plus UC vs sham training (no resistance) plus UC (most
likely after discharge from usual hospital care approximately 18 months post-stroke)
Randomisation: software used to randomise participants
Allocation concealment: information not included
Blinding: information not included
ITT: not completed but no losses
Measurements: before and end of intervention (6 weeks)


Son 2014 (Continued)

Withdrawals: none reported

Participants 28 participants randomised: 14 to intervention, 14 to control

Intervention: 14 participants; 8 men, 6 women; mean age: (combined men and women) 57.4 years (SD

not given); type of stroke: ischaemic 7, haemorrhagic 7; paretic side: right 6 , left 8; time since stroke onset: 17.9 months

Control: 14 participants; 7 men 7 women: mean age: (combined men and women) 56.6 years (SD not given); type of stroke: ischaemic 8 , haemorrhagic 6 ; paretic side: right 7 , left 7 ; time since stroke onset: 18.7 months

Inclusion criteria: a Brunnstrom $\geq$ stage 3, ability to independently walk $10 \mathrm{~m}$ with or without supervision or an aid or orthosis, and a minimum score of 20 on the Korean-MMSE

Exclusion criteria: joint contracture, pain, or fracture of the musculoskeletal system, and hemianopia

Interventions

Intervention: participants in the training group $(n=14)$ received lower-limb resistance training for 30 min, 5 times/week for 6 weeks. Warm-up included 1 set of 4 reps of resisted knee extension at 25\% 1RM, followed by 3 sets of $8-10$ reps of resisted knee extension at 70\% 1-RM. Resistance load was progressed weekly via reassessment of 1-RM

Control: participants in the control group $(n=14)$ received sham lower limb training with no resistance for $30 \mathrm{~min}, 5$ times/week for 6 weeks. Warm-up included 1 set of 4 reps of knee extension at no resistance, followed by 3 sets of $8-10$ reps of knee extension at no resistance

All participants received conservative physical therapy for $30 \mathrm{~min} /$ day, 5 days/week, for a period of 6 weeks. Conservative physical therapy consisted of joint mobilisation, muscle strengthening, and balance training

Setting: unclear; community-based project

Outcomes Included outcomes: balance outcomes including antero-posterior (A-P), medio-lateral (M-L) sway distances, and the BBS; TUG times

Notes

$-$

\section{Risk of bias}

\begin{tabular}{lll}
\hline Bias & Authors' judgement & Support for judgement \\
\hline $\begin{array}{l}\text { Random sequence genera- } \\
\text { tion (selection bias) }\end{array}$ & Low risk & Software-generated randomisation \\
\hline $\begin{array}{l}\text { Allocation concealment } \\
\text { (selection bias) }\end{array}$ & Unclear risk & Not reported \\
\hline $\begin{array}{l}\text { Blinding (performance } \\
\text { bias and detection bias) } \\
\text { All outcomes }\end{array}$ & Unclear risk & Not reported although there was a suitable attention control \\
\hline $\begin{array}{l}\text { Blinding of outcome as- } \\
\text { sessment (detection bias) } \\
\text { All outcomes }\end{array}$ & Unclear risk & Not reported \\
\hline $\begin{array}{l}\text { Incomplete outcome data } \\
\text { (attrition bias) } \\
\text { End of intervention }\end{array}$ & Low risk & $\begin{array}{l}\text { All 3 criteria for ITT analysis have not been met - methods suggest a per-proto- } \\
\text { col analysis; however there were no losses }\end{array}$ \\
\hline $\begin{array}{l}\text { Selective reporting (re- } \\
\text { porting bias) }\end{array}$ & Unclear risk & \begin{tabular}{l} 
Trial register or protocol not available \\
\hline
\end{tabular}
\end{tabular}


Son 2014 (Continued)

\begin{tabular}{lll} 
Other bias & Low risk & None known \\
\hline Imbalanced exposure & Low risk & Balanced exposure (sham training) \\
\hline
\end{tabular}

\section{Takami 2010}

Methods
Randomised: envelope method
Allocation concealment: unknown
Blinding: unknown
ITT: not reported
Measurements: end of intervention (3 weeks)
Withdrawals: 2 participants from backward walking group and 1 participant from forward walking
group due to family reasons

Participants

Randomised: 36 participants

Intervention 1: 12 participants in backward walking group; 6 men and 6 women; mean age 66.1 years (SD 6.3); 13.2 days post-stroke (SD 8.4)

Intervention 2: 12 participants in forward walking group; 9 men and 3 women; mean age 71.1 years (SD 10.6); 14.7 days post-stroke (SD 8.1)

Control: 12 participants; 5 men and 7 women; mean age 66.9 years (SD 10.6); 13.7 days post-stroke (SD 8.9)

Inclusion criteria: ability to walk $10 \mathrm{~m}$ using aids; post-stroke period of < 5 weeks; FIM-Locomotion score of $\leq 5$; perfect BBS and RMI scores

Exclusion criteria: unknown

\begin{tabular}{|c|c|c|}
\hline \multirow[t]{4}{*}{ Interventions } & \multicolumn{2}{|c|}{$\begin{array}{l}\text { Invention groups: BWSTT for } 30 \text { min then } 10 \text { min of either: backward or forward walking } 6 \text { times/week } \\
\text { for } 3 \text { weeks }\end{array}$} \\
\hline & \multicolumn{2}{|c|}{ Treadmill speed was progressed each week $(0.8,1.0$, and $1.3 \mathrm{~km} / \mathrm{h})$} \\
\hline & \multicolumn{2}{|c|}{$\begin{array}{l}\text { Control group: conventional training overground walking }(150-200 \mathrm{~m}) \text { for } 40 \mathrm{~min} 6 \text { times/week for } 3 \\
\text { weeks }\end{array}$} \\
\hline & \multicolumn{2}{|c|}{ Setting: rehabilitation unit and community settings } \\
\hline Outcomes & \multicolumn{2}{|c|}{$\begin{array}{l}\text { Included outcomes: BBS; RMI; 10-metre maximum walking speed; walking ratios during } 10 \text { metre for- } \\
\text { ward walking and } 5 \text { metre backward walking; Motricity Index; FIM-Locomotion }\end{array}$} \\
\hline Notes & \multicolumn{2}{|l|}{-} \\
\hline \multicolumn{3}{|l|}{ Risk of bias } \\
\hline Bias & Authors' judgement & Support for judgement \\
\hline $\begin{array}{l}\text { Random sequence genera- } \\
\text { tion (selection bias) }\end{array}$ & Unclear risk & Described only as "envelope method" \\
\hline
\end{tabular}


Takami 2010 (Continued)

\begin{tabular}{lll}
$\begin{array}{l}\text { Allocation concealment } \\
\text { (selection bias) }\end{array}$ & Unclear risk & Nature of envelopes not described \\
\hline $\begin{array}{l}\text { Blinding (performance } \\
\text { bias and detection bias) }\end{array}$ & Unclear risk & Attention control is incorporated \\
All outcomes & &
\end{tabular}

\begin{tabular}{lll}
\hline $\begin{array}{l}\text { Blinding of outcome as- } \\
\text { sessment (detection bias) } \\
\text { All outcomes }\end{array}$ & Unclear risk & Not reported \\
\hline $\begin{array}{l}\text { Incomplete outcome data } \\
\text { (attrition bias) }\end{array}$ & Unclear risk & ITT not reported \\
End of intervention & $\begin{array}{l}3 / 36(8 \%) \text { losses at the end of intervention; } 2 \text { participants from backward walk- } \\
\text { ing training group and } 1 \text { participant from forward walking training group due } \\
\text { to family reasons }\end{array}$ \\
\hline
\end{tabular}

\begin{tabular}{lll}
\hline $\begin{array}{l}\text { Selective reporting (re- } \\
\text { porting bias) }\end{array}$ & Unclear risk & Protocol not available \\
\hline Other bias & Low risk & Not known \\
\hline Imbalanced exposure & Low risk & Balanced exposure \\
\hline
\end{tabular}

Taylor-Pilliae 2014

\begin{tabular}{|c|c|}
\hline \multirow[t]{8}{*}{ Methods } & $\begin{array}{l}\text { Design: community-based RCT, } 3 \text { groups; Tai Chi, 'SilverSneakers' group exercise, or non-exercise inter- } \\
\text { vention }\end{array}$ \\
\hline & $\begin{array}{l}\text { Only Silversneakers and control groups were included giving strength training vs non-exercise interven- } \\
\text { tion - after UC }\end{array}$ \\
\hline & $\begin{array}{l}\text { Randomisation: simple randomisation; participants drew a slip of paper from a non-transparent con- } \\
\text { tainer }\end{array}$ \\
\hline & $\begin{array}{l}\text { Allocation concealment: allocation was concealed; participants were handed an opaque, sealed enve- } \\
\text { lope matching the slip of paper taken from the container, and instructed to open the envelope when } \\
\text { they returned home }\end{array}$ \\
\hline & Blinding: assessor blinded to group allocation \\
\hline & ITT: ITT analyses were used \\
\hline & Measurements: baseline and end of intervention (12 weeks) \\
\hline & $\begin{array}{l}\text { Withdrawals: intervention } n=6 \text { (refuse group allocation } n=5 \text {, lack of time } n=1) \text {; control } n=3 \text { (refuse } \\
\text { group allocation } n=3 \text { ) }\end{array}$ \\
\hline \multirow[t]{4}{*}{ Participants } & $\begin{array}{l}\text { Randomised: } n=92 \text { : intervention (Silversneakers mixed training) } n=44 \text {; control group } n=48 . N=145 \\
\text { randomised to study as a whole }\end{array}$ \\
\hline & $\begin{array}{l}\text { Intervention: } 44 \text { participants; } 20 \text { men (45.5\%) and } 24 \text { women (54.5\%); mean age: } 69.6 \text { (SD 9.4); days af- } \\
\text { ter stroke not reported }\end{array}$ \\
\hline & $\begin{array}{l}\text { Control: } 48 \text { participants; } 23 \text { men (47.9\%) and } 25 \text { women (52.1\%); mean age: } 68.2 \text { (SD 10.3); days after } \\
\text { stroke not reported }\end{array}$ \\
\hline & $\begin{array}{l}\text { Inclusion criteria: based on pre-screening using standardised tests: functional disability (mRS), physical } \\
\text { function (Short Physical Performance Battery), cognitive impairment (MMSE) }\end{array}$ \\
\hline
\end{tabular}


Taylor-Pilliae 2014 (Continued)

Exclusion criteria: no disability (e.g. no post-stroke sequela), severe disability (e.g. bedridden), or a serious medical condition (e.g. active cancer treatment) that would interfere with study participation

Interventions

Intervention: SilverSneakers is a national fitness programme for older adults offering different types of group-based exercise classes (e.g. aerobics, strength and range of movement, water aerobics, yoga). In this particular intervention muscular strength and range of movement classes were taught by a certified instructor at local community fitness centres. $1 \mathrm{~h} /$ day, 3 days/week for 12 weeks. Each class approximately consisted of a 10-min warm-up period, 40-min of exercise, and a 10-min cool-down period

Control: received resources encouraging participation in community-based physical activity plus a weekly phone call about their health

Setting: community setting

\begin{tabular}{|c|c|}
\hline Outcomes & $\begin{array}{l}\text { Included outcomes: physical function (SPBB including balance using timed balance test, gait speed us- } \\
\text { ing 4-metre walk test, lower body strength using chair stand test), patient-reported fall rates, aerobic } \\
\text { endurance (2-min step test), QoL (SF-36) physical health and mental health composite scores, depres- } \\
\text { sive symptoms (Center for Epidemiologic Studies Depression Scale) }\end{array}$ \\
\hline & Other outcomes: sleep quality (Pittsburgh Sleep Quality Index) \\
\hline Notes & $\begin{array}{l}\text { The study author indicated that the 'SilverSneakers Classic' intervention was used, which is focused on } \\
\text { muscular strength and range of motion exercises, and this has no substantive cardiorespiratory train- } \\
\text { ing }\end{array}$ \\
\hline
\end{tabular}

\section{Risk of bias}

\begin{tabular}{lll}
\hline Bias & Authors' judgement & Support for judgement \\
\hline $\begin{array}{l}\text { Random sequence genera- } \\
\text { tion (selection bias) }\end{array}$ & Unclear risk & $\begin{array}{l}\text { Simple randomisation; the study authors reference Taylor-Pilliae 2012 in } \\
\text { which the following was reported "subjects drew a slip of paper from a non- } \\
\text { transparent container" }\end{array}$ \\
\hline
\end{tabular}

\begin{tabular}{lll}
\hline $\begin{array}{l}\text { Allocation concealment } \\
\text { (selection bias) }\end{array}$ & Unclear risk & Concealed allocation identified, no further details provided \\
\hline $\begin{array}{l}\text { Blinding (performance } \\
\text { bias and detection bias) }\end{array}$ & High risk & $\begin{array}{l}\text { No specific details provided. Study staff supervised intervention groups. Un- } \\
\text { likely blinding for performance bias. Participants dropped out after knowing } \\
\text { group allocation; no participant blinding as such and no balanced attention } \\
\text { control dose }\end{array}$ \\
\hline
\end{tabular}

\begin{tabular}{lll}
\hline $\begin{array}{l}\text { Blinding of outcome as- } \\
\text { sessment (detection bias) } \\
\text { All outcomes }\end{array}$ & Low risk & Assessor blinded to allocation of group \\
\hline $\begin{array}{l}\text { Incomplete outcome data } \\
\text { (attrition bias) } \\
\text { End of intervention }\end{array}$ & Low risk & Dropouts; Intervention 6/44 (14\%) control 3/48 (7\%), ITT analysis employed \\
\hline
\end{tabular}

Incomplete outcome data Low risk
(attrition bias)
End of follow-up

\begin{tabular}{lll}
\hline $\begin{array}{l}\text { Selective reporting (re- } \\
\text { porting bias) }\end{array}$ & Unclear risk & No trial registry details available \\
\hline Other bias & Unclear risk & Range of motion (passive) exercises included in strength intervention \\
\hline Imbalanced exposure & High risk & Attention control exposure not matched for dose \\
\hline
\end{tabular}


Teixeira 1999

\begin{tabular}{|c|c|}
\hline Methods & $\begin{array}{l}\text { Design: randomised trial of mixed training vs no intervention - after UC } \\
\text { First iteration only of a lag control design; participants randomly allocated to immediate or delayed - } \\
\text { participants allocated delayed intervention initially received no intervention } \\
\text { Randomisation mechanism: unknown; method: unclear ("balanced blocks") } \\
\text { Allocation concealment: unknown } \\
\text { Blinding: unknown } \\
\text { ITT: no } \\
\text { Measurements: end of intervention (10 weeks) } \\
\text { Withdrawals: none }\end{array}$ \\
\hline Participants & $\begin{array}{l}\text { Randomised: } 13 \text { participants } \\
\text { Intervention: } 6 \text { participants; } 1 \text { man and } 5 \text { women; age } 65.9 \text { years (SD 10.2); } 9.15 \text { years post-stroke (SD } \\
\text { 12.7) } \\
\text { Control: } 7 \text { participants; } 1 \text { man and } 6 \text { women; age } 69.4 \text { years (SD 8.85); } 6.4 \text { years post-stroke (SD 6.2) } \\
\text { All participants had unilateral stroke resulting in residual weakness or abnormal muscle tone or both } \\
\text { Inclusion criteria: at least } 9 \text { months post-stroke; independently ambulatory with or without walking } \\
\text { aids; no comprehensive aphasia } \\
\text { Exclusion criteria: non-stroke-related disability }\end{array}$ \\
\hline Interventions & $\begin{array}{l}\text { Intervention: mixed training: cardiorespiratory and lower extremity strength training } 60-90 \mathrm{~min} / \text { day } 3 \\
\text { days/week for } 10 \text { weeks; cardiorespiratory training: graded walking plus stepping or cycling progress- } \\
\text { ing from } 10-20 \text { min/day and from 50\%-70\% of maximal cycling work rate over first } 5 \text { weeks; strength } \\
\text { training: } 7 \text { exercises involving use of body weight and progressive resistive exercise using different } \\
\text { masses and elastic bands (TheraBand), each performed as } 3 \times 10 \text { repetitions and progressing from } \\
50 \%-80 \% \text { of 1-RM; warm-up and warm-down } 10-20 \text { min/day } \\
\text { Control: no intervention } \\
\text { Setting: unclear }\end{array}$ \\
\hline
\end{tabular}

\begin{tabular}{ll}
\hline Outcomes & Included outcomes: gait preferred speed (22 m); Adjusted Activity Score; NHP \\
Other outcomes: insufficient data to compare lower limb muscle strength (peak torque Nm); muscle \\
tone assessment; and stair climbing
\end{tabular}

Notes - -

\section{Risk of bias}

\begin{tabular}{lll}
\hline Bias & Authors' judgement & Support for judgement \\
\hline $\begin{array}{ll}\text { Random sequence genera- } \\
\text { tion (selection bias) }\end{array}$ & Unclear risk & Unclear \\
& & $\begin{array}{l}\text { Quote: "randomly assigned to one of the two groups (treatment and control) } \\
\text { with equal probability and balanced into similar blocks" }\end{array}$
\end{tabular}

\begin{tabular}{|c|c|c|}
\hline $\begin{array}{l}\text { Allocation concealment } \\
\text { (selection bias) }\end{array}$ & Unclear risk & Not reported \\
\hline $\begin{array}{l}\text { Blinding (performance } \\
\text { bias and detection bias) } \\
\text { All outcomes }\end{array}$ & High risk & No attention control \\
\hline $\begin{array}{l}\text { Blinding of outcome as- } \\
\text { sessment (detection bias) } \\
\text { All outcomes }\end{array}$ & Unclear risk & Not reported \\
\hline $\begin{array}{l}\text { Incomplete outcome data } \\
\text { (attrition bias) }\end{array}$ & Low risk & ITT not reported \\
\hline
\end{tabular}


Teixeira 1999 (Continued)

End of intervention

No losses

Selective reporting (re- Unclear risk $\quad$ Protocol not available
porting bias)

\begin{tabular}{lll}
\hline Other bias & High risk & Recruitment via advertisements \\
\hline Imbalanced exposure & High risk & Imbalanced exposure \\
\hline
\end{tabular}

Toledano-Zarhi 2011

Mesign: mixed training plus non-exercise intervention vs non-exercise intervention after UC
Randomisation mechanism: mechanism not reported
Allocation concealment: unknown
Blinding: unknown
ITT: yes (LOCF)
Measurements: end of intervention (6 weeks)
Withdrawals: 1 from intervention group (discontinued intervention)
Randomised: 28 participants
Intervention: 14 participants; 11 men and 3 women; age 65 years (SD 10); 1-3 weeks post-stroke
Control: 14 participants; 10 men and 4 women; age 65 years (SD 12); 1-3 weeks post-stroke
All participants had very minor ischaemic stroke
Participants
Exclusion criteria: SBP > 200 mmHg; DBP > 110 mmHg; unstable angina; arrhythmia; congestive heart
failure; ST depression $\geq 2$ mm on resting ECG; arterioventricular block with no pacemaker; severe pe-
ripheral vascular disease; severe lung disease; orthopaedic or neurological disability; dementia or ma-
jor depression

Interventions

Intervention: mixed training; 2 days/week for total of $3 \mathrm{~h} /$ week for 6 weeks. Twice/week 35-55 min of treadmill, hand bike, and cycle ergometer at 50\%-70\% HR maximum. Once/week 45-55 min of group strength, flexibility, and co-ordination

Control: home-based booklet with guidance on strength and flexibility and encouragement to continue with usual community routine

Setting: hospital Included outcomes: 6-MWT; Four Square Step Test; stair ascending and descending; treadmill perfor-
mance (Bruce protocol); BP

Notes Described as 'aerobic' training but this is mixed training

\section{Risk of bias}

\begin{tabular}{lll}
\hline Bias & Authors' judgement & Support for judgement \\
\hline $\begin{array}{l}\text { Random sequence genera- } \\
\text { tion (selection bias) }\end{array}$ & Unclear risk & Method not reported \\
\hline $\begin{array}{l}\text { Allocation concealment } \\
\text { (selection bias) }\end{array}$ & Unclear risk & Not reported \\
\hline $\begin{array}{l}\text { Blinding (performance } \\
\text { bias and detection bias) } \\
\text { All outcomes }\end{array}$ & High risk & No suitable attention control \\
\hline
\end{tabular}


Toledano-Zarhi 2011 (Continued)

$\begin{array}{lll}\text { Blinding of outcome as- } & \text { Unclear risk } & \text { Not reported } \\ \text { sessment (detection bias) } & \\ \text { All outcomes } & \end{array}$

\begin{tabular}{lll}
\hline $\begin{array}{l}\text { Incomplete outcome data } \\
\text { (attrition bias) } \\
\text { End of intervention }\end{array}$ & Low risk & ITT \\
\hline $\begin{array}{l}\text { Selective reporting (re- } \\
\text { porting bias) }\end{array}$ & Unclear risk & No protocol available \\
\hline Other bias & Low risk & None known \\
\hline Imbalanced exposure & High risk & Imbalanced exposure \\
\hline
\end{tabular}

\section{Topcuoglu 2015}

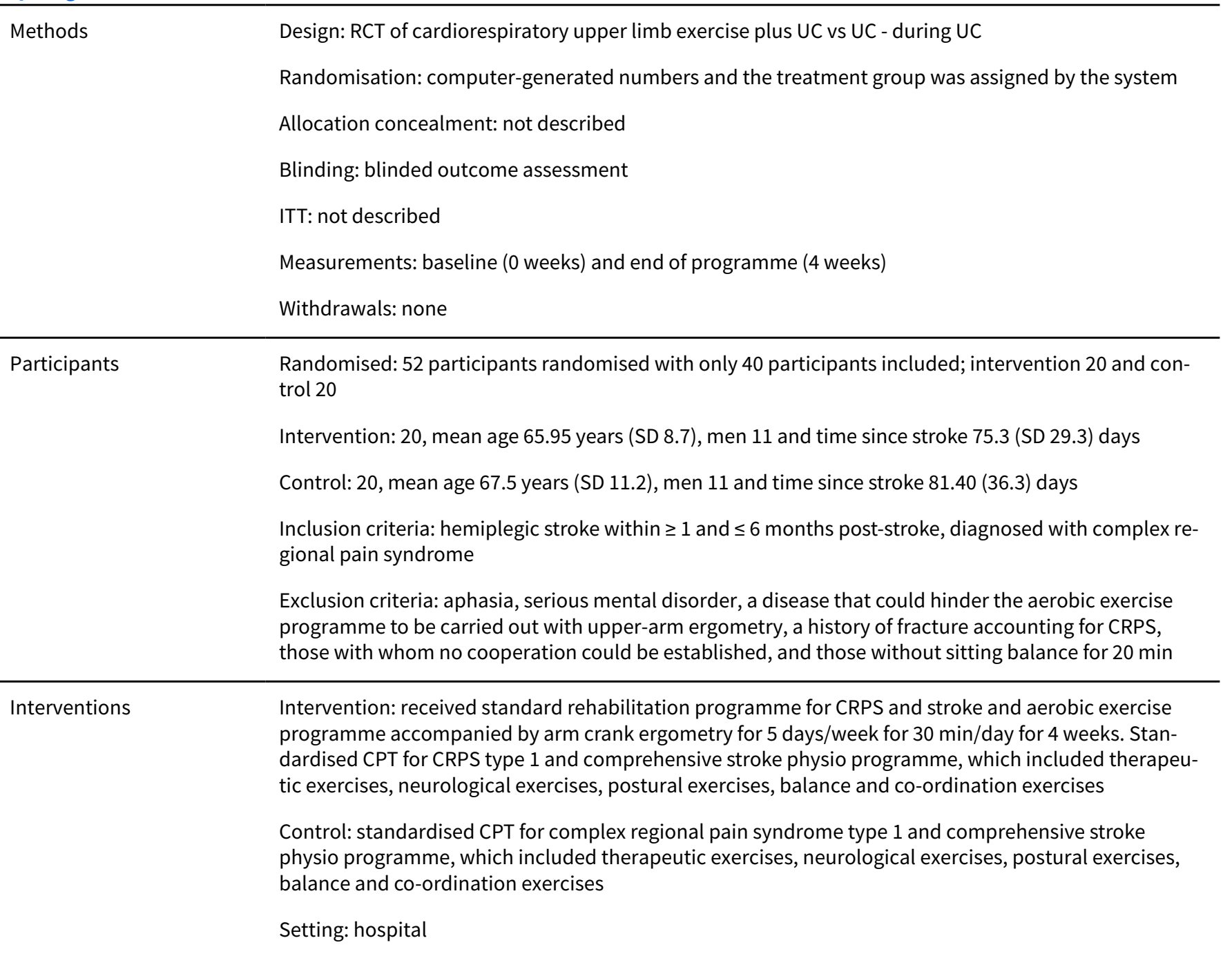

Outcomes Included outcomes: independence (FIM), QoL (NHP), mood (BDI)


Topcuoglu 2015 (Continued)

Other outcomes: motor level using Brunnstrom's staging for the upper extremity and the hand Spasticity (Modified Ashworth scale), pain

\begin{tabular}{|c|c|c|}
\hline Notes & Included outcomes ha & e no available data \\
\hline \multicolumn{3}{|l|}{ Risk of bias } \\
\hline Bias & Authors' judgement & Support for judgement \\
\hline $\begin{array}{l}\text { Random sequence genera- } \\
\text { tion (selection bias) }\end{array}$ & Low risk & Computer-generated numbers, treatment group assigned by the system \\
\hline $\begin{array}{l}\text { Allocation concealment } \\
\text { (selection bias) }\end{array}$ & Unclear risk & Not described \\
\hline $\begin{array}{l}\text { Blinding (performance } \\
\text { bias and detection bias) } \\
\text { All outcomes }\end{array}$ & High risk & No attention control and no description of blinding of other study personnel \\
\hline $\begin{array}{l}\text { Blinding of outcome as- } \\
\text { sessment (detection bias) } \\
\text { All outcomes }\end{array}$ & Low risk & $\begin{array}{l}\text { Assessment provided by same physician pre- and post- who were not aware of } \\
\text { participants' group assignment }\end{array}$ \\
\hline $\begin{array}{l}\text { Incomplete outcome data } \\
\text { (attrition bias) } \\
\text { End of intervention }\end{array}$ & Low risk & ITT not described but no dropouts \\
\hline $\begin{array}{l}\text { Selective reporting (re- } \\
\text { porting bias) }\end{array}$ & Unclear risk & No trial registry available \\
\hline Other bias & Low risk & None known \\
\hline Imbalanced exposure & High risk & No attention control \\
\hline
\end{tabular}

Van de Port 2012

Design: multicentre randomised trial of mixed training vs usual outpatient care - after UC
Randomised: online minimisation procedure
Allocation concealment: unknown
Blinding: assessors blinded to group allocation
ITT: yes
Measurements: end of intervention (12 weeks) and follow-up (24 weeks)
Withdrawals: intervention group (4 participants did not start intervention, 1 participant withdrew with-
out reason); control (1 participant at the end of intervention missing assessment, 2 participants died
from cancer, 2 participants had recurrent stroke, 2 participants withdrew without reason)

Participants

Randomised: 250 participants

Intervention: 124 participants; 82 men and 42 women; mean age 56 years (SD 10); time post-stroke 80.9 days (SD 13.0) 
Van de Port 2012 (Continued)

Control: 126 participants; 80 men and 46 women; mean age 58 years (SD 10); time post-stroke 77.8 days (SD 15.0)

Inclusion criteria: verified stroke (according to WHO definition); able to walk a minimum of $10 \mathrm{~m}$ unassisted; discharged home from rehabilitation centre; requirement to continue PT during outpatient care

Exclusion criteria: cognitive deficits (MMSE < 24 score); unable to communicate; lived $>30 \mathrm{~km}$ from rehabilitation centre

\begin{tabular}{|c|c|}
\hline \multirow[t]{3}{*}{ Interventions } & $\begin{array}{l}\text { Invention group: circuit training programme for } 90 \mathrm{~min} \text { twice/week for } 12 \text { weeks. Training included } 8 \\
\text { stations intended to improve walking competency. Each station exercise was performed for } 3 \text { min with } \\
3 \text { min recovery }\end{array}$ \\
\hline & $\begin{array}{l}\text { Control group: outpatient CPT care, no restriction or detail given regarding time or duration of these } \\
\text { sessions }\end{array}$ \\
\hline & Setting: rehabilitation outpatient centre \\
\hline Outcomes & $\begin{array}{l}\text { Included outcomes: mobility domain of SIS; RMI; falls efficacy scale; NEADL; HADS; fatigue severity } \\
\text { scale; Motricity index; 6-MWT; 5-metre comfortable walking speed test; timed balance test; TUG; modi- } \\
\text { fied stair test }\end{array}$ \\
\hline Notes & - \\
\hline
\end{tabular}

\section{Risk of bias}

\begin{tabular}{lll}
\hline Bias & Authors' judgement & Support for judgement \\
\hline $\begin{array}{l}\text { Random sequence genera- } \\
\text { tion (selection bias) }\end{array}$ & Low risk & $\begin{array}{l}\text { Participants stratified by rehabilitation centre using an online minimisation } \\
\text { procedure }\end{array}$ \\
\hline $\begin{array}{l}\text { Allocation concealment } \\
\text { (selection bias) }\end{array}$ & Low risk & $\begin{array}{l}\text { Risk removed due to online dynamic allocation mechanism: i.e. there is no al- } \\
\text { location list to conceal }\end{array}$ \\
\hline
\end{tabular}

Blinding (performance $\quad$ Unclear risk $\quad$ Some degree of attention control
bias and detection bias)
All outcomes

\begin{tabular}{|c|c|c|}
\hline $\begin{array}{l}\text { Blinding of outcome as- } \\
\text { sessment (detection bias) } \\
\text { All outcomes }\end{array}$ & Low risk & $\begin{array}{l}\text { Blinded outcome assessment. The efficacy of blinding was confirmed through } \\
\text { statistical analysis of guesses of allocation }\end{array}$ \\
\hline
\end{tabular}

\begin{tabular}{ll}
\hline $\begin{array}{l}\text { Incomplete outcome data } \\
\text { (attrition bias) }\end{array}$ & Low risk \\
$\begin{array}{l}\text { End of intervention } \\
\text { ITT analysis used }\end{array}$ & $\begin{array}{l}8 / 250(3 \%) \text { losses. Slight imbalance in losses in the control group } 7 / 124 \text { and } \\
\text { training group } 1 / 126\end{array}$ \\
& $\begin{array}{l}\text { Intervention group (4 participants did not start intervention, } 1 \text { participant } \\
\text { withdrew without reason); control group (1 participant at the end of interven- } \\
\text { tion missing assessment, 2 participants died from cancer, 2 participants had } \\
\text { recurrent stroke, 2 participants withdrew without reason) }\end{array}$ \\
\hline
\end{tabular}

\begin{tabular}{lll}
\hline $\begin{array}{l}\text { Incomplete outcome data } \\
\text { (attrition bias) } \\
\text { End of follow-up }\end{array}$ & Low risk & ITT analysis used \\
$8 / 250(3 \%)$ overall losses
\end{tabular}


Van de Port 2012 (Continued)

tional ambulation categories (included in review) and the Letter Cancellation

Task (but this is not included in this review)

\begin{tabular}{|c|c|c|}
\hline Other bias & Low risk & None known \\
\hline \multirow[t]{2}{*}{ Imbalanced exposure } & High risk & Imbalanced exposure \\
\hline & & $\begin{array}{l}\text { Quote: "The circuit training group received } 4461 \text { treatment sessions compared } \\
\text { with } 4378 \text { for the UC group. The average treatment time/session was } 72 \text { (SD } \\
\text { 39) mins for the intervention group compared with } 34 \text { (SD 10) mins for the con- } \\
\text { trol group }(P<0.05) \text {." }\end{array}$ \\
\hline
\end{tabular}

Vanroy 2017

\section{Methods}

Design: randomised trial. Cardiorespiratory training (cycling) plus UC vs non-exercise intervention plus UC - commenced during UC with some participants discharged home. A second phase in which the intervention group were allocated to 2 different exposures will be excluded from this review

Randomisation: permuted block randomisation using computer. Stratified on stroke type, motor function and cardiorespiratory fitness

Allocation concealment: allocation schedule held offsite

Blinding: assessor blinded to group allocation

ITT: unclear what was done

Measurements: at the end of intervention (3 months). We excluded data at 6 - and 12-month follow-up as other exposures started at the end of the 3-month exercise intervention

Withdrawals: 2/33 (6\%) lost at 3 months in the intervention group (1 high BP, 1 refusal). 1/26 (4\%) lost at 3 months in the control group (discharged home)

\section{Participants}

Randomised: total 59 participants. 33 participants were randomised to intervention group, 26 to control group

Intervention: 33 participants; 20 men (61\%) and 13 women (39\%); mean age: 66.7 (SD 8.8); 50.5 (SD 19.8) days after stroke

Control: 26 participants; 18 men (69\%) and 8 women (31\%); mean age: 63.8 (SD 11.8); 48.5 (SD 19.2) days after stroke

Inclusion criteria: first-ever stroke; age $\leq 80$ years; $3-10$ weeks post stroke; able to carry out simple instructions; able to pedal a MOTOmed cycling leg trainer at 50 revolutions/min

Exclusion criteria: pre-existing neurologic disorders with impaired functionality; existing pre-stroke; pre-stroke $\mathrm{Bl}<50$; absolute contraindications for exercise testing

Interventions

Intervention: cardiorespiratory cycling exercise using a МОTOmed seated cycling ergometer. Sessions were in intervals weeks 1-8, and continuous weeks 9-12 all performed at 50 pedal revolutions/min or at a self-chosen frequency but maintaining the target HR training zone. Intensity progressed from $60 \%-75 \%$ HR reserve. Frequency of intervention: $30 \mathrm{~min} /$ day for 3 days/week for 3 months. Total session time starting at $51 \mathrm{~min}$ reduced to $40 \mathrm{~min}$ as intensity increased.

The intervention group also received 4 educational sessions (weeks 3, 6, 8, 12) about stroke and risk factors, importance of an active lifestyle, how to increase physical activity and cardiorespiratory capacity during and after the study

Control: passive, supine mobilisation therapy of paretic hip and knee: $30 \mathrm{~min} /$ day for 3 days/week for 3 months 
Vanroy 2017 (Continued)

Setting: inpatient rehabilitation centre or at home if discharged

Outcomes Included outcomes: cardiorespiratory fitness (peak workload and oxygen uptake), mobility (FAC, preferred walking speed $(10 \mathrm{~m})$, maximum walking speed $(10 \mathrm{~m})$

Other outcomes: knee extension strength (omitted as a handheld dynamometer was used). In second paper measures of physical activity (steps, energy expenditure SenseWear) and also self-reported physical activity (diary, Physical Activity Scale for Individuals with Physical Disabilities). Also Baecke Questionnaire of Habitual Physical Activity only used at 12 months which is not end of intervention

Notes Originally included as 2017 ePub; no trial registry entry

\section{Risk of bias}

\begin{tabular}{|c|c|c|}
\hline Bias & Authors' judgement & Support for judgement \\
\hline $\begin{array}{l}\text { Random sequence genera- } \\
\text { tion (selection bias) }\end{array}$ & Low risk & Computer-based permuted block method \\
\hline $\begin{array}{l}\text { Allocation concealment } \\
\text { (selection bias) }\end{array}$ & Low risk & Allocation schedule was offsite \\
\hline $\begin{array}{l}\text { Blinding (performance } \\
\text { bias and detection bias) } \\
\text { All outcomes }\end{array}$ & High risk & $\begin{array}{l}\text { Attention control balances the groups however participants were aware of the } \\
\text { different intervention programmes }\end{array}$ \\
\hline $\begin{array}{l}\text { Blinding of outcome as- } \\
\text { sessment (detection bias) } \\
\text { All outcomes }\end{array}$ & Low risk & $\begin{array}{l}\text { The outcome assessors were blinded. Participants were aware of different pro- } \\
\text { grammes but instructed not to inform the assessor }\end{array}$ \\
\hline $\begin{array}{l}\text { Incomplete outcome data } \\
\text { (attrition bias) } \\
\text { End of intervention }\end{array}$ & Low risk & ITT not described but very low attrition \\
\hline $\begin{array}{l}\text { Selective reporting (re- } \\
\text { porting bias) }\end{array}$ & Unclear risk & $\begin{array}{l}\text { All outcomes specified in the methods were reported, however no pre-pub- } \\
\text { lished protocol documents or trial registry entries }\end{array}$ \\
\hline Other bias & Low risk & $\begin{array}{l}\text { The intervention group also received } 4 \text { educational sessions (weeks } 3,6,8 \text {, } \\
\text { 12) relating to continuing the intervention after it had finished, since this is fo- } \\
\text { cused on the period after our outcome of interest at the end of the interven- } \\
\text { tion it is unlikely that it led to bias }\end{array}$ \\
\hline Imbalanced exposure & Low risk & Balanced exposure \\
\hline
\end{tabular}

\section{Verheyden 2009}

Design: randomised trial of functional strength training plus UC vs UC
Randomisation: simple randomisation
Allocation concealment: information not included
Blinding: assessor blinded
ITT: not completed
Measurements: before and end of intervention (5 weeks)
Withdrawals: none, however 2 participants received fewer hours of intervention therapy due to ear-
ly discharge. In the control group, 3 participants were discharged early and therefore received fewer
hours of UC. All participants were evaluated before discharge and included in analysis


Verheyden 2009 (Continued)

Intervention: 17 participants; 11 men, 6 women; mean age: (combined men and women) 55 years (SD 11); mean height: unknown; mean weight: unknown; type of stroke: ischaemic 15 , haemorrhagic 2 ; paretic side: right 9, left 8; time since stroke onset: 53 (SD 24) days

Control: 16 participants; 9 men, 7 women; mean age: (combined men and women) 62 years (SD 14); mean height: unknown; mean weight: unknown; type of stroke: ischaemic 13, haemorrhagic 3; paretic side: right 7, left 9; time since stroke onset: 49 (SD 28) days

Inclusion criteria: stroke-related hemiparesis. Full recovery from an earlier stroke

Exclusion criteria: an age of $\geq 80$ years; unable to understand instructions; other disorders that could affect motor performance or an ability to obtain maximum trunk performance

Interventions

Intervention: participants in the training group $(n=17)$ received resistance training for the trunk for 30 min, 4 times/week for 5 weeks. Seated exercises included selective movements of the upper and lower part of the trunk in supine and in sitting. In addition conventional multidisciplinary stroke rehabilitation, such as neuro-developmental treatment and motor learning strategies, was provided. No other details were reported.

Control: participants in the control group $(n=16)$ received conventional multidisciplinary stroke rehabilitation, such as neuro-developmental treatment and motor learning strategies. No other details were reported

Setting: rehabilitation centre

Included outcomes: Trunk Impairment Scale
Other outcomes: Tinetti Scale (only reported at baseline)

Notes -

\section{Risk of bias}

Bias Authors' judgement Support for judgement

Random sequence genera- Unclear risk Method described only as simple randomisation by personnel not involved in tion (selection bias) the study

\begin{tabular}{lll}
\hline $\begin{array}{l}\text { Allocation concealment } \\
\text { (selection bias) }\end{array}$ & Unclear risk & Not reported in paper \\
\hline $\begin{array}{l}\text { Blinding (performance } \\
\text { bias and detection bias) } \\
\text { All outcomes }\end{array}$ & High risk & $\begin{array}{l}\text { "...patients nor the physiotherapists who delivered the interventions were } \\
\text { blinded" }\end{array}$ \\
\hline
\end{tabular}

All outcomes

Blinding of outcome as- Low risk Assessor blinded

sessment (detection bias)

All outcomes

Incomplete outcome data Low risk

(attrition bias)

End of intervention

\begin{abstract}
A total of 2 participants from the intervention group completed 3 and $4 \mathrm{~h}$ fewer of additional therapy due to early discharge. The participants were evaluated before discharge and included in the analysis. A total of 3 participants from the control group were discharged early after 21,23 , and 25 days respectively and therefore received fewer $h$ of CPT (number of fewer $h$ not reported)
\end{abstract}

Although ITT was not referred to specifically there were "no dropouts during the course" of the study, and "... all participants were evaluated before discharge."
Selective reporting (re-
Unclear risk
Trial register or protocol not available porting bias)

\begin{tabular}{lll}
\hline Other bias $\quad$ Low risk None known \\
\hline
\end{tabular}


Verheyden 2009 (Continued)

Imbalanced exposure High risk Imbalanced exposure

Wang 2014

Methods Design: randomised trial of cardiovascular training plus UC vs UC - during UC

Randomisation: sealed card selection by participants

Allocation concealment: information not included

Blinding: investigators were not blinded, outcome assessors and therapists were blinded

ITT: not completed

Measurements: before and end of intervention (6 weeks)

Withdrawals: in the intervention group, unclear if up to 9 participants withdrew due to lower leg dis-

comfort during intervention, transfer to another hospital or withdrawal from study

Participants

54 participants randomised: 27 were randomised to intervention, 27 to control

Intervention: 23 participants; 19 men, 8 women; mean age: (combined men and women) 54 years (SD

7.2); mean height: unknown; mean weight: $71.1 \mathrm{~kg}$ (SD 10.2); type of stroke: ischaemic 15, haemorrhag-

ic 12; paretic side: right 15 , left 12; time since stroke onset: 1-6 months

Control: 27 participants; 17 men, 10 women: mean age: (combined men and women) 52 years (SD

12.1); mean height: unknown; mean weight: $75.2 \mathrm{~kg}$ (SD8.1); type of stroke: ischaemic 16, haemorrhagic

11; paretic side: right 18, left 9; time since stroke onset: 1-6 months

Inclusion criteria: time since stroke onset of 1-6 months; age > 45 years; severely impaired with the affected leg marked $\leq 3$ on the 7-point Chedoke-McMaster Stroke Assessment scale; unable to walk even with walk aids; the unaffected leg can move against normal resistance; fasting glucose $<7 \mathrm{mmol} / \mathrm{L}$; no physician diagnosed diabetes; never using medications that may significantly alter HR and blood glucose level; and able to understand the purpose and content of the study

Exclusion criteria: signs and symptoms of subarachnoid haemorrhage, transient ischaemic attack, severe cerebral oedema, $\mathrm{O}_{2}$ dependence, angina, unstable cardiac conditions, peripheral arterial occlusive disease, abnormal high fever, severe pneumonia, high BP > 200/110 mmHg, dementia, aphasia operationally defined as incapacity to follow 2-point commands, untreated major depression, and other medical conditions that precluded participation in exercise training

Interventions

Intervention: participants in the training group $(n=23)$ received conventional stroke rehabilitation 5 days/week for 6 weeks, ( 3 × 40-min physical therapy sessions; $2 \times 15$-min OT sessions; 1 × 30-min acupuncture or traditional Chinese manipulation session; and $1 \times 30$-min physical agents therapy session). 1 x 40-min physical training session was replaced by low-intensity aerobic cardiovascular training 3 days a week for 6 weeks using a cycle ergometer. Cycling training consisted of 30 min sessions including: 5 min warm-up; 30 min active pedaling at an intensity based on an incremental graded exercise test ( $2.5 \mathrm{~W}$ ramp every $3 \mathrm{~min}$ maintaining $50 \mathrm{rpm}$ until exhaustion); followed by 5 -min cool down. Target HR was calculated as ((peak HR in graded exercise test - resting HR) x 50\% to 70\%) + resting HR

Control: participants in the training group $(n=27)$ received conventional stroke rehabilitation 5 days/ week for 6 weeks, ( 3 x 40-min physical therapy sessions; 2 × 15-min OT sessions; 1 × 30-min acupuncture or traditional Chinese manipulation session; and $1 \times 30$-min physical agents therapy session)

Setting: rehabilitation centre

Outcomes

Included outcomes: BI; exercise test time; glucose tolerance variables (fasting glucose; fasting insulin; 2-h plasma glucose; homeostasis model assessment-insulin resistance index), and serum lipid profiles (total triglycerides, HDL cholesterol, LDL cholesterol)

Other outcomes: Fugl-Meyer motor scores; peak and resting HR 
Wang 2014 (Continued)

Bias Authors' judgement Support for judgement

Random sequence genera- Unclear risk 1 of 2 cards selected from a sealed envelope by participants

tion (selection bias)

\begin{tabular}{ll}
\hline $\begin{array}{l}\text { Allocation concealment } \\
\text { (selection bias) }\end{array}$ & Unclear risk $\quad$ Not reported in paper
\end{tabular}

\begin{tabular}{lll}
\hline $\begin{array}{l}\text { Blinding (performance } \\
\text { bias and detection bias) } \\
\text { All outcomes }\end{array}$ & High risk & Therapists blinded but there was imbalanced intervention exposure \\
\hline $\begin{array}{l}\text { Blinding of outcome as- } \\
\text { sessment (detection bias) } \\
\text { All outcomes }\end{array}$ & Low risk & Outcome measures administered by a blinded rater \\
\hline $\begin{array}{l}\text { Incomplete outcome data } \\
\text { (attrition bias) } \\
\text { End of intervention }\end{array}$ & High risk & $\begin{array}{l}\text { 9/54 (17\%) overall losses } \\
\text { A total of up to 9 participants withdrew due to discomfort, hospital transfer, } \\
\text { and general withdrawal from study. Unclear how these withdrawals were dis- } \\
\text { tributed between intervention and control groups. No details given of whether } \\
\text { pre-intervention assessment data were included in analysis }\end{array}$ \\
\hline $\begin{array}{l}\text { Selective reporting (re- } \\
\text { porting bias) }\end{array}$ & Unclear risk & $\begin{array}{l}\text { All 3 criteria for ITT analysis have not been met - methods suggest a per-proto- } \\
\text { col analysis }\end{array}$ \\
\hline $\begin{array}{l}\text { Other bias } \\
\text { Imbalanced exposure }\end{array}$ & High risk & $\begin{array}{l}\text { Trial register or protocol not available } \\
\text { Intervention group was exposed to an additional exposure volume on top of } \\
\text { UC PT }\end{array}$ \\
\hline
\end{tabular}

\section{Winstein 2004}

Design: randomised trial of resistance training plus UC vs UC - during and after UC
Randomisation mechanism: unknown; method: stratified on Orpington Prognostic Scale (1.6-1.4 and
$4.2-6.8$ )
Allocation concealment: sealed envelopes
Blinding: principal investigator but not outcome assessor
ITT: no
Measurements: end of intervention (4-6 weeks) and 9-month post-stroke follow-up
Withdrawals: before end of intervention: 1 (treatment group, medical complications), 1 (control group,
lost interest); before end of follow-up: 9 (treatment group 4, control group 5 - moved away or lost con-
tact)
Randomised: 42 participants
Intervention: 21 participants; 12 men and 8 women; time since stroke 17.3 days (SD 10.6)
Control: 20 participants; 2 men and 8 women; time since stroke 15.4 days (SD 5.5)
Age: $29-76$ years, most 35-75 years
Inclusion criteria: first stroke; 2 -35 days post-stroke; FIM score
Exclusion criteria: peripheral nerve or orthopaedic condition limiting arm movement; function limited
by cardiac disease; subarachnoid haemorrhage without infarction; progressive hydrocephalus; history
of brain injury; severe aphasia, neglect, agitation or depression, which could limit participation


Winstein 2004 (Continued)

Interventions

Intervention: upper limb movements resisted by gravity, free weights, TheraBand and grip devices for fingers, $60 \mathrm{~min} /$ day 5 days/week for 4-6 weeks, high-intensity for 3 days/week and low-intensity higher velocity for 2 days/week, training target $20 \mathrm{~h}$ total

Control: standard care delivered by OT, included muscle facilitation exercises using neuro-developmental approach, electrical stimulation, stretching, $A D L$ and caregiver training; activities included use of upper limbs Setting: inpatient rehabilitation hospital and outpatient clinic

\section{Outcomes}

Included outcomes: FIM (mobility and self-care scores); FTHUE; composite measure of strength (sum of torque from extension and flexion of the wrist elbow and shoulder); grip and pinch force

Other outcomes: Fugl-Meyer scores

Notes Change from baseline scores reported and analysed

\section{Risk of bias}

\begin{tabular}{|c|c|c|}
\hline Bias & Authors' judgement & Support for judgement \\
\hline $\begin{array}{l}\text { Random sequence genera- } \\
\text { tion (selection bias) }\end{array}$ & Unclear risk & $\begin{array}{l}\text { Mechanism unknown; stratification based on Orpington Prognostic Scale } \\
\text { (1.6-1.4 and 4.2-6.8) }\end{array}$ \\
\hline $\begin{array}{l}\text { Allocation concealment } \\
\text { (selection bias) }\end{array}$ & Unclear risk & Envelopes; opaque, sealed, numbered is unknown \\
\hline $\begin{array}{l}\text { Blinding (performance } \\
\text { bias and detection bias) } \\
\text { All outcomes }\end{array}$ & High risk & $\begin{array}{l}\text { No suitable attention control } \\
\text { Quote: "This treatment regimen was separate (i.e. it was added to the stan- } \\
\text { dard dose of occupational and physical therapy)." }\end{array}$ \\
\hline $\begin{array}{l}\text { Blinding of outcome as- } \\
\text { sessment (detection bias) } \\
\text { All outcomes }\end{array}$ & High risk & $\begin{array}{l}\text { Outcome assessor not blinded } \\
\text { Outcome measures related to muscle strength are biased due to use of a } \\
\text { hand-held dynamometer }\end{array}$ \\
\hline $\begin{array}{l}\text { Incomplete outcome data } \\
\text { (attrition bias) } \\
\text { End of intervention }\end{array}$ & Low risk & $\begin{array}{l}\text { ITT not reported } \\
2 / 42(5 \%) \text { losses at the end of intervention: } 1 \text { treatment group (medical com- } \\
\text { plications), } 1 \text { control group (lost interest) }\end{array}$ \\
\hline $\begin{array}{l}\text { Incomplete outcome data } \\
\text { (attrition bias) } \\
\text { End of follow-up }\end{array}$ & High risk & $\begin{array}{l}\text { ITT not reported } \\
11 / 42(26 \%) \text { losses at the end of follow-up: } 4 \text { intervention group; } 5 \text { control } \\
\text { group (moved away or lost contact) }\end{array}$ \\
\hline $\begin{array}{l}\text { Selective reporting (re- } \\
\text { porting bias) }\end{array}$ & Unclear risk & Protocol not available \\
\hline Other bias & Low risk & None known \\
\hline Imbalanced exposure & High risk & Imbalanced exposure \\
\hline
\end{tabular}

Yang 2006

Design: randomised trial of mixed training vs no intervention - after UC
Randomisation mechanism: picking envelopes
Allocation concealment: sealed envelopes
Blinding: investigator


Yang 2006 (Continued)

\section{ITT: unknown}

Measurements: end of intervention (4 weeks)

Withdrawals: none

\begin{tabular}{ll}
\hline Participants & Randomised: 48 participants \\
Intervention: 24 participants; 16 men and 8 women; age 56.8 years (SD 10.2); time since stroke $>1$ year \\
Control: 24 participants; 18 men and 8 women; age 60 years (SD 10.4); time since stroke $>1$ year \\
Inclusion criteria: first stroke $\geq 1$ year ago; not receiving rehabilitation; ambulatory, independent with \\
no aids; medically stable to participate; able to understand instructions and follow commands \\
Exclusion criteria: medical condition preventing participation; uncontrolled health condition for which \\
exercise was contraindicated
\end{tabular}

Interventions Intervention: mixed training performed as a circuit $30 \mathrm{~min} /$ day 3 days/week for 4 weeks; circuit comprised $6 \times 5$-min lower extremity workstations (standing and reaching, sit-to-stand from chair, stepping forwards and backwards onto blocks, stepping sideways onto blocks, forward step-up onto blocks), participants encouraged to work hard, progression achieved by increasing number of repetitions in each 5-min block, and increasing step and chair height, and the complexity of task; extended periods (5-min) warrant acknowledgement of a cardiorespiratory component despite the author's title (progressive resistance strength training)

Control: no intervention

Included outcomes: gait endurance (6-MWT - outcome assessor not blinded); gait speed preferred (10
m); 3 metre TUG; step test; isometric strength of knee and hip ankle extension and flexion; and ankle
dorsi-flexion and plantar-flexion (using handheld dynamometer)
Other outcomes: gait cadence and stride length

Notes

Study authors stated "strength training" but intervention was actually mixed training. Data reported as absolute and change scores

\section{Risk of bias}

\begin{tabular}{|c|c|c|}
\hline Bias & Authors' judgement & Support for judgement \\
\hline $\begin{array}{l}\text { Random sequence genera- } \\
\text { tion (selection bias) }\end{array}$ & Unclear risk & $\begin{array}{l}\text { Quote: "...independent person who picked one of the sealed envelopes } 30 \mathrm{~min} \\
\text { before the start of the intervention." }\end{array}$ \\
\hline $\begin{array}{l}\text { Allocation concealment } \\
\text { (selection bias) }\end{array}$ & Unclear risk & Sealed envelopes; opaque and numbered not reported \\
\hline $\begin{array}{l}\text { Blinding (performance } \\
\text { bias and detection bias) } \\
\text { All outcomes }\end{array}$ & High risk & No attention control \\
\hline $\begin{array}{l}\text { Blinding of outcome as- } \\
\text { sessment (detection bias) } \\
\text { All outcomes }\end{array}$ & Low risk & Investigator blinded \\
\hline $\begin{array}{l}\text { Incomplete outcome data } \\
\text { (attrition bias) } \\
\text { End of intervention }\end{array}$ & Low risk & $\begin{array}{l}\text { ITT not reported } \\
\text { No losses }\end{array}$ \\
\hline $\begin{array}{l}\text { Selective reporting (re- } \\
\text { porting bias) }\end{array}$ & Unclear risk & Protocol not available \\
\hline Other bias & Low risk & None known \\
\hline Imbalanced exposure & High risk & Imbalanced exposure \\
\hline
\end{tabular}




Methods
tient)
Randomisation: computer-generated randomisation
Allocation concealment: sealed envelopes
Blinding: outcome assessor
ITT: not completed

Measurements: before and end (4 weeks) of intervention

Withdrawals: in the intervention group, 1 participant withdrew due to a fall at home

\section{Participants}

30 participants randomised: 15 participants were randomised to intervention, 15 to control Intervention: 15 participants; 9 men, 6 women; mean age: (combined men and women) 53.9 years (SD 10.5); mean height: unknown; mean weight: unknown; type of stroke: ischaemic 9 , haemorrhagic 11 ; paretic side: right 11, left 4; time since stroke onset: 11.1 months (SD 8.1)

Control: 15 participants; 13 men, 2 women: mean age: (combined men and women) 54.5 years (SD 8.0); mean height: unknown mean weight: unknown; type of stroke: ischaemic 8, haemorrhagic 7; paretic side: right 8, left 7; time since stroke onset: 11.1 months (SD 9.7)

Inclusion criteria: first ever stroke; stroke onset > 3 months; unilateral hemiplegia; between 18-70 years of age; ability to walk $10 \mathrm{~m}$ with or without assistance; zero score of 3 levels of the National Institute of Health Stroke Scale

Exclusion criteria: patients with aphasia who were unable to follow instructions; blindness or visual impairment; musculoskeletal disorders; cardiac disorders and peripheral neuropathy $1 \mathrm{~h} \mathrm{OT}$ ). In addition extra cardiovascular training was given for $30 \mathrm{~min}, 5$ times/week for 4 weeks. Cycling training consisted of 15 min sessions each of forward and backward cycling including: 150-s passive warm-up; 10-min active pedaling at 50-70 rpm at an intensity of stage 13 of the Borg scale; 150 seconds of passive cool-down

Control: 15 participants in the control group received conventional stroke rehabilitation (1 h PT; $1 \mathrm{~h} \mathrm{OT)}$

Setting: rehabilitation centre - usual outpatient care

Outcomes Included outcomes: 6-MWT; comfortable walking speed using 10-metre walk test Other outcomes: lower limb subscale of Fugl-Meyer assessment (LE-FMA); modified Ashworth scale

Notes The first iteration of this cross-over study is equivalent to a RCT

The data in this paper, which can be analysed as a 2-group RCT, correspond to 'Group A' (intervention n

$=15$ per protocol) and 'Group B' (control $n=15)$ at the end of the first iteration ('T2')

Authors indicate the 10-MWT was at a comfortable speed not a maximal speed

\section{Risk of bias}

\begin{tabular}{lll}
\hline Bias & Authors' judgement & Support for judgement \\
\hline $\begin{array}{l}\text { Random sequence genera- } \\
\text { tion (selection bias) }\end{array}$ & Low risk & Quote: "... computer-generated random numbers" \\
\hline $\begin{array}{l}\text { Allocation concealment } \\
\text { (selection bias) }\end{array}$ & Unclear risk & $\begin{array}{l}\text { Some evidence of concealment but the description is inadequate; only report- } \\
\text { ed as Quote: "... held in sealed envelopes by an independent individual" }\end{array}$ \\
\hline
\end{tabular}


Yang 2014 (Continued)
Blinding (performance
High risk
Imbalanced exposure
bias and detection bias)

All outcomes

\begin{tabular}{|c|c|c|}
\hline $\begin{array}{l}\text { Blinding of outcome as- } \\
\text { sessment (detection bias) } \\
\text { All outcomes }\end{array}$ & Low risk & Blinded rater used \\
\hline
\end{tabular}

\begin{tabular}{|c|c|c|}
\hline $\begin{array}{l}\text { Incomplete outcome data } \\
\text { (attrition bias) } \\
\text { End of intervention }\end{array}$ & Low risk & $\begin{array}{l}\text { 1/30 (3\%) total dropouts } \\
\text { A total of } 1 \text { participant withdrew due to a fall at home; no details given of } \\
\text { whether pre-intervention assessment data were included in analysis } \\
\text { All } 3 \text { criteria for ITT analysis have not been met - methods suggest a per-proto- } \\
\text { col analysis, however only } 1 \text { participant affected }\end{array}$ \\
\hline $\begin{array}{l}\text { Selective reporting (re- } \\
\text { porting bias) }\end{array}$ & Unclear risk & Trial register or protocol not available \\
\hline Other bias & Low risk & None known \\
\hline Imbalanced exposure & High risk & Imbalanced exposure \\
\hline
\end{tabular}

Zedlitz 2012

Methods

Design: multicentre randomised trial of mixed training plus non-exercise intervention vs non-exercise intervention - after UC

Randomised: block randomisation; implemented individually but also as a cluster when numbers were low

Allocation concealment: sealed envelopes

Blinding: assessor blind to group allocation

ITT: yes

Measurements: end of intervention (12 weeks) and end of 6-month follow-up

Withdrawals: 1 participant withdrew consent before allocation into group; intervention group (5 participants, 3 withdrew consent before end of intervention, 1 participant withdrew due to poor health before end of intervention; 1 participant withdrew due to recurrent stroke before follow-up); control group ( 6 participants, 3 withdrew consent, 1 got new job; 1 family emergency, 1 participant recurrent stroke all before end of intervention; 4 participants lost to follow-up)

Participants

Randomised: 84 participants

Intervention: 38 participants ( 1 withdrew consent); 22 men and 23 women; mean age 54.8 years (SD 9.1); 4.4 years post-stroke (SD 4.2)

Control: 45 participants; 21 men and 17 women; mean age 55.6 years (SD 8.8); 3.3 years post-stroke (SD 3.9)

Inclusion criteria: sustained stroke > 4 months; reported severe fatigue; between ages 18-70 years; able to walk independently

Exclusion criteria: severe cognitive deficits; severe comorbidity (cardiac disease, pulmonary disease); depression 
Zedlitz 2012 (Continued)

Interventions

Invention group: treadmill walking and strength training ranging from 40\%-70\% maximum $\mathrm{HR}$ for $2 \mathrm{~h}$ twice/week for 12 weeks

Control group: non-exercise control intervention (cognitive therapy)

Setting: 8 rehabilitation centres

\begin{tabular}{ll}
\hline Outcomes & Included outcomes: Checklist Individual Strength-subscale Fatigue; HADS; SIS; 6-MWT \\
\hline Notes & - \\
\hline
\end{tabular}

\section{Risk of bias}

\begin{tabular}{lll}
\hline Bias & Authors' judgement & Support for judgement \\
\hline $\begin{array}{l}\text { Random sequence genera- } \\
\text { tion (selection bias) }\end{array}$ & Unclear risk & $\begin{array}{l}\text { Randomisation implemented (individually) in groups of } 8 \text { in each centre by } \\
\text { picking } 1 \text { of } 8 \text { sealed envelopes. If only 4 participants were available in } 1 \text { centre } \\
\text { then they were allocated as a group (cluster) }\end{array}$ \\
\hline $\begin{array}{l}\text { Allocation concealment } \\
\text { (selection bias) }\end{array}$ & Unclear risk & Full nature and use of envelopes is unclear \\
\hline $\begin{array}{l}\text { Blinding (performance } \\
\text { bias and detection bias) } \\
\text { All outcomes }\end{array}$ & High risk & No attention control \\
\hline
\end{tabular}

\begin{tabular}{|c|c|c|}
\hline $\begin{array}{l}\text { Blinding of outcome as- } \\
\text { sessment (detection bias) } \\
\text { All outcomes }\end{array}$ & Low risk & Blinded assessors used \\
\hline $\begin{array}{l}\text { Incomplete outcome data } \\
\text { (attrition bias) }\end{array}$ & Unclear risk & ITT analyses used \\
\hline End of intervention & & $\begin{array}{l}\text { 11/84 (13\%) losses: intervention group ( } 5 \text { participants, } 3 \text { withdrew consent be- } \\
\text { fore end of intervention, } 1 \text { participant withdrew due to poor health before end } \\
\text { of intervention); control group ( } 6 \text { participants, } 3 \text { withdrew consent, } 1 \text { got new } \\
\text { job; } 1 \text { family emergency, } 1 \text { participant recurrent stroke all before end of inter- } \\
\text { vention) }\end{array}$ \\
\hline
\end{tabular}

\begin{tabular}{ll}
\hline $\begin{array}{l}\text { Incomplete outcome data } \\
\text { (attrition bias) }\end{array}$ & High risk \\
End of follow-up & ITT analyses used \\
& $\begin{array}{l}16 / 84(19 \%) \text { total losses: intervention group (1 participant withdrew due to } \\
\text { recurrent stroke before follow-up); control group (4 participants lost to fol- } \\
\text { low-up) }\end{array}$
\end{tabular}

\begin{tabular}{|c|c|c|}
\hline $\begin{array}{l}\text { Selective reporting (re- } \\
\text { porting bias) }\end{array}$ & Low risk & $\begin{array}{l}\text { Included outcomes correspond to trial registry NTR2704. Some proposed cog- } \\
\text { nitive outcomes not present in publication }\end{array}$ \\
\hline
\end{tabular}

\begin{tabular}{lll}
\hline Other bias & High risk & Self-report questionnaires used \\
& $\begin{array}{l}\text { Monitoring period before randomisation to identify those with potentially } \\
\text { poor compliance } \\
\text { Risk of self-selection bias as newspaper adverts used for recruitment }\end{array}$ \\
\hline Imbalanced exposure & High risk & Imbalanced exposure \\
\hline
\end{tabular}


Methods
Design: RCT. Resistance training vs attention control - after UC

Randomisation: randomised by selection of sealed envelopes. Block sampling to ensure similar group sizes

Allocation concealment: sealed envelopes used (not clear if sequentially numbered or opaque)

Blinding: study investigators not blinded, outcome assessors blinded, participants may have understood nature of group assignments

ITT: no pre-planned ITT analysis; outcome presented per-protocol

Measurements: at the end of intervention ( 8 weeks)

Withdrawals: $2 / 28$ (7\%) participants from the intervention group (2 withdrew due to muscle fatigue). $3 / 28$ (11\%) participants from the control group (3 due to moving to another location)
Interventions
Randomised: 56 participants were randomised

Intervention: 28 participants; 13 men (46\%) and 15 women (54\%); mean age: 52.3 (SD 6.9); 15 (SD 6.2) months after stroke

Control: 28 participants; 9 men (32\%) and 19 women (68\%); mean age: 51.4 (SD 7.2); 8 (SD 5.1) months after stroke

Inclusion criteria: age $<60$ years, nondiabetic, $>6$ months after stroke onset disabled stroke patients who had completed CPT. Baseline characteristics indicate at least 3 weeks of rehabilitation training and BI score $>60$

Exclusion criteria: diabetes, heart failure, unstable angina, dementia, and aphasia

Intervention: resistance training; 3 sets of 15 repetitions of leg press, leg extension and leg curl using resistance training machines. Legs were trained unilaterally and alternately. Resistance was progressed every 2 weeks. Frequency of intervention: $40 \mathrm{~min} /$ day for 3 days/week for 8 weeks

Control: active and passive upper and lower body stretching exercises. Frequency of intervention: 40 $\mathrm{min} /$ day for 3 days/week for 8 weeks

Setting: outpatient clinics of 2 hospitals

Outcomes

Included outcomes: risk factors (blood glucose control indices, serum lipids, BMI); muscle strength; Fugl-Meyer scores

Other outcomes: none

Notes No pre-published trial registry entry

\section{Risk of bias}

\begin{tabular}{lll}
\hline Bias & Authors' judgement & Support for judgement \\
\hline $\begin{array}{l}\text { Random sequence genera- } \\
\text { tion (selection bias) }\end{array}$ & Unclear risk & Physical method suggested but no mechanism actually described \\
\hline $\begin{array}{l}\text { Allocation concealment } \\
\text { (selection bias) }\end{array}$ & High risk & $\begin{array}{l}\text { Sealed envelopes not stated as opaque (or numbered) plus unblinded investi- } \\
\text { gators could make this vulnerable }\end{array}$ \\
\hline $\begin{array}{l}\text { Blinding (performance } \\
\text { bias and detection bias) } \\
\text { All outcomes }\end{array}$ & Unclear risk & $\begin{array}{l}\text { Those delivering intervention were blinded to baseline data and group assign- } \\
\text { ment. Attention control was used but study authors indicated participants } \\
\text { may have known which group they belonged to }\end{array}$ \\
\hline
\end{tabular}


Zou 2015 (Continued)

Blinding of outcome as- Low risk Outcome assessors and therapists were blinded to baseline data and group assessment (detection bias) signment

All outcomes

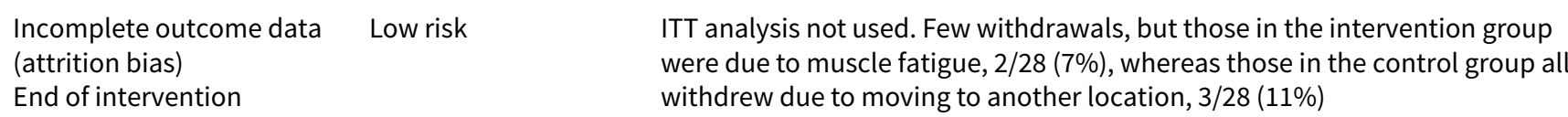

End of intervention withdrew due to moving to another location, $3 / 28(11 \%)$

$\begin{aligned} & \text { Selective reporting (re- } \\ & \text { porting bias) }\end{aligned}$
Unclear risk $\quad$ Trial registry or protocol not available

\begin{tabular}{|c|c|c|}
\hline Other bias & Unclear risk & $\begin{array}{l}\text { Not all participants completed all training content some of the stretches were } \\
\text { active as well as passive }\end{array}$ \\
\hline
\end{tabular}

Imbalanced exposure Low risk Balanced attention control

6-MWT: 6-Minute Walk Test; 9-HPT: 9-Hole Peg Test; 12-MWT: 12-minute walk test; ABCS: Activities-Specific Balance Confidence Scale; ACSM: American College of Sports Medicine; ADL: activities of daily living; AQoL: Assessment of Quality of Life Instrument; ARAT: Action Research Arm Test; BBS: Berg Balance scale; BDI: Beck Depression Inventory; BI: Barthel Index; BMI: body mass index; BP: blood pressure; bpm: beats per minute;BWSTT: body weight supported treadmill training; CES-D: Centre for Epidemiological Studies Depression scale; CRPS: complex regional pain syndrome; CT: computerised tomography; CPT: conventional physiotherapy; DBP: diastolic blood pressure; ECG: electrocardiogram; EMS: Elderly Mobility Scale; FAC: Functional Ambulation Classification; FAI: Frenchay Activity Index; FAME: familymediated exercise; FAPS: Functional Ambulation Profile Score; FIM: Functional Independence Measure; FST: functional strength training; FTHUE: Functional Test of the Hemiparetic Upper Extremity; HADS: Hospital Anxiety and Depression Scale; HDL: high-density lipoprotein; HR: heart rate; HRQoL: health-related quality of life; IQR: interquartile range; ITT: intention-to-treat; LDL: low-density lipoprotein; LOCF: last observation carried forward; LOT-R: Life Orientation Test - Revised; MAS: Motor Assessment Scale; MI: myocardial infarction; MMSE: Mini Mental State Examination; MRI: magnetic resonance imaging; mRS: modified Rankin Scale; NEADL: Nottingham Extended Activities of Daily Living; NHP: Nottingham Health Profile; NHS: National Health Service; OT: occupational therapy; PADS: Peripheral Arterial Diseases Walking Impairment questionnaire; PT: physiotherapy; QoL: quality of life; RCT: randomised controlled trial; RLOC: Recovery Locus of Control Scale; RM: repetition maximum; RMA: Rivermead Motor Assessment; RMI: Rivermead Mobility Index; RPE: rate of perceived exertion; RPM: revolutions per minute; SBP: systolic blood pressure; SD: standard deviation; SF-12: Short Form-12 Health Survey Questionnaire; SF-36: Short Form 36 Health Survey; SIS: Stroke Impact Scale; SSS: Scandinavian Stroke Scale; SWLS: Satisfaction with Life Scale; TUG: timed up and go test; UC: usual care; WHO: World Health Organization

Characteristics of excluded studies [ordered by study ID]

\begin{tabular}{ll}
\hline Study & Reason for exclusion \\
\hline Alabdulwahab 2015 & Wrong control group \\
\hline Askim 2018 & Co-intervention \\
\hline Awad 2015 & Wrong control group \\
\hline Baer 2018 & Wrong type of intervention \\
\hline Bang 2014 & Wrong type of intervention \\
\hline Bernhardt 2018 & Wrong type of intervention \\
\hline Boss 2017 & Co-intervention \\
\hline Brauer 2018 & Co-intervention \\
\hline Brouwer 2018 & Wrong type of intervention \\
\hline
\end{tabular}




\begin{tabular}{|c|c|}
\hline Study & Reason for exclusion \\
\hline Buyukavci 2016 & Wrong type of intervention \\
\hline Cabanas-Valdés 2017 & Wrong type of intervention \\
\hline Choi 2015 & Wrong type of intervention \\
\hline Choi 2017 & Wrong type of intervention \\
\hline Chua 2016 & Wrong type of intervention \\
\hline Dong Hyun 2016 & Wrong type of intervention \\
\hline Dubey 2018 & Wrong type of intervention \\
\hline English 2015 & Wrong type of intervention \\
\hline Faulkner 2014 & Co-intervention \\
\hline Faulkner 2015 & Co-intervention \\
\hline Faulkner 2017a & Co-intervention \\
\hline Graef 2016 & Wrong control group \\
\hline Gunnes 2017 & Cointervention \\
\hline Hahn 2015 & Wrong type of intervention \\
\hline Haruyama 2017 & Wrong type of intervention \\
\hline Hendrey 2018 & Wrong control group \\
\hline Heron 2017 & Wrong type of intervention \\
\hline Hillier 2014 & Wrong type of intervention \\
\hline Hornby 2016 & Wrong control group \\
\hline Hubbard 2015 & Wrong type of intervention \\
\hline Hunter 2018 & Wrong control group \\
\hline Immink 2014 & Wrong type of intervention \\
\hline Kim 2016b & Wrong type of intervention \\
\hline Kim 2017b & Wrong control group \\
\hline Kwon 2015 & Wrong type of intervention \\
\hline Lee 2015 & Wrong control group \\
\hline Lee 2016 & Wrong type of intervention \\
\hline Lee $2017 a$ & Wrong type of intervention \\
\hline
\end{tabular}




\begin{tabular}{|c|c|}
\hline Study & Reason for exclusion \\
\hline Lee $2017 b$ & Wrong type of intervention \\
\hline Lee $2018 a$ & Wrong type of intervention \\
\hline Lee $2018 b$ & Wrong type of intervention \\
\hline Lim 2016 & Wrong type of intervention \\
\hline Lim 2017 & Wrong type of intervention \\
\hline Lin 2015 & Wrong control group \\
\hline Linder 2017 & Wrong control group \\
\hline Lund 2018 & Wrong control group \\
\hline Malagoni 2016 & Wrong control group \\
\hline Malik 2018 & Wrong type of intervention \\
\hline Marryam 2017 & Wrong type of intervention \\
\hline Martins 2017 & Wrong type of intervention \\
\hline McDonnell 2017 & Wrong type of intervention \\
\hline Meng 2018 & Wrong type of intervention \\
\hline Ordahan 2015 & Wrong type of intervention \\
\hline Pandian 2015 & Cointervention \\
\hline Park 2015a & Wrong type of intervention \\
\hline Park 2015b & Wrong control group \\
\hline Park 2015c & Wrong control group \\
\hline Park 2016a & Wrong type of intervention \\
\hline Park 2016b & Wrong type of intervention \\
\hline Park 2016c & Wrong type of intervention \\
\hline Park 2016d & Wrong control group \\
\hline Park 2017a & Wrong type of intervention \\
\hline Park 2017b & Wrong control group \\
\hline Paul 2016 & Wrong type of intervention \\
\hline Rand 2015 & Wrong control group \\
\hline Redzuan 2012 & Wrong type of intervention \\
\hline
\end{tabular}




\begin{tabular}{|c|c|}
\hline Study & Reason for exclusion \\
\hline Ribeiro 2017 & Wrong control group \\
\hline Roh 2016 & Wrong type of intervention \\
\hline Rose 2017 & Wrong control group \\
\hline Rose 2018 & Wrong type of intervention \\
\hline Ru 2017 & Co-intervention \\
\hline Schachten 2015 & Wrong type of intervention \\
\hline Seo 2015 & Wrong control group \\
\hline Sharma 2017 & Wrong control group \\
\hline Shin 2016 & Wrong type of intervention \\
\hline Sun 2016 & Wrong control group \\
\hline Tang 2014 & Wrong control group \\
\hline Vahlberg 2017 & Co-intervention \\
\hline Valkenborghs 2016 & Wrong control group \\
\hline Valkenborghs 2017 & Wrong control group \\
\hline Van Criekinge 2017 & Wrong control group \\
\hline Vasileva 2017 & Wrong type of intervention \\
\hline Wang 2015 & Co-intervention \\
\hline Wright 2018 & Wrong control group \\
\hline Zhang 2016 & Wrong control group \\
\hline Zhiyan 2017 & Wrong type of intervention \\
\hline Zhu 2016 & Wrong control group \\
\hline
\end{tabular}

Characteristics of studies awaiting assessment [ordered by study ID]

Brands Guendling 2017

\begin{tabular}{ll}
\hline Methods & RCT \\
\hline Participants & Stroke $\mathrm{n}=20$ \\
\hline Interventions & Arm and leg training \\
\hline Outcomes & PT \\
\hline
\end{tabular}


Brands Guendling 2017 (Continued)
Notes
Abstract only

Chan 2017

\begin{tabular}{ll}
\hline Methods & RCT \\
\hline Participants & Stroke $\mathrm{n}=32$ \\
\hline Interventions & Aquatic exercise + land exercise vs land exercise only \\
\hline Outcomes & BBS, Community Balance and Mobility Score, TUG, 2-MWT \\
\hline Notes & Not clear if land training is outpatient UC \\
\hline
\end{tabular}

Chan 2018

\begin{tabular}{ll}
\hline Methods & RCT \\
\hline Participants & Stroke $\mathrm{n}=$ unclear \\
\hline Interventions & Conventional training arm of study (Tai Chi arm excluded) vs no intervention \\
\hline Outcomes & Stroop test, mobility dual task performance \\
\hline Notes & $\begin{array}{l}\text { Conventional training unclear on content and progression; } 2 \text { connected studies with different re- } \\
\text { ported sample sizes }\end{array}$ \\
\hline
\end{tabular}

Chen 2014

\begin{tabular}{ll}
\hline Methods & RCT \\
\hline Participants & Stroke $n=77$ \\
\hline Interventions & Lower limb exercise vs conventional rehabilitation \\
\hline Outcomes & Muscle power, balance, NIHSS, mRS, BI, number of falls \\
\hline Notes & Abstract only \\
\hline
\end{tabular}

\section{Deshpande 2018}

\begin{tabular}{ll}
\hline Methods & RCT \\
\hline Participants & Stroke $\mathrm{n}=$ unknown \\
\hline Interventions & Task-oriented circuit training \\
\hline Outcomes & Upper limb function \\
\hline \hline
\end{tabular}


Deshpande 2018 (Continued)
Notes
Abstract only

Ellis 2018

\begin{tabular}{ll}
\hline Methods & RCT \\
\hline Participants & Stroke $\mathrm{n}=32$ \\
\hline Interventions & Robotic resistance training \\
\hline Outcomes & Upper limb function, strength, clinical battery \\
\hline Notes & Robot device involved; unclear if this represents physical fitness training \\
\hline
\end{tabular}

Faulkner 2017b

\begin{tabular}{ll}
\hline Methods & RCT \\
\hline Participants & Mixed stroke $n=16 ;$ TIA $n=31$ \\
\hline Interventions & Cardiorespiratory training \\
\hline Outcomes & Secondary prevention UC + education \\
\hline Notes & Subgroup of stroke-only data sought from study authors \\
\hline
\end{tabular}

Floel 2018

\begin{tabular}{ll}
\hline Methods & RCT \\
\hline Participants & Stroke $\mathrm{n}=200$ \\
\hline Interventions & Cardiorespiratory treadmill exercise vs relaxation \\
\hline Outcomes & Gait speed, $\mathrm{BI}$ \\
\hline Notes & Abstract only \\
\hline
\end{tabular}

Frimpong 2014

\begin{tabular}{ll}
\hline Methods & RCT \\
\hline Participants & Stroke $n=20$ \\
\hline Interventions & Circuit training + UC vs UC alone \\
\hline Outcomes & 10-MWT, 6-MWT, FAC \\
\hline
\end{tabular}


Frimpong 2014 (Continued)

Notes

Contacted study author about progression model of exercise intervention

Gezer 2018

\begin{tabular}{ll}
\hline Methods & RCT \\
\hline Participants & Stroke $\mathrm{n}=42$ \\
\hline Interventions & Aerobic exercise \\
\hline Outcomes & Exercise tolerance, respiratory function, 6-MWT, FIM, NHP, BDI, and Pittsburgh Sleep Quality Index \\
\hline Notes & May not be randomised; study author contacted \\
\hline
\end{tabular}

Hwang 2015

\begin{tabular}{ll}
\hline Methods & Randomised cross-over study \\
\hline Participants & Stroke $n=11$ \\
\hline Interventions & Aquatic exercise \\
\hline Outcomes & $\begin{array}{l}\text { Resting HR, mean arterial pressure, respiratory variables, normal gait speed, TUG, psychological } \\
\text { variables relating to affect }\end{array}$ \\
\hline Notes & Need cross-over data from first iteration; study author contacted \\
\hline
\end{tabular}

Jeon 2016

\begin{tabular}{ll}
\hline Methods & RCT \\
\hline Participants & Stroke $n=12$ \\
\hline Interventions & Strength training \\
\hline Outcomes & Range of motion, pain, modified motor assessment score \\
\hline Notes & Nature of control group unclear \\
\hline
\end{tabular}

Kim 2015

\begin{tabular}{ll}
\hline Methods & RCT \\
\hline Participants & Stroke $\mathrm{n}=27$ \\
\hline Interventions & Cycle ergometer \\
\hline Outcomes & BBS, TUG test, 10-MWT \\
\hline \hline
\end{tabular}


Kim 2015 (Continued)
Notes
Not known whether any progression of intervention

Kim 2017c

\begin{tabular}{ll}
\hline Methods & RCT \\
\hline Participants & Stroke $\mathrm{n}=30$ \\
\hline Interventions & Circuit training + UC vs UC + co-intervention? \\
\hline Outcomes & BBS, timed up and go test, FAC, 6-MWT \\
\hline Notes & The control group may incorporate an exercise co-intervention that is not UC \\
\hline
\end{tabular}

\section{Koç 2015}

\begin{tabular}{ll}
\hline Methods & RCT \\
\hline Participants & Stroke $\mathrm{n}=134$ \\
\hline Interventions & Home-based mixed training \\
\hline Outcomes & BI \\
\hline Notes & Progression and intervention content unclear; sought from study authors \\
\hline
\end{tabular}

Kumaran 2016

\begin{tabular}{ll}
\hline Methods & RCT \\
\hline Participants & Stroke $n=62$ \\
\hline Interventions & Context-based exercise programme \\
\hline Outcomes & SIS, 5-MWT, 6-MWT \\
\hline Notes & Abstract only \\
\hline
\end{tabular}

Lawal 2016

\begin{tabular}{ll}
\hline Methods & RCT \\
\hline Participants & Stroke $n=91$ \\
\hline Interventions & Circuit class \\
\hline Outcomes & MMT, Modified Tardieu Scale \\
\hline
\end{tabular}


Lawal 2016 (Continued)
Notes
Abstract only

Maheshwari 2018

\begin{tabular}{ll}
\hline Methods & RCT \\
\hline Participants & Stroke $\mathrm{n}=47$ \\
\hline Interventions & Task-specific exercise with or without strength training \\
\hline Outcomes & Fugl Meyer, MAL, ARAT \\
\hline Notes & Abstract only \\
\hline
\end{tabular}

Matsumoto 2016

\begin{tabular}{ll}
\hline Methods & RCT \\
\hline Participants & Stroke $n=120$ \\
\hline Interventions & Aquatic exercise \\
\hline Outcomes & 10-MWT, Modified Ashworth Scale, SF-36, lower-extremity function, QoL \\
\hline Notes & Unclear if intervention is progressive fitness training \\
\hline
\end{tabular}

Oh 2016

\begin{tabular}{ll}
\hline Methods & RCT \\
\hline Participants & Stroke $\mathrm{n}=40$ \\
\hline Interventions & Lumbar stabilisation exercise group vs general physical therapy \\
\hline Outcomes & Pulmonary function \\
\hline Notes & Unclear if intervention is progressive \\
\hline
\end{tabular}

Opara 2016

\begin{tabular}{ll}
\hline Methods & RCT \\
\hline Participants & Stroke $\mathrm{n}=60$ \\
\hline Interventions & BWSTT \\
\hline Outcomes & Functional Index Repty, FAC, TUG Test, 10-MWT \\
\hline
\end{tabular}


Opara 2016 (Continued)
Notes
Abstract only

Ploughman 2017

\begin{tabular}{ll}
\hline Methods & RCT \\
\hline Participants & Stroke $n=60$ \\
\hline Interventions & Cardiorespiratory exercise vs activity intervention vs 2 non-exercise control groups \\
\hline Outcomes & Cognition outcomes \\
\hline Notes & Abstract only \\
\hline
\end{tabular}

Pudipeddi 2016

\begin{tabular}{ll}
\hline Methods & Controlled study; randomisation unclear \\
\hline Participants & Stroke $\mathrm{n}=30$ \\
\hline Interventions & Strength training + UC vs UC \\
\hline Outcomes & Strength, spasticity \\
\hline Notes & Abstract only \\
\hline
\end{tabular}

Ruescas-Nicolau 2015

\begin{tabular}{ll}
\hline Methods & RCT \\
\hline Participants & Stroke $\mathrm{n}=13$ \\
\hline Interventions & Cardiovascular/task oriented interval training vs control \\
\hline Outcomes & 6-MWT, TUG, Tinetti-walking subscale \\
\hline Notes & Abstract only \\
\hline
\end{tabular}

Sanchez Sanchez 2015

\begin{tabular}{ll}
\hline Methods & RCT \\
\hline Participants & Stroke $\mathrm{n}=12$ \\
\hline Interventions & Strength training vs conventional rehabilitation \\
\hline Outcomes & Fugl-Meyer Assessment, WMFT \\
\hline
\end{tabular}


Sanchez Sanchez 2015 (Continued)
Notes
Abstract only

Song 2015a

\begin{tabular}{ll}
\hline Methods & RCT \\
\hline Participants & Stroke $n=40$ \\
\hline Interventions & Sliding machine exercise \\
\hline Outcomes & Balance \\
\hline Notes & Need more information about the nature of the exercise \\
\hline
\end{tabular}

Song 2015b

\begin{tabular}{ll}
\hline Methods & RCT \\
\hline Participants & Stroke $\mathrm{n}=30$ \\
\hline Interventions & Individual circuit training vs class circuit training vs conventional PT \\
\hline Outcomes & Walking velocity, cadence, and 2-MWT \\
\hline Notes & Unclear whether the intervention is progressive fitness training \\
\hline
\end{tabular}

\section{Szczygiel 2015}

\begin{tabular}{ll}
\hline Methods & RCT \\
\hline Participants & Stroke $n=60$ \\
\hline Interventions & Partial body weight support walking \\
\hline Outcomes & 10-MWT, 3-D assessment of gait \\
\hline Notes & Abstract only \\
\hline
\end{tabular}

Van den Berg 2016

\begin{tabular}{ll}
\hline Methods & RCT \\
\hline Participants & Stroke $n=63$ \\
\hline Interventions & Unclear; mixed training possibly \\
\hline Outcomes & Mobility section of the SIS; other sections of the SIS; mobility, RMI, BI, NEADL, TUG test, mRS, Fugl \\
Meyer lower extremity, Motricity Index, BBS, HADS
\end{tabular}


Van den Berg 2016 (Continued)

Other outcomes: General Self-Efficacy Scale; and Fatigue Severity Scale. Caregivers also completed the HADS, Carer QoL and the Expanded Caregiver Strain Index, length of stay, number of readmissions, caregiver satisfaction with the intervention

Notes

Vij 2015

\begin{tabular}{ll}
\hline Methods & RCT \\
\hline Participants & Stroke $n=40$ \\
\hline Interventions & Strength training, gait training \\
\hline Outcomes & Step length, stride length, cadence, gait velocity, spasticity, foot pressure, Wisconsin Gait Scale \\
\hline Notes & Abstract only \\
\hline
\end{tabular}

Wu 2017

\begin{tabular}{ll}
\hline Methods & RCT \\
\hline Participants & Stroke, $\mathrm{n}=60$ \\
\hline Interventions & Bodybuilding exercise (resistance training?) \\
\hline Outcomes & Anxiety, depression, patient satisfaction with care, re-bleeding \\
\hline Notes & Abstract only; full text only in Chinese \\
\hline
\end{tabular}

Xu 2015

\begin{tabular}{ll}
\hline Methods & RCT \\
\hline Participants & Stroke $\mathrm{n}=120$ \\
\hline Interventions & Exercise vs 3 non-exercise comparisons \\
\hline Outcomes & Fugl-Meyer Motor Function Scale, NIHSS, and modified BI \\
\hline Notes & Full text in Chinese \\
\hline
\end{tabular}

Yang 2018

\begin{tabular}{ll}
\hline Methods & RCT \\
\hline Participants & Stroke $n=60$ \\
\hline
\end{tabular}


Yang 2018 (Continued)

\begin{tabular}{ll} 
Interventions & Strength training vs Bobath vs robot rehabilitation \\
\hline Outcomes & Peak torque of knee joint, Fugl-Meyer Score, FIM, BBS \\
\hline Notes & Abstract only
\end{tabular}

Yelnik 2017

\begin{tabular}{ll}
\hline Methods & RCT \\
\hline Participants & Stroke $n=104$ \\
\hline Interventions & Physical therapy including training \\
\hline Outcomes & $\begin{array}{l}\text { Fugl-Meyer Score, number of days to walking } 10 \mathrm{~m} \text { unassisted, balance, autonomy, QoL, unexpect- } \\
\text { ed medical events }\end{array}$ \\
\hline Notes & Unclear intervention content \\
\hline
\end{tabular}

Zhang 2015

\begin{tabular}{ll}
\hline Methods & RCT \\
\hline Participants & Stroke $\mathrm{n}=40$ \\
\hline Interventions & Sling exercise therapy \\
\hline Outcomes & Lower-limb motor function \\
\hline Notes & Full text in Chinese \\
\hline
\end{tabular}

2-MWT: 2 minute walk test; 5-MWT: 5-Metre Walk Test; 6-MWT: 6-Minute Walk Test; 10-MWT: 10-Metre Walking Test; ARAT: Action Research Arm Test; BBS: Berg Balance Scale; BI: Barthel Index; BDI: Beck Depression Inventory; BWSTT: body weight supported treadmill training; FAC: Functional Ambulation Classification; FIM: Functional Independence Measure; HADS: Hospital Anxiety and Depression Scale; HR: heart rate; MAL: Motor Activity Log; MMT: Manual Muscle Test; mRS: modified Rankin Scale; NEADL: Nottingham Extended Activities of Daily Living; NHP: Nottingham Health Profile; NIHSS: National Institutes of Health Stroke Scale; PT: physiotherapy; QoL: quality of life; RCT: randomised controlled trial; RMI: Rivermead Mobility Index; SIS: Stroke Impact Scale; TIA: transient ischaemic attack; TUG: Timed Up-and Go Test; UC: usual care; WMFT: Wolf Motor Function Test

Characteristics of ongoing studies [ordered by study ID]

\section{ACTRN12615000728538}

\begin{tabular}{ll}
\hline Trial name or title & Home-based, tailored intervention for reducing falls after stroke: the Falls After Stroke Trial (FAST) \\
\hline Methods & RCT \\
\hline Participants & $\mathrm{N}=270$ \\
\hline Interventions & Mixed training \\
\hline
\end{tabular}


ACTRN12615000728538 (Continued)

Outcomes

Self-report falls incidence, BBS, 6MWT, self-report and accelerometer-measured physical activity, community participation (Later-Life Function and Disability Instrument), HRQoL, Step test, Health care utilisation and costs, gait speed

\begin{tabular}{ll}
\hline Starting date & Unknown \\
\hline Contact information & $\begin{array}{l}\text { Prof Catherine Dean, Ground Floor } 75 \text { Talavera Road, Macquarie University, NSW 2109, Australia; } \\
+61298506620 ; \text { catherine.dean@mq.edu.au }\end{array}$ \\
\hline
\end{tabular}

Notes

\section{ACTRN12616000391471}

\begin{tabular}{ll}
\hline Trial name or title & $\begin{array}{l}\text { A study on benefit of circuit class therapy on mobility, balance, reintegration into normal life and } \\
\text { quality of life of people with stroke }\end{array}$ \\
\hline Methods & RCT \\
\hline Participants & Mixed training \\
\hline Interventions & TUG, BBS, Reintegration to Normal Living Index, HRQoL \\
\hline Outcomes & 9 March 2015 \\
\hline Starting date & $\begin{array}{l}\text { Dr Nor Azlin Mohd. Nordin, Physiotherapy Programme, School of Rehabilitation Sciences, Facul- } \\
\text { ty of Health Sciences, Jalan Raja Muda Aziz, Universiti Kebangsaan Malaysia, 50300 Kuala Lumpur, } \\
\text { Malaysia; +6019 359 4418, +603 26878038; norazlin8@ukm.edu.my }\end{array}$ \\
\hline Contact information & Recruitment completed \\
\hline Notes &
\end{tabular}

\section{ACTRN12616001288415}

Trial name or title

A comparison of two forms of physiotherapy on functional performance after acquired brain injury: a pilot randomised controlled trial

\begin{tabular}{ll}
\hline Methods & $\mathrm{RCT}$ \\
\hline Participants & $\mathrm{N}=30$ \\
\hline Interventions & Mixed training \\
\hline Outcomes & $\begin{array}{l}\text { Global Impression of Change scale, STS item of the Mobility Scale for Acute Stroke Patients (MSAS), } \\
\text { muscle strength, goal attainment, subjective ranking of functional task performance }\end{array}$ \\
\hline Starting date & 22 September 2016 \\
\hline Contact information & $\begin{array}{l}\text { Mr Davide de Sousa, Physiotherapy Department, Graythwaite Rehabilitation Centre, Ryde Hospi- } \\
\text { tal, Denistone Road, Eastwood NSW 2122, Australia; +61298587144; davide.desousa@ @health.n- } \\
\text { sw.gov.au }\end{array}$ \\
\hline
\end{tabular}


ACTRN12616001288415 (Continued)

Notes

Details of functional tasks and strategies used in the intervention are not presented

ACTRN12617000452392

Trial name or title Core muscles strengthening for balance and gait performance in individuals with chronic stroke

\begin{tabular}{ll}
\hline Methods & RCT \\
\hline Participants & Stroke \\
\hline Interventions & Core strength training \\
\hline Outcomes & Conventional training \\
\hline
\end{tabular}

Starting date

Contact information

Notes Not clear if progressive in nature

\section{ACTRN12617000746336}

\begin{tabular}{ll}
\hline Trial name or title & Walking away fatigue and disease after stroke \\
\hline Methods & $\mathrm{RCT}$ \\
\hline Participants & $\mathrm{N}=30$ \\
\hline Interventions & Cardiorespiratory training \\
\hline Outcomes & $\begin{array}{l}\text { Fatigue Severity Scale, feasibility, daily step count, fasting glucose, resting BP, BMI, gait speed, } \\
\text { blood lipids, daily time spent in moderate intensity activity, gait endurance, MAS }\end{array}$ \\
\hline Starting date & Unknown \\
\hline Contact information & $\begin{array}{l}\text { Dr Niru Mahendran, 12D47, Faculty of Health, University of Canberra, } 1 \text { University Drive, Bruce ACT } \\
\text { 2617, Australia; +612 6206 8302; niru.mahendran@canberra.edu.au }\end{array}$
\end{tabular}

Notes

\section{ChiCTR ICR 15006362}

Trial name or title

Effect of early low-intensity aerobic training with ergometer on the activities of daily living among severely impaired post-stroke hemiplegic patients: a pilot study

\begin{tabular}{ll}
\hline Methods & RCT \\
\hline Participants & $N=42$
\end{tabular}


ChiCTR ICR 15006362 (Continued)

\begin{tabular}{ll} 
Interventions & Cardiorespiratory training \\
\hline Outcomes & $\begin{array}{l}\text { BI, FAC scale, Frequency Activities Index, Fugl-Meyer motor score, exercise test duration, peak heart } \\
\text { rate }\end{array}$ \\
\hline Starting date & Unknown \\
\hline Contact information & $\begin{array}{l}\text { Huaping Pan, 168 Gushan Rd, Jiangning District, Nanjing, Jiangsu, +86 18901588339, heap- } \\
\text { ing_pan70@163.com }\end{array}$ \\
\hline
\end{tabular}

Notes

\section{ChicTR-IOR-17010821}

\begin{tabular}{ll}
\hline Trial name or title & Effect of sling exercise training on the balance function of stroke patients with hemiplegia \\
\hline Methods & Intervention vs control \\
\hline Participants & $\mathrm{N}=50$ \\
\hline Interventions & Strength training \\
\hline Outcomes & BBS, Fugl-Meyer, TUG, Modified Ashworth Scale, BI \\
\hline Starting date & Unknown \\
\hline Contact information & Liu Jing \\
\hline Notes & U10505633@qq.com \\
\hline
\end{tabular}

\section{CTRI/2016/09/007258}

\begin{tabular}{ll}
\hline Trial name or title & Exercise using all four limbs in half-side paralysis \\
\hline Methods & $\mathrm{RCT}$ \\
\hline Participants & $\mathrm{N}=50$ \\
\hline Interventions & Cardiorespiratory training \\
\hline Outcomes & FAC, RVGA, Fugl-Meyer, mRS \\
\hline Starting date & 1 January 2016 \\
\hline Contact information & Dr Kamal Narayan Arya, Pt. Deendayal Upadhyaya Intitute for the Physically Handicapped, 4 VD \\
\hline Marg, New Delhi, Central, DELHI 110002 India; 9899897408; kamalnarya@yahoo.com
\end{tabular}


CTRI/2016/10/007337

\begin{tabular}{ll}
\hline Trial name or title & Effectiveness of physiotherapy training to improve control of sitting on floor \\
\hline Methods & $\mathrm{RCT}$ \\
\hline Participants & $\mathrm{N}=50$ \\
\hline Interventions & Task-orientated exercise \\
\hline Outcomes & $\begin{array}{l}\text { Attainment of squatting and sitting on the floor using goal attainment scale, lower limb strength } \\
\text { using Motricity Index (lower limb strength), Fugl Meyer Scale (lower limb motor recovery), short fall } \\
\text { efficacy scale (fear of falling, Neuro QoL-Short form (ability to participate in social roles and activi- } \\
\text { ties)) }\end{array}$ \\
\hline Starting date & \begin{tabular}{l} 
11 September 2015 \\
\hline Contact information
\end{tabular} \\
\hline prakashvaidhiyalingam@gmail.com
\end{tabular}

\section{CTRI/2017/03/008061}

\begin{tabular}{ll}
\hline Trial name or title & $\begin{array}{l}\text { Comparison of home exercises verses exercises given in hospital set up in improving functional re- } \\
\text { covery of upper limbs in post stroke patients }\end{array}$ \\
\hline Methods & $\mathrm{RCT}$ \\
\hline Participants & Home-based exercises by care givers with routine therapy given at hospital setup \\
\hline Interventions & $\begin{array}{l}\text { Fugl-Meyer MAS (upper extremity level of impairment), WMFT (upper extremity function), SIS (par- } \\
\text { ticipation level) }\end{array}$ \\
\hline Outcomes & 29 January 2016 \\
\hline Starting date & abraham.joshua@manipal.edu \\
\hline Contact information & \\
\hline Notes &
\end{tabular}

\section{CTRI/2018/01/011543}

Trial name or title $\quad$ Efficacy of task-oriented training approach on trunk and hip musculature to improve balance in
stroke subjects: a randomised controlled trial

\begin{tabular}{ll}
\hline Methods & RCT \\
\hline Participants & $\mathrm{N}=40$ \\
\hline Interventions & Task-oriented training approach for trunk and hip abductor \\
\hline
\end{tabular}


CTRI/2018/01/011543 (Continued)

Outcomes

Trunk Impairment Scale, Brunel Balance Assessment, Tinetti performance oriented mobility assessment scale, stroke rehabilitation assessment of movement measures

\begin{tabular}{ll}
\hline Starting date & 1 March 2018 \\
\hline Contact information & abraham.joshua@manipal.edu
\end{tabular}

Notes

IRCT20150721023277N2

Trial name or title The efficacy of occupation-based and exercise-based interventions on performance components and areas of occupation in subjects with chronic stroke

\begin{tabular}{ll}
\hline Methods & RCT \\
\hline Participants & $\mathrm{N}=45$ \\
\hline Interventions & $\begin{array}{l}\text { Structured exercise protocol including the range of motion and strengthening exercises, balance } \\
\text { and endurance training and functional use of the upper extremity }\end{array}$
\end{tabular}

Outcomes

Participation in occupational areas 2. Performance components including upper limb function, balance and functional mobility and cognition

\section{Starting date}

22 December 2017

Contact information

lajevardi.l@iums.ac.ir

Notes

\section{IRCT2016102430477N1}

\begin{tabular}{ll}
\hline Trial name or title & Effect of rehabilitation in stroke recovery \\
\hline Methods & $\mathrm{RCT}$ \\
\hline Participants & $\mathrm{N}=20$ \\
\hline Interventions & Resistance training \\
\hline Outcomes & $\begin{array}{l}\text { Neurophysiological parameters (rest motor threshold, active motor threshold, recruitment curve, } \\
\text { cortical silent period and ipsilateral silent period) recorded by transcranial magnetic stimulation, } \\
\text { Modified Bl, Modified Ashworth Scale, Fugl-Meyer }\end{array}$ \\
\hline Starting date & Unknown \\
\hline Contact information & Fahimeh Kamali \\
\hline Notes & fahimehkamali@hotmail.com \\
\hline
\end{tabular}


IRCT20171105037256N4

\begin{tabular}{ll}
\hline Trial name or title & $\begin{array}{l}\text { The effect of dual-task exercises on balance, falling and activities of daily living in stroke } \\
\text { patients }\end{array}$ \\
\hline Methods & Intervention vs control \\
\hline Participants & Unknown \\
\hline Interventions & Mixed training \\
\hline Outcomes & TUG \\
\hline Starting date & Unknown \\
\hline Contact information & Unknown \\
\hline Notes & Possibility it may not be progressive in nature
\end{tabular}

\section{JPRN-UMIN000019380}

\begin{tabular}{ll}
\hline Trial name or title & The effect of walking exercise by Nordic pole on the aerobic performance for stroke patients \\
\hline Methods & $\mathrm{RCT}$ \\
\hline Participants & $\mathrm{N}=40$ \\
\hline Interventions & Cardiorespiratory \\
\hline Outcomes & Gait speed, gait distance, HR and $\mathrm{O}_{2}$ consumption during 6-MWT \\
\hline Starting date & Unknown \\
\hline Contact information & Hidetoshi Takahashi \\
& taka1959@saitama-med.ac.jp \\
\hline
\end{tabular}

Notes

\section{Miller 2014}

\begin{tabular}{ll} 
Trial name or title & $\begin{array}{l}\text { Protocol for a randomised controlled clinical trial investigating the effectiveness of Fast muscle Ac- } \\
\text { tivation and Stepping Training (FAST) for improving balance and mobility in sub-acute stroke }\end{array}$ \\
\hline Methods & $\mathrm{RCT}$ \\
\hline Participants & $\mathrm{N}=60$ \\
\hline Interventions & Mixed training \\
\hline Outcomes & $\begin{array}{l}\text { Chedoke-McMaster Stroke Assessment, BBS, 10-MWT, maximum voluntary contraction, gait speed, } \\
\text { Physiological Balance Test }\end{array}$
\end{tabular}


Miller 2014 (Continued)

\begin{tabular}{ll} 
Starting date & November 2012 \\
\hline Contact information & S Jayne Garland \\
& jayne.garland@ubc.ca
\end{tabular}

Notes

\section{NCT01161329}

Trial name or title

Description of physical and psychosocial problems one year after stroke and the effect of intensified physical activity for patients with stroke - a combined physical and behavioural approach

\begin{tabular}{ll}
\hline Methods & RCT \\
\hline Participants & $\mathrm{N}=67$ \\
\hline Interventions & High intense functional exercise programme \\
\hline Outcomes & BBS \\
& Short Physical Performance Battery \\
\hline Starting date & September 2009 (completed 2016) \\
\hline Contact information & karin.hellstrom@neuro.uu.se \\
\hline Notes & \\
\hline
\end{tabular}

\section{NCT01915368}

\begin{tabular}{ll}
\hline Trial name or title & Determining Optimal post-Stroke Exercise (DOSE) \\
\hline Methods & $\mathrm{RCT}$ \\
\hline Participants & Physical exercise \\
\hline Interventions & $\begin{array}{l}\text { Ambulatory function measured by the 6-MWT, ambulatory function from the 5-Meter Walk Test, bal- } \\
\text { ance function from the BBS, ambulatory function from the FAC, QoL measured with EuroQol, cog- } \\
\text { nition measured by the Montreal Cognitive Assessment, cognition measured by the Digit Symbols } \\
\text { Substitution Test, cognition measured by the Trail Making Test, depression measured by Patient } \\
\text { Health Questionnaire-9, HR measured during the intervention sessions, step count measured dur- } \\
\text { ing the intervention sessions }\end{array}$ \\
\hline Starting date & $\begin{array}{l}\text { September 2013 } \\
\text { Contact information }\end{array}$ \\
\hline Notes & chihya.hung@ubc.ca \\
\hline
\end{tabular}


NCT01916486

\begin{tabular}{ll}
\hline Trial name or title & Vitality: promoting cognitive function in older adults with chronic stroke (Vitality) \\
\hline Methods & $\mathrm{RCT}$ \\
\hline Participants & $\mathrm{N}=119$ \\
\hline Interventions & Mixed training \\
\hline Outcomes & $\begin{array}{l}\text { Alzheimer's Disease Assessment Scale Cognitive Subscale Plus, IADL Scale, Fatigue Severity Scale, } \\
\text { Short Physical Performance Battery, 6-MWT, TUG, quadriceps strength, grip strength, European } \\
\text { QoL }\end{array}$ \\
\hline Starting date & November 2013 \\
\hline Contact information & Janice J Eng \\
\hline Notes & \\
\hline
\end{tabular}

\section{NCT02272426} $\begin{array}{ll}\text { Trial name or title } & \begin{array}{l}\text { The safety and tolerability of an aerobic and resistance exercise program with cognitive training } \\ \text { post-stroke }\end{array}\end{array}$

\begin{tabular}{ll}
\hline Methods & RCT \\
\hline Participants & $\mathrm{N}=132$ \\
\hline Interventions & Mixed training \\
\hline Outcomes & $\begin{array}{l}\text { Adverse events related to interventions, training programme adherence, Cognitive Assessment } \\
\text { Battery, Depression Scale, QoL }\end{array}$ \\
\hline Starting date & November 2014 \\
\hline Contact information & Unknown \\
\hline Notes & Possibility of co-intervention with cognitive training
\end{tabular}

\section{NCT02437006}

\begin{tabular}{ll} 
Trial name or title & $\begin{array}{l}\text { Early intervention with a low-intensity leg cycling exercise program for individuals after } \\
\text { stroke }\end{array}$ \\
\hline Methods & $\mathrm{RCT}$ \\
\hline Participants & $\mathrm{N}=120$ \\
\hline Interventions & Cardiorespiratory training \\
\hline Outcomes & Exercise capacity, sympathetic nerve tests \\
\hline
\end{tabular}


NCT02437006 (Continued)

\begin{tabular}{ll} 
Starting date & March 2014 \\
\hline Contact information & Miao-Ju Hsu \\
& mjhsu@kmu.edu.tw
\end{tabular}

Notes

\section{NCT02494518}

\begin{tabular}{ll}
\hline Trial name or title & Forced aerobic exercise for stroke rehabilitation \\
\hline Methods & $\mathrm{RCT}$ \\
\hline Participants & $\mathrm{N}=34$ \\
\hline Interventions & Mixed training \\
\hline Outcomes & Fugl Meyer Assessment, WMFT, SIS, Metabolic Stress Test, ARAT, 6-MWT \\
\hline Starting date & July 2015 \\
\hline Contact information & Susan Linder \\
\hline Notes & \\
\hline
\end{tabular}

\section{NCT02550015}

\begin{tabular}{ll}
\hline Trial name or title & High intensity interval training after stroke \\
\hline Methods & RCT \\
\hline Participants & $\mathrm{N}=70$ \\
\hline Interventions & Cardiorespiratory training \\
\hline Outcomes & Maximal Oxygen Uptake, change in BP, 10-MWT, TUG, BBS, FIM, International Physical Activity \\
& Questionnaire, Montreal Cognitive Assessment, 6-MWT \\
\hline Starting date & September 2015 \\
\hline Contact information & Torunn Askim \\
\hline Notes & \\
\hline
\end{tabular}

\section{NCT02550990}

Trial name or title

Synergistic effects of aerobic exercise and cognitive training on cognition in stroke patients with cognitive decline 
NCT02550990 (Continued)

\begin{tabular}{ll} 
Methods & $\mathrm{RCT}$ \\
\hline Participants & $\mathrm{N}=75$ \\
\hline Interventions & Cardiorespiratory training \\
\hline Outcomes & $\begin{array}{l}\text { Montreal Cognitive Assessment, Wechsler Memory Scale, Wechsler Adult Intelligence Scale, FIM, } \\
\text { Lawton IADL Scale, SIS, TUG, 6-MWT, mobility, Fugl-Meyer Assessment, RMI, Geriatric Depression } \\
\text { Scale, MVC knee extension strength }\end{array}$ \\
\hline Starting date & September 2015 \\
\hline Contact information & Ching-Yi Wu \\
& cywu@mail.cgu.edu.tw \\
\hline
\end{tabular}

Notes

NCT02619110 \begin{tabular}{ll}
\hline Trial name or title & $\begin{array}{l}\text { The effect of backward walking treadmill training on balance in patient with chronic } \\
\text { stroke }\end{array}$
\end{tabular}

\begin{tabular}{ll}
\hline Methods & RCT \\
\hline Participants & $\mathrm{N}=30$ \\
\hline Interventions & Cardiorespiratory training \\
\hline Outcomes & BBS, pulmonary function test, 10-MWT, 6-MWT, TUG \\
\hline Starting date & February 2014 \\
\hline Contact information & Lan Y Guo \\
\hline
\end{tabular}

Notes

NCT02703805

\begin{tabular}{ll}
\hline Trial name or title & FIT for FUNCTION \\
\hline Methods & RCT \\
\hline Participants & $\mathrm{N}=216$ \\
\hline Interventions & Mixed training - unclear \\
\hline Outcomes & $\begin{array}{l}\text { Reintegration to Normal Living Index, Rapid Assessment of Physical Activity, BBS, Short Physical } \\
\text { Performance Battery, 6-MWT, grip and knee strength, European QoL 5-Dimension Questionnaire, } \\
\text { cardiovascular risk factors, self-efficacy for physical activity (Stanford 6-item Scale), level of par- } \\
\text { ticipant knowledge, skill, and confidence for self-management (Patient Activation Measure) and } \\
\text { healthcare utilisation and cost evaluation }\end{array}$ \\
\hline
\end{tabular}


NCT02703805 (Continued)

\begin{tabular}{ll} 
Starting date & May 2014 \\
\hline Contact information & Julie Richardson, PhD McMaster University \\
\hline Notes & NCT02703805
\end{tabular}

NCT02710773

\begin{tabular}{ll}
\hline Trial name or title & Backward treadmill training in patients with chronic stroke \\
\hline Methods & $\mathrm{RCT}$ \\
\hline Participants & $\mathrm{N}=20$ \\
\hline Interventions & Cardiorespiratory training \\
\hline Outcomes & 10-MWT, gait analysis, SIS, Modified Ashworth Scale \\
\hline Starting date & April 2016 \\
\hline Contact information & Alessandro Picelli \\
& alessandro.picelli@univr.it \\
\hline Notes & \\
\hline
\end{tabular}

\section{NCT02717715}

\begin{tabular}{ll}
\hline Trial name or title & SunRISe Study - Stroke Rehabilitation In Suriname \\
\hline Methods & RCT \\
\hline Participants & $\mathrm{N}=20$ \\
\hline Interventions & Cardiorespiratory training \\
\hline Outcomes & Change in peak oxygen uptake \\
\hline Starting date & March 2016 \\
\hline Contact information & Anton De Kom \\
\hline Notes & Possibility that training could be mixed, details unknown \\
\hline
\end{tabular}

\section{NCT02731235}

\begin{tabular}{ll}
\hline Trial name or title & The effect of aerobic exercise in patients with minor stroke \\
\hline Methods & RCT \\
\hline
\end{tabular}


NCT02731235 (Continued)

\begin{tabular}{ll} 
Participants & $\mathrm{N}=84$ \\
\hline Interventions & Cardiorespiratory training \\
\hline Outcomes & $\begin{array}{l}\text { Graded Cycling Test with Talk Test, Physical Activity Scale, Short time activity measurements, WHO- } \\
\text { five Well-being Index, Montreal Cognitive Assessment, Major Depression Inventory, Multidimen- } \\
\text { sional Fatigue Inventory }\end{array}$ \\
\hline Starting date & January 2016 \\
\hline Contact information & Christina Kruuse \\
& christina.kruuse.01@regionh.dk \\
\hline
\end{tabular}

Notes

NCT02753322

\begin{tabular}{ll}
\hline Trial name or title & Training dual-task balance and walking in people with stroke \\
\hline Methods & $\mathrm{RCT}$ \\
\hline Participants & $\mathrm{N}=84$ \\
\hline Interventions & Dual-task training, single-task training, limbs exercise \\
\hline Outcomes & $\begin{array}{l}\text { Degree of the dual-task interference, standing balance (with eyes open and closed), 10-MWT, ob- } \\
\text { stacle crossing, Chinese version of Activities-specific Balance Confidence Scale, Chedoke Arm and } \\
\text { Hand Activity Inventory, incidence of falls }\end{array}$
\end{tabular}

\begin{tabular}{ll}
\hline Starting date & April 2016 \\
\hline Contact information & Margaret WY Poon \\
& Email: pwy751@ha.org.hk \\
\hline Notes & \\
\hline
\end{tabular}

\section{NCT02855424}

\begin{tabular}{ll}
\hline Trial name or title & $\begin{array}{l}\text { The effect of leg cycling exercise program at low or moderate intensity for individuals with suba- } \\
\text { cute stroke }\end{array}$ \\
\hline Methods & $\mathrm{RCT}$ \\
\hline Participants & $\mathrm{N}=90$ \\
\hline Interventions & Low-intensity exercise vs moderate-intensity exercise vs traditional rehabilitation \\
\hline Outcomes & $\begin{array}{l}\text { Symptom-limit exercise tolerance tests, changed activity of autonomic nervous system, 10-MWT, } \\
\text { Bl, Stroke-Specific QoL Scale, Fugl-Meyer (lower extremity), Multi-dimensional Fatigue Inventory, } \\
\text { performance of cycling }\end{array}$
\end{tabular}


NCT02855424 (Continued)

\begin{tabular}{ll} 
Starting date & August 2016 \\
\hline Contact information & Miao-Ju Hsu \\
& Email: mjhsu@kmu.edu.tw
\end{tabular}

Notes

\section{NCT02902367}

Trial name or title

SMS-guided training after acute stroke or transient ischemic attack - a randomised controlled trial (SMS/TIA)

\begin{tabular}{ll}
\hline Methods & $\mathrm{RCT}$ \\
\hline Participants & $\mathrm{N}=80$ \\
\hline Interventions & Outdoor walking and strength exercise vs UC \\
\hline Outcomes & $\begin{array}{l}\text { 6-MWT, Short Physical Performance Battery, 10-MWT, hand-dynamometer, body composition, car- } \\
\text { diometabolic risk markers, mortality, health-related QoL }\end{array}$ \\
\hline Starting date & November 2016 \\
\hline Contact information & Birgit Vahlberg \\
& Email: birgit.vahlberg@pubcare.uu.se \\
\hline
\end{tabular}

Notes

\section{NCT02923765}

\begin{tabular}{ll}
\hline Trial name or title & Stepper aerobic training on fitness, disability, inflammation and thrombosis in stroke patients \\
\hline Methods & $\mathrm{RCT}$ \\
\hline Participants & $\mathrm{N}=170$ \\
\hline Interventions & Aerobic training by a stepper vs usual rehabilitation vs healthy participants \\
\hline Outcomes & $\begin{array}{l}\text { Cardiopulmonary Exercise Test, FIM, thrombosis and coagulation activities of blood samples by } \\
\text { flow cytometry, dynamic TG assay, and enzyme-linked Immunosont assay }\end{array}$ \\
\hline Starting date & October 2017 \\
\hline Contact information & Shu-Chun Huang \\
\hline Notes & Email: mr7171@cgmh.org.tw \\
\hline
\end{tabular}


NCT02937480

\begin{tabular}{|c|c|}
\hline Trial name or title & Efficacy of task-specific training in physical activity level post-stroke \\
\hline Methods & $\mathrm{RCT}$ \\
\hline Participants & $N=38$ \\
\hline Interventions & Task-specific training vs sham training \\
\hline Outcomes & $\begin{array}{l}\text { Physical activity levels (physical activity monitor, Human Activity Profile), gait speed, TUG, Upper } \\
\text { Extremity Performance Test (TEMPA), handgrip strength, 6-MWT, SS-QOL }\end{array}$ \\
\hline Starting date & August 2016 \\
\hline \multirow[t]{2}{*}{ Contact information } & Christina Danielli Coelho de Morais Faria \\
\hline & Email: chrismoraisf@yahoo.com \\
\hline \multicolumn{2}{|l|}{ Notes } \\
\hline \multicolumn{2}{|l|}{ NCT02938000 } \\
\hline Trial name or title & E-rehabilitation: aerobic resistance training for stroke survivors \\
\hline Methods & $\mathrm{RCT}$ \\
\hline Participants & $N=3$ \\
\hline Interventions & TheraBand with exercise video vs UC with advice to exercise \\
\hline Outcomes & $\begin{array}{l}\text { Peak VO2 using 6-MWT, BBS, adherence to exercise programme, Patient Health Questionnaire - } 9 \\
\text { (PHQ 9) }\end{array}$ \\
\hline Starting date & August 2016 \\
\hline \multirow[t]{2}{*}{ Contact information } & William P Neil \\
\hline & Email:William.P.Neil@kp.org \\
\hline Notes & Reconsider when full text is available \\
\hline
\end{tabular}

\section{NCT02948725}

\begin{tabular}{ll}
\hline Trial name or title & Clinical application of cross-education during stroke rehabilitation (X-Ed-Stroke01) \\
\hline Methods & $\mathrm{RCT}$ \\
\hline Participants & $\mathrm{N}=24$ \\
\hline Interventions & Cross-education and standard rehabilitation vs standard rehabilitation \\
\hline Outcomes & Fugl-Meyer Assessment, grip and wrist strength, volume of motor cortex activation
\end{tabular}


NCT02948725 (Continued)

\begin{tabular}{ll} 
Starting date & November 2016 \\
\hline Contact information & Jon Farthing \\
& Email: jon.farthing@usask.ca
\end{tabular}

Notes

\section{NCT03122626}

Trial name or title

Does participation in a group, task-oriented community-based exercise program improve the ability to do everyday activities among people with stroke?

\begin{tabular}{ll}
\hline Methods & $\mathrm{RCT}$ \\
\hline Participants & $\mathrm{N}=60$ \\
\hline Interventions & Together In Movement and Exercise (TIME) Program vs wait-listed control group \\
\hline Outcomes & Subjective Index of Physical and Social Outcome, NEADL (multiple secondary outcomes) \\
\hline Starting date & February 2017 \\
\hline Contact information & Nancy Salbach \\
\hline Notes & Email: nancy.salbach@utoronto.ca \\
\hline
\end{tabular}

\section{NCT03259932}

\section{Trial name or title}

Comparison of fatigue and recovery after stroke depending on the usual management with or without physical training (FRAM)

\begin{tabular}{ll}
\hline Methods & RCT \\
\hline Participants & $\mathrm{N}=160$ \\
\hline Interventions & $\begin{array}{l}\text { Physical activity - aerobic exercise, muscle building in circuit training, balance and flexibility exer- } \\
\text { cises }\end{array}$ \\
\hline Outcomes & Fatigue relief (fatigue severity scale) \\
\hline Starting date & September 2017 \\
\hline Contact information & Vincent.gremeaux@chu-dijon.fr \\
\hline Notes & \\
\hline
\end{tabular}


NCT03425890

\begin{tabular}{ll}
\hline Trial name or title & $\begin{array}{l}\text { Effect of self-empowered upper limb repetitive engagement (SURE) program on upper limb recov- } \\
\text { ery after stroke }\end{array}$ \\
\hline Methods & $\mathrm{RCT}$ \\
\hline Participants & $\mathrm{N}=20$ \\
\hline Interventions & $\begin{array}{l}\text { SURE Program - individualised daily self exercise and functional use of the arm and hand on their } \\
\text { own outside of therapy } 60 \text { min a day } 6 \text { days a week for } 4 \text { weeks. Strengthening and exercise exercis- } \\
\text { es. Control group received no program, only an educational booklet on stroke }\end{array}$ \\
\hline Outcomes & $\begin{array}{l}\text { Upper limb Fugl Meyer Scale (primary outcome), ARAT, rating of everyday arm-use in the communi- } \\
\text { ty and home, Stanford Fatigue Visual Numeric Scale, VAS scale for pain, Modified Ashworth Scale, } \\
\text { duration of affected upper limb activity in h, bilateral motor cortex brain activation }\end{array}$ \\
\hline Starting date & February 2018 \\
\hline Contact information & Lay_fong_chin@ttsh.com.sg \\
\hline Notes &
\end{tabular}

\section{NCT03458884}

\begin{tabular}{ll}
\hline Trial name or title & Does cardiorespiratory interval training improve post-stroke fatigue? \\
\hline Methods & RCT \\
\hline Participants & $\mathrm{N}=50$ \\
\hline Interventions & Cardiorespiratory interval training (3 days a week, 8 weeks) \\
\hline Outcomes & $\begin{array}{l}\text { Post-stroke fatigue, peak o2 consumption, VO2 peak, feasibility (fidelity, adherence and adverse } \\
\text { events) }\end{array}$ \\
\hline Starting date & March 2018 \\
\hline Contact information & Anna.brandal@ume.se \\
\hline Notes & \\
\hline
\end{tabular}

\section{NCT03479632}

\begin{tabular}{ll}
\hline Trial name or title & Aerobic exercise for non-ambulatory stroke survivors \\
\hline Methods & $\mathrm{RCT}$ \\
\hline Participants & $\mathrm{N}=40$ \\
\hline Interventions & $\begin{array}{l}\text { Aerobic walking programme using a treadmill with body-weight support system. Control receive } \\
\text { only standard care }\end{array}$ \\
\hline Outcomes & Resting heart rate, resting BP, change in vital capacity and change in forced vital capacity \\
\hline
\end{tabular}


NCT03479632 (Continued)

\begin{tabular}{ll} 
Starting date & March 2018 \\
\hline Contact information & aalqahtani@kumc.edu
\end{tabular}

Notes

NCT03528148

\begin{tabular}{ll} 
Trial name or title & The effect of elliptical cross training bike for stroke patients \\
\hline Methods & RCT \\
\hline Participants & $\mathrm{N}=50$ \\
\hline Interventions & Active cycling group, conventional physical therapy \\
\hline Outcomes & Walking speed and step length \\
\hline Starting date & August 2018 \\
\hline Contact information & chouliwe@mail.cmuh.org.tw \\
\hline
\end{tabular}

Notes

\section{NCT03548090}

\begin{tabular}{ll}
\hline Trial name or title & The effect of plantarflexion training in people with chronic stroke \\
\hline Methods & RCT \\
\hline Participants & $\mathrm{N}=50$ \\
\hline Interventions & Skateboard exercise at an incline angle with different bodyweight $(50 \%, 75 \%, 90 \%)$ \\
\hline Outcomes & Step length asymmetry ratio, EMG of gastrocnemius, soleus, plantarflexion torque, walking speed, \\
\hline Starting date & SIS \\
\hline Contact information & October 2018 \\
\hline Notes & Daniel.wingard@uth.tmc.edu \\
\hline
\end{tabular}

PACTR201511001359344

\begin{tabular}{ll}
\hline Trial name or title & The Nigerian stroke aerobic study (NISAS) \\
\hline Methods & $\mathrm{RCT}$ \\
\hline Participants & $\mathrm{N}=56$ \\
\hline \hline
\end{tabular}


PACTR201511001359344 (Continued)

Interventions Continuous aerobic training (cycling) and interval training (cycling)

\begin{tabular}{ll}
\hline Outcomes & VO2 max, gait speed, motor function, balance, lipid profile, fasting blood sugar, red and white \\
blood count, packed cell volume, anthropometric (e.g. BMI), biochemical (e.g. urea), cardiovascular \\
(BP etc)
\end{tabular}

\begin{tabular}{ll}
\hline Starting date & 1 October 2018 \\
\hline Contact information & Nelson.ekechukwu@unn.edu.ng \\
\hline
\end{tabular}

\section{Notes}

\section{PACTR201712002689193}

\begin{tabular}{ll}
\hline Trial name or title & Effect of 6 weeks task-orientated circuit training on balance and quality of life of stroke \\
\hline Methods & $\mathrm{RCT}$ \\
\hline Participants & $\mathrm{N}=30$ \\
\hline Interventions & $\begin{array}{l}\text { Task-orientated circuit training (balance training, double leg standing, tandem walking, standing } \\
\text { and walking, squat exercises }\end{array}$ \\
\hline Outcomes & BBS, SS-QoL Scale \\
\hline Starting date & Not yet recruiting \\
\hline Contact information & Sinda4life@gmail.com \\
\hline Notes &
\end{tabular}

RBR-26q4z9

\begin{tabular}{ll}
\hline Trial name or title & $\begin{array}{l}\text { Effect of exercises with action observation and aquatic physical therapy in arm recov- } \\
\text { ery after stroke }\end{array}$ \\
\hline Methods & Not listed \\
\hline Participants & $\mathrm{N}=60$ \\
\hline Interventions & Action observation training \\
\hline Outcomes & Fugl-Meyer Scale \\
\hline Starting date & Not listed \\
\hline Contact information & Not listed \\
\hline Notes & \\
\hline
\end{tabular}


RBR-2mf595

\begin{tabular}{|c|c|}
\hline Trial name or title & Serious game for evaluation and treatment in stroke \\
\hline Methods & $\mathrm{RCT}$ \\
\hline Participants & $N=24$ \\
\hline Interventions & Resistance training \\
\hline Outcomes & $\begin{array}{l}\text { MVC, TUG, gait speed, Modified Ashworth Scale, Fugl Meyer, Modified BI, NHP, motor con- } \\
\text { trol }\end{array}$ \\
\hline Starting date & Unknown \\
\hline Contact information & Unknown \\
\hline Notes & Unclear if randomised \\
\hline
\end{tabular}

\section{RBR-4g6fhf}

\begin{tabular}{ll}
\hline Trial name or title & The effects of exercise with cycle ergometer in patients after acute stroke \\
\hline Methods & $\mathrm{RCT}$ \\
\hline Participants & $\mathrm{N}=24$ \\
\hline Interventions & Cardiorespiratory training \\
\hline Outcomes & $\begin{array}{l}\text { Dynamometry for assessment of upper and lower limb muscle strength, Ordinal Scale of Muscle } \\
\text { Tone Assessment in Adults for evaluation of muscle tone, 10-MWT for gait evaluation, BBS for bal- } \\
\text { ance evaluation, Manovacuometry, Spirometry, Mental State Minisease for Cognitive Assessment, } \\
\text { mRS, and Scandinavian Stroke Scale }\end{array}$ \\
\hline Starting date & Unknown \\
\hline Contact information & douglaspinheirodarosa@yahoo.com.br \\
\hline Notes & \\
\hline
\end{tabular}

\section{RBR-4wk4b3}

\begin{tabular}{ll}
\hline Trial name or title & $\begin{array}{l}\text { Influence of an exercise program on cardiac remodeling and functional capacity of patients with } \\
\text { stroke }\end{array}$ \\
\hline Methods & $\mathrm{RCT}$ \\
\hline Participants & $\mathrm{N}=40$ \\
\hline Interventions & Cardiorespiratory training \\
\hline Outcomes & $\begin{array}{l}\text { 6-MWT, neurological assessment, nutritional assessment, ambulatory BP monitoring, transthoracic } \\
\text { echocardiography, and assessment of QoL }\end{array}$ \\
\hline
\end{tabular}


RBR-4wk4b3 (Continued)

\begin{tabular}{ll} 
Starting date & 2016 \\
\hline Contact information & sgzanati@fmb.unesp.br \\
\hline
\end{tabular}

Notes

\section{RBR-4wz3w3}

\begin{tabular}{ll}
\hline Trial name or title & $\begin{array}{l}\text { Task-oriented group therapy and home-based exercise increase in the amount of practice in the } \\
\text { subacute phase after Stroke - randomised clinical trial }\end{array}$ \\
\hline Methods & $\mathrm{RCT}$ \\
\hline Participants & Unclear; mixed training \\
\hline Interventions & Fugl-Meyer, grip strength, box and block test, 6-MWT, TUG test \\
\hline Outcomes & March 2017 \\
\hline Starting date & michaelsenstella@hotmail.com \\
\hline Contact information & \\
\hline Notes
\end{tabular}

\section{RBR-5thjgv}

\begin{tabular}{ll}
\hline Trial name or title & $\begin{array}{l}\text { Effects of physical training on metabolic, hemodynamic, autonomic and inflammatory parameters } \\
\text { of post-stroke individuals }\end{array}$ \\
\hline Methods & $\mathrm{RCT}$ \\
\hline Participants & $\mathrm{N}=30$ \\
\hline Interventions & Resistance training \\
\hline Outcomes & Muscle strength, 10-MWT, TUG test, HR variability, haemodynamic and inflammatory markers \\
\hline Starting date & August 2017 \\
\hline Contact information & prof.brodrigues@gmail.com \\
\hline Notes & RBR-5thjgv
\end{tabular}

\section{RBR-7699xz}

\section{Trial name or title}

Multidisciplinary analysis of a motor rehabilitation protocol with partial weight support for post stroke patients: a randomised clinical trial

\begin{tabular}{ll}
\hline Methods & RCT \\
\hline
\end{tabular}


RBR-7699xz (Continued)

\begin{tabular}{ll} 
Participants & $\mathrm{N}=50$ \\
\hline Interventions & Cardiorespiratory training \\
\hline Outcomes & BBS, stride velocity, gait kinematics, BI \\
\hline Starting date & 2014 \\
\hline Contact information & elisangela.manffra@pucpr.br \\
\hline Notes & \\
\hline
\end{tabular}

\section{RBR-7hqk8t}

\begin{tabular}{ll}
\hline Trial name or title & $\begin{array}{l}\text { Efficacy of task-specific training in changing neurotrophic factors and impact on clinical out- } \\
\text { comes in individuals with stroke: a randomised controlled trial }\end{array}$ \\
\hline Methods & $\mathrm{RCT}$ \\
\hline Participants & $\mathrm{N}=36$ \\
\hline Interventions & Mixed training \\
\hline Outcomes & Neurotropic factor concentration, 10-MWT, 6-MWT, TUG test \\
\hline Starting date & February 2018 \\
\hline Contact information & scalzopl@gmail.com \\
\hline Notes & \\
\hline
\end{tabular}

\section{TCTR20160601005}

Trial name or title

Effectiveness of antigravity treadmill training in improving walking capacity and balance in hemiparetic ischemic stroke patients

\begin{tabular}{ll}
\hline Methods & $\mathrm{RCT}$ \\
\hline Participants & $\mathrm{N}=34$ \\
\hline Interventions & Cardiorespiratory - unclear \\
\hline Outcomes & 6-MWT, FAC, balance, muscle EMG, satisfaction after antigravity treadmill training \\
\hline Starting date & July 2016 \\
\hline Contact information & kwanyupa@gmail.com \\
\hline Notes &
\end{tabular}




\section{TCTR20170615002}

Trial name or title Effects of task-oriented training on upper extremity functional performance in patients with subacute stroke: a randomised controlled trial

\begin{tabular}{ll}
\hline Methods & $\mathrm{RCT}$ \\
\hline Participants & $\mathrm{N}=28$ \\
\hline Interventions & Resistance training - unclear \\
\hline Outcomes & Upper extremity functional performance and recovery, SIS \\
\hline Starting date & August 2017 \\
\hline Contact information & ayeayethantumt@gmail.com \\
\hline
\end{tabular}

Notes

Vahlberg 2018

\begin{tabular}{ll}
\hline Trial name or title & $\begin{array}{l}\text { Protocol and pilot study of a short message service-guided training after acute stroke/transient is- } \\
\text { chemic attack to increase walking capacity and physical activity }\end{array}$ \\
\hline Methods & $\mathrm{RCT}$ \\
\hline Participants & Mixed training \\
\hline Interventions & $\begin{array}{l}\text { 6-MWT, chair-rising, mobility, gait speed, handgrip strength, body composition (fat mass and mus- } \\
\text { cle mass), biochemical risk-markers, health-related QoL, and cardiovascular events }\end{array}$ \\
\hline Outcomes & November 2016 \\
\hline Starting date & birgit.vahlberg@pubcare.uu.se \\
\hline Contact information & NCT02720276
\end{tabular}

10-MWT: 10-Metre Walk Test; 6-MWT: 6-Minute Walk Test; ARAT: Action Research Arm Test; BBS: Berg Balance Scale; BMI: body mass index; BI: Barthel Index; BP: blood pressure; EMG: electromyography; FAC: Functional Ambulation Classification; FIM: Functional Independence Measure; HRQoL: health-related quality of life; IADL: Instrumental Activities of Daily Living; MAS: Motor Assessment Scale; mRS: modified Rankin Scale; MVC: maximal voluntary contraction; NEADL: Nottingham Extended Activities of Daily Living Scale; NHP: Nottingham Health Profile; QoL: quality of life; RCT: randomised controlled trial; RMI: Rivermead Mobility Index; RVGA: Rivermead Visual Gait Assessment; SIS: Stroke Impact Scale; SS-QoL: Stroke Specific Quality of Life Scale; STS: sit to stand; TIA: transient ischaemic attack; TUG: Timed Up and Go Test; UC: usual care; VAS: Visual Analogue Scale; WHO: World Health Organization; WMFT: Wolf Motor Function Test

\section{DATA AND ANALYSES}


Comparison 1. Cardiorespiratory training versus control - end of intervention

\begin{tabular}{|c|c|c|c|c|}
\hline Outcome or subgroup title & $\begin{array}{l}\text { No. of } \\
\text { studies }\end{array}$ & $\begin{array}{l}\text { No. of } \\
\text { partici- } \\
\text { pants }\end{array}$ & Statistical method & Effect size \\
\hline 1 Death & 32 & 1631 & $\begin{array}{l}\text { Risk Difference }(\mathrm{M}-\mathrm{H} \text {, Random, } \\
95 \% \mathrm{Cl})\end{array}$ & $0.0[-0.01,0.01]$ \\
\hline 1.1 During usual care & 16 & 698 & $\begin{array}{l}\text { Risk Difference (M-H, Random, } \\
95 \% \mathrm{CI})\end{array}$ & $0.0[-0.02,0.02]$ \\
\hline 1.2 After usual care & 16 & 933 & $\begin{array}{l}\text { Risk Difference (M-H, Random, } \\
95 \% \mathrm{CI})\end{array}$ & $0.0[-0.02,0.02]$ \\
\hline $\begin{array}{l}2 \text { Disability - Functional Independence } \\
\text { Measure }\end{array}$ & 3 & 162 & $\begin{array}{l}\text { Std. Mean Difference (IV, Random, } \\
95 \% \mathrm{Cl})\end{array}$ & $0.21[-0.10,0.52]$ \\
\hline 2.1 During usual care & 1 & 52 & $\begin{array}{l}\text { Std. Mean Difference (IV, Random, } \\
95 \% \mathrm{CI} \text { ) }\end{array}$ & $0.23[-0.32,0.78]$ \\
\hline 2.2 After usual care & 2 & 110 & $\begin{array}{l}\text { Std. Mean Difference (IV, Random, } \\
95 \% \mathrm{CI} \text { ) }\end{array}$ & $0.17[-0.29,0.63]$ \\
\hline 3 Disability - Barthel Index & 3 & 243 & $\begin{array}{l}\text { Mean Difference (IV, Random, } \\
95 \% \mathrm{CI} \text { ) }\end{array}$ & $6.65[-0.67,13.98]$ \\
\hline 3.1 During usual care & 2 & 115 & $\begin{array}{l}\text { Mean Difference (IV, Random, } \\
95 \% \mathrm{CI} \text { ) }\end{array}$ & $10.48[-11.83,32.80]$ \\
\hline 3.2 After usual care & 1 & 128 & $\begin{array}{l}\text { Mean Difference (IV, Random, } \\
95 \% \mathrm{CI})\end{array}$ & $2.60[-0.15,5.35]$ \\
\hline $\begin{array}{l}4 \text { Disability - Rivermead Mobility Index } \\
\text { (scale } 0 \text { to } 15 \text { ) }\end{array}$ & 3 & 146 & $\begin{array}{l}\text { Mean Difference (IV, Random, } \\
95 \% \mathrm{CI})\end{array}$ & $1.56[0.20,2.92]$ \\
\hline 4.1 During usual care & 2 & 110 & $\begin{array}{l}\text { Mean Difference (IV, Random, } \\
95 \% \mathrm{CI})\end{array}$ & $1.43[-0.62,3.49]$ \\
\hline 4.2 After usual care & 1 & 36 & $\begin{array}{l}\text { Mean Difference (IV, Random, } \\
95 \% \mathrm{CI})\end{array}$ & $2.0[0.53,3.47]$ \\
\hline 5 Disability - combined disability scales & 8 & 462 & $\begin{array}{l}\text { Std. Mean Difference (IV, Random, } \\
95 \% \mathrm{CI})\end{array}$ & $0.52[0.19,0.84]$ \\
\hline 5.1 During usual care & 3 & 130 & $\begin{array}{l}\text { Std. Mean Difference (IV, Random, } \\
95 \% \mathrm{Cl})\end{array}$ & $0.88[0.08,1.68]$ \\
\hline 5.2 After usual care & 5 & 332 & $\begin{array}{l}\text { Std. Mean Difference (IV, Random, } \\
95 \% \mathrm{CI} \text { ) }\end{array}$ & $0.33[0.11,0.55]$ \\
\hline 6 Risk factors - blood pressure, systolic & 5 & 318 & $\begin{array}{l}\text { Mean Difference (IV, Random, } \\
95 \% \mathrm{CI})\end{array}$ & $-0.20[-4.00,5.60]$ \\
\hline 6.1 During usual care & 1 & 12 & $\begin{array}{l}\text { Mean Difference (IV, Random, } \\
95 \% \mathrm{CI})\end{array}$ & $26.33[1.95,50.71]$ \\
\hline 6.2 After usual care & 4 & 306 & $\begin{array}{l}\text { Mean Difference (IV, Random, } \\
95 \% \mathrm{CI} \text { ) }\end{array}$ & $-1.41[-5.25,2.43]$ \\
\hline
\end{tabular}




\begin{tabular}{|c|c|c|c|c|}
\hline Outcome or subgroup title & $\begin{array}{l}\text { No. of } \\
\text { studies }\end{array}$ & $\begin{array}{l}\text { No. of } \\
\text { partici- } \\
\text { pants }\end{array}$ & Statistical method & Effect size \\
\hline 7 Risk factors - blood pressure, diastolic & 5 & 318 & $\begin{array}{l}\text { Mean Difference (IV, Random, } \\
95 \% \mathrm{CI})\end{array}$ & $-0.15[-2.28,1.98]$ \\
\hline 7.1 During usual care & 1 & 12 & $\begin{array}{l}\text { Mean Difference (IV, Random, } \\
95 \% \mathrm{CI})\end{array}$ & $1.0[-10.46,12.46]$ \\
\hline 7.2 After usual care & 4 & 306 & $\begin{array}{l}\text { Mean Difference (IV, Random, } \\
95 \% \mathrm{CI} \text { ) }\end{array}$ & $-0.19[-2.35,1.98]$ \\
\hline 8 Risk factors - body mass index (BMI) & 2 & 174 & $\begin{array}{l}\text { Mean Difference (IV, Random, } \\
95 \% \mathrm{CI})\end{array}$ & $1.19[-0.38,2.76]$ \\
\hline 8.1 During usual care & 0 & 0 & $\begin{array}{l}\text { Mean Difference (IV, Random, } \\
95 \% \mathrm{CI})\end{array}$ & $0.0[0.0,0.0]$ \\
\hline 8.2 After usual care & 2 & 174 & $\begin{array}{l}\text { Mean Difference (IV, Random, } \\
95 \% \mathrm{CI})\end{array}$ & $1.19[-0.38,2.76]$ \\
\hline 9 Physical fitness - peak $\mathrm{VO}_{2}$ & 9 & 438 & $\begin{array}{l}\text { Mean Difference (IV, Random, } \\
95 \% \mathrm{CI})\end{array}$ & $3.40[2.98,3.83]$ \\
\hline 9.1 During usual care & 3 & 121 & $\begin{array}{l}\text { Mean Difference (IV, Random, } \\
95 \% \mathrm{CI} \text { ) }\end{array}$ & $2.28[0.75,3.81]$ \\
\hline 9.2 After usual care & 6 & 317 & $\begin{array}{l}\text { Mean Difference (IV, Random, } \\
95 \% \mathrm{CI} \text { ) }\end{array}$ & $3.51[3.06,3.96]$ \\
\hline $\begin{array}{l}10 \text { Physical fitness - maximum cycling } \\
\text { work rate }\end{array}$ & 6 & 336 & $\begin{array}{l}\text { Mean Difference (IV, Random, } \\
95 \% \mathrm{CI})\end{array}$ & $10.60[1.88,19.33]$ \\
\hline 10.1 During usual care & 3 & 148 & $\begin{array}{l}\text { Mean Difference (IV, Random, } \\
95 \% \mathrm{CI})\end{array}$ & $3.48[-5.23,12.19]$ \\
\hline 10.2 After usual care & 3 & 188 & $\begin{array}{l}\text { Mean Difference (IV, Random, } \\
95 \% \mathrm{CI})\end{array}$ & $13.37[8.55,18.19]$ \\
\hline $\begin{array}{l}11 \text { Mobility - functional ambulation cat- } \\
\text { egories }\end{array}$ & 2 & 73 & $\begin{array}{l}\text { Mean Difference (IV, Random, } \\
95 \% \mathrm{CI})\end{array}$ & $0.53[0.21,0.85]$ \\
\hline 11.1 During usual care & 2 & 73 & $\begin{array}{l}\text { Mean Difference (IV, Random, } \\
95 \% \mathrm{CI})\end{array}$ & $0.53[0.21,0.85]$ \\
\hline 11.2 After usual care & 0 & 0 & $\begin{array}{l}\text { Mean Difference (IV, Random, } \\
95 \% \mathrm{CI} \text { ) }\end{array}$ & $0.0[0.0,0.0]$ \\
\hline $\begin{array}{l}12 \text { Mobility - walking maximal speed } \\
\text { (over } 5 \text { to } 10 \text { metres) }\end{array}$ & 17 & 782 & $\begin{array}{l}\text { Mean Difference (IV, Random, } \\
95 \% \mathrm{CI})\end{array}$ & $7.66[3.65,11.68]$ \\
\hline 12.1 During usual care & 10 & 383 & $\begin{array}{l}\text { Mean Difference (IV, Random, } \\
95 \% \mathrm{Cl} \text { ) }\end{array}$ & $5.20[0.48,9.92]$ \\
\hline 12.2 After usual care & 7 & 399 & $\begin{array}{l}\text { Mean Difference (IV, Random, } \\
95 \% \mathrm{CI} \text { ) }\end{array}$ & $10.94[7.13,14.76]$ \\
\hline
\end{tabular}




\begin{tabular}{|c|c|c|c|c|}
\hline Outcome or subgroup title & $\begin{array}{l}\text { No. of } \\
\text { studies }\end{array}$ & $\begin{array}{l}\text { No. of } \\
\text { partici- } \\
\text { pants }\end{array}$ & Statistical method & Effect size \\
\hline 13 Mobility - walking preferred speed & 12 & 588 & $\begin{array}{l}\text { Mean Difference (IV, Random, } \\
95 \% \mathrm{CI})\end{array}$ & $4.47[2.07,6.87]$ \\
\hline 13.1 During usual care & 6 & 211 & $\begin{array}{l}\text { Mean Difference (IV, Random, } \\
95 \% \mathrm{CI})\end{array}$ & $3.90[-1.25,9.05]$ \\
\hline 13.2 After usual care & 6 & 377 & $\begin{array}{l}\text { Mean Difference (IV, Random, } \\
95 \% \mathrm{CI} \text { ) }\end{array}$ & $4.69[1.57,7.80]$ \\
\hline $\begin{array}{l}14 \text { Mobility - walking capacity (6-MWT } \\
\text { metres) }\end{array}$ & 16 & 882 & $\begin{array}{l}\text { Mean Difference (IV, Random, } \\
95 \% \mathrm{CI})\end{array}$ & $33.41[19.04,47.78]$ \\
\hline 14.1 During usual care & 7 & 225 & $\begin{array}{l}\text { Mean Difference (IV, Random, } \\
95 \% \mathrm{CI})\end{array}$ & $32.10[10.11,54.10]$ \\
\hline 14.2 After usual care & 9 & 657 & $\begin{array}{l}\text { Mean Difference (IV, Random, } \\
95 \% \mathrm{CI})\end{array}$ & $37.03[15.54,58.51]$ \\
\hline 15 Mobility - walking capacity (m/min) & 3 & 154 & $\begin{array}{l}\text { Mean Difference (IV, Random, } \\
95 \% \mathrm{CI})\end{array}$ & $8.87[1.35,16.40]$ \\
\hline 15.1 During usual care & 2 & 63 & $\begin{array}{l}\text { Mean Difference (IV, Random, } \\
95 \% \mathrm{CI})\end{array}$ & $12.24[-3.41,27.89]$ \\
\hline 15.2 After usual care & 1 & 91 & $\begin{array}{l}\text { Mean Difference (IV, Random, } \\
95 \% \mathrm{CI} \text { ) }\end{array}$ & $6.60[-2.66,15.86]$ \\
\hline 16 Mobility - community walk (min) & 2 & 47 & $\begin{array}{l}\text { Mean Difference (IV, Random, } \\
95 \% \mathrm{CI})\end{array}$ & $-10.54[-14.11,-6.98]$ \\
\hline 16.1 During usual care & 2 & 47 & $\begin{array}{l}\text { Mean Difference (IV, Random, } \\
95 \% \mathrm{CI})\end{array}$ & $-10.54[-14.11,-6.98]$ \\
\hline 16.2 After usual care & 0 & 0 & $\begin{array}{l}\text { Mean Difference (IV, Random, } \\
95 \% \mathrm{CI})\end{array}$ & $0.0[0.0,0.0]$ \\
\hline $\begin{array}{l}17 \text { Physical function - Berg Balance } \\
\text { Scale (score } 0 \text { to 56) }\end{array}$ & 8 & 471 & $\begin{array}{l}\text { Mean Difference (IV, Random, } \\
95 \% \mathrm{CI})\end{array}$ & $1.92[0.16,3.68]$ \\
\hline 17.1 During usual care & 3 & 160 & $\begin{array}{l}\text { Mean Difference (IV, Random, } \\
95 \% \mathrm{CI})\end{array}$ & $0.79[-2.01,3.59]$ \\
\hline 17.2 After usual care & 5 & 311 & $\begin{array}{l}\text { Mean Difference (IV, Random, } \\
95 \% \mathrm{CI} \text { ) }\end{array}$ & $2.67[0.07,5.26]$ \\
\hline $\begin{array}{l}18 \text { Physical function - Timed Up and Go } \\
\text { (sec) }\end{array}$ & 5 & 223 & $\begin{array}{l}\text { Mean Difference (IV, Random, } \\
95 \% \mathrm{CI})\end{array}$ & $-3.42[-4.78,-2.05]$ \\
\hline 18.1 During usual care & 1 & 20 & $\begin{array}{l}\text { Mean Difference (IV, Random, } \\
95 \% \mathrm{Cl} \text { ) }\end{array}$ & $-2.10[-6.27,2.07]$ \\
\hline 18.2 After usual care & 4 & 203 & $\begin{array}{l}\text { Mean Difference (IV, Random, } \\
95 \% \mathrm{CI} \text { ) }\end{array}$ & $-3.58[-5.02,-2.13]$ \\
\hline
\end{tabular}




\begin{tabular}{|c|c|c|c|c|}
\hline Outcome or subgroup title & $\begin{array}{l}\text { No. of } \\
\text { studies }\end{array}$ & $\begin{array}{l}\text { No. of } \\
\text { partici- } \\
\text { pants }\end{array}$ & Statistical method & Effect size \\
\hline $\begin{array}{l}19 \text { Health-related QoL - SF- } 36 \text { \& SF-12 } \\
\text { Physical Health Component }\end{array}$ & 2 & 164 & $\begin{array}{l}\text { Std. Mean Difference (IV, Random, } \\
95 \% \mathrm{CI} \text { ) }\end{array}$ & $0.51[0.20,0.82]$ \\
\hline 19.1 During usual care & 0 & 0 & $\begin{array}{l}\text { Std. Mean Difference (IV, Random, } \\
95 \% \mathrm{CI} \text { ) }\end{array}$ & $0.0[0.0,0.0]$ \\
\hline 19.2 After usual care & 2 & 164 & $\begin{array}{l}\text { Std. Mean Difference (IV, Random, } \\
95 \% \mathrm{CI} \text { ) }\end{array}$ & $0.51[0.20,0.82]$ \\
\hline $\begin{array}{l}20 \text { Health-related QoL - SF- } 36 \text { \& SF-12 } \\
\text { Mental Health Component }\end{array}$ & 2 & 164 & $\begin{array}{l}\text { Std. Mean Difference (IV, Random, } \\
95 \% \mathrm{CI} \text { ) }\end{array}$ & $0.58[-0.52,1.68]$ \\
\hline 20.1 During usual care & 0 & 0 & $\begin{array}{l}\text { Std. Mean Difference (IV, Random, } \\
95 \% \mathrm{CI} \text { ) }\end{array}$ & $0.0[0.0,0.0]$ \\
\hline 20.2 After usual care & 2 & 164 & $\begin{array}{l}\text { Std. Mean Difference (IV, Random, } \\
95 \% \mathrm{CI} \text { ) }\end{array}$ & $0.58[-0.52,1.68]$ \\
\hline 21 Health-related QoL - EuroQol EQ-5D & 2 & 158 & $\begin{array}{l}\text { Mean Difference (IV, Random, } \\
95 \% \mathrm{CI} \text { ) }\end{array}$ & $6.55[-1.36,14.47]$ \\
\hline 21.1 During usual care & 0 & 0 & $\begin{array}{l}\text { Mean Difference (IV, Random, } \\
95 \% \mathrm{CI} \text { ) }\end{array}$ & $0.0[0.0,0.0]$ \\
\hline 21.2 After usual care & 2 & 158 & $\begin{array}{l}\text { Mean Difference (IV, Random, } \\
95 \% \mathrm{CI} \text { ) }\end{array}$ & $6.55[-1.36,14.47]$ \\
\hline 22 Mood - Beck Depression Index & 2 & 56 & $\begin{array}{l}\text { Mean Difference (IV, Random, } \\
95 \% \mathrm{CI} \text { ) }\end{array}$ & $-1.22[-5.62,3.19]$ \\
\hline 22.1 During usual care & 0 & 0 & $\begin{array}{l}\text { Mean Difference (IV, Random, } \\
95 \% \mathrm{Cl} \text { ) }\end{array}$ & $0.0[0.0,0.0]$ \\
\hline 22.2 After usual care & 2 & 56 & $\begin{array}{l}\text { Mean Difference (IV, Random, } \\
95 \% \mathrm{CI} \text { ) }\end{array}$ & $-1.22[-5.62,3.19]$ \\
\hline
\end{tabular}

Analysis 1.1. Comparison 1 Cardiorespiratory training versus control - end of intervention, Outcome 1 Death.

\begin{tabular}{|c|c|c|c|c|c|}
\hline Study or subgroup & $\begin{array}{c}\text { Training } \\
\mathrm{n} / \mathrm{N}\end{array}$ & $\begin{array}{c}\text { Control } \\
n / N\end{array}$ & $\begin{array}{c}\text { Risk Difference } \\
\text { M-H, Random, 95\% Cl }\end{array}$ & Weight & $\begin{array}{c}\text { Risk Difference } \\
\text { M-H, Random, 95\% Cl }\end{array}$ \\
\hline \multicolumn{6}{|c|}{ 1.1.1 During usual care } \\
\hline Bateman 2001 & $0 / 40$ & $0 / 44$ & + & $7.19 \%$ & $0[-0.05,0.05]$ \\
\hline da Cunha 2002 & $0 / 7$ & $0 / 8$ & $\longrightarrow$ & $0.29 \%$ & $0[-0.22,0.22]$ \\
\hline Eich 2004 & $0 / 25$ & $0 / 25$ & + & $2.67 \%$ & $0[-0.07,0.07]$ \\
\hline Glasser 1986 & $0 / 10$ & $0 / 10$ & $\longrightarrow$ & $0.49 \%$ & $0[-0.17,0.17]$ \\
\hline Kim 2014 & $0 / 13$ & $0 / 13$ & $\longrightarrow$ & $0.79 \%$ & $0[-0.14,0.14]$ \\
\hline Kuys 2011 & $0 / 15$ & $0 / 15$ & -1 & $1.02 \%$ & $0[-0.12,0.12]$ \\
\hline MacKay-Lyons 2013 & $0 / 24$ & $0 / 26$ & + & $2.65 \%$ & $0[-0.07,0.07]$ \\
\hline
\end{tabular}




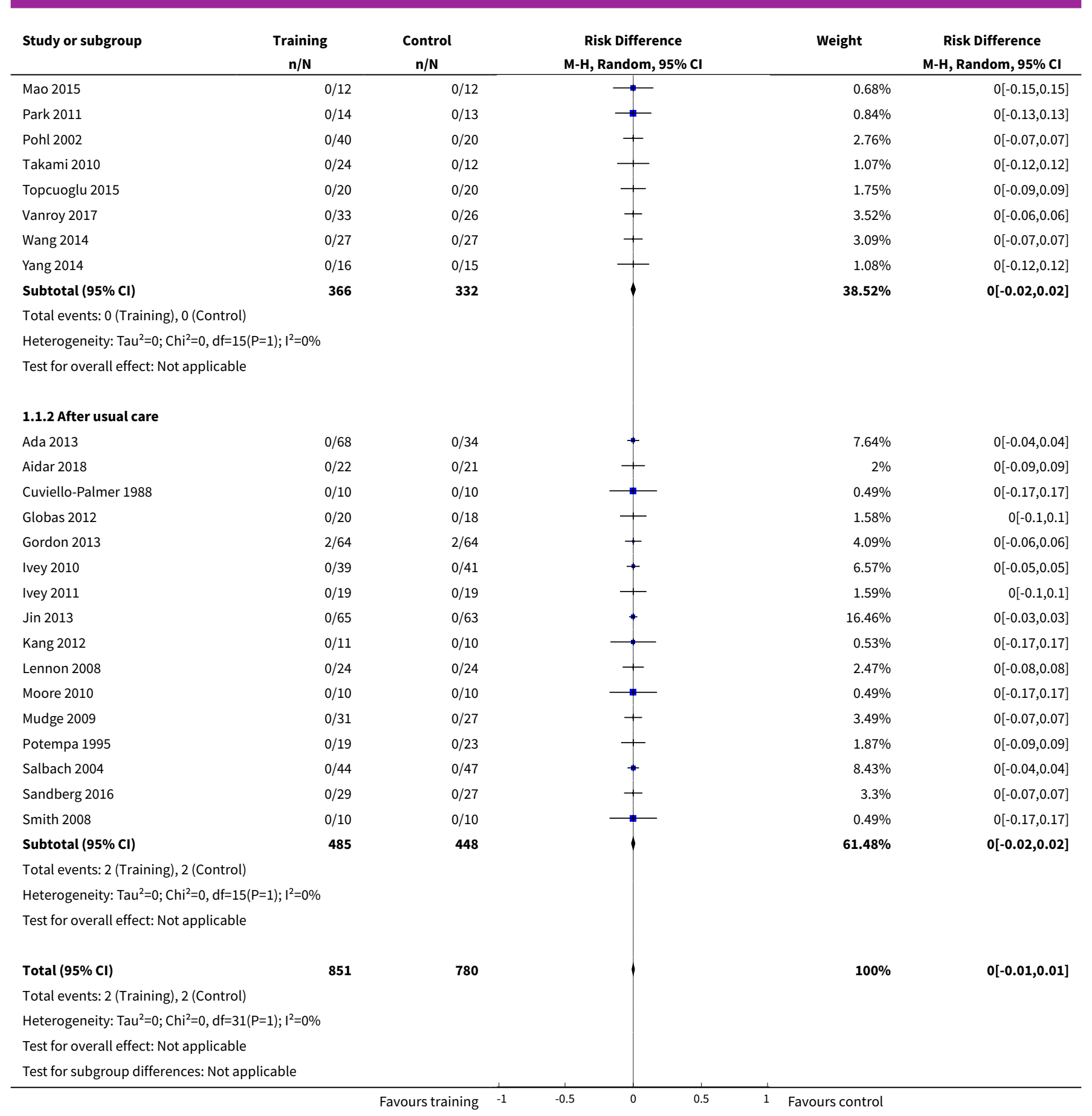

Analysis 1.2. Comparison 1 Cardiorespiratory training versus control - end of intervention, Outcome 2 Disability - Functional Independence Measure.

\begin{tabular}{|c|c|c|c|c|c|c|c|c|c|}
\hline \multirow[t]{2}{*}{ Study or subgroup } & \multicolumn{2}{|c|}{ Training } & \multicolumn{2}{|c|}{ Control } & \multirow{2}{*}{\multicolumn{3}{|c|}{$\begin{array}{c}\text { Std. Mean Difference } \\
\text { Random, } 95 \% \mathrm{CI}\end{array}$}} & \multirow[t]{2}{*}{ Weight } & \multirow{2}{*}{$\begin{array}{c}\text { Std. Mean Difference } \\
\text { Random, } 95 \% \mathrm{CI}\end{array}$} \\
\hline & $\mathbf{N}$ & Mean(SD) & $\mathbf{N}$ & Mean(SD) & & & & & \\
\hline Bateman 2001 & 23 & $104.7(17.7)$ & 29 & $100.4(18.9)$ & & & $\#$ & $31.91 \%$ & $0.23[-0.32,0.78]$ \\
\hline
\end{tabular}




\begin{tabular}{|c|c|c|c|c|c|c|c|}
\hline \multirow[t]{2}{*}{ Study or subgroup } & \multicolumn{2}{|c|}{ Training } & \multicolumn{2}{|c|}{ Control } & \multirow{2}{*}{$\begin{array}{c}\text { Std. Mean Difference } \\
\text { Random, } 95 \% \mathrm{CI}\end{array}$} & \multirow[t]{2}{*}{ Weight } & \multirow{2}{*}{$\begin{array}{c}\text { Std. Mean Difference } \\
\text { Random, } 95 \% \mathrm{Cl}\end{array}$} \\
\hline & $\mathbf{N}$ & Mean(SD) & $\mathbf{N}$ & Mean(SD) & & & \\
\hline Subtotal $* \star \star$ & 23 & & 29 & & & $31.91 \%$ & $0.23[-0.32,0.78]$ \\
\hline \multicolumn{8}{|c|}{ Heterogeneity: Not applicable } \\
\hline \multicolumn{8}{|c|}{ Test for overall effect: $Z=0.83(P=0.4)$} \\
\hline \multicolumn{8}{|l|}{ 1.2.2 After usual care } \\
\hline Cuviello-Palmer 1988 & 10 & $44.8(8.8)$ & 10 & $47.2(9.9)$ & $\rightarrow$ & $12.41 \%$ & $-0.25[-1.13,0.64]$ \\
\hline Katz-Leurer 2003 & 46 & $105.8(12.5)$ & 44 & $101.4(16)$ & + & $55.68 \%$ & $0.3[-0.11,0.72]$ \\
\hline Subtotal *** & 56 & & 54 & & 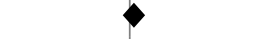 & $68.09 \%$ & $0.17[-0.29,0.63]$ \\
\hline \multicolumn{8}{|c|}{ Heterogeneity: $\mathrm{Tau}^{2}=0.03 ; \mathrm{Chi}^{2}=1.22, \mathrm{df}=1(\mathrm{P}=0.27) ; \mathrm{I}^{2}=18.31 \%$} \\
\hline \multicolumn{8}{|c|}{ Test for overall effect: $\mathrm{Z}=0.73(\mathrm{P}=0.46)$} \\
\hline Total $\star \star \star$ & 79 & & 83 & & $\checkmark$ & $100 \%$ & $0.21[-0.1,0.52]$ \\
\hline \multicolumn{8}{|c|}{ Heterogeneity: $\operatorname{Tau}^{2}=0 ; \mathrm{Chi}^{2}=1.23, \mathrm{df}=2(\mathrm{P}=0.54) ; \mathrm{I}^{2}=0 \%$} \\
\hline \multicolumn{8}{|c|}{ Test for overall effect: $Z=1.35(P=0.18)$} \\
\hline \multicolumn{8}{|c|}{ Test for subgroup differences: $\mathrm{Chi}^{2}=0.03, \mathrm{df}=1(\mathrm{P}=0.87), \mathrm{I}^{2}=0 \%$} \\
\hline & & & & urs control & -2.5 & Favours & ing \\
\hline
\end{tabular}

Analysis 1.3. Comparison 1 Cardiorespiratory training versus control - end of intervention, Outcome 3 Disability - Barthel Index.

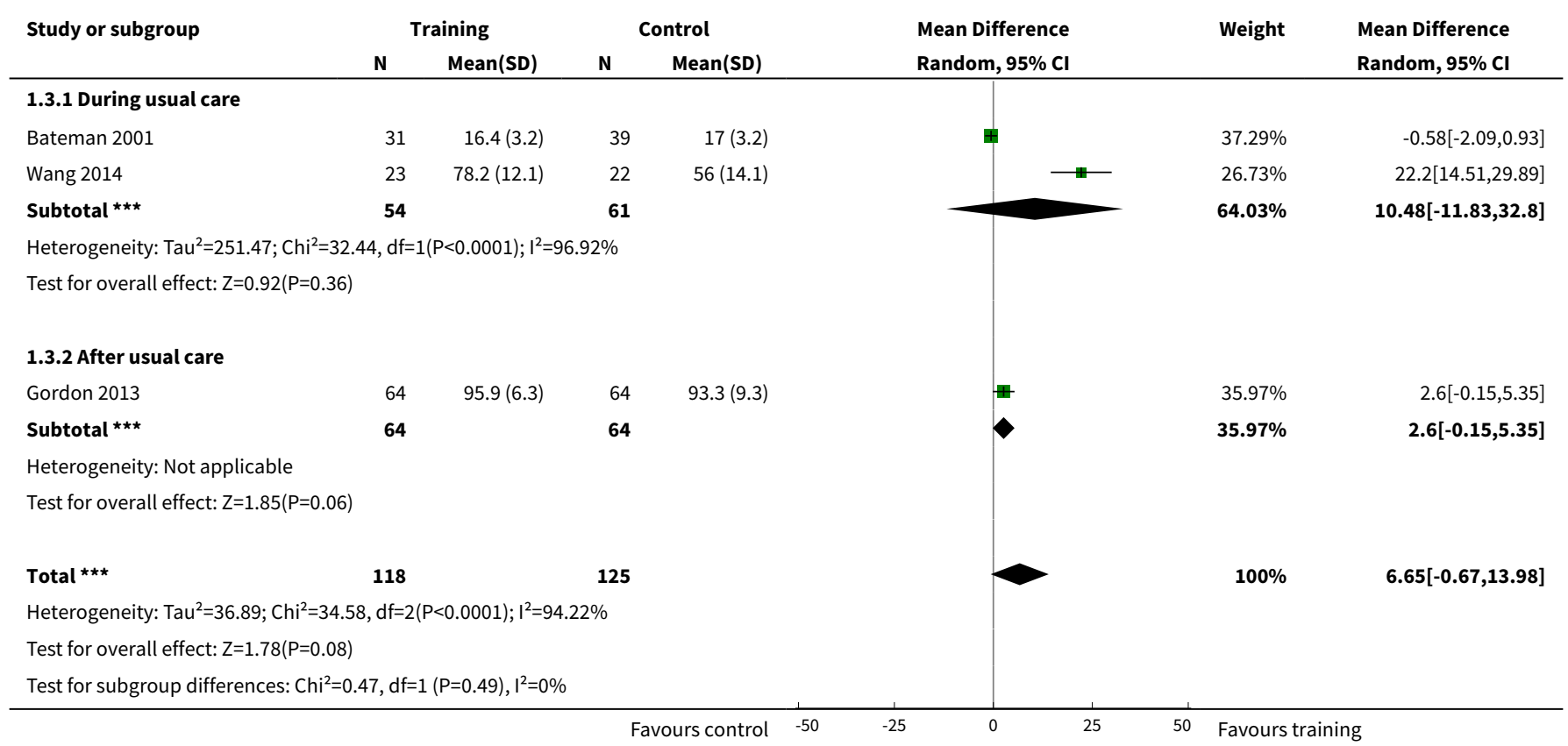

Analysis 1.4. Comparison 1 Cardiorespiratory training versus control - end of intervention, Outcome 4 Disability - Rivermead Mobility Index (scale 0 to 15).

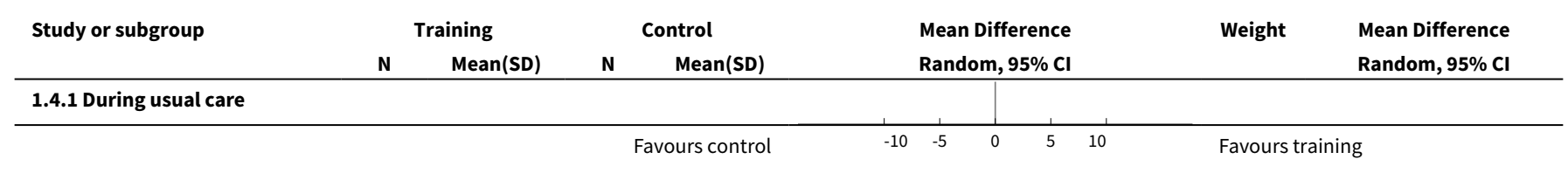




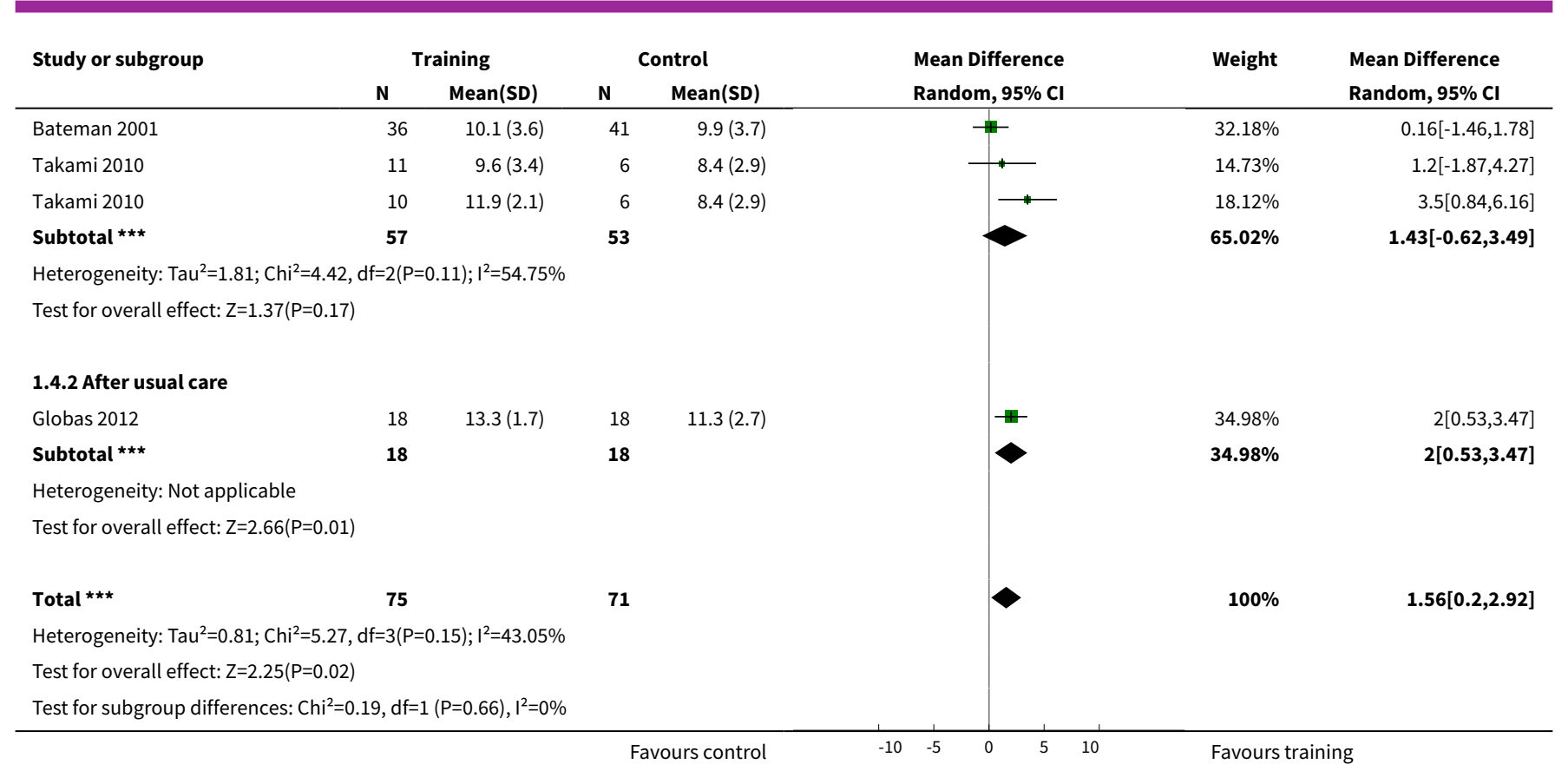

Analysis 1.5. Comparison 1 Cardiorespiratory training versus control end of intervention, Outcome 5 Disability - combined disability scales.

\begin{tabular}{|c|c|c|c|c|c|c|c|}
\hline \multirow[t]{2}{*}{ Study or subgroup } & \multicolumn{2}{|c|}{ Training } & \multicolumn{2}{|c|}{ Control } & \multirow{2}{*}{$\begin{array}{c}\text { Std. Mean Difference } \\
\text { Random, } 95 \% \mathrm{Cl}\end{array}$} & \multirow[t]{2}{*}{ Weight } & \multirow{2}{*}{$\begin{array}{c}\text { Std. Mean Difference } \\
\text { Random, } 95 \% \mathrm{CI}\end{array}$} \\
\hline & $\mathbf{N}$ & $\operatorname{Mean}(S D)$ & $\mathbf{N}$ & Mean(SD) & & & \\
\hline \multicolumn{5}{|c|}{ 1.5.1 During usual care } & & & \\
\hline Bateman 2001 & 23 & $104.7(17.7)$ & 29 & $100.4(18.9)$ & $\longrightarrow$ & $12.84 \%$ & $0.23[-0.32,0.78]$ \\
\hline Takami 2010 & 10 & $11.9(2.1)$ & 6 & $8.4(2.9)$ & + & $5.75 \%$ & $1.37[0.22,2.52]$ \\
\hline Takami 2010 & 11 & $9.6(3.4)$ & 6 & $8.4(2.9)$ & $\longrightarrow$ & $6.93 \%$ & $0.35[-0.65,1.36]$ \\
\hline Wang 2014 & 23 & $78.2(12.1)$ & 22 & $56(14.1)$ & & $10.66 \%$ & $1.66[0.98,2.35]$ \\
\hline Subtotal $\star \star \star$ & 67 & & 63 & & & $36.18 \%$ & $0.88[0.08,1.68]$ \\
\hline \multicolumn{8}{|c|}{ Heterogeneity: $\operatorname{Tau}^{2}=0.48 ; \mathrm{Chi}^{2}=11.87, \mathrm{df}=3(\mathrm{P}=0.01) ; \mathrm{I}^{2}=74.72 \%$} \\
\hline \multicolumn{8}{|c|}{ Test for overall effect: $Z=2.17(P=0.03)$} \\
\hline \multicolumn{8}{|l|}{ 1.5.2 After usual care } \\
\hline Cuviello-Palmer 1988 & 10 & $44.8(8.8)$ & 10 & $47.2(9.9)$ & & $8.16 \%$ & $-0.25[-1.13,0.64]$ \\
\hline Globas 2012 & 18 & $13.3(1.7)$ & 18 & $11.3(2.7)$ & 1 & $10.65 \%$ & $0.87[0.18,1.55]$ \\
\hline Katz-Leurer 2003 & 46 & $105.8(12.5)$ & 44 & $101.4(16)$ & $\longrightarrow$ & $15.21 \%$ & $0.3[-0.11,0.72]$ \\
\hline Mudge 2009 & 31 & $77.8(55.7)$ & 27 & $60.9(67.2)$ & 4 & $13.38 \%$ & $0.27[-0.25,0.79]$ \\
\hline 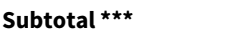 & 169 & & 163 & & & $63.82 \%$ & $0.33[0.11,0.55]$ \\
\hline \multicolumn{8}{|c|}{ Heterogeneity: $\mathrm{Tau}^{2}=0 ; \mathrm{Chi}^{2}=4.05, \mathrm{df}=4(\mathrm{P}=0.4) ; \mathrm{I}^{2}=1.16 \%$} \\
\hline \multicolumn{8}{|c|}{ Test for overall effect: $Z=2.95(P=0)$} \\
\hline 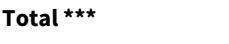 & 236 & & 226 & & & $100 \%$ & $0.52[0.19,0.84]$ \\
\hline \multicolumn{8}{|c|}{ Heterogeneity: $\mathrm{Tau}^{2}=0.14 ; \mathrm{Chi}^{2}=20.31, \mathrm{df}=8(\mathrm{P}=0.01) ; \mathrm{I}^{2}=60.61 \%$} \\
\hline \multicolumn{8}{|c|}{ Test for overall effect: $Z=3.12(P=0)$} \\
\hline Test for subgroup diffe & $2, \mathrm{df}=$ & $(P=0.19), I^{2}=4$ & & & & & \\
\hline
\end{tabular}


Analysis 1.6. Comparison 1 Cardiorespiratory training versus control end of intervention, Outcome 6 Risk factors - blood pressure, systolic.

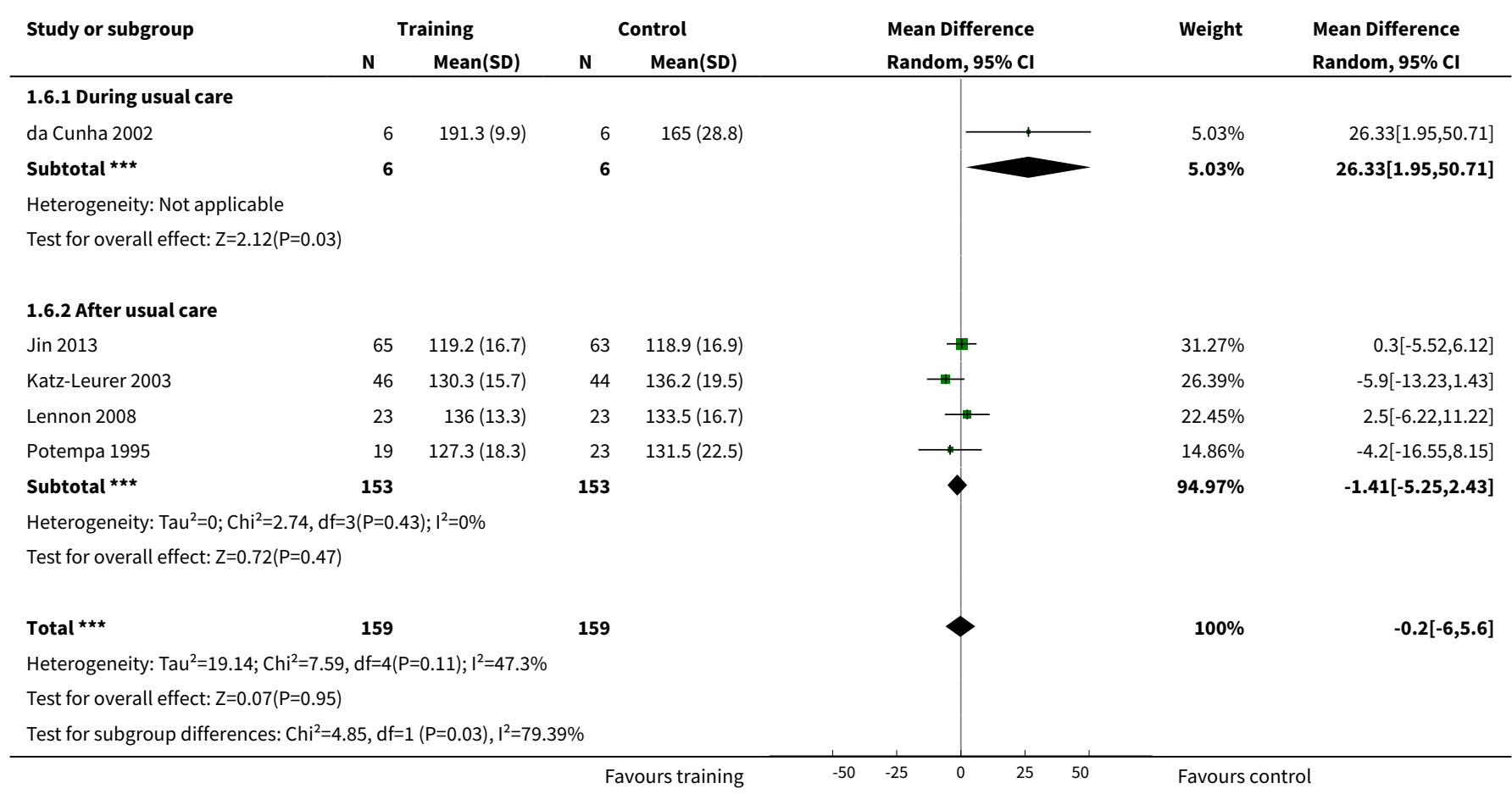

Analysis 1.7. Comparison 1 Cardiorespiratory training versus control end of intervention, Outcome 7 Risk factors - blood pressure, diastolic.

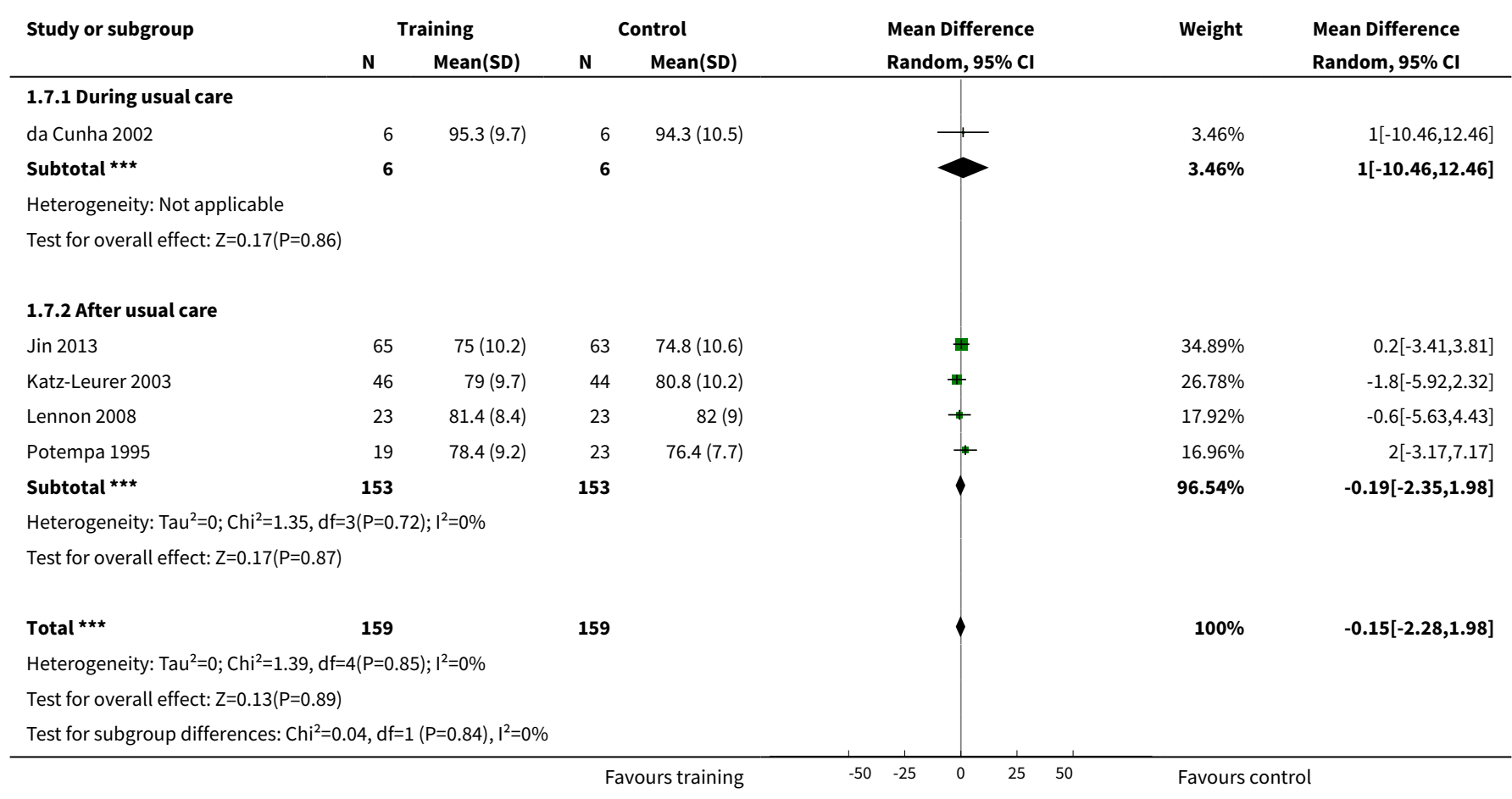


Analysis 1.8. Comparison 1 Cardiorespiratory training versus control - end of intervention, Outcome 8 Risk factors - body mass index (BMI).

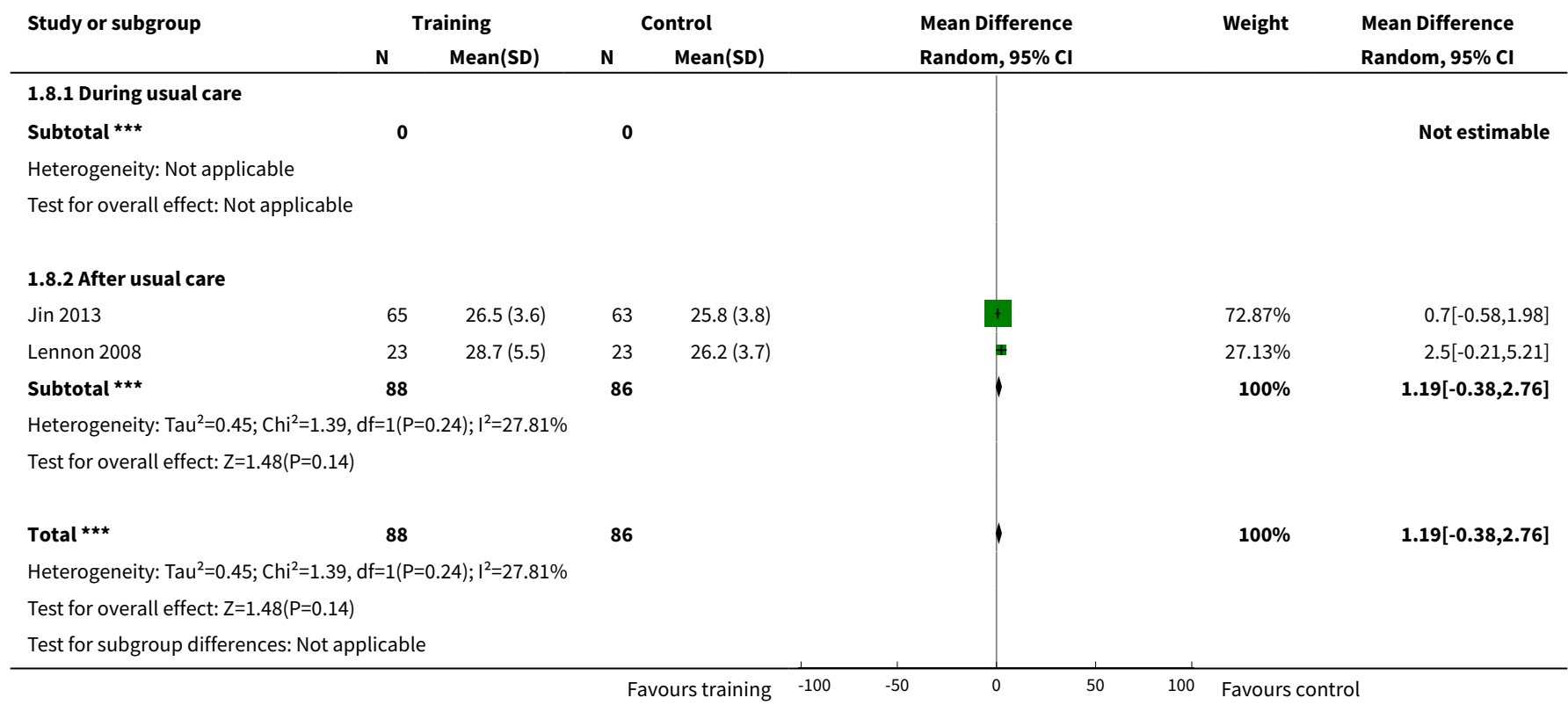

Analysis 1.9. Comparison 1 Cardiorespiratory training versus control - end of intervention, Outcome 9 Physical fitness - peak $\mathrm{VO}_{2}$.

\begin{tabular}{|c|c|c|c|c|c|c|c|c|}
\hline \multirow[t]{2}{*}{ Study or subgroup } & \multicolumn{2}{|c|}{ Training } & \multicolumn{2}{|c|}{ Control } & \multirow{2}{*}{\multicolumn{2}{|c|}{$\begin{array}{l}\text { Mean Difference } \\
\text { Random, } 95 \% \mathrm{Cl}\end{array}$}} & \multirow[t]{2}{*}{ Weight } & \multirow{2}{*}{$\begin{array}{l}\text { Mean Difference } \\
\text { Random, } 95 \% \mathrm{Cl}\end{array}$} \\
\hline & $\mathbf{N}$ & Mean(SD) & $\mathbf{N}$ & $\operatorname{Mean}(S D)$ & & & & \\
\hline \multicolumn{9}{|c|}{ 1.9.1 During usual care } \\
\hline da Cunha 2002 & 6 & $11.6(2.8)$ & 6 & $8.1(2.3)$ & & $\longrightarrow$ & $2.21 \%$ & $3.43[0.56,6.3]$ \\
\hline MacKay-Lyons 2013 & 24 & $18.4(4)$ & 26 & $15.6(4.2)$ & & 1 & $3.54 \%$ & $2.8[0.53,5.07]$ \\
\hline Vanroy 2017 & 33 & $16.1(4.6)$ & 26 & $15.3(5.1)$ & & -1 & $2.91 \%$ & $0.77[-1.74,3.28]$ \\
\hline Subtotal $\star \star \star$ & 63 & & 58 & & & & $8.65 \%$ & $2.28[0.75,3.81]$ \\
\hline \multicolumn{9}{|c|}{ Test for overall effect: $Z=2.92(P=0)$} \\
\hline \multicolumn{9}{|c|}{ 1.9.2 After usual care } \\
\hline Potempa 1995 & 19 & $18.8(4.8)$ & 23 & $15.2(4.3)$ & & 1 & $2.36 \%$ & $3.6[0.82,6.38]$ \\
\hline Moore 2010 & 10 & $18(5.4)$ & 10 & $16(7.1)$ & & & $0.6 \%$ & $2[-3.53,7.53]$ \\
\hline Ivey 2010 & 29 & $16.6(5.6)$ & 24 & $12.8(24)$ & & & $0.19 \%$ & $3.8[-6.02,13.62]$ \\
\hline Globas 2012 & 18 & $24.4(6.6)$ & 18 & $20.9(7.8)$ & & \#- & $0.82 \%$ & $3.5[-1.22,8.22]$ \\
\hline Jin 2013 & 65 & $16.8(1.6)$ & 63 & $13.3(1)$ & & + & $86.08 \%$ & $3.5[3.04,3.96]$ \\
\hline Subtotal $\star \star \star$ & 160 & & 157 & & & $\diamond$ & $91.35 \%$ & $3.51[3.06,3.96]$ \\
\hline \multicolumn{9}{|c|}{ Heterogeneity: $\mathrm{Tau}^{2}=0 ; \mathrm{Chi}^{2}=0.62, \mathrm{df}=5(\mathrm{P}=0.99) ; \mathrm{I}^{2}=0 \%$} \\
\hline \multicolumn{9}{|c|}{ Test for overall effect: $Z=15.38(P<0.0001)$} \\
\hline Total ${ }^{\star \star \star}$ & 223 & & 215 & & & $\diamond$ & $100 \%$ & $3.4[2.98,3.83]$ \\
\hline \multicolumn{9}{|c|}{ Heterogeneity: $\operatorname{Tau}^{2}=0 ; \mathrm{Chi}^{2}=5.35, \mathrm{df}=8(\mathrm{P}=0.72) ; \mathrm{I}^{2}=0 \%$} \\
\hline \multicolumn{9}{|c|}{ Test for overall effect: $Z=15.6(P<0.0001)$} \\
\hline & & & & urs control & -10 & -5 & Favours & \\
\hline
\end{tabular}




\begin{tabular}{|c|c|c|c|c|c|c|c|c|c|}
\hline \multirow[t]{2}{*}{ Study or subgroup } & Training & \multicolumn{2}{|c|}{ Control } & & \multirow{2}{*}{\multicolumn{2}{|c|}{$\begin{array}{l}\text { Mean Difference } \\
\text { Random, } 95 \% \mathrm{Cl}\end{array}$}} & & \multirow[t]{2}{*}{ Weight } & \multirow{2}{*}{$\begin{array}{l}\text { Mean Difference } \\
\text { Random, } 95 \% \mathrm{Cl}\end{array}$} \\
\hline & Mean(SD) & $\mathbf{N}$ & Mean(SD) & & & & & & \\
\hline \multicolumn{10}{|c|}{ Test for subgroup differences: $\mathrm{Chi}^{2}=2.28, \mathrm{df}=1(\mathrm{P}=0.13), \mathrm{I}^{2}=56.06 \%$} \\
\hline
\end{tabular}

Analysis 1.10. Comparison 1 Cardiorespiratory training versus control - end of intervention, Outcome 10 Physical fitness - maximum cycling work rate.

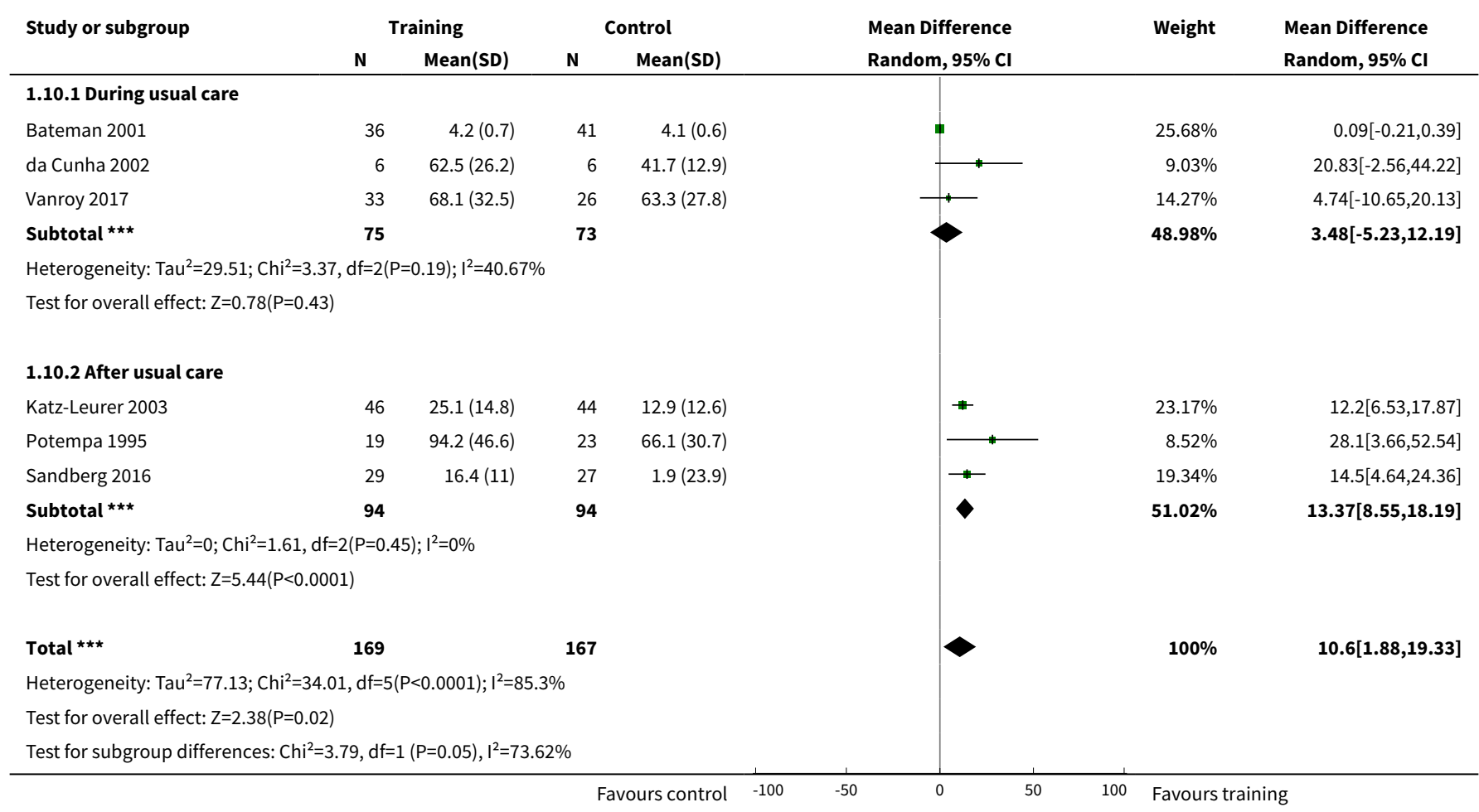

\section{Analysis 1.11. Comparison 1 Cardiorespiratory training versus control - end of intervention, Outcome 11 Mobility - functional ambulation categories.}

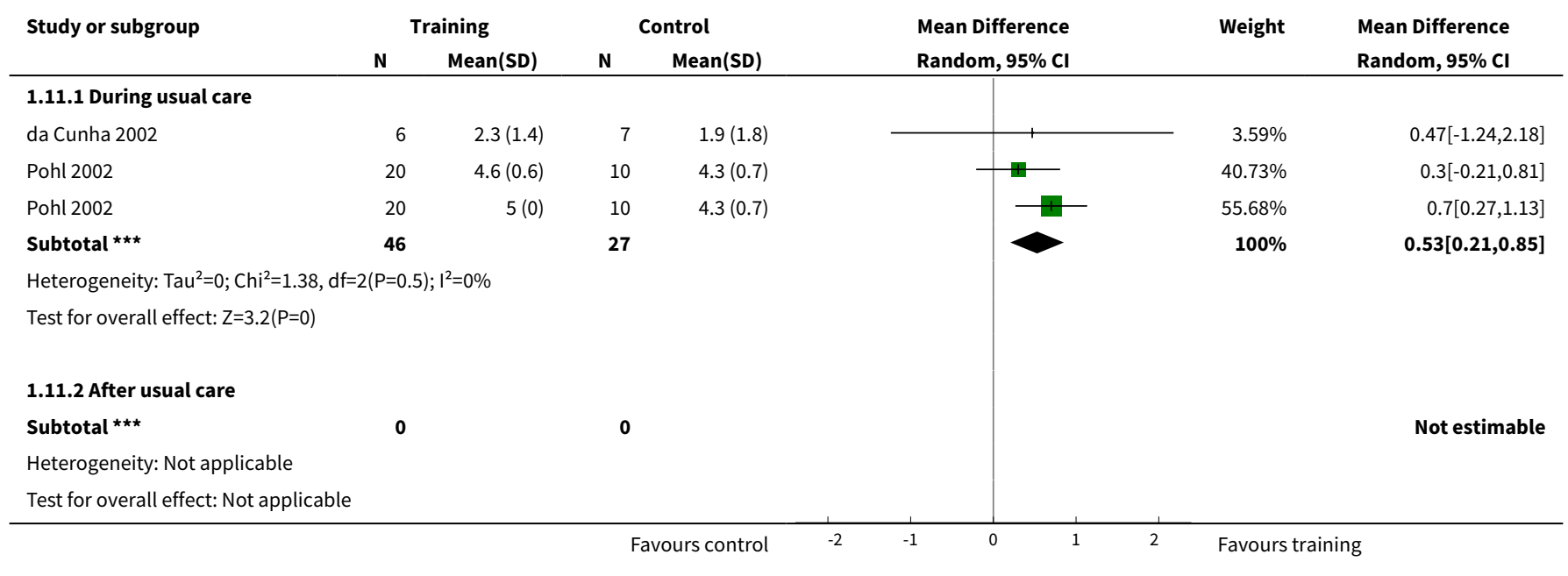




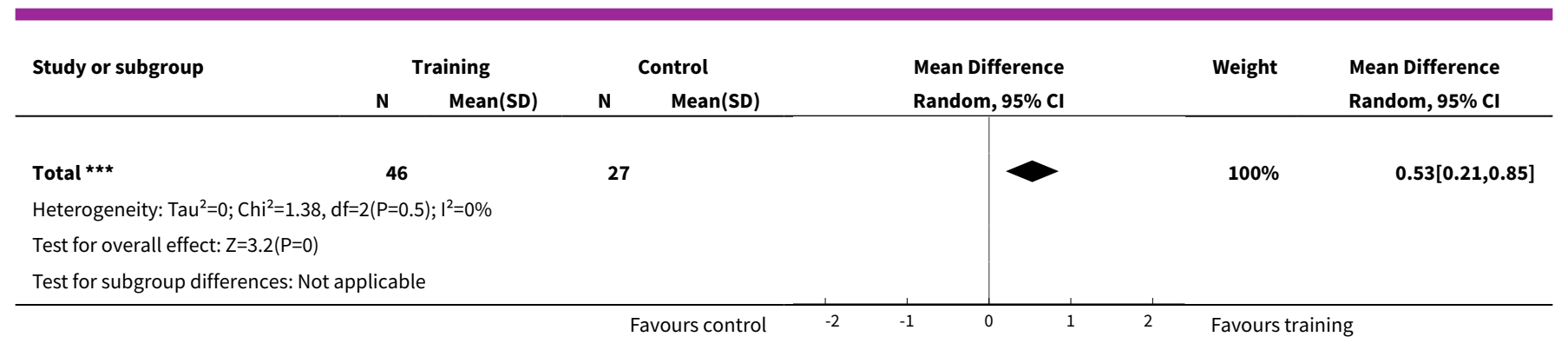

Analysis 1.12. Comparison 1 Cardiorespiratory training versus control - end of intervention, Outcome 12 Mobility - walking maximal speed (over 5 to 10 metres).

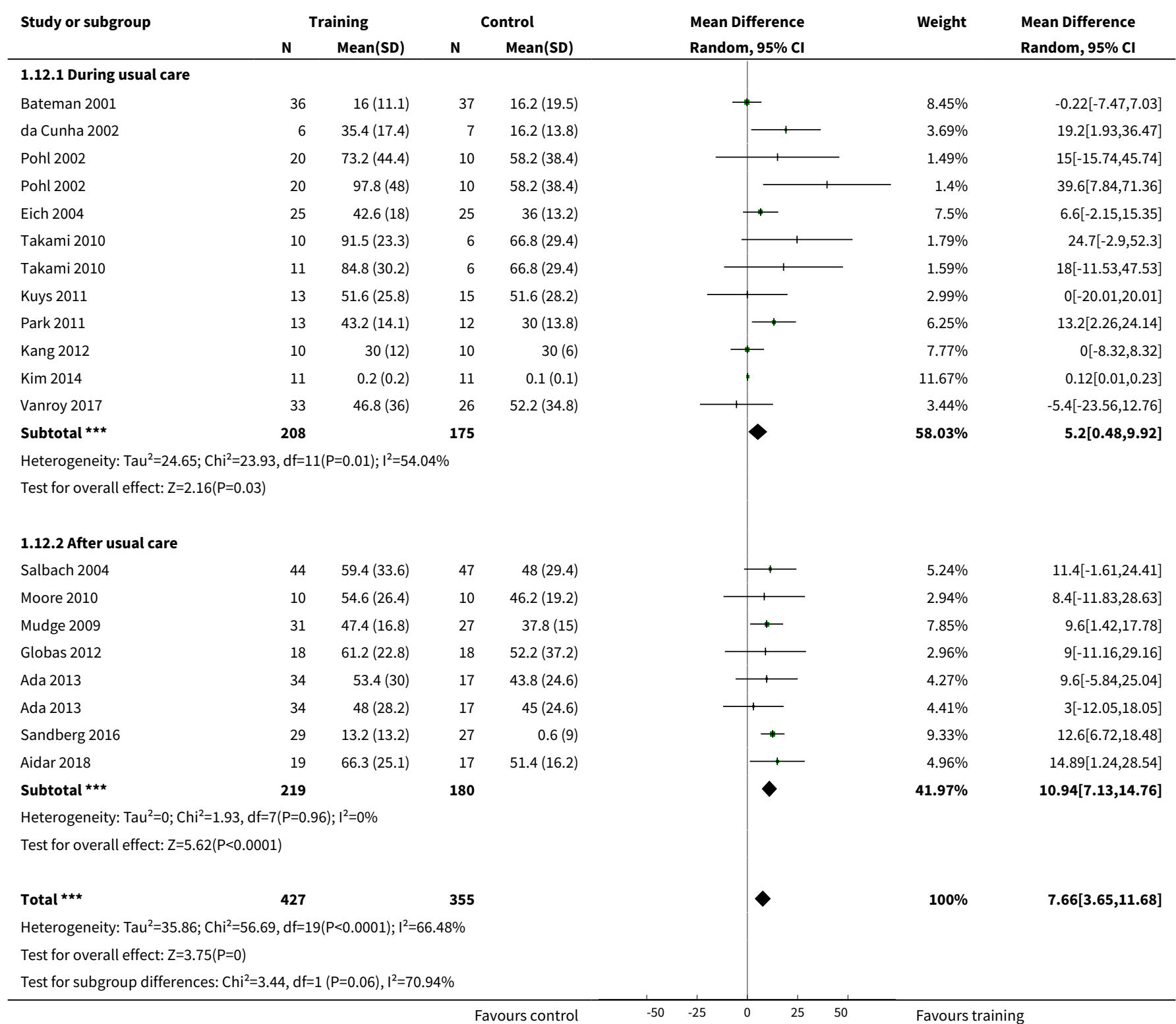


Analysis 1.13. Comparison 1 Cardiorespiratory training versus control - end of intervention, Outcome 13 Mobility - walking preferred speed.

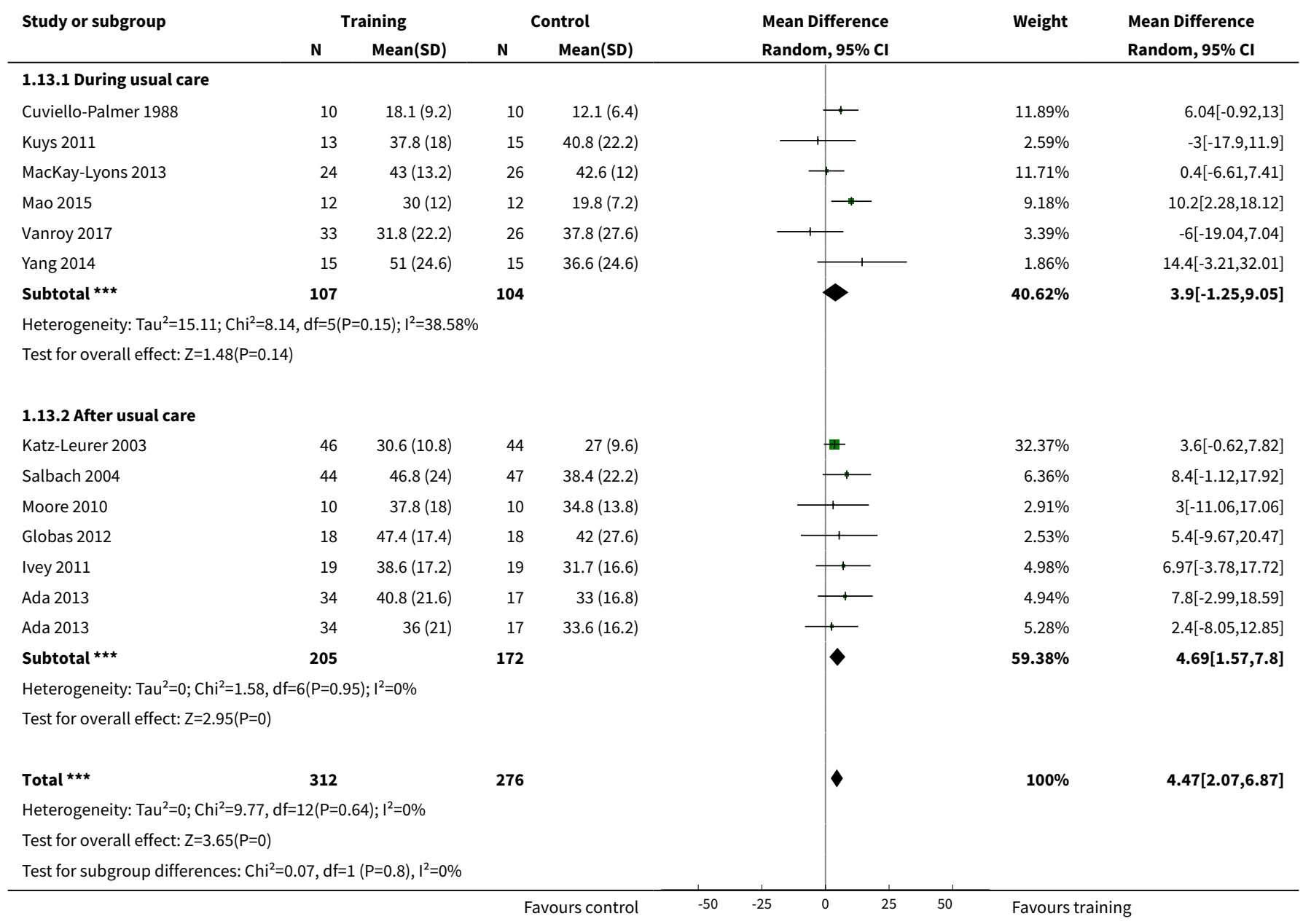

Analysis 1.14. Comparison 1 Cardiorespiratory training versus control - end of intervention, Outcome 14 Mobility - walking capacity (6-MWT metres).

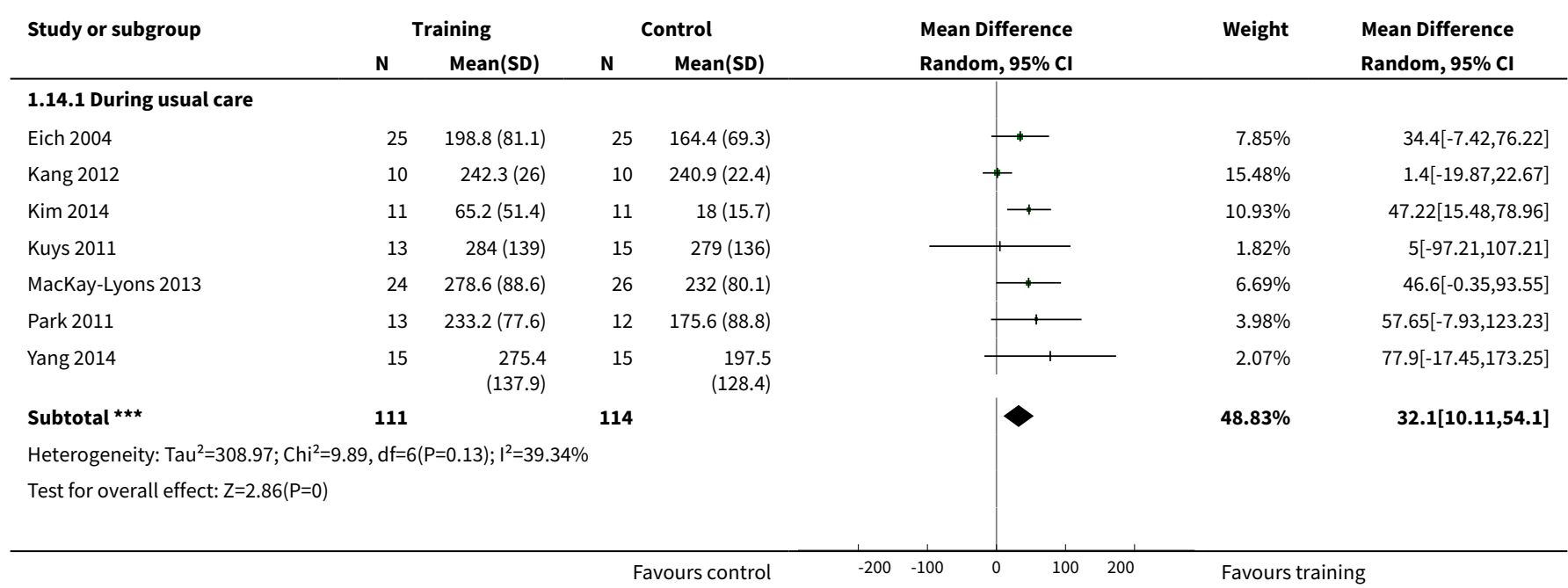




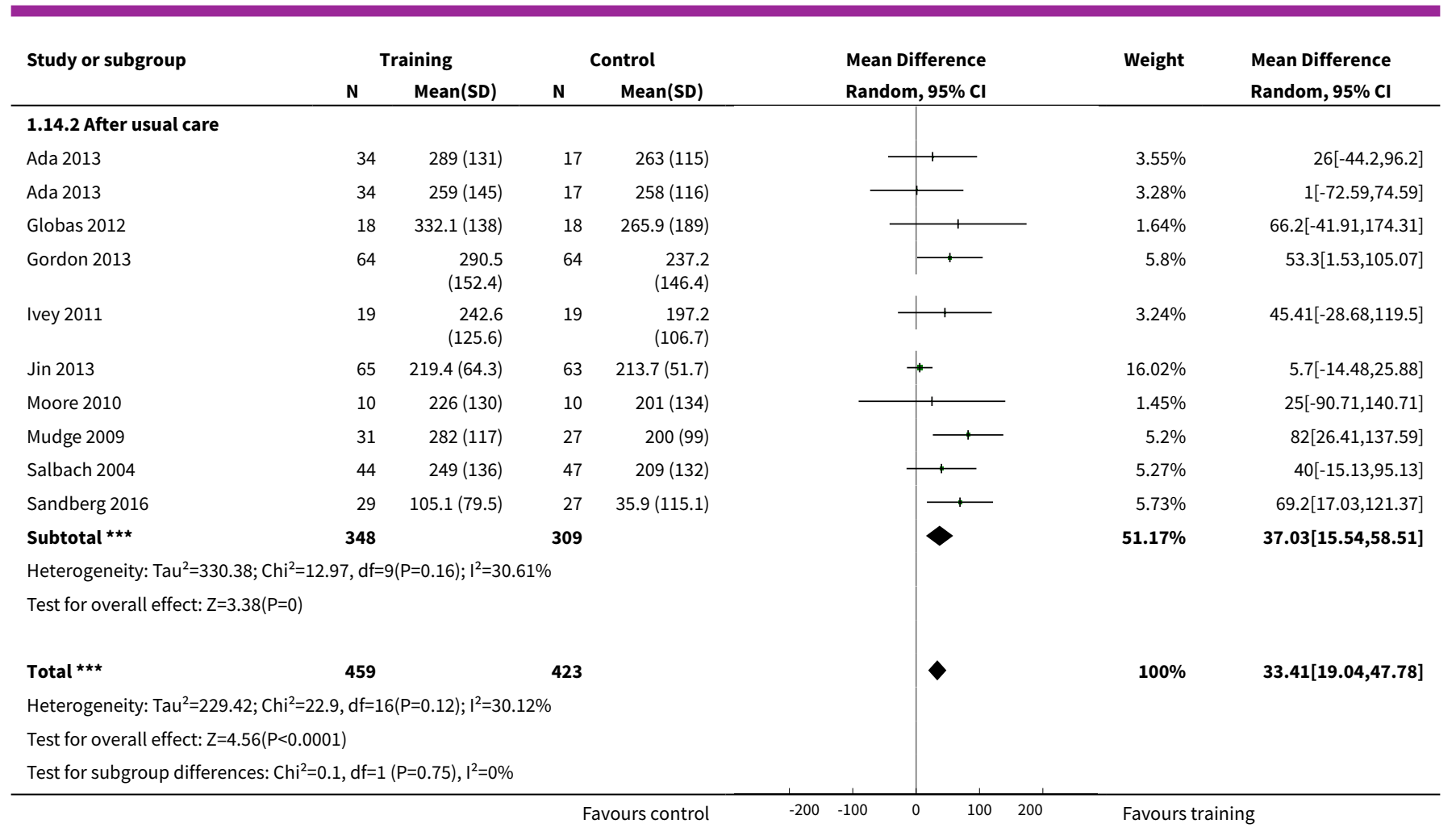

Analysis 1.15. Comparison 1 Cardiorespiratory training versus control - end of intervention, Outcome 15 Mobility - walking capacity ( $/ \mathrm{m} / \mathrm{min})$.

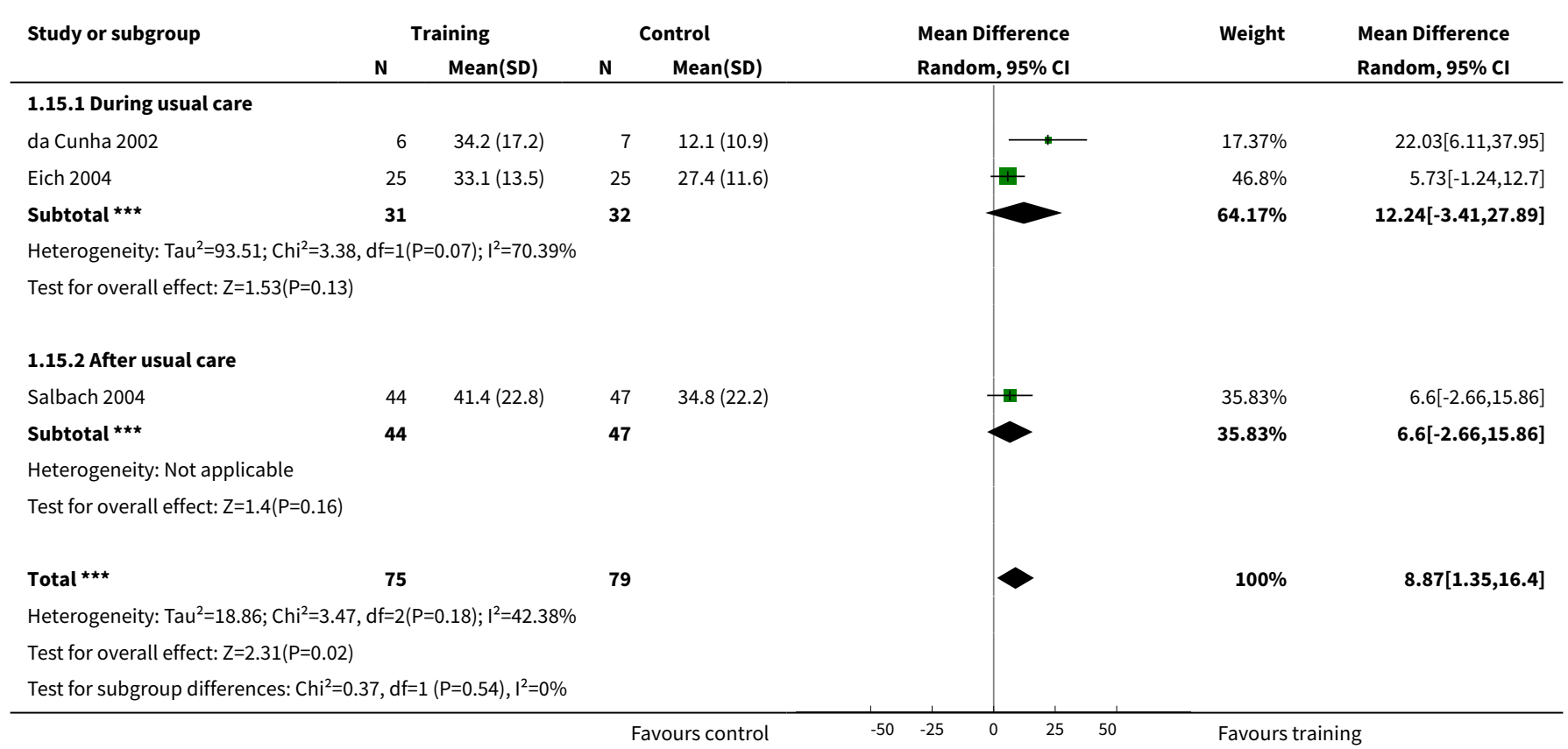


Analysis 1.16. Comparison 1 Cardiorespiratory training versus control - end of intervention, Outcome 16 Mobility - community walk (min).

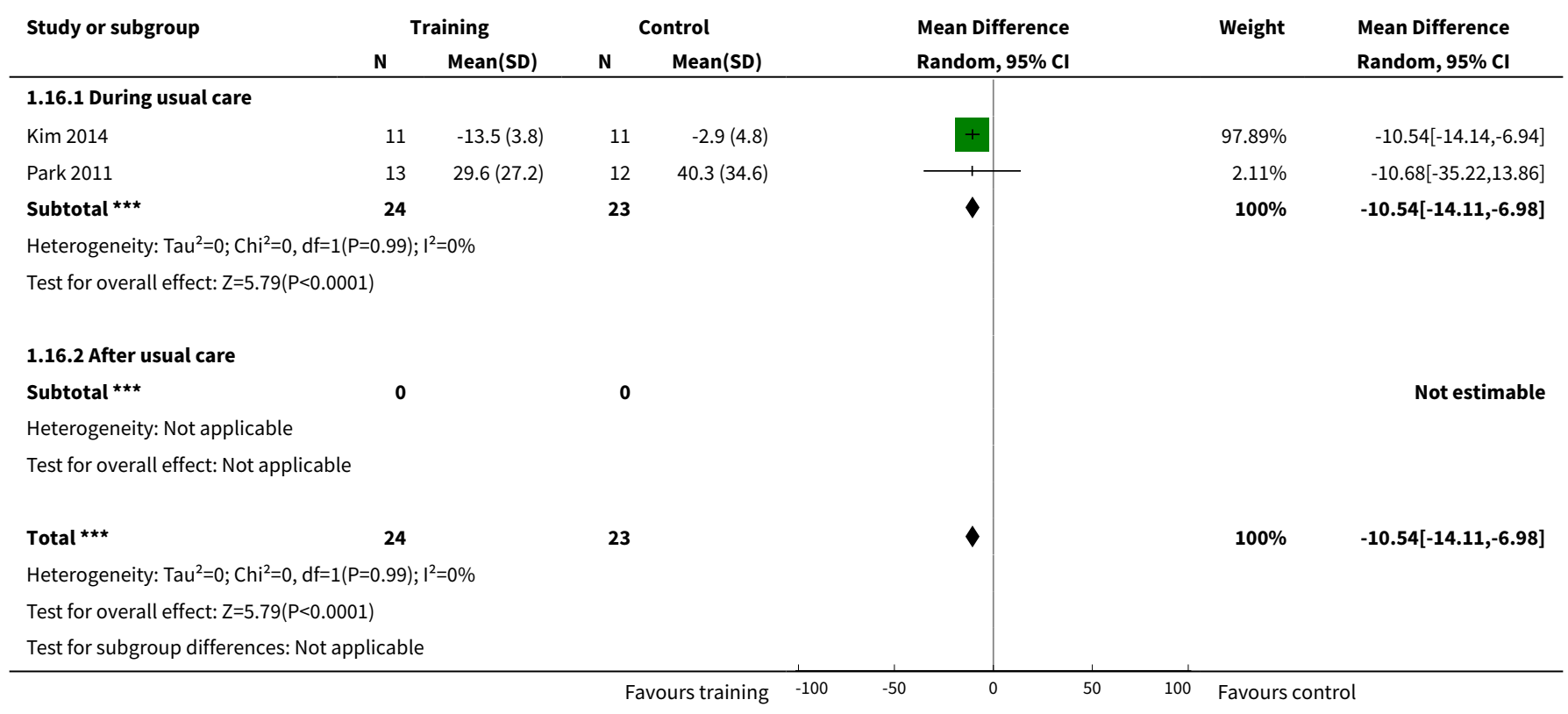

Analysis 1.17. Comparison 1 Cardiorespiratory training versus control - end of intervention, Outcome 17 Physical function - Berg Balance Scale (score 0 to 56).

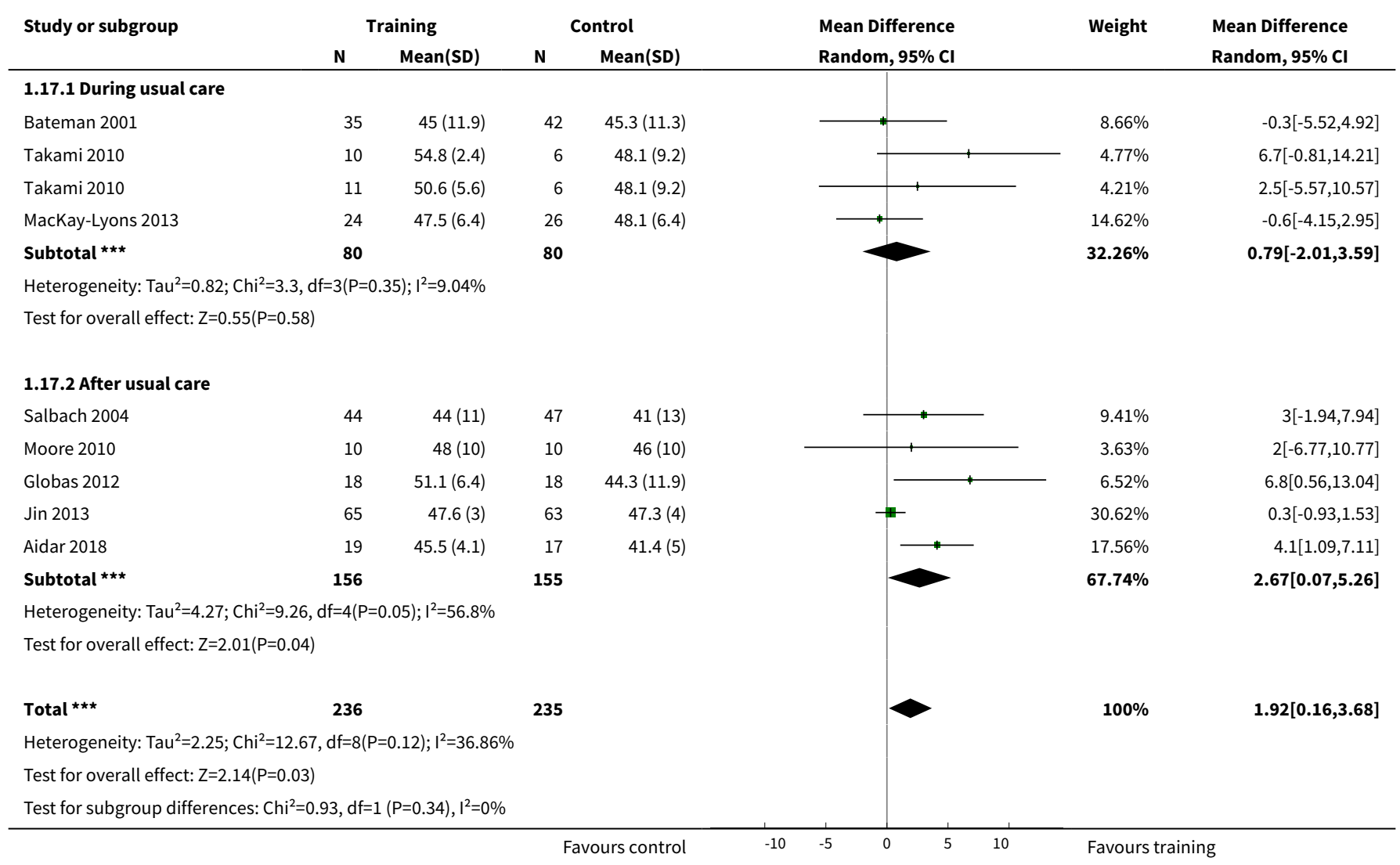


Analysis 1.18. Comparison 1 Cardiorespiratory training versus control end of intervention, Outcome 18 Physical function - Timed Up and Go (sec).

\begin{tabular}{|c|c|c|c|c|c|c|c|}
\hline \multirow[t]{2}{*}{ Study or subgroup } & \multicolumn{2}{|c|}{ Training } & \multicolumn{2}{|c|}{ Control } & \multirow{2}{*}{$\begin{array}{l}\text { Mean Difference } \\
\text { Random, } 95 \% \mathrm{Cl}\end{array}$} & \multirow[t]{2}{*}{ Weight } & \multirow{2}{*}{$\begin{array}{l}\text { Mean Difference } \\
\text { Random, } 95 \% \mathrm{CI}\end{array}$} \\
\hline & $\mathbf{N}$ & Mean(SD) & $\mathbf{N}$ & Mean(SD) & & & \\
\hline \multicolumn{8}{|c|}{ 1.18.1 During usual care } \\
\hline Kang 2012 & 10 & $17.9(4.5)$ & 10 & $20(5)$ & + & $10.69 \%$ & $-2.1[-6.27,2.07]$ \\
\hline Subtotal $\star \star \star$ & 10 & & 10 & & & $10.69 \%$ & $-2.1[-6.27,2.07]$ \\
\hline \multicolumn{8}{|c|}{ Heterogeneity: Not applicable } \\
\hline \multicolumn{8}{|c|}{ Test for overall effect: $Z=0.99(P=0.32)$} \\
\hline \multicolumn{8}{|c|}{ 1.18.2 After usual care } \\
\hline Aidar 2018 & 19 & $9.8(3.9)$ & 17 & $13.5(4.1)$ & \# & $27.04 \%$ & $-3.7[-6.32,-1.08]$ \\
\hline Moore 2010 & 10 & $20(12)$ & 10 & $24(16)$ & 1 & $1.21 \%$ & $-4[-16.4,8.4]$ \\
\hline Salbach 2004 & 44 & $23.2(20.6)$ & 47 & $27.1(27.1)$ & 1 & $1.91 \%$ & $-3.9[-13.75,5.95]$ \\
\hline Sandberg 2016 & 29 & $-4.2(4.5)$ & 27 & $-0.7(1.8)$ & + & $59.14 \%$ & $-3.5[-5.27,-1.73]$ \\
\hline 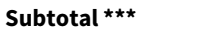 & 102 & & 101 & & 1 & $89.31 \%$ & $-3.58[-5.02,-2.13]$ \\
\hline \multicolumn{8}{|c|}{ Test for overall effect: $\mathrm{Z}=4.86(\mathrm{P}<0.0001)$} \\
\hline Total $\star \star \star$ & 112 & & 111 & & 1 & $100 \%$ & $-3.42[-4.78,-2.05]$ \\
\hline \multicolumn{8}{|c|}{ Heterogeneity: $\operatorname{Tau}^{2}=0 ; \mathrm{Chi}^{2}=0.45, \mathrm{df}=4(\mathrm{P}=0.98) ; \mathrm{I}^{2}=0 \%$} \\
\hline \multicolumn{8}{|c|}{ Test for overall effect: $Z=4.91(P<0.0001)$} \\
\hline Test for subgroup dif & $43, \mathrm{df}=$ & $P=0.51), I^{2}=0 \%$ & & & & & \\
\hline
\end{tabular}

Analysis 1.19. Comparison 1 Cardiorespiratory training versus control - end of intervention, Outcome 19 Health-related QoL - SF-36 \& SF-12 Physical Health Component.

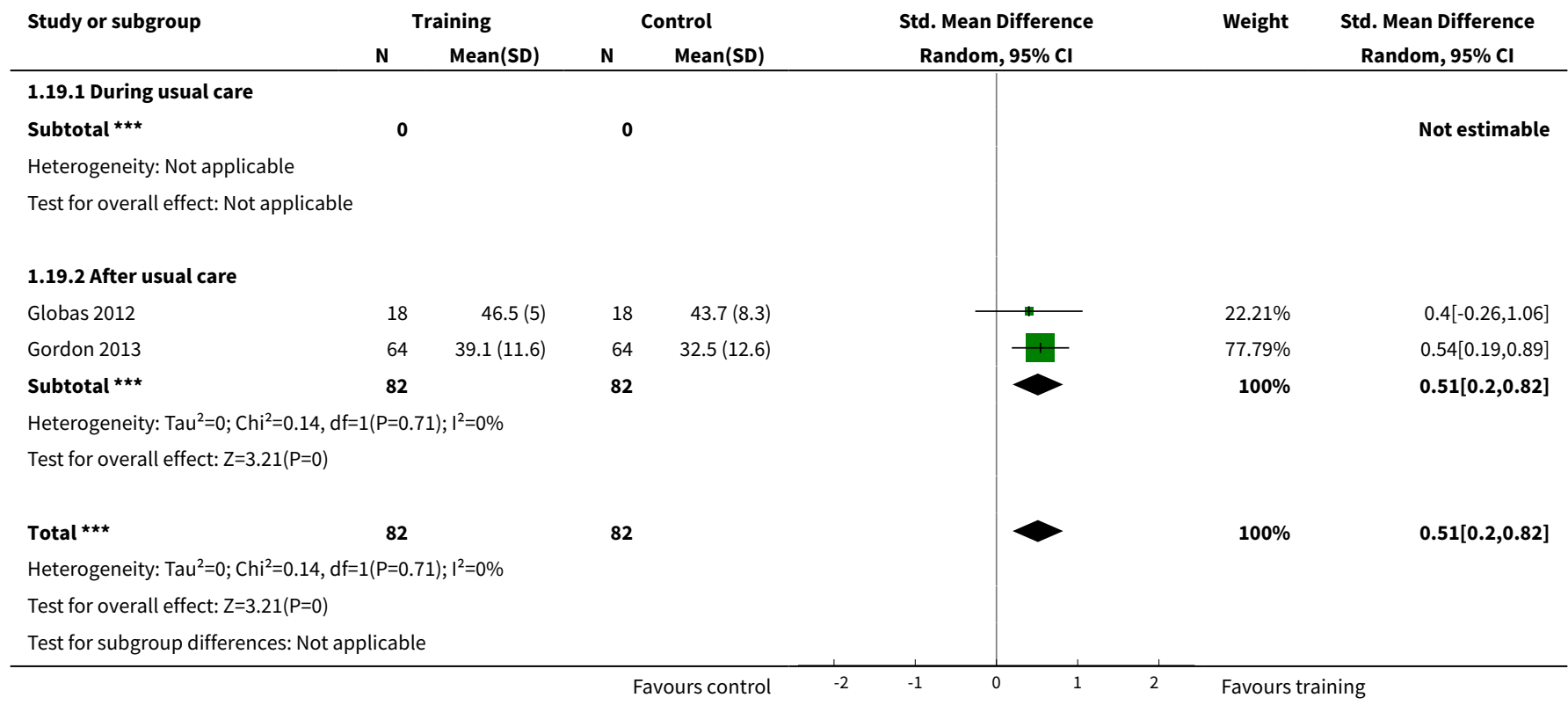


Analysis 1.20. Comparison 1 Cardiorespiratory training versus control - end of intervention, Outcome 20 Health-related QoL - SF-36 \& SF-12 Mental Health Component.

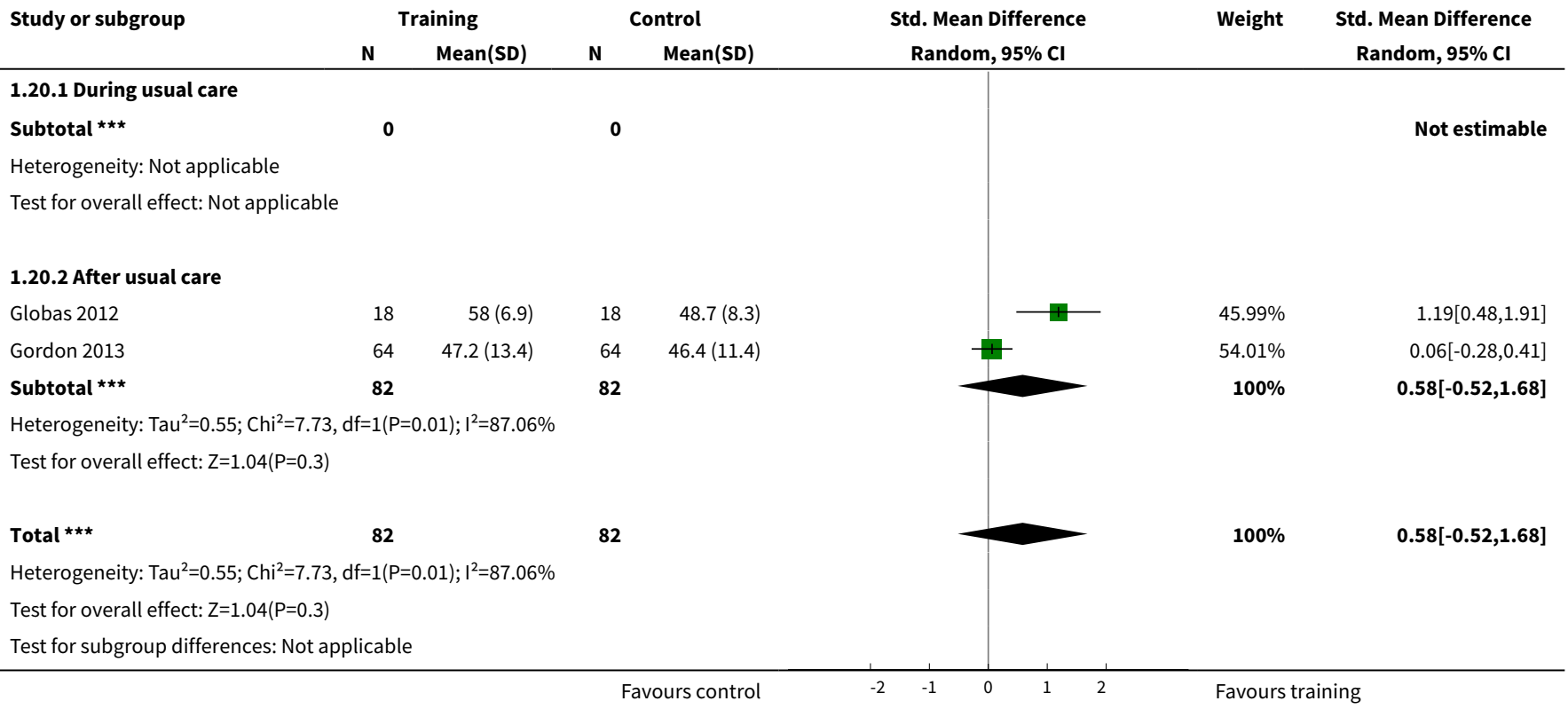

Analysis 1.21. Comparison 1 Cardiorespiratory training versus control - end of intervention, Outcome 21 Health-related QoL - EuroQol EQ-5D.

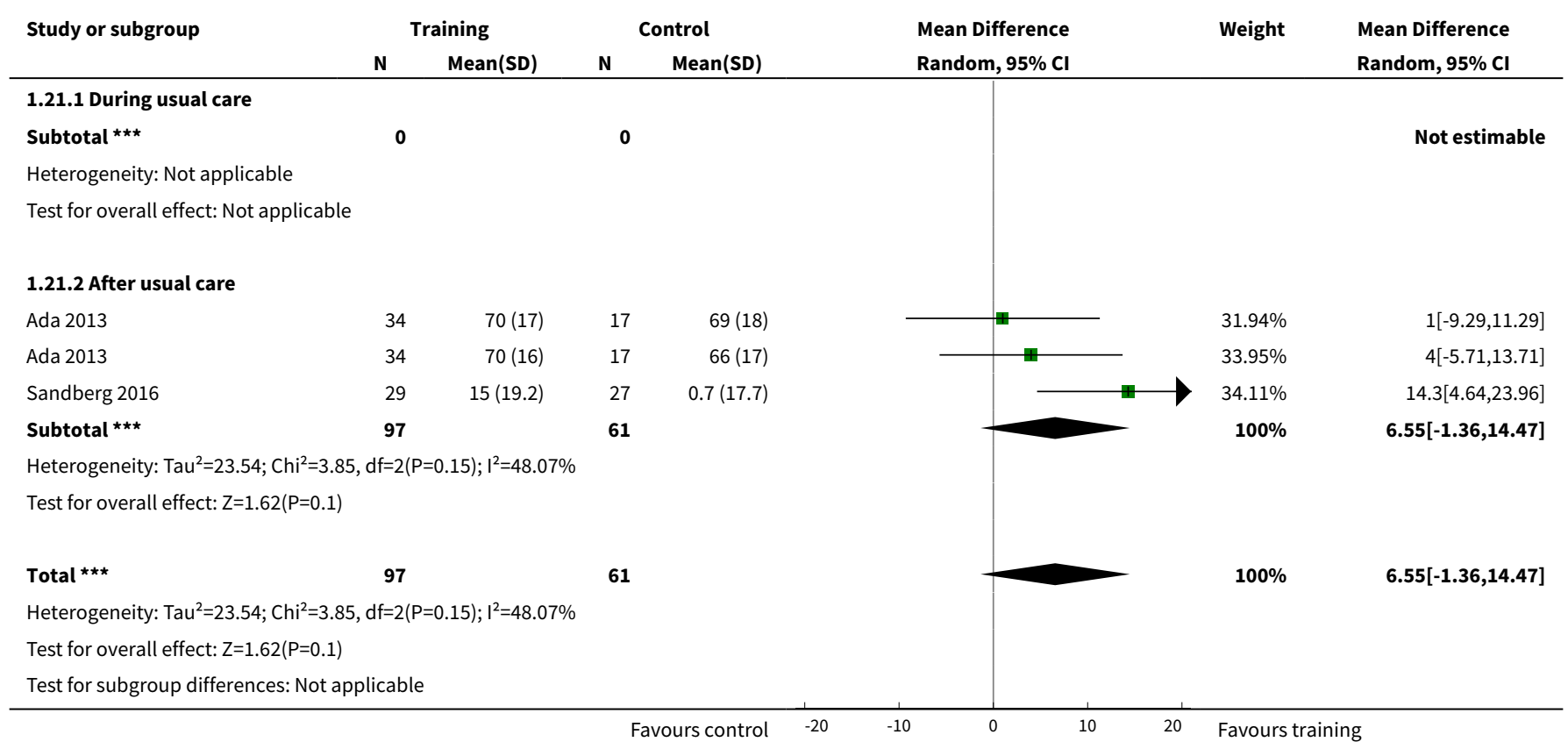


Analysis 1.22. Comparison 1 Cardiorespiratory training versus control - end of intervention, Outcome 22 Mood - Beck Depression Index.

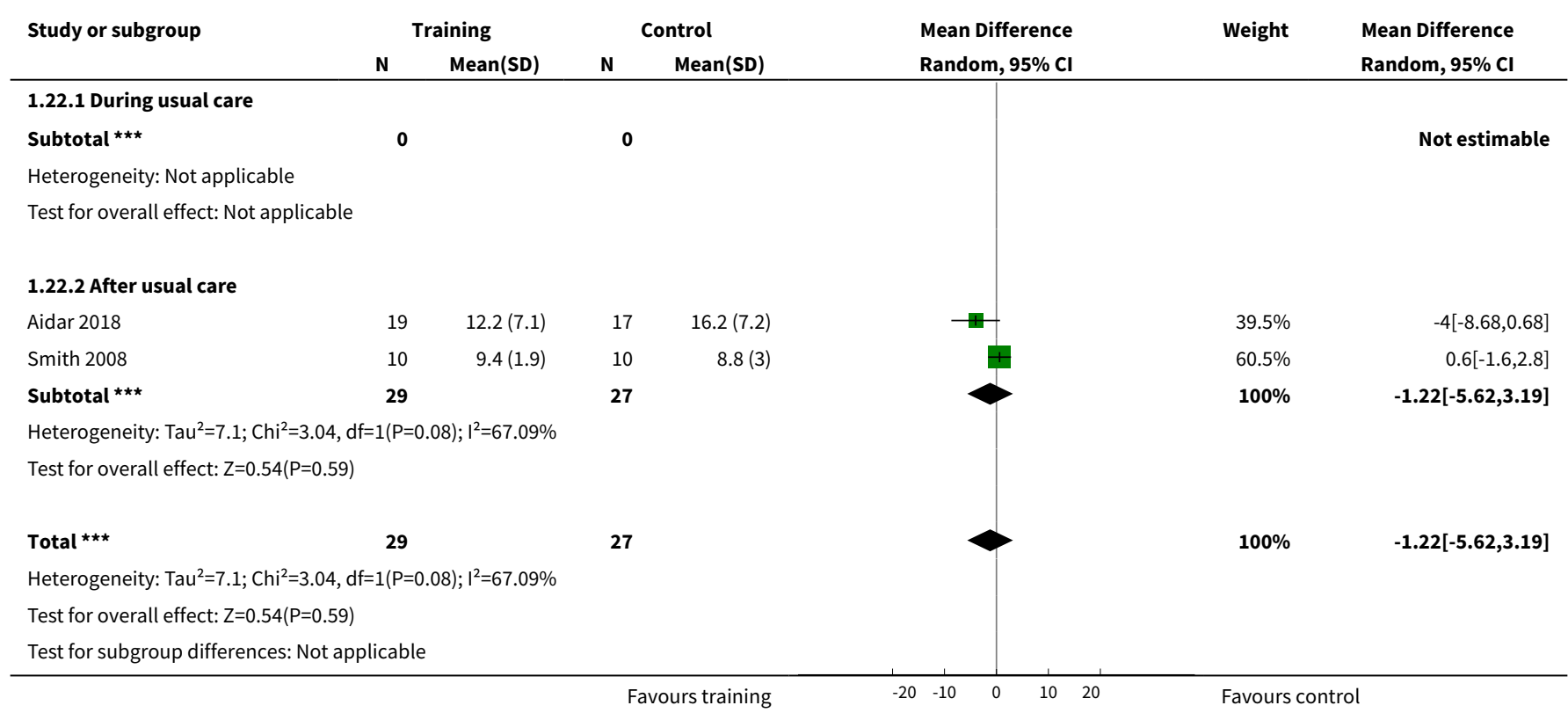

Comparison 2. Cardiorespiratory training versus control - end of retention follow-up

\begin{tabular}{|c|c|c|c|c|}
\hline Outcome or subgroup title & $\begin{array}{l}\text { No. of } \\
\text { studies }\end{array}$ & $\begin{array}{l}\text { No. of } \\
\text { partici- } \\
\text { pants }\end{array}$ & Statistical method & Effect size \\
\hline 1 Death & 6 & 360 & $\begin{array}{l}\text { Risk Difference (M-H, Random, } \\
95 \% \mathrm{Cl} \text { ) }\end{array}$ & $0.0[-0.03,0.03]$ \\
\hline 1.1 During usual care & 3 & 226 & $\begin{array}{l}\text { Risk Difference (M-H, Random, } \\
95 \% \mathrm{Cl} \text { ) }\end{array}$ & $0.0[-0.03,0.03]$ \\
\hline 1.2 After usual care & 3 & 134 & $\begin{array}{l}\text { Risk Difference (M-H, Random, } \\
95 \% \mathrm{Cl} \text { ) }\end{array}$ & $0.0[-0.05,0.05]$ \\
\hline 2 Disability - combined disability scales & 3 & 220 & $\begin{array}{l}\text { Std. Mean Difference (IV, Random, } \\
95 \% \mathrm{CI} \text { ) }\end{array}$ & $0.20[-0.07,0.46]$ \\
\hline 2.1 During usual care & 1 & 83 & $\begin{array}{l}\text { Std. Mean Difference (IV, Random, } \\
95 \% \mathrm{CI} \text { ) }\end{array}$ & $0.18[-0.26,0.61]$ \\
\hline 2.2 After usual care & 2 & 137 & $\begin{array}{l}\text { Std. Mean Difference (IV, Random, } \\
95 \% \mathrm{CI} \text { ) }\end{array}$ & $0.21[-0.12,0.55]$ \\
\hline $\begin{array}{l}3 \text { Mobility - walking maximal speed }(\mathrm{m} / \\
\mathrm{min})\end{array}$ & 5 & 312 & $\begin{array}{l}\text { Mean Difference (IV, Random, } 95 \% \\
\mathrm{CI} \text { ) }\end{array}$ & $6.71[2.40,11.02]$ \\
\hline 3.1 During usual care & 3 & 152 & $\begin{array}{l}\text { Mean Difference (IV, Random, 95\% } \\
\mathrm{CI} \text { ) }\end{array}$ & $7.92[2.01,13.83]$ \\
\hline
\end{tabular}




\begin{tabular}{|c|c|c|c|c|}
\hline Outcome or subgroup title & $\begin{array}{l}\text { No. of } \\
\text { studies }\end{array}$ & $\begin{array}{l}\text { No. of } \\
\text { partici- } \\
\text { pants }\end{array}$ & Statistical method & Effect size \\
\hline 3.2 After usual care & 2 & 160 & $\begin{array}{l}\text { Mean Difference (IV, Random, 95\% } \\
\mathrm{CI} \text { ) }\end{array}$ & $5.33[-0.96,11.63]$ \\
\hline $\begin{array}{l}4 \text { Mobility - walking preferred speed } \\
\text { (m/min) }\end{array}$ & 3 & 176 & $\begin{array}{l}\text { Mean Difference (IV, Random, 95\% } \\
\mathrm{CI} \text { ) }\end{array}$ & $1.67[-3.27,6.62]$ \\
\hline 4.1 During usual care & 2 & 74 & $\begin{array}{l}\text { Mean Difference (IV, Random, 95\% } \\
\mathrm{Cl} \text { ) }\end{array}$ & $2.54[-3.65,8.73]$ \\
\hline 4.2 After usual care & 1 & 102 & $\begin{array}{l}\text { Mean Difference (IV, Random, 95\% } \\
\mathrm{CI} \text { ) }\end{array}$ & $0.14[-8.08,8.37]$ \\
\hline $\begin{array}{l}5 \text { Mobility - walking capacity (6-MWT } \\
\text { metres) }\end{array}$ & 5 & 283 & $\begin{array}{l}\text { Mean Difference (IV, Random, 95\% } \\
\mathrm{CI} \text { ) }\end{array}$ & $38.29[7.19,69.39]$ \\
\hline 5.1 During usual care & 3 & 123 & $\begin{array}{l}\text { Mean Difference (IV, Random, 95\% } \\
\mathrm{CI})\end{array}$ & $50.76[19.09,82.43]$ \\
\hline 5.2 After usual care & 2 & 160 & $\begin{array}{l}\text { Mean Difference (IV, Random, 95\% } \\
\mathrm{CI} \text { ) }\end{array}$ & $22.34[-44.02,88.69]$ \\
\hline $\begin{array}{l}6 \text { Physical function - Berg Balance } \\
\text { scale }\end{array}$ & 2 & 134 & $\begin{array}{l}\text { Mean Difference (IV, Random, 95\% } \\
\mathrm{Cl} \text { ) }\end{array}$ & $0.04[-2.48,2.56]$ \\
\hline 6.1 During usual care & 2 & 134 & $\begin{array}{l}\text { Mean Difference (IV, Random, 95\% } \\
\mathrm{Cl} \text { ) }\end{array}$ & $0.04[-2.48,2.56]$ \\
\hline 6.2 After usual care & 0 & 0 & $\begin{array}{l}\text { Mean Difference (IV, Random, 95\% } \\
\mathrm{Cl} \text { ) }\end{array}$ & $0.0[0.0,0.0]$ \\
\hline 7 Health-related QoL - EuroQol EQ-5D & 2 & 150 & $\begin{array}{l}\text { Mean Difference (IV, Random, 95\% } \\
\mathrm{CI} \text { ) }\end{array}$ & $-4.25[-8.00,1.49]$ \\
\hline 7.1 During usual care & 0 & 0 & $\begin{array}{l}\text { Mean Difference (IV, Random, 95\% } \\
\mathrm{CI} \text { ) }\end{array}$ & $0.0[0.0,0.0]$ \\
\hline 7.2 After usual care & 2 & 150 & $\begin{array}{l}\text { Mean Difference (IV, Random, 95\% } \\
\mathrm{CI})\end{array}$ & $-4.25[-8.00,1.49]$ \\
\hline
\end{tabular}

\section{Analysis 2.1. Comparison 2 Cardiorespiratory training versus control - end of retention follow-up, Outcome 1 Death.}

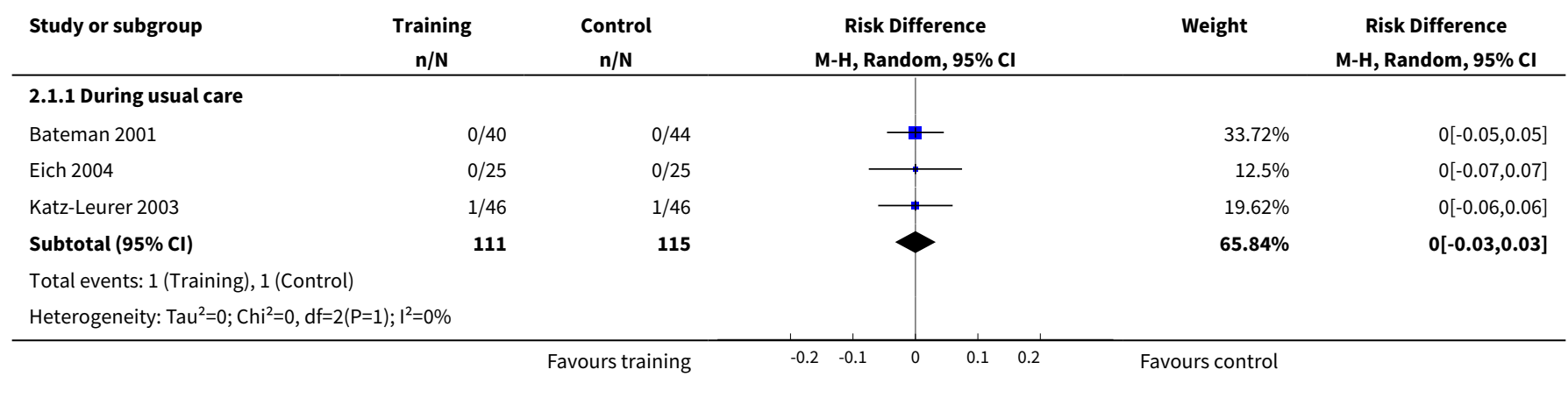




\begin{tabular}{|c|c|c|c|c|c|}
\hline Study or subgroup & $\begin{array}{c}\text { Training } \\
\mathbf{n} / \mathbf{N}\end{array}$ & $\begin{array}{c}\text { Control } \\
n / N\end{array}$ & $\begin{array}{c}\text { Risk Difference } \\
\text { M-H, Random, } 95 \% \mathrm{CI}\end{array}$ & Weight & $\begin{array}{c}\text { Risk Difference } \\
\text { M-H, Random, } 95 \% \mathrm{Cl}\end{array}$ \\
\hline \multicolumn{6}{|c|}{ Test for overall effect: Not applicable } \\
\hline \multicolumn{6}{|l|}{ 2.1.2 After usual care } \\
\hline Mudge 2009 & $0 / 31$ & $0 / 27$ & & $16.39 \%$ & $0[-0.07,0.07]$ \\
\hline Sandberg 2016 & $0 / 29$ & $0 / 27$ & & $15.47 \%$ & $0[-0.07,0.07]$ \\
\hline Smith 2008 & $0 / 10$ & $0 / 10$ & & $2.3 \%$ & $0[-0.17,0.17]$ \\
\hline Subtotal $(95 \% \mathrm{Cl})$ & 70 & 64 & & $34.16 \%$ & $0[-0.05,0.05]$ \\
\hline \multicolumn{6}{|c|}{ Total events: 0 (Training), 0 (Control) } \\
\hline \multicolumn{6}{|c|}{ Heterogeneity: $\operatorname{Tau}^{2}=0 ; \mathrm{Chi}^{2}=0, \mathrm{df}=2(\mathrm{P}=1) ; \mathrm{I}^{2}=0 \%$} \\
\hline \multicolumn{6}{|c|}{ Test for overall effect: Not applicable } \\
\hline Total $(95 \% \mathrm{Cl})$ & 181 & 179 & & $100 \%$ & $0[-0.03,0.03]$ \\
\hline \multicolumn{6}{|c|}{ Total events: 1 (Training), 1 (Control) } \\
\hline \multicolumn{6}{|c|}{ Heterogeneity: Tau $^{2}=0 ; \mathrm{Chi}^{2}=0, \mathrm{df}=5(\mathrm{P}=1) ; \mathrm{I}^{2}=0 \%$} \\
\hline \multicolumn{6}{|c|}{ Test for overall effect: Not applicable } \\
\hline \multicolumn{6}{|c|}{ Test for subgroup differences: Not applicable } \\
\hline
\end{tabular}

\section{Analysis 2.2. Comparison 2 Cardiorespiratory training versus control - end of retention follow-up, Outcome 2 Disability - combined disability scales.}

\begin{tabular}{|c|c|c|c|c|c|c|c|}
\hline \multirow[t]{2}{*}{ Study or subgroup } & \multicolumn{2}{|c|}{ Training } & \multicolumn{2}{|c|}{ Control } & \multirow{2}{*}{$\begin{array}{c}\text { Std. Mean Difference } \\
\text { Random, } 95 \% \mathrm{Cl}\end{array}$} & \multirow[t]{2}{*}{ Weight } & \multirow{2}{*}{$\begin{array}{c}\text { Std. Mean Difference } \\
\text { Random, } 95 \% \mathrm{Cl}\end{array}$} \\
\hline & $\mathbf{N}$ & Mean(SD) & $\mathbf{N}$ & Mean(SD) & & & \\
\hline \multicolumn{8}{|c|}{ 2.2.1 During usual care } \\
\hline Bateman 2001 & 39 & $36.8(17.7)$ & 44 & $33.6(17.6)$ & - & $37.76 \%$ & $0.18[-0.26,0.61]$ \\
\hline Subtotal $* \star \star$ & 39 & & 44 & & & $37.76 \%$ & $0.18[-0.26,0.61]$ \\
\hline \multicolumn{8}{|c|}{ Heterogeneity: Not applicable } \\
\hline \multicolumn{8}{|c|}{ Test for overall effect: $\mathrm{Z}=0.8(\mathrm{P}=0.43)$} \\
\hline \multicolumn{8}{|c|}{ 2.2.2 After usual care } \\
\hline Katz-Leurer 2003 & 41 & $27(6.5)$ & 38 & $26(5)$ & 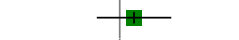 & $36.03 \%$ & $0.17[-0.27,0.61]$ \\
\hline Mudge 2009 & 31 & $82.1(72.8)$ & 27 & $62.2(72.5)$ & - & $26.21 \%$ & $0.27[-0.25,0.79]$ \\
\hline Subtotal $\star \star \star ~$ & 72 & & 65 & & & $62.24 \%$ & $0.21[-0.12,0.55]$ \\
\hline \multicolumn{8}{|c|}{ Heterogeneity: $\operatorname{Tau}^{2}=0 ; \mathrm{Chi}^{2}=0.08, \mathrm{df}=1(\mathrm{P}=0.77) ; \mathrm{I}^{2}=0 \%$} \\
\hline \multicolumn{8}{|c|}{ Test for overall effect: $Z=1.24(P=0.22)$} \\
\hline 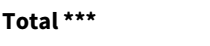 & 111 & & 109 & & & $100 \%$ & $0.2[-0.07,0.46]$ \\
\hline \multicolumn{8}{|c|}{ Heterogeneity: Tau $^{2}=0 ; \mathrm{Chi}^{2}=0.1, \mathrm{df}=2(\mathrm{P}=0.95) ; \mathrm{I}^{2}=0 \%$} \\
\hline \multicolumn{8}{|c|}{ Test for overall effect: $Z=1.46(P=0.14)$} \\
\hline \multicolumn{8}{|c|}{ Test for subgroup differences: $\mathrm{Chi}^{2}=0.02, \mathrm{df}=1(\mathrm{P}=0.9), \mathrm{I}^{2}=0 \%$} \\
\hline
\end{tabular}


Analysis 2.3. Comparison 2 Cardiorespiratory training versus control - end of retention follow-up, Outcome 3 Mobility - walking maximal speed ( $\mathrm{m} / \mathrm{min})$.

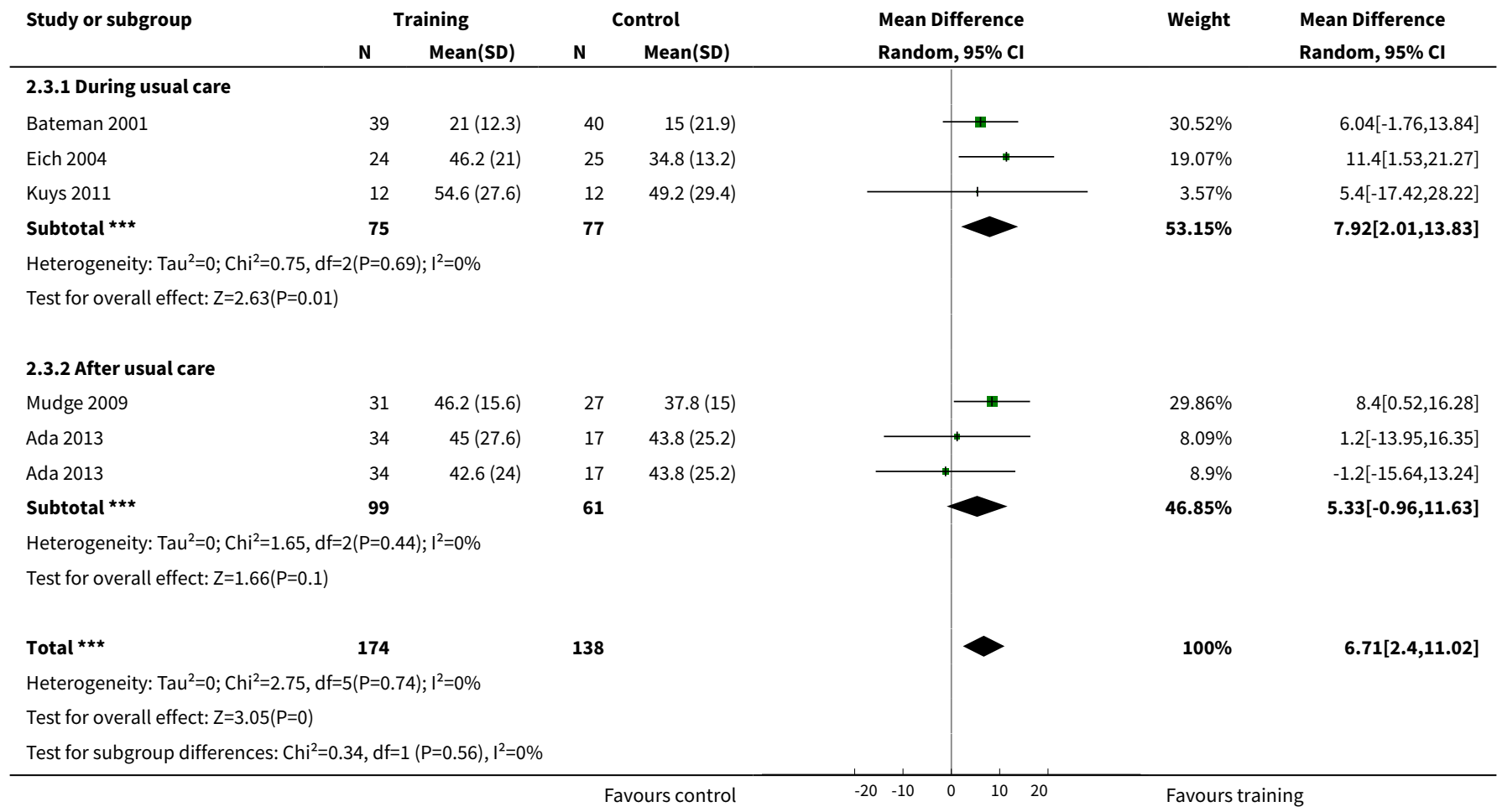

Analysis 2.4. Comparison 2 Cardiorespiratory training versus control - end of retention follow-up, Outcome 4 Mobility - walking preferred speed ( $/ \mathrm{min})$.

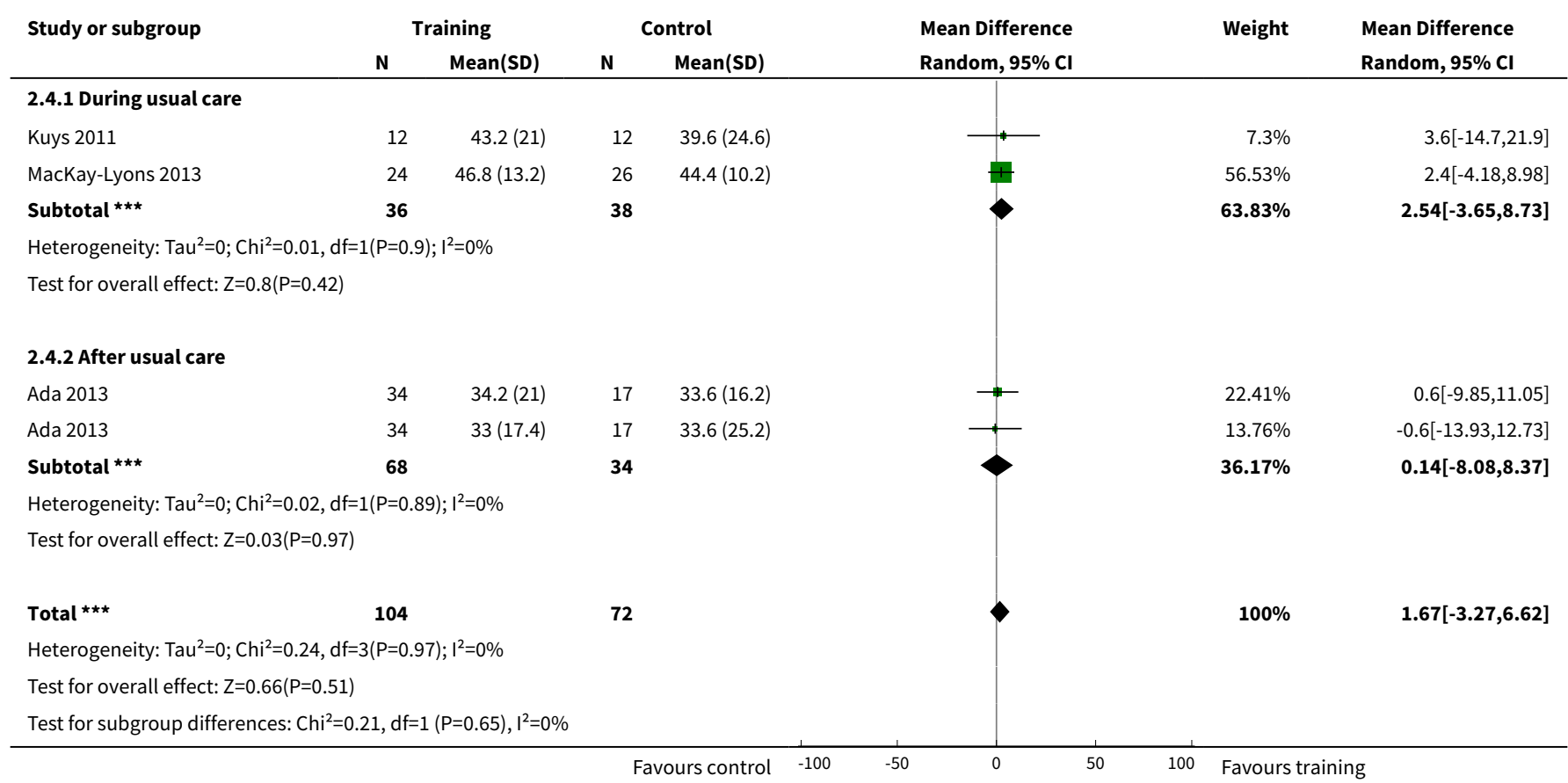


Analysis 2.5. Comparison 2 Cardiorespiratory training versus control - end of retention follow-up, Outcome 5 Mobility - walking capacity (6-MWT metres).

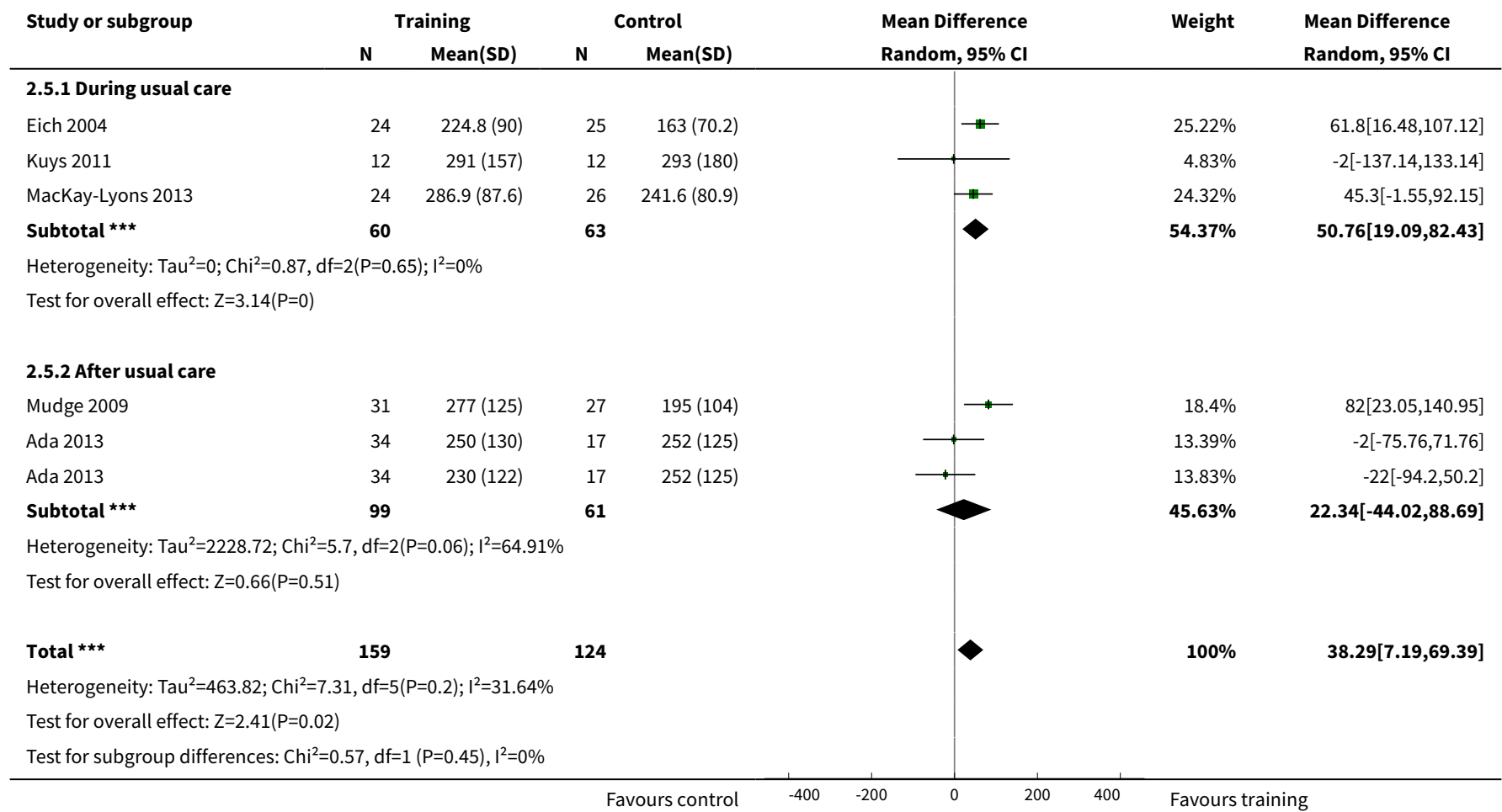

Analysis 2.6. Comparison 2 Cardiorespiratory training versus control - end of retention follow-up, Outcome 6 Physical function - Berg Balance scale.

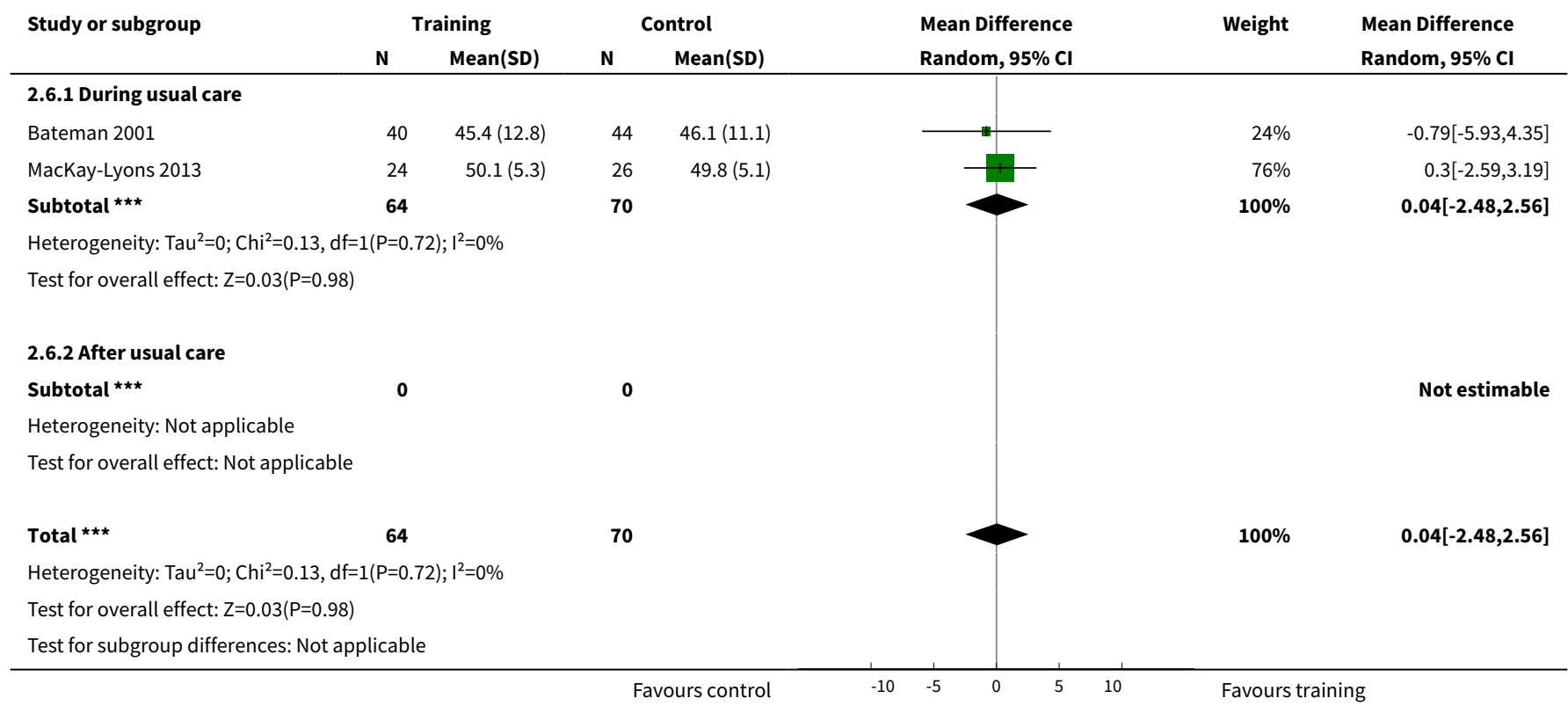




\section{Analysis 2.7. Comparison 2 Cardiorespiratory training versus control - end of retention follow-up, Outcome 7 Health-related QoL - EuroQol EQ-5D.}

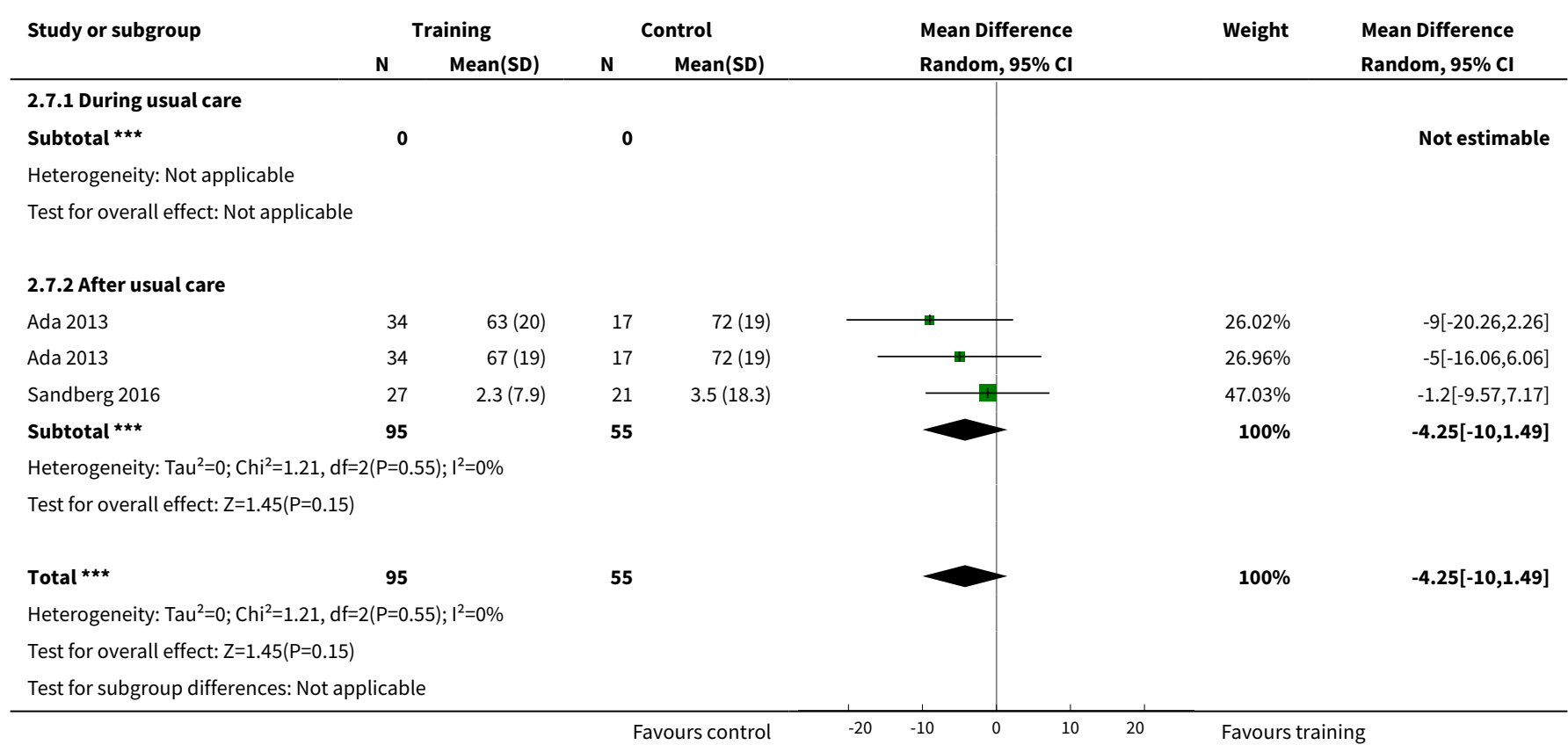

Comparison 3. Resistance training versus control - end of intervention

\begin{tabular}{|c|c|c|c|c|}
\hline Outcome or subgroup title & $\begin{array}{l}\text { No. of } \\
\text { studies }\end{array}$ & $\begin{array}{l}\text { No. of } \\
\text { partici- } \\
\text { pants }\end{array}$ & Statistical method & Effect size \\
\hline 1 Death & 20 & 803 & $\begin{array}{l}\text { Risk Difference (M-H, Random, } \\
95 \% \mathrm{Cl})\end{array}$ & $0.00[-0.02,0.02]$ \\
\hline 1.1 During usual care & 7 & 236 & $\begin{array}{l}\text { Risk Difference (M-H, Random, } \\
95 \% \mathrm{Cl})\end{array}$ & $0.0[-0.04,0.04]$ \\
\hline 1.2 After usual care & 13 & 567 & $\begin{array}{l}\text { Risk Difference (M-H, Random, } \\
95 \% \mathrm{Cl})\end{array}$ & $0.00[-0.02,0.02]$ \\
\hline $\begin{array}{l}2 \text { Physical fitness - composite measure of } \\
\text { muscle strength }\end{array}$ & 2 & 60 & $\begin{array}{l}\text { Std. Mean Difference (IV, Ran- } \\
\text { dom, } 95 \% \mathrm{CI} \text { ) }\end{array}$ & $0.58[0.06,1.10]$ \\
\hline 2.1 During usual care & 0 & 0 & $\begin{array}{l}\text { Std. Mean Difference (IV, Ran- } \\
\text { dom, } 95 \% \mathrm{CI} \text { ) }\end{array}$ & $0.0[0.0,0.0]$ \\
\hline 2.2 During and after usual care & 1 & 40 & $\begin{array}{l}\text { Std. Mean Difference (IV, Ran- } \\
\text { dom, } 95 \% \mathrm{CI} \text { ) }\end{array}$ & $0.47[-0.16,1.10]$ \\
\hline 2.3 After usual care & 1 & 20 & $\begin{array}{l}\text { Std. Mean Difference (IV, Ran- } \\
\text { dom, } 95 \% \mathrm{CI} \text { ) }\end{array}$ & $0.84[-0.09,1.76]$ \\
\hline $\begin{array}{l}3 \text { Physical fitness - muscle strength, paretic } \\
\text { knee flexion }\end{array}$ & 3 & 93 & $\begin{array}{l}\text { Std. Mean Difference (IV, Ran- } \\
\text { dom, } 95 \% \mathrm{CI} \text { ) }\end{array}$ & $0.72[0.10,1.34]$ \\
\hline
\end{tabular}




\begin{tabular}{|c|c|c|c|c|}
\hline Outcome or subgroup title & $\begin{array}{l}\text { No. of } \\
\text { studies }\end{array}$ & $\begin{array}{l}\text { No. of } \\
\text { partici- } \\
\text { pants }\end{array}$ & Statistical method & Effect size \\
\hline 3.1 During usual care & 1 & 18 & $\begin{array}{l}\text { Std. Mean Difference (IV, Ran- } \\
\text { dom, 95\% Cl) }\end{array}$ & $0.03[-0.90,0.96]$ \\
\hline 3.2 After usual care & 2 & 75 & $\begin{array}{l}\text { Std. Mean Difference (IV, Ran- } \\
\text { dom, } 95 \% \mathrm{CI} \text { ) }\end{array}$ & $1.01[0.52,1.50]$ \\
\hline $\begin{array}{l}4 \text { Physical fitness - muscle strength, paretic } \\
\text { knee extension }\end{array}$ & 3 & 93 & $\begin{array}{l}\text { Std. Mean Difference (IV, Ran- } \\
\text { dom, } 95 \% \mathrm{Cl} \text { ) }\end{array}$ & $1.09[-0.23,2.41]$ \\
\hline 4.1 During usual care & 1 & 18 & $\begin{array}{l}\text { Std. Mean Difference (IV, Ran- } \\
\text { dom, } 95 \% \mathrm{Cl} \text { ) }\end{array}$ & $-0.06[-0.99,0.87]$ \\
\hline 4.2 After usual care & 2 & 75 & $\begin{array}{l}\text { Std. Mean Difference (IV, Ran- } \\
\text { dom, 95\% Cl) }\end{array}$ & $1.66[0.53,2.79]$ \\
\hline $\begin{array}{l}5 \text { Mobility - walking maximal speed (m/ } \\
\text { min) }\end{array}$ & 7 & 274 & $\begin{array}{l}\text { Mean Difference (IV, Random, } \\
95 \% \mathrm{CI})\end{array}$ & $2.83[-0.49,6.14]$ \\
\hline 5.1 During usual care & 1 & 18 & $\begin{array}{l}\text { Mean Difference (IV, Random, } \\
95 \% \mathrm{CI})\end{array}$ & $8.40[2.82,13.98]$ \\
\hline 5.2 After usual care & 6 & 256 & $\begin{array}{l}\text { Mean Difference (IV, Random, } \\
95 \% \mathrm{CI})\end{array}$ & $1.80[-1.52,5.12]$ \\
\hline $\begin{array}{l}6 \text { Mobility - walking preferred speed (m/ } \\
\text { min) }\end{array}$ & 5 & 203 & $\begin{array}{l}\text { Mean Difference (IV, Random, } \\
95 \% \mathrm{CI})\end{array}$ & $2.15[-3.57,7.87]$ \\
\hline 6.1 During usual care & 1 & 18 & $\begin{array}{l}\text { Mean Difference (IV, Random, } \\
95 \% \mathrm{CI})\end{array}$ & $9.0[3.42,14.58]$ \\
\hline 6.2 After usual care & 4 & 185 & $\begin{array}{l}\text { Mean Difference (IV, Random, } \\
95 \% \mathrm{CI})\end{array}$ & $-0.18[-4.99,4.63]$ \\
\hline $\begin{array}{l}7 \text { Mobility - walking capacity (6-MWT me- } \\
\text { tres) }\end{array}$ & 5 & 238 & $\begin{array}{l}\text { Mean Difference (IV, Random, } \\
95 \% \mathrm{CI})\end{array}$ & $24.98[11.98,37.98]$ \\
\hline 7.1 During usual care & 0 & 0 & $\begin{array}{l}\text { Mean Difference (IV, Random, } \\
95 \% \mathrm{CI})\end{array}$ & $0.0[0.0,0.0]$ \\
\hline 7.2 After usual care & 5 & 238 & $\begin{array}{l}\text { Mean Difference (IV, Random, } \\
95 \% \mathrm{CI})\end{array}$ & $24.98[11.98,37.98]$ \\
\hline $\begin{array}{l}8 \text { Physical function - Berg Balance Scale } \\
\text { (score } 0 \text { to } 56 \text { ) }\end{array}$ & 5 & 220 & $\begin{array}{l}\text { Mean Difference (IV, Random, } \\
95 \% \mathrm{CI})\end{array}$ & $3.27[2.15,4.38]$ \\
\hline 8.1 During usual care & 1 & 20 & $\begin{array}{l}\text { Mean Difference (IV, Random, } \\
95 \% \mathrm{CI})\end{array}$ & $3.70[0.11,7.29]$ \\
\hline 8.2 After usual care & 4 & 200 & $\begin{array}{l}\text { Mean Difference (IV, Random, } \\
95 \% \mathrm{CI} \text { ) }\end{array}$ & $3.22[2.04,4.39]$ \\
\hline $\begin{array}{l}9 \text { Physical function - stair climbing, maxi- } \\
\text { mal (sec/step) }\end{array}$ & 2 & 91 & $\begin{array}{l}\text { Mean Difference (IV, Random, } \\
95 \% \mathrm{CI})\end{array}$ & $-2.07[-3.18,-0.96]$ \\
\hline
\end{tabular}




\begin{tabular}{|c|c|c|c|c|}
\hline Outcome or subgroup title & $\begin{array}{l}\text { No. of } \\
\text { studies }\end{array}$ & $\begin{array}{l}\text { No. of } \\
\text { partici- } \\
\text { pants }\end{array}$ & Statistical method & Effect size \\
\hline 9.1 During usual care & 1 & 50 & $\begin{array}{l}\text { Mean Difference (IV, Random, } \\
95 \% \mathrm{Cl} \text { ) }\end{array}$ & $-2.0[-3.12,-0.88]$ \\
\hline 9.2 After usual care & 1 & 41 & $\begin{array}{l}\text { Mean Difference (IV, Random, } \\
95 \% \mathrm{CI} \text { ) }\end{array}$ & $-5.36[-13.13,2.41]$ \\
\hline $\begin{array}{l}10 \text { Physical function - Timed Up and Go } \\
\text { (sec) }\end{array}$ & 5 & 224 & $\begin{array}{l}\text { Mean Difference (IV, Random, } \\
95 \% \mathrm{CI} \text { ) }\end{array}$ & $-3.46[-6.94,0.02]$ \\
\hline 10.1 During usual care & 1 & 50 & $\begin{array}{l}\text { Mean Difference (IV, Random, } \\
95 \% \mathrm{CI} \text { ) }\end{array}$ & $-2.0[-3.12,-0.88]$ \\
\hline 10.2 After usual care & 4 & 174 & $\begin{array}{l}\text { Mean Difference (IV, Random, } \\
95 \% \mathrm{CI} \text { ) }\end{array}$ & $-5.72[-7.92,-3.52]$ \\
\hline $\begin{array}{l}11 \text { Health-related QoL - SF- } 36 \text { physical } \\
\text { functioning (PF) scale }\end{array}$ & 3 & 70 & $\begin{array}{l}\text { Mean Difference (IV, Random, } \\
95 \% \mathrm{CI} \text { ) }\end{array}$ & $5.72[-5.26,16.70]$ \\
\hline 11.1 During usual care & 0 & 0 & $\begin{array}{l}\text { Mean Difference (IV, Random, } \\
95 \% \mathrm{CI} \text { ) }\end{array}$ & $0.0[0.0,0.0]$ \\
\hline 11.2 After usual care & 3 & 70 & $\begin{array}{l}\text { Mean Difference (IV, Random, } \\
95 \% \mathrm{CI} \text { ) }\end{array}$ & $5.72[-5.26,16.70]$ \\
\hline $\begin{array}{l}12 \text { Health-related QoL - SF- } 36 \text { mental } \\
\text { health }(\mathrm{MH}) \text { scale }\end{array}$ & 3 & 70 & $\begin{array}{l}\text { Mean Difference (IV, Random, } \\
95 \% \mathrm{CI} \text { ) }\end{array}$ & $7.69[1.56,13.83]$ \\
\hline 12.1 During usual care & 0 & 0 & $\begin{array}{l}\text { Mean Difference (IV, Random, } \\
95 \% \mathrm{CI} \text { ) }\end{array}$ & $0.0[0.0,0.0]$ \\
\hline 12.2 After usual care & 3 & 70 & $\begin{array}{l}\text { Mean Difference (IV, Random, } \\
95 \% \mathrm{CI} \text { ) }\end{array}$ & $7.69[1.56,13.83]$ \\
\hline $\begin{array}{l}13 \text { Mood - Centre for Epidemiologic Studies } \\
\text { for Depression scale (CES-D) }\end{array}$ & 2 & 180 & $\begin{array}{l}\text { Mean Difference (IV, Random, } \\
95 \% \mathrm{CI} \text { ) }\end{array}$ & $-3.76[-6.98,-0.54]$ \\
\hline 13.1 During usual care & 0 & 0 & $\begin{array}{l}\text { Mean Difference (IV, Random, } \\
95 \% \mathrm{CI} \text { ) }\end{array}$ & $0.0[0.0,0.0]$ \\
\hline 13.2 After usual care & 2 & 180 & $\begin{array}{l}\text { Mean Difference (IV, Random, } \\
95 \% \mathrm{CI} \text { ) }\end{array}$ & $-3.76[-6.98,-0.54]$ \\
\hline 14 Mood - combined depression scales & 3 & 209 & $\begin{array}{l}\text { Std. Mean Difference (IV, Ran- } \\
\text { dom, } 95 \% \mathrm{Cl} \text { ) }\end{array}$ & $-0.36[-0.64,-0.09]$ \\
\hline 14.1 During usual care & 0 & 0 & $\begin{array}{l}\text { Std. Mean Difference (IV, Ran- } \\
\text { dom, } 95 \% \mathrm{Cl} \text { ) }\end{array}$ & $0.0[0.0,0.0]$ \\
\hline 14.2 After usual care & 3 & 209 & $\begin{array}{l}\text { Std. Mean Difference (IV, Ran- } \\
\text { dom, } 95 \% \mathrm{Cl} \text { ) }\end{array}$ & $-0.36[-0.64,-0.09]$ \\
\hline
\end{tabular}


Analysis 3.1. Comparison 3 Resistance training versus control - end of intervention, Outcome 1 Death.

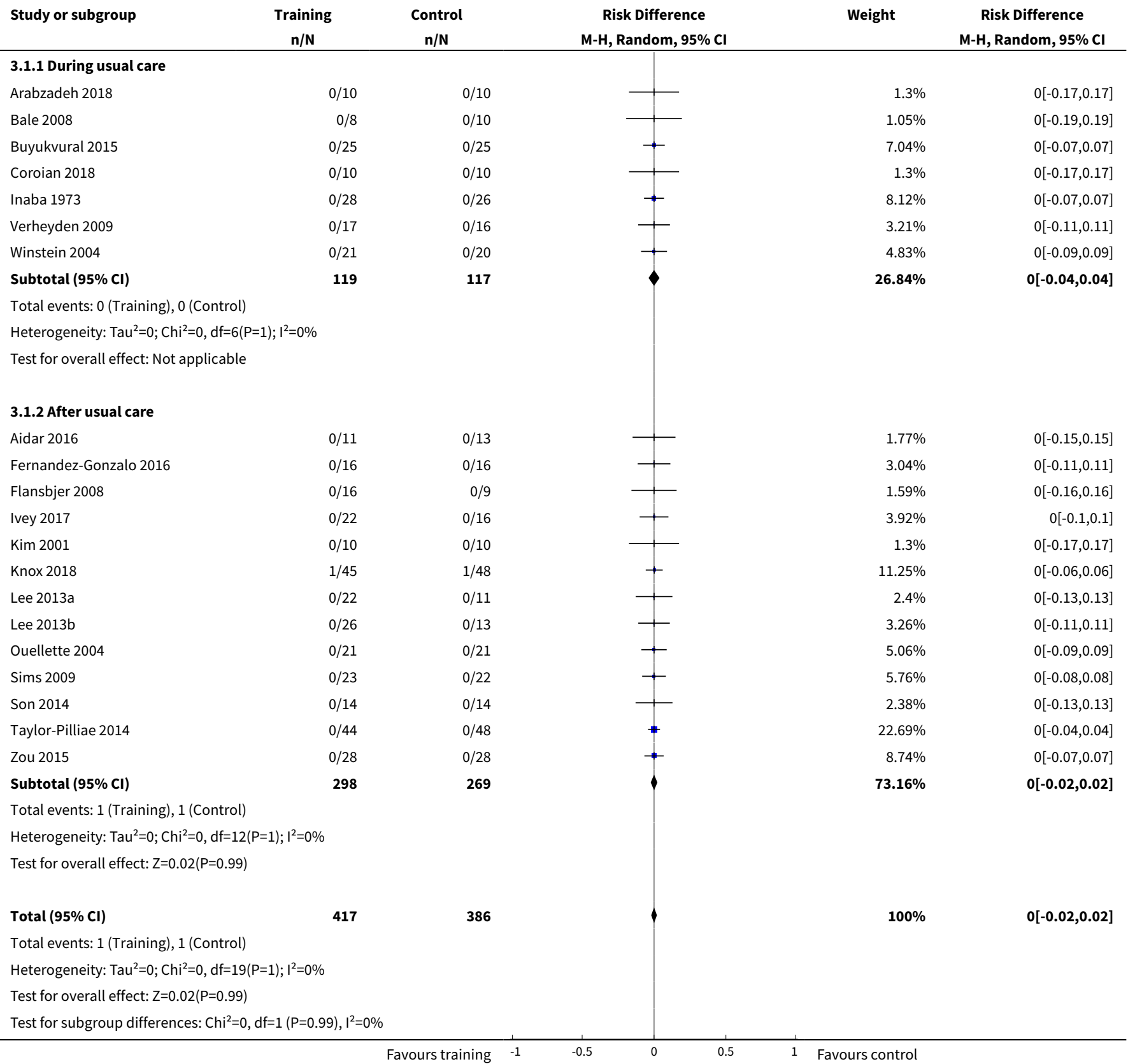

Analysis 3.2. Comparison 3 Resistance training versus control - end of intervention, Outcome 2 Physical fitness - composite measure of muscle strength.

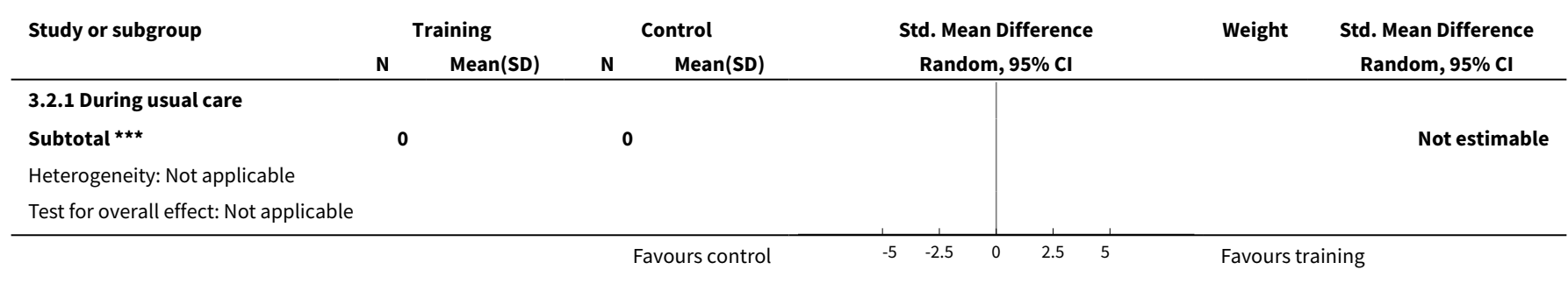




\begin{tabular}{|c|c|c|c|c|c|c|c|}
\hline \multirow[t]{2}{*}{ Study or subgroup } & \multicolumn{2}{|c|}{ Training } & \multicolumn{2}{|c|}{ Control } & \multirow{2}{*}{$\begin{array}{c}\text { Std. Mean Difference } \\
\text { Random, } 95 \% \mathrm{Cl}\end{array}$} & \multirow[t]{2}{*}{ Weight } & \multirow{2}{*}{$\begin{array}{c}\text { Std. Mean Difference } \\
\text { Random, } 95 \% \mathrm{Cl}\end{array}$} \\
\hline & $\mathbf{N}$ & Mean(SD) & $\mathbf{N}$ & Mean(SD) & & & \\
\hline \multicolumn{8}{|c|}{ 3.2.2 During and after usual care } \\
\hline Winstein 2004 & 20 & $\begin{array}{r}353.5 \\
(296.3)\end{array}$ & 20 & $\begin{array}{r}220.6 \\
(260.3)\end{array}$ & & $68.28 \%$ & $0.47[-0.16,1.1]$ \\
\hline Subtotal ${ }^{\star \star \star}$ & 20 & & 20 & & & $68.28 \%$ & $0.47[-0.16,1.1]$ \\
\hline \multicolumn{8}{|c|}{ Heterogeneity: $\mathrm{Tau}^{2}=0 ; \mathrm{Chi}^{2}=0, \mathrm{df}=0(\mathrm{P}<0.0001) ; \mathrm{I}^{2}=100 \%$} \\
\hline \multicolumn{8}{|c|}{ Test for overall effect: $Z=1.46(P=0.15)$} \\
\hline \multicolumn{8}{|c|}{ 3.2.3 After usual care } \\
\hline Kim 2001 & 10 & $507(559)$ & 10 & $142(193)$ & + & $31.72 \%$ & $0.84[-0.09,1.76]$ \\
\hline Subtotal $* \star \star$ & 10 & & 10 & & & $31.72 \%$ & $0.84[-0.09,1.76]$ \\
\hline \multicolumn{8}{|c|}{ Heterogeneity: Not applicable } \\
\hline \multicolumn{8}{|c|}{ Test for overall effect: $\mathrm{Z}=1.78(\mathrm{P}=0.08)$} \\
\hline Total $\star \star \star$ & 30 & & 30 & & $>$ & $100 \%$ & $0.58[0.06,1.1]$ \\
\hline \multicolumn{8}{|c|}{ Heterogeneity: $\operatorname{Tau}^{2}=0 ; \mathrm{Chi}^{2}=0.42, \mathrm{df}=1(\mathrm{P}=0.52) ;\left.\right|^{2}=0 \%$} \\
\hline \multicolumn{8}{|c|}{ Test for overall effect: $Z=2.2(P=0.03)$} \\
\hline \multicolumn{8}{|c|}{ Test for subgroup differences: $\mathrm{Chi}^{2}=0.42, \mathrm{df}=1(\mathrm{P}=0.52), \mathrm{I}^{2}=0 \%$} \\
\hline & & & & urs control & $\begin{array}{lll}-5 & -2.5 & 0\end{array}$ & Favours & ing \\
\hline
\end{tabular}

Analysis 3.3. Comparison 3 Resistance training versus control - end of intervention, Outcome 3 Physical fitness - muscle strength, paretic knee flexion.

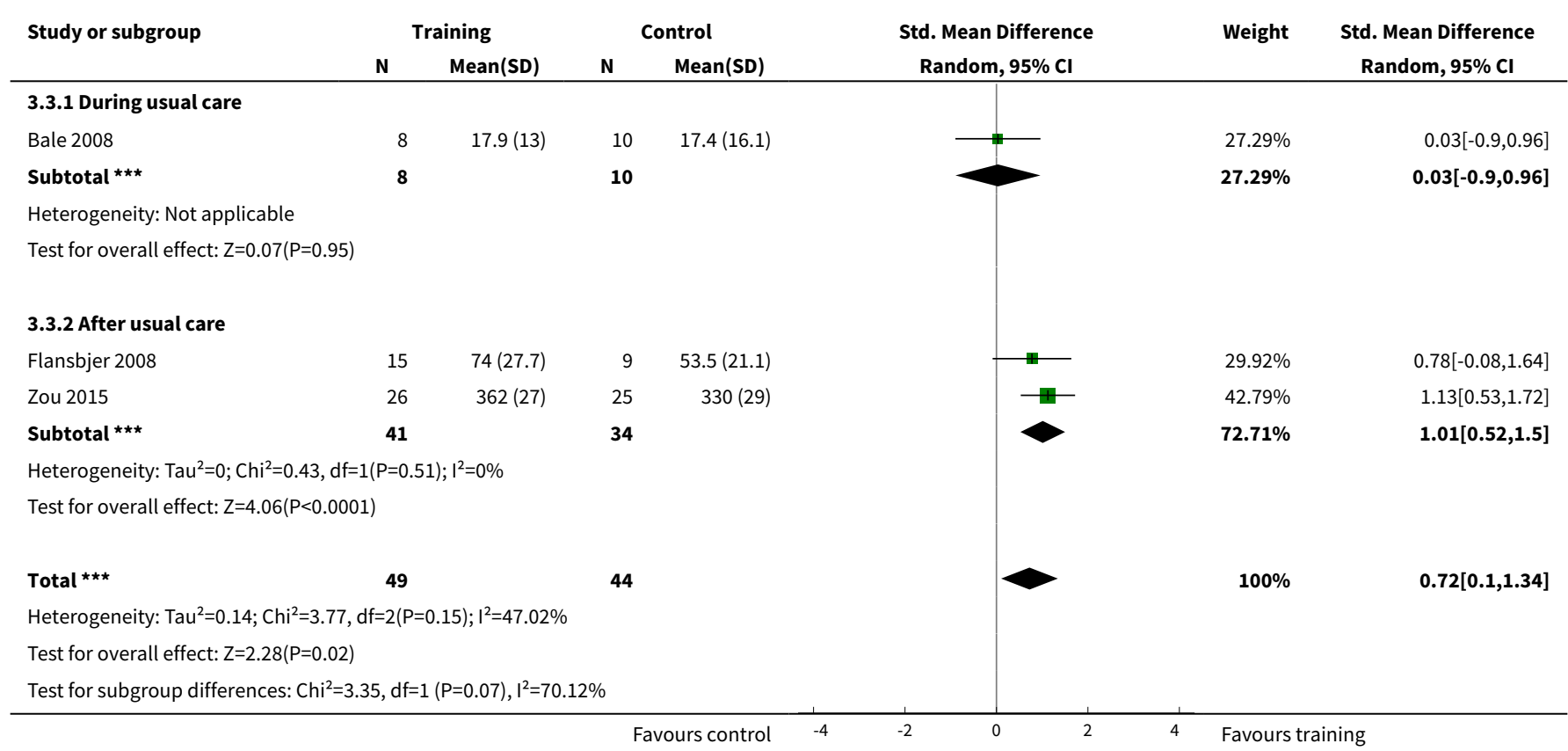


Analysis 3.4. Comparison 3 Resistance training versus control - end of intervention, Outcome 4 Physical fitness - muscle strength, paretic knee extension.

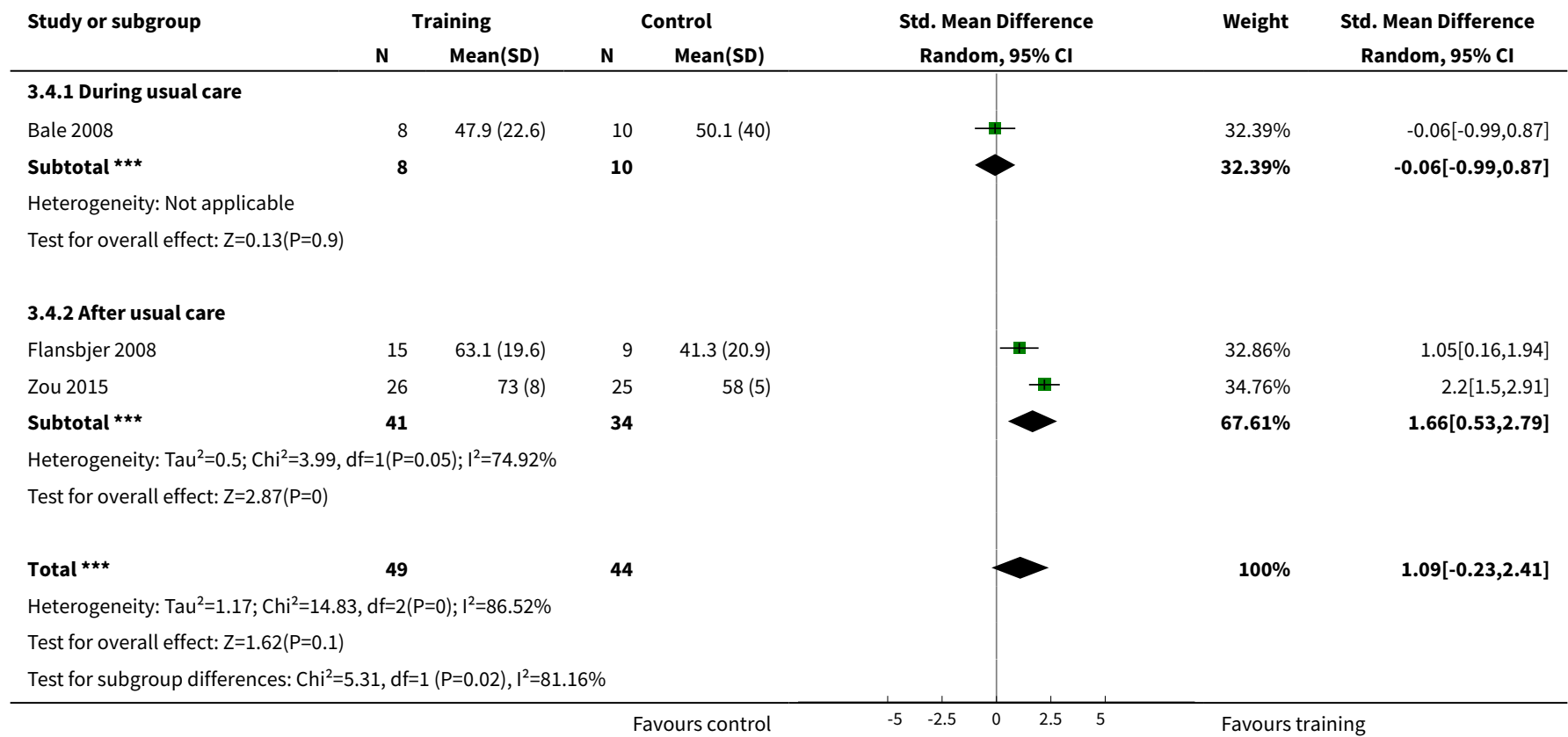

Analysis 3.5. Comparison 3 Resistance training versus control - end of intervention, Outcome 5 Mobility - walking maximal speed $(\mathrm{m} / \mathrm{min})$.

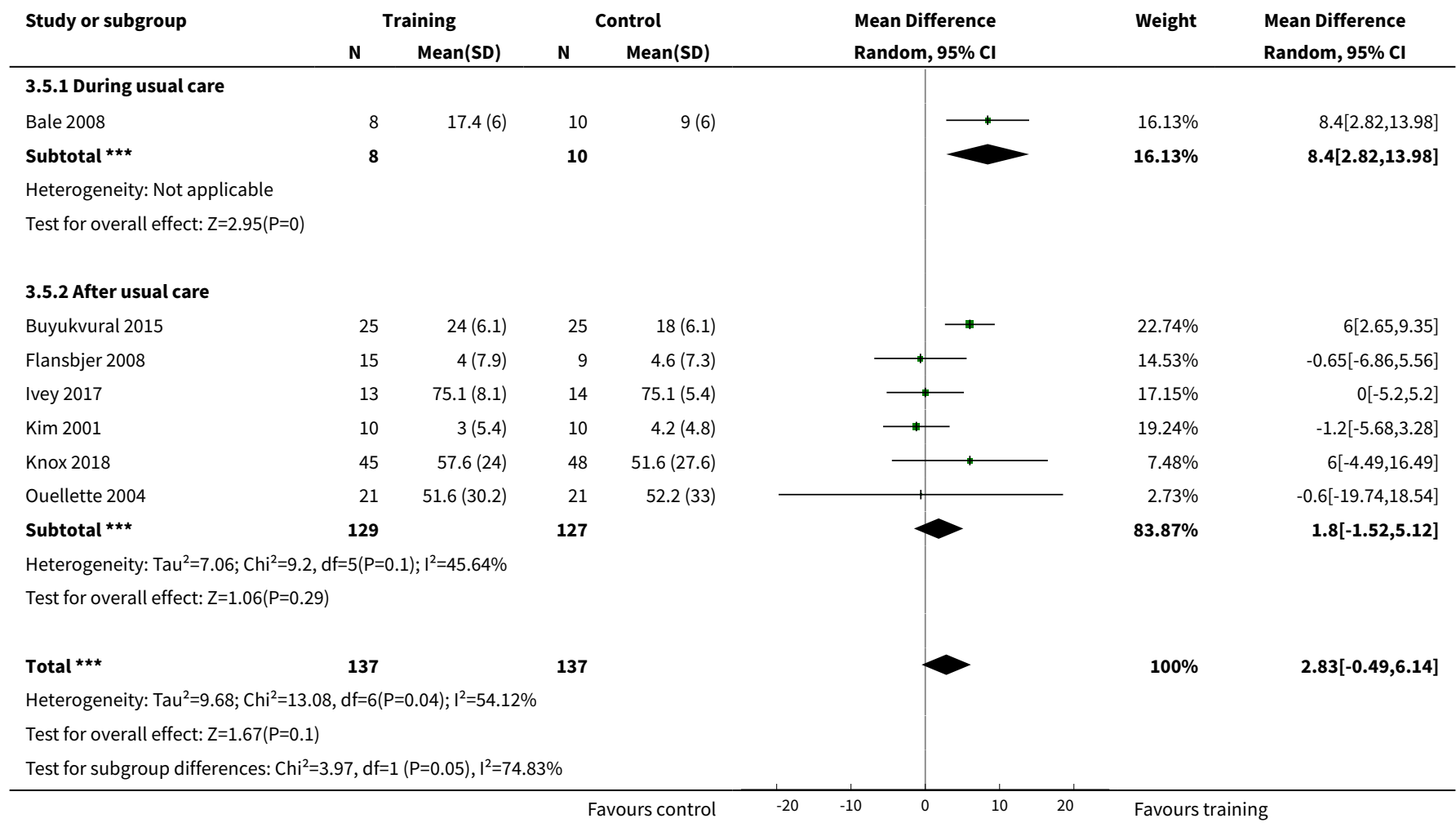


Analysis 3.6. Comparison 3 Resistance training versus control - end of intervention, Outcome 6 Mobility - walking preferred speed $(\mathrm{m} / \mathrm{min})$.

\begin{tabular}{|c|c|c|c|c|c|c|c|}
\hline \multirow[t]{2}{*}{ Study or subgroup } & \multicolumn{2}{|c|}{ Training } & \multicolumn{2}{|c|}{ Control } & \multirow{2}{*}{$\begin{array}{l}\text { Mean Difference } \\
\text { Random, 95\% Cl }\end{array}$} & \multirow[t]{2}{*}{ Weight } & \multirow{2}{*}{$\begin{array}{l}\text { Mean Difference } \\
\text { Random, } 95 \% \mathrm{Cl}\end{array}$} \\
\hline & $\mathbf{N}$ & Mean(SD) & $\mathbf{N}$ & Mean(SD) & & & \\
\hline \multicolumn{8}{|c|}{ 3.6.1 During usual care } \\
\hline Bale 2008 & 8 & $13.8(6)$ & 10 & $4.8(6)$ & $\rightarrow$ & $22.51 \%$ & $9[3.42,14.58]$ \\
\hline 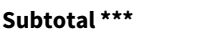 & 8 & & 10 & & & $22.51 \%$ & $9[3.42,14.58]$ \\
\hline \multicolumn{8}{|c|}{ Heterogeneity: Not applicable } \\
\hline \multicolumn{8}{|c|}{ Test for overall effect: $Z=3.16(P=0)$} \\
\hline \multicolumn{8}{|l|}{ 3.6.2 After usual care } \\
\hline Ivey 2017 & 14 & $51(5.4)$ & 16 & $53.6(5.4)$ & $\#$ & $25.36 \%$ & $-2.68[-6.52,1.16]$ \\
\hline Kim 2001 & 10 & $2.4(7.8)$ & 10 & $5.4(4.2)$ & $\rightarrow$ & $22.66 \%$ & $-3[-8.49,2.49]$ \\
\hline Knox 2018 & 45 & $46.2(17.4)$ & 48 & $38.4(19.8)$ & 世- & $19.08 \%$ & $7.8[0.24,15.36]$ \\
\hline Ouellette 2004 & 21 & $38.4(22)$ & 21 & $38.4(24.8)$ & & $10.39 \%$ & $0[-14.16,14.16]$ \\
\hline Subtotal $\star \star \star$ & 90 & & 95 & & & $77.49 \%$ & $-0.18[-4.99,4.63]$ \\
\hline \multicolumn{8}{|c|}{ Test for overall effect: $Z=0.07(P=0.94)$} \\
\hline Total $\star \star \star ~$ & 98 & & 105 & & & $100 \%$ & $2.15[-3.57,7.87]$ \\
\hline \multicolumn{8}{|c|}{ Heterogeneity: Tau $^{2}=29.74 ; \mathrm{Chi}^{2}=16.58, \mathrm{df}=4(\mathrm{P}=0) ; \mathrm{I}^{2}=75.87 \%$} \\
\hline \multicolumn{8}{|c|}{ Test for overall effect: $\mathrm{Z}=0.74(\mathrm{P}=0.46)$} \\
\hline Test for subgroup dif & $97, \mathrm{df}=$ & $P=0.01), I^{2}=83$ & & & & & \\
\hline
\end{tabular}

Analysis 3.7. Comparison 3 Resistance training versus control - end of intervention, Outcome 7 Mobility - walking capacity (6-MWT metres).

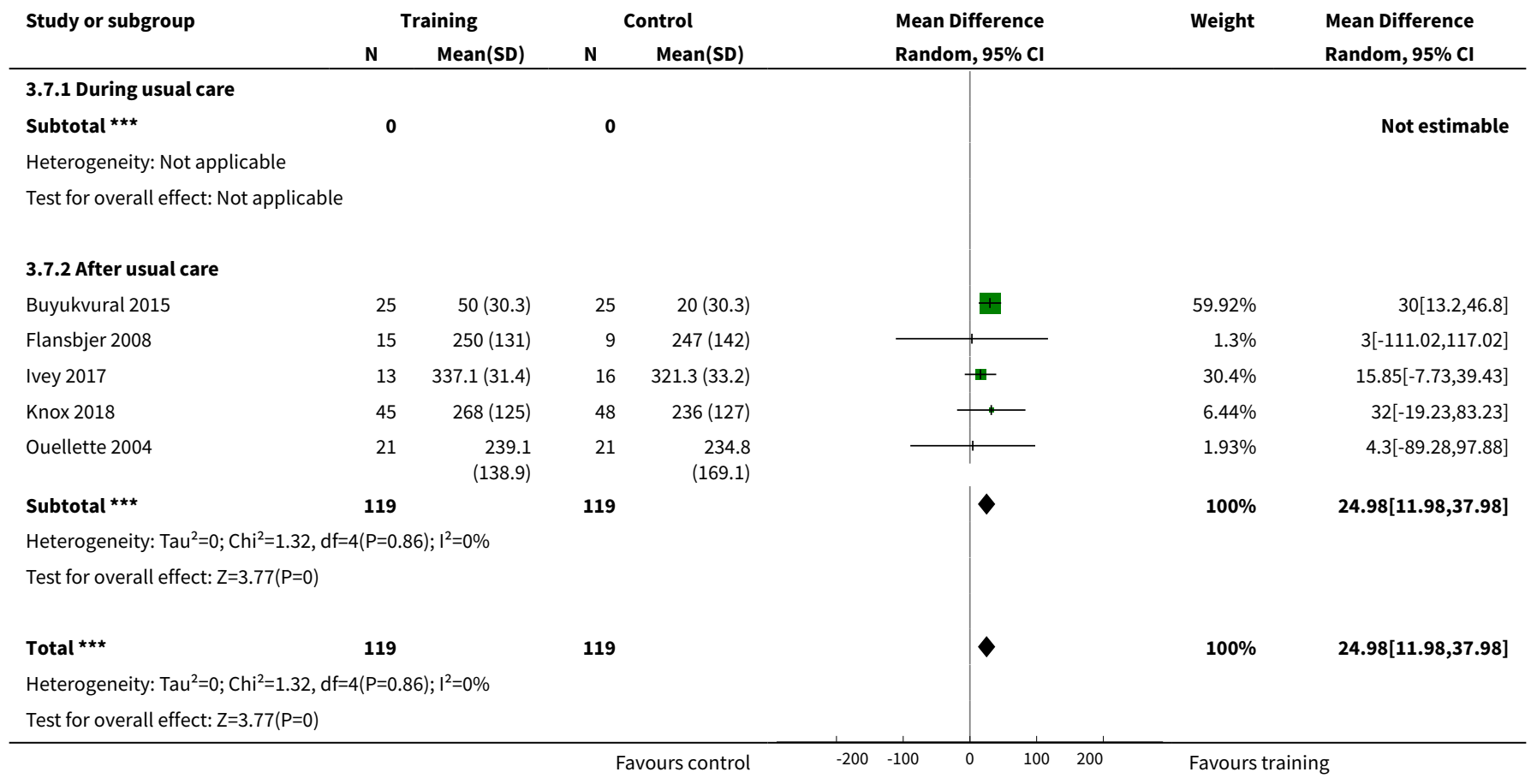




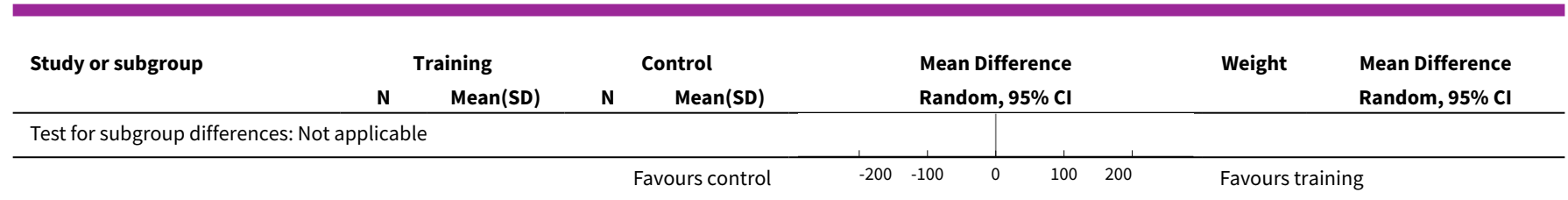

Analysis 3.8. Comparison 3 Resistance training versus control - end of intervention, Outcome 8 Physical function - Berg Balance Scale (score 0 to 56).

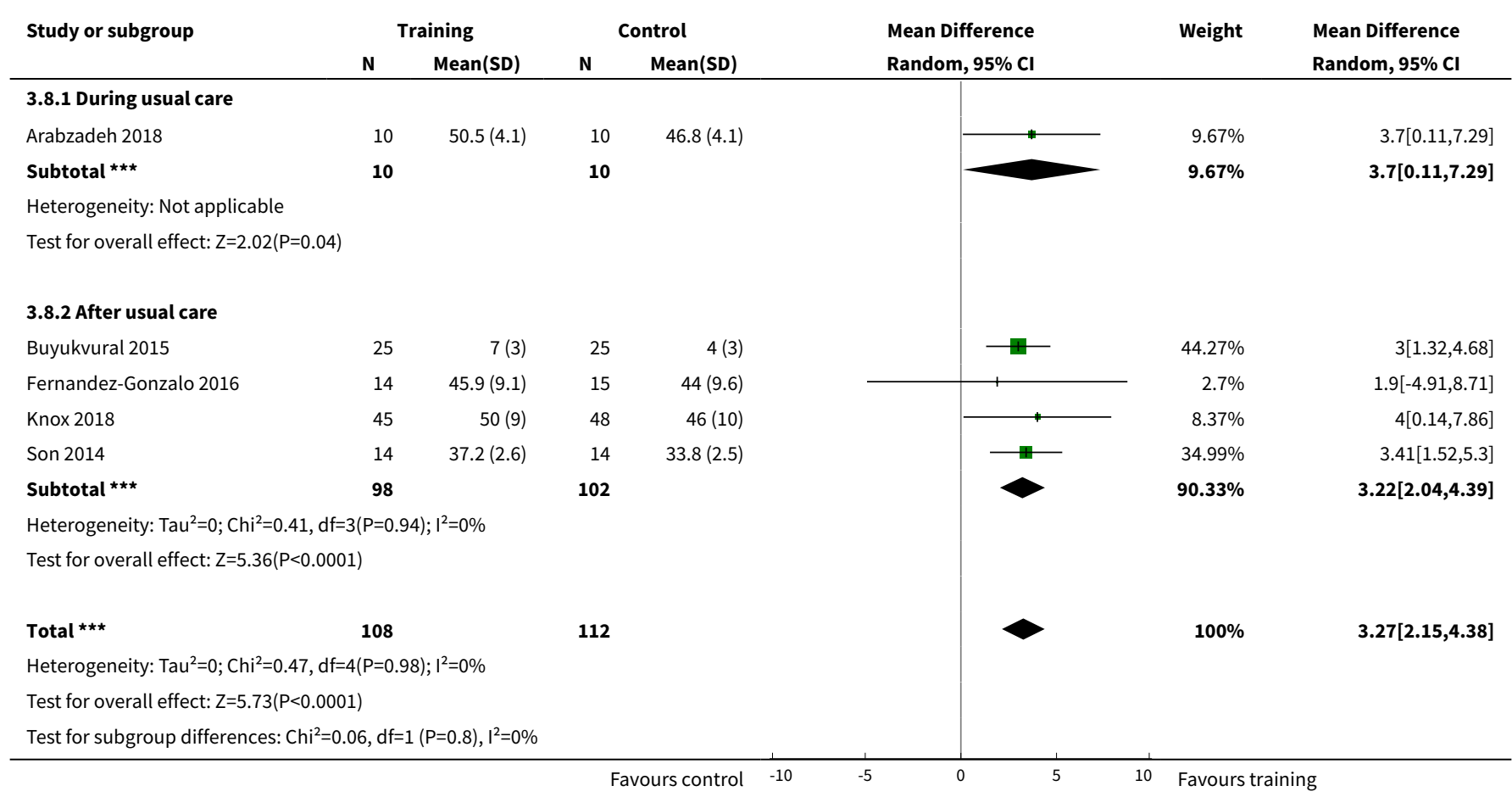

Analysis 3.9. Comparison 3 Resistance training versus control - end of intervention, Outcome 9 Physical function - stair climbing, maximal (sec/step).

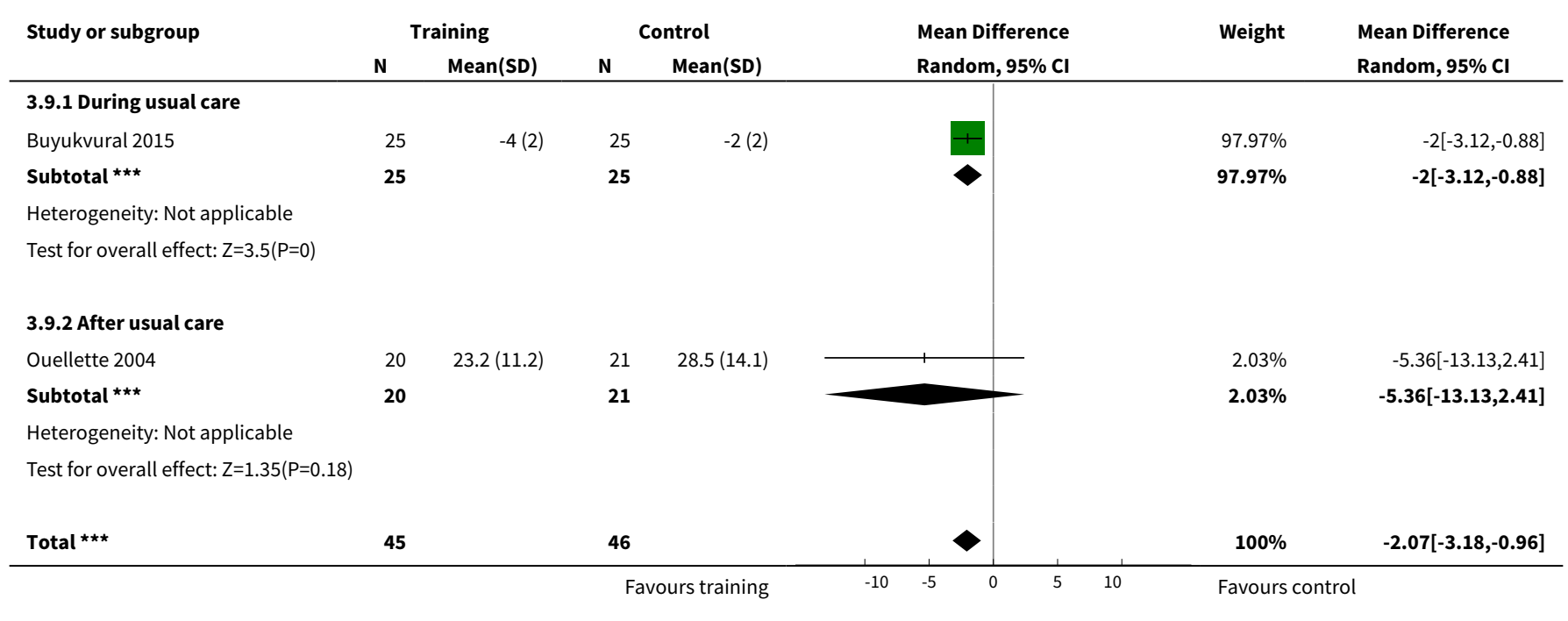




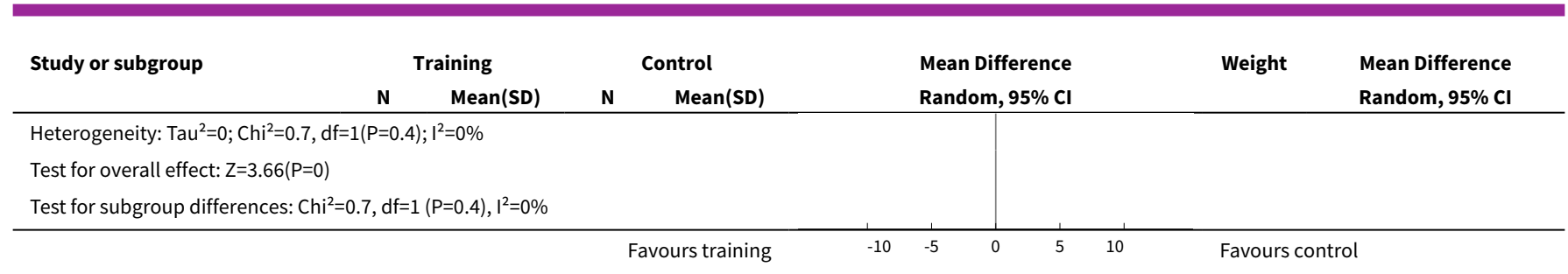

Analysis 3.10. Comparison 3 Resistance training versus control - end of intervention, Outcome 10 Physical function - Timed Up and Go (sec).

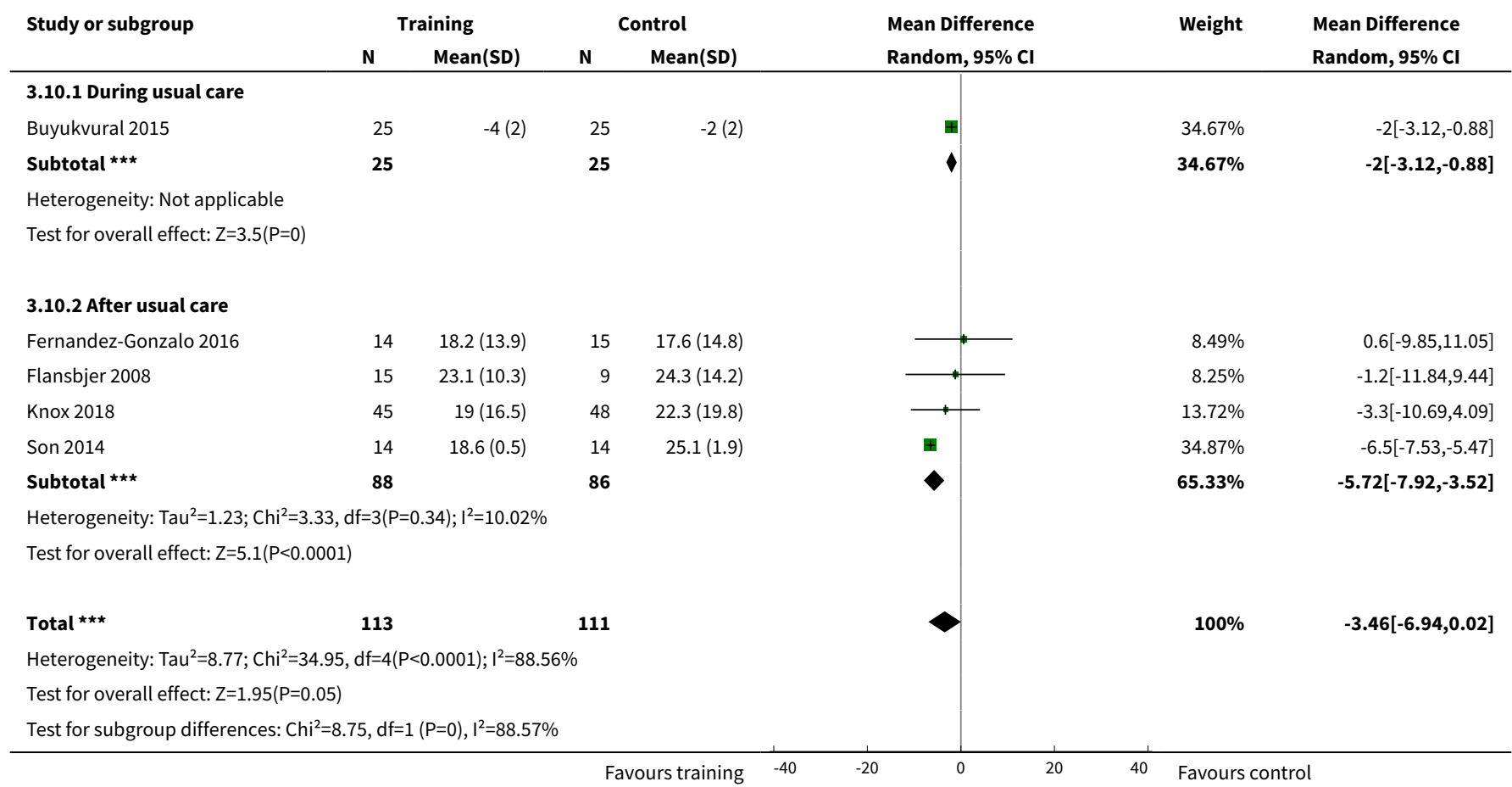

Analysis 3.11. Comparison 3 Resistance training versus control - end of intervention, Outcome 11 Health-related QoL - SF-36 physical functioning (PF) scale.

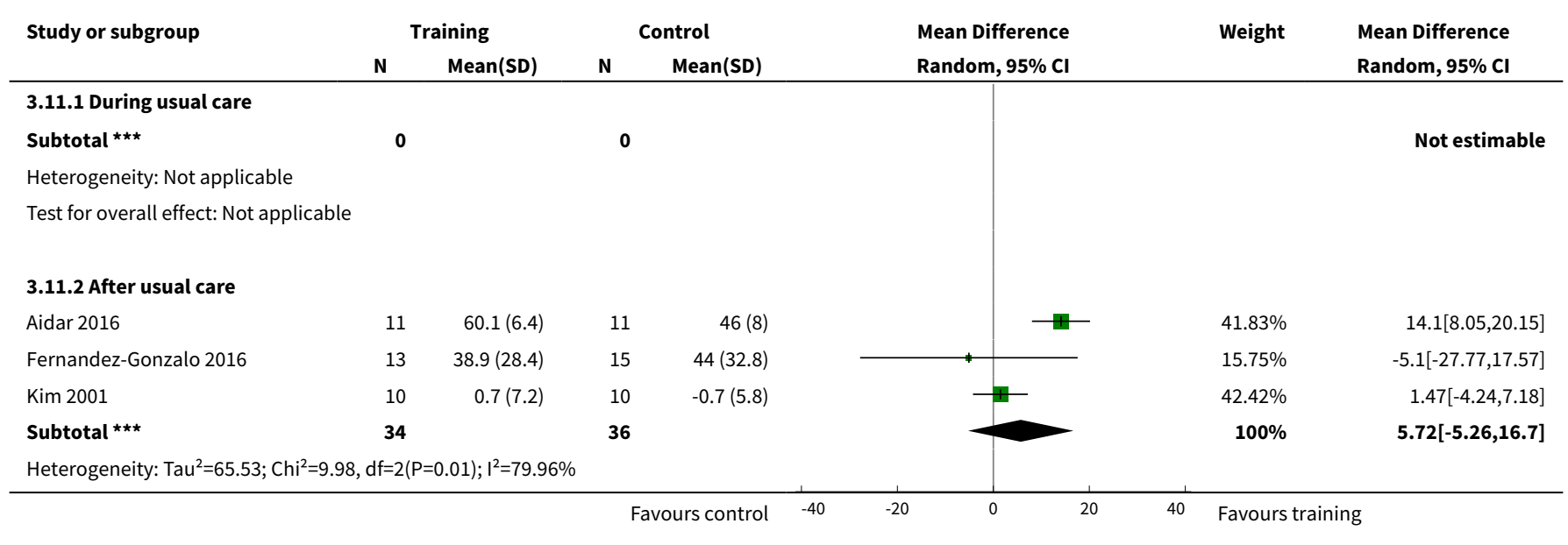




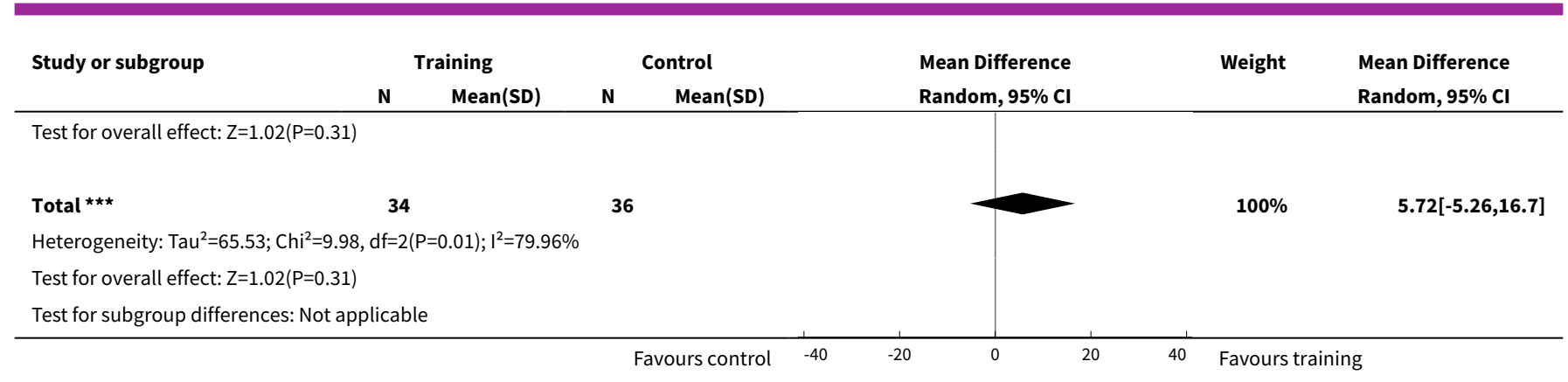

Analysis 3.12. Comparison 3 Resistance training versus control - end of intervention, Outcome 12 Health-related QoL - SF-36 mental health (MH) scale.

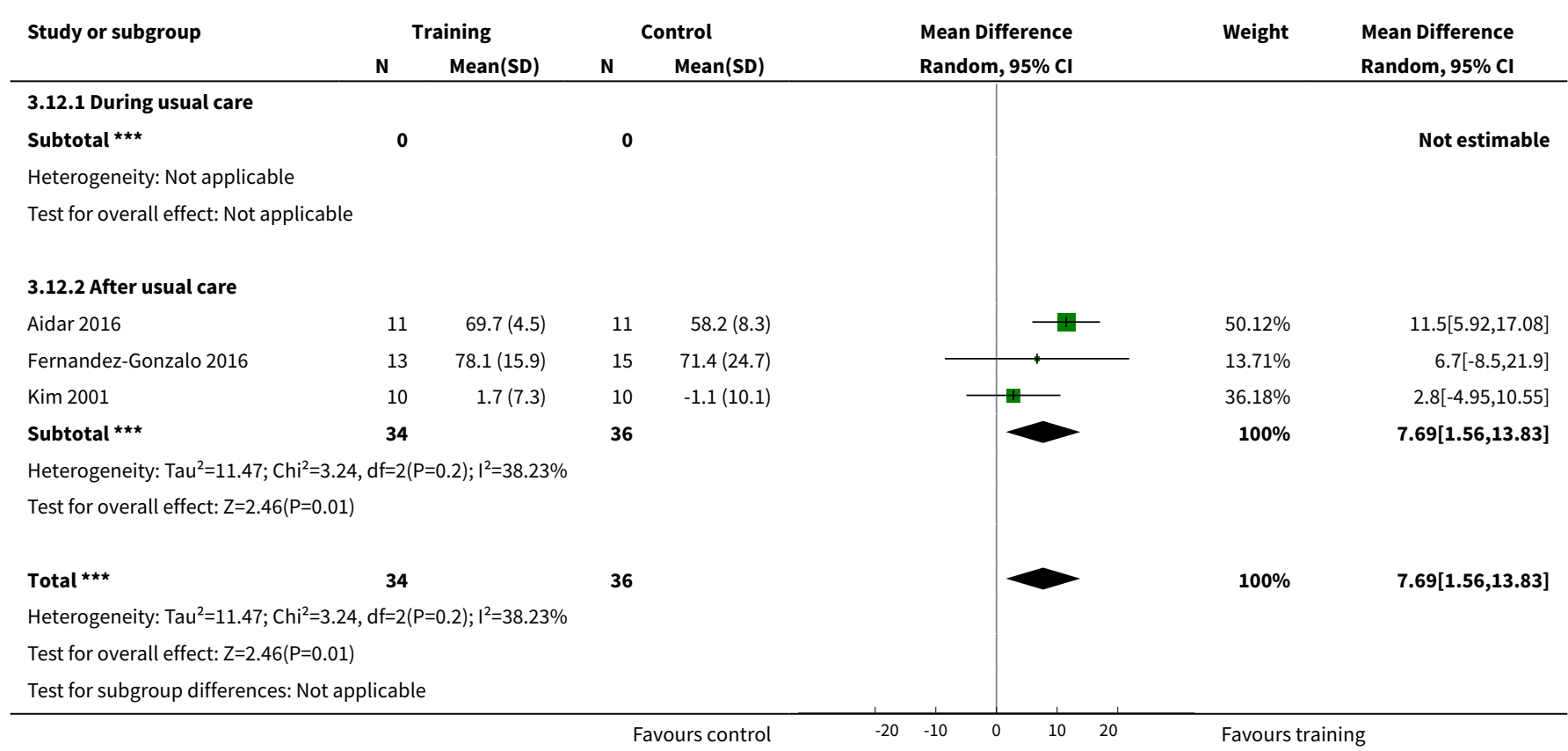

Analysis 3.13. Comparison 3 Resistance training versus control - end of intervention, Outcome 13 Mood - Centre for Epidemiologic Studies for Depression scale (CES-D).

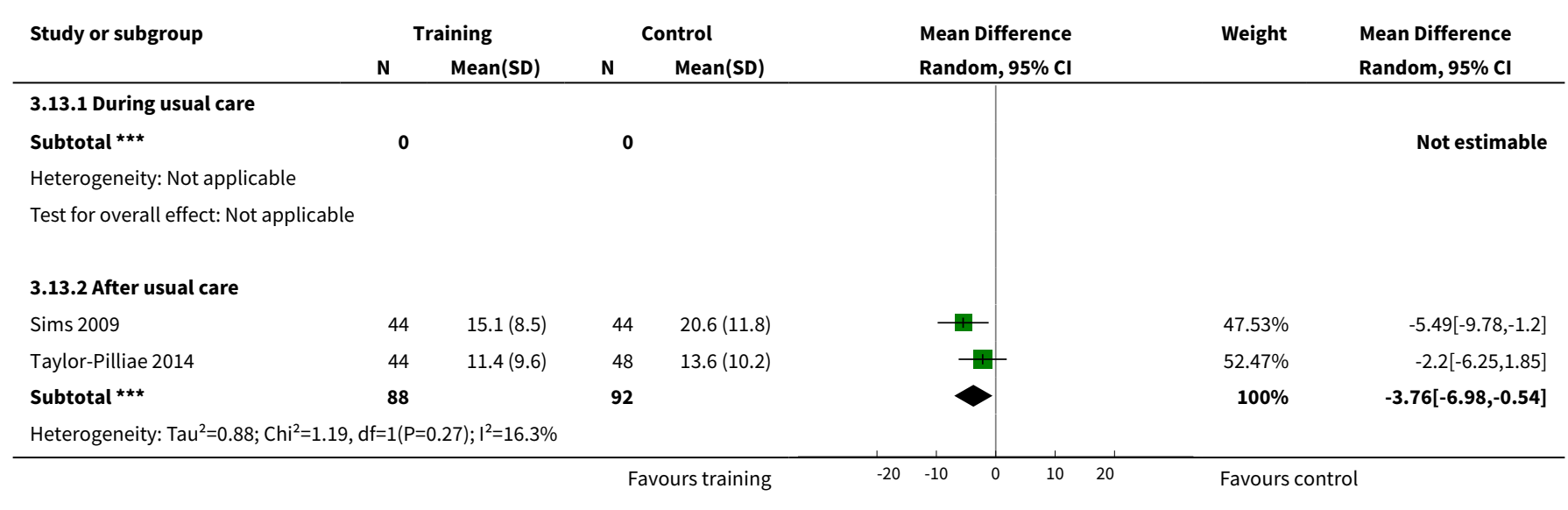




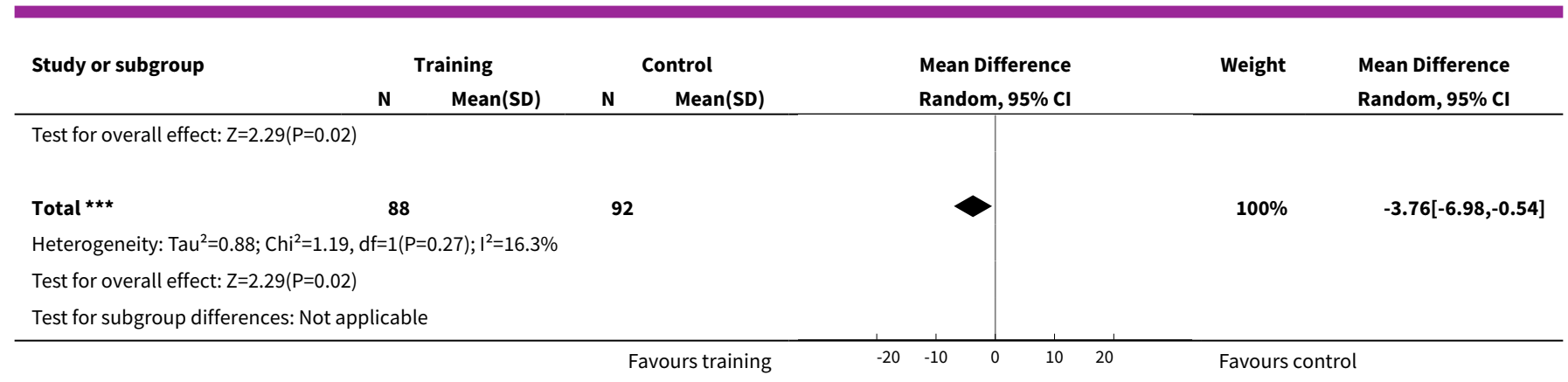

\section{Analysis 3.14. Comparison 3 Resistance training versus control - end of intervention, Outcome 14 Mood - combined depression scales.}

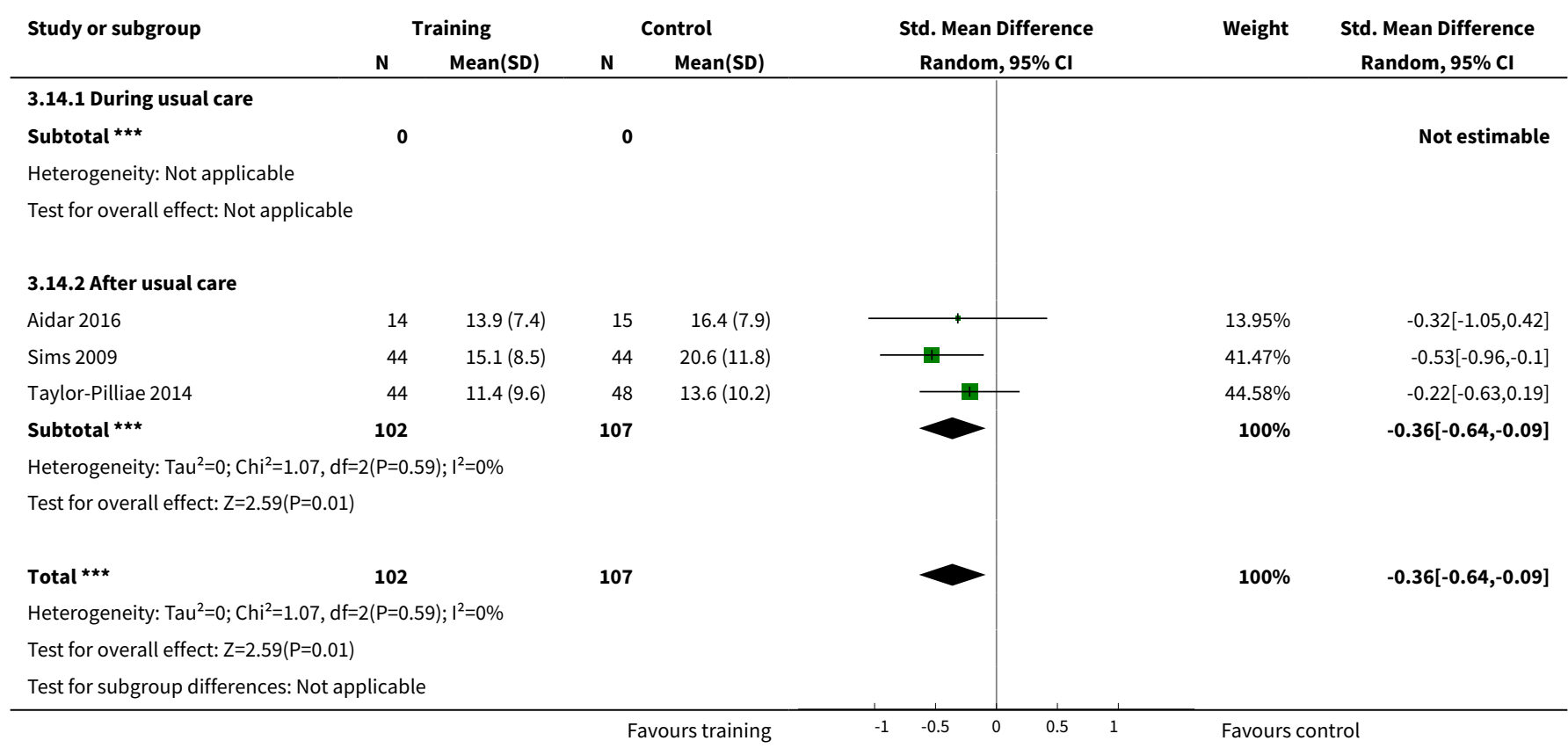

\section{Comparison 4. Resistance training versus control - end of retention follow-up}

\begin{tabular}{|c|c|c|c|c|}
\hline Outcome or subgroup title & $\begin{array}{l}\text { No. of } \\
\text { studies }\end{array}$ & $\begin{array}{l}\text { No. of } \\
\text { partici- } \\
\text { pants }\end{array}$ & Statistical method & Effect size \\
\hline 1 Death & 5 & 251 & $\begin{array}{l}\text { Risk Difference (M-H, Random, 95\% } \\
\mathrm{Cl})\end{array}$ & $0.00[-0.04,0.04]$ \\
\hline 1.1 During usual care & 3 & 115 & $\begin{array}{l}\text { Risk Difference (M-H, Random, 95\% } \\
\mathrm{Cl})\end{array}$ & $0.0[-0.05,0.05]$ \\
\hline 1.2 After usual care & 2 & 136 & $\begin{array}{l}\text { Risk Difference (M-H, Random, 95\% } \\
\mathrm{Cl} \text { ) }\end{array}$ & $0.00[-0.06,0.06]$ \\
\hline $\begin{array}{l}2 \text { Mobility - walking maximal speed } \\
\text { (m/min) }\end{array}$ & 2 & 117 & $\begin{array}{l}\text { Mean Difference (IV, Random, 95\% } \\
\mathrm{CI})\end{array}$ & $7.80[-3.32,18.91]$ \\
\hline
\end{tabular}




\begin{tabular}{|c|c|c|c|c|}
\hline Outcome or subgroup title & $\begin{array}{l}\text { No. of } \\
\text { studies }\end{array}$ & $\begin{array}{l}\text { No. of } \\
\text { partici- } \\
\text { pants }\end{array}$ & Statistical method & Effect size \\
\hline 2.1 During usual care & 0 & 0 & $\begin{array}{l}\text { Mean Difference (IV, Random, 95\% } \\
\mathrm{CI})\end{array}$ & $0.0[0.0,0.0]$ \\
\hline 2.2 After usual care & 2 & 117 & $\begin{array}{l}\text { Mean Difference (IV, Random, 95\% } \\
\mathrm{CI})\end{array}$ & $7.80[-3.32,18.91]$ \\
\hline $\begin{array}{l}3 \text { Mobility - walking capacity (6-MWT } \\
\text { metres) }\end{array}$ & 2 & 117 & $\begin{array}{l}\text { Mean Difference (IV, Random, 95\% } \\
\mathrm{CI})\end{array}$ & $22.41[-27.87,72.69]$ \\
\hline 3.1 During usual care & 0 & 0 & $\begin{array}{l}\text { Mean Difference (IV, Random, 95\% } \\
\mathrm{CI})\end{array}$ & $0.0[0.0,0.0]$ \\
\hline 3.2 After usual care & 2 & 117 & $\begin{array}{l}\text { Mean Difference (IV, Random, 95\% } \\
\mathrm{CI})\end{array}$ & $22.41[-27.87,72.69]$ \\
\hline $\begin{array}{l}4 \text { Physical function - Timed Up and } \\
\text { Go (sec) }\end{array}$ & 2 & 117 & $\begin{array}{l}\text { Mean Difference (IV, Random, 95\% } \\
\mathrm{CI})\end{array}$ & $-2.64[-9.24,3.95]$ \\
\hline 4.1 During usual care & 0 & 0 & $\begin{array}{l}\text { Mean Difference (IV, Random, 95\% } \\
\mathrm{CI})\end{array}$ & $0.0[0.0,0.0]$ \\
\hline 4.2 After usual care & 2 & 117 & $\begin{array}{l}\text { Mean Difference (IV, Random, 95\% } \\
\mathrm{Cl} \text { ) }\end{array}$ & $-2.64[-9.24,3.95]$ \\
\hline
\end{tabular}

\section{Analysis 4.1. Comparison 4 Resistance training versus control - end of retention follow-up, Outcome 1 Death.}

\begin{tabular}{|c|c|c|c|c|c|}
\hline Study or subgroup & $\begin{array}{c}\text { Training } \\
\mathrm{n} / \mathrm{N}\end{array}$ & $\begin{array}{l}\text { Control } \\
\mathrm{n} / \mathrm{N}\end{array}$ & $\begin{array}{c}\text { Risk Difference } \\
\text { M-H, Random, } 95 \% \mathrm{CI}\end{array}$ & Weight & $\begin{array}{c}\text { Risk Difference } \\
\text { M-H, Random, } 95 \% \mathrm{Cl} \\
\end{array}$ \\
\hline \multicolumn{6}{|c|}{ 4.1.1 During usual care } \\
\hline Coroian 2018 & $0 / 10$ & $0 / 10$ & & $5.12 \%$ & $0[-0.17,0.17]$ \\
\hline Inaba 1973 & $0 / 28$ & $0 / 26$ & & $32.14 \%$ & $0[-0.07,0.07]$ \\
\hline Winstein 2004 & $0 / 21$ & $0 / 20$ & & $19.09 \%$ & $0[-0.09,0.09]$ \\
\hline Subtotal (95\% CI) & 59 & 56 & & $56.35 \%$ & $0[-0.05,0.05]$ \\
\hline \multicolumn{6}{|c|}{ Total events: 0 (Training), 0 (Control) } \\
\hline \multicolumn{6}{|c|}{ Heterogeneity: Tau $^{2}=0 ; \mathrm{Chi}^{2}=0, \mathrm{df}=2(\mathrm{P}=1) ; \mathrm{I}^{2}=0 \%$} \\
\hline \multicolumn{6}{|c|}{ Test for overall effect: Not applicable } \\
\hline \multicolumn{6}{|l|}{ 4.1.2 After usual care } \\
\hline Knox 2018 & $2 / 45$ & $2 / 48$ & & $22.76 \%$ & $0[-0.08,0.09]$ \\
\hline Sims 2009 & $0 / 21$ & $0 / 22$ & & $20.89 \%$ & $0[-0.09,0.09]$ \\
\hline Subtotal $(95 \% \mathrm{Cl})$ & 66 & 70 & & $43.65 \%$ & $0[-0.06,0.06]$ \\
\hline \multicolumn{6}{|c|}{ Total events: 2 (Training), 2 (Control) } \\
\hline \multicolumn{6}{|c|}{ Heterogeneity: $\operatorname{Tau}^{2}=0 ; \mathrm{Chi}^{2}=0, \mathrm{df}=1(\mathrm{P}=0.96) ; \mathrm{I}^{2}=0 \%$} \\
\hline \multicolumn{6}{|c|}{ Test for overall effect: $\mathrm{Z}=0.05(\mathrm{P}=0.96)$} \\
\hline Total $(95 \% \mathrm{Cl})$ & 125 & 126 & & $100 \%$ & $0[-0.04,0.04]$ \\
\hline \multicolumn{6}{|c|}{ Total events: 2 (Training), 2 (Control) } \\
\hline \multicolumn{6}{|c|}{ Heterogeneity: $\operatorname{Tau}^{2}=0 ; \mathrm{Chi}^{2}=0, \mathrm{df}=4(\mathrm{P}=1) ; \mathrm{I}^{2}=0 \%$} \\
\hline \multicolumn{6}{|c|}{ Test for overall effect: $Z=0.03(P=0.97)$} \\
\hline
\end{tabular}




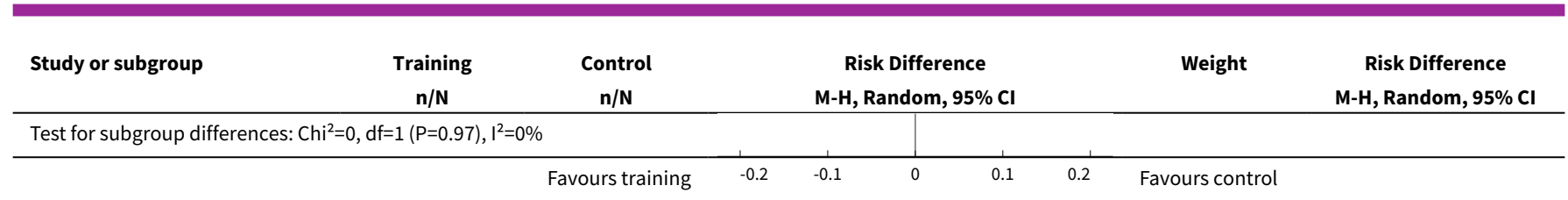

Analysis 4.2. Comparison 4 Resistance training versus control - end of retention follow-up, Outcome 2 Mobility - walking maximal speed ( $\mathrm{m} / \mathrm{min})$.

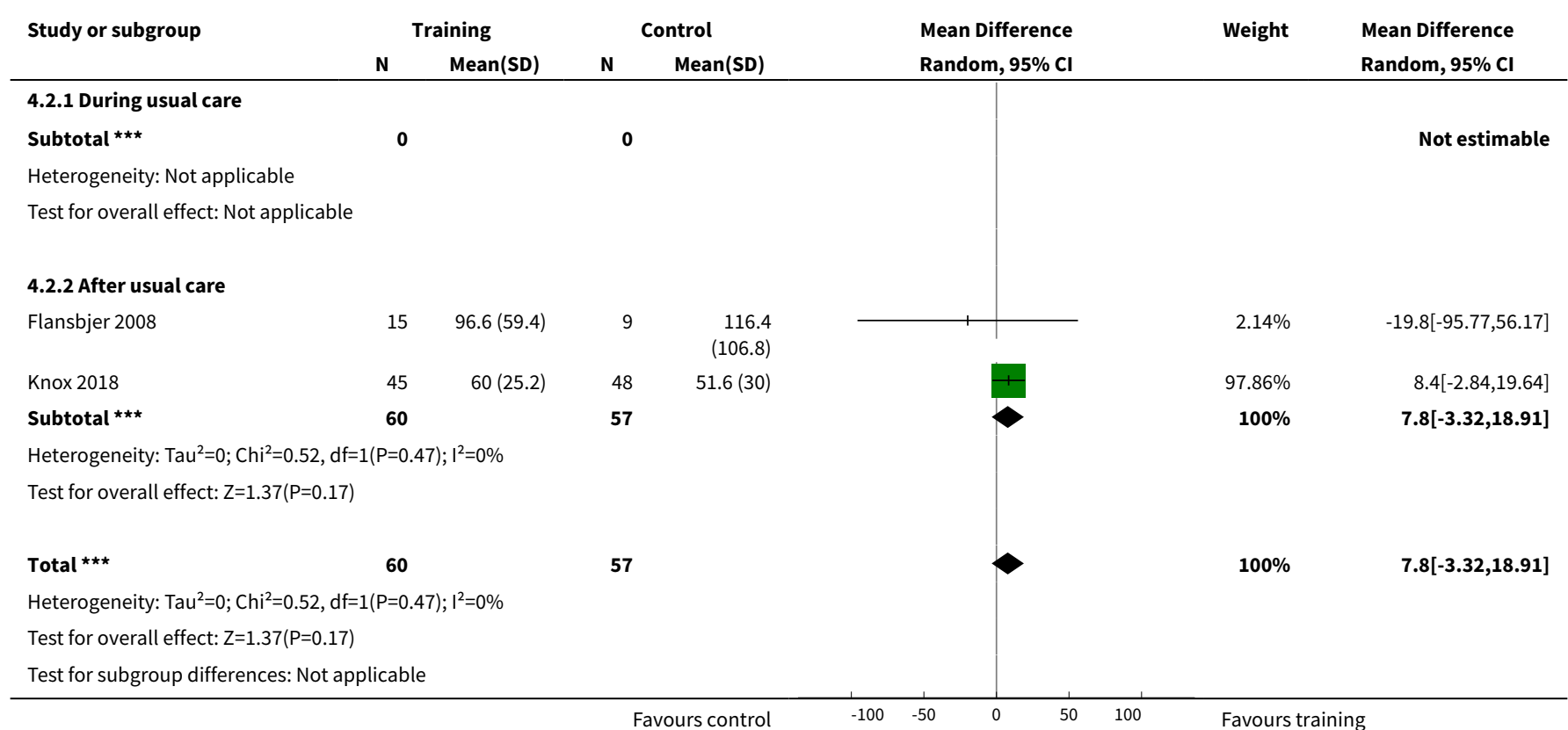

Analysis 4.3. Comparison 4 Resistance training versus control - end of retention follow-up, Outcome 3 Mobility - walking capacity (6-MWT metres).

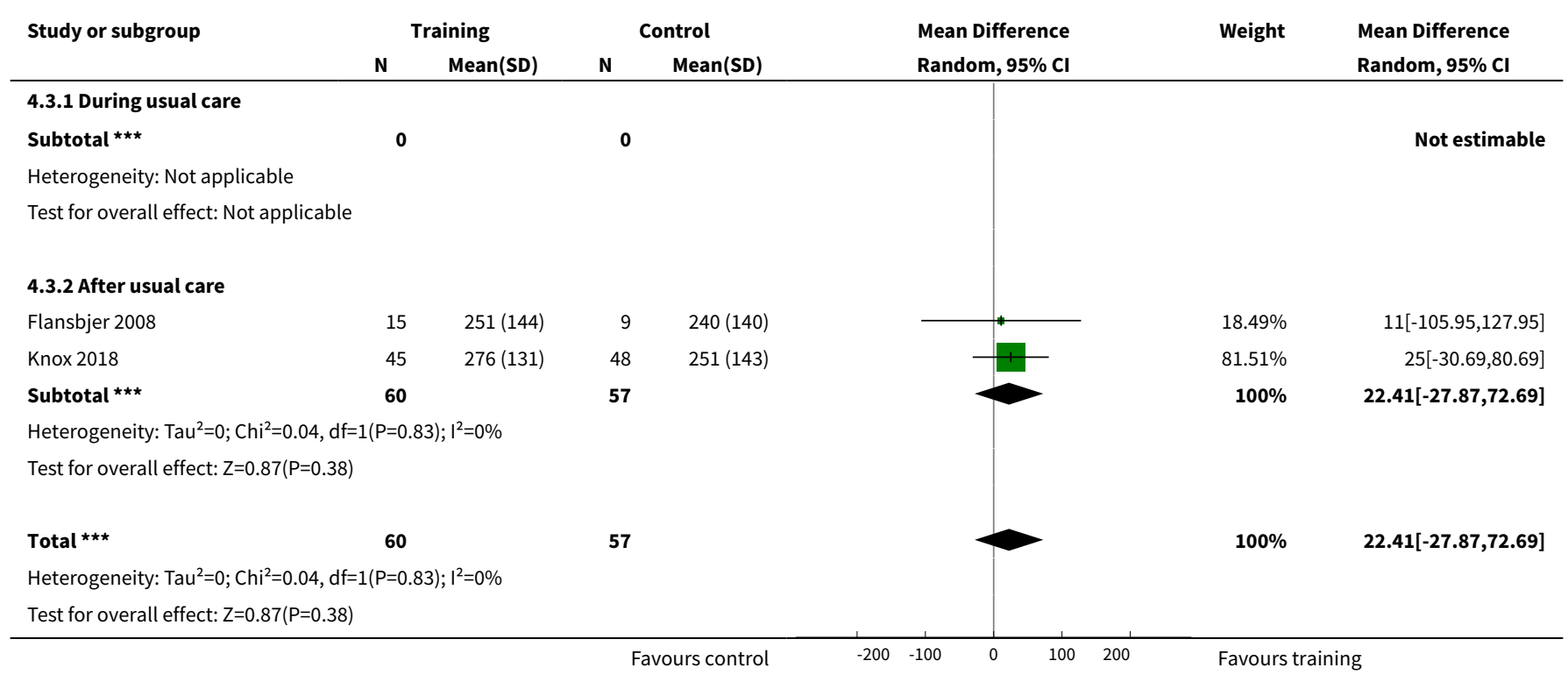




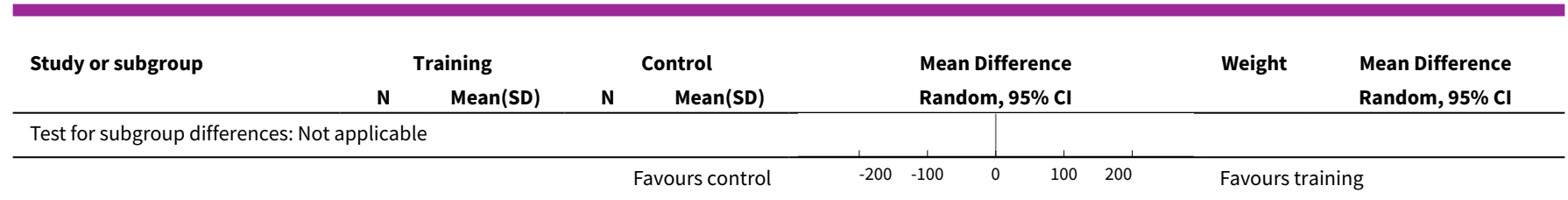

Analysis 4.4. Comparison 4 Resistance training versus control - end of retention follow-up, Outcome 4 Physical function - Timed Up and Go (sec).

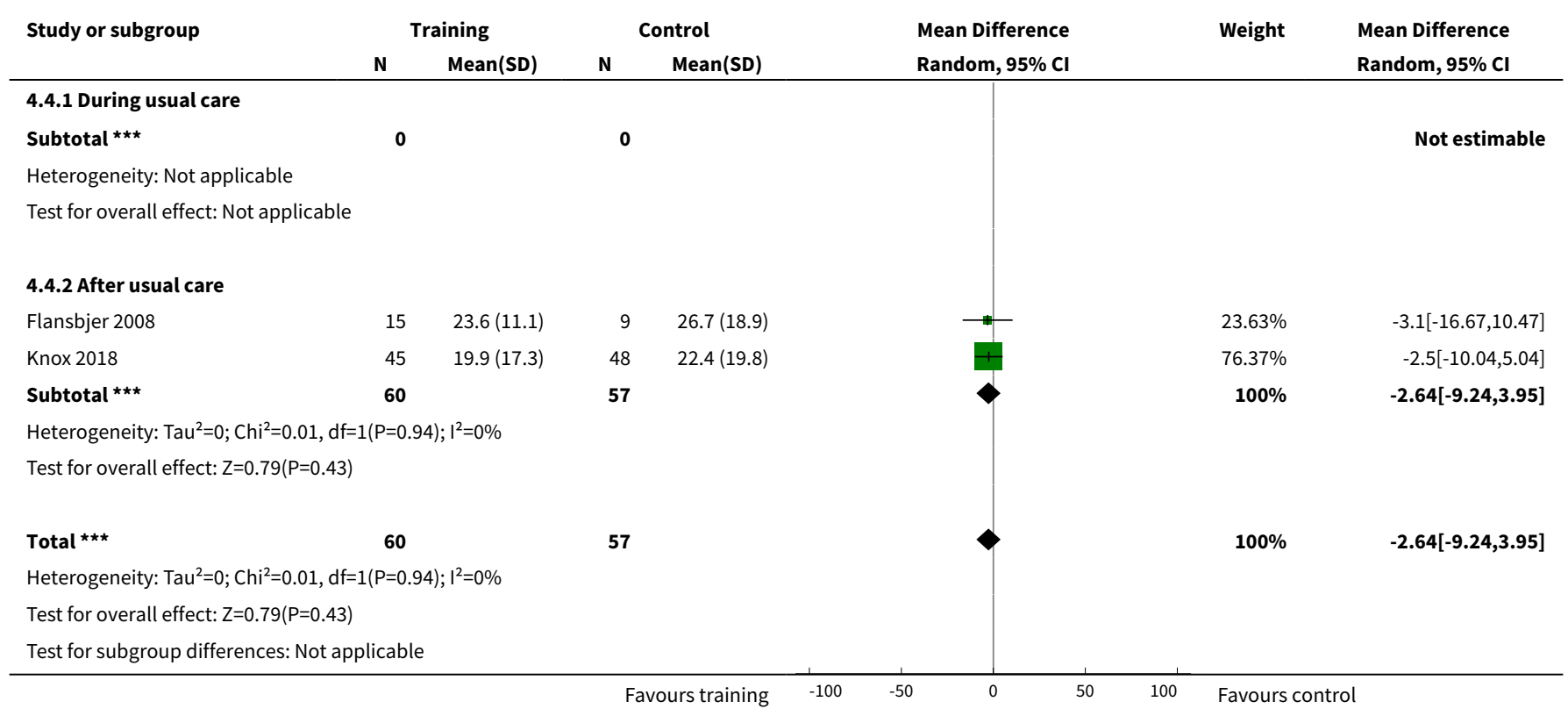

\section{Comparison 5. Mixed training versus control - end of intervention}

\begin{tabular}{|c|c|c|c|c|}
\hline Outcome or subgroup title & $\begin{array}{l}\text { No. of } \\
\text { studies }\end{array}$ & $\begin{array}{l}\text { No. of } \\
\text { partici- } \\
\text { pants }\end{array}$ & Statistical method & Effect size \\
\hline 1 Death & 23 & 1231 & $\begin{array}{l}\text { Risk Difference (M-H, Random, } \\
95 \% \mathrm{Cl})\end{array}$ & $-0.00[-0.02,0.01]$ \\
\hline 1.1 During usual care & 10 & 344 & $\begin{array}{l}\text { Risk Difference (M-H, Random, } \\
95 \% \mathrm{Cl})\end{array}$ & $0.0[-0.03,0.03]$ \\
\hline 1.2 After usual care & 13 & 887 & $\begin{array}{l}\text { Risk Difference (M-H, Random, } \\
95 \% \mathrm{Cl})\end{array}$ & $-0.01[-0.02,0.01]$ \\
\hline 2 Disability - Barthel Index (BI) & 6 & 256 & $\begin{array}{l}\text { Mean Difference (IV, Random, } \\
95 \% \mathrm{CI})\end{array}$ & $2.84[-0.48,6.17]$ \\
\hline 2.1 During usual care & 3 & 78 & $\begin{array}{l}\text { Mean Difference (IV, Random, } \\
95 \% \mathrm{CI} \text { ) }\end{array}$ & $5.44[-2.12,13.00]$ \\
\hline 2.2 After usual care & 3 & 178 & $\begin{array}{l}\text { Mean Difference (IV, Random, } \\
95 \% \mathrm{Cl} \text { ) }\end{array}$ & $1.99[-2.32,6.29]$ \\
\hline
\end{tabular}




\begin{tabular}{|c|c|c|c|c|}
\hline Outcome or subgroup title & $\begin{array}{l}\text { No. of } \\
\text { studies }\end{array}$ & $\begin{array}{l}\text { No. of } \\
\text { partici- } \\
\text { pants }\end{array}$ & Statistical method & Effect size \\
\hline 3 Disability - Lawton IADL & 2 & 113 & $\begin{array}{l}\text { Mean Difference (IV, Random, } \\
95 \% \mathrm{CI})\end{array}$ & $0.83[-0.51,2.17]$ \\
\hline 3.1 During usual care & 0 & 0 & $\begin{array}{l}\text { Mean Difference (IV, Random, } \\
95 \% \mathrm{CI})\end{array}$ & $0.0[0.0,0.0]$ \\
\hline 3.2 After usual care & 2 & 113 & $\begin{array}{l}\text { Mean Difference (IV, Random, } \\
95 \% \mathrm{CI})\end{array}$ & $0.83[-0.51,2.17]$ \\
\hline $\begin{array}{l}4 \text { Disability - Rivermead Mobility Index } \\
\text { (RMI) }\end{array}$ & 3 & 348 & $\begin{array}{l}\text { Mean Difference (IV, Random, } \\
95 \% \mathrm{CI})\end{array}$ & $0.41[-0.02,0.84]$ \\
\hline 4.1 During usual care & 0 & 0 & $\begin{array}{l}\text { Mean Difference (IV, Random, } \\
95 \% \mathrm{CI})\end{array}$ & $0.0[0.0,0.0]$ \\
\hline 4.2 After usual care & 3 & 348 & $\begin{array}{l}\text { Mean Difference (IV, Random, } \\
95 \% \mathrm{CI})\end{array}$ & $0.41[-0.02,0.84]$ \\
\hline 5 Disability - combined disability scales & 9 & 604 & $\begin{array}{l}\text { Std. Mean Difference (IV, Ran- } \\
\text { dom, } 95 \% \mathrm{CI} \text { ) }\end{array}$ & $0.23[0.03,0.42]$ \\
\hline 5.1 During usual care & 3 & 78 & $\begin{array}{l}\text { Std. Mean Difference (IV, Ran- } \\
\text { dom, 95\% CI) }\end{array}$ & $0.39[-0.06,0.84]$ \\
\hline 5.2 After usual care & 6 & 526 & $\begin{array}{l}\text { Std. Mean Difference (IV, Ran- } \\
\text { dom, 95\% CI) }\end{array}$ & $0.17[-0.09,0.43]$ \\
\hline 6 Risk factors - blood pressure, systolic & 2 & 68 & $\begin{array}{l}\text { Mean Difference (IV, Random, } \\
95 \% \mathrm{CI})\end{array}$ & $4.98[-2.70,12.66]$ \\
\hline 6.1 During usual care & 0 & 0 & $\begin{array}{l}\text { Mean Difference (IV, Random, } \\
95 \% \mathrm{CI})\end{array}$ & $0.0[0.0,0.0]$ \\
\hline 6.2 After usual care & 2 & 68 & $\begin{array}{l}\text { Mean Difference (IV, Random, } \\
95 \% \mathrm{CI} \text { ) }\end{array}$ & $4.98[-2.70,12.66]$ \\
\hline 7 Risk factors - blood pressure, diastolic & 2 & 68 & $\begin{array}{l}\text { Mean Difference (IV, Random, } \\
95 \% \mathrm{CI})\end{array}$ & $-3.49[-9.51,2.53]$ \\
\hline 7.1 During usual care & 0 & 0 & $\begin{array}{l}\text { Mean Difference (IV, Random, } \\
95 \% \mathrm{CI})\end{array}$ & $0.0[0.0,0.0]$ \\
\hline 7.2 After usual care & 2 & 68 & $\begin{array}{l}\text { Mean Difference (IV, Random, } \\
95 \% \mathrm{CI} \text { ) }\end{array}$ & $-3.49[-9.51,2.53]$ \\
\hline 8 Physical fitness - peak $\mathrm{VO}_{2}(\mathrm{~mL} / \mathrm{kg} / \mathrm{min})$ & 2 & 140 & $\begin{array}{l}\text { Mean Difference (IV, Random, } \\
95 \% \mathrm{CI})\end{array}$ & $1.40[-0.19,2.99]$ \\
\hline 8.1 During usual care & 0 & 0 & $\begin{array}{l}\text { Mean Difference (IV, Random, } \\
95 \% \mathrm{CI})\end{array}$ & $0.0[0.0,0.0]$ \\
\hline 8.2 After usual care & 2 & 140 & $\begin{array}{l}\text { Mean Difference (IV, Random, } \\
95 \% \mathrm{CI})\end{array}$ & $1.40[-0.19,2.99]$ \\
\hline
\end{tabular}




\begin{tabular}{|c|c|c|c|c|}
\hline Outcome or subgroup title & $\begin{array}{l}\text { No. of } \\
\text { studies }\end{array}$ & $\begin{array}{l}\text { No. of } \\
\text { partici- } \\
\text { pants }\end{array}$ & Statistical method & Effect size \\
\hline $\begin{array}{l}9 \text { Physical fitness - gait economy, } \mathrm{VO}_{2}(\mathrm{~mL} / \\
\mathrm{kg} / \text { metre) }\end{array}$ & 1 & 66 & $\begin{array}{l}\text { Mean Difference (IV, Random, } \\
95 \% \mathrm{Cl} \text { ) }\end{array}$ & $-0.01[-0.03,-0.00]$ \\
\hline 9.1 During usual care & 0 & 0 & $\begin{array}{l}\text { Mean Difference (IV, Random, } \\
95 \% \mathrm{CI} \text { ) }\end{array}$ & $0.0[0.0,0.0]$ \\
\hline 9.2 After usual care & 1 & 66 & $\begin{array}{l}\text { Mean Difference (IV, Random, } \\
95 \% \mathrm{CI} \text { ) }\end{array}$ & $-0.01[-0.03,-0.00]$ \\
\hline $\begin{array}{l}10 \text { Physical fitness - muscle strength, ankle } \\
\text { dorsiflexion* }\end{array}$ & 2 & 148 & $\begin{array}{l}\text { Std. Mean Difference (IV, Ran- } \\
\text { dom, } 95 \% \mathrm{CI} \text { ) }\end{array}$ & $0.80[-0.82,2.41]$ \\
\hline 10.1 During usual care & 0 & 0 & $\begin{array}{l}\text { Std. Mean Difference (IV, Ran- } \\
\text { dom, } 95 \% \mathrm{CI} \text { ) }\end{array}$ & $0.0[0.0,0.0]$ \\
\hline 10.2 After usual care & 2 & 148 & $\begin{array}{l}\text { Std. Mean Difference (IV, Ran- } \\
\text { dom, } 95 \% \mathrm{CI} \text { ) }\end{array}$ & $0.80[-0.82,2.41]$ \\
\hline $\begin{array}{l}11 \text { Physical fitness - muscle strength, knee } \\
\text { extension* }\end{array}$ & 3 & 202 & $\begin{array}{l}\text { Std. Mean Difference (IV, Ran- } \\
\text { dom, } 95 \% \mathrm{Cl} \text { ) }\end{array}$ & $0.33[0.05,0.61]$ \\
\hline 11.1 During usual care & 1 & 54 & $\begin{array}{l}\text { Std. Mean Difference (IV, Ran- } \\
\text { dom, } 95 \% \mathrm{CI} \text { ) }\end{array}$ & $0.29[-0.25,0.83]$ \\
\hline 11.2 After usual care & 2 & 148 & $\begin{array}{l}\text { Std. Mean Difference (IV, Ran- } \\
\text { dom, } 95 \% \mathrm{CI} \text { ) }\end{array}$ & $0.36[-0.02,0.73]$ \\
\hline $\begin{array}{l}12 \text { Physical fitness - muscle strength, grip } \\
\text { strength (paretic hand) }\end{array}$ & 3 & 147 & $\begin{array}{l}\text { Mean Difference (IV, Random, } \\
95 \% \mathrm{CI})\end{array}$ & $0.32[-0.88,1.52]$ \\
\hline 12.1 During usual care & 2 & 47 & $\begin{array}{l}\text { Mean Difference (IV, Random, } \\
95 \% \mathrm{CI} \text { ) }\end{array}$ & $0.32[-1.12,1.76]$ \\
\hline 12.2 After usual care & 1 & 100 & $\begin{array}{l}\text { Mean Difference (IV, Random, } \\
95 \% \mathrm{Cl} \text { ) }\end{array}$ & $0.32[-1.85,2.49]$ \\
\hline 13 Mobility - walking maximum speed & 3 & 168 & $\begin{array}{l}\text { Mean Difference (IV, Random, } \\
95 \% \mathrm{CI} \text { ) }\end{array}$ & $8.48[1.76,15.20]$ \\
\hline 13.1 During usual care & 1 & 29 & $\begin{array}{l}\text { Mean Difference (IV, Random, } \\
95 \% \mathrm{CI} \text { ) }\end{array}$ & $8.00[-5.06,21.06]$ \\
\hline 13.2 After usual care & 2 & 139 & $\begin{array}{l}\text { Mean Difference (IV, Random, } \\
95 \% \mathrm{CI} \text { ) }\end{array}$ & $8.65[0.82,16.48]$ \\
\hline $\begin{array}{l}14 \text { Mobility - walking preferred speed (m/ } \\
\text { min) }\end{array}$ & 10 & 738 & $\begin{array}{l}\text { Mean Difference (IV, Random, } \\
95 \% \mathrm{CI} \text { ) }\end{array}$ & $4.71[1.32,8.10]$ \\
\hline 14.1 During usual care & 3 & 153 & $\begin{array}{l}\text { Mean Difference (IV, Random, } \\
95 \% \mathrm{CI} \text { ) }\end{array}$ & $3.37[-2.63,9.37]$ \\
\hline 14.2 After usual care & 7 & 585 & $\begin{array}{l}\text { Mean Difference (IV, Random, } \\
95 \% \mathrm{CI} \text { ) }\end{array}$ & $5.13[1.16,9.10]$ \\
\hline
\end{tabular}




\begin{tabular}{|c|c|c|c|c|}
\hline Outcome or subgroup title & $\begin{array}{l}\text { No. of } \\
\text { studies }\end{array}$ & $\begin{array}{l}\text { No. of } \\
\text { partici- } \\
\text { pants }\end{array}$ & Statistical method & Effect size \\
\hline $\begin{array}{l}15 \text { Mobility - walking capacity (6-MWT me- } \\
\text { tres) }\end{array}$ & 10 & 720 & $\begin{array}{l}\text { Mean Difference (IV, Random, } \\
95 \% \mathrm{Cl} \text { ) }\end{array}$ & $35.00[15.91,54.09]$ \\
\hline 15.1 During usual care & 2 & 60 & $\begin{array}{l}\text { Mean Difference (IV, Random, } \\
95 \% \mathrm{CI} \text { ) }\end{array}$ & $13.21[-75.07,101.49]$ \\
\hline 15.2 After usual care & 8 & 660 & $\begin{array}{l}\text { Mean Difference (IV, Random, } \\
95 \% \mathrm{CI} \text { ) }\end{array}$ & $40.37[24.82,55.92]$ \\
\hline $\begin{array}{l}16 \text { Mobility - Community Ambulation } \\
\text { Speed }(>0.8 \mathrm{~m} / \mathrm{sec})\end{array}$ & 3 & 232 & $\begin{array}{l}\text { Odds Ratio (M-H, Random, 95\% } \\
\mathrm{Cl} \text { ) }\end{array}$ & $1.38[0.78,2.42]$ \\
\hline 16.1 During usual care & 1 & 67 & $\begin{array}{l}\text { Odds Ratio (M-H, Random, 95\% } \\
\mathrm{Cl} \text { ) }\end{array}$ & $1.75[0.46,6.65]$ \\
\hline 16.2 After usual care & 2 & 165 & $\begin{array}{l}\text { Odds Ratio (M-H, Random, 95\% } \\
\mathrm{Cl} \text { ) }\end{array}$ & $1.31[0.70,2.44]$ \\
\hline $\begin{array}{l}17 \text { Physical function - balance - Berg Bal- } \\
\text { ance scale }\end{array}$ & 9 & 419 & $\begin{array}{l}\text { Mean Difference (IV, Random, } \\
95 \% \mathrm{CI} \text { ) }\end{array}$ & $2.12[0.82,3.41]$ \\
\hline 17.1 During usual care & 5 & 160 & $\begin{array}{l}\text { Mean Difference (IV, Random, } \\
95 \% \mathrm{CI} \text { ) }\end{array}$ & $0.50[-3.00,4.01]$ \\
\hline 17.2 After usual care & 4 & 259 & $\begin{array}{l}\text { Mean Difference (IV, Random, } \\
95 \% \mathrm{CI})\end{array}$ & $2.64[1.34,3.95]$ \\
\hline $\begin{array}{l}18 \text { Physical function - balance - functional } \\
\text { reach }\end{array}$ & 2 & 166 & $\begin{array}{l}\text { Std. Mean Difference (IV, Ran- } \\
\text { dom, } 95 \% \mathrm{CI} \text { ) }\end{array}$ & $0.14[-0.22,0.50]$ \\
\hline 18.1 During usual care & 0 & 0 & $\begin{array}{l}\text { Std. Mean Difference (IV, Ran- } \\
\text { dom, } 95 \% \mathrm{CI} \text { ) }\end{array}$ & $0.0[0.0,0.0]$ \\
\hline 18.2 After usual care & 2 & 166 & $\begin{array}{l}\text { Std. Mean Difference (IV, Ran- } \\
\text { dom, } 95 \% \mathrm{CI} \text { ) }\end{array}$ & $0.14[-0.22,0.50]$ \\
\hline $\begin{array}{l}19 \text { Physical function - balance - combined } \\
\text { outcome data }\end{array}$ & 12 & 755 & $\begin{array}{l}\text { Std. Mean Difference (IV, Ran- } \\
\text { dom, } 95 \% \mathrm{CI} \text { ) }\end{array}$ & $0.28[0.11,0.45]$ \\
\hline 19.1 During usual care & 5 & 160 & $\begin{array}{l}\text { Std. Mean Difference (IV, Ran- } \\
\text { dom, } 95 \% \mathrm{CI} \text { ) }\end{array}$ & $0.10[-0.23,0.43]$ \\
\hline 19.2 After usual care & 7 & 595 & $\begin{array}{l}\text { Std. Mean Difference (IV, Ran- } \\
\text { dom, } 95 \% \mathrm{CI} \text { ) }\end{array}$ & $0.35[0.15,0.54]$ \\
\hline $\begin{array}{l}20 \text { Physical function - Timed Up and Go } \\
\text { (sec) }\end{array}$ & 7 & 586 & $\begin{array}{l}\text { Mean Difference (IV, Random, } \\
95 \% \mathrm{CI})\end{array}$ & $-2.21[-4.43,0.02]$ \\
\hline 20.1 During usual care & 2 & 91 & $\begin{array}{l}\text { Mean Difference (IV, Random, } \\
95 \% \mathrm{CI} \text { ) }\end{array}$ & $-8.17[-20.66,4.33]$ \\
\hline 20.2 After usual care & 5 & 495 & $\begin{array}{l}\text { Mean Difference (IV, Random, } \\
95 \% \mathrm{CI})\end{array}$ & $-1.45[-2.66,-0.24]$ \\
\hline
\end{tabular}




\begin{tabular}{|c|c|c|c|c|}
\hline Outcome or subgroup title & $\begin{array}{l}\text { No. of } \\
\text { studies }\end{array}$ & $\begin{array}{l}\text { No. of } \\
\text { partici- } \\
\text { pants }\end{array}$ & Statistical method & Effect size \\
\hline $\begin{array}{l}21 \text { Health-related QoL - SF-36 physical } \\
\text { functioning }\end{array}$ & 2 & 112 & $\begin{array}{l}\text { Std. Mean Difference (IV, Ran- } \\
\text { dom, } 95 \% \mathrm{CI} \text { ) }\end{array}$ & $0.48[0.10,0.85]$ \\
\hline 21.1 During usual care & 0 & 0 & $\begin{array}{l}\text { Std. Mean Difference (IV, Ran- } \\
\text { dom, } 95 \% \mathrm{CI} \text { ) }\end{array}$ & $0.0[0.0,0.0]$ \\
\hline 21.2 After usual care & 2 & 112 & $\begin{array}{l}\text { Std. Mean Difference (IV, Ran- } \\
\text { dom, } 95 \% \mathrm{CI} \text { ) }\end{array}$ & $0.48[0.10,0.85]$ \\
\hline $\begin{array}{l}22 \text { Health-related QoL - SF-36 physical role } \\
\text { functioning }\end{array}$ & 3 & 178 & $\begin{array}{l}\text { Std. Mean Difference (IV, Ran- } \\
\text { dom, } 95 \% \mathrm{CI} \text { ) }\end{array}$ & $0.56[0.26,0.86]$ \\
\hline 22.1 During usual care & 0 & 0 & $\begin{array}{l}\text { Std. Mean Difference (IV, Ran- } \\
\text { dom, } 95 \% \mathrm{CI} \text { ) }\end{array}$ & $0.0[0.0,0.0]$ \\
\hline 22.2 After usual care & 3 & 178 & $\begin{array}{l}\text { Std. Mean Difference (IV, Ran- } \\
\text { dom, } 95 \% \mathrm{CI} \text { ) }\end{array}$ & $0.56[0.26,0.86]$ \\
\hline $\begin{array}{l}23 \text { Health-related QoL - SF-36 social role } \\
\text { functioning }\end{array}$ & 2 & 112 & $\begin{array}{l}\text { Std. Mean Difference (IV, Ran- } \\
\text { dom, } 95 \% \mathrm{Cl} \text { ) }\end{array}$ & $0.48[-0.22,1.17]$ \\
\hline 23.1 During usual care & 0 & 0 & $\begin{array}{l}\text { Std. Mean Difference (IV, Ran- } \\
\text { dom, } 95 \% \mathrm{CI} \text { ) }\end{array}$ & $0.0[0.0,0.0]$ \\
\hline 23.2 After usual care & 2 & 112 & $\begin{array}{l}\text { Std. Mean Difference (IV, Ran- } \\
\text { dom, } 95 \% \mathrm{CI} \text { ) }\end{array}$ & $0.48[-0.22,1.17]$ \\
\hline $\begin{array}{l}24 \text { Mood - Hospital Anxiety and Depression } \\
\text { Scale (HADS) - anxiety score }\end{array}$ & 3 & 391 & $\begin{array}{l}\text { Mean Difference (IV, Random, } \\
95 \% \mathrm{CI})\end{array}$ & $-0.28[-0.95,0.40]$ \\
\hline 24.1 During usual care & 0 & 0 & $\begin{array}{l}\text { Mean Difference (IV, Random, } \\
95 \% \mathrm{CI} \text { ) }\end{array}$ & $0.0[0.0,0.0]$ \\
\hline 24.2 After usual care & 3 & 391 & $\begin{array}{l}\text { Mean Difference (IV, Random, } \\
95 \% \mathrm{CI})\end{array}$ & $-0.28[-0.95,0.40]$ \\
\hline $\begin{array}{l}25 \text { Mood - Hospital Anxiety and Depression } \\
\text { Scale (HADS) - depression score }\end{array}$ & 3 & 391 & $\begin{array}{l}\text { Mean Difference (IV, Random, } \\
95 \% \mathrm{CI})\end{array}$ & $0.59[-0.08,1.26]$ \\
\hline 25.1 During usual care & 0 & 0 & $\begin{array}{l}\text { Mean Difference (IV, Random, } \\
95 \% \mathrm{CI})\end{array}$ & $0.0[0.0,0.0]$ \\
\hline 25.2 After usual care & 3 & 391 & $\begin{array}{l}\text { Mean Difference (IV, Random, } \\
95 \% \mathrm{CI} \text { ) }\end{array}$ & $0.59[-0.08,1.26]$ \\
\hline $\begin{array}{l}26 \text { Mood - Stroke Impact Scale emotion } \\
\text { score }\end{array}$ & 2 & 335 & $\begin{array}{l}\text { Mean Difference (IV, Random, } \\
95 \% \mathrm{CI} \text { ) }\end{array}$ & $2.87[-3.40,9.14]$ \\
\hline 26.1 During usual care & 0 & 0 & $\begin{array}{l}\text { Mean Difference (IV, Random, } \\
95 \% \mathrm{CI} \text { ) }\end{array}$ & $0.0[0.0,0.0]$ \\
\hline 26.2 After usual care & 2 & 335 & $\begin{array}{l}\text { Mean Difference (IV, Random, } \\
95 \% \mathrm{CI} \text { ) }\end{array}$ & $2.87[-3.40,9.14]$ \\
\hline
\end{tabular}




\begin{tabular}{|c|c|c|c|c|}
\hline Outcome or subgroup title & $\begin{array}{l}\text { No. of } \\
\text { studies }\end{array}$ & $\begin{array}{l}\text { No. of } \\
\text { partici- } \\
\text { pants }\end{array}$ & Statistical method & Effect size \\
\hline 27 Mood - combined depression scales & 4 & 484 & $\begin{array}{l}\text { Std. Mean Difference (IV, Ran- } \\
\text { dom, } 95 \% \mathrm{CI} \text { ) }\end{array}$ & $-0.01[-0.39,0.37]$ \\
\hline 27.1 During usual care & 0 & 0 & $\begin{array}{l}\text { Std. Mean Difference (IV, Ran- } \\
\text { dom, } 95 \% \mathrm{CI} \text { ) }\end{array}$ & $0.0[0.0,0.0]$ \\
\hline 27.2 After usual care & 4 & 484 & $\begin{array}{l}\text { Std. Mean Difference (IV, Ran- } \\
\text { dom, } 95 \% \mathrm{Cl} \text { ) }\end{array}$ & $-0.01[-0.39,0.37]$ \\
\hline 28 Cognitive function - FIM cognitive score & 2 & 159 & $\begin{array}{l}\text { Mean Difference (IV, Random, } \\
95 \% \mathrm{Cl} \text { ) }\end{array}$ & $-0.08[-0.47,0.31]$ \\
\hline 28.1 During usual care & 0 & 0 & $\begin{array}{l}\text { Mean Difference (IV, Random, } \\
95 \% \mathrm{Cl} \text { ) }\end{array}$ & $0.0[0.0,0.0]$ \\
\hline 28.2 After usual care & 2 & 159 & $\begin{array}{l}\text { Mean Difference (IV, Random, } \\
95 \% \mathrm{Cl} \text { ) }\end{array}$ & $-0.08[-0.47,0.31]$ \\
\hline $\begin{array}{l}29 \text { Cognitive function - SIS memory and } \\
\text { thinking }\end{array}$ & 2 & 133 & $\begin{array}{l}\text { Mean Difference (IV, Random, } \\
95 \% \mathrm{Cl} \text { ) }\end{array}$ & $1.57[-10.56,13.70]$ \\
\hline 29.1 During usual care & 0 & 0 & $\begin{array}{l}\text { Mean Difference (IV, Random, } \\
95 \% \mathrm{CI} \text { ) }\end{array}$ & $0.0[0.0,0.0]$ \\
\hline 29.2 After usual care & 2 & 133 & $\begin{array}{l}\text { Mean Difference (IV, Random, } \\
95 \% \mathrm{CI} \text { ) }\end{array}$ & $1.57[-10.56,13.70]$ \\
\hline 30 Cognitive function - SIS communication & 2 & 133 & $\begin{array}{l}\text { Mean Difference (IV, Random, } \\
95 \% \mathrm{CI})\end{array}$ & $-1.19[-12.06,9.67]$ \\
\hline 30.1 During usual care & 0 & 0 & $\begin{array}{l}\text { Mean Difference (IV, Random, } \\
95 \% \mathrm{Cl} \text { ) }\end{array}$ & $0.0[0.0,0.0]$ \\
\hline 30.2 After usual care & 2 & 133 & $\begin{array}{l}\text { Mean Difference (IV, Random, } \\
95 \% \mathrm{CI})\end{array}$ & $-1.19[-12.06,9.67]$ \\
\hline
\end{tabular}

Analysis 5.1. Comparison 5 Mixed training versus control - end of intervention, Outcome 1 Death.

\begin{tabular}{|c|c|c|c|c|c|}
\hline Study or subgroup & $\begin{array}{c}\text { Training } \\
\mathrm{n} / \mathrm{N}\end{array}$ & $\begin{array}{c}\text { Control } \\
n / N\end{array}$ & $\begin{array}{c}\text { Risk Difference } \\
\text { M-H, Random, 95\% Cl }\end{array}$ & Weight & $\begin{array}{c}\text { Risk Difference } \\
\text { M-H, Random, 95\% Cl }\end{array}$ \\
\hline \multicolumn{6}{|c|}{ 5.1.1 During usual care } \\
\hline Cooke 2010 & $0 / 36$ & $0 / 38$ & $\longrightarrow$ & $7.97 \%$ & $0[-0.05,0.05]$ \\
\hline Donaldson 2009 & $0 / 10$ & $0 / 10$ & & $0.69 \%$ & $0[-0.17,0.17]$ \\
\hline Furnari 2014 & $0 / 20$ & $0 / 20$ & & $2.46 \%$ & $0[-0.09,0.09]$ \\
\hline Galvin 2011 & $0 / 20$ & $0 / 20$ & & $2.46 \%$ & $0[-0.09,0.09]$ \\
\hline Kim 2017a & $0 / 15$ & $0 / 15$ & & $1.44 \%$ & $0[-0.12,0.12]$ \\
\hline Letombe 2010 & $0 / 9$ & $0 / 9$ & & $0.57 \%$ & $0[-0.19,0.19]$ \\
\hline Richards 1993 & $0 / 10$ & $0 / 8$ & & $0.56 \%$ & $0[-0.19,0.19]$ \\
\hline
\end{tabular}




\begin{tabular}{|c|c|c|c|c|c|}
\hline Study or subgroup & $\begin{array}{l}\text { Training } \\
\mathbf{n} / \mathbf{N}\end{array}$ & $\begin{array}{c}\text { Control } \\
n / N\end{array}$ & $\begin{array}{c}\text { Risk Difference } \\
\text { M-H, Random, } 95 \% \mathrm{Cl}\end{array}$ & Weight & $\begin{array}{c}\text { Risk Difference } \\
\text { M-H, Random, } 95 \% \mathrm{Cl}\end{array}$ \\
\hline Richards 2004 & $0 / 32$ & $0 / 31$ & $\longrightarrow$ & $5.85 \%$ & $0[-0.06,0.06]$ \\
\hline Shin 2011 & $0 / 11$ & $0 / 10$ & & $0.75 \%$ & $0[-0.17,0.17]$ \\
\hline Subtotal $(95 \% \mathrm{Cl})$ & 173 & 171 & & $23.45 \%$ & $0[-0.03,0.03]$ \\
\hline \multicolumn{6}{|c|}{ Total events: 0 (Training), 0 (Control) } \\
\hline \multicolumn{6}{|c|}{ Heterogeneity: Tau $^{2}=0 ; \mathrm{Chi}^{2}=0, \mathrm{df}=9(\mathrm{P}=1) ; \mathrm{I}^{2}=0 \%$} \\
\hline \multicolumn{6}{|c|}{ Test for overall effect: Not applicable } \\
\hline \multicolumn{6}{|l|}{ 5.1.2 After usual care } \\
\hline Dean 2018 & $0 / 23$ & $0 / 22$ & 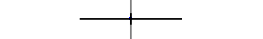 & $3.07 \%$ & $0[-0.08,0.08]$ \\
\hline Duncan 1998 & $0 / 50$ & $0 / 50$ & + & $14.33 \%$ & $0[-0.04,0.04]$ \\
\hline Duncan 2003 & $0 / 10$ & $0 / 10$ & & $0.69 \%$ & $0[-0.17,0.17]$ \\
\hline James 2002 & $0 / 10$ & $0 / 10$ & & $0.69 \%$ & $0[-0.17,0.17]$ \\
\hline Knox 2018 & $3 / 51$ & $1 / 48$ & 1 & $3.61 \%$ & $0.04[-0.04,0.11]$ \\
\hline Langhammer 2007 & $1 / 35$ & $6 / 40$ & & $1.37 \%$ & $-0.12[-0.25,0]$ \\
\hline Mead 2007 & $0 / 32$ & $0 / 34$ & $\longleftarrow$ & $6.39 \%$ & $0[-0.06,0.06]$ \\
\hline Moore 2015 & $0 / 20$ & $0 / 20$ & - & $2.46 \%$ & $0[-0.09,0.09]$ \\
\hline Teixeira 1999 & $0 / 6$ & $0 / 7$ & & $0.32 \%$ & $0[-0.25,0.25]$ \\
\hline Toledano-Zarhi 2011 & $0 / 14$ & $0 / 14$ & & $1.27 \%$ & $0[-0.13,0.13]$ \\
\hline Van de Port 2012 & $0 / 126$ & $2 / 124$ & $\rightarrow$ & $29.07 \%$ & $-0.02[-0.04,0.01]$ \\
\hline Yang 2006 & $0 / 24$ & $0 / 24$ & - & $3.48 \%$ & $0[-0.08,0.08]$ \\
\hline Zedlitz 2012 & $0 / 38$ & $0 / 45$ & $\longrightarrow$ & $9.77 \%$ & $0[-0.05,0.05]$ \\
\hline Subtotal $(95 \% \mathrm{Cl})$ & 439 & 448 & & $76.55 \%$ & $-0.01[-0.02,0.01]$ \\
\hline \multicolumn{6}{|c|}{ Total events: 4 (Training), 9 (Control) } \\
\hline \multicolumn{6}{|c|}{ Heterogeneity: $\mathrm{Tau}^{2}=0 ; \mathrm{Chi}^{2}=5.68, \mathrm{df}=12(\mathrm{P}=0.93) ; \mathrm{I}^{2}=0 \%$} \\
\hline \multicolumn{6}{|c|}{ Test for overall effect: $Z=0.77(P=0.44)$} \\
\hline Total $(95 \% \mathrm{Cl})$ & 612 & 619 & 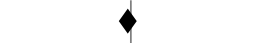 & $100 \%$ & $-0[-0.02,0.01]$ \\
\hline \multicolumn{6}{|c|}{ Total events: 4 (Training), 9 (Control) } \\
\hline \multicolumn{6}{|c|}{ Heterogeneity: $\mathrm{Tau}^{2}=0 ; \mathrm{Chi}^{2}=5.72, \mathrm{df}=22(\mathrm{P}=1) ; \mathrm{I}^{2}=0 \%$} \\
\hline \multicolumn{6}{|c|}{ Test for overall effect: $Z=0.67(P=0.5)$} \\
\hline Test for subgroup diff & $d f=1(P=0.71)$, & & & & \\
\hline
\end{tabular}

Favours training

Analysis 5.2. Comparison 5 Mixed training versus control end of intervention, Outcome 2 Disability - Barthel Index (BI).

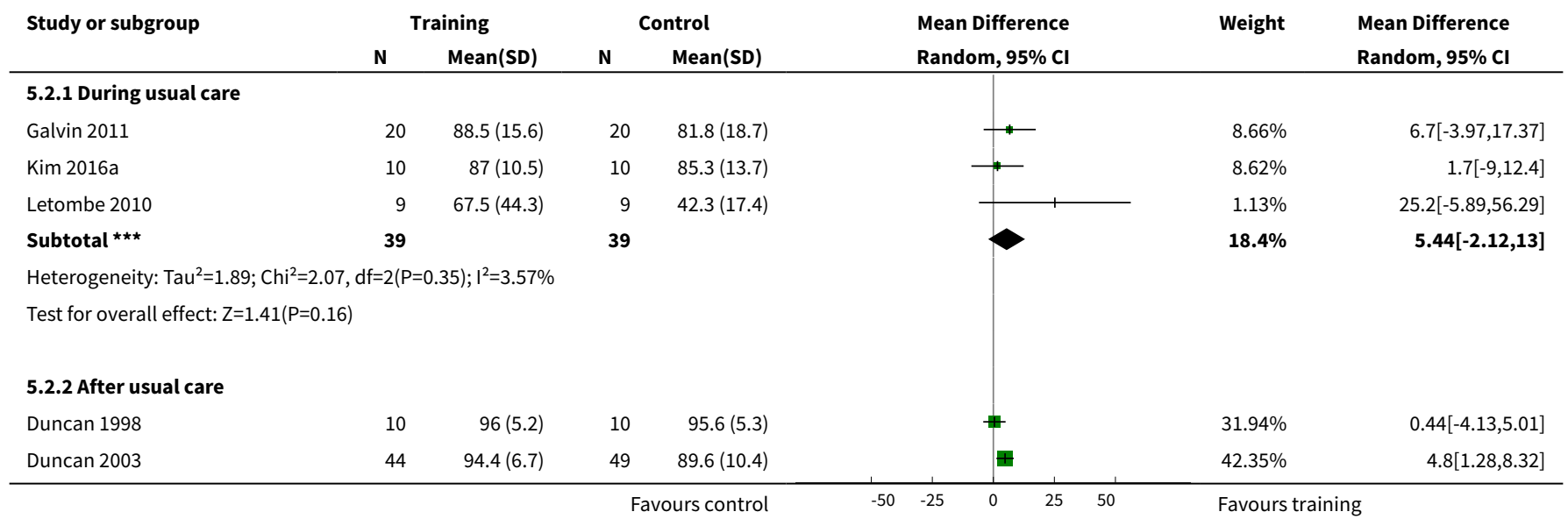




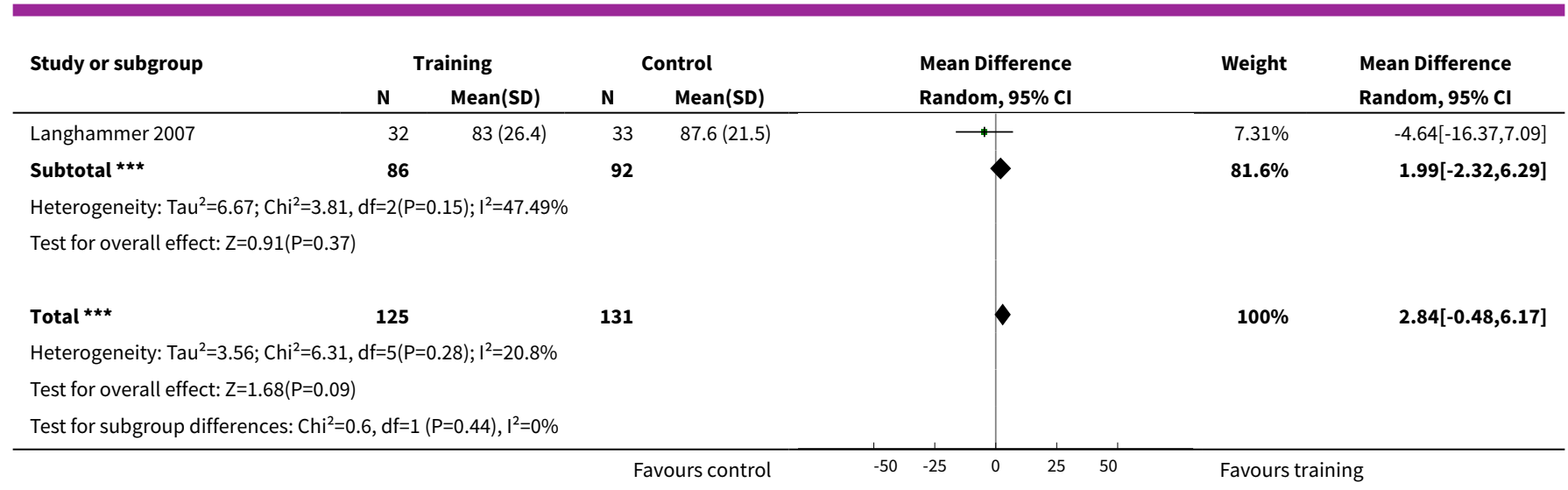

Analysis 5.3. Comparison 5 Mixed training versus control - end of intervention, Outcome 3 Disability - Lawton IADL.

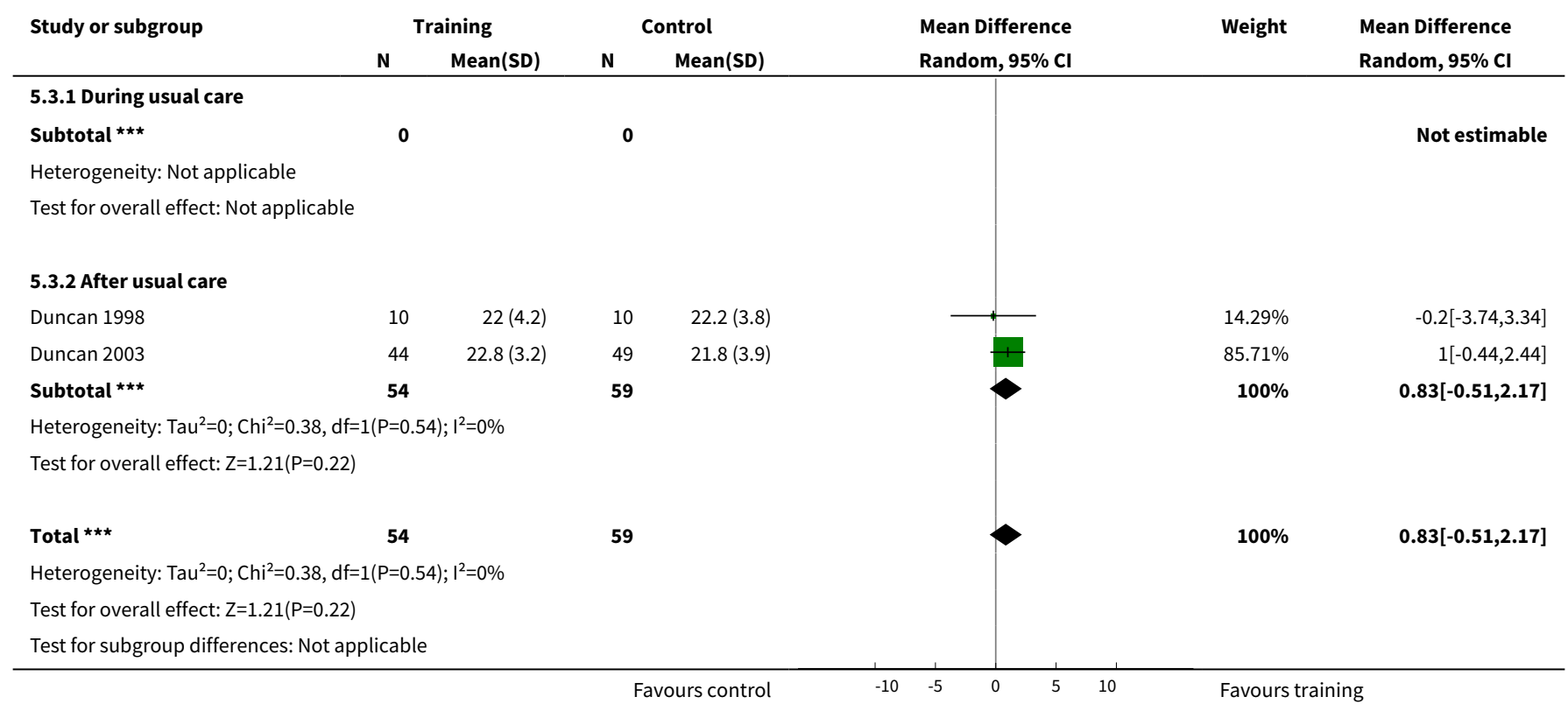

Analysis 5.4. Comparison 5 Mixed training versus control - end of intervention, Outcome 4 Disability - Rivermead Mobility Index (RMI).

\begin{tabular}{|c|c|c|c|c|c|c|c|}
\hline \multirow[t]{2}{*}{ Study or subgroup } & \multicolumn{2}{|c|}{ Training } & \multicolumn{2}{|c|}{ Control } & \multirow{2}{*}{$\begin{array}{l}\text { Mean Difference } \\
\text { Random, } 95 \% \mathrm{Cl}\end{array}$} & \multirow[t]{2}{*}{ Weight } & \multirow{2}{*}{$\begin{array}{l}\text { Mean Difference } \\
\text { Random, } 95 \% \mathrm{Cl}\end{array}$} \\
\hline & $\mathbf{N}$ & $\operatorname{Mean}(S D)$ & $\mathbf{N}$ & $\operatorname{Mean}(S D)$ & & & \\
\hline \multicolumn{8}{|c|}{ 5.4.1 During usual care } \\
\hline 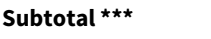 & 0 & & 0 & & & & Not estimable \\
\hline \multicolumn{8}{|c|}{ Heterogeneity: Not applicable } \\
\hline \multicolumn{8}{|c|}{ Test for overall effect: Not applicable } \\
\hline \multicolumn{8}{|c|}{ 5.4.2 After usual care } \\
\hline Dean 2018 & 21 & $12.1(2.7)$ & 19 & $12.5(1.9)$ & $\rightarrow$ & $8.27 \%$ & $-0.33[-1.77,1.11]$ \\
\hline Mead 2007 & 32 & $13.2(1.3)$ & 34 & $13(1.3)$ & \#- & $35.33 \%$ & $0.2[-0.41,0.81]$ \\
\hline Van de Port 2012 & 125 & $13.5(1.4)$ & 117 & $12.8(1.9)$ & + & $56.4 \%$ & $0.65[0.22,1.08]$ \\
\hline
\end{tabular}




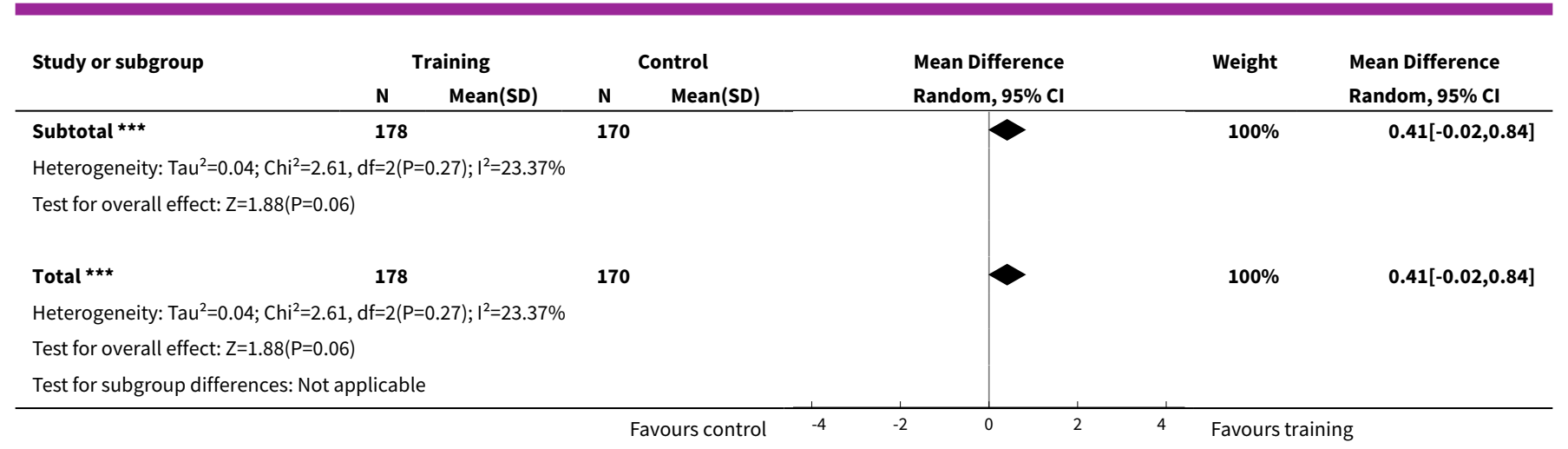

Analysis 5.5. Comparison 5 Mixed training versus control - end of intervention, Outcome 5 Disability - combined disability scales.

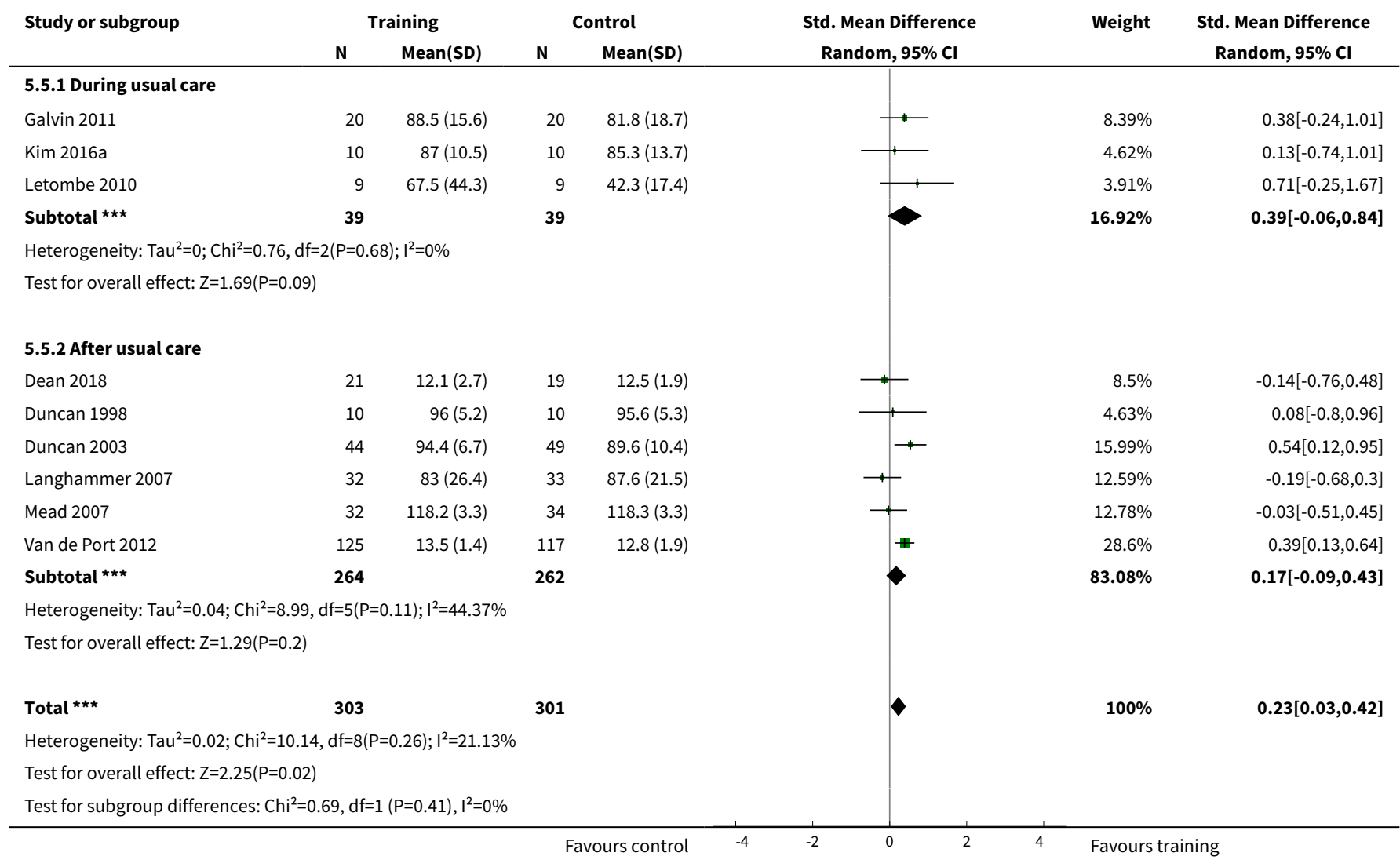

Analysis 5.6. Comparison 5 Mixed training versus control - end of intervention, Outcome 6 Risk factors - blood pressure, systolic.

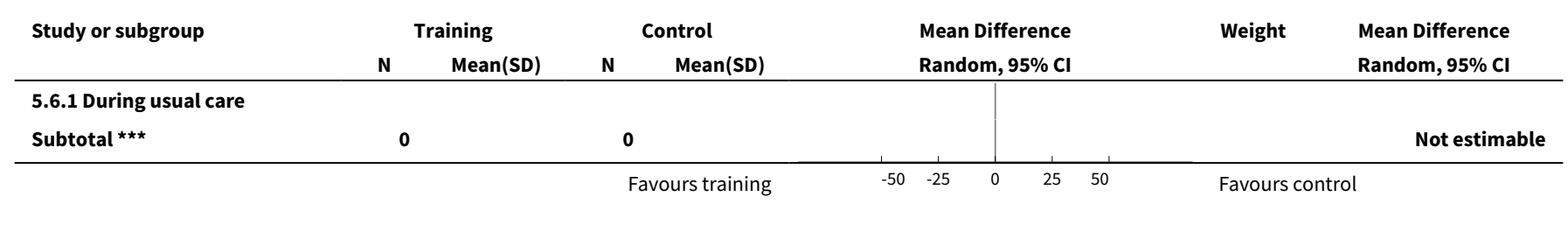




\begin{tabular}{|c|c|c|c|c|c|c|c|}
\hline \multirow[t]{2}{*}{ Study or subgroup } & \multicolumn{2}{|c|}{ Training } & \multicolumn{2}{|c|}{ Control } & \multirow{2}{*}{$\begin{array}{l}\text { Mean Difference } \\
\text { Random, } 95 \% \mathrm{Cl}\end{array}$} & \multirow[t]{2}{*}{ Weight } & \multirow{2}{*}{$\begin{array}{l}\text { Mean Difference } \\
\text { Random, } 95 \% \mathrm{CI}\end{array}$} \\
\hline & $\mathbf{N}$ & Mean(SD) & $\mathbf{N}$ & Mean(SD) & & & \\
\hline \multicolumn{8}{|c|}{ Heterogeneity: Not applicable } \\
\hline \multicolumn{8}{|c|}{ Test for overall effect: Not applicable } \\
\hline \multicolumn{8}{|l|}{ 5.6.2 After usual care } \\
\hline Moore 2015 & 20 & $143(19)$ & 20 & $137(11)$ & & $63.7 \%$ & $6[-3.62,15.62]$ \\
\hline Toledano-Zarhi 2011 & 14 & $131(16.8)$ & 14 & $127.8(17.6)$ & 4 & $36.3 \%$ & $3.2[-9.55,15.95]$ \\
\hline Subtotal $\star \star \star$ & 34 & & 34 & & & $100 \%$ & $4.98[-2.7,12.66]$ \\
\hline \multicolumn{8}{|c|}{ Heterogeneity: $\operatorname{Tau}^{2}=0 ; \mathrm{Chi}^{2}=0.12, \mathrm{df}=1(\mathrm{P}=0.73) ; \mathrm{I}^{2}=0 \%$} \\
\hline \multicolumn{8}{|c|}{ Test for overall effect: $Z=1.27(P=0.2)$} \\
\hline 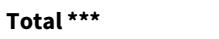 & 34 & & 34 & & & $100 \%$ & $4.98[-2.7,12.66]$ \\
\hline \multicolumn{8}{|c|}{ Heterogeneity: $\mathrm{Tau}^{2}=0 ; \mathrm{Chi}^{2}=0.12, \mathrm{df}=1(\mathrm{P}=0.73) ; \mathrm{I}^{2}=0 \%$} \\
\hline \multicolumn{8}{|c|}{ Test for overall effect: $Z=1.27(P=0.2)$} \\
\hline Test for subgroup diff & olicabl & & & & & & \\
\hline
\end{tabular}

Analysis 5.7. Comparison 5 Mixed training versus control - end of intervention, Outcome 7 Risk factors - blood pressure, diastolic.

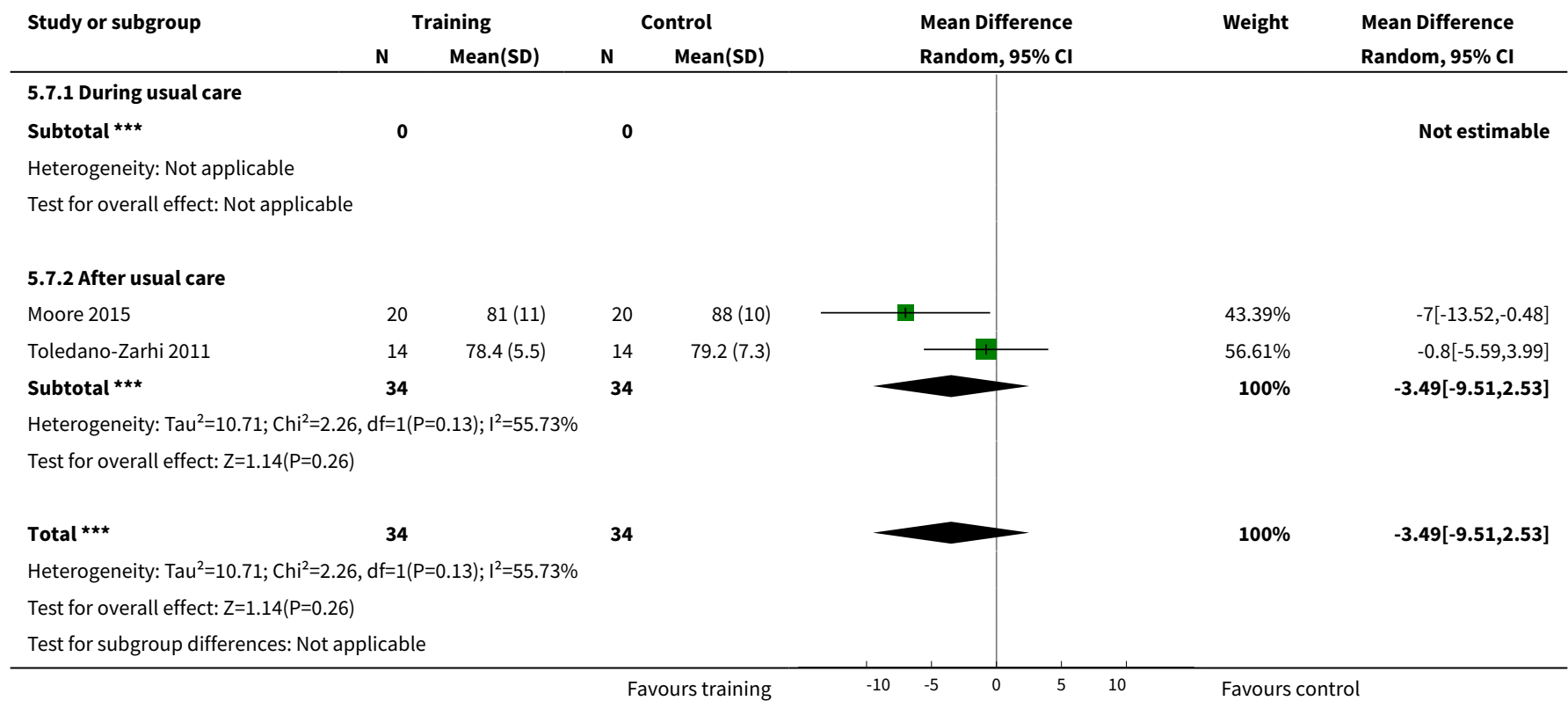

Analysis 5.8. Comparison 5 Mixed training versus control - end of intervention, Outcome 8 Physical fitness - peak $\mathrm{VO}_{2}(\mathrm{~mL} / \mathrm{kg} / \mathrm{min})$.

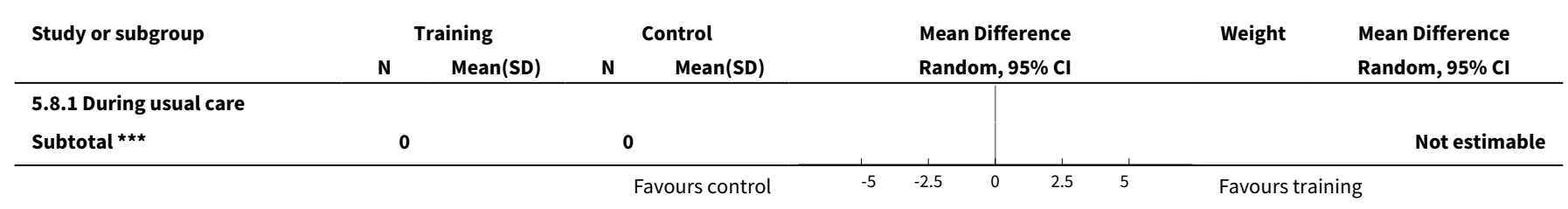




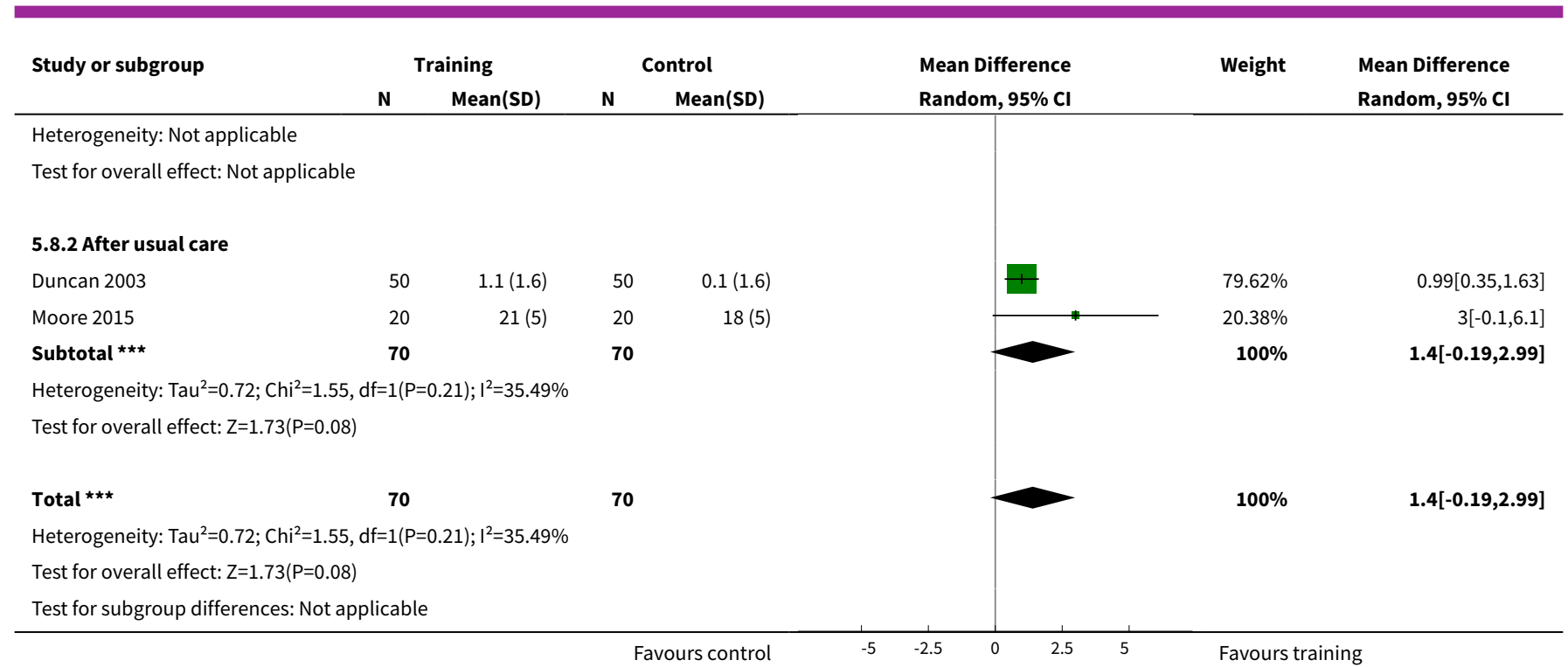

Analysis 5.9. Comparison 5 Mixed training versus control - end of intervention, Outcome 9 Physical fitness - gait economy, $\mathrm{VO}_{2}(\mathrm{~mL} / \mathrm{kg} / \mathrm{metre})$.

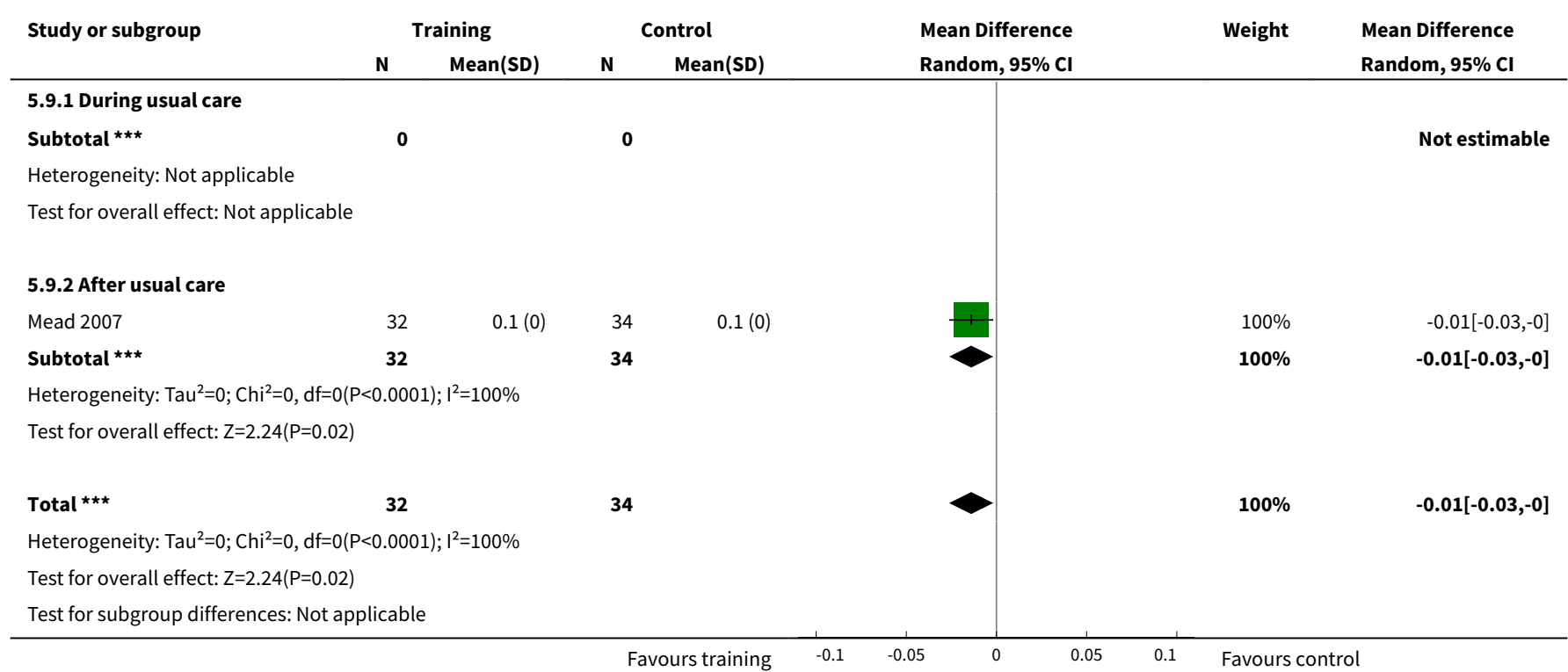

Analysis 5.10. Comparison 5 Mixed training versus control - end of intervention, Outcome 10 Physical fitness - muscle strength, ankle dorsiflexion*.

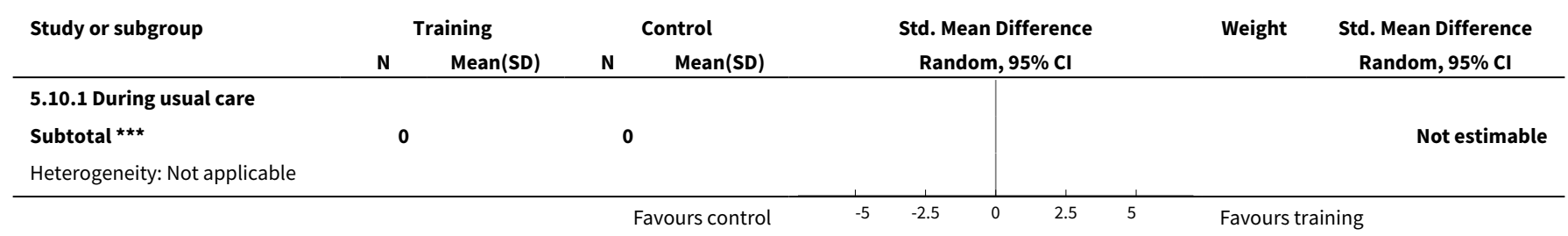




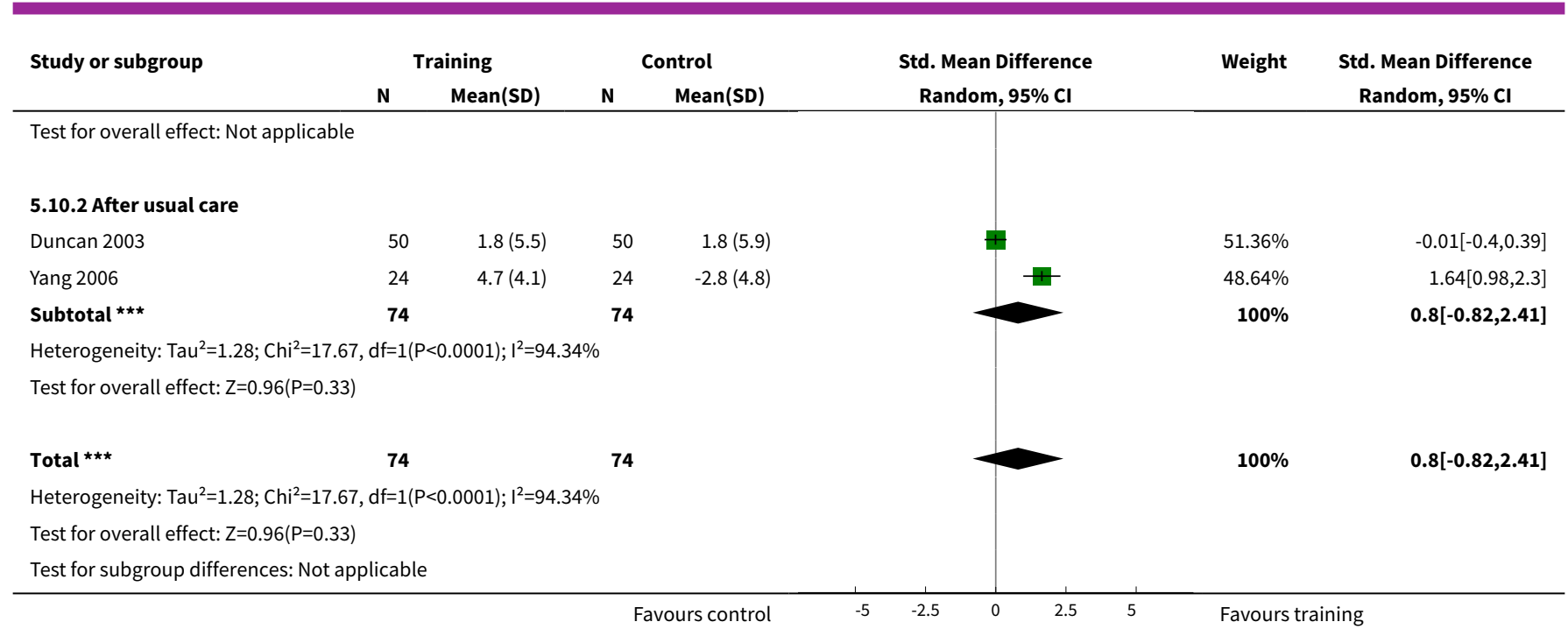

Analysis 5.11. Comparison 5 Mixed training versus control - end of intervention, Outcome 11 Physical fitness - muscle strength, knee extension*.

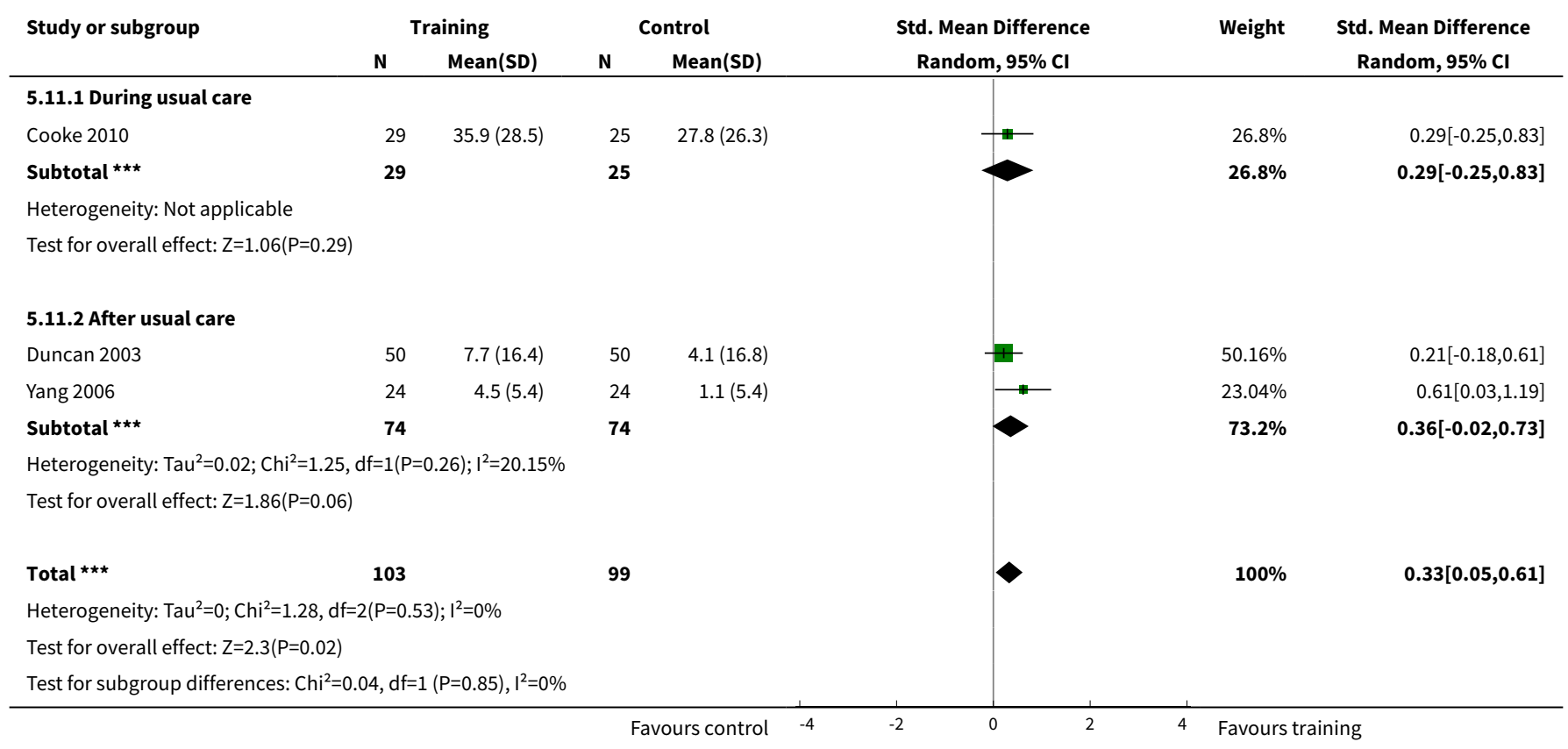

Analysis 5.12. Comparison 5 Mixed training versus control - end of intervention, Outcome 12 Physical fitness - muscle strength, grip strength (paretic hand).

\begin{tabular}{|c|c|c|c|c|c|c|c|c|c|}
\hline \multirow[t]{2}{*}{ Study or subgroup } & \multicolumn{2}{|c|}{ Training } & \multicolumn{2}{|c|}{ Control } & & \multirow{2}{*}{\multicolumn{2}{|c|}{$\begin{array}{l}\text { Mean Difference } \\
\text { Random, } 95 \% \mathrm{CI}\end{array}$}} & \multirow[t]{2}{*}{ Weight } & \multirow{2}{*}{$\begin{array}{l}\text { Mean Difference } \\
\text { Random, } 95 \% \mathrm{Cl}\end{array}$} \\
\hline & $\mathbf{N}$ & $\operatorname{Mean}(S D)$ & $\mathbf{N}$ & $\operatorname{Mean}(S D)$ & & & & & \\
\hline \multicolumn{10}{|c|}{ 5.12.1 During usual care } \\
\hline Kim 2017a & 14 & $1.1(1.8)$ & 15 & $0.7(2.3)$ & & & & $62.9 \%$ & $0.42[-1.1,1.94]$ \\
\hline
\end{tabular}




\begin{tabular}{|c|c|c|c|c|c|c|c|}
\hline \multirow[t]{2}{*}{ Study or subgroup } & \multicolumn{2}{|c|}{ Training } & \multicolumn{2}{|c|}{ Control } & \multirow{2}{*}{$\begin{array}{l}\text { Mean Difference } \\
\text { Random, } 95 \% \mathrm{Cl}\end{array}$} & \multirow[t]{2}{*}{ Weight } & \multirow{2}{*}{$\begin{array}{l}\text { Mean Difference } \\
\text { Random, } 95 \% \mathrm{CI}\end{array}$} \\
\hline & $\mathbf{N}$ & $\operatorname{Mean}(S D)$ & $\mathbf{N}$ & Mean(SD) & & & \\
\hline Subtotal $\star \star \star$ & 24 & & 23 & & & $69.43 \%$ & $0.32[-1.12,1.76]$ \\
\hline \multicolumn{8}{|c|}{ Heterogeneity: $\operatorname{Tau}^{2}=0 ; \mathrm{Chi}^{2}=0.18, \mathrm{df}=1(\mathrm{P}=0.67) ; \mathrm{I}^{2}=0 \%$} \\
\hline \multicolumn{8}{|c|}{ Test for overall effect: $Z=0.44(P=0.66)$} \\
\hline \multicolumn{8}{|c|}{ 5.12.2 After usual care } \\
\hline Duncan 2003 & 50 & $2.1(5)$ & 50 & $1.8(6.1)$ & 世- & $30.57 \%$ & $0.32[-1.85,2.49]$ \\
\hline Subtotal $\star \star \star$ & 50 & & 50 & & & $30.57 \%$ & $0.32[-1.85,2.49]$ \\
\hline \multicolumn{8}{|c|}{ Heterogeneity: $\operatorname{Tau}^{2}=0 ; \mathrm{Chi}^{2}=0, \mathrm{df}=0(\mathrm{P}<0.0001) ; \mathrm{I}^{2}=100 \%$} \\
\hline \multicolumn{8}{|c|}{ Test for overall effect: $Z=0.29(P=0.77)$} \\
\hline 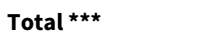 & 74 & & 73 & & & $100 \%$ & $0.32[-0.88,1.52]$ \\
\hline \multicolumn{8}{|c|}{ Heterogeneity: $\mathrm{Tau}^{2}=0 ; \mathrm{Chi}^{2}=0.18, \mathrm{df}=2(\mathrm{P}=0.92) ; \mathrm{I}^{2}=0 \%$} \\
\hline \multicolumn{8}{|c|}{ Test for overall effect: $Z=0.52(P=0.6)$} \\
\hline \multicolumn{8}{|c|}{ Test for subgroup differences: $\mathrm{Chi}^{2}=0, \mathrm{df}=1(\mathrm{P}=1), \mathrm{I}^{2}=0 \%$} \\
\hline
\end{tabular}

Analysis 5.13. Comparison 5 Mixed training versus control - end of intervention, Outcome 13 Mobility - walking maximum speed.

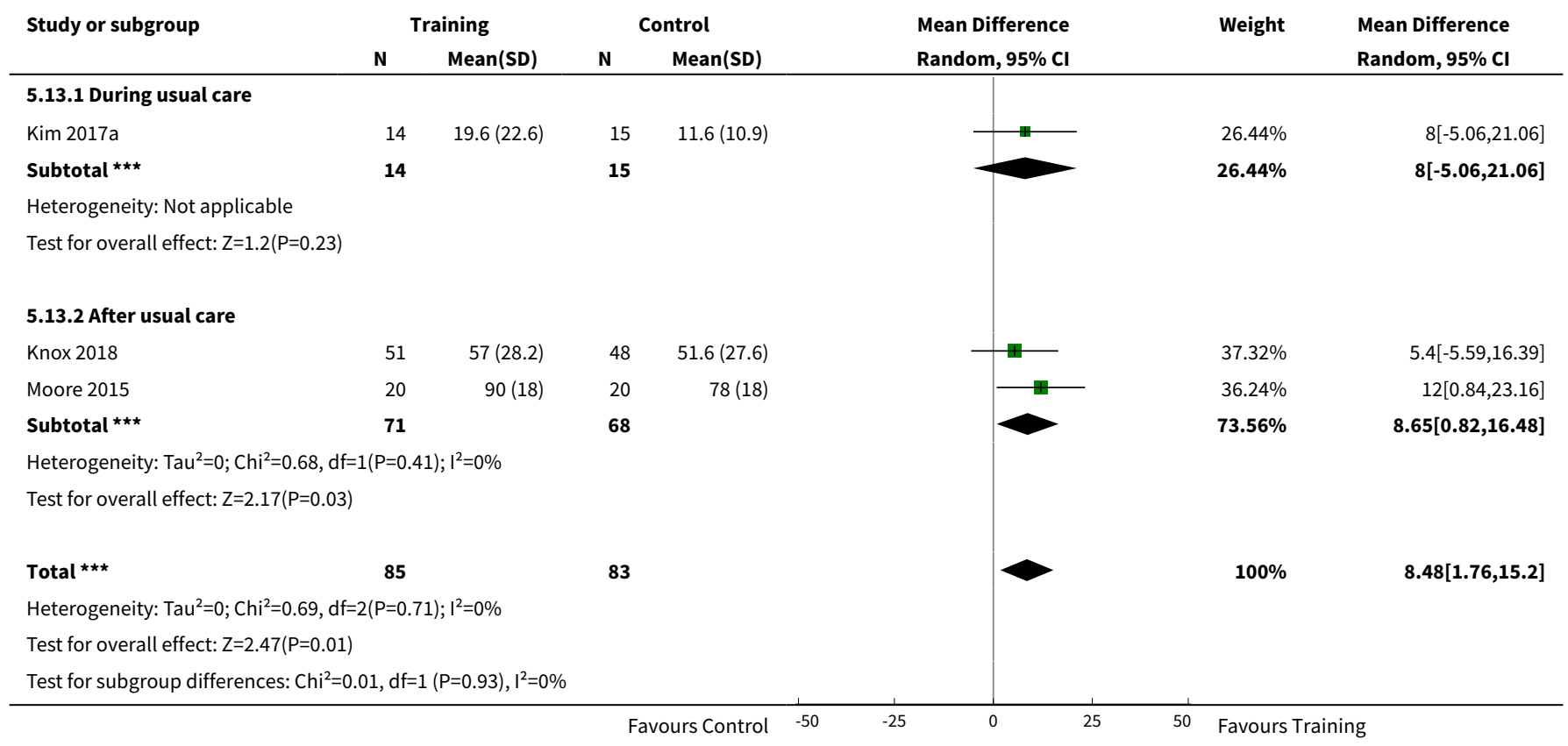

Analysis 5.14. Comparison 5 Mixed training versus control - end of intervention, Outcome 14 Mobility - walking preferred speed $(\mathrm{m} / \mathrm{min})$.

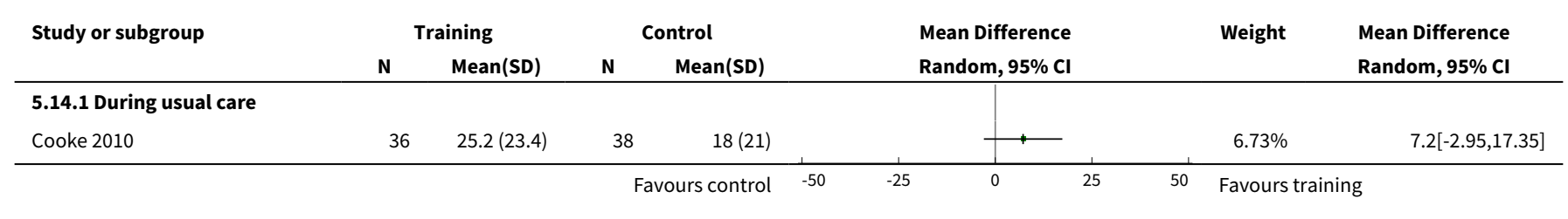




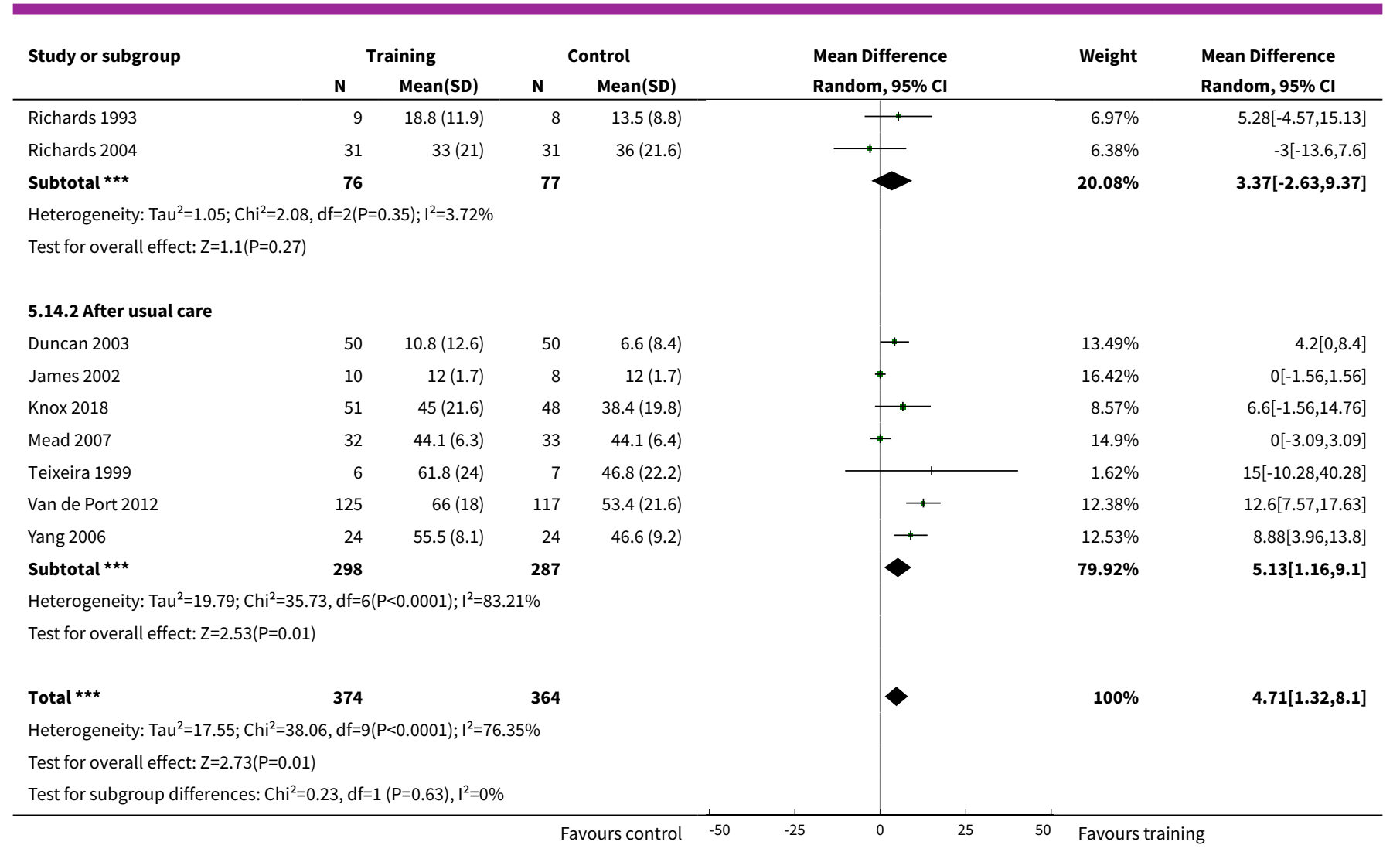

Analysis 5.15. Comparison 5 Mixed training versus control - end of intervention, Outcome 15 Mobility - walking capacity (6-MWT metres).

\begin{tabular}{|c|c|c|c|c|c|c|c|c|c|}
\hline \multirow[t]{2}{*}{ Study or subgroup } & \multicolumn{2}{|c|}{ Training } & \multicolumn{2}{|c|}{ Control } & \multirow{2}{*}{\multicolumn{2}{|c|}{$\begin{array}{l}\text { Mean Difference } \\
\text { Random, } 95 \% \mathrm{Cl}\end{array}$}} & & \multirow[t]{2}{*}{ Weight } & \multirow{2}{*}{$\begin{array}{l}\text { Mean Difference } \\
\text { Random, 95\% Cl }\end{array}$} \\
\hline & $\mathbf{N}$ & Mean(SD) & $\mathbf{N}$ & Mean(SD) & & & & & \\
\hline \multicolumn{10}{|c|}{ 5.15.1 During usual care } \\
\hline Galvin 2011 & 20 & $\begin{array}{r}231.8 \\
(131.3)\end{array}$ & 20 & $\begin{array}{r}165.5 \\
(146.1)\end{array}$ & & & & $4.36 \%$ & $66.3[-19.79,152.39]$ \\
\hline Kim 2016a & 10 & $93.5(56.3)$ & 10 & $118.5(50.4)$ & & $\cdots$ & & $11.63 \%$ & $-25[-71.82,21.82]$ \\
\hline Subtotal $\star \star \star$ & 30 & & 30 & & & & & $15.99 \%$ & $13.21[-75.07,101.49]$ \\
\hline \multicolumn{10}{|c|}{ Heterogeneity: $\mathrm{Tau}^{2}=2917.84 ; \mathrm{Chi}^{2}=3.33, \mathrm{df}=1(\mathrm{P}=0.07) ; \mathrm{I}^{2}=70.01 \%$} \\
\hline \multicolumn{10}{|c|}{ Test for overall effect: $Z=0.29(P=0.77)$} \\
\hline \multicolumn{10}{|c|}{ 5.15.2 After usual care } \\
\hline Duncan 1998 & 10 & $\begin{array}{r}209.1 \\
(110.6)\end{array}$ & 10 & $\begin{array}{r}204.5 \\
(121.4)\end{array}$ & & & & $3.22 \%$ & $4.64[-97.15,106.43]$ \\
\hline Duncan 2003 & 50 & $61.6(70.5)$ & 50 & $33.6(51.8)$ & & - & & $23.83 \%$ & $28.02[3.77,52.27]$ \\
\hline Knox 2018 & 51 & $260(136)$ & 48 & $236(127)$ & & + & & $10.05 \%$ & $24[-27.81,75.81]$ \\
\hline Moore 2015 & 20 & $513(131)$ & 20 & $441(126)$ & & & & $5 \%$ & $72[-7.66,151.66]$ \\
\hline Toledano-Zarhi 2011 & 14 & $\begin{array}{r}469.2 \\
(189.5)\end{array}$ & 14 & $\begin{array}{r}484.2 \\
(122.7)\end{array}$ & & & & $2.44 \%$ & $-15[-133.26,103.26]$ \\
\hline Van de Port 2012 & 125 & $412(117)$ & 117 & $354(145)$ & & $\rightarrow-$ & & $17.76 \%$ & $58[24.67,91.33]$ \\
\hline Yang 2006 & 24 & $392.8(54.2)$ & 24 & $\begin{array}{r}341.3 \\
(126.8)\end{array}$ & & + & & $9.15 \%$ & $51.5[-3.67,106.67]$ \\
\hline Zedlitz 2012 & 38 & $504(94)$ & 45 & $444(112)$ & & $\longrightarrow$ & & $12.54 \%$ & $60[15.68,104.32]$ \\
\hline Subtotal $\star \star \star ~$ & 332 & & 328 & & & $>$ & & $84.01 \%$ & $40.37[24.82,55.92]$ \\
\hline \multicolumn{10}{|c|}{ Heterogeneity: $\mathrm{Tau}^{2}=0 ; \mathrm{Chi}^{2}=5.29, \mathrm{df}=7(\mathrm{P}=0.63) ; \mathrm{I}^{2}=0 \%$} \\
\hline
\end{tabular}




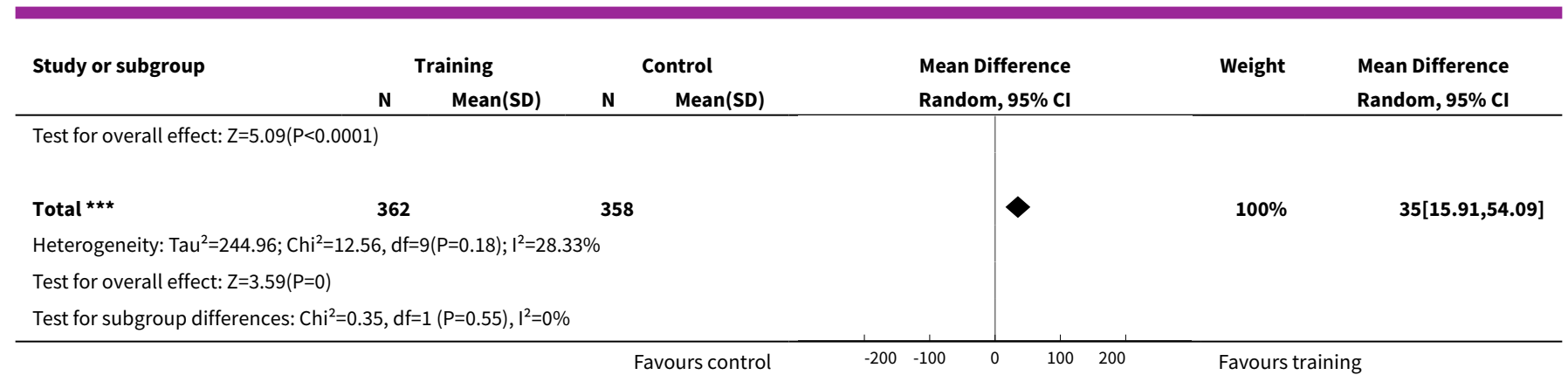

Analysis 5.16. Comparison 5 Mixed training versus control - end of intervention, Outcome 16 Mobility - Community Ambulation Speed (>0.8 m/sec).

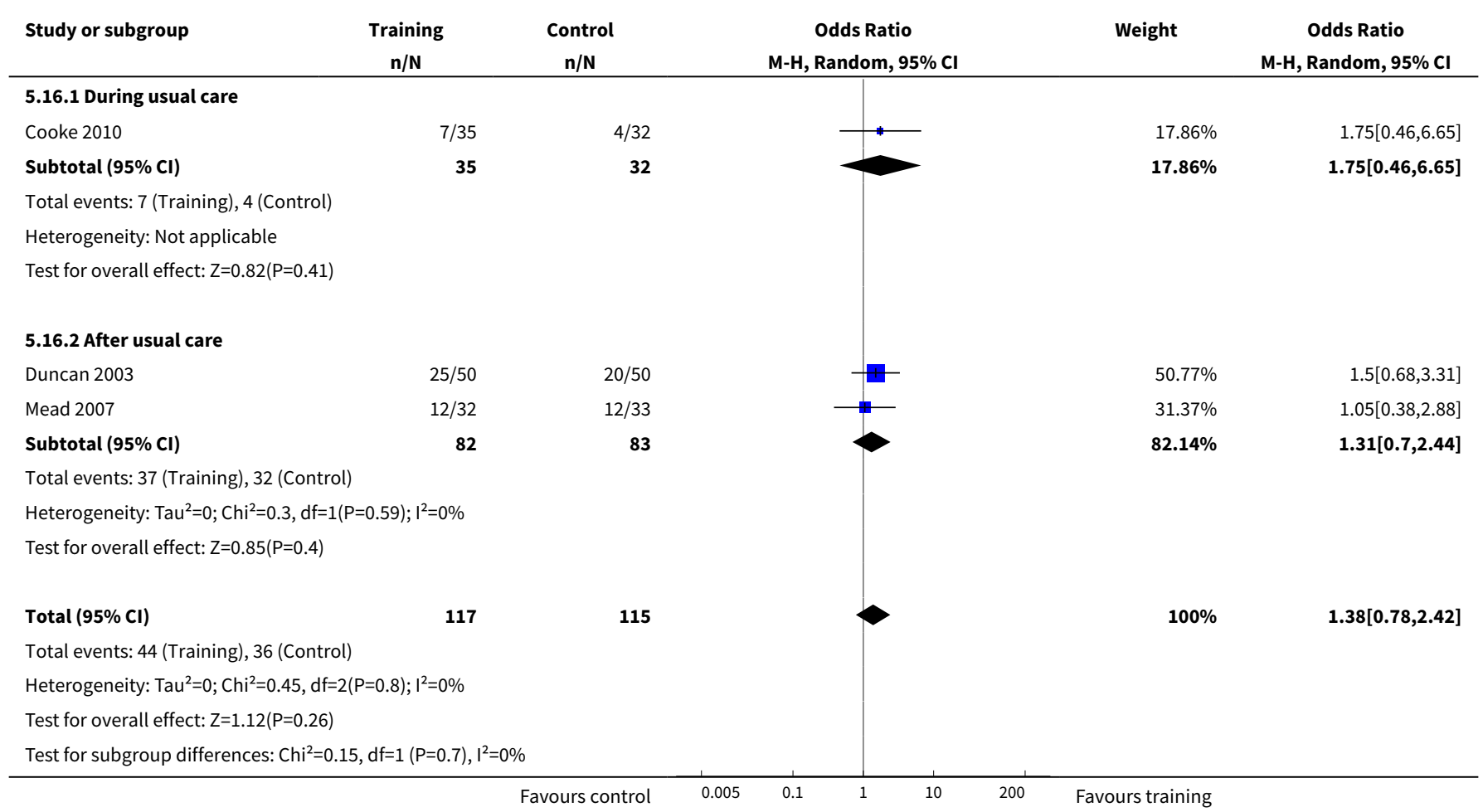

Analysis 5.17. Comparison 5 Mixed training versus control - end of intervention, Outcome 17 Physical function - balance - Berg Balance scale.

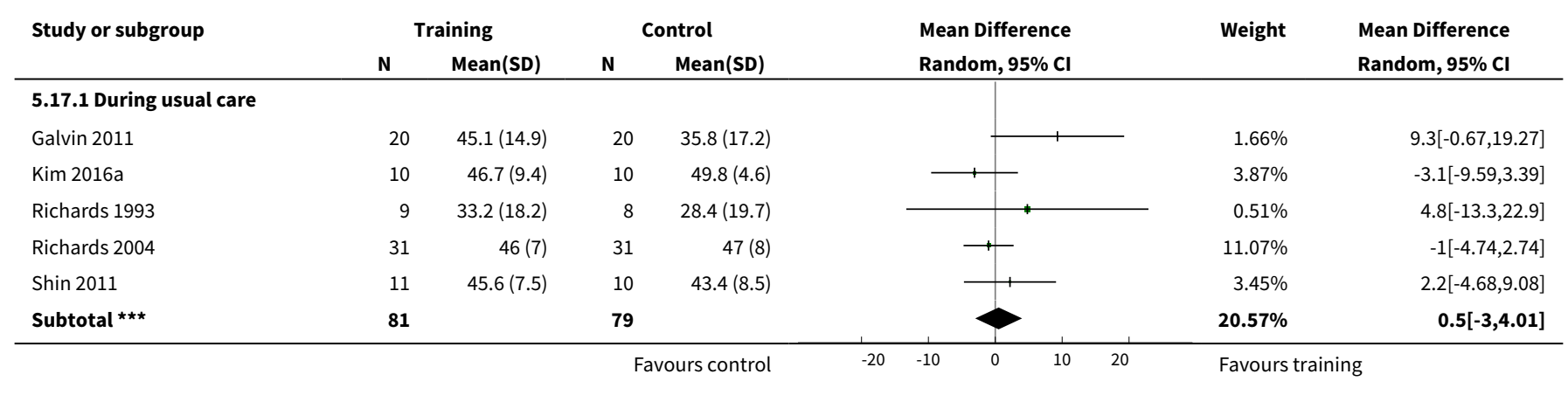




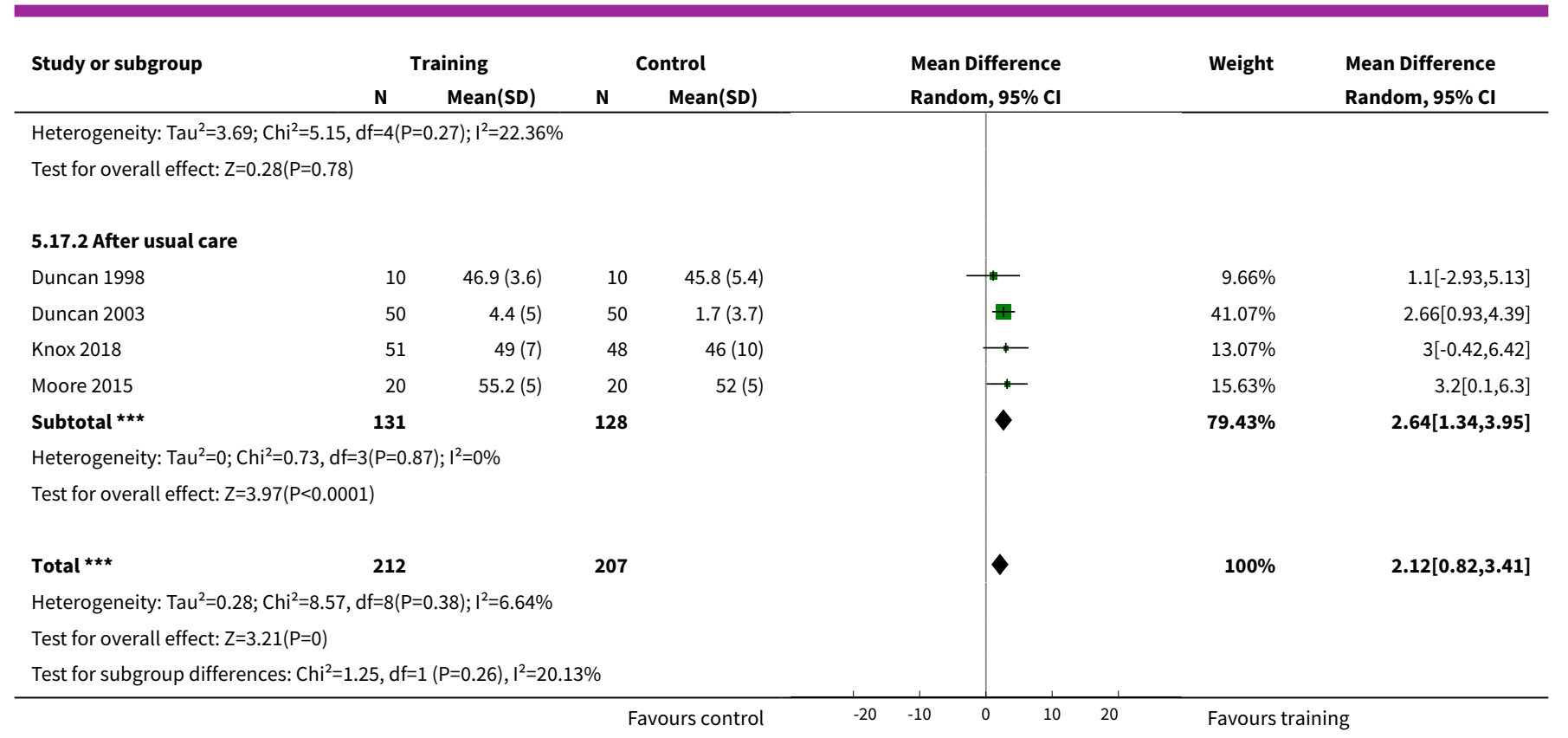

Analysis 5.18. Comparison 5 Mixed training versus control - end of intervention, Outcome 18 Physical function - balance - functional reach.

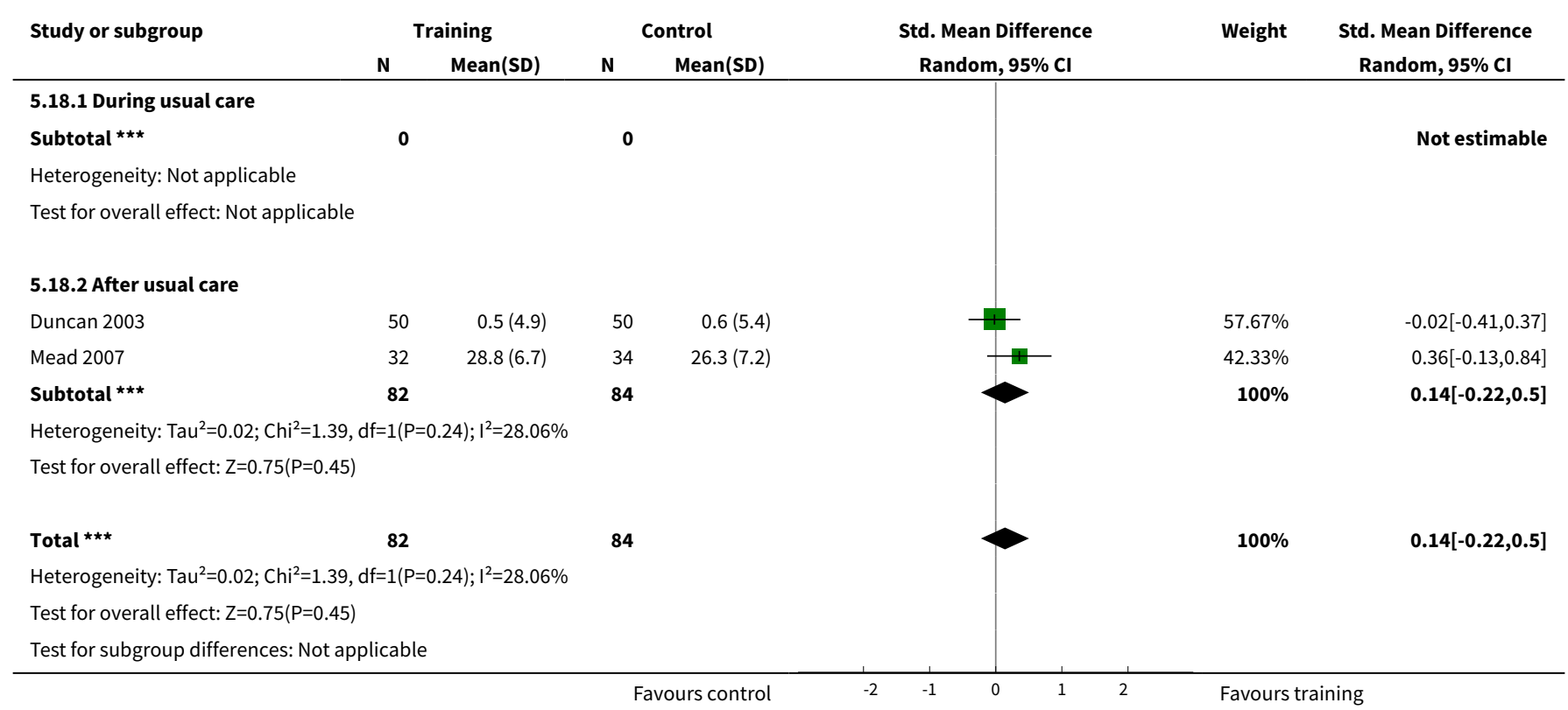

Analysis 5.19. Comparison 5 Mixed training versus control - end of intervention, Outcome 19 Physical function - balance - combined outcome data.

\begin{tabular}{|c|c|c|c|c|c|c|c|c|c|}
\hline \multirow[t]{2}{*}{ Study or subgroup } & \multicolumn{2}{|c|}{ Training } & \multicolumn{2}{|c|}{ Control } & \multirow{2}{*}{\multicolumn{3}{|c|}{$\begin{array}{c}\text { Std. Mean Difference } \\
\text { Random, } 95 \% \mathrm{Cl}\end{array}$}} & \multirow[t]{2}{*}{ Weight } & \multirow{2}{*}{$\begin{array}{c}\text { Std. Mean Difference } \\
\text { Random, } 95 \% \mathrm{Cl}\end{array}$} \\
\hline & $\mathbf{N}$ & Mean(SD) & $\mathbf{N}$ & Mean(SD) & & & & & \\
\hline \multicolumn{10}{|l|}{ 5.19.1 During usual care } \\
\hline
\end{tabular}




\begin{tabular}{|c|c|c|c|c|c|c|c|}
\hline \multirow{3}{*}{$\begin{array}{l}\text { Study or subgroup } \\
\text { Galvin } 2011\end{array}$} & \multicolumn{2}{|c|}{ Training } & \multicolumn{2}{|c|}{ Control } & \multirow{2}{*}{$\begin{array}{c}\text { Std. Mean Difference } \\
\text { Random, } 95 \% \mathrm{CI}\end{array}$} & \multirow[t]{2}{*}{ Weight } & \multirow{2}{*}{$\begin{array}{c}\text { Std. Mean Difference } \\
\text { Random, } 95 \% \mathrm{Cl}\end{array}$} \\
\hline & $\mathbf{N}$ & Mean(SD) & $\mathbf{N}$ & Mean(SD) & & & \\
\hline & 20 & $45.1(14.9)$ & 20 & $35.8(17.2)$ & + & $6.41 \%$ & $0.57[-0.07,1.2]$ \\
\hline Kim 2016a & 10 & $46.7(9.4)$ & 10 & $49.8(4.6)$ & \begin{tabular}{l|l}
+ & \\
1 &
\end{tabular} & $3.51 \%$ & $-0.4[-1.29,0.49]$ \\
\hline Richards 1993 & 9 & $33.2(18.2)$ & 8 & $28.4(19.7)$ & & $3.06 \%$ & $0.24[-0.72,1.2]$ \\
\hline Richards 2004 & 31 & $46(7)$ & 31 & $47(8)$ & $\longrightarrow$ & $9.51 \%$ & $-0.13[-0.63,0.37]$ \\
\hline Shin 2011 & 11 & $45.6(7.5)$ & 10 & $43.4(8.5)$ & & $3.72 \%$ & $0.26[-0.6,1.13]$ \\
\hline Subtotal $* \star \star$ & 81 & & 79 & & & $26.21 \%$ & $0.1[-0.23,0.43]$ \\
\hline \multicolumn{8}{|c|}{ Heterogeneity: $\mathrm{Tau}^{2}=0.01 ; \mathrm{Chi}^{2}=4.36, \mathrm{df}=4(\mathrm{P}=0.36) ; \mathrm{I}^{2}=8.24 \%$} \\
\hline \multicolumn{8}{|c|}{ Test for overall effect: $Z=0.6(P=0.55)$} \\
\hline \multicolumn{8}{|c|}{ 5.19.2 After usual care } \\
\hline Duncan 1998 & 10 & $46.9(3.6)$ & 10 & $45.8(5.4)$ & & $3.57 \%$ & $0.23[-0.65,1.11]$ \\
\hline Duncan 2003 & 50 & $4.4(5)$ & 50 & $1.7(3.7)$ & $\longrightarrow$ & $13.16 \%$ & $0.6[0.2,1]$ \\
\hline Knox 2018 & 51 & $49(7)$ & 48 & $46(10)$ & + & $13.34 \%$ & $0.35[-0.05,0.74]$ \\
\hline Mead 2007 & 32 & $28.8(6.7)$ & 34 & $26.3(7.2)$ & *- & $9.87 \%$ & $0.36[-0.13,0.84]$ \\
\hline Moore 2015 & 20 & $55.2(5)$ & 20 & $52(5)$ & + & $6.36 \%$ & $0.63[-0.01,1.26]$ \\
\hline Toledano-Zarhi 2011 & 14 & $-11.7(7.6)$ & 14 & $-8.7(2.6)$ & 1 & $4.72 \%$ & $-0.51[-1.27,0.24]$ \\
\hline Van de Port 2012 & 125 & $4.1(1)$ & 117 & $3.7(1.1)$ & $\rightarrow$ & $22.79 \%$ & $0.31[0.05,0.56]$ \\
\hline 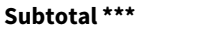 & 302 & & 293 & & & $73.79 \%$ & $0.35[0.15,0.54]$ \\
\hline \multicolumn{8}{|c|}{ Heterogeneity: $\mathrm{Tau}^{2}=0.01 ; \mathrm{Chi}^{2}=7.42, \mathrm{df}=6(\mathrm{P}=0.28) ; \mathrm{I}^{2}=19.15 \%$} \\
\hline \multicolumn{8}{|c|}{ Test for overall effect: $Z=3.51(P=0)$} \\
\hline Total $\star \star \star ~$ & 383 & & 372 & & 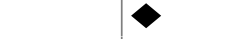 & $100 \%$ & $0.28[0.11,0.45]$ \\
\hline \multicolumn{8}{|c|}{ Heterogeneity: $\mathrm{Tau}^{2}=0.02 ; \mathrm{Chi}^{2}=13.72, \mathrm{df}=11(\mathrm{P}=0.25) ; \mathrm{I}^{2}=19.82 \%$} \\
\hline \multicolumn{8}{|c|}{ Test for overall effect: $Z=3.18(P=0)$} \\
\hline Test for subgroup diff & $6, \mathrm{df}=$ & $P=0.21), I^{2}=35$ & & & & & \\
\hline
\end{tabular}

Analysis 5.20. Comparison 5 Mixed training versus control - end of intervention, Outcome 20 Physical function - Timed Up and Go (sec).

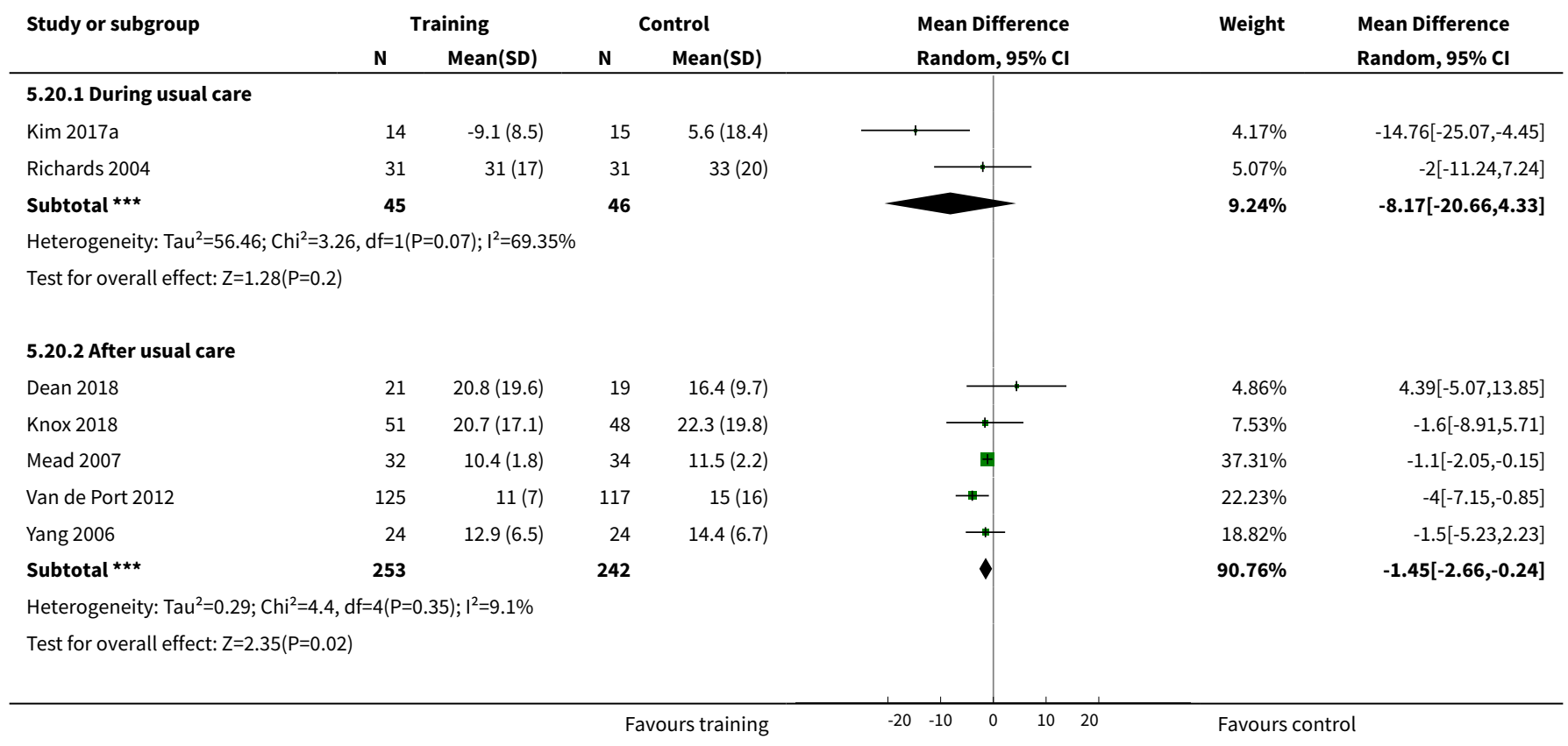




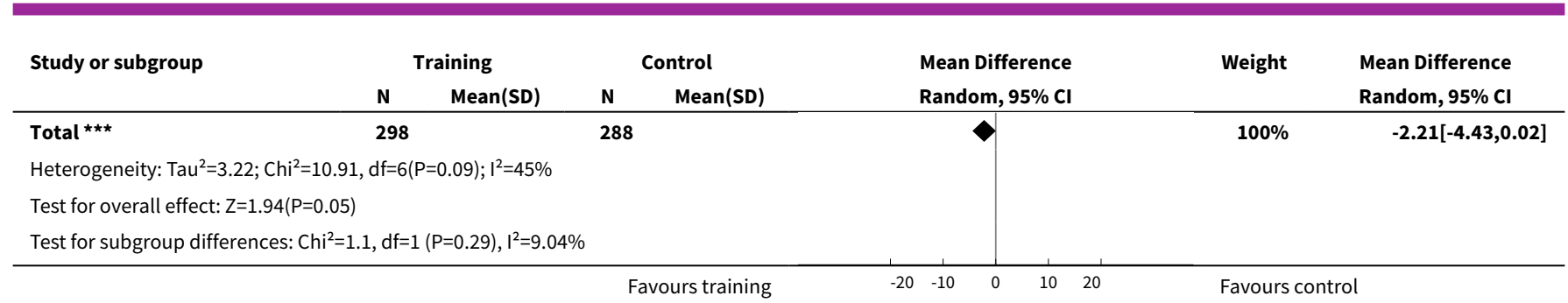

Analysis 5.21. Comparison 5 Mixed training versus control - end of intervention, Outcome 21 Health-related QoL - SF-36 physical functioning.

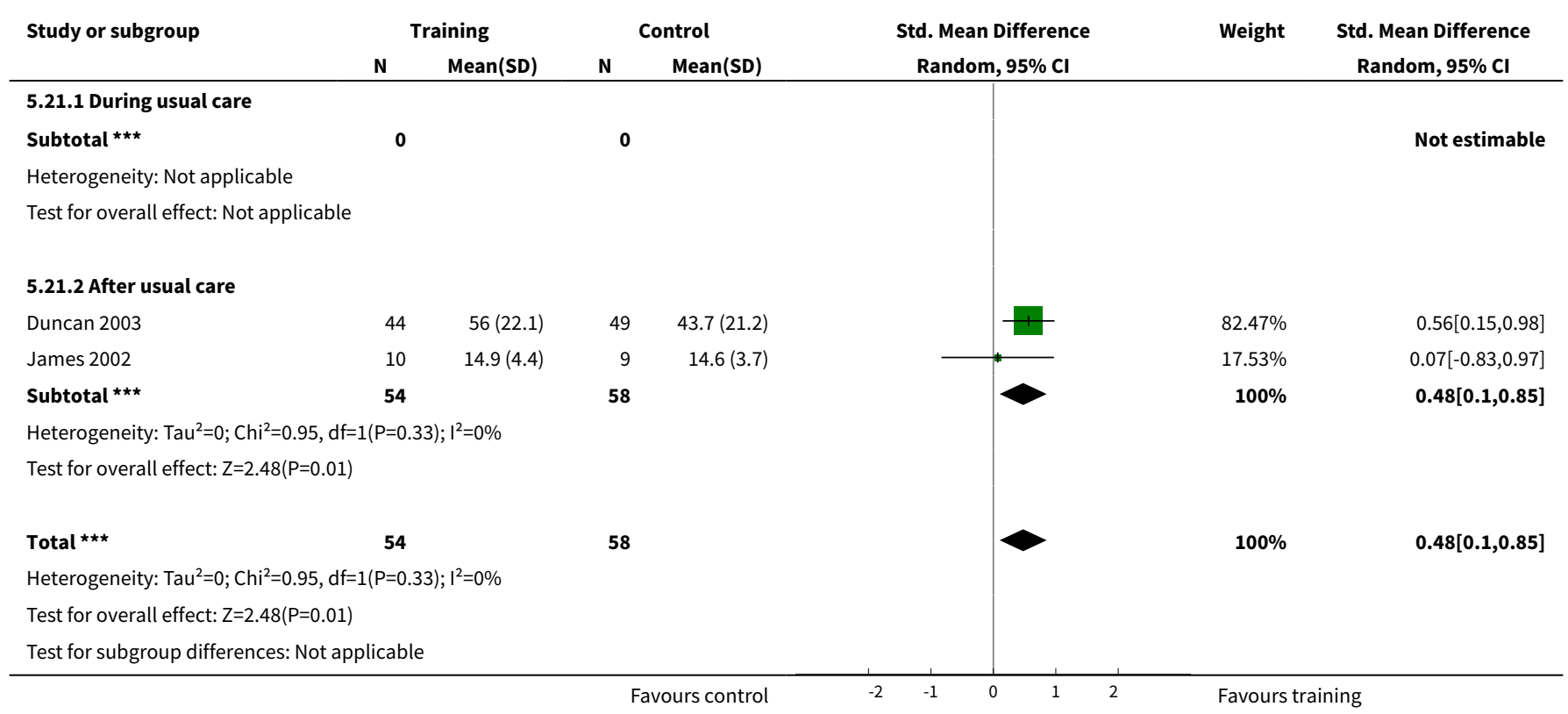

Analysis 5.22. Comparison 5 Mixed training versus control - end of intervention, Outcome 22 Health-related QoL - SF-36 physical role functioning.

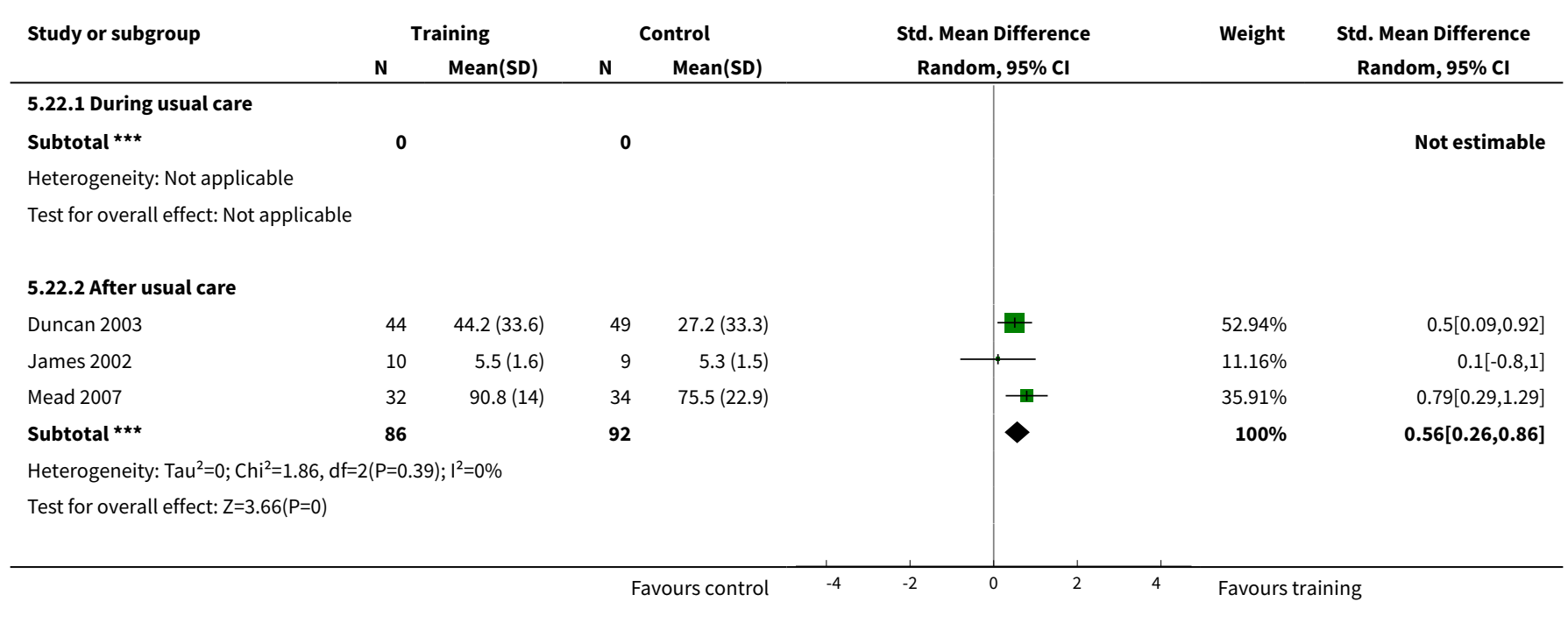




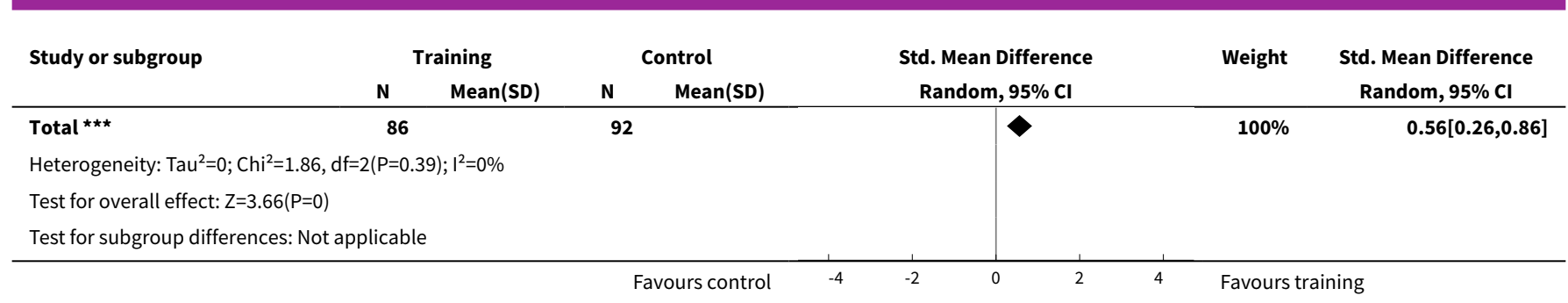

Analysis 5.23. Comparison 5 Mixed training versus control - end of intervention, Outcome 23 Health-related QoL - SF-36 social role functioning.

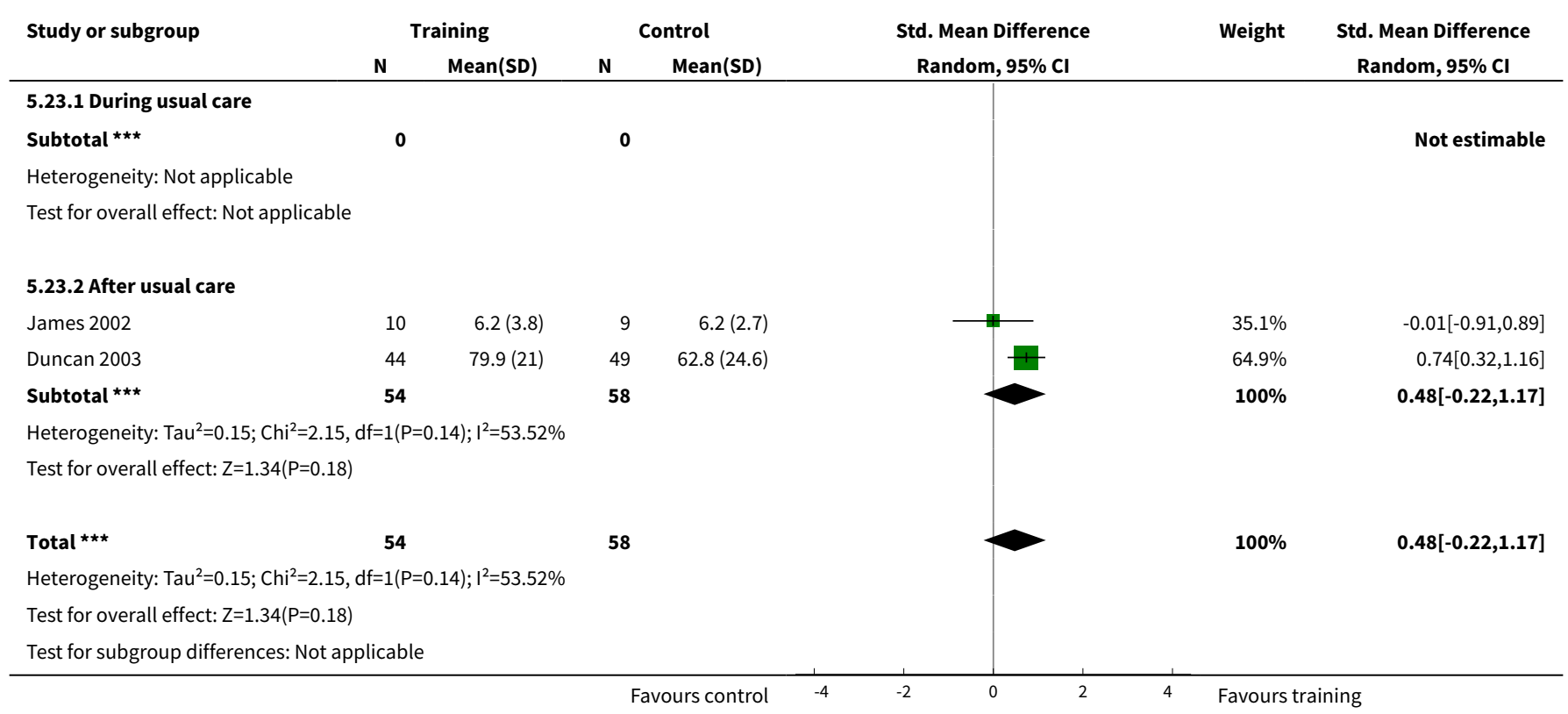

Analysis 5.24. Comparison 5 Mixed training versus control - end of intervention, Outcome 24 Mood - Hospital Anxiety and Depression Scale (HADS) - anxiety score.

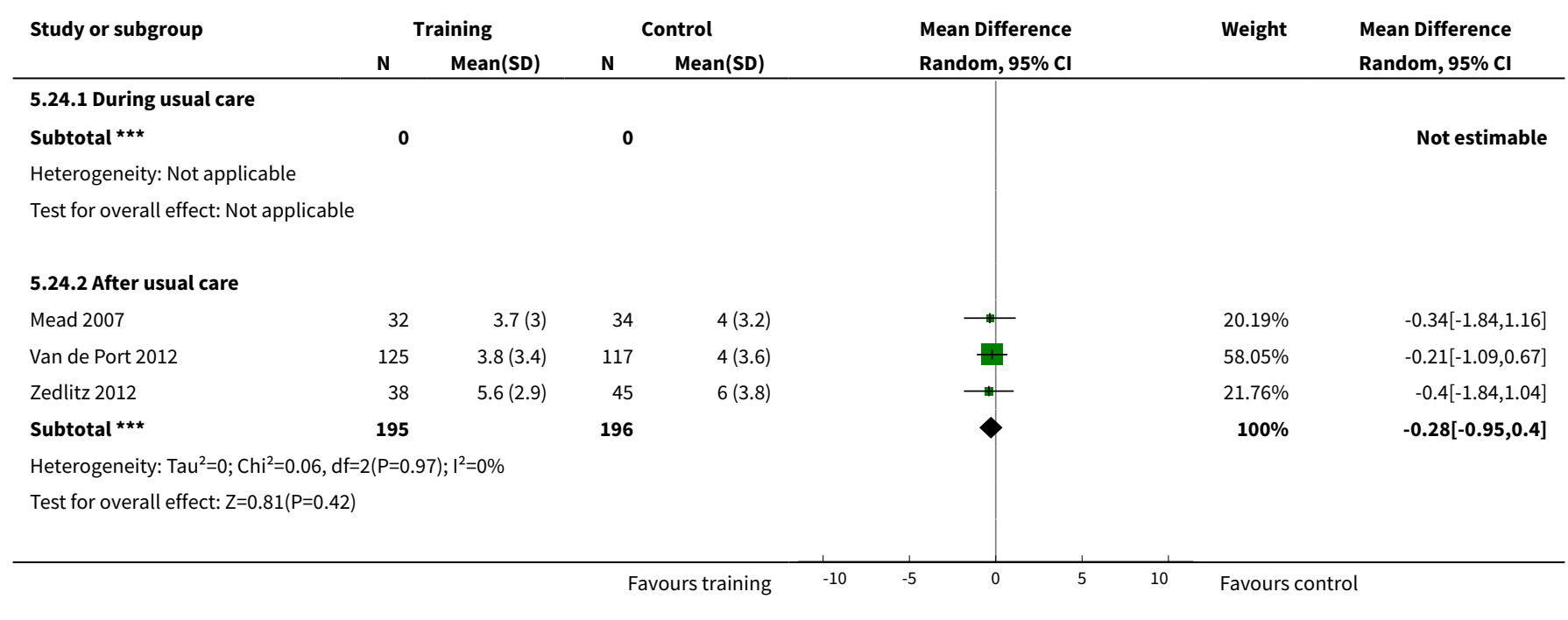




\begin{tabular}{|c|c|c|c|c|c|}
\hline \multirow[t]{2}{*}{ Study or subgroup } & Training & Control & \multirow{2}{*}{$\begin{array}{l}\text { Mean Difference } \\
\text { Random, 95\% Cl }\end{array}$} & \multirow[t]{2}{*}{ Weight } & \multirow{2}{*}{$\begin{array}{l}\text { Mean Difference } \\
\text { Random, } 95 \% \mathrm{CI}\end{array}$} \\
\hline & Mean(SD) & Mean(SD) & & & \\
\hline 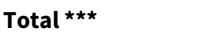 & 195 & 196 & $\gamma$ & $100 \%$ & $-0.28[-0.95,0.4]$ \\
\hline \multicolumn{6}{|c|}{ Heterogeneity: $\operatorname{Tau}^{2}=0 ; \mathrm{Chi}^{2}=0.06, \mathrm{df}=2(\mathrm{P}=0.97) ; \mathrm{I}^{2}=0 \%$} \\
\hline \multicolumn{6}{|c|}{ Test for overall effect: $Z=0.81(P=0.42)$} \\
\hline \multicolumn{6}{|c|}{ Test for subgroup differences: Not applicable } \\
\hline & & Favours training & -10 & Favours & \\
\hline
\end{tabular}

Analysis 5.25. Comparison 5 Mixed training versus control - end of intervention, Outcome 25 Mood - Hospital Anxiety and Depression Scale (HADS) - depression score.

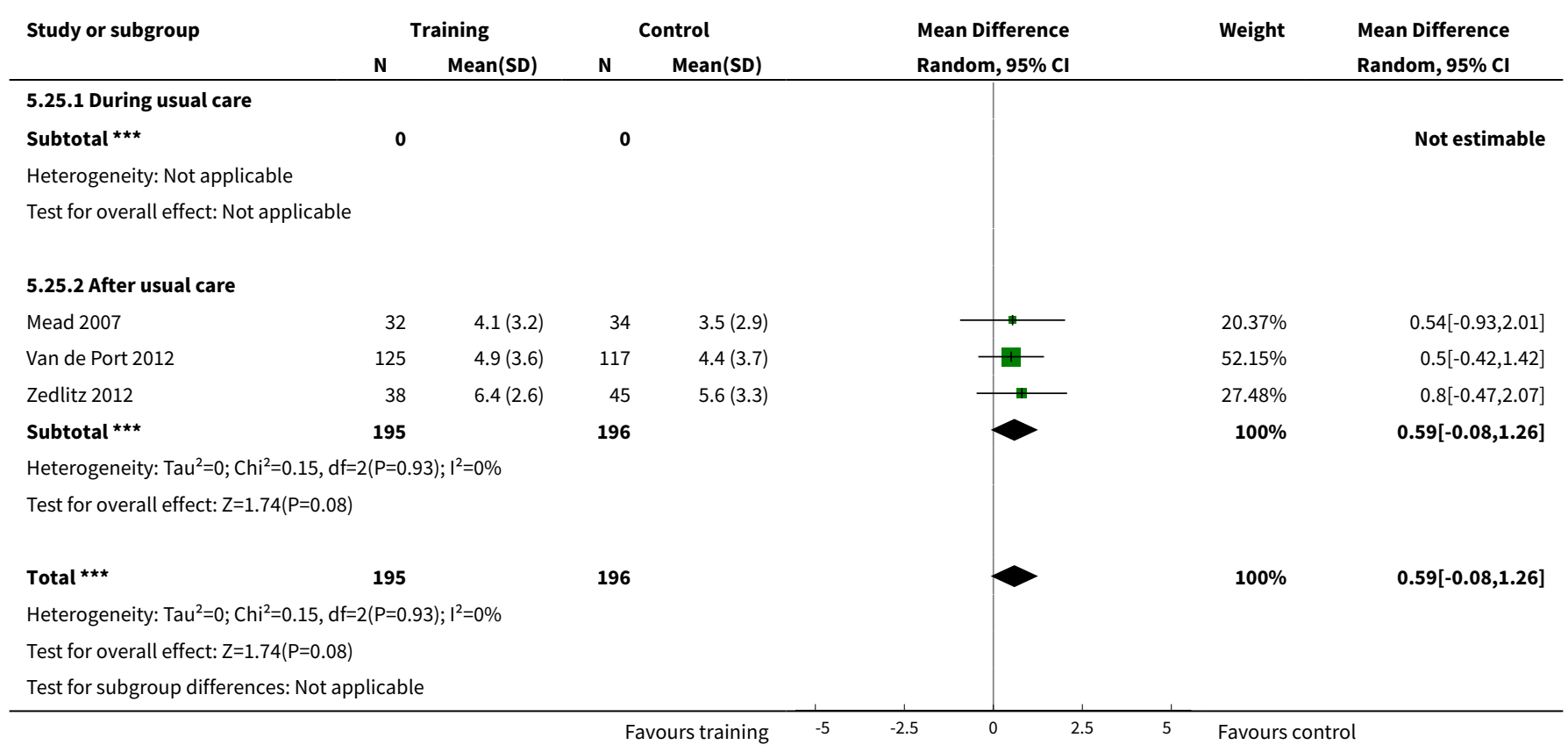

Analysis 5.26. Comparison 5 Mixed training versus control - end of intervention, Outcome 26 Mood - Stroke Impact Scale emotion score.

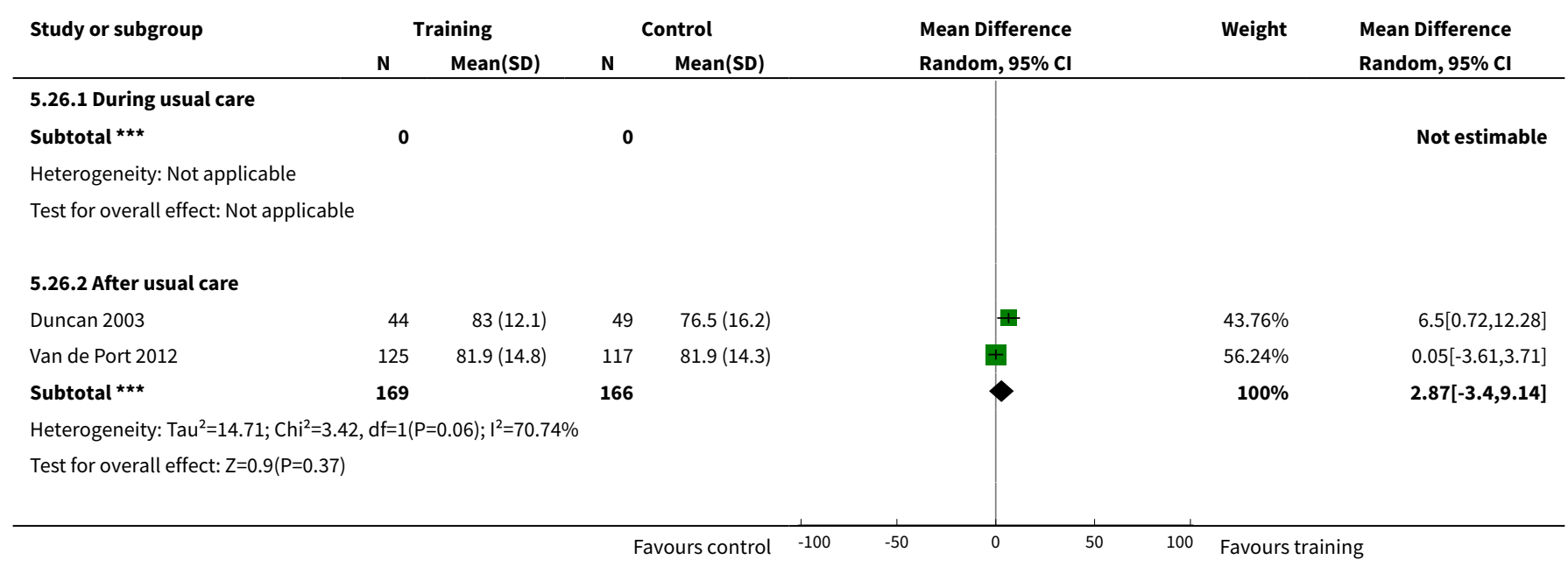




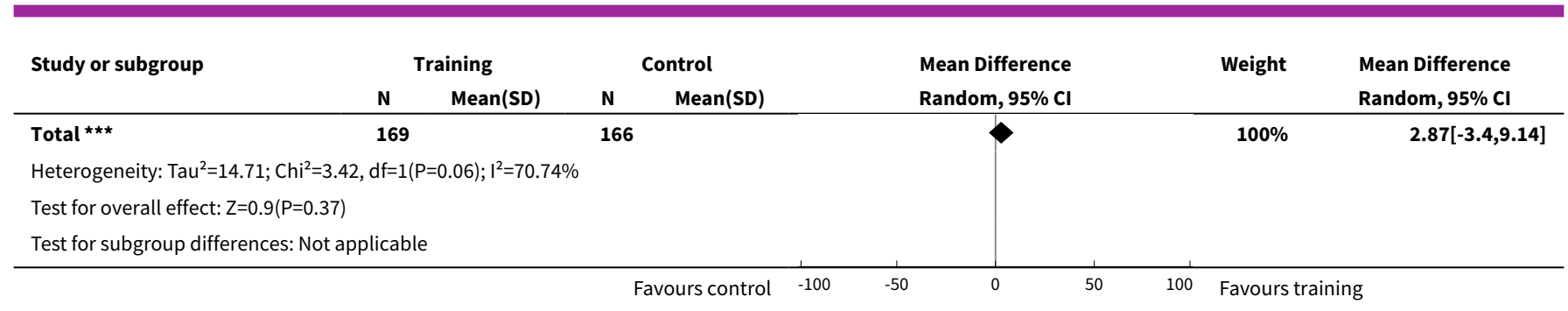

Analysis 5.27. Comparison 5 Mixed training versus control - end of intervention, Outcome 27 Mood - combined depression scales.

\begin{tabular}{|c|c|c|c|c|c|c|c|}
\hline \multirow[t]{2}{*}{ Study or subgroup } & \multicolumn{2}{|c|}{ Training } & \multicolumn{2}{|c|}{ Control } & \multirow{2}{*}{$\begin{array}{c}\text { Std. Mean Difference } \\
\text { Random, } 95 \% \mathrm{Cl}\end{array}$} & \multirow[t]{2}{*}{ Weight } & \multirow{2}{*}{$\begin{array}{c}\text { Std. Mean Difference } \\
\text { Random, } 95 \% \mathrm{Cl}\end{array}$} \\
\hline & $\mathbf{N}$ & Mean(SD) & $\mathbf{N}$ & $\operatorname{Mean}(\mathrm{SD})$ & & & \\
\hline \multicolumn{8}{|c|}{ 5.27.1 During usual care } \\
\hline Subtotal $\star \star \star$ & 0 & & 0 & & & & Not estimable \\
\hline \multicolumn{8}{|c|}{ Heterogeneity: Not applicable } \\
\hline \multicolumn{8}{|c|}{ Test for overall effect: Not applicable } \\
\hline \multicolumn{8}{|c|}{ 5.27.2 After usual care } \\
\hline Duncan 2003 & 44 & $2.5(2.5)$ & 49 & $4.4(3.4)$ & I & $24.31 \%$ & $-0.63[-1.04,-0.21]$ \\
\hline Mead 2007 & 32 & $4.1(3.2)$ & 34 & $3.5(2.9)$ & - & $22.09 \%$ & $0.18[-0.31,0.66]$ \\
\hline Van de Port 2012 & 125 & $4.9(3.6)$ & 117 & $4.4(3.7)$ & & $29.85 \%$ & $0.14[-0.12,0.39]$ \\
\hline Zedlitz 2012 & 38 & $6.4(2.6)$ & 45 & $5.6(3.3)$ & " & $23.75 \%$ & $0.26[-0.17,0.7]$ \\
\hline 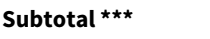 & 239 & & 245 & & & $100 \%$ & $-0.01[-0.39,0.37]$ \\
\hline \multicolumn{8}{|c|}{ Heterogeneity: $\operatorname{Tau}^{2}=0.11 ; \mathrm{Chi}^{2}=11.64, \mathrm{df}=3(\mathrm{P}=0.01) ; \mathrm{I}^{2}=74.23 \%$} \\
\hline 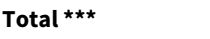 & 239 & & 245 & & & $100 \%$ & $-0.01[-0.39,0.37]$ \\
\hline \multicolumn{8}{|c|}{ Heterogeneity: $\mathrm{Tau}^{2}=0.11 ; \mathrm{Chi}^{2}=11.64, \mathrm{df}=3(\mathrm{P}=0.01) ; \mathrm{I}^{2}=74.23 \%$} \\
\hline \multicolumn{8}{|c|}{ Test for overall effect: $Z=0.05(P=0.96)$} \\
\hline Test for subgroup dif & licable & & & & & & \\
\hline
\end{tabular}

Analysis 5.28. Comparison 5 Mixed training versus control - end of intervention, Outcome 28 Cognitive function - FIM cognitive score.

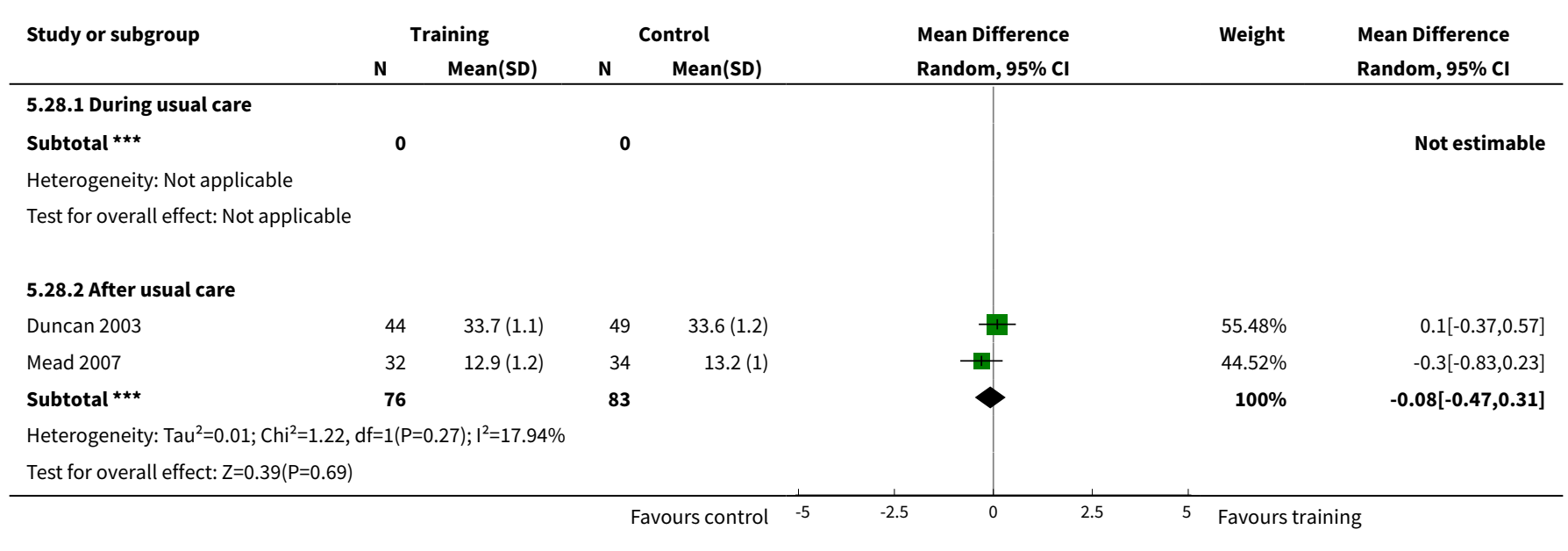




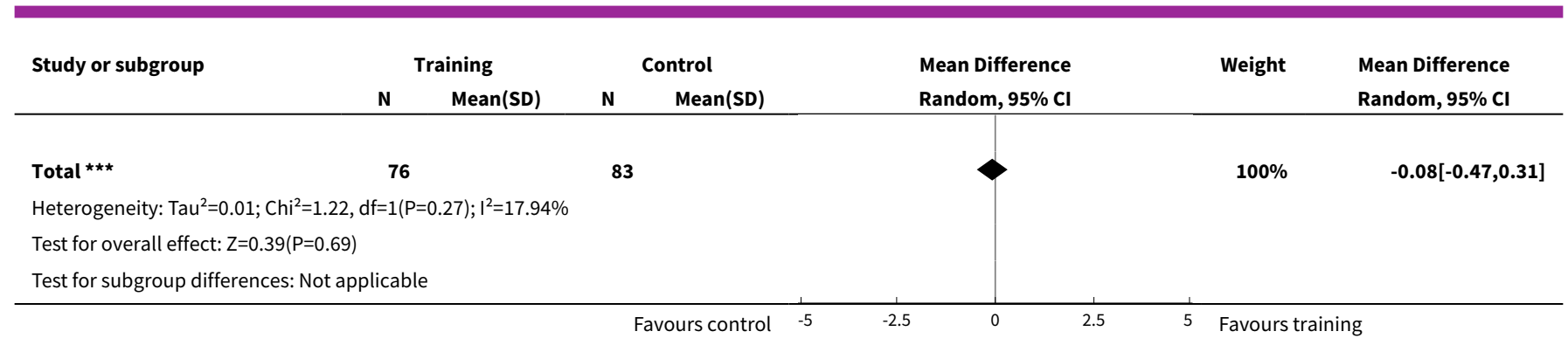

Analysis 5.29. Comparison 5 Mixed training versus control - end of intervention, Outcome 29 Cognitive function - SIS memory and thinking.

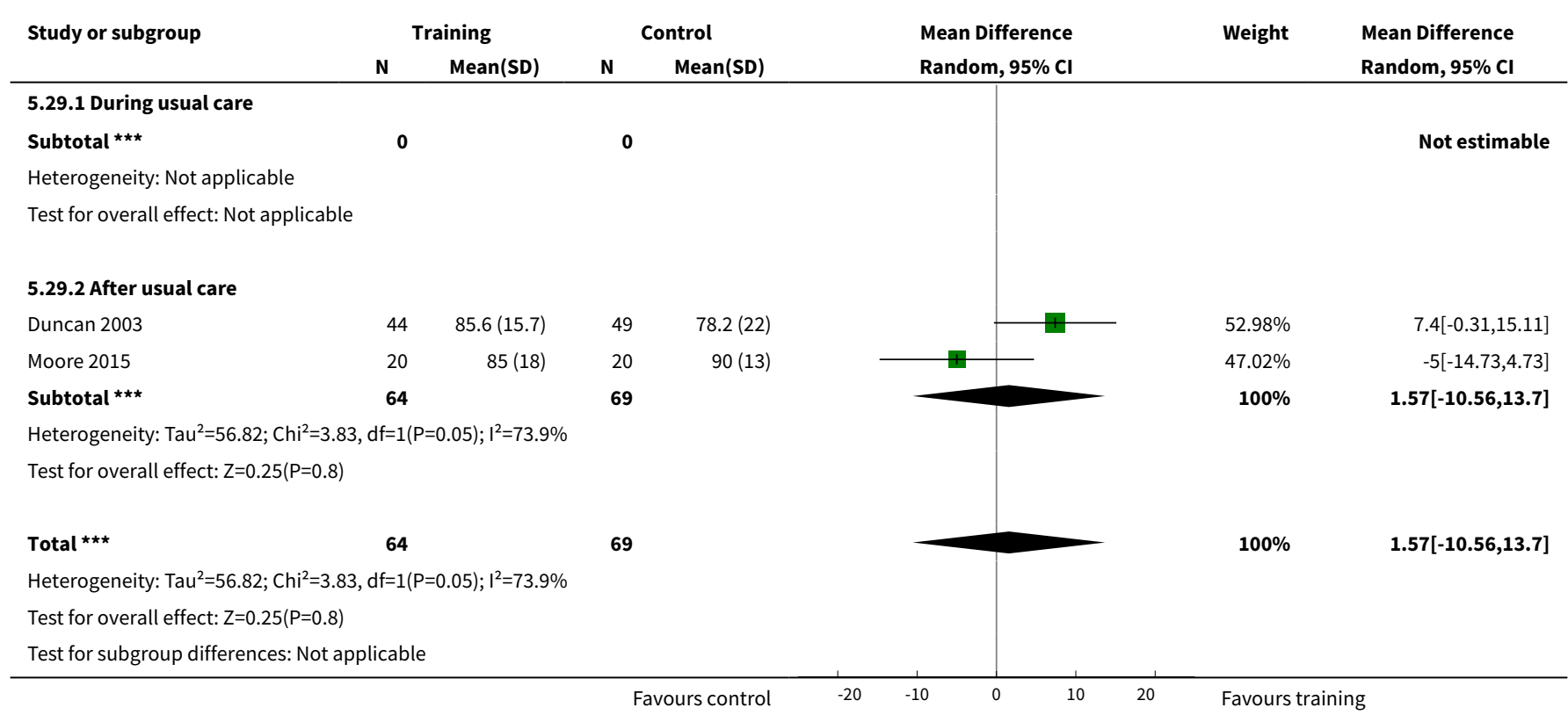

Analysis 5.30. Comparison 5 Mixed training versus control - end of intervention, Outcome 30 Cognitive function - SIS communication.

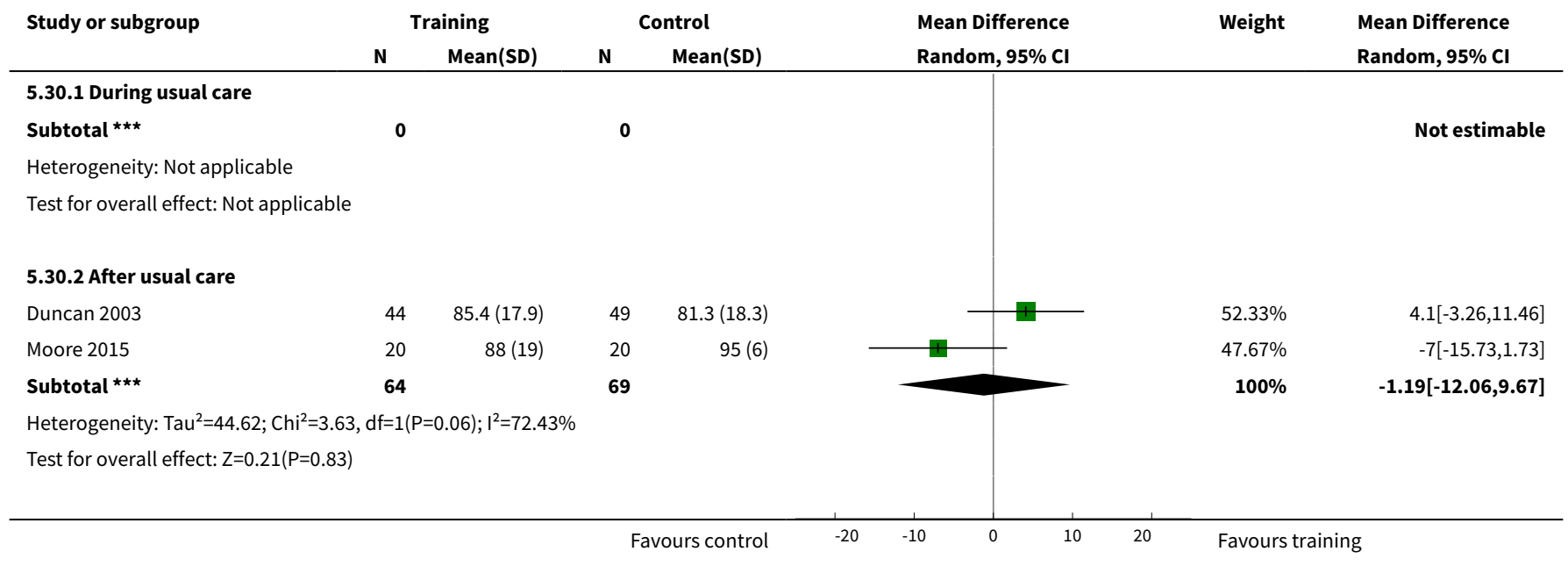




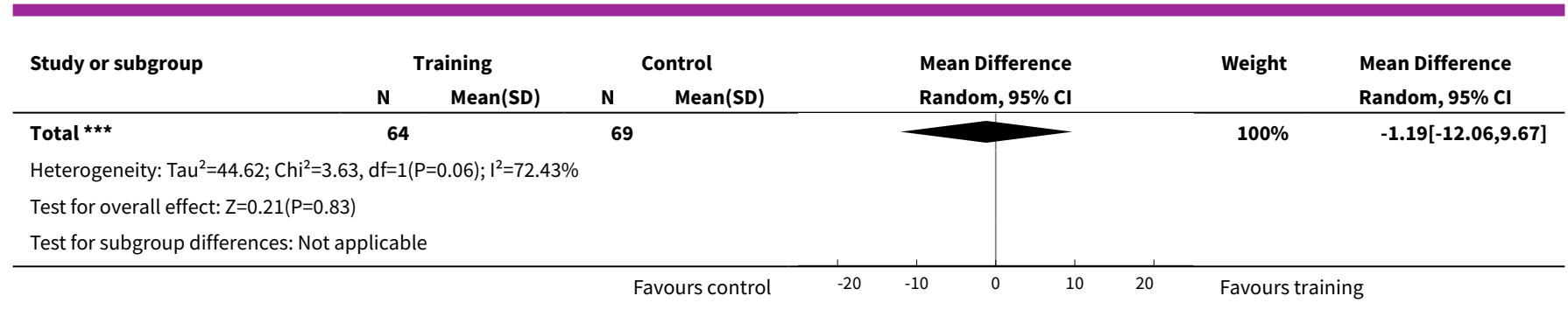

\section{Comparison 6. Mixed training versus control - end of retention follow-up}

\begin{tabular}{|c|c|c|c|c|}
\hline Outcome or subgroup title & $\begin{array}{l}\text { No. of } \\
\text { studies }\end{array}$ & $\begin{array}{l}\text { No. of } \\
\text { partici- } \\
\text { pants }\end{array}$ & Statistical method & Effect size \\
\hline 1 Death & 13 & 906 & $\begin{array}{l}\text { Risk Difference (M-H, Random, } \\
95 \% \mathrm{Cl} \text { ) }\end{array}$ & $-0.01[-0.03,0.01]$ \\
\hline 1.1 During usual care & 6 & 243 & $\begin{array}{l}\text { Risk Difference (M-H, Random, } \\
95 \% \mathrm{Cl} \text { ) }\end{array}$ & $-0.02[-0.06,0.02]$ \\
\hline 1.2 After usual care & 7 & 663 & $\begin{array}{l}\text { Risk Difference (M-H, Random, } \\
95 \% \mathrm{Cl} \text { ) }\end{array}$ & $-0.01[-0.03,0.01]$ \\
\hline 2 Disability - Barthel Index (BI) & 2 & 103 & $\begin{array}{l}\text { Mean Difference (IV, Random, } \\
95 \% \mathrm{CI} \text { ) }\end{array}$ & $1.82[-13.69,17.33]$ \\
\hline 2.1 During usual care & 1 & 40 & $\begin{array}{l}\text { Mean Difference (IV, Random, } \\
95 \% \mathrm{CI} \text { ) }\end{array}$ & $9.0[-1.29,19.29]$ \\
\hline 2.2 After usual care & 1 & 63 & $\begin{array}{l}\text { Mean Difference (IV, Random, } \\
95 \% \mathrm{CI} \text { ) }\end{array}$ & $-6.90[-21.05,7.25]$ \\
\hline 3 Disability - Nottingham Extended ADL & 2 & 106 & $\begin{array}{l}\text { Mean Difference (IV, Random, } \\
95 \% \mathrm{CI} \text { ) }\end{array}$ & $3.10[-5.20,11.40]$ \\
\hline 3.1 During usual care & 1 & 40 & $\begin{array}{l}\text { Mean Difference (IV, Random, } \\
95 \% \mathrm{CI} \text { ) }\end{array}$ & $9.5[-1.83,20.83]$ \\
\hline 3.2 After usual care & 1 & 66 & $\begin{array}{l}\text { Mean Difference (IV, Random, } \\
95 \% \mathrm{Cl} \text { ) }\end{array}$ & $0.30[-0.93,1.53]$ \\
\hline $\begin{array}{l}4 \text { Disability - Rivermead Mobility Index } \\
\text { (RMI) }\end{array}$ & 3 & 349 & $\begin{array}{l}\text { Mean Difference (IV, Random, } \\
95 \% \mathrm{CI} \text { ) }\end{array}$ & $0.35[0.02,0.69]$ \\
\hline 4.1 During usual care & 0 & 0 & $\begin{array}{l}\text { Mean Difference (IV, Random, } \\
95 \% \mathrm{CI})\end{array}$ & $0.0[0.0,0.0]$ \\
\hline 4.2 After usual care & 3 & 349 & $\begin{array}{l}\text { Mean Difference (IV, Random, } \\
95 \% \mathrm{CI} \text { ) }\end{array}$ & $0.35[0.02,0.69]$ \\
\hline 5 Disability - combined disability scales & 5 & 452 & $\begin{array}{l}\text { Std. Mean Difference (IV, Ran- } \\
\text { dom, } 95 \% \mathrm{CI} \text { ) }\end{array}$ & $0.10[-0.17,0.37]$ \\
\hline 5.1 During usual care & 1 & 40 & $\begin{array}{l}\text { Std. Mean Difference (IV, Ran- } \\
\text { dom, } 95 \% \mathrm{CI} \text { ) }\end{array}$ & $0.53[-0.10,1.16]$ \\
\hline
\end{tabular}




\begin{tabular}{|c|c|c|c|c|}
\hline Outcome or subgroup title & $\begin{array}{l}\text { No. of } \\
\text { studies }\end{array}$ & $\begin{array}{l}\text { No. of } \\
\text { partici- } \\
\text { pants }\end{array}$ & Statistical method & Effect size \\
\hline 5.2 After usual care & 4 & 412 & $\begin{array}{l}\text { Std. Mean Difference (IV, Ran- } \\
\text { dom, } 95 \% \mathrm{CI} \text { ) }\end{array}$ & $0.04[-0.25,0.32]$ \\
\hline $\begin{array}{l}6 \text { Mobility - Functional Ambulation Cate- } \\
\text { gories }\end{array}$ & 1 & 242 & $\begin{array}{l}\text { Mean Difference (IV, Random, } \\
95 \% \mathrm{CI} \text { ) }\end{array}$ & $0.11[0.00,0.22]$ \\
\hline 6.1 During usual care & 0 & 0 & $\begin{array}{l}\text { Mean Difference (IV, Random, } \\
95 \% \mathrm{CI} \text { ) }\end{array}$ & $0.0[0.0,0.0]$ \\
\hline 6.2 After usual care & 1 & 242 & $\begin{array}{l}\text { Mean Difference (IV, Random, } \\
95 \% \mathrm{Cl} \text { ) }\end{array}$ & $0.11[0.00,0.22]$ \\
\hline $\begin{array}{l}7 \text { Mobility - walking preferred speed (m/ } \\
\text { min) }\end{array}$ & 5 & 542 & $\begin{array}{l}\text { Mean Difference (IV, Random, } \\
95 \% \mathrm{CI})\end{array}$ & $2.54[-3.65,8.72]$ \\
\hline 7.1 During usual care & 2 & 136 & $\begin{array}{l}\text { Mean Difference (IV, Random, } \\
95 \% \mathrm{CI})\end{array}$ & $-1.02[-8.64,6.60]$ \\
\hline 7.2 After usual care & 3 & 406 & $\begin{array}{l}\text { Mean Difference (IV, Random, } \\
95 \% \mathrm{CI})\end{array}$ & $4.29[-4.46,13.05]$ \\
\hline $\begin{array}{l}8 \text { Mobility - walking capacity (6-MWT me- } \\
\text { tres) }\end{array}$ & 4 & 464 & $\begin{array}{l}\text { Mean Difference (IV, Random, } \\
95 \% \mathrm{CI} \text { ) }\end{array}$ & $47.48[23.72,71.23]$ \\
\hline 8.1 During usual care & 1 & 40 & $\begin{array}{l}\text { Mean Difference (IV, Random, } \\
95 \% \mathrm{CI} \text { ) }\end{array}$ & $109.50[17.12,201.88]$ \\
\hline 8.2 After usual care & 3 & 424 & $\begin{array}{l}\text { Mean Difference (IV, Random, } \\
95 \% \mathrm{CI})\end{array}$ & $43.09[18.50,67.67]$ \\
\hline $\begin{array}{l}9 \text { Mobility - community ambulation speed } \\
\text { (>0.8 m/sec) }\end{array}$ & 3 & 217 & $\begin{array}{l}\text { Odds Ratio (M-H, Random, 95\% } \\
\mathrm{Cl} \text { ) }\end{array}$ & $1.33[0.70,2.53]$ \\
\hline 9.1 During usual care & 1 & 52 & $\begin{array}{l}\text { Odds Ratio (M-H, Random, 95\% } \\
\mathrm{Cl})\end{array}$ & $2.14[0.56,8.12]$ \\
\hline 9.2 After usual care & 2 & 165 & $\begin{array}{l}\text { Odds Ratio (M-H, Random, 95\% } \\
\mathrm{Cl})\end{array}$ & $1.15[0.48,2.76]$ \\
\hline $\begin{array}{l}10 \text { Physical function - balance - Berg Bal- } \\
\text { ance Scale }\end{array}$ & 3 & 201 & $\begin{array}{l}\text { Mean Difference (IV, Random, } \\
95 \% \mathrm{CI} \text { ) }\end{array}$ & $1.86[-3.05,6.78]$ \\
\hline 10.1 During usual care & 2 & 102 & $\begin{array}{l}\text { Mean Difference (IV, Random, } \\
95 \% \mathrm{CI})\end{array}$ & $2.22[-7.79,12.22]$ \\
\hline 10.2 After usual care & 1 & 99 & $\begin{array}{l}\text { Mean Difference (IV, Random, } \\
95 \% \mathrm{CI} \text { ) }\end{array}$ & $3.0[-0.54,6.54]$ \\
\hline $\begin{array}{l}11 \text { Physical function - balance - functional } \\
\text { reach }\end{array}$ & 1 & 66 & $\begin{array}{l}\text { Mean Difference (IV, Random, } \\
95 \% \mathrm{CI} \text { ) }\end{array}$ & $2.5[-0.97,5.97]$ \\
\hline 11.1 During usual care & 0 & 0 & $\begin{array}{l}\text { Mean Difference (IV, Random, } \\
95 \% \mathrm{CI} \text { ) }\end{array}$ & $0.0[0.0,0.0]$ \\
\hline
\end{tabular}




\begin{tabular}{|c|c|c|c|c|}
\hline Outcome or subgroup title & $\begin{array}{l}\text { No. of } \\
\text { studies }\end{array}$ & $\begin{array}{l}\text { No. of } \\
\text { partici- } \\
\text { pants }\end{array}$ & Statistical method & Effect size \\
\hline 11.2 After usual care & 1 & 66 & $\begin{array}{l}\text { Mean Difference (IV, Random, } \\
95 \% \mathrm{Cl} \text { ) }\end{array}$ & $2.5[-0.97,5.97]$ \\
\hline $\begin{array}{l}12 \text { Physical function - Timed Up and Go } \\
\text { (sec) }\end{array}$ & 5 & 510 & $\begin{array}{l}\text { Mean Difference (IV, Random, } \\
95 \% \mathrm{CI})\end{array}$ & $-1.41[-3.74,0.92]$ \\
\hline 12.1 During usual care & 1 & 62 & $\begin{array}{l}\text { Mean Difference (IV, Random, } \\
95 \% \mathrm{CI} \text { ) }\end{array}$ & $0.0[-6.97,6.97]$ \\
\hline 12.2 After usual care & 4 & 448 & $\begin{array}{l}\text { Mean Difference (IV, Random, } \\
95 \% \mathrm{CI})\end{array}$ & $-1.61[-4.39,1.17]$ \\
\hline $\begin{array}{l}13 \text { Health-related QoL - SF-36 physical } \\
\text { functioning }\end{array}$ & 2 & 146 & $\begin{array}{l}\text { Mean Difference (IV, Random, } \\
95 \% \mathrm{CI})\end{array}$ & $2.46[-7.20,12.11]$ \\
\hline 13.1 During usual care & 0 & 0 & $\begin{array}{l}\text { Mean Difference (IV, Random, } \\
95 \% \mathrm{CI})\end{array}$ & $0.0[0.0,0.0]$ \\
\hline 13.2 After usual care & 2 & 146 & $\begin{array}{l}\text { Mean Difference (IV, Random, } \\
95 \% \mathrm{CI})\end{array}$ & $2.46[-7.20,12.11]$ \\
\hline $\begin{array}{l}14 \text { Health-related QoL - SF-36 physical role } \\
\text { functioning }\end{array}$ & 2 & 146 & $\begin{array}{l}\text { Mean Difference (IV, Random, } \\
95 \% \mathrm{CI})\end{array}$ & $11.61[2.38,20.84]$ \\
\hline 14.1 During usual care & 0 & 0 & $\begin{array}{l}\text { Mean Difference (IV, Random, } \\
95 \% \mathrm{CI} \text { ) }\end{array}$ & $0.0[0.0,0.0]$ \\
\hline 14.2 After usual care & 2 & 146 & $\begin{array}{l}\text { Mean Difference (IV, Random, } \\
95 \% \mathrm{CI})\end{array}$ & $11.61[2.38,20.84]$ \\
\hline $\begin{array}{l}15 \text { Mood - Stroke Impact Scale emotion } \\
\text { score }\end{array}$ & 2 & 322 & $\begin{array}{l}\text { Mean Difference (IV, Random, } \\
95 \% \mathrm{CI} \text { ) }\end{array}$ & $0.13[-3.26,3.51]$ \\
\hline 15.1 During usual care & 0 & 0 & $\begin{array}{l}\text { Mean Difference (IV, Random, } \\
95 \% \mathrm{CI})\end{array}$ & $0.0[0.0,0.0]$ \\
\hline 15.2 After usual care & 2 & 322 & $\begin{array}{l}\text { Mean Difference (IV, Random, } \\
95 \% \mathrm{CI})\end{array}$ & $0.13[-3.26,3.51]$ \\
\hline 16 Mood - Geriatric Depression Scale & 1 & 80 & $\begin{array}{l}\text { Mean Difference (IV, Random, } \\
95 \% \mathrm{CI})\end{array}$ & $-1.4[-2.54,-0.26]$ \\
\hline 16.1 During usual care & 0 & 0 & $\begin{array}{l}\text { Mean Difference (IV, Random, } \\
95 \% \mathrm{CI} \text { ) }\end{array}$ & $0.0[0.0,0.0]$ \\
\hline 16.2 After usual care & 1 & 80 & $\begin{array}{l}\text { Mean Difference (IV, Random, } \\
95 \% \mathrm{CI} \text { ) }\end{array}$ & $-1.4[-2.54,-0.26]$ \\
\hline $\begin{array}{l}17 \text { Mood - Hospital Anxiety and Depression } \\
\text { Scale (HADS) - anxiety score }\end{array}$ & 3 & 391 & $\begin{array}{l}\text { Mean Difference (IV, Random, } \\
95 \% \mathrm{CI} \text { ) }\end{array}$ & $-0.11[-0.78,0.57]$ \\
\hline 17.1 During usual care & 0 & 0 & $\begin{array}{l}\text { Mean Difference (IV, Random, } \\
95 \% \mathrm{CI} \text { ) }\end{array}$ & $0.0[0.0,0.0]$ \\
\hline
\end{tabular}




\begin{tabular}{|c|c|c|c|c|}
\hline Outcome or subgroup title & $\begin{array}{l}\text { No. of } \\
\text { studies }\end{array}$ & $\begin{array}{l}\text { No. of } \\
\text { partici- } \\
\text { pants }\end{array}$ & Statistical method & Effect size \\
\hline 17.2 After usual care & 3 & 391 & $\begin{array}{l}\text { Mean Difference (IV, Random, } \\
95 \% \mathrm{Cl})\end{array}$ & $-0.11[-0.78,0.57]$ \\
\hline $\begin{array}{l}18 \text { Mood - Hospital Anxiety and Depression } \\
\text { Scale (HADS) - depression score }\end{array}$ & 3 & 391 & $\begin{array}{l}\text { Mean Difference (IV, Random, } \\
95 \% \mathrm{CI})\end{array}$ & $0.26[-0.43,0.96]$ \\
\hline 18.1 During usual care & 0 & 0 & $\begin{array}{l}\text { Mean Difference (IV, Random, } \\
95 \% \mathrm{Cl} \text { ) }\end{array}$ & $0.0[0.0,0.0]$ \\
\hline 18.2 After usual care & 3 & 391 & $\begin{array}{l}\text { Mean Difference (IV, Random, } \\
95 \% \mathrm{Cl} \text { ) }\end{array}$ & $0.26[-0.43,0.96]$ \\
\hline 19 Mood - combined depression scales & 4 & 471 & $\begin{array}{l}\text { Std. Mean Difference (IV, Ran- } \\
\text { dom, } 95 \% \mathrm{Cl} \text { ) }\end{array}$ & $-0.06[-0.33,0.22]$ \\
\hline 19.1 During usual care & 0 & 0 & $\begin{array}{l}\text { Std. Mean Difference (IV, Ran- } \\
\text { dom, } 95 \% \mathrm{CI} \text { ) }\end{array}$ & $0.0[0.0,0.0]$ \\
\hline 19.2 After usual care & 4 & 471 & $\begin{array}{l}\text { Std. Mean Difference (IV, Ran- } \\
\text { dom, } 95 \% \mathrm{CI} \text { ) }\end{array}$ & $-0.06[-0.33,0.22]$ \\
\hline 20 Cognitive function - FIM cognitive score & 1 & 93 & $\begin{array}{l}\text { Mean Difference (IV, Random, } \\
95 \% \mathrm{CI} \text { ) }\end{array}$ & $0.40[-0.19,0.99]$ \\
\hline 20.1 During usual care & 0 & 0 & $\begin{array}{l}\text { Mean Difference (IV, Random, } \\
95 \% \mathrm{Cl} \text { ) }\end{array}$ & $0.0[0.0,0.0]$ \\
\hline 20.2 After usual care & 1 & 93 & $\begin{array}{l}\text { Mean Difference (IV, Random, } \\
95 \% \mathrm{Cl} \text { ) }\end{array}$ & $0.40[-0.19,0.99]$ \\
\hline $\begin{array}{l}21 \text { Cognitive function - SIS memory and } \\
\text { thinking }\end{array}$ & 1 & 93 & $\begin{array}{l}\text { Mean Difference (IV, Random, } \\
95 \% \mathrm{CI} \text { ) }\end{array}$ & $4.30[-3.32,11.92]$ \\
\hline 21.1 During usual care & 0 & 0 & $\begin{array}{l}\text { Mean Difference (IV, Random, } \\
95 \% \mathrm{CI} \text { ) }\end{array}$ & $0.0[0.0,0.0]$ \\
\hline 21.2 After usual care & 1 & 93 & $\begin{array}{l}\text { Mean Difference (IV, Random, } \\
95 \% \mathrm{Cl} \text { ) }\end{array}$ & $4.30[-3.32,11.92]$ \\
\hline 22 Cognitive function - SIS communication & 1 & 93 & $\begin{array}{l}\text { Mean Difference (IV, Random, } \\
95 \% \mathrm{Cl} \text { ) }\end{array}$ & $2.90[-4.16,9.96]$ \\
\hline 22.1 During usual care & 0 & 0 & $\begin{array}{l}\text { Mean Difference (IV, Random, } \\
95 \% \mathrm{CI})\end{array}$ & $0.0[0.0,0.0]$ \\
\hline 22.2 After usual care & 1 & 93 & $\begin{array}{l}\text { Mean Difference (IV, Random, } \\
95 \% \mathrm{Cl} \text { ) }\end{array}$ & $2.90[-4.16,9.96]$ \\
\hline
\end{tabular}


Analysis 6.1. Comparison 6 Mixed training versus control - end of retention follow-up, Outcome 1 Death.

\begin{tabular}{|c|c|c|c|c|c|}
\hline Study or subgroup & $\begin{array}{c}\text { Training } \\
\mathrm{n} / \mathrm{N}\end{array}$ & $\begin{array}{c}\text { Control } \\
\mathrm{n} / \mathrm{N}\end{array}$ & $\begin{array}{c}\text { Risk Difference } \\
\text { M-H, Random, } 95 \% \mathrm{Cl}\end{array}$ & Weight & $\begin{array}{c}\text { Risk Difference } \\
\text { M-H, Random, } 95 \% \mathrm{Cl}\end{array}$ \\
\hline \multicolumn{6}{|c|}{ 6.1.1 During usual care } \\
\hline Cooke 2010 & $0 / 36$ & $2 / 38$ & - & $4.18 \%$ & $-0.05[-0.14,0.03]$ \\
\hline Donaldson 2009 & $0 / 10$ & $0 / 10$ & & $1.01 \%$ & $0[-0.17,0.17]$ \\
\hline Galvin 2011 & $0 / 20$ & $2 / 20$ & + & $1.3 \%$ & $-0.1[-0.25,0.05]$ \\
\hline Richards 1993 & $0 / 10$ & $0 / 8$ & & $0.81 \%$ & $0[-0.19,0.19]$ \\
\hline Toledano-Zarhi 2011 & $0 / 14$ & $0 / 14$ & & $1.85 \%$ & $0[-0.13,0.13]$ \\
\hline Subtotal $(95 \% \mathrm{CI})$ & 122 & 121 & & $17.67 \%$ & $-0.02[-0.06,0.02]$ \\
\hline \multicolumn{6}{|c|}{ Total events: 0 (Training), 4 (Control) } \\
\hline \multicolumn{6}{|c|}{ Heterogeneity: $\operatorname{Tau}^{2}=0 ; \mathrm{Chi}^{2}=2.58, \mathrm{df}=5(\mathrm{P}=0.76) ; \mathrm{I}^{2}=0 \%$} \\
\hline \multicolumn{6}{|c|}{ Test for overall effect: $Z=0.94(P=0.35)$} \\
\hline Dean 2018 & $0 / 23$ & $0 / 22$ & & $4.48 \%$ & $0[-0.08,0.08]$ \\
\hline Duncan 1998 & $0 / 10$ & $0 / 10$ & & $1.01 \%$ & $0[-0.17,0.17]$ \\
\hline Duncan 2003 & $1 / 50$ & $2 / 50$ & & $6.85 \%$ & $-0.02[-0.09,0.05]$ \\
\hline Knox 2018 & $3 / 51$ & $2 / 48$ & - & $4.14 \%$ & $0.02[-0.07,0.1]$ \\
\hline Mead 2007 & $0 / 32$ & $0 / 34$ & & $9.3 \%$ & $0[-0.06,0.06]$ \\
\hline Van de Port 2012 & $0 / 126$ & $2 / 124$ & \# & $42.33 \%$ & $-0.02[-0.04,0.01]$ \\
\hline Zedlitz 2012 & $0 / 38$ & $0 / 45$ & $\longrightarrow$ & $14.23 \%$ & $0[-0.05,0.05]$ \\
\hline Subtotal $(95 \% \mathrm{Cl})$ & 330 & 333 & & $82.33 \%$ & $-0.01[-0.03,0.01]$ \\
\hline \multicolumn{6}{|c|}{ Total events: 4 (Training), 6 (Control) } \\
\hline \multicolumn{6}{|c|}{ Heterogeneity: $\operatorname{Tau}^{2}=0 ; \mathrm{Chi}^{2}=1.09, \mathrm{df}=6(\mathrm{P}=0.98) ; \mathrm{I}^{2}=0 \%$} \\
\hline \multicolumn{6}{|c|}{ Test for overall effect: $\mathrm{Z}=0.93(\mathrm{P}=0.35)$} \\
\hline Total $(95 \% \mathrm{Cl})$ & 452 & 454 & & $100 \%$ & $-0.01[-0.03,0.01]$ \\
\hline \multicolumn{6}{|c|}{ Heterogeneity: $\mathrm{Tau}^{2}=0 ; \mathrm{Chi}^{2}=3.54, \mathrm{df}=12(\mathrm{P}=0.99) ; \mathrm{I}^{2}=0 \%$} \\
\hline \multicolumn{6}{|c|}{ Test for overall effect: $Z=1.23(P=0.22)$} \\
\hline Test for subgroup diff & , $d f=1(P=0.65)$, & & & & \\
\hline
\end{tabular}

Analysis 6.2. Comparison 6 Mixed training versus control - end of retention follow-up, Outcome 2 Disability - Barthel Index (BI).

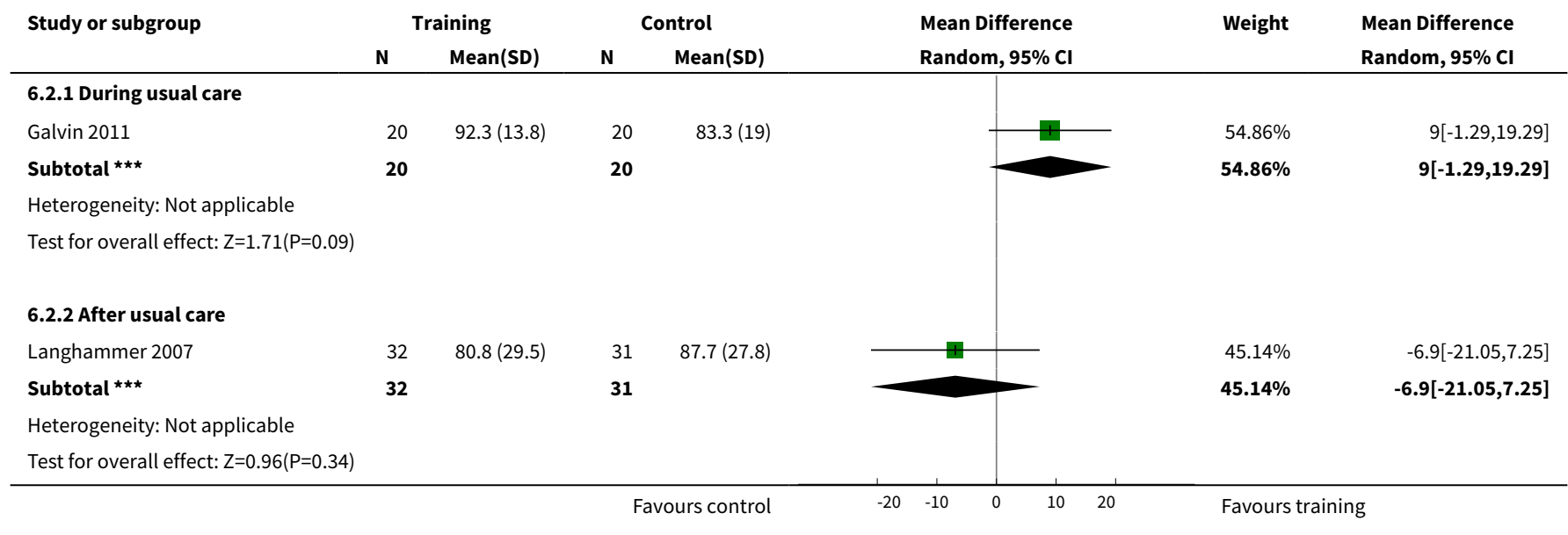




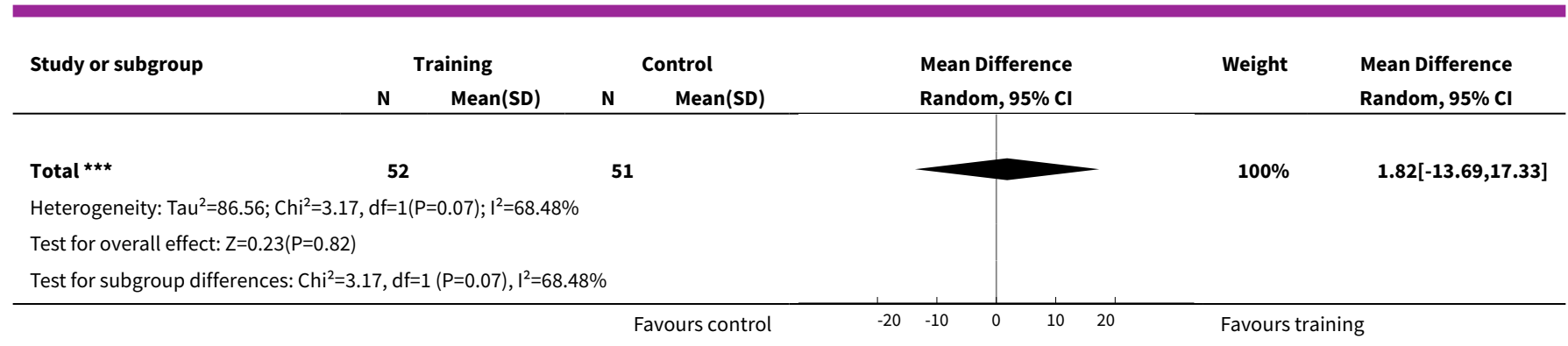

Analysis 6.3. Comparison 6 Mixed training versus control - end of retention follow-up, Outcome 3 Disability - Nottingham Extended ADL.

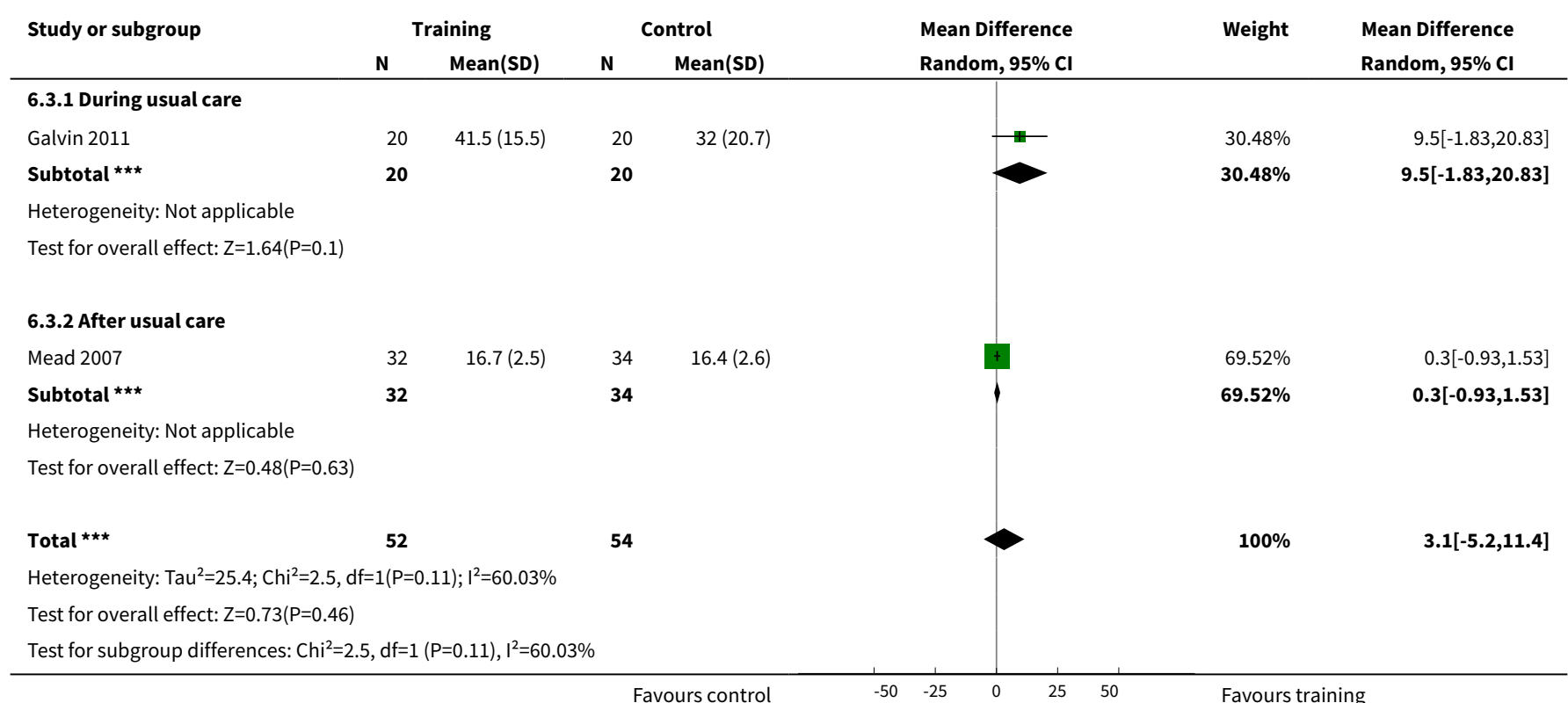

Analysis 6.4. Comparison 6 Mixed training versus control - end of retention follow-up, Outcome 4 Disability - Rivermead Mobility Index (RMI).

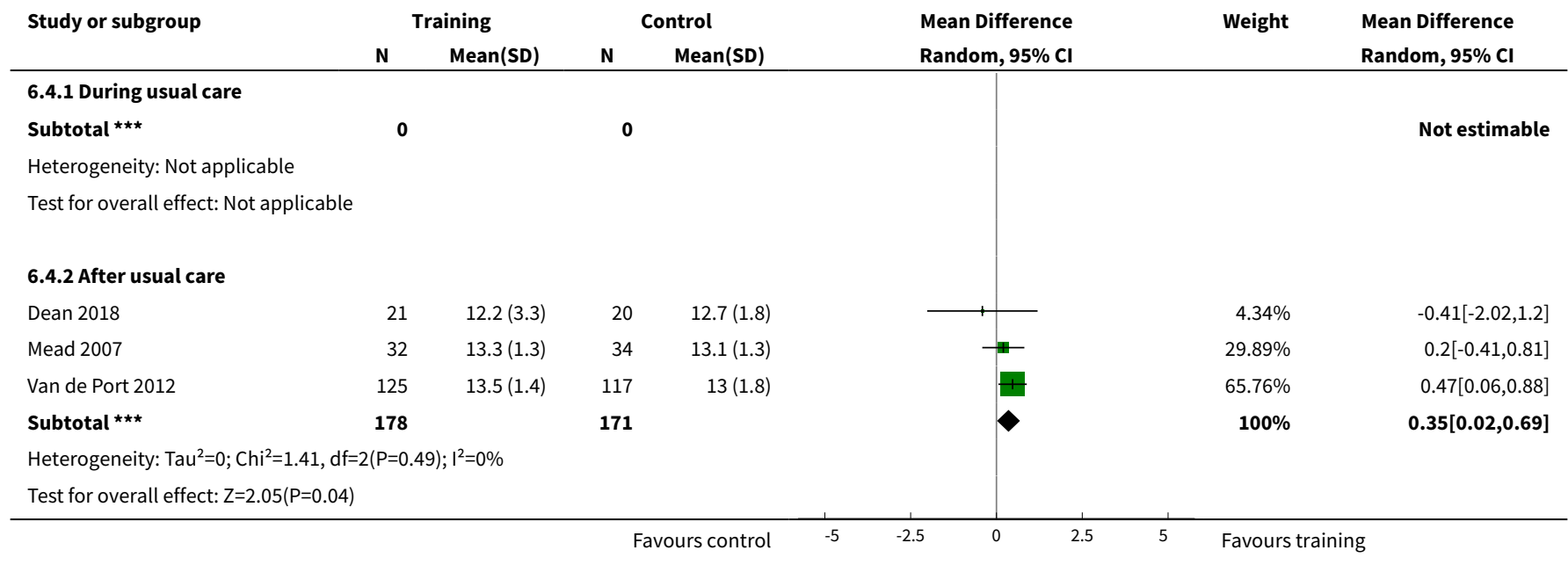




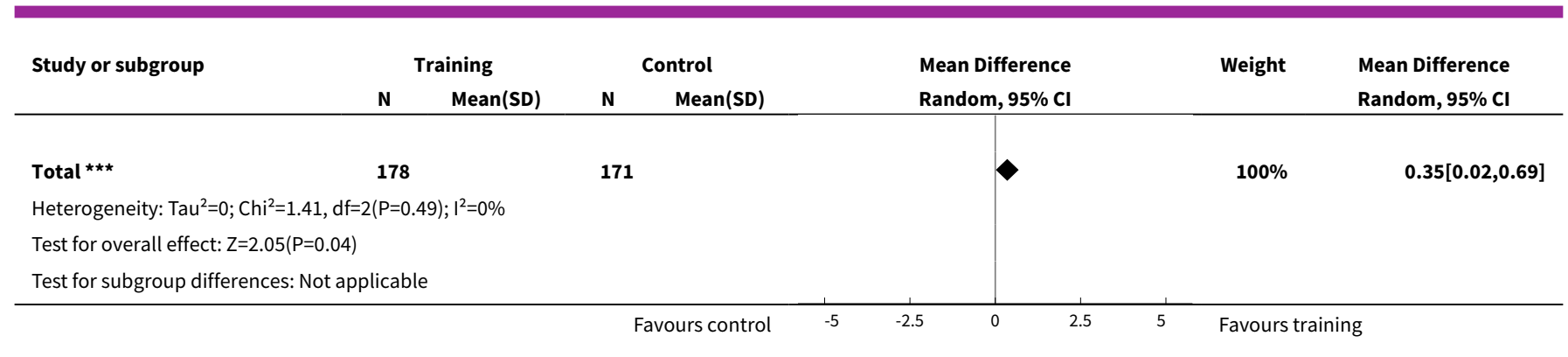

Analysis 6.5. Comparison 6 Mixed training versus control - end of retention follow-up, Outcome 5 Disability - combined disability scales.

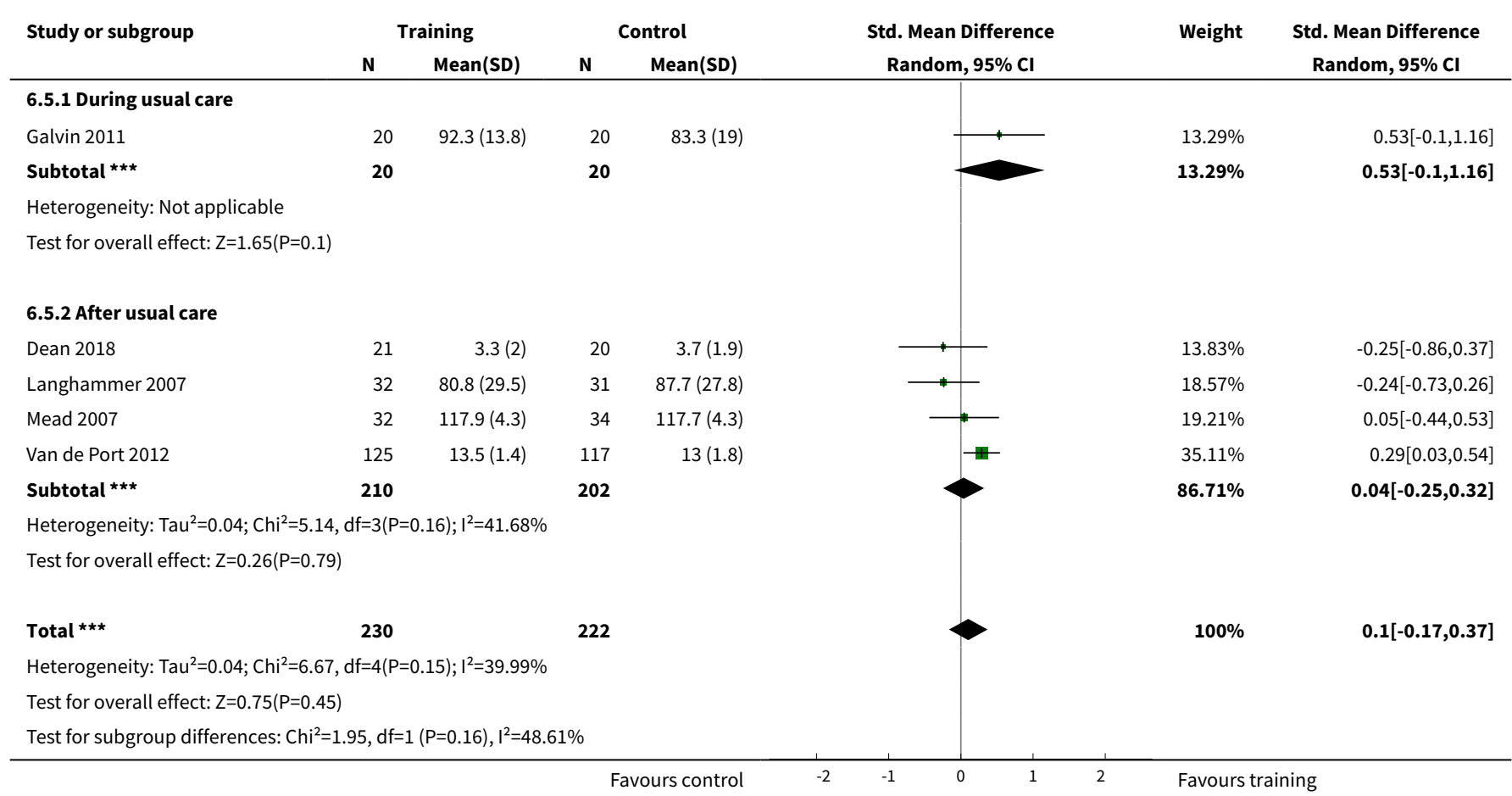

Analysis 6.6. Comparison 6 Mixed training versus control - end of retention follow-up, Outcome 6 Mobility - Functional Ambulation Categories.

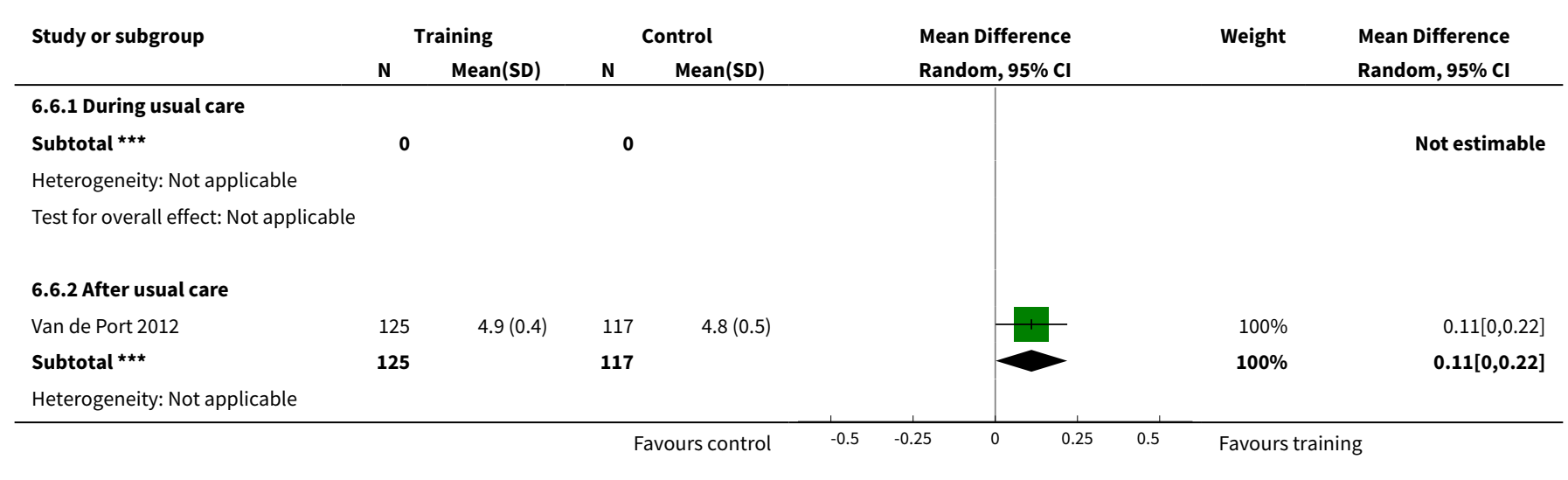




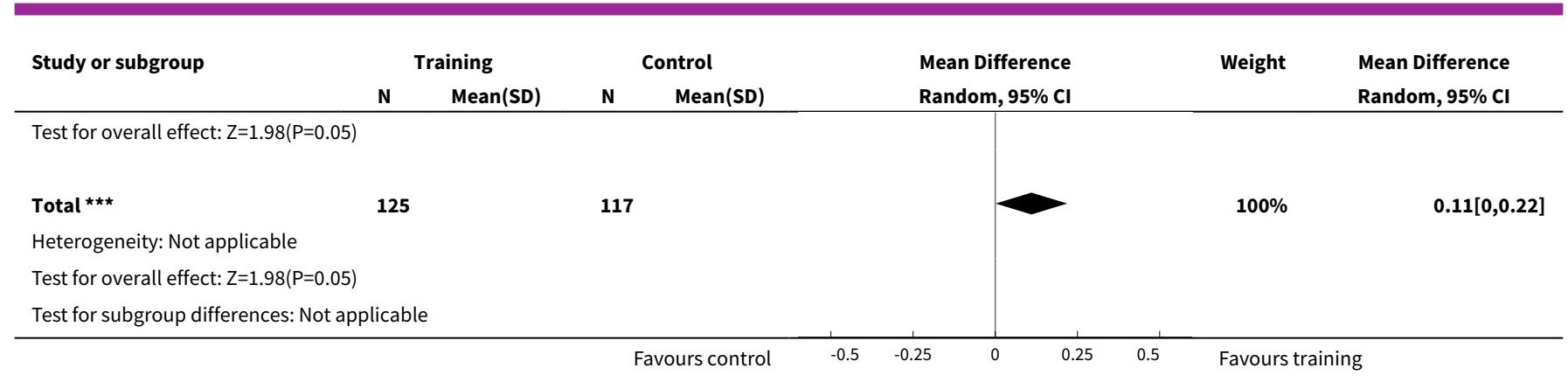

Analysis 6.7. Comparison 6 Mixed training versus control - end of retention follow-up, Outcome 7 Mobility - walking preferred speed ( $\mathrm{m} / \mathrm{min}$ ).

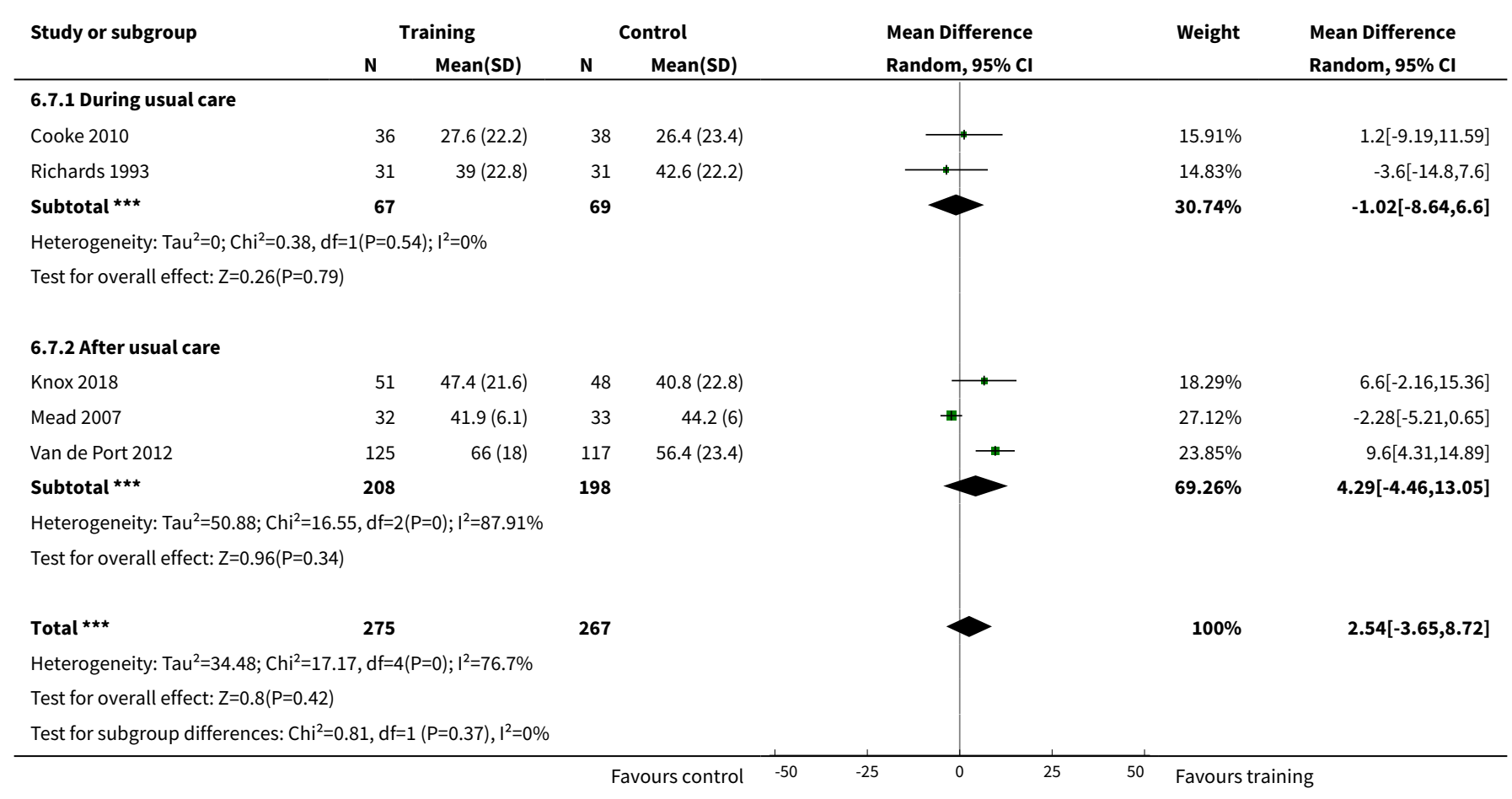

Analysis 6.8. Comparison 6 Mixed training versus control - end of retention follow-up, Outcome 8 Mobility - walking capacity (6-MWT metres).

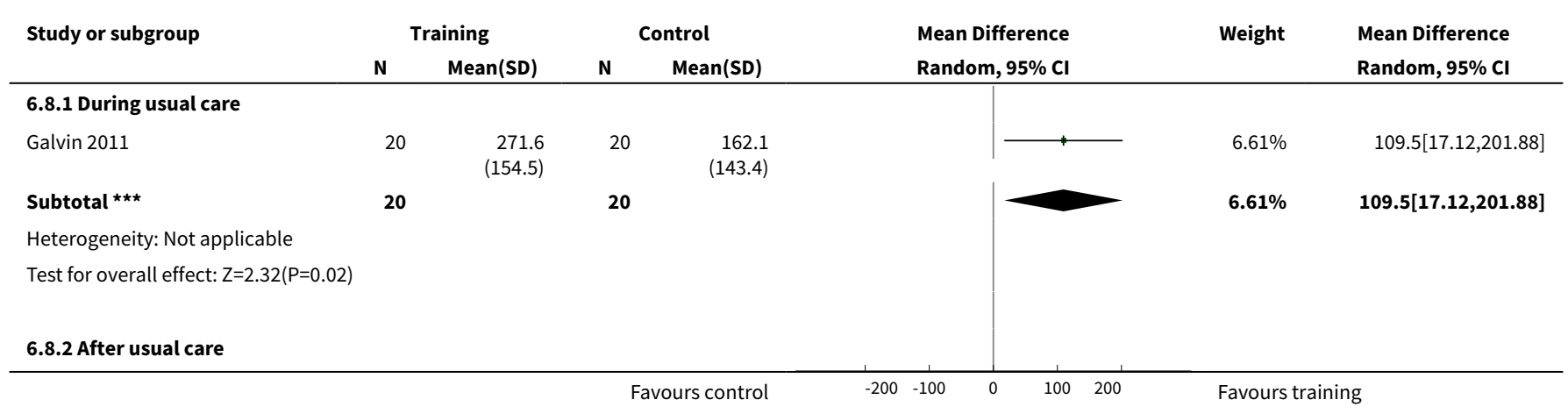




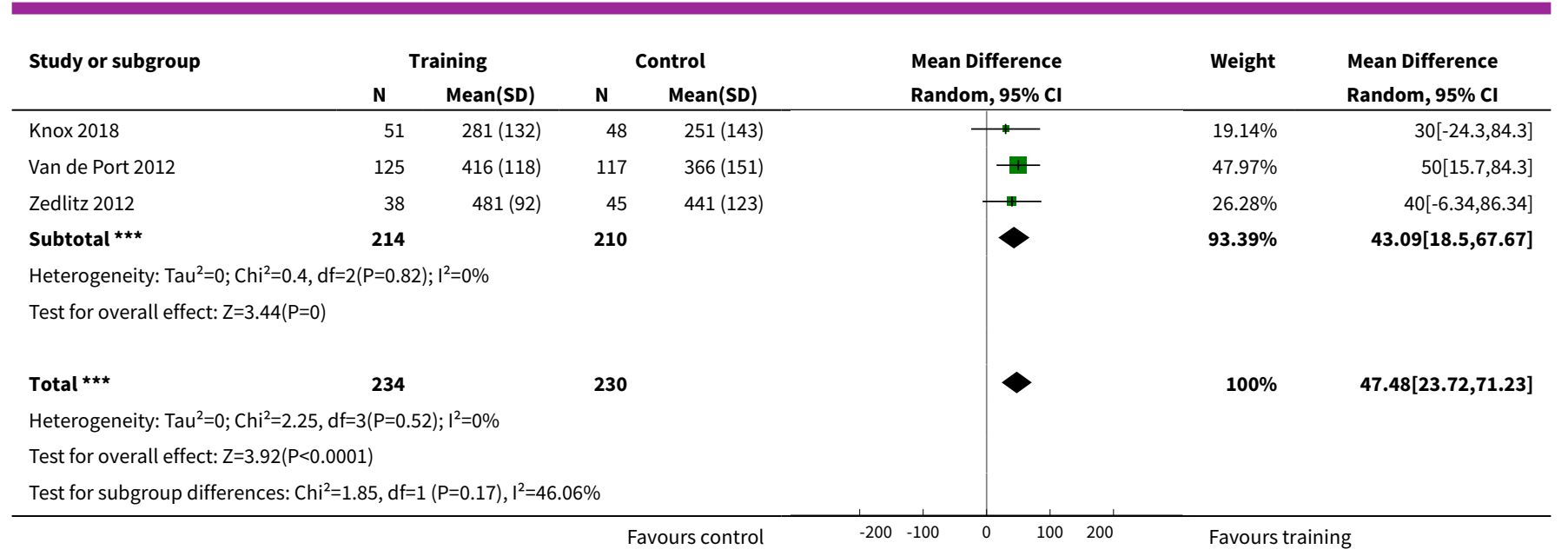

Analysis 6.9. Comparison 6 Mixed training versus control - end of retention follow-up, Outcome 9 Mobility - community ambulation speed ( $>0.8 \mathrm{~m} / \mathrm{sec}$ ).

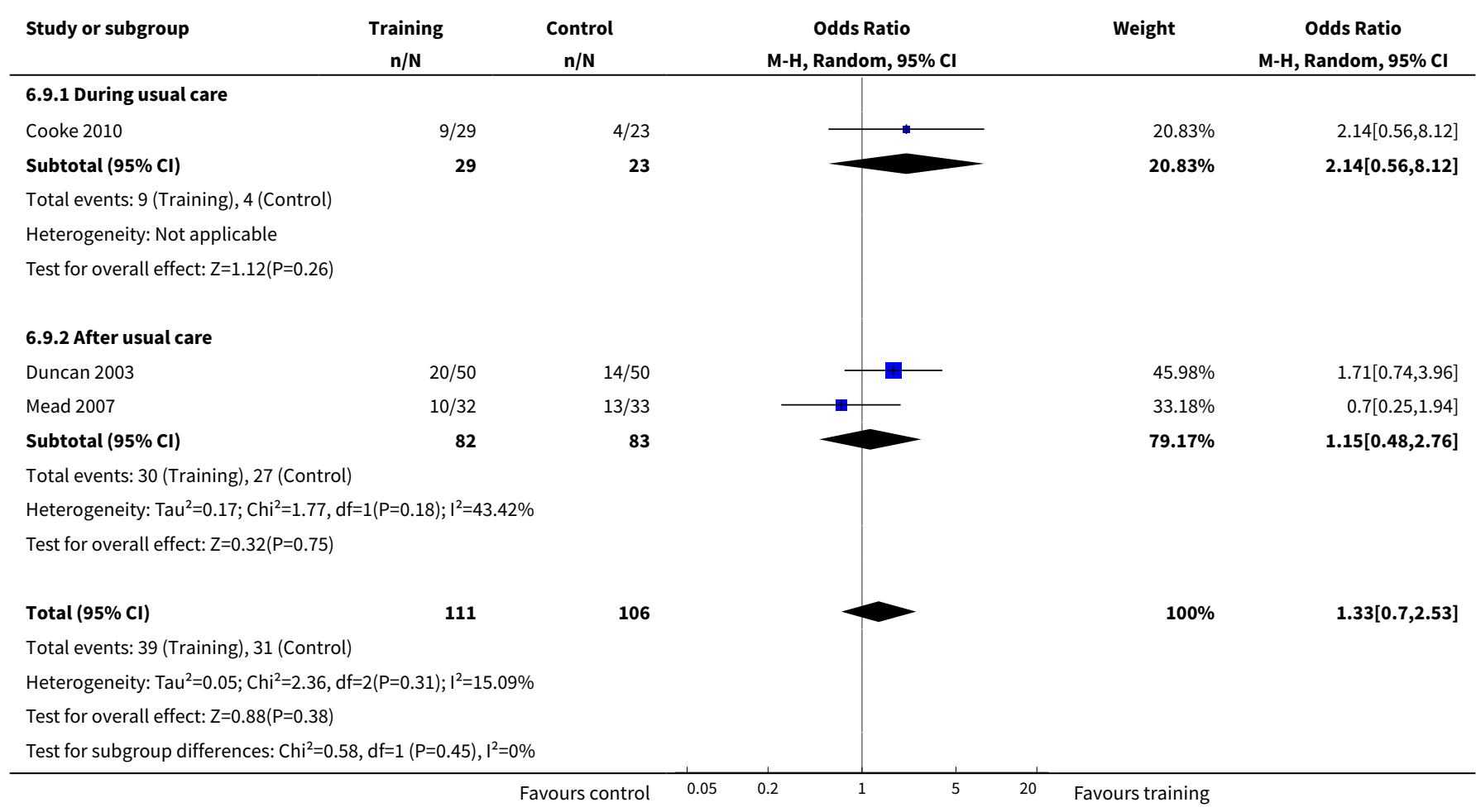

Analysis 6.10. Comparison 6 Mixed training versus control - end of retention follow-up, Outcome 10 Physical function - balance - Berg Balance Scale.

\begin{tabular}{lllllllll} 
Study or subgroup & \multicolumn{2}{c}{$\begin{array}{c}\text { Training } \\
\text { N }\end{array}$} & Mean(SD) & N & $\begin{array}{c}\text { Control } \\
\text { Mean(SD) }\end{array}$ & $\begin{array}{l}\text { Mean Difference } \\
\text { Random, 95\% Cl }\end{array}$ & $\begin{array}{l}\text { Wean Difference } \\
\text { Random, 95\% Cl }\end{array}$ \\
\hline $\begin{array}{l}\text { 6.10.1 During usual care } \\
\text { Galvin } 2011\end{array}$ & 20 & $46(14.2)$ & 20 & $37.6(16.2)$ & & & & \\
\hline
\end{tabular}




\begin{tabular}{|c|c|c|c|c|c|c|c|}
\hline \multirow[t]{2}{*}{ Study or subgroup } & \multicolumn{2}{|c|}{ Training } & \multicolumn{2}{|c|}{ Control } & \multirow{2}{*}{$\begin{array}{l}\text { Mean Difference } \\
\text { Random, 95\% Cl }\end{array}$} & \multirow[t]{2}{*}{ Weight } & \multirow{2}{*}{$\begin{array}{l}\text { Mean Difference } \\
\text { Random, 95\% Cl }\end{array}$} \\
\hline & $\mathbf{N}$ & $\operatorname{Mean}(S D)$ & $\mathbf{N}$ & Mean(SD) & & & \\
\hline Richards 2004 & 31 & $47(7)$ & 31 & $49(6)$ & -1 & $41.85 \%$ & $-2[-5.25,1.25]$ \\
\hline Subtotal $\star \star \star ~$ & 51 & & 51 & & & $59.56 \%$ & $2.22[-7.79,12.22]$ \\
\hline \multicolumn{8}{|c|}{ Heterogeneity: $\mathrm{Tau}^{2}=41.11 ; \mathrm{Chi}^{2}=4.17, \mathrm{df}=1(\mathrm{P}=0.04) ; \mathrm{I}^{2}=76.01 \%$} \\
\hline \multicolumn{8}{|c|}{ Test for overall effect: $Z=0.43(P=0.66)$} \\
\hline \multicolumn{8}{|c|}{ 6.10.2 After usual care } \\
\hline Knox 2018 & 51 & $50(6.7)$ & 48 & $47(10.7)$ & +1 & $40.44 \%$ & $3[-0.54,6.54]$ \\
\hline 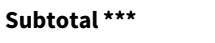 & 51 & & 48 & & & $40.44 \%$ & $3[-0.54,6.54]$ \\
\hline \multicolumn{8}{|c|}{ Heterogeneity: Not applicable } \\
\hline \multicolumn{8}{|c|}{ Test for overall effect: $Z=1.66(P=0.1)$} \\
\hline Total ${ }^{\star \star \star}$ & 102 & & 99 & & & $100 \%$ & $1.86[-3.05,6.78]$ \\
\hline \multicolumn{8}{|c|}{ Heterogeneity: Tau $^{2}=12.26 ; \mathrm{Chi}^{2}=6.83, \mathrm{df}=2(\mathrm{P}=0.03) ; \mathrm{I}^{2}=70.72 \%$} \\
\hline \multicolumn{8}{|c|}{ Test for overall effect: $Z=0.74(P=0.46)$} \\
\hline \multicolumn{8}{|c|}{ Test for subgroup differences: $\mathrm{Chi}^{2}=0.02, \mathrm{df}=1(\mathrm{P}=0.88), \mathrm{I}^{2}=0 \%$} \\
\hline & & & & urs control & $-10 \quad-5$ & Favours & \\
\hline
\end{tabular}

Analysis 6.11. Comparison 6 Mixed training versus control - end of retention follow-up, Outcome 11 Physical function - balance - functional reach.

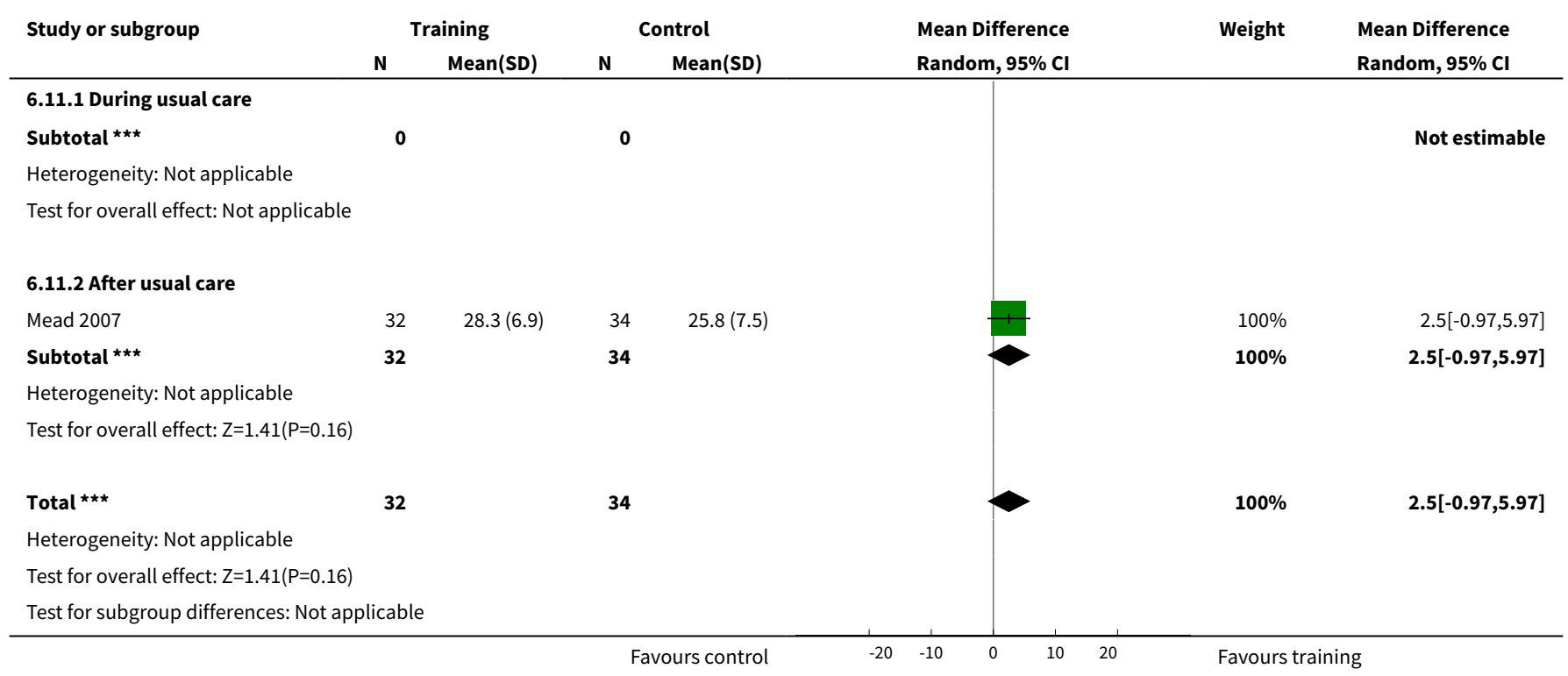

Analysis 6.12. Comparison 6 Mixed training versus control - end of retention follow-up, Outcome 12 Physical function - Timed Up and Go (sec).

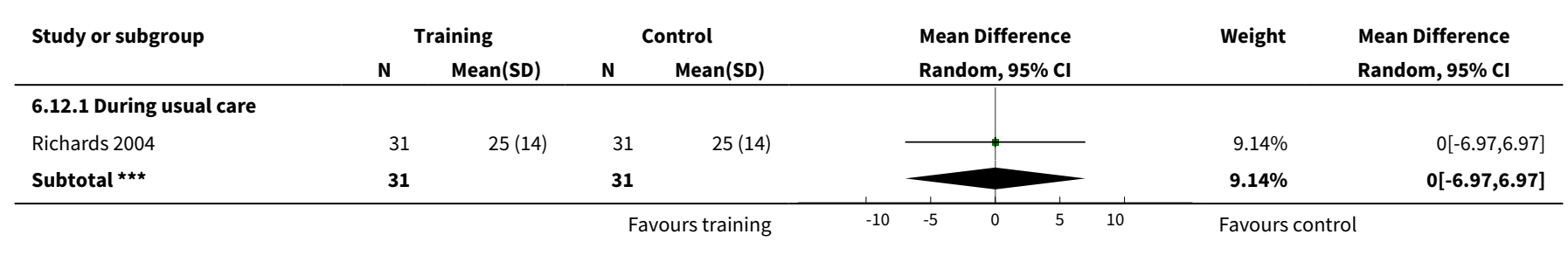




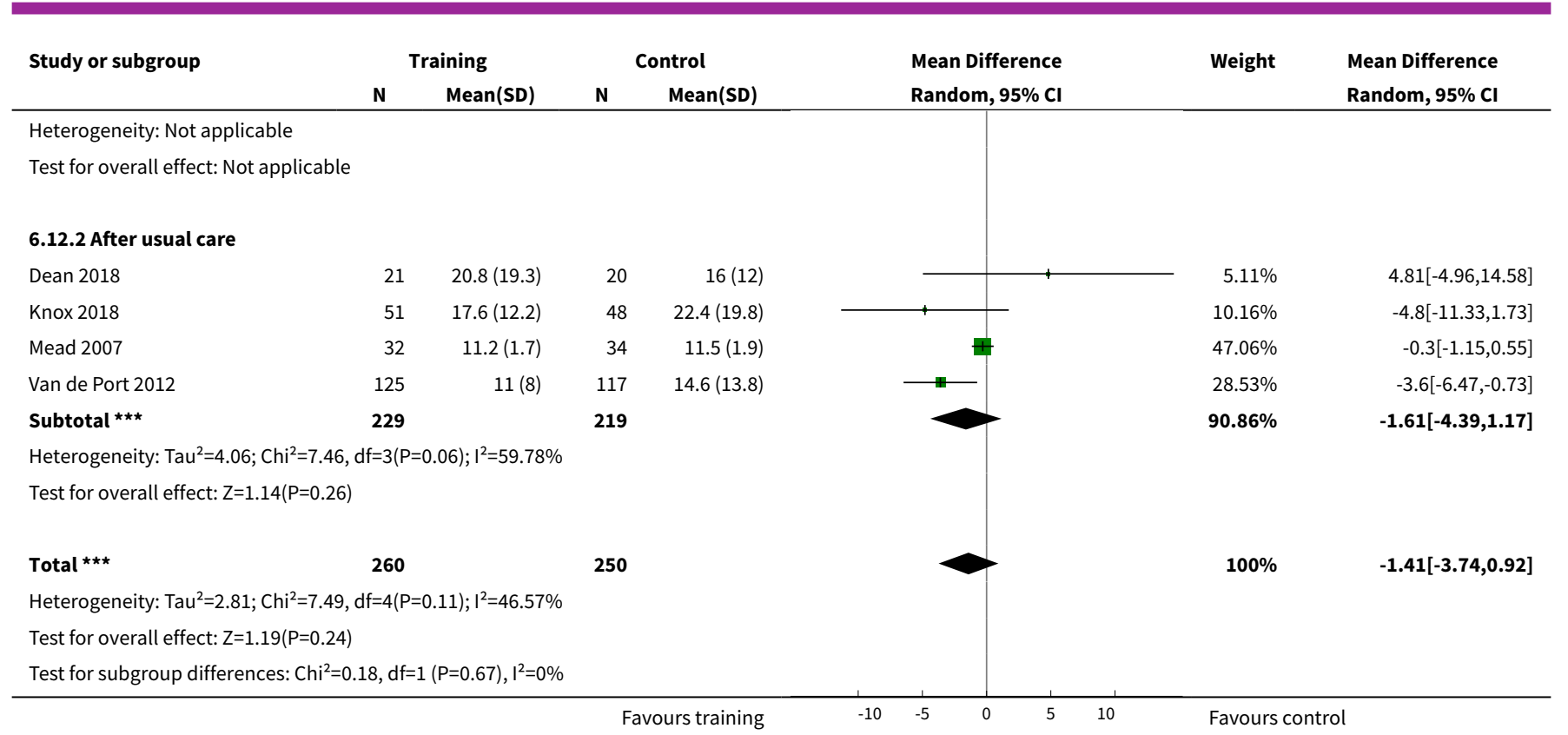

Analysis 6.13. Comparison 6 Mixed training versus control - end of retention follow-up, Outcome 13 Health-related QoL - SF-36 physical functioning.

\begin{tabular}{|c|c|c|c|c|c|c|c|}
\hline \multirow[t]{2}{*}{ Study or subgroup } & \multicolumn{2}{|c|}{ Training } & \multicolumn{2}{|c|}{ Control } & \multirow{2}{*}{$\begin{array}{l}\text { Mean Difference } \\
\text { Random, 95\% Cl }\end{array}$} & \multirow[t]{2}{*}{ Weight } & \multirow{2}{*}{$\begin{array}{l}\text { Mean Difference } \\
\text { Random, } 95 \% \mathrm{Cl}\end{array}$} \\
\hline & $\mathbf{N}$ & $\operatorname{Mean}(S D)$ & $\mathbf{N}$ & $\operatorname{Mean}(S D)$ & & & \\
\hline \multicolumn{8}{|c|}{ 6.13.1 During usual care } \\
\hline Subtotal $\star \star \star ~$ & 0 & & 0 & & & & Not estimable \\
\hline \multicolumn{8}{|c|}{ Heterogeneity: Not applicable } \\
\hline \multicolumn{8}{|c|}{ Test for overall effect: Not applicable } \\
\hline Duncan 2003 & 40 & $58.9(22.7)$ & 40 & $51(22.9)$ & 1 & $45.01 \%$ & $7.9[-2.09,17.89]$ \\
\hline Mead 2007 & 32 & $55.8(16.4)$ & 34 & $57.8(16.3)$ & & $54.99 \%$ & $-2[-9.89,5.89]$ \\
\hline Subtotal $\star \star \star ~$ & 72 & & 74 & & & $100 \%$ & $2.46[-7.2,12.11]$ \\
\hline \multicolumn{8}{|c|}{ Heterogeneity: $\mathrm{Tau}^{2}=27.9 ; \mathrm{Chi}^{2}=2.32, \mathrm{df}=1(\mathrm{P}=0.13) ; \mathrm{I}^{2}=56.93 \%$} \\
\hline \multicolumn{8}{|c|}{ Test for overall effect: $Z=0.5(P=0.62)$} \\
\hline \multicolumn{8}{|c|}{ Heterogeneity: $\mathrm{Tau}^{2}=27.9 ; \mathrm{Chi}^{2}=2.32, \mathrm{df}=1(\mathrm{P}=0.13) ; \mathrm{I}^{2}=56.93 \%$} \\
\hline \multicolumn{8}{|c|}{ Test for overall effect: $Z=0.5(P=0.62)$} \\
\hline \multicolumn{8}{|c|}{ Test for subgroup differences: Not applicable } \\
\hline & & & & urs control & $-20-10$ & Favourst & \\
\hline
\end{tabular}

Analysis 6.14. Comparison 6 Mixed training versus control - end of retention follow-up, Outcome 14 Health-related QoL - SF-36 physical role functioning.

\begin{tabular}{|c|c|c|c|c|c|c|c|c|c|}
\hline \multirow{2}{*}{$\begin{array}{l}\text { Study or subgroup } \\
\text { 6.14.1 During usual care }\end{array}$} & \multicolumn{2}{|c|}{ Training } & \multicolumn{2}{|c|}{ Control } & \multirow{2}{*}{\multicolumn{3}{|c|}{$\begin{array}{l}\text { Mean Difference } \\
\text { Random, } 95 \% \mathrm{Cl}\end{array}$}} & \multirow[t]{2}{*}{ Weight } & \multirow{2}{*}{$\begin{array}{l}\text { Mean Difference } \\
\text { Random, } 95 \% \mathrm{Cl}\end{array}$} \\
\hline & $\mathbf{N}$ & Mean(SD) & $\mathbf{N}$ & $\operatorname{Mean}(\mathrm{SD})$ & & & & & \\
\hline
\end{tabular}




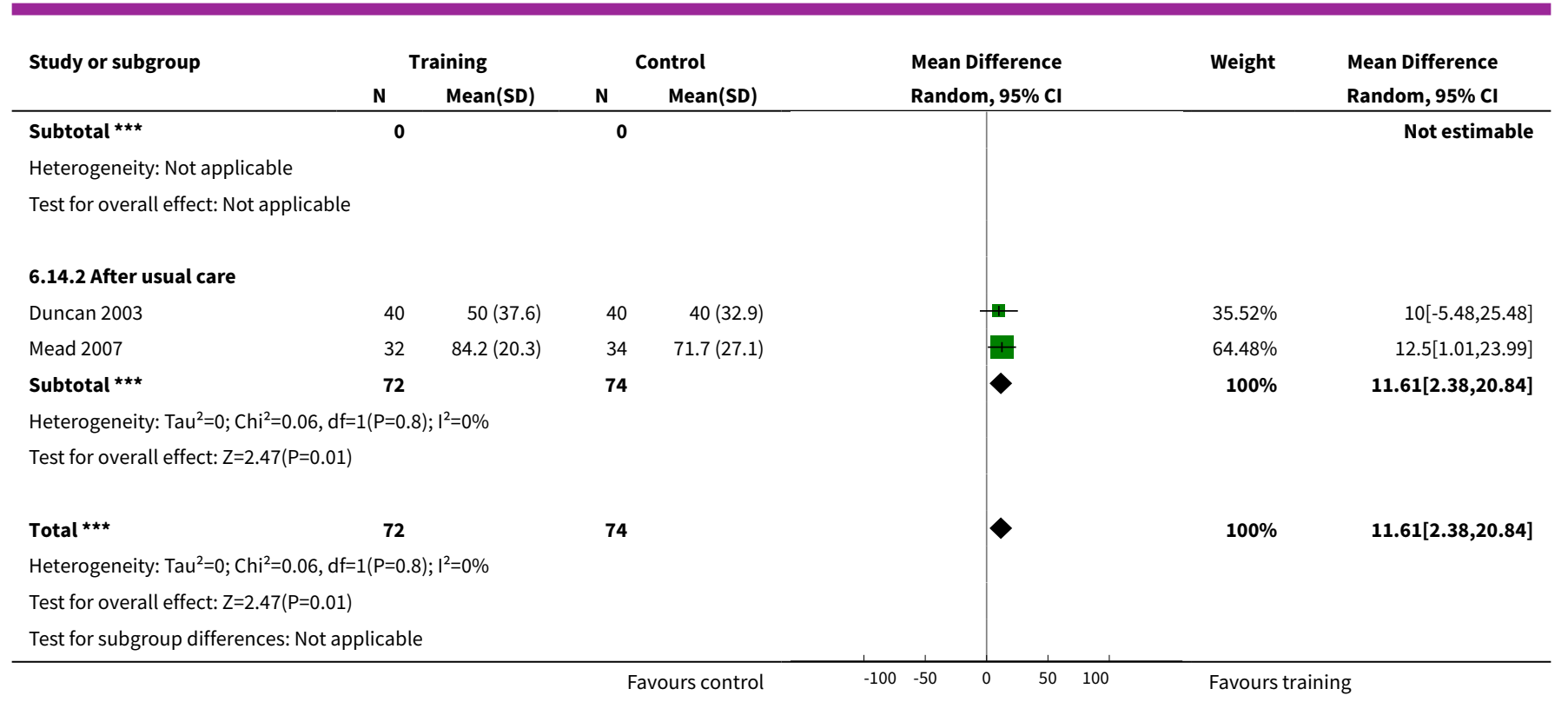

Analysis 6.15. Comparison 6 Mixed training versus control - end of retention follow-up, Outcome 15 Mood - Stroke Impact Scale emotion score.

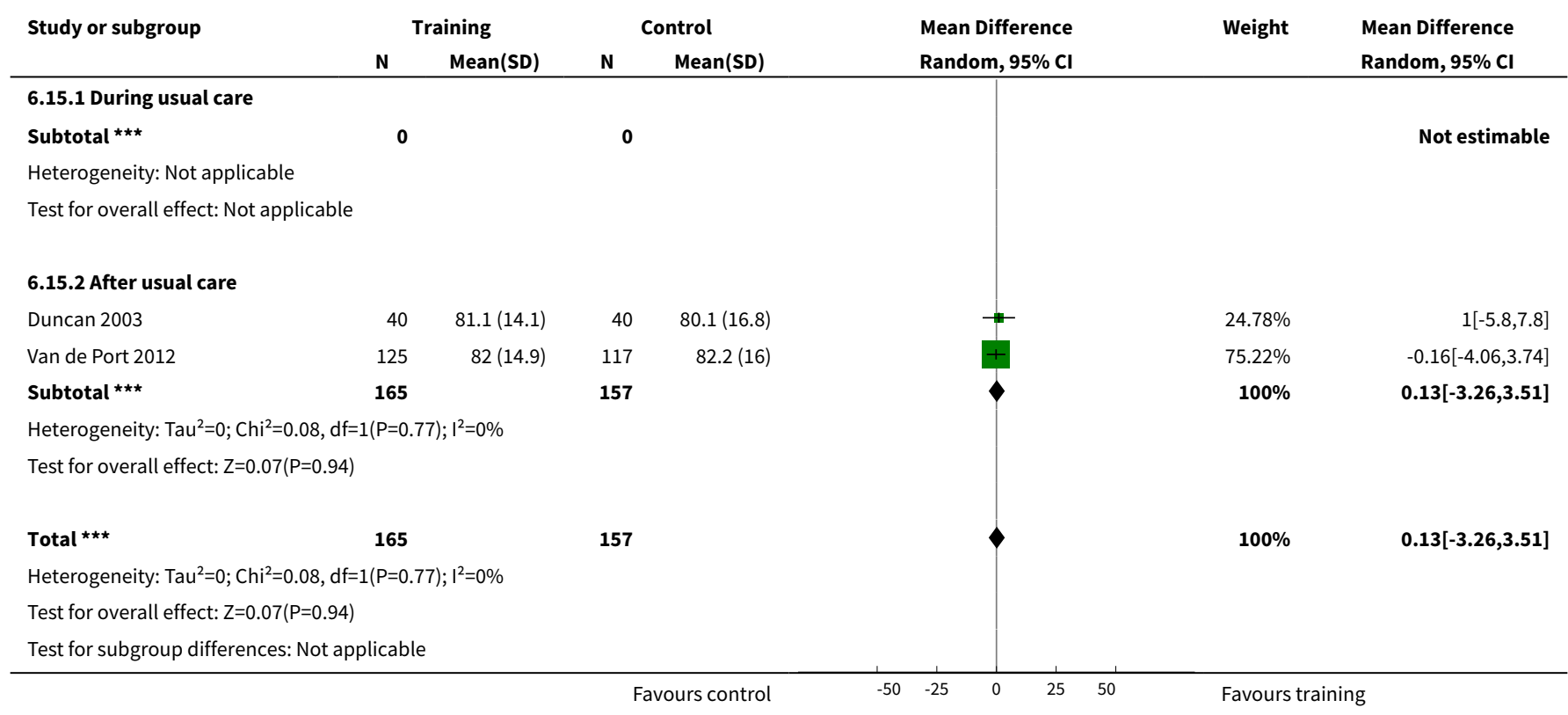

Analysis 6.16. Comparison 6 Mixed training versus control - end of retention follow-up, Outcome 16 Mood - Geriatric Depression Scale.

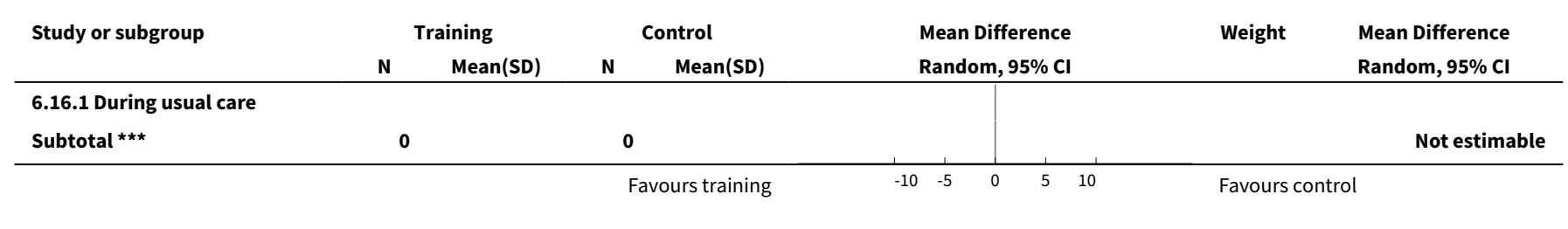




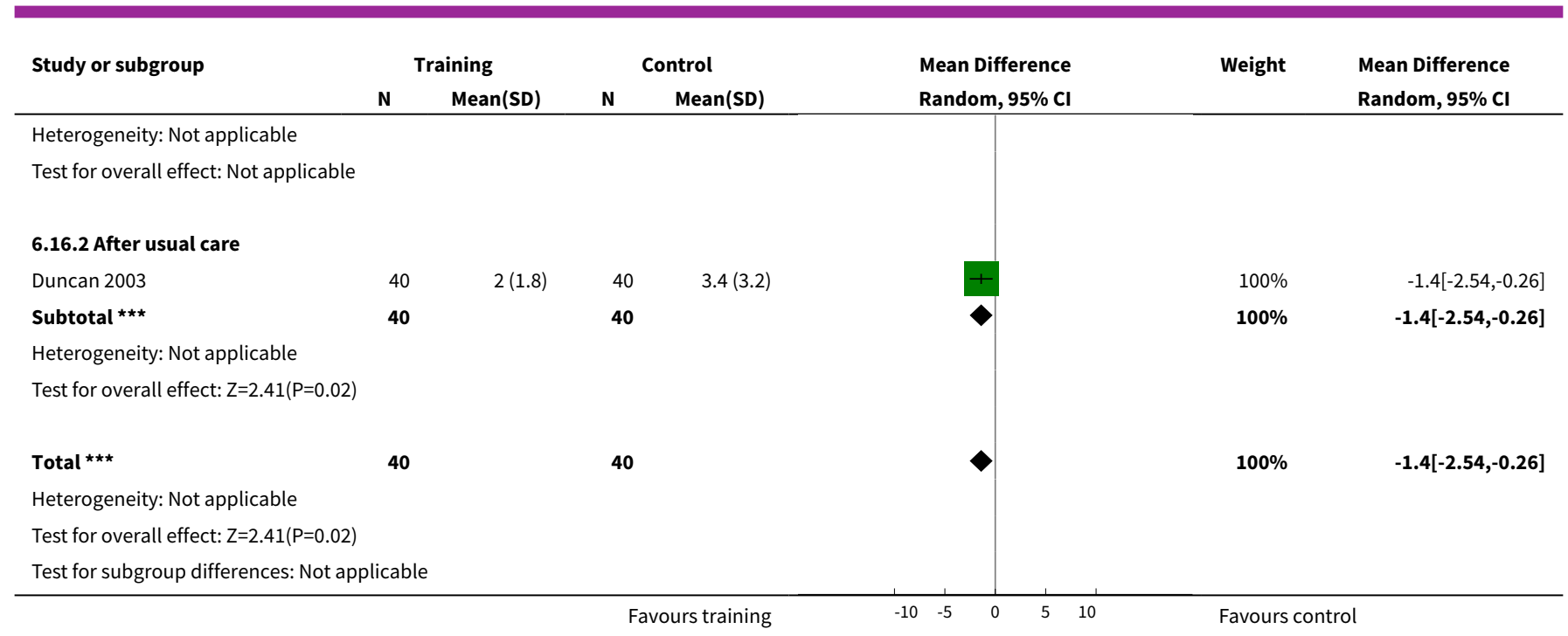

Analysis 6.17. Comparison 6 Mixed training versus control - end of retention followup, Outcome 17 Mood - Hospital Anxiety and Depression Scale (HADS) - anxiety score.

\begin{tabular}{|c|c|c|c|c|c|c|c|}
\hline \multirow[t]{2}{*}{ Study or subgroup } & \multicolumn{2}{|c|}{ Training } & \multicolumn{2}{|c|}{ Control } & \multirow{2}{*}{$\begin{array}{l}\text { Mean Difference } \\
\text { Random, } 95 \% \mathrm{Cl} \\
\end{array}$} & \multirow[t]{2}{*}{ Weight } & \multirow{2}{*}{$\begin{array}{l}\text { Mean Difference } \\
\text { Random, } 95 \% \mathrm{Cl}\end{array}$} \\
\hline & $\mathbf{N}$ & Mean(SD) & $\mathbf{N}$ & Mean(SD) & & & \\
\hline \multicolumn{8}{|c|}{ 6.17.1 During usual care } \\
\hline Subtotal $\star \star \star$ & 0 & & 0 & & & & Not estimable \\
\hline \multicolumn{8}{|c|}{ Heterogeneity: Not applicable } \\
\hline \multicolumn{8}{|c|}{ Test for overall effect: Not applicable } \\
\hline Mead 2007 & 32 & $4(3.2)$ & 34 & $4.2(3.2)$ & 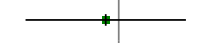 & $19.18 \%$ & $-0.25[-1.79,1.29]$ \\
\hline Van de Port 2012 & 125 & $3.7(3.1)$ & 117 & $3.7(3.6)$ & & $63.78 \%$ & $-0.01[-0.86,0.84]$ \\
\hline Zedlitz 2012 & 38 & $5.8(3.6)$ & 45 & $6.1(4)$ & & $17.04 \%$ & $-0.3[-1.94,1.34]$ \\
\hline Subtotal $* \star \star$ & 195 & & 196 & & & $100 \%$ & $-0.11[-0.78,0.57]$ \\
\hline \multicolumn{8}{|c|}{ Heterogeneity: $\operatorname{Tau}^{2}=0 ; \mathrm{Chi}^{2}=0.14, \mathrm{df}=2(\mathrm{P}=0.93) ; \mathrm{I}^{2}=0 \%$} \\
\hline \multicolumn{8}{|c|}{ Test for overall effect: $Z=0.31(P=0.76)$} \\
\hline Total $\star \star \star$ & 195 & & 196 & & & $100 \%$ & $-0.11[-0.78,0.57]$ \\
\hline \multicolumn{8}{|c|}{ Heterogeneity: $\operatorname{Tau}^{2}=0 ; \mathrm{Chi}^{2}=0.14, \mathrm{df}=2(\mathrm{P}=0.93) ; \mathrm{I}^{2}=0 \%$} \\
\hline \multicolumn{8}{|c|}{ Test for overall effect: $Z=0.31(P=0.76)$} \\
\hline \multicolumn{8}{|c|}{ Test for subgroup differences: Not applicable } \\
\hline & & & & rs training & -2 & Favours & \\
\hline
\end{tabular}

Analysis 6.18. Comparison 6 Mixed training versus control - end of retention followup, Outcome 18 Mood - Hospital Anxiety and Depression Scale (HADS) - depression score.

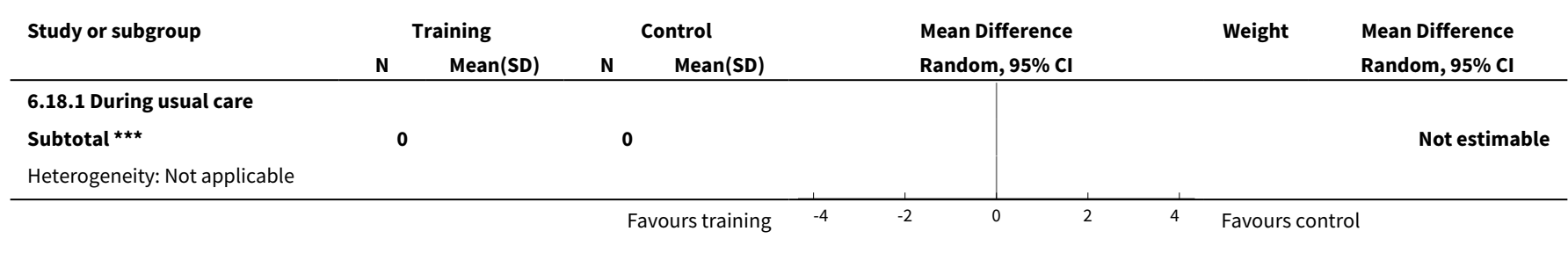




\begin{tabular}{|c|c|c|c|c|c|c|c|}
\hline \multirow[t]{2}{*}{ Study or subgroup } & \multicolumn{2}{|c|}{ Training } & \multicolumn{2}{|c|}{ Control } & \multirow{2}{*}{$\begin{array}{l}\text { Mean Difference } \\
\text { Random, } 95 \% \mathrm{CI}\end{array}$} & \multirow[t]{2}{*}{ Weight } & \multirow{2}{*}{$\begin{array}{l}\text { Mean Difference } \\
\text { Random, } 95 \% \mathrm{CI}\end{array}$} \\
\hline & $\mathbf{N}$ & Mean(SD) & $\mathbf{N}$ & Mean(SD) & & & \\
\hline \multicolumn{8}{|c|}{ Test for overall effect: Not applicable } \\
\hline \multicolumn{8}{|c|}{ 6.18.2 After usual care } \\
\hline Mead 2007 & 32 & $4.2(3)$ & 34 & $4(3)$ & & $23.22 \%$ & $0.18[-1.27,1.63]$ \\
\hline Van de Port 2012 & 125 & $4.5(3.5)$ & 117 & $4.3(4)$ & & $53.62 \%$ & $0.24[-0.71,1.19]$ \\
\hline Zedlitz 2012 & 38 & $6.1(3.4)$ & 45 & $5.7(3.3)$ & & $23.16 \%$ & $0.4[-1.05,1.85]$ \\
\hline Subtotal $\star \star \star ~$ & 195 & & 196 & & & $100 \%$ & $0.26[-0.43,0.96]$ \\
\hline \multicolumn{8}{|c|}{ Heterogeneity: $\operatorname{Tau}^{2}=0 ; \mathrm{Chi}^{2}=0.05, \mathrm{df}=2(\mathrm{P}=0.98) ; \mathrm{I}^{2}=0 \%$} \\
\hline \multicolumn{8}{|c|}{ Test for overall effect: $Z=0.74(P=0.46)$} \\
\hline 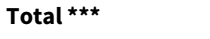 & 195 & & 196 & & & $100 \%$ & $0.26[-0.43,0.96]$ \\
\hline \multicolumn{8}{|c|}{ Heterogeneity: $\operatorname{Tau}^{2}=0 ; \mathrm{Chi}^{2}=0.05, \mathrm{df}=2(\mathrm{P}=0.98) ; \mathrm{I}^{2}=0 \%$} \\
\hline \multicolumn{8}{|c|}{ Test for overall effect: $Z=0.74(P=0.46)$} \\
\hline \multicolumn{8}{|c|}{ Test for subgroup differences: Not applicable } \\
\hline
\end{tabular}

Analysis 6.19. Comparison 6 Mixed training versus control - end of retention follow-up, Outcome 19 Mood - combined depression scales.

\begin{tabular}{|c|c|c|c|c|c|c|c|}
\hline \multirow[t]{2}{*}{ Study or subgroup } & \multicolumn{2}{|c|}{ Training } & \multicolumn{2}{|c|}{ Control } & \multirow{2}{*}{$\begin{array}{c}\text { Std. Mean Difference } \\
\text { Random, } 95 \% \mathrm{Cl}\end{array}$} & \multirow[t]{2}{*}{ Weight } & \multirow{2}{*}{$\begin{array}{c}\text { Std. Mean Difference } \\
\text { Random, } 95 \% \mathrm{Cl}\end{array}$} \\
\hline & $\mathbf{N}$ & $\operatorname{Mean}(\mathrm{SD})$ & $\mathbf{N}$ & $\operatorname{Mean}(S D)$ & & & \\
\hline \multicolumn{8}{|c|}{ 6.19.1 During usual care } \\
\hline Subtotal $\star \star \star$ & 0 & & 0 & & & & Not estimable \\
\hline \multicolumn{8}{|c|}{ Heterogeneity: Not applicable } \\
\hline \multicolumn{8}{|c|}{ Test for overall effect: Not applicable } \\
\hline \multicolumn{8}{|c|}{ 6.19.2 After usual care } \\
\hline Duncan 2003 & 40 & $2(1.8)$ & 40 & $3.4(3.2)$ & $*$ & $21.84 \%$ & $-0.53[-0.98,-0.09]$ \\
\hline Mead 2007 & 32 & $4.2(3)$ & 34 & $4(3)$ & + & $19.91 \%$ & $0.06[-0.42,0.54]$ \\
\hline Van de Port 2012 & 125 & $4.5(3.5)$ & 117 & $4.3(4)$ & 中 & $35.61 \%$ & $0.06[-0.19,0.32]$ \\
\hline Zedlitz 2012 & 38 & $6.1(3.4)$ & 45 & $5.7(3.3)$ & + & $22.64 \%$ & $0.12[-0.31,0.55]$ \\
\hline Subtotal $\star \star \star$ & 235 & & 236 & & $\checkmark$ & $100 \%$ & $-0.06[-0.33,0.22]$ \\
\hline \multicolumn{8}{|c|}{ Heterogeneity: $\mathrm{Tau}^{2}=0.04 ; \mathrm{Chi}^{2}=6.01, \mathrm{df}=3(\mathrm{P}=0.11) ;\left.\right|^{2}=50.09 \%$} \\
\hline Total $\star \star \star$ & 235 & & 236 & & 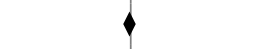 & $100 \%$ & $-0.06[-0.33,0.22]$ \\
\hline \multicolumn{8}{|c|}{ Heterogeneity: $\mathrm{Tau}^{2}=0.04 ; \mathrm{Chi}^{2}=6.01, \mathrm{df}=3(\mathrm{P}=0.11) ; \mathrm{I}^{2}=50.09 \%$} \\
\hline \multicolumn{8}{|c|}{ Test for overall effect: $Z=0.39(P=0.7)$} \\
\hline \multicolumn{8}{|c|}{ Test for subgroup differences: Not applicable } \\
\hline & & & & urs training & -5 & Favours & \\
\hline
\end{tabular}

Analysis 6.20. Comparison 6 Mixed training versus control - end of retention follow-up, Outcome 20 Cognitive function - FIM cognitive score.

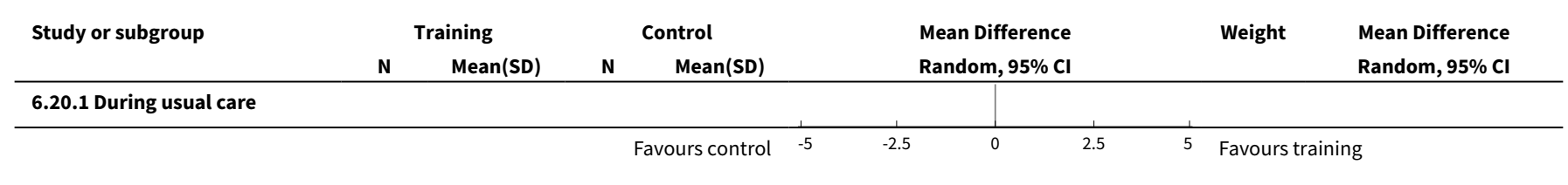




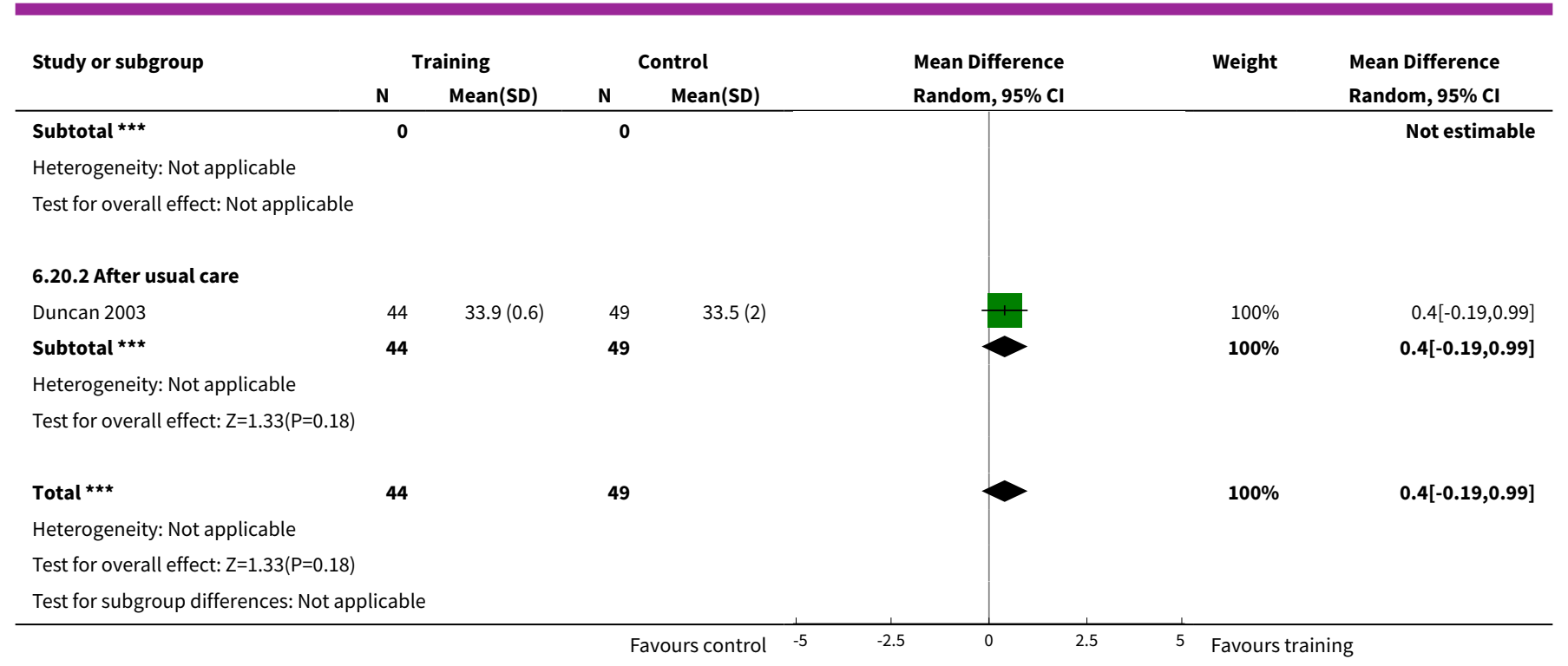

Analysis 6.21. Comparison 6 Mixed training versus control - end of retention follow-up, Outcome 21 Cognitive function - SIS memory and thinking.

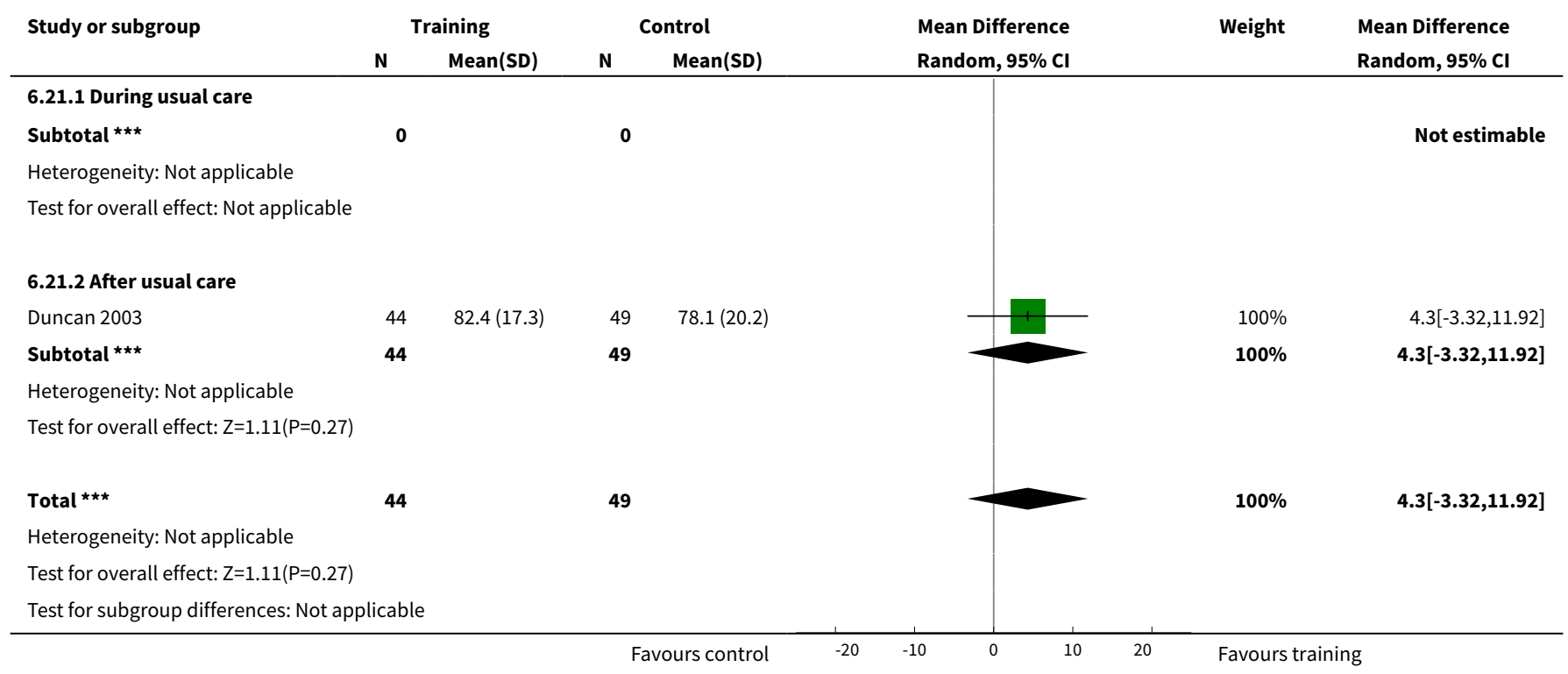

Analysis 6.22. Comparison 6 Mixed training versus control - end of retention follow-up, Outcome 22 Cognitive function - SIS communication.

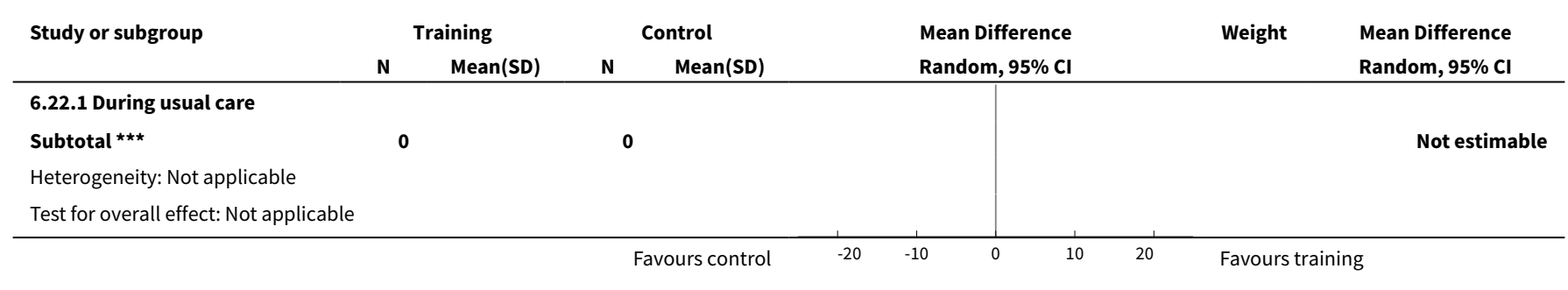




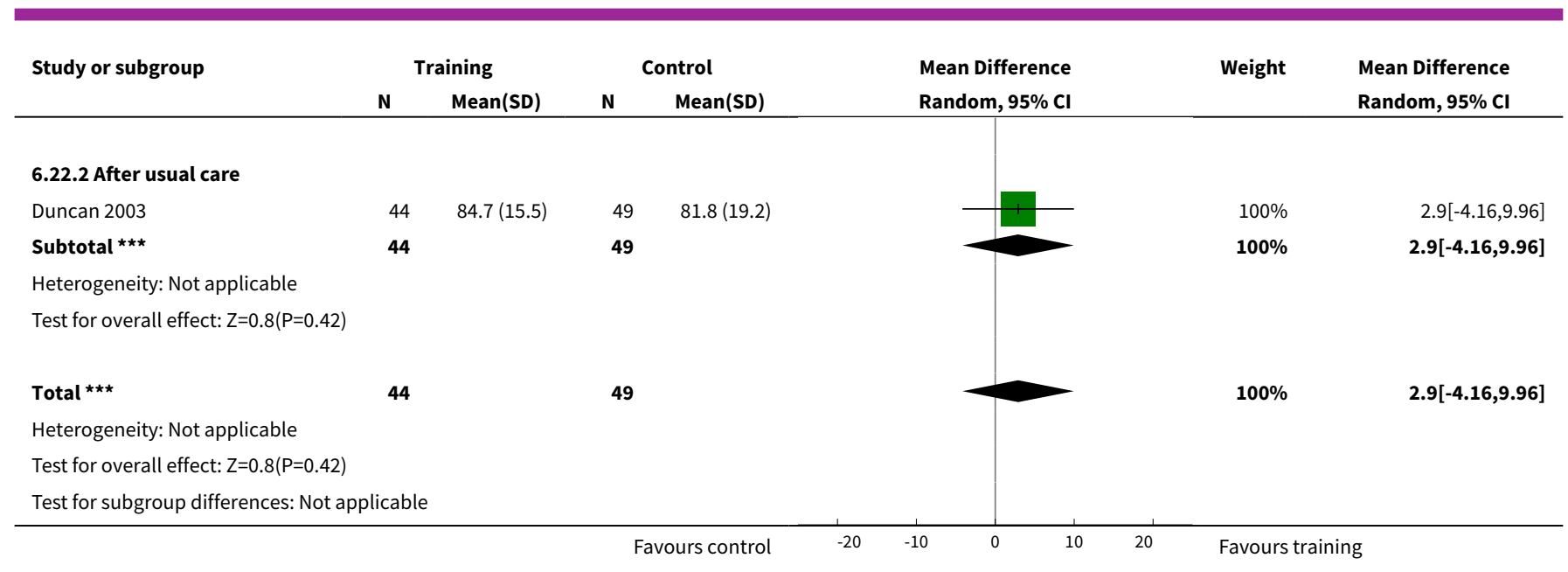

\section{Comparison 7. Cardiorespiratory versus resistance versus mixed training}

\begin{tabular}{|c|c|c|c|c|}
\hline Outcome or subgroup title & $\begin{array}{l}\text { No. of } \\
\text { studies }\end{array}$ & $\begin{array}{l}\text { No. of } \\
\text { partici- } \\
\text { pants }\end{array}$ & Statistical method & Effect size \\
\hline 1 Disability - combined disability scales & 18 & & $\begin{array}{l}\text { Std. Mean Difference (IV, Random, } \\
95 \% \mathrm{Cl})\end{array}$ & Subtotals only \\
\hline 1.1 Cardiorespiratory training & 8 & 462 & $\begin{array}{l}\text { Std. Mean Difference (IV, Random, } \\
95 \% \mathrm{Cl} \text { ) }\end{array}$ & $0.52[0.19,0.84]$ \\
\hline 1.2 Resistance training & 1 & 42 & $\begin{array}{l}\text { Std. Mean Difference (IV, Random, } \\
95 \% \mathrm{CI})\end{array}$ & $0.12[-0.48,0.73]$ \\
\hline 1.3 Mixed training & 9 & 604 & $\begin{array}{l}\text { Std. Mean Difference (IV, Random, } \\
95 \% \mathrm{CI})\end{array}$ & $0.23[0.03,0.42]$ \\
\hline 2 Mobility - walking maximal speed & 26 & 1176 & $\begin{array}{l}\text { Mean Difference (IV, Random, 95\% } \\
\text { CI) }\end{array}$ & $5.60[3.11,8.08]$ \\
\hline 2.1 Cardiorespiratory training & 17 & 782 & $\begin{array}{l}\text { Mean Difference (IV, Random, 95\% } \\
\text { CI) }\end{array}$ & $7.66[3.65,11.68]$ \\
\hline 2.2 Resistance training & 7 & 250 & $\begin{array}{l}\text { Mean Difference (IV, Random, 95\% } \\
\text { Cl) }\end{array}$ & $2.75[-0.61,6.12]$ \\
\hline 2.3 Mixed training & 3 & 144 & $\begin{array}{l}\text { Mean Difference (IV, Random, 95\% } \\
\text { CI) }\end{array}$ & $8.92[1.74,16.10]$ \\
\hline 3 Mobility - walking preferred speed & 26 & 1481 & $\begin{array}{l}\text { Mean Difference (IV, Random, 95\% } \\
\mathrm{Cl} \text { ) }\end{array}$ & $3.98[1.96,6.01]$ \\
\hline 3.1 Cardiorespiratory training & 12 & 588 & $\begin{array}{l}\text { Mean Difference (IV, Random, 95\% } \\
\text { CI) }\end{array}$ & $4.47[2.07,6.87]$ \\
\hline 3.2 Resistance training & 5 & 179 & $\begin{array}{l}\text { Mean Difference (IV, Random, 95\% } \\
\mathrm{CI} \text { ) }\end{array}$ & $1.97[-3.76,7.71]$ \\
\hline
\end{tabular}




\begin{tabular}{|c|c|c|c|c|}
\hline Outcome or subgroup title & $\begin{array}{l}\text { No. of } \\
\text { studies }\end{array}$ & $\begin{array}{l}\text { No. of } \\
\text { partici- } \\
\text { pants }\end{array}$ & Statistical method & Effect size \\
\hline 3.3 Mixed training & 10 & 714 & $\begin{array}{l}\text { Mean Difference (IV, Random, 95\% } \\
\mathrm{Cl})\end{array}$ & $4.68[1.26,8.09]$ \\
\hline $\begin{array}{l}4 \text { Mobility - walking capacity (6-MWT dis- } \\
\text { tance) }\end{array}$ & 30 & 1792 & $\begin{array}{l}\text { Mean Difference (IV, Random, 95\% } \\
\mathrm{Cl})\end{array}$ & $30.14[21.27,39.00]$ \\
\hline 4.1 Cardiorespiratory training & 16 & 882 & $\begin{array}{l}\text { Mean Difference (IV, Random, 95\% } \\
\mathrm{CI})\end{array}$ & $33.41[19.04,47.78]$ \\
\hline 4.2 Resistance training & 5 & 214 & $\begin{array}{l}\text { Mean Difference (IV, Random, 95\% } \\
\mathrm{CI})\end{array}$ & $24.83[11.68,37.97]$ \\
\hline 4.3 Mixed training & 10 & 696 & $\begin{array}{l}\text { Mean Difference (IV, Random, 95\% } \\
\mathrm{CI})\end{array}$ & $35.30[15.88,54.71]$ \\
\hline $\begin{array}{l}5 \text { Physical Function - Balance - Berg Bal- } \\
\text { ance Scale }\end{array}$ & 21 & 1062 & $\begin{array}{l}\text { Mean Difference (IV, Random, 95\% } \\
\mathrm{CI})\end{array}$ & $2.29[1.42,3.17]$ \\
\hline 5.1 Cardiorespiratory training & 8 & 471 & $\begin{array}{l}\text { Mean Difference (IV, Random, 95\% } \\
\mathrm{CI})\end{array}$ & $1.92[0.16,3.68]$ \\
\hline 5.2 Resistance training & 5 & 196 & $\begin{array}{l}\text { Mean Difference (IV, Random, 95\% } \\
\mathrm{CI})\end{array}$ & $3.24[2.11,4.38]$ \\
\hline 5.3 Mixed training & 9 & 395 & $\begin{array}{l}\text { Mean Difference (IV, Random, 95\% } \\
\mathrm{Cl} \text { ) }\end{array}$ & $2.10[0.73,3.48]$ \\
\hline 6 Physical function - Timed up and go & 16 & 1033 & $\begin{array}{l}\text { Mean Difference (IV, Random, 95\% } \\
\mathrm{Cl} \text { ) }\end{array}$ & $-3.04[-4.62,-1.45]$ \\
\hline 6.1 Cardiorespiratory training & 5 & 223 & $\begin{array}{l}\text { Mean Difference (IV, Random, 95\% } \\
\mathrm{Cl} \text { ) }\end{array}$ & $-3.42[-4.78,-2.05]$ \\
\hline 6.2 Resistance training & 5 & 224 & $\begin{array}{l}\text { Mean Difference (IV, Random, 95\% } \\
\mathrm{Cl})\end{array}$ & $-3.46[-6.94,0.02]$ \\
\hline 6.3 Mixed training & 7 & 586 & $\begin{array}{l}\text { Mean Difference (IV, Random, 95\% } \\
\mathrm{Cl})\end{array}$ & $-2.21[-4.43,0.02]$ \\
\hline
\end{tabular}

\begin{tabular}{|c|c|c|c|c|c|c|c|}
\hline \multirow{3}{*}{ Study or subgroup } & \multicolumn{7}{|c|}{$\begin{array}{l}\text { Analysis 7.1. Comparison } 7 \text { Cardiorespiratory versus resistance versus } \\
\text { mixed training, Outcome } 1 \text { Disability - combined disability scales. }\end{array}$} \\
\hline & \multicolumn{2}{|c|}{ Training } & \multicolumn{2}{|c|}{ Control } & \multirow{2}{*}{$\begin{array}{l}\text { Std. Mean Difference } \\
\text { Random, } 95 \% \mathrm{Cl}\end{array}$} & \multirow[t]{2}{*}{ Weight } & \multirow{2}{*}{$\begin{array}{c}\text { Std. Mean Difference } \\
\text { Random, } 95 \% \mathrm{Cl}\end{array}$} \\
\hline & $\mathbf{N}$ & Mean(SD) & $\mathbf{N}$ & Mean(SD) & & & \\
\hline \multicolumn{8}{|c|}{ 7.1.1 Cardiorespiratory training } \\
\hline Bateman 2001 & 23 & $104.7(17.7)$ & 29 & $100.4(18.9)$ & 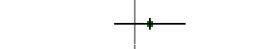 & $12.84 \%$ & $0.23[-0.32,0.78]$ \\
\hline Cuviello-Palmer 1988 & 10 & $44.8(8.8)$ & 10 & 47.2 (9.9) & 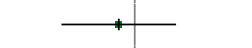 & $8.16 \%$ & $-0.25[-1.13,0.64]$ \\
\hline Globas 2012 & 18 & $13.3(1.7)$ & 18 & $11.3(2.7)$ & 1 & $10.65 \%$ & $0.87[0.18,1.55]$ \\
\hline Gordon 2013 & 64 & $95.9(6.3)$ & 64 & $93.3(9.3)$ & 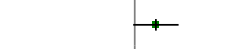 & $16.42 \%$ & $0.33[-0.02,0.67]$ \\
\hline Katz-Leurer 2003 & 46 & $105.8(12.5)$ & 44 & $101.4(16)$ & $\longrightarrow$ & $15.21 \%$ & $0.3[-0.11,0.72]$ \\
\hline
\end{tabular}




\begin{tabular}{|c|c|c|c|c|c|c|c|}
\hline \multirow[t]{2}{*}{ Study or subgroup } & \multicolumn{2}{|c|}{ Training } & \multicolumn{2}{|c|}{ Control } & \multirow{2}{*}{$\begin{array}{c}\text { Std. Mean Difference } \\
\text { Random, } 95 \% \mathrm{Cl}\end{array}$} & \multirow[t]{2}{*}{ Weight } & \multirow{2}{*}{$\begin{array}{c}\text { Std. Mean Difference } \\
\text { Random, } 95 \% \mathrm{Cl}\end{array}$} \\
\hline & $\mathbf{N}$ & $\operatorname{Mean}(\mathrm{SD})$ & $\mathbf{N}$ & Mean(SD) & & & \\
\hline Mudge 2009 & 31 & $77.8(55.7)$ & 27 & $60.9(67.2)$ & 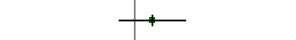 & $13.38 \%$ & $0.27[-0.25,0.79]$ \\
\hline Takami 2010 & 10 & $11.9(2.1)$ & 6 & $8.4(2.9)$ & $\longrightarrow$ & $5.75 \%$ & $1.37[0.22,2.52]$ \\
\hline Takami 2010 & 11 & $9.6(3.4)$ & 6 & $8.4(2.9)$ & $\rightarrow$ & $6.93 \%$ & $0.35[-0.65,1.36]$ \\
\hline Wang 2014 & 23 & $78.2(12.1)$ & 22 & $56(14.1)$ & 1 & $10.66 \%$ & $1.66[0.98,2.35]$ \\
\hline Subtotal $\star \star \star$ & 236 & & 226 & & & $100 \%$ & $0.52[0.19,0.84]$ \\
\hline \multicolumn{8}{|c|}{ Heterogeneity: $\operatorname{Tau}^{2}=0.14 ; \mathrm{Chi}^{2}=20.31, \mathrm{df}=8(\mathrm{P}=0.01) ; \mathrm{I}^{2}=60.61 \%$} \\
\hline \multicolumn{8}{|c|}{ Test for overall effect: $Z=3.12(P=0)$} \\
\hline \multicolumn{8}{|c|}{ 7.1.2 Resistance training } \\
\hline Ouellette 2004 & 21 & $57.1(10.5)$ & 21 & $55.8(10.1)$ & & $100 \%$ & $0.12[-0.48,0.73]$ \\
\hline Subtotal $\star \star \star$ & 21 & & 21 & & & $100 \%$ & $0.12[-0.48,0.73]$ \\
\hline \multicolumn{8}{|c|}{ Heterogeneity: $\mathrm{Tau}^{2}=0 ; \mathrm{Chi}^{2}=0, \mathrm{df}=0(\mathrm{P}<0.0001) ; \mathrm{I}^{2}=100 \%$} \\
\hline \multicolumn{8}{|c|}{ Test for overall effect: $Z=0.4(P=0.69)$} \\
\hline \multicolumn{8}{|l|}{ 7.1.3 Mixed training } \\
\hline Dean 2018 & 21 & $12.1(2.7)$ & 19 & $12.5(1.9)$ & $\longrightarrow$ & $8.5 \%$ & $-0.14[-0.76,0.48]$ \\
\hline Duncan 1998 & 10 & $96(5.2)$ & 10 & $95.6(5.3)$ & & $4.63 \%$ & $0.08[-0.8,0.96]$ \\
\hline Duncan 2003 & 44 & $94.4(6.7)$ & 49 & $89.6(10.4)$ & $\rightarrow$ & $15.99 \%$ & $0.54[0.12,0.95]$ \\
\hline Galvin 2011 & 20 & $88.5(15.6)$ & 20 & $81.8(18.7)$ & $\rightarrow$ & $8.39 \%$ & $0.38[-0.24,1.01]$ \\
\hline Kim 2016a & 10 & $87(10.5)$ & 10 & $85.3(13.7)$ & 5 & $4.62 \%$ & $0.13[-0.74,1.01]$ \\
\hline Langhammer 2007 & 32 & $83(26.4)$ & 33 & $87.6(21.5)$ & $\longrightarrow$ & $12.59 \%$ & $-0.19[-0.68,0.3]$ \\
\hline Letombe 2010 & 9 & $67.5(44.3)$ & 9 & $42.3(17.4)$ & 1 & $3.91 \%$ & $0.71[-0.25,1.67]$ \\
\hline Mead 2007 & 32 & $118.2(3.3)$ & 34 & $118.3(3.3)$ & $\longrightarrow$ & $12.78 \%$ & $-0.03[-0.51,0.45]$ \\
\hline Van de Port 2012 & 125 & $13.5(1.4)$ & 117 & $12.8(1.9)$ & + & $28.6 \%$ & $0.39[0.13,0.64]$ \\
\hline Subtotal $* \star \star$ & 303 & & 301 & & 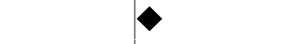 & $100 \%$ & $0.23[0.03,0.42]$ \\
\hline \multicolumn{8}{|c|}{ Heterogeneity: $\operatorname{Tau}^{2}=0.02 ; \mathrm{Chi}^{2}=10.14, \mathrm{df}=8(\mathrm{P}=0.26) ; \mathrm{I}^{2}=21.13 \%$} \\
\hline \multicolumn{8}{|c|}{ Test for overall effect: $Z=2.25(P=0.02)$} \\
\hline \multicolumn{8}{|c|}{ Test for subgroup differences: $\mathrm{Chi}^{2}=2.58, \mathrm{df}=1(\mathrm{P}=0.28), \mathrm{I}^{2}=22.4 \%$} \\
\hline
\end{tabular}

Analysis 7.2. Comparison 7 Cardiorespiratory versus resistance versus mixed training, Outcome 2 Mobility - walking maximal speed.

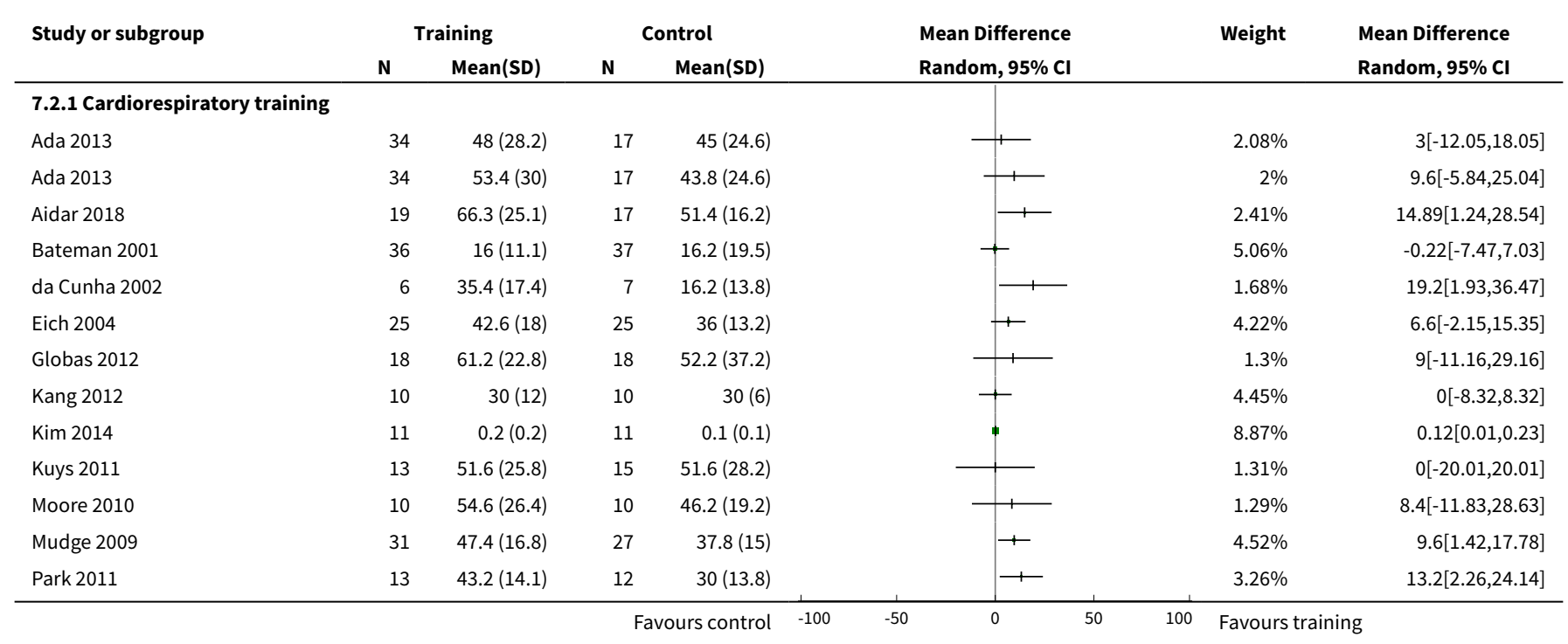




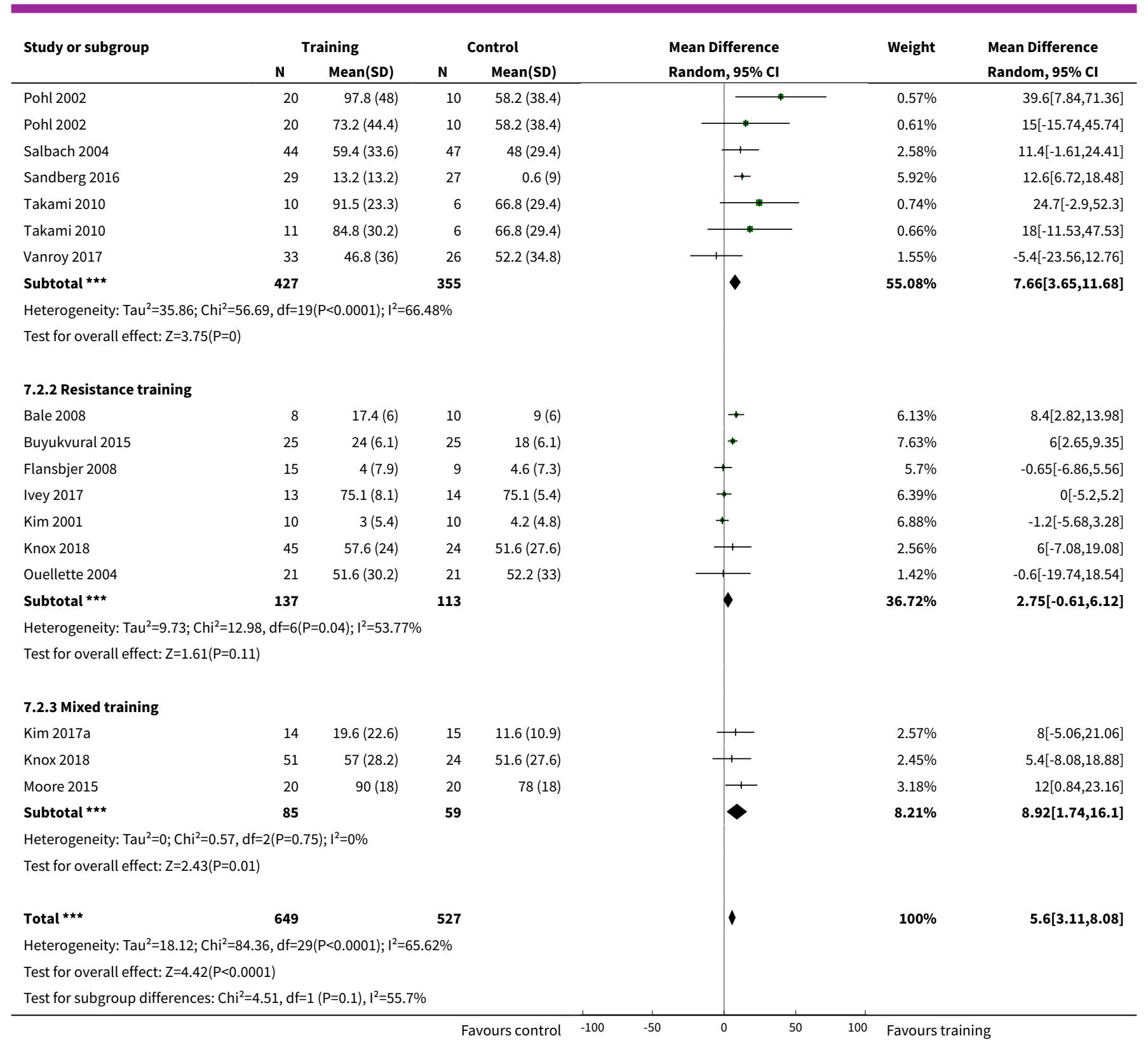

Analysis 7.3. Comparison 7 Cardiorespiratory versus resistance versus mixed training, Outcome 3 Mobility - walking preferred speed.

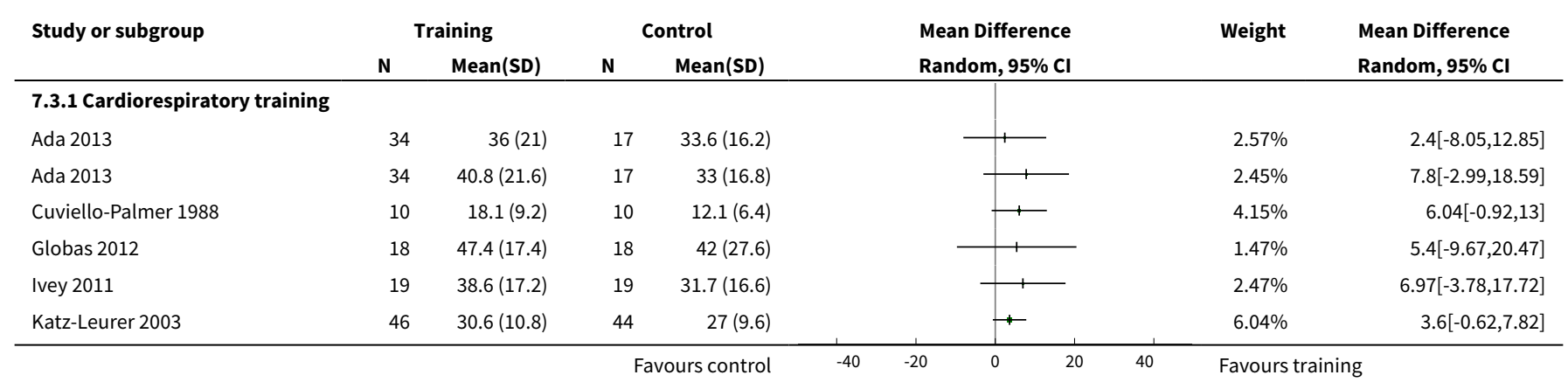




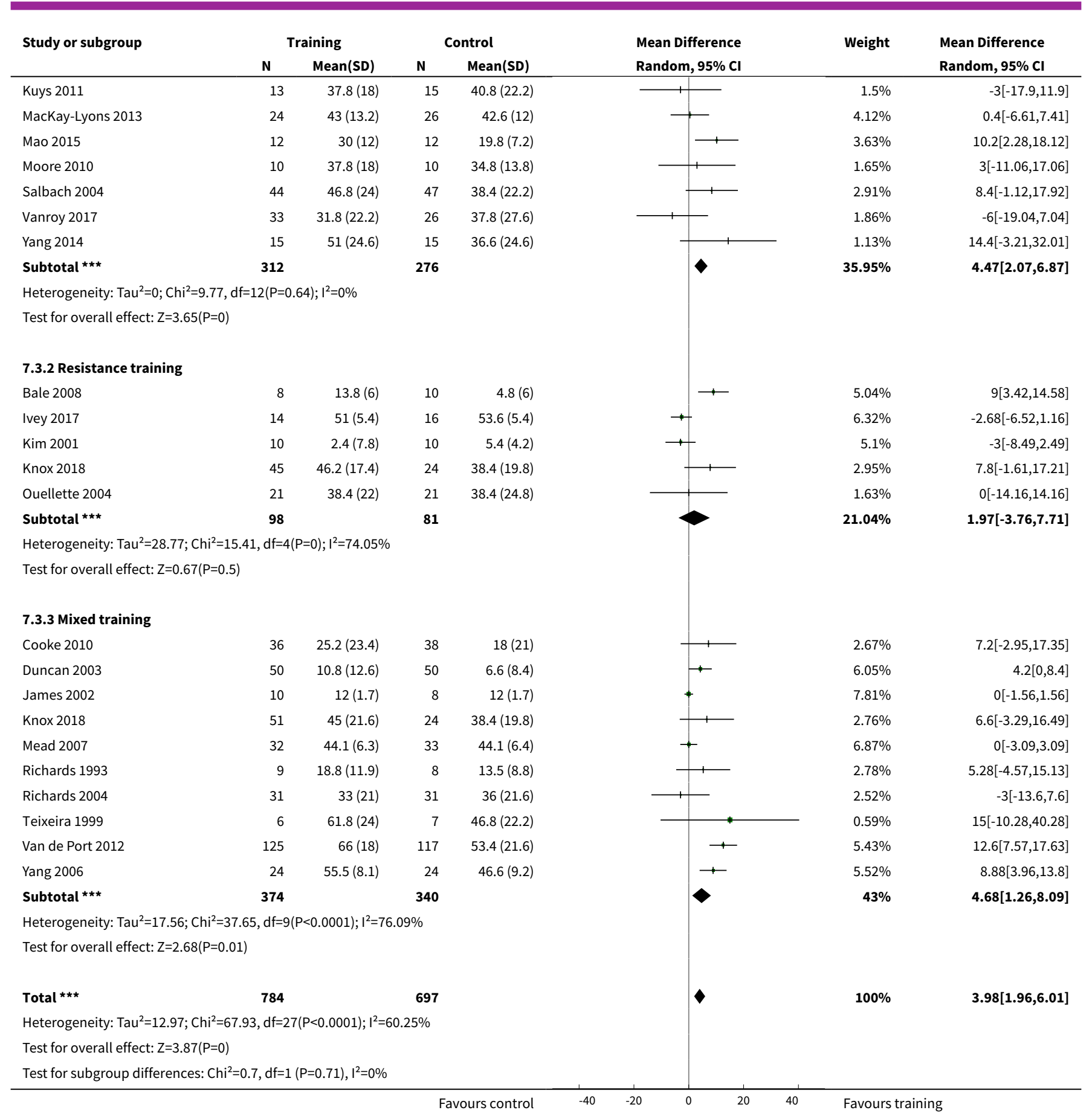

Analysis 7.4. Comparison 7 Cardiorespiratory versus resistance versus mixed training, Outcome 4 Mobility - walking capacity (6-MWT distance).

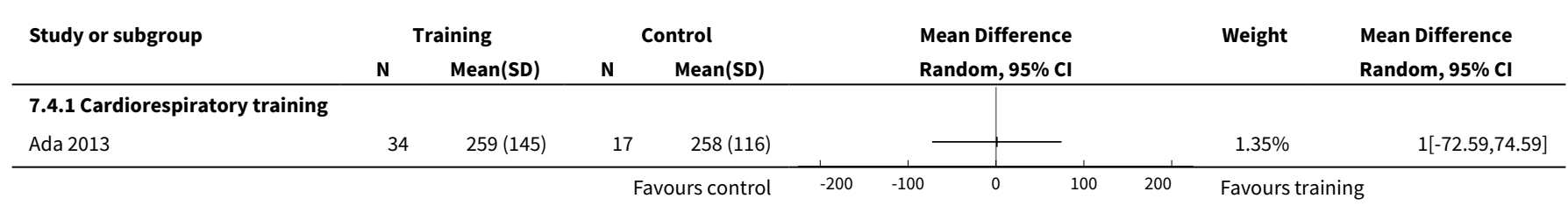




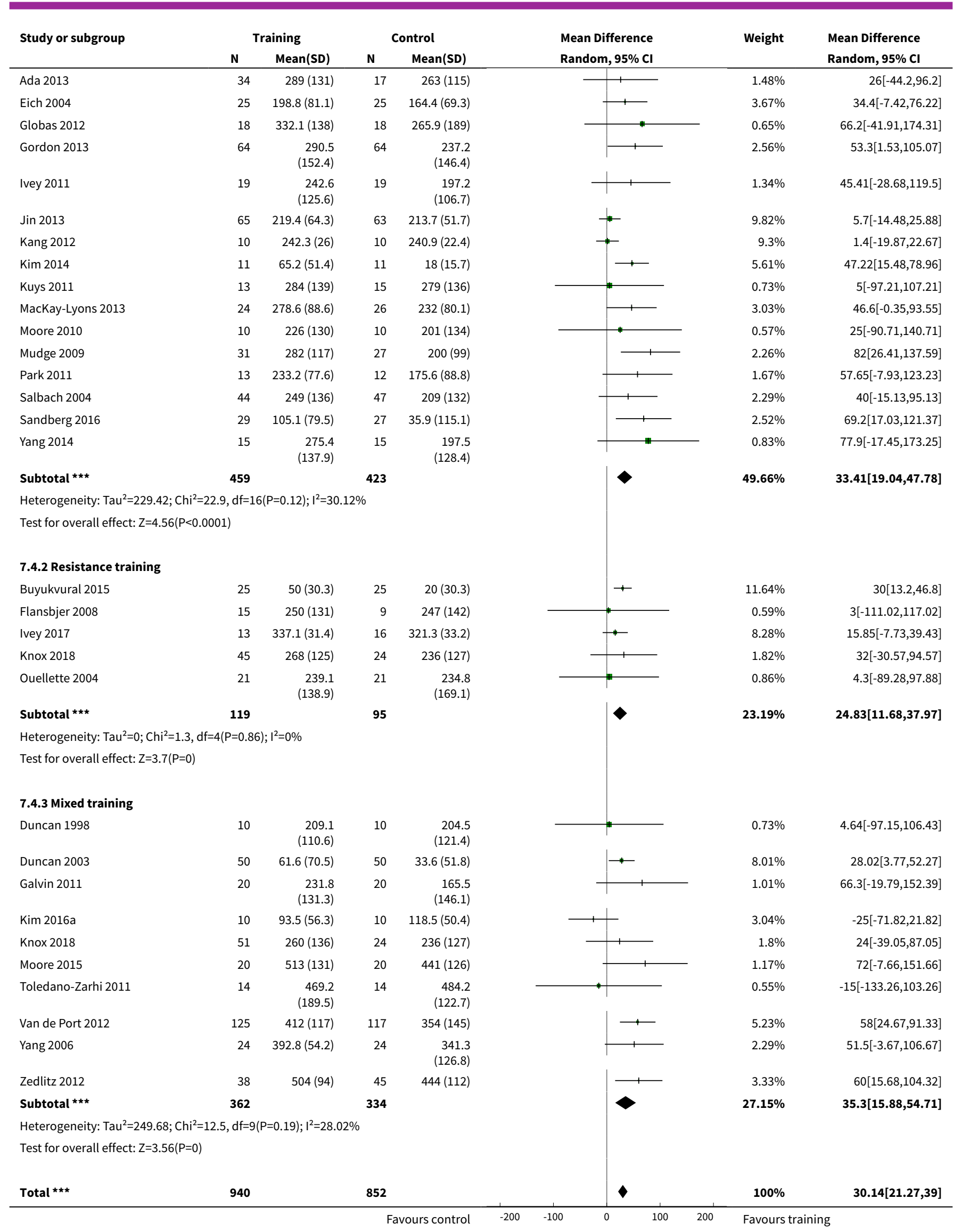




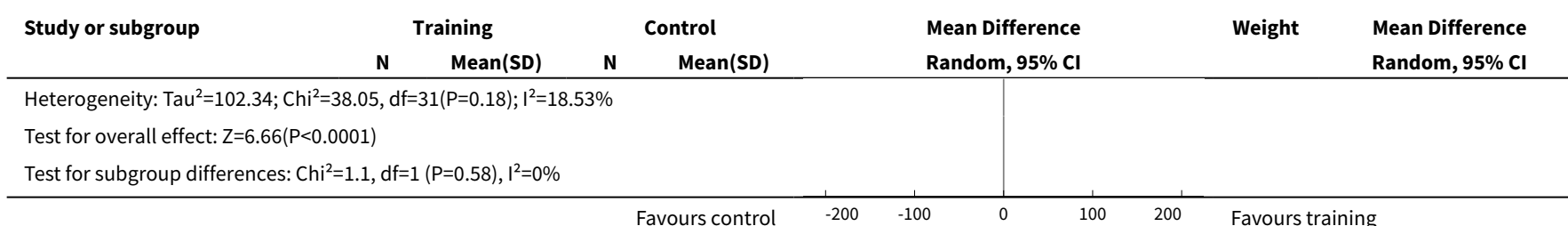

Analysis 7.5. Comparison 7 Cardiorespiratory versus resistance versus mixed training, Outcome 5 Physical Function - Balance - Berg Balance Scale.

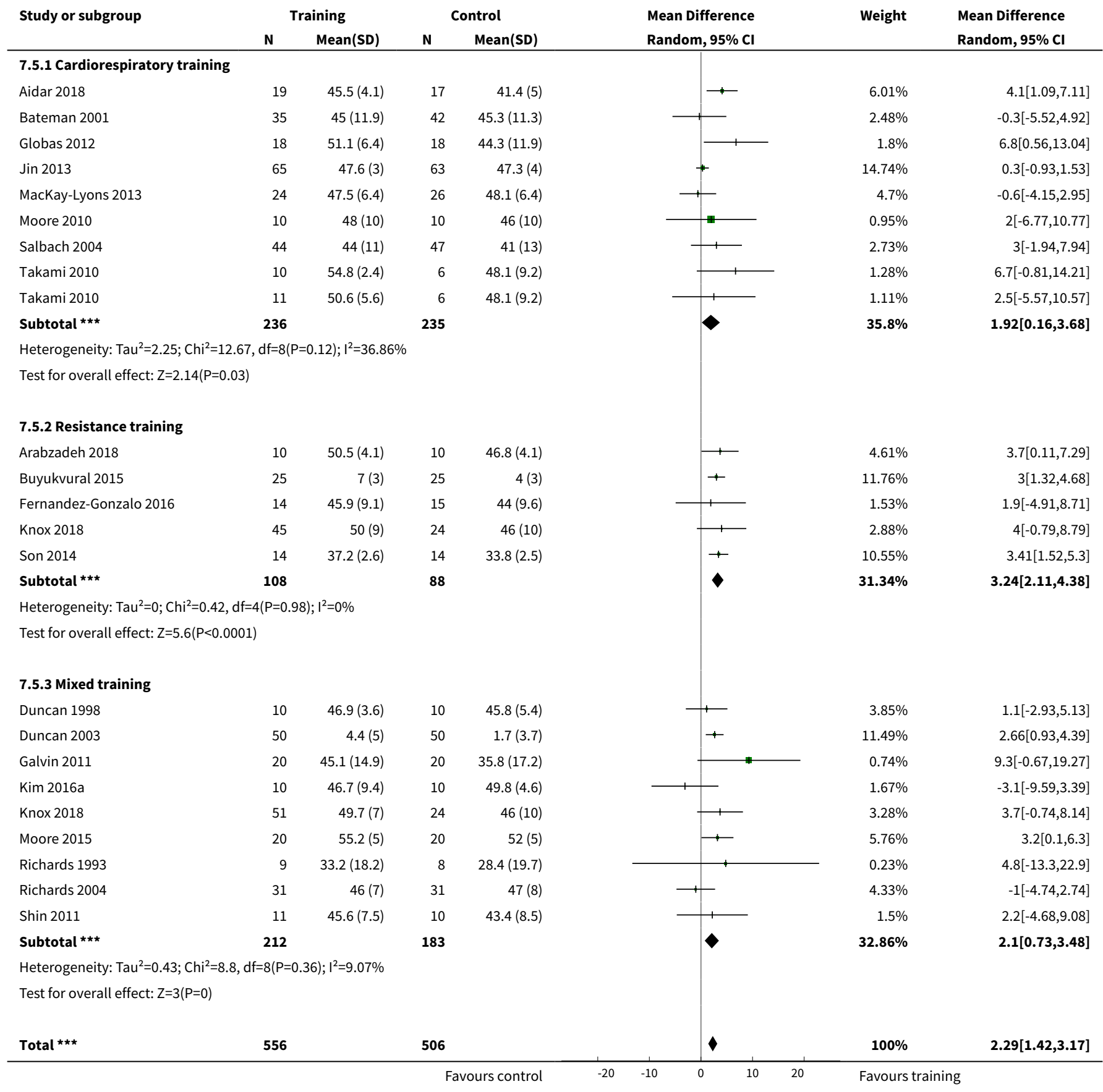




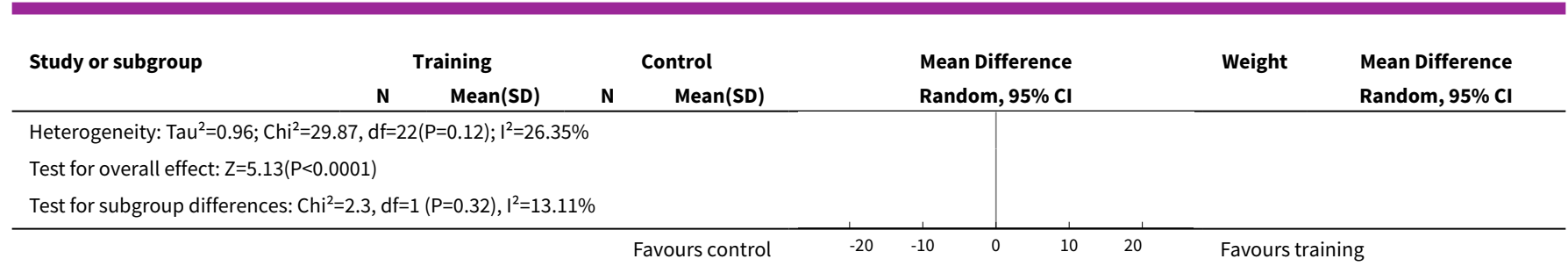

Favours control

Favours training

Analysis 7.6. Comparison 7 Cardiorespiratory versus resistance versus mixed training, Outcome 6 Physical function - Timed up and go.

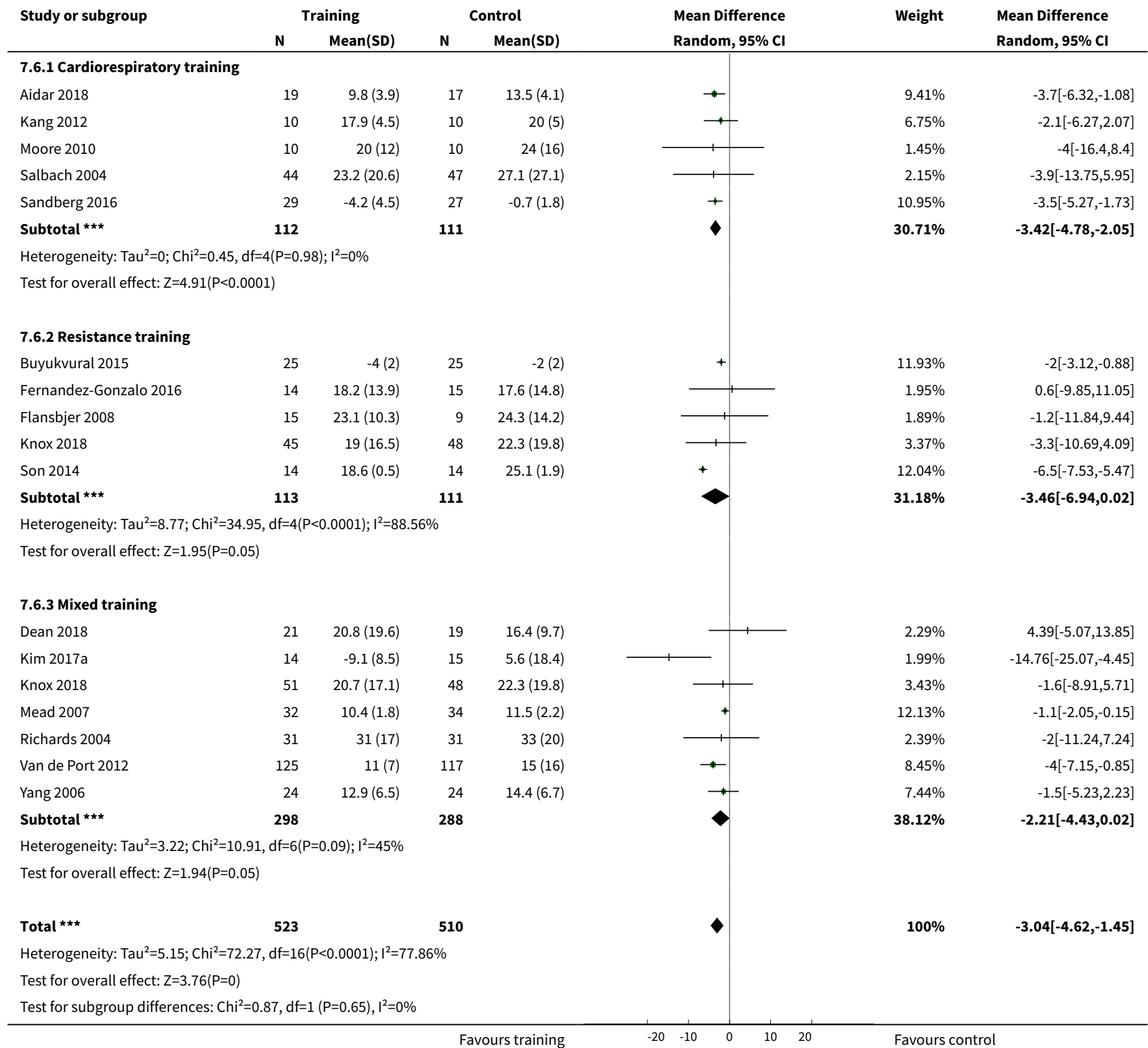

Physical fitness training for stroke patients (Review) 
ADDITIONAL TABLES 


\begin{tabular}{|c|c|c|c|c|c|c|c|c|}
\hline Study ID & Mode of training & $\begin{array}{l}\text { During or } \\
\text { after usu- } \\
\text { al care }\end{array}$ & $\begin{array}{l}\text { Upper } \\
\text { or lower } \\
\text { body }\end{array}$ & $\begin{array}{l}\text { Specific } \\
\text { training }\end{array}$ & Intensity & $\begin{array}{l}\text { Duration } \\
\text { (minutes) }\end{array}$ & $\begin{array}{l}\text { Frequen- } \\
\text { cy } \\
\text { (days) }\end{array}$ & $\begin{array}{l}\text { Pro- } \\
\text { gramme } \\
\text { length } \\
\text { (weeks) }\end{array}$ \\
\hline Ada 2013 & Treadmill + overground walking & After & $\begin{array}{l}\text { Lower } \\
\text { body }\end{array}$ & Yes & Unknown & $30 \mathrm{~min}$ & 3 & $\begin{array}{l}\text { Group } 1= \\
16 \\
\text { Group } 2= \\
8\end{array}$ \\
\hline Aidar 2018 & Water training & After & Both & Yes & Unknown & $45-60$ & 2 & 12 \\
\hline $\begin{array}{l}\text { Bateman } \\
2001\end{array}$ & Cycle ergometer & During & $\begin{array}{l}\text { Lower } \\
\text { body }\end{array}$ & No & $\begin{array}{l}60 \%-80 \% \text { age-related heart rate } \\
\text { maximum }\end{array}$ & $\leq 30$ & 3 & 12 \\
\hline $\begin{array}{l}\text { Cuviel- } \\
\text { lo-Palmer } \\
1988\end{array}$ & Kinetron & During & $\begin{array}{l}\text { Lower } \\
\text { body }\end{array}$ & No & Heart rate $<$ resting +20 beats $/ \mathrm{min}$ & $7-17$ & 5 & 3 \\
\hline $\begin{array}{l}\text { da Cunha } \\
2002\end{array}$ & $\begin{array}{l}\text { Treadmill gait training with body } \\
\text { weight support }\end{array}$ & During & $\begin{array}{l}\text { Lower } \\
\text { body }\end{array}$ & Yes & Unknown & 20 & 5 & $2-3$ \\
\hline Eich 2004 & Treadmill gait training & During & $\begin{array}{l}\text { Lower } \\
\text { body }\end{array}$ & Yes & $60 \%$ heart rate reserve & 30 & 5 & 6 \\
\hline $\begin{array}{l}\text { Glasser } \\
1986\end{array}$ & Kinetron & During & $\begin{array}{l}\text { Lower } \\
\text { body }\end{array}$ & No & Unknown & $20-60$ & 5 & 3 \\
\hline $\begin{array}{l}\text { Globas } \\
2012\end{array}$ & Treadmill & After & $\begin{array}{l}\text { Lower } \\
\text { body }\end{array}$ & Yes & $\begin{array}{l}40 \%-50 \% \text { progressing to } 60 \%-80 \% \\
\text { heart rate reserve }\end{array}$ & $\begin{array}{l}10-20 \text { min } \\
\text { increasing } \\
\text { to } 30-50 \\
\text { min }\end{array}$ & 3 & 12 \\
\hline $\begin{array}{l}\text { Gordon } \\
2013\end{array}$ & $\begin{array}{l}\text { Overground community-based } \\
\text { walking }\end{array}$ & After & $\begin{array}{l}\text { Lower } \\
\text { body }\end{array}$ & Yes & $\begin{array}{l}\text { Target heart rate was } 60 \%-85 \% \text { of } \\
\text { age-predicted maximum heart rate } \\
\text { (220-age). }\end{array}$ & $\begin{array}{l}15 \mathrm{~min} \\
\text { progress- } \\
\text { ing by }+5 \\
\text { min per } \\
\text { week }\end{array}$ & 3 & 12 \\
\hline Ivey 2010 & Treadmill & After & $\begin{array}{l}\text { Lower } \\
\text { body }\end{array}$ & Yes & $\begin{array}{l}40 \%-50 \% \text { progressing to } 60 \%-70 \% \\
\text { heart rate reserve }\end{array}$ & $\begin{array}{l}10-20 \mathrm{~min} \\
\text { increasing } \\
\text { to } 40 \mathrm{~min}\end{array}$ & 3 & $\begin{array}{l}24 \\
\text { (6 months) }\end{array}$ \\
\hline
\end{tabular}




\begin{tabular}{|c|c|c|c|c|c|c|c|c|}
\hline Ivey 2011 & Treadmill & After & $\begin{array}{l}\text { Lower } \\
\text { body }\end{array}$ & Yes & $\begin{array}{l}40 \%-50 \% \text { progressing to } 60 \%-70 \% \\
\text { heart rate reserve }\end{array}$ & $\begin{array}{l}10-20 \mathrm{~min} \\
\text { increasing } \\
\text { to } 40 \mathrm{~min}\end{array}$ & 3 & $\begin{array}{l}24 \\
\text { (6 months) }\end{array}$ \\
\hline Jin 2013 & Cycle ergometry & During & Lower & No & $\begin{array}{l}\text { Commencing at } 40 \%-50 \% \text { heart rate } \\
\text { reserve progressing } 5 \% \text { heart rate re- } \\
\text { serve every } 2 \text { weeks up to } 70 \% \text { heart } \\
\text { rate reserve }\end{array}$ & 40 & 5 & 12 \\
\hline Kang 2012 & Treadmill & After & $\begin{array}{l}\text { Lower } \\
\text { body }\end{array}$ & Yes & Unknown & 30 & 3 & 4 \\
\hline $\begin{array}{l}\text { Katz-Leur- } \\
\text { er } 2003\end{array}$ & Cycle ergometer & After & $\begin{array}{l}\text { Lower } \\
\text { body }\end{array}$ & No & $\leq 60 \%$ heart rate reserve & 20 then 30 & 5 then 3 & $\begin{array}{l}2 \text { then } 6 \\
\text { (total } 8 \text { ) }\end{array}$ \\
\hline Kim 2014 & Community walking programme & During & Lower & Yes & Unclear & 60 & 5 & 4 \\
\hline & & & & & $\begin{array}{l}\text { The walking environment was made } \\
\text { more challenging with increased ex- } \\
\text { posure to uneven ground, gradients } \\
\text { and stairs }\end{array}$ & & & \\
\hline Kuys 2011 & Treadmill & After & $\begin{array}{l}\text { Lower } \\
\text { body }\end{array}$ & Yes & $\begin{array}{l}40 \% \text { progressing to } 60 \% \text { heart rate } \\
\text { reserve }\end{array}$ & 30 & 3 & 6 \\
\hline $\begin{array}{l}\text { Lennon } \\
2008\end{array}$ & $\begin{array}{l}\text { Cycle ergometer (cardiac rehabil- } \\
\text { itation programme) }\end{array}$ & After & Both & No & $50 \%-60 \%$ maximum heart rate & 30 & 2 & 10 \\
\hline $\begin{array}{l}\text { Mack- } \\
\text { ay-Lyons } \\
2013\end{array}$ & $\begin{array}{l}\text { Body weight supported treadmill } \\
\text { training }\end{array}$ & During & Both & Yes & $\begin{array}{l}\text { Target heart rates corresponding to } \\
60 \%-75 \% \text { of baseline } \mathrm{VO}_{2} \text { peak } \\
\text { Initially treadmill speed } 80 \%-90 \% \text { of } \\
\text { self-paced overground speed with } \\
20 \%-30 \% \text { body weight supported } \\
\text { for ambulatory independent partic- } \\
\text { ipants and } 70 \%-80 \% \text { of overground } \\
\text { speed with } 40 \% \text { body weight sup- } \\
\text { ported for ambulatory dependent } \\
\text { participants }\end{array}$ & 60 & $\begin{array}{l}5 / \text { week for } \\
6 \text { weeks } \\
\text { then } 3 / \\
\text { week for } 6 \\
\text { weeks }\end{array}$ & 12 \\
\hline Mao 2015 & $\begin{array}{l}\text { Body weight supported treadmill } \\
\text { training }\end{array}$ & During & Lower & Yes & $\begin{array}{l}\text { Treadmill walking with } 30 \%-40 \% \\
\text { body weight support. Body support } \\
\text { was decreased and treadmill speed } \\
\text { increased. No further detail for per- }\end{array}$ & 20 up to 40 & 5 & 3 \\
\hline
\end{tabular}




\begin{tabular}{|c|c|c|c|c|c|c|c|c|}
\hline & & & & & $\begin{array}{l}\text { centage assisted body support was } \\
\text { provided. Speed initially } 0.5 \mathrm{miles} / \mathrm{h} \\
(0.8 \mathrm{~km} / \mathrm{h}) \text { for } 20 \mathrm{~min} \text { progressing to } \\
2.5 \mathrm{miles} / \mathrm{h}(4.0 \mathrm{~km} / \mathrm{h}) \text { for } 40 \mathrm{~min}\end{array}$ & & & \\
\hline $\begin{array}{l}\text { Moore } \\
2010\end{array}$ & $\begin{array}{l}\text { Treadmill gait training with over- } \\
\text { head harness }\end{array}$ & After & $\begin{array}{l}\text { Lower } \\
\text { body }\end{array}$ & Yes & $\begin{array}{l}80 \%-85 \% \text { age-predicted maximum } \\
\text { heart rate }\end{array}$ & Unknown & $2-5$ & 4 \\
\hline $\begin{array}{l}\text { Mudge } \\
2009\end{array}$ & Circuit training & After & $\begin{array}{l}\text { Lower } \\
\text { body }\end{array}$ & Yes & Unknown & 30 & 3 & 4 \\
\hline Park 2011 & $\begin{array}{l}\text { Overground community-based } \\
\text { walking }\end{array}$ & During & Lower & Yes & Unknown & 60 & 3 & 4 \\
\hline Pohl 2002 & $\begin{array}{l}\text { Treadmill gait training } \\
\text { Group 1: structured speed-de- } \\
\text { pendent treadmill training } \\
\text { Group 2: limited progressive } \\
\text { treadmill training }\end{array}$ & During & $\begin{array}{l}\text { Lower } \\
\text { body }\end{array}$ & Yes & Unknown & 30 & 3 & 4 \\
\hline $\begin{array}{l}\text { Potempa } \\
1995\end{array}$ & Cycle ergometer & After & $\begin{array}{l}\text { Lower } \\
\text { body }\end{array}$ & No & $30 \%-50 \%$ maximum effort & 30 & 3 & 10 \\
\hline $\begin{array}{l}\text { Salbach } \\
2004\end{array}$ & Circuit training & After & $\begin{array}{l}\text { Lower } \\
\text { body }\end{array}$ & Yes & Unknown & 55 & 3 & 6 \\
\hline $\begin{array}{l}\text { Sandberg } \\
2016\end{array}$ & Cycling (main exercise element) & After & Lower & No & $\begin{array}{l}\text { Class included } 2 \times 8 \text {-min periods of } \\
\text { high-intensity exercise (14-15 RPE; } \\
75 \% \text { maximum oxygen consumption; } \\
80 \% \text { maximum heart rate) }\end{array}$ & 60 & 2 & 12 \\
\hline $\begin{array}{l}\text { Smith } \\
2008\end{array}$ & Treadmill gait training & After & $\begin{array}{l}\text { Lower } \\
\text { body }\end{array}$ & Yes & $\mathrm{RPE} \leq 13$ & 20 & 3 & 4 \\
\hline \multirow[t]{3}{*}{$\begin{array}{l}\text { Takami } \\
2010\end{array}$} & $\begin{array}{l}\text { Treadmill gait training with body } \\
\text { weight support }\end{array}$ & During & $\begin{array}{l}\text { Lower } \\
\text { body }\end{array}$ & Yes & Unknown & 10 & 6 & 3 \\
\hline & $\begin{array}{l}\text { Group 1: backward walking } \\
\text { group }\end{array}$ & & & & & & & \\
\hline & Group 2: forward walking group & & & & & & & \\
\hline
\end{tabular}




\begin{tabular}{|c|c|c|c|c|c|c|c|c|}
\hline $\begin{array}{l}\text { Topcuoglu } \\
2015\end{array}$ & Arm-cranking ergometer & During & Upper & No & Intensity 10 watts/minute & 30 & 5 & 4 \\
\hline $\begin{array}{l}\text { Vanroy } \\
2017\end{array}$ & $\begin{array}{l}\text { MOTOmed seated cycling er- } \\
\text { gometer }\end{array}$ & $\begin{array}{l}\text { Com- } \\
\text { menced } \\
\text { during } \\
\text { (some dis- } \\
\text { charged } \\
\text { home) }\end{array}$ & Lower & No & $\begin{array}{l}\text { Intensity progressed from } 60 \%-75 \% \\
\text { heart rate reserve }\end{array}$ & $\begin{array}{l}30 \\
\text { (Total ses- } \\
\text { sion } 51 \\
\text { min reduc- } \\
\text { ing to } 40 \\
\text { min) }\end{array}$ & 3 & $\begin{array}{l}12 \text { (3 } \\
\text { months) }\end{array}$ \\
\hline Wang 2014 & $\begin{array}{l}\text { Wheelchair-seated pedaling er- } \\
\text { gometry }\end{array}$ & During & Lower & Yes & $\begin{array}{l}\text { Cycling training consisted of } 30-\mathrm{min} \\
\text { sessions including: } 5 \text {-min warm-up; } \\
30 \text {-min active pedaling at an intensi- } \\
\text { ty based on an incremental graded } \\
\text { exercise test ( } 2.5 \mathrm{~W} \text { ramp every } 3 \mathrm{~min} \\
\text { maintaining } 50 \text { rpm until exhaus- } \\
\text { tion); followed by 5-min cool down. } \\
\text { Target heart rate was calculated as } \\
\text { ((peak heart rate in graded exercise } \\
\text { test - resting heart rate) } \times 50 \%-70 \%) \\
\text { + resting heart rate }\end{array}$ & 30 & 3 & 6 \\
\hline Yang 2014 & Cycle ergometer & During & Lower & Yes & $\begin{array}{l}\text { Cycling training consisted of } 15 \text {-min } \\
\text { sessions each of forward and back- } \\
\text { ward cycling including: } 150 \text {-s passive } \\
\text { warm-up; } 10 \text {-min active pedaling at } \\
50-70 \text { rpm at an intensity of stage } \\
13 \text { of the Borg scale; } 150 \text { s of passive } \\
\text { cool-down }\end{array}$ & 30 & 5 & 4 \\
\hline
\end{tabular}

mph: miles per hour; RPE: rate of perceived exertion;RPM: revolutions per minute

Table 2. Outline of the studies that focused on resistance training interventions

\begin{tabular}{|c|c|c|c|c|c|c|c|c|}
\hline Study ID & Mode of training & $\begin{array}{l}\text { During/af- } \\
\text { ter usual } \\
\text { care }\end{array}$ & $\begin{array}{l}\text { Upper } \\
\text { or lower } \\
\text { body }\end{array}$ & $\begin{array}{l}\text { Specific } \\
\text { training }\end{array}$ & Intensity & $\begin{array}{l}\text { Duration } \\
\text { (minutes) }\end{array}$ & $\begin{array}{l}\text { Frequen- } \\
\text { cy (days) }\end{array}$ & $\begin{array}{l}\text { Pro- } \\
\text { gramme } \\
\text { length } \\
\text { (weeks) }\end{array}$ \\
\hline
\end{tabular}




\begin{tabular}{|c|c|c|c|c|c|c|c|c|}
\hline Aidar 2016 & Resistance training; machine weights & After & Both & No & $\begin{array}{l}\text { OMNI Resistance } \\
\text { Exercise Scale }\end{array}$ & $45-60$ & 3 & 12 \\
\hline $\begin{array}{l}\text { Arabzadeh } \\
2018\end{array}$ & $\begin{array}{l}\text { Task-oriented circuit with added weights and some } \\
\text { balance activities }\end{array}$ & During & Lower & Yes & $\begin{array}{l}\text { Unknown; tailored } \\
\text { to individual ca- } \\
\text { pacity }\end{array}$ & 50 & 3 & 4 \\
\hline Bale 2008 & Resistance training; weights & During & $\begin{array}{l}\text { Lower } \\
\text { body }\end{array}$ & No & $\begin{array}{l}10-15 \text { repetitions to } \\
\text { achieve moderate } \\
\text { fatigue }\end{array}$ & 50 & 3 & 4 \\
\hline $\begin{array}{l}\text { Buyukvur- } \\
\text { al } 2015\end{array}$ & Isokinetic dynamometer training & During & Lower & No & Unclear & Unclear & 5 & 3 \\
\hline $\begin{array}{l}\text { Coroian } \\
2018\end{array}$ & Isokinetic dynamometer training & During & Upper & No & $\begin{array}{l}6 \text { sets of } 8 \text { repeti- } \\
\text { tions increasing } \\
\text { from } 40 \%-70 \% \text { of } \\
\text { maximal baseline } \\
\text { torque }\end{array}$ & 45 & 3 & 5 \\
\hline $\begin{array}{l}\text { Fernan- } \\
\text { dez-Gon- } \\
\text { zalo } 2016\end{array}$ & $\begin{array}{l}\text { Unilateral explosive resistance training of the more } \\
\text { affected leg }\end{array}$ & After & Lower & No & Maximal effort & Unclear & 2 & 12 \\
\hline $\begin{array}{l}\text { Flansbjer } \\
2008\end{array}$ & $\begin{array}{l}\text { Dynamic and isokinetic resistance training (leg ex- } \\
\text { tension/curl rehab exercise machine) }\end{array}$ & After & $\begin{array}{l}\text { Lower } \\
\text { body }\end{array}$ & Yes & $\begin{array}{l}6 \text {-10 repetitions } \\
\text { equivalent to } 80 \% \\
\text { of maximum load }\end{array}$ & 90 & Unknown & 10 \\
\hline Inaba 1973 & Resistance training & During & $\begin{array}{l}\text { Lower } \\
\text { body }\end{array}$ & No & $\begin{array}{l}50 \% \text { and } 100 \% \\
\text { maximum weight }\end{array}$ & Unknown & 'Daily' & $4-8$ \\
\hline Ivey 2017 & Pneumatic resistance machines & After & Lower & No & $\begin{array}{l}10-15 \text {, decreasing } \\
\text { to } 20 \text { repetition } \\
\text { maximum across } \\
\text { sets }\end{array}$ & Unclear & 3 & 12 \\
\hline Kim 2001 & Resistance training; isokinetic dynamometer & After & $\begin{array}{l}\text { Lower } \\
\text { body }\end{array}$ & No & $\begin{array}{l}\text { Maximal effort } \\
3 \times 10 \text { repetitions }\end{array}$ & 30 & 3 & 6 \\
\hline Knox 2018 & Gravity, free weights, elastic bands and balls & After & Lower & No & $\begin{array}{l}3 \text { set of } 10 \text { repeti- } \\
\text { tions; progressed } \\
\text { individually }\end{array}$ & 60 & 6 sessions & 12 \\
\hline
\end{tabular}




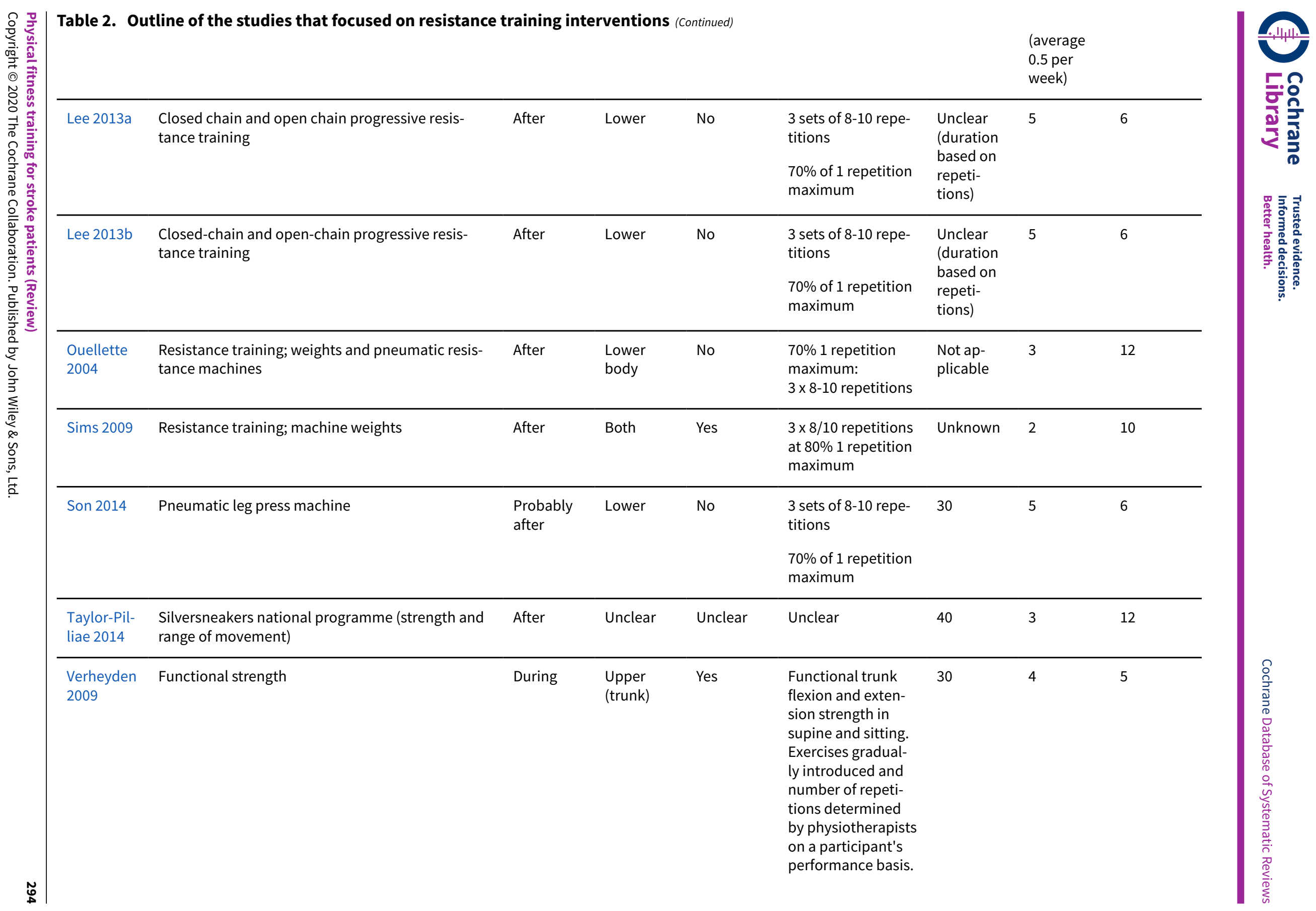




\begin{tabular}{|c|c|c|c|c|c|c|c|c|c|c|}
\hline & & & & & & & $\begin{array}{l}\text { No further details } \\
\text { reported }\end{array}$ & & & \\
\hline $\begin{array}{l}\text { Winstein } \\
2004\end{array}$ & \multicolumn{3}{|l|}{$\begin{array}{l}\text { Resistance training; weights; } \\
\text { TheraBand and grip devices }\end{array}$} & During & $\begin{array}{l}\text { Upper } \\
\text { body }\end{array}$ & No & Unknown & 60 & $\begin{array}{l}3 \text { high } \\
2 \text { slow }\end{array}$ & $\begin{array}{l}4-6 \text { (target } \\
\text { of } 20 \text { ses- } \\
\text { sions) }\end{array}$ \\
\hline Zou 2015 & \multicolumn{3}{|l|}{ Resistance training machines } & After & Lower & No & $\begin{array}{l}3 \text { sets of } 15 \text { repeti- } \\
\text { tions; initial inten- } \\
\text { sity causing failure } \\
10-12 \text { repetitions, } \\
\text { then reduce to al- } \\
\text { low completion of } \\
15\end{array}$ & 40 & 3 & 8 \\
\hline Study ID & Mode of training & $\begin{array}{l}\text { During or } \\
\text { after usu- } \\
\text { al care }\end{array}$ & $\begin{array}{l}\text { Upper } \\
\text { or lower } \\
\text { body }\end{array}$ & $\begin{array}{l}\text { Specific } \\
\text { training }\end{array}$ & \multicolumn{2}{|c|}{ Intensity } & & $\begin{array}{l}\text { Duration } \\
\text { (minutes) }\end{array}$ & $\begin{array}{l}\text { Frequen- } \\
\text { cy } \\
\text { (days) }\end{array}$ & $\begin{array}{l}\text { Pro- } \\
\text { gramme } \\
\text { length } \\
\text { (weeks) }\end{array}$ \\
\hline $\begin{array}{l}\text { Cooke } \\
2010\end{array}$ & $\begin{array}{l}\text { Resistance training }+ \\
\text { treadmill training }\end{array}$ & During & $\begin{array}{l}\text { Lower } \\
\text { body }\end{array}$ & Yes & \multicolumn{2}{|c|}{ Unknown } & & 60 & 4 & 6 \\
\hline Dean 2018 & $\begin{array}{l}\text { Group circuit training (0-3 } \\
\text { months) + home training } \\
\text { (0-6 months) }\end{array}$ & After & Both & Yes & \multicolumn{2}{|c|}{ Unclear } & & Unclear & $\begin{array}{l}2 \text { classes } \\
\text { (+ home } \\
\text { exercise }\end{array}$ & $\begin{array}{l}6 \\
\text { (0-3 } \\
\text { months } \\
\text { group ex- } \\
\text { ercise; 0-6 } \\
\text { months } \\
\text { home ex- } \\
\text { ercise) }\end{array}$ \\
\hline $\begin{array}{l}\text { Donaldson } \\
2009\end{array}$ & $\begin{array}{l}\text { Paretic upper limb exer- } \\
\text { cises and hand grip activi- } \\
\text { ties }\end{array}$ & During & $\begin{array}{l}\text { Upper } \\
\text { body }\end{array}$ & Yes & \multicolumn{2}{|c|}{ Unknown } & & 60 & 4 & 6 \\
\hline $\begin{array}{l}\text { Duncan } \\
1998\end{array}$ & $\begin{array}{l}\text { Walking or cycle ergome- } \\
\text { try; elastic-resisted con- } \\
\text { tractions }\end{array}$ & After & Both & Yes & \multicolumn{2}{|c|}{ Unknown } & & 90 & 3 & 12 \\
\hline
\end{tabular}




\begin{tabular}{|c|c|c|c|c|c|c|c|c|}
\hline $\begin{array}{l}\text { Duncan } \\
2003\end{array}$ & Circuit training & After & $\begin{array}{l}\text { Lower } \\
\text { body }\end{array}$ & Yes & $50 \%-60 \%$ heart rate reserve & $90-120$ & 3 & 4 \\
\hline $\begin{array}{l}\text { Furnari } \\
2014\end{array}$ & Aquatic exercise & During & $\begin{array}{l}\text { Upper } \\
\text { or lower } \\
\text { body }\end{array}$ & Yes & Unclear; difficulty progressed weekly & 60 & 3 & 8 \\
\hline $\begin{array}{l}\text { Galvin } \\
2011\end{array}$ & $\begin{array}{l}\text { Family-mediated gait and } \\
\text { strength training }\end{array}$ & During & Lower & Yes & Unknown & 35 & 7 & 8 \\
\hline $\begin{array}{l}\text { James } \\
2002\end{array}$ & Circuit training & After & Both & Yes & Unknown & 90 & 3 & $\begin{array}{l}12-14 \text { (to- } \\
\text { tal of } 36 \\
\text { sessions) }\end{array}$ \\
\hline Kim 2016a & Circuit training & During & Both & Yes & $\begin{array}{l}\text { Treadmill speed/gradient } \\
\text { TheraBand repetitions/load }\end{array}$ & 90 & 5 & 4 \\
\hline Kim 2017a & $\begin{array}{l}\text { Handgrip resistance train- } \\
\text { ing + treadmill walking } \\
\text { with some added load to } \\
\text { unaffected leg }\end{array}$ & During & Both & Yes & $\begin{array}{l}\text { Resistance increase } \\
\text { Treadmill speed increase }\end{array}$ & 30 & 3 & 6 \\
\hline Knox 2018 & $\begin{array}{l}\text { Task-oriented circuit train- } \\
\text { ing (+ home-based walk- } \\
\text { ing) }\end{array}$ & After & $\begin{array}{l}\text { Lower } \\
\text { (+upper?) }\end{array}$ & $\begin{array}{l}\text { Yes (walk- } \\
\text { ing) }\end{array}$ & $\begin{array}{l}\text { Reduced support and increased complexity } \\
\text { and more demanding home-based walking }\end{array}$ & 60 & $\begin{array}{l}6 \text { sessions } \\
\text { (average } \\
0.5 \text { per } \\
\text { week) }\end{array}$ & 12 \\
\hline $\begin{array}{l}\text { Langham- } \\
\text { mer } 2007\end{array}$ & $\begin{array}{l}\text { Walking, stationary bicy- } \\
\text { cling, stair walking, tread- } \\
\text { mill, and resistance train- } \\
\text { ing }\end{array}$ & After & Both & Yes & $\begin{array}{l}70 \%-80 \% \text { maximum pulse (cardiorespira- } \\
\text { tory component); } 50 \%-60 \% \text { one repetition } \\
\text { maximum (strength component) }\end{array}$ & 45 & $2 / 3$ & $\begin{array}{l}\text { Unclear. } \\
\text { Minimum } \\
20 \text { hours } \\
\text { every third } \\
\text { month in } \\
\text { the first } \\
\text { year after } \\
\text { stroke }\end{array}$ \\
\hline $\begin{array}{l}\text { Letombe } \\
2010\end{array}$ & $\begin{array}{l}\text { Cycle ergometry, treadmill } \\
\text { walking, and isokinetic re- } \\
\text { sistance training }\end{array}$ & During & $\begin{array}{l}\text { Both in- } \\
\text { cluding } \\
\text { trunk }\end{array}$ & $\begin{array}{l}\text { Yes (walk- } \\
\text { ing) }\end{array}$ & $\begin{array}{l}\text { Cardiorespiratory training: } 70 \%-80 \% \text { maxi- } \\
\text { mal cycling power } \\
\text { Strength training; } 6 \times 10 \text { repetitions at } \\
50 \%-60 \% \text { maximum }\end{array}$ & $40-60$ & 4 & 4 \\
\hline
\end{tabular}




\begin{tabular}{|c|c|c|c|c|c|c|c|c|}
\hline Mead 2007 & $\begin{array}{l}\text { Circuit including walking, } \\
\text { stepping, cycle ergometry; } \\
\text { resistance training body } \\
\text { mass, weights, and elastic }\end{array}$ & After & Both & Yes & Rating of perceived exertion: $13-16$ & $40-75$ & 3 & $\begin{array}{l}12-14 \text { (to- } \\
\text { tal of } 36 \\
\text { sessions) }\end{array}$ \\
\hline $\begin{array}{l}\text { Moore } \\
2015\end{array}$ & $\begin{array}{l}\text { Community-based group } \\
\text { classes including warm- } \\
\text { up, stretching, functional } \\
\text { strengthening, balance, } \\
\text { agility and cardiorespira- } \\
\text { tory training }\end{array}$ & After & Both & Yes & $\begin{array}{l}\text { Increasing load and repetitions } \\
40 \%-50 \% \text { maximum heart rate increasing to } \\
70 \%-80 \%\end{array}$ & $40-60$ & 3 & 19 \\
\hline $\begin{array}{l}\text { Richards } \\
1993\end{array}$ & $\begin{array}{l}\text { Treadmill + Kinetron + tilt } \\
\text { table }\end{array}$ & During & $\begin{array}{l}\text { Lower } \\
\text { body }\end{array}$ & Yes & Unknown & 104 & 5 & 5 \\
\hline $\begin{array}{l}\text { Richards } \\
2004\end{array}$ & $\begin{array}{l}\text { Treadmill + Kinetron + } \\
\text { limb load monitor }\end{array}$ & During & $\begin{array}{l}\text { Lower } \\
\text { body }\end{array}$ & Yes & Unknown & 60 & 5 & 8 \\
\hline Shin 2011 & $\begin{array}{l}\text { Functional strength train- } \\
\text { ing (bridging and step- } \\
\text { ping) + treadmill and cycle } \\
\text { ergometry }\end{array}$ & During & Lower & $\begin{array}{l}\text { Yes (walk- } \\
\text { ing and } \\
\text { stepping) }\end{array}$ & $\begin{array}{l}\text { Cardiorespiratory progressive but }<40 \% \\
\text { heart rate reserve } \\
\text { Strength training described only as 'medi- } \\
\text { um intensity' of 5-15 repetitions }\end{array}$ & 60 & 5 & 4 \\
\hline $\begin{array}{l}\text { Teixeira } \\
1999\end{array}$ & $\begin{array}{l}\text { Walking and stepping or } \\
\text { cycle ergometry; } \\
\text { resistance training body } \\
\text { mass, weights and elastic }\end{array}$ & After & $\begin{array}{l}\text { Lower } \\
\text { body }\end{array}$ & Yes & $\begin{array}{l}50 \%-70 \% \text { maximum work rate (cardiores- } \\
\text { piratory component) 50\%-80\% } 1 \text { repetition } \\
\text { maximum, } 3 \times 10 \text { repetitions (strength com- } \\
\text { ponent) }\end{array}$ & $60-90$ & 3 & 10 \\
\hline $\begin{array}{l}\text { Toledano- } \\
\text { Zarhi } 2011\end{array}$ & $\begin{array}{l}\text { Treadmill, hand bike, cy- } \\
\text { cle ergometer + group ex- } \\
\text { ercise for strength, bal- } \\
\text { ance and co-ordination } \\
\text { exercise }\end{array}$ & During & Both & $\begin{array}{l}\text { Yes (tread- } \\
\text { mill) }\end{array}$ & $\begin{array}{l}\text { Cardiorespiratory } 50 \%-70 \% \text { of maximal } \\
\text { heart rate }\end{array}$ & $\begin{array}{l}\text { Cardiores- } \\
\text { piratory } 90 \\
\text { min } \\
\text { Group } \\
45-55 \text { min }\end{array}$ & $\begin{array}{l}\text { Cardiores- } \\
\text { piratory 2/ } \\
\text { week } \\
\text { Group 1/ } \\
\text { week }\end{array}$ & 6 \\
\hline $\begin{array}{l}\text { Van de } \\
\text { Port } 2012\end{array}$ & $\begin{array}{l}\text { Task-orientated circuit } \\
\text { training. } 8 \text { workstations } \\
\text { targeting balance, stair } \\
\text { walking, turning, transfers } \\
\text { and speed walking }\end{array}$ & After & Lower & $\begin{array}{l}\text { Yes (task- } \\
\text { orientat- } \\
\text { ed) }\end{array}$ & Unknown & 90 & 2 & 12 \\
\hline Yang 2006 & $\begin{array}{l}\text { Functional stepping and } \\
\text { chair rising }\end{array}$ & After & $\begin{array}{l}\text { Lower } \\
\text { body }\end{array}$ & Yes & Unknown & 30 & 3 & 4 \\
\hline
\end{tabular}


Table 3. Outline of the studies that focused on mixed training interventions (Continued)

Zedlitz Treadmill walking,

strength training, and

After

Both

Yes (walk-

Cardiorespiratory and strength progressed

ments

Table 4. Pooled walking data for cardiorespiratory training, resistance training, and mixed training at the end of the training period and at follow-up End of intervention

\begin{tabular}{|c|c|c|c|c|c|c|c|}
\hline $\begin{array}{l}\text { Interven- } \\
\text { tion }\end{array}$ & Walking outcome & $\begin{array}{l}\text { Studies } \\
\text { (number of } \\
\text { participants) }\end{array}$ & $\begin{array}{l}\text { MD } \\
(95 \% \mathrm{Cl})\end{array}$ & $\begin{array}{l}\text { Significance } \\
\text { level }\end{array}$ & $\begin{array}{l}\text { Studies } \\
\text { (number } \\
\text { of partici- } \\
\text { pants) }\end{array}$ & $\begin{array}{l}\text { MD } \\
(95 \% \mathrm{Cl})\end{array}$ & $\begin{array}{l}\text { Signifi- } \\
\text { cance level }\end{array}$ \\
\hline $\begin{array}{l}\text { Cardiores- } \\
\text { piratory }\end{array}$ & Maximal gait speed & $17(782)$ & $\begin{array}{l}7.66 \mathrm{~m} / \mathrm{min} \text { (3.65 to } \\
11.68)\end{array}$ & $P=0.0002$ & $5(312)$ & $6.71 \mathrm{~m} / \mathrm{min}$ ( 2.40 to 11.02 ) & $P=0.002$ \\
\hline \multirow[t]{2}{*}{ training } & Preferred gait speed & $12(588)$ & $4.47 \mathrm{~m} / \mathrm{min}$ (2.07 to 6.87$)$ & $P=0.0003$ & $3(176)$ & $1.67 \mathrm{~m} / \mathrm{min}(-3.27$ to 6.62$)$ & NS \\
\hline & 6-Minute Walk Test & $16(882)$ & $33.41 \mathrm{~m}$ (19.04 to 47.78$)$ & $P=0.00001$ & $5(283)$ & $38.29 \mathrm{~m}$ (7.19 to 69.39$)$ & $P=0.02$ \\
\hline \multirow[t]{3}{*}{$\begin{array}{l}\text { Resistance } \\
\text { training }\end{array}$} & Maximal gait speed & $6(274)$ & $\begin{array}{l}2.83 \mathrm{~m} / \mathrm{min}(-0.49 \text { to } \\
6.14)\end{array}$ & NS & $2(117)$ & $7.80 \mathrm{~m} / \mathrm{min}(-3.32$ to 18.91$)$ & NS \\
\hline & Preferred gait speed & $5(203)$ & $\begin{array}{l}2.15 \mathrm{~m} / \mathrm{min}(-3.57 \text { to } \\
7.87)\end{array}$ & NS & - & - & - \\
\hline & 6-Minute Walk Test & $5(238)$ & $24.98 \mathrm{~m}$ (11.98 to 37.98$)$ & $P$ value 0.0002 & $2(117)$ & $22.41 \mathrm{~m}(-27.87$ to 72.69$)$ & NS \\
\hline \multirow{3}{*}{$\begin{array}{l}\text { Mixed } \\
\text { training }\end{array}$} & Maximal gait speed & $3(168)$ & $\begin{array}{l}8.48 \mathrm{~m} / \mathrm{min}(1.76 \text { to } \\
15.20)\end{array}$ & $P=0.01$ & - & - & - \\
\hline & Preferred gait speed & $10(738)$ & $4.71 \mathrm{~m} / \mathrm{min}$ (1.32 to 8.10$)$ & $P=0.006$ & $5(542)$ & $2.54 \mathrm{~m} / \mathrm{min}(-3.65$ to 8.72$)$ & NS \\
\hline & 6-Minute Walk Test & $10(720)$ & $35.00 \mathrm{~m}$ (15.91 to 54.09$)$ & $\begin{array}{l}\mathrm{P} \text { value }< \\
0.0003\end{array}$ & $4(464)$ & $47.48 \mathrm{~m}$ (23.72 to 71.23$)$ & $P=0.0001$ \\
\hline
\end{tabular}

Cl: confidence interval; MD: mean difference; NS: no statistically significant difference 


\section{AP PE N DICES}

\section{Appendix 1. CENTRAL search strategy (the Cochrane Library)}

\#1[mh ^"cerebrovascular disorders"] or [mh "basal ganglia cerebrovascular disease"] or [mh "brain ischemia"] or [mh "carotid artery diseases"] or [mh "intracranial arterial diseases"] or [mh "intracranial arteriovenous malformations"] or [mh "intracranial embolism and thrombosis"] or [mh "intracranial hemorrhages"] or [mh^stroke] or [mh "brain infarction"] or [mh^"stroke, lacunar"] or [mh^"vasospasm, intracranial"] or [mh ^"vertebral artery dissection"] or [mh^"brain injuries"] or [mh^^"brain injury, chronic"] 15776

\#2(stroke or poststroke or post-stroke or cerebrovasc* or brain next vasc* or cerebral next vasc* or cva* or apoplex* or SAH):ti,ab,kw (Word variations have been searched) 48705

\#3((brain* or cerebr ${ }^{\star}$ or cerebell ${ }^{\star}$ or intracran* or intracerebral) near/5 (isch*emi* or infarct* or thrombo* or emboli* or occlus $\left.{ }^{\star}\right)$ ):ti,ab,kw (Word variations have been searched) 11213

\#4((brain* or cerebr* or cerebell* or intracerebral or intracranial or subarachnoid) near/5 (haemorrhage* or hemorrhage* or haematoma* or hematoma* or bleed*)):ti,ab,kw (Word variations have been searched) 7971

\#5[mh ${ }^{\wedge}$ hemiplegia] or [mh paresis] 1338

\#6(hempar* or hemipleg ${ }^{\star}$ or brain next injur $\left.{ }^{\star}\right)$ :ti,ab,kw (Word variations have been searched)6443

\#7[mh ^ "Gait Disorders, Neurologic"] 555

\#8\#1 or \#2 or \#3 or \#4 or \#5 or \#6 or \#7 62227

\#9[mh exercise] 21418

\#10[mh "exercise therapy"] 11971

$\# 11$ [mh sports] 14247

\#12[mh^"muscle strength"] or [mh "physical endurance"] or [mh^^"physical exertion"] or [mh^"physical fitness"] 13586

\#13exercis*:ti,ab,kw (Word variations have been searched)70858

\#14train*:ti,ab,kw (Word variations have been searched) 67624

\#15conditioning:ti,ab,kw (Word variations have been searched) 84620

\#16strengthen*:ti,ab,kw (Word variations have been searched) 4564

\#17\{or\#9-\#16\} 189900

$\# 18 \# 8$ and \#17 10540

\section{Appendix 2. MEDLINE (Ovid) search strategy}

\section{SAUNDERSV5_MEDLINE_2018}

Physical fitness training for stroke patients_July2018_Final

1. cerebrovascular disorders/ or exp basal ganglia cerebrovascular disease/ or exp brain ischemia/ or exp carotid artery diseases/ or exp intracranial arterial diseases/ or exp intracranial arteriovenous malformations/or exp "intracranial embolism and thrombosis"/ or exp intracranial hemorrhages/ or stroke/ or exp brain infarction/ or stroke, lacunar/ or vasospasm, intracranial/ or vertebral artery dissection/ or brain injuries/ or brain injury, chronic/

2. (stroke or poststroke or post-stroke or cerebrovasc\$ or brain vasc\$ or cerebral vasc\$ or cva\$ or apoplex\$ or SAH).tw.

3. ((brain $\$$ or cerebr\$ or cerebell\$ or intracran\$ or intracerebral) adj5 (isch?emi\$ or infarct\$ or thrombo $\$$ or emboli\$ or occlus\$)).tw.

4. ((brain $\$$ or cerebr\$ or cerebell\$ or intracerebral or intracranial or subarachnoid) adj5 (haemorrhage $\$$ or hemorrhage $\$$ or haematoma

\$ or hematoma\$ or bleed\$)).tw.

5. hemiplegia/ or exp paresis/

6. (hempar\$ or hemipleg\$ or brain injur\$).tw.

7. Gait Disorders, Neurologic/

8. or/1-7

9. exp exercise/ or exp exercise therapy/

10. exp sports/

11. muscle strength/ or exp physical endurance/ or physical exertion/ or physical fitness/

12. exercis\$.tw.

13. train\$.tw.

14. conditioning.tw.

15. strengthen\$.tw.

16. or/9-15

17. randomized controlled trial.pt.

18. controlled clinical trial.pt.

19. randomized.ab.

20. placebo.ab.

21. randomly.ab.

22. trial.ab.

23. groups.ab.

24. or/17-23

25. exp animals/ not humans.sh. 
26.8 and 16 and 24

27.26 not 25

28. limit 27 to $y r=" 2015$-Current"

\section{Appendix 3. Embase (Ovid) search strategy}

\section{SAUNDERSv5_Embase_2018}

Physical fitness training for stroke patients_March2018_Saunders suggestions

1. cerebrovascular disease/ or exp basal ganglion hemorrhage/ or exp brain hematoma/ or exp brain hemorrhage/ or exp brain infarction/ or exp brain ischemia/ or exp carotid artery disease/ or cerebral artery disease/ or exp cerebrovascular accident/ or exp intracranial aneurysm/ or exp occlusive cerebrovascular disease/

2. stroke patient/ or stroke unit/

3. (stroke or poststroke or post-stroke or cerebrovasc\$ or brain vasc\$ or cerebral vasc\$ or cva\$ or apoplex\$ or SAH).tw.

4. ((brain $\$$ or cerebr\$ or cerebell\$ or intracran\$ or intracerebral) adj5 (isch?emi\$ or infarct $\$$ or thrombo $\$$ or emboli\$ or occlus\$)).tw.

5. ((brain\$ or cerebr\$ or cerebell\$ or intracerebral or intracranial or subarachnoid) adj5 (haemorrhage\$ or hemorrhage\$ or haematoma

\$ or hematoma\$ or bleed\$)).tw.

6. brain injury/ or acquired brain injury/

7. hemiparesis/ or hemiplegia/ or paresis/ or neurologic gait disorder/ or hemiplegic gait/

8. (hempar\$ or hemipleg\$ or brain injur\$).tw.

9. or/1-8

10. exp exercise/

11. exp kinesiotherapy/

12. exp sport/

13. muscle strength/

14. endurance/

15. fitness/

16. exercis\$.tw.

17. train\$.tw.

18. conditioning.tw.

19. strengthen\$.tw.

20. or/10-19

21. Randomized Controlled Trial/ or "randomized controlled trial (topic)"/

22. Randomization/

23. Controlled clinical trial/ or "controlled clinical trial (topic)"/

24. control group/ or controlled study/

25. clinical trial/ or "clinical trial (topic)"/ or phase 1 clinical trial/ or phase 2 clinical trial/ or phase $3 \mathrm{clinical}$ trial/ or phase $4 \mathrm{clinical}$ trial/

26. Crossover Procedure/

27. Double Blind Procedure/

28. Single Blind Procedure/ or triple blind procedure/

29. placebo/ or placebo effect/

30. (random\$ or RCT or RCTs).tw.

31. (controlled adj5 (trial\$ or stud\$)).tw.

32. (clinical\$ adj5 trial\$).tw.

33. ((control or treatment or experiment\$ or intervention) adj5 (group\$ or subject\$ or patient\$)).tw.

34. (quasi-random $\$$ or quasi random\$ or pseudo-random $\$$ or pseudo random\$).tw.

35. ((control or experiment $\$$ or conservative) adj5 (treatment or therapy or procedure or manage $\$)$ ).tw.

36. ((singl\$ or doubl\$ or tripl\$ or trebl\$) adj5 (blind\$ or mask\$)).tw.

37. (cross-over or cross over or crossover).tw.

38. (placebo $\$$ or sham).tw.

39. trial.ti.

40. (assign\$ or allocat\$).tw.

41. controls.tw.

42. or/21-41

43.9 and 20 and 42

44. (exp animals/or exp invertebrate/ or animal experiment/ or animal model/or animal tissue/ or animal cell/ or nonhuman/) not (human/ or normal human/ or human cell/)

45.43 not 44

46. limit 45 to $y r=" 2015$-Current"

\section{Appendix 4. CINAHL (EBSCO) search strategy}

S1(MH "Cerebrovascular Disorders+") or (MH "stroke patients") or (MH "stroke units") 
$\mathrm{S} 2 \mathrm{TI}$ ( stroke or poststroke or post-stroke or cerebrovasc* or brain vasc* or cerebral vasc or cva or apoplex or SAH ) or AB ( $\mathrm{stroke}$ or poststroke or post-stroke or cerebrovasc* or brain vasc* or cerebral vasc or cva or apoplex or SAH )

S3TI ( brain* or cerebr* or cerebell* or intracran* or intracerebral ) or AB ( brain* or cerebr ${ }^{\star}$ or cerebell* or intracran* or intracerebral )

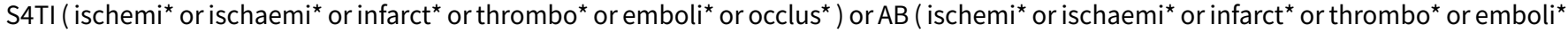
or occlus $\left.{ }^{\star}\right)$

S5S3 and S4

S6TI ( brain* or cerebr ${ }^{\star}$ or cerebell* or intracerebral or intracranial or subarachnoid ) or AB ( brain* or cerebr ${ }^{\star}$ or cerebell ${ }^{\star}$ or intracerebral or intracranial or subarachnoid)

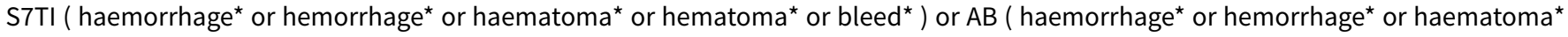
or hematoma* or bleed* ${ }^{\star}$

$\mathrm{S} 8 \mathrm{~S} 6$ and $\mathrm{S} 7$

S9(MH "Hemiplegia")

S10TI ( hemipleg* or hemipar ${ }^{\star}$ or paresis or paretic ) or AB ( hemipleg* or hemipar* or paresis or paretic )

S11(MH "Gait Disorders, Neurologic+")

$\mathrm{S} 12 \mathrm{~S} 1$ or $\mathrm{S} 2$ or $\mathrm{S} 5$ or $\mathrm{S} 8$ or $\mathrm{S} 9$ or $\mathrm{S} 10$ or $\mathrm{S} 11$

S13(MH "Exercise+")

S14(MH "Therapeutic Exercise+")

S15(MH "Sports+")

S16(MH "Physical Endurance+") OR (MH "Exertion+") OR (MH "Muscle Strengthening+")

S17TI exercis* OR AB exercis*

S18TI train* OR AB train*

S19TI conditioning OR AB conditioning

S20TI strengthen* OR AB strengthen*

S21S13 OR S14 OR S15 OR S16 OR S17 OR S18 OR S19 OR S20

S22MH Random Assignment or MH Single-blind Studies or MH Double-blind Studies or MH Triple-blind Studies or MH Crossover design or MH Factorial Design

S23TI ("multicentre study" or "multicenter study" or "multi-centre study" or "multi-center study") or AB ("multicentre study" or "multicenter study" or "multi-centre study" or "multi-center study") or SU ("multicentre study" or "multicenter study" or "multi-centre study" or "multi-center study")

S24TI random* or AB random*

S25AB "latin square" or TI "latin square"

S26TI (crossover or cross-over) or AB (crossover or cross-over) or SU (crossover or cross-over)

S27MH Placebos

S28TI ( ((singl* or doubl* or trebl* or tripl*) N3 (blind* or mask $\left.\left.{ }^{\star}\right)\right)$ ) OR AB ( ((singl* or doubl* or trebl* or tripl*) N3 (blind ${ }^{\star}$ or mask $\left.\left.^{\star}\right)\right)$ )

S29TI Placebo* or AB Placebo* or SU Placebo*

S30MH Clinical Trials

S31TI (Clinical AND Trial) or AB (Clinical AND Trial) or SU (Clinical AND Trial)

S32S22 OR S23 OR S24 OR S25 OR S26 OR S27 OR S28 OR S29 OR S30 OR S31

\section{Appendix 5. SPORTDiscus (EBSCO) search strategy}

S1. DE "CEREBROVASCULAR disease" or DE "BRAIN Hemorrhage" or DE "CEREBRAL embolism \& thrombosis"

S2. $\mathrm{TI}$ ( stroke or poststroke or post-stroke or cerebrovasc ${ }^{\star}$ or brain vasc* or cerebral vasc or cva or apoplex or SAH ) or AB ( stroke or poststroke or post-stroke or cerebrovasc ${ }^{\star}$ or brain vasc* or cerebral vasc or cva or apoplex or SAH )

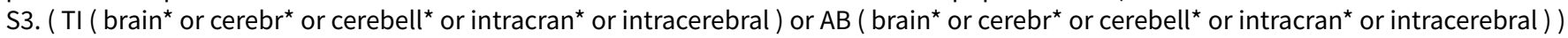
and ( $\mathrm{TI}$ ( ischemi ${ }^{\star}$ or ischaemi ${ }^{\star}$ or infarct ${ }^{\star}$ or thrombo* or emboli ${ }^{\star}$ or occlus ${ }^{\star}$ ) or AB (ischemi ${ }^{\star}$ or ischaemi ${ }^{\star}$ or infarct $^{\star}$ or thrombo $^{\star}$ or emboli* or occlus*))

S4. ( TI ( brain* or cerebr* or cerebell* or intracerebral or intracranial or subarachnoid) or AB ( brain ${ }^{\star}$ or cerebr $^{\star}$ or cerebell ${ }^{\star}$ or intracerebral

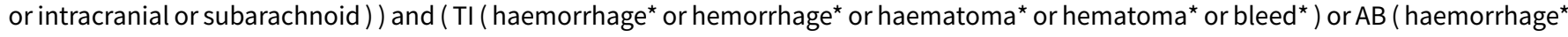
or hemorrhage* or haematoma* or hematoma* or bleed*))

S5. DE "HEMIPLEGIA"

S6. TI ( hemipleg* or hemipar* or paresis or paretic ) or AB ( hemipleg* or hemipar* or paresis or paretic)

$\mathrm{S} 7 . \mathrm{S} 1$ or $\mathrm{S} 2$ or $\mathrm{S} 3$ or $\mathrm{S} 4$ or $\mathrm{S} 5$ or $\mathrm{S} 6$

S8. TI random* or AB random*

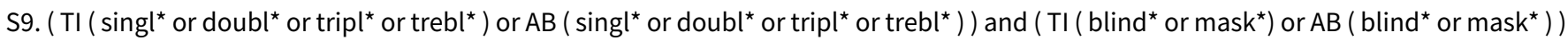
S10. TI (crossover or cross-over or placebo* or control* or factorial or sham ) or AB (crossover or cross-over or placebo* or control* or factorial or sham )

S11. ( TI ( clin* or intervention* or compar ${ }^{\star}$ or experiment* or preventive or therapeutic) or AB ( clin* or intervention* or compar* or experiment* or preventive or therapeutic ) ) and ( TI trial* or AB trial*)

S12. $\mathrm{Tl}$ ( counterbalance* or multiple baseline ${ }^{\star}$ or $A B A B$ design ) or $A B$ ( counterbalance ${ }^{\star}$ or multiple baseline ${ }^{\star}$ or $A B A B$ design )

S13. TI ( meta analysis $^{\star}$ or metaanlaysis or meta-anlaysis or systematic review ${ }^{\star}$ ) or AB ( meta analysis* or metaanlaysis or meta-anlaysis or systematic review*) 
S14. SU ( random* or trial or crossover or cross-over or placebo* or control* or factorial or sham or counterbalance* or multiple baseline* or ABAB design or meta analysis* or metaanlaysis or meta-anlaysis or systematic review*) or KW ( random $^{\star}$ or trial or crossover or crossover or placebo* or control ${ }^{\star}$ or factorial or sham or counterbalance* or multiple baseline ${ }^{\star}$ or ABAB design or meta analysis* or metaanlaysis or meta-anlaysis or systematic review* ${ }^{*}$

$\mathrm{S} 15 . \mathrm{S} 8$ or $\mathrm{S} 9$ or $\mathrm{S} 10$ or $\mathrm{S} 11$ or $\mathrm{S} 12$ or $\mathrm{S} 13$ or $\mathrm{S} 14$

S16. (S7 and S15)

\section{Appendix 6. PsycINFO (Ovid) search strategy}

1. cerebrovascular disorders/ or cerebral hemorrhage/ or exp cerebral ischemia/ or cerebrovascular accidents/ or subarachnoid hemorrhage/

2. (stroke $\$$ or post stroke or poststroke or post-stroke or apoplex\$ or cerebral vasc $\$$ or cerebrovasc $\$$ or $\mathrm{cva}$ or SAH).tw.

3. ((brain\$ or cerebr\$ or cerebell\$ or vertebrobasil\$ or hemispher\$ or intracran\$ or intracerebral or infratentorial or supratentorial or middle cerebral artery or MCA\$ or anterior circulation or posterior circulation or basilar artery or vertebral artery or space-occupying) adj3 (isch?emi\$ or infarct\$ or thrombo or emboli\$ or occlus\$ or hypoxi\$)).tw.

4. ((brain\$ or cerebr\$ or cerebell\$ or intracerebral or intracran\$ or parenchymal or intraparenchymal or intraventricular or infratentorial or supratentorial or basal gangli\$ or putaminal or putamen or posterior fossa or hemispher\$ or subarachnoid) adj3 (h?emorrhag\$ or $\mathrm{h}$ ? ematoma\$ or bleed\$)).tw.

5. hemiparesis/ or hemiplegia/

6. (hemipleg\$ or hemipar\$ or paresis or paretic).tw.

7. or/1-6

8. exp exercise/

9. exp sports/

10. physical strength/ or physical endurance/ or physical fitness/

11. exercis\$.tw.

12. train\$.tw.

13. conditioning.tw.

14. strengthen\$.tw.

15. or/8-14

16. clinical trials/ or treatment effectiveness evaluation/ or placebo/

17. (random\$ or RCT or RCTs).tw.

18. (controlled adj5 (trial\$ or stud\$)).tw.

19. (clinical\$ adj5 trial\$).tw.

20. ((control or treatment or experiment\$ or intervention) adj5 (group\$ or subject\$ or patient\$)).tw.

21. (quasi-random\$ or quasi random\$ or pseudo-random\$ or pseudo random\$).tw.

22. ((control or experiment\$ or conservative) adj5 (treatment or therapy or procedure or manage\$)).tw.

23. ((singl\$ or doubl\$ or tripl\$ or trebl\$) adj5 (blind\$ or mask\$)).tw.

24. (cross-over or cross over or crossover).tw.

25. (placebo $\$$ or sham).tw.

26. trial.ti.

27. (assign\$ or allocat\$).tw.

28. controls.tw.

29. or $/ 16-28$

30.7 and 15 and 29

\section{Appendix 7. Conference Procedings (WoS)}

\# 20\#19 AND \#10 AND \#5

Indexes=SCI-EXPANDED, CPCI-S Timespan=2015-2018

\# 19\#18 OR \#17 OR \#16 OR \#15 OR \#14 OR \#13 OR \#12 OR \#11

Indexes=SCI-EXPANDED, CPCI-S Timespan=2015-2018

\# 18TS=(placebo* or sham)

Indexes=SCI-EXPANDED, CPCI-S Timespan=2015-2018

\# 17TS=(cross-over or cross over or crossover)

Indexes $=$ SCI-EXPANDED, CPCI-S Timespan $=2015-2018$

\# 16TS $=\left(\left(\right.\right.$ singl ${ }^{\star}$ or doubl ${ }^{*}$ or tripl ${ }^{*}$ or trebl $\left.{ }^{\star}\right)$ NEAR/5 $\left(\right.$ blind $^{\star}$ or mask $\left.\left.{ }^{\star}\right)\right)$

Indexes=SCI-EXPANDED, CPCI-S Timespan=2015-2018

\# 15TS=((control or experiment ${ }^{\star}$ or conservative) NEAR/5 (treatment or therapy or procedure or manage $\left.\left.{ }^{\star}\right)\right)$

Indexes=SCI-EXPANDED, CPCI-S Timespan=2015-2018

\# 14TS=((control or treatment or experiment ${ }^{\star}$ or intervention) NEAR/5 (group* ${ }^{\star}$ r subject ${ }^{\star}$ or patient $\left.{ }^{\star}\right)$ )

Indexes=SCI-EXPANDED, CPCI-S Timespan=2015-2018

\# 13TS=(clinical ${ }^{*}$ NEAR/5 trial $\left.^{\star}\right)$

Indexes=SCI-EXPANDED, CPCI-S Timespan=2015-2018

Physical fitness training for stroke patients (Review) 
\# 12TS=(controlled NEAR/5 $\left(\right.$ trial $^{*}$ or stud $\left.\left.{ }^{*}\right)\right)$

Indexes=SCI-EXPANDED, CPCI-S Timespan=2015-2018

\# 11TS=random*

Indexes=SCI-EXPANDED, CPCI-S Timespan $=2015-2018$

\# 10\#9 OR \#8 OR \#7 OR \#6

Indexes=SCI-EXPANDED, CPCI-S Timespan $=2015-2018$

\# 9TS=strengthen ${ }^{*}$

Indexes=SCI-EXPANDED, CPCI-S Timespan $=2015-2018$

\# 8TS=conditioning

Indexes=SCI-EXPANDED, CPCI-S Timespan=2015-2018

\# 7TS=train*

Indexes=SCI-EXPANDED, CPCI-S Timespan $=2015-2018$

\# 6TS=exercis ${ }^{*}$

Indexes=SCI-EXPANDED, CPCI-S Timespan $=2015-2018$

\# 5\#4 OR \#3 OR \#2 OR \#1

Indexes=SCI-EXPANDED, CPCI-S Timespan $=2015-2018$

\# 4TS=(hemipleg* or hemipar ${ }^{\star}$ or bain injur ${ }^{\star}$ )

Indexes=SCI-EXPANDED, CPCI-S Timespan=2015-2018

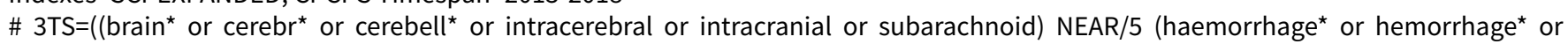
haematoma* or hematoma* or bleed $\left.{ }^{\star}\right)$ )

Indexes=SCI-EXPANDED, CPCI-S Timespan=2015-2018

\# 2TS=((brain ${ }^{\star}$ or cerebr ${ }^{\star}$ or cerebell ${ }^{\star}$ or intracran ${ }^{\star}$ or intracerebral) NEAR/5 (isch\$emi ${ }^{\star}$ or infarct ${ }^{\star}$ or thrombo ${ }^{\star}$ or emboli $^{\star}$ or occlus $\left.\left.^{\star}\right)\right)$

Indexes=SCI-EXPANDED, CPCI-S Timespan=2015-2018

\# 1TS=(stroke or poststroke or post-stroke or cerebrovasc ${ }^{*}$ or brain vasc* or cerebral vasc* or cva* or apoplex* or SAH)

Indexes=SCI-EXPANDED, CPCI-S Timespan=2015-2018

\section{Appendix 8. PEDro search strategy}

Two separate searches were performed using the PEDRO advanced search

Search \#1

1. Title and Abstract $=$ "Stroke"

2. Therapy = "FITNESS TRAINING"

3. Problem $=$ [No appropriate value in this field]

4. Body Part $=$ [No appropriate value in this field $]$

5. Sub Discipline $=[$ No appropriate value in this field $]$

6. Topic $=[$ No appropriate value in this field $]$

7. Method = "Clinical Trial"

8. New Records Added Since $=01 / 01 / 2015$

Match all search terms (AND)

Search \#2

1. Title and Abstract = "Stroke"

2. Therapy = "STRENGTH TRAINING"

3. Problem $=$ [No appropriate value in this field]

4. Body Part $=$ [No appropriate value in this field $]$

5. Sub Discipline $=[$ No appropriate value in this field $]$

6. Topic $=[$ No appropriate value in this field $]$

7. Method = "Clinical Trial"

8. New Records Added Since $=01 / 01 / 2015$

Match all search terms (AND)

\section{Appendix 9. Searching other resources}

The additional resources were searched using thew following simple search approach

STROKE AND (FITNESS OR EXERCISE OR TRAINING)

WHAT'S NEW 


\begin{tabular}{ll}
\hline Date & Event \\
\hline 7 July 2019 & $\begin{array}{l}\text { New citation required and conclusions } \\
\text { have changed }\end{array}$
\end{tabular}

\section{Description}

In this update there are now more studies containing resistance training either as a standalone intervention or as part of mixed training (resistance plus cardiorespiratory). This has clarified that resistance training content within programmes of training can benefit balance after stroke.

There are new co-authors with experience of exercise and activity after stroke.

7 July $2019 \quad$ New search has been performed

We have updated all main electronic search strategies to July 2018; we have rewritten the syntax of all the search strategies including the addition of new resources (PsycINFO). Layout of the methods now mirrors that in a related review (Interventions to reduce sedentary behaviour after stroke).

We have included 17 additional randomised controlled trials, bringing the total number of included studies to 75 , involving 3617 participants.

\section{H I S T O R Y}

Protocol first published: Issue 4, 2001

Review first published: Issue 1, 2004

\begin{tabular}{lll}
\hline Date & Event & Description \\
\hline 12 November 2015 & $\begin{array}{l}\text { New citation required and conclusions } \\
\text { have changed }\end{array}$ & $\begin{array}{l}\text { New trials have changed where significant benefits emerge. Im- } \\
\text { provements in global indices of disability are apparent now for } \\
\text { mixed training as well as cardiorespiratory training. Improve- } \\
\text { ments in balance are now only apparent among trials of mixed } \\
\text { training. We have added a new patient-important outcome (cog- } \\
\text { nitive function) but there is a lack of evidence and this highlights } \\
\text { an important knowledge gap. }\end{array}$ \\
& & \\
\end{tabular}

29 October $2015 \quad$ New search has been performed

We have updated all main electronic search strategies to February 2015. We have included 13 additional randomised controlled trials, bringing the total number of included trials to 58 , involving 2797 participants. We have added a cognitive function outcome to the review because this has been identified as a research priority. Secondly an application to carry out a Cochrane review of exercise interventions for cognition after stroke was judged to overlap and to be more efficiently combined with this review of fitness training interventions after stroke. We checked all previously included trials for cognitive outcomes as well as those in the updated searches. We have added two new co-authors.

\begin{tabular}{lll}
\hline 5 July 2013 & $\begin{array}{l}\text { New citation required and conclusions } \\
\text { have changed }\end{array}$ & $\begin{array}{l}\text { Additional co-author. We have revised the main text and conclu- } \\
\text { sions of the review according to the findings of the new included } \\
\text { trials. }\end{array}$ \\
\hline 28 January 2013 New search has been performed & $\begin{array}{l}\text { We have updated all main electronic search strategies to January } \\
2013 . \text { We have included } 13 \text { additional randomised clinical trials, } \\
\text { bringing the total number of included trials to 45, involving 2188 } \\
\text { participants. We have incorporated 'Risk of bias' tables. }\end{array}$
\end{tabular}




\begin{tabular}{lll}
\hline Date & Event & Description \\
\hline 22 November 2010 & New search has been performed & $\begin{array}{l}\text { We have updated all main electronic search strategies to March } \\
\text { 2010. We have included 11 additional randomised clinical trials } \\
\text { and } 7 \text { ongoing trials. We have better clarified our inclusion crite- } \\
\text { ria and objectives. }\end{array}$
\end{tabular}

\begin{tabular}{lll}
\hline 22 November 2010 & $\begin{array}{l}\text { New citation required and conclusions } \\
\text { have changed }\end{array}$ & $\begin{array}{l}\text { New first author. We have revised the main text and conclusions } \\
\text { of the review according to the findings of the new included trials. }\end{array}$ \\
\hline 2 March 2009 & New search has been performed & $\begin{array}{l}\text { We updated the search of the Cochrane Stroke Group Trials Reg- } \\
\text { ister in March 2009. }\end{array}$
\end{tabular}

3 November $2008 \quad$ New citation required and conclusions have changed

There is sufficient evidence to incorporate cardiorespiratory training, using walking as a mode of exercise, into the rehabilitation of patients with stroke in order to improve speed, tolerance, and independence during walking, but further trials are needed to determine the optimal exercise prescription after stroke and to establish whether any long-term benefits exist.

3 November $2008 \quad$ New search has been performed

We updated the searches to March 2007. There are now 24 trials, involving 1147 participants, included in the review; 12 more trials than in the previous version. The text of the review has been revised throughout.

23 October $2008 \quad$ Amended Converted to new review format.

\section{CONTRIBUTIONS OF AUTHORS}

\section{Original review}

DH Saunders, CA Greig, GE Mead, and A Young contributed to writing the review protocol.

DH Saunders developed and ran searches, selected studies, extracted and interpreted data, performed the analyses, and co-wrote the review.

CA Greig and GE Mead selected studies, extracted and interpreted data, performed the analyses, and co-wrote the review.

A Young provided comments on interim drafts of the review.

\section{For this update}

DH Saunders developed and ran searches, selected studies, extracted and interpreted data, performed the analyses, and wrote the review. MF Sanderson, S Hayes, L Johnson, S Kramer, D Carter, and H Jarvis selected studies, extracted and interpreted data, and contributed to writing the review.

M Brazzelli advised on the methodology, interpreted data, and contributed to writing the review. GE Mead helped select studies and provided comments on a draft version of the review.

\section{DECLARATIONS OF INTEREST}

DH Saunders was a co-author of one included study (Mead 2007).

M Sanderson has no declarations of interest.

S Hayes has no declarations of interest.

L Johnson has no declarations of interest.

S Kramer has no declarations of interest.

D Carter has no declarations of interest.

$\mathrm{H}$ Jarvis has no declarations of interest. 
M Brazzelli has no declarations of interest.

GE Mead receives royalties (about GBP 100 a year) for a book about fitness training after stroke (Exercise and Fitness Training after stroke) a handbook for evidence-based practice. Ed Gillian Mead and Frederike van Wijck. These royalties are used to support further research. She has led a study of exercise after stroke that is included in the review (Mead 2007).

\section{SOURCES OF SUPPORT}

\section{Internal sources}

- No sources of support supplied

\section{External sources}

- National Institute for Health Research (NIHR), UK.

Cochrane Review Incentive Scheme 2012

\section{DIFFERENCES BETWEEN PROTOCOL AND REVIEW}

Subgroup analyses were, on the whole, not possible as there were too few studies within the meta-analyses, too many other influential factors, and the scope and breadth of the review makes these unmanageable.

The subgroups in the previous version have been removed:

- type of control interventions (no intervention versus non-exercise intervention versus other intervention); no intervention is explored in depth anyway (studies confounded for exposure time);

- duration of training (less than 12 weeks versus 12 weeks or more); duration is still discussed in the current results;

- severity of stroke (mild symptoms versus severe symptoms); most participants recruited in exercise studies are high-functioning.

\section{INDEX TERMS}

\section{Medical Subject Headings (MeSH)}

${ }^{\star}$ Physical Fitness; *Stroke Rehabilitation; Activities of Daily Living; Exercise Therapy [*methods]; Randomized Controlled Trials as Topic; Resistance Training; Stroke [mortality]; Walking [ ${ }^{\star}$ physiology]

\section{MeSH check words}

Humans 